\title{
Zur Intonation von Modus und Fokus im Deutschen
}

Herausgegeben von

Hans Altmann, Anton Batliner

und Wilhelm Oppenrieder

Max Niemeyer Verlag Tübingen 1989 


\section{Universitäts- \\ Bibliothek \\ München}

CIP-Titelaufnahme der Deutschen Bibliothek

Zur Intonation von Modus und Fokus im Deutschen / hrsg. von Hans Altmann ... Tübingen : Niemeyer, 1989

(Linguistische Arbeiten ; 234)

NE: Altmann, Hans [Hrsg.]; GT

\section{ISBN 3-484-30234-8 ISSN 0344-6727}

(C) Max Niemeyer Verlag, Tübingen 1989

Das Werk einschließlich aller seiner Teile ist urheberrechtlich geschützt. Jede Verwertung außerhalb der engen Grenzen des Urheberrechtsgesetzes ist ohne Zustimmung des Verlages unzulässig und strafbar. Das gilt insbesondere für Vervielfältigungen, Übersetzungen, Mikroverfilmungen und die Einspeicherung und Verarbeitung in elektronischen Systemen. Printed in Germany.

Druck: Weihert-Druck GmbH, Darmstadt 
In diesem Band sollen die Ergebnisse des DFG-Projekts 'Modus-FokusIntonation' zusammengefaßt und, soweit noch nicht an anderer stelle geschehen, dokumentiert werden. Das Projekt wurde im Rahmen des DFG-Schwerpunkts "Formen und Funktionen der Intonation" von 1984 bis 1988 gefördert. Unser Dank gilt zuallererst der Deutschen Forschungsgemeinschaft und den Gutachtern im Schwerpunkt für diese Förderung.

Projektarbeit ist Teamarbeit. Allen, die im Projekt tätig waren, danken wir hiermit herzlich: Als wissenschaptliche Mitarbeiter Leila Luukko-Vinchenzo, Klaus Netter, Ulrike Scholz, Jörg Schorradt und Anna Winner; als studentische Hilfskräfte Marion Bludszuweit, Ute Hofmann, Kris Langmeier und Andrea Stephan.

Wir profitierten von Diskussionen mit den anderen Projekten im Schwerpunkt, sowohl bei den beiden im Schwerpunkt veranstalteten Kolloquien als auch bei informellen Treffen.

Insbesondere den folgenden Kolleginnen und Kollegen möchten wir für ihre Unterstützung und Diskussionsbereitschaft danken: Eva Breindl, Joachim Jacobs, Bernd Pompino-Marschall, Elmar Nöth, Lisa Schiefer, Maria Thurmair, Eva-Maria Willkop und Dietmar Zaefferer.

Für technische, organisatorische und institutionelle Hilfe danken wir Hans G. Tillmann und Theo Vennemann.

Schließlich gilt unser Dank dem Verlag und den Herausgebern für die Aufnahme in diese Reihe und - zuletzt aber überhaupt nicht zuletzt - Michael Hinterdobler und Claudia Weiand für die Hilfe und die (aupbauende) Kritik beim Zustandekommen dieses Bandes.

München, im Mai 1989

Hans Altmann Anton Batliner Wilhelm Oppenrieder 

INHALTSVERZEICHNIS

DAS PROJEKT 'MODUS-FOKUS-INTONATION'.

AUSGANGSPUNKT, KONZEPTION UND RESULTATE IM OBERBLICK.

Hans Altmann, Anton Batliner und Wilhelm Oppenrieder

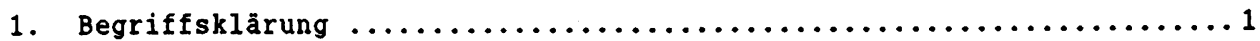

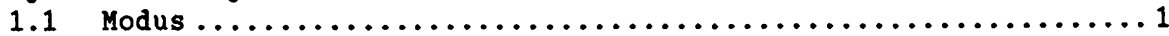

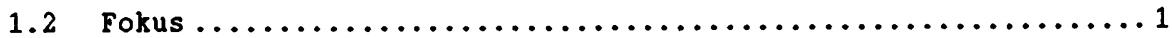

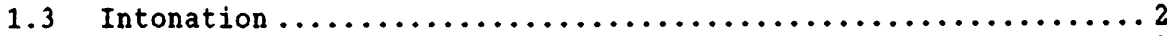

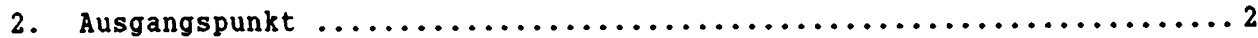

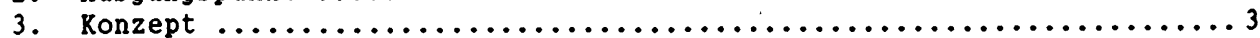

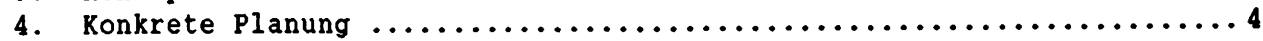

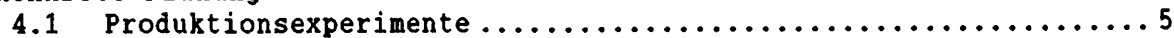

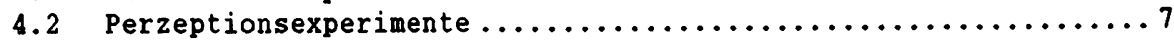

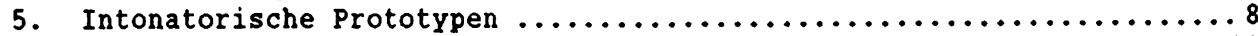

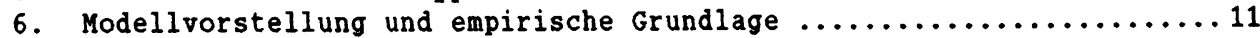

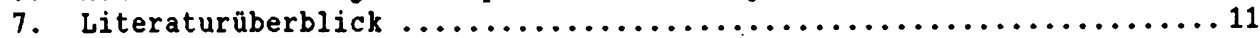

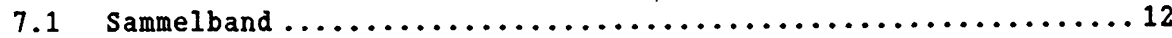

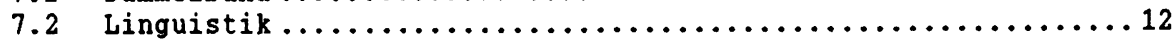

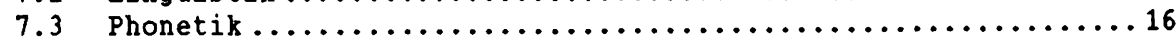

FOKUS, MODUS UND DIE GROSSE ZAHL.

ZUR INTONATORISCHEN INDIZIERUNG DES FOKUS IM DEUTSCHEN.

\section{Anton Batliner}

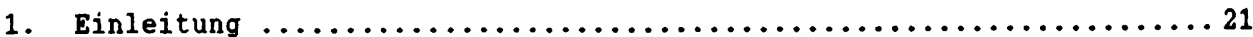

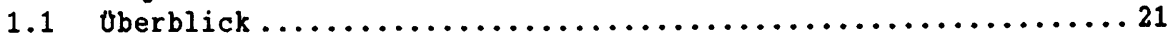

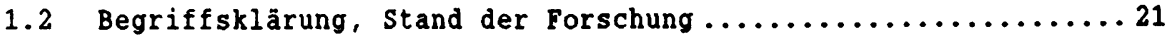

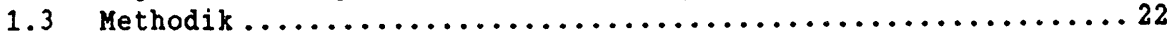

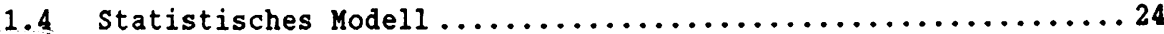

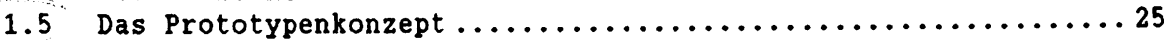

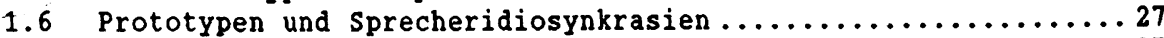

1.7 Unterschiedliche Gewinnung der Prototypen ............... 27

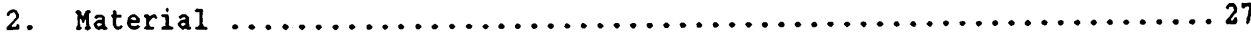

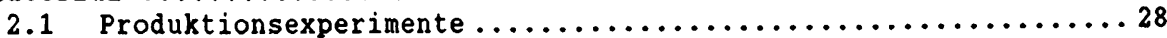

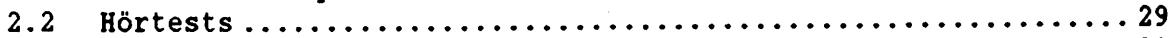

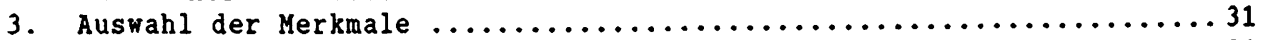

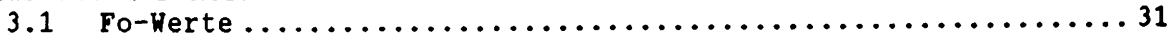

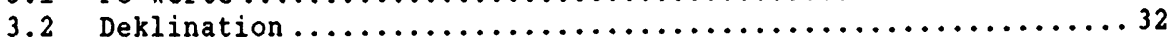

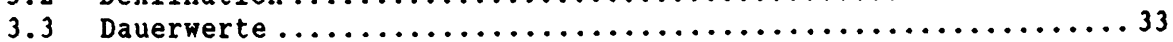

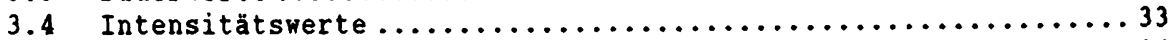

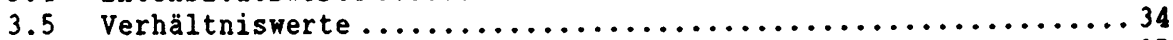

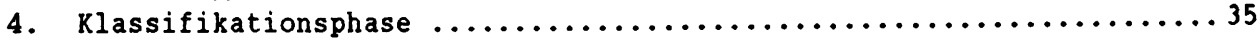


5. Relevante Merkmale: sprecherübergreifend oder sprecherspezifisch? ............................. 44

6. Abschließende Bemerkungen zur statistischen Analyse $\ldots \ldots \ldots \ldots \ldots \ldots 47$

7. Gewinnung der Prototypen über die große 2 ahl $\ldots \ldots \ldots \ldots \ldots \ldots \ldots \ldots$

8. Gewinnung der Prototypen über Hörerurteile $\ldots \ldots \ldots \ldots \ldots \ldots \ldots \ldots$

9. Fehleranalyse der Klassifikation durch

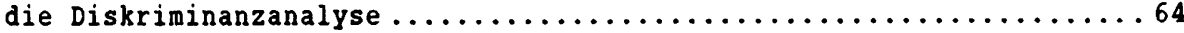

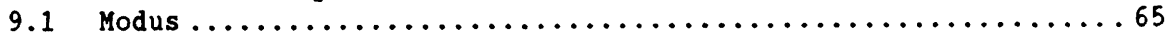

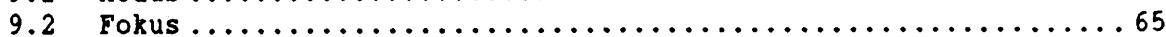

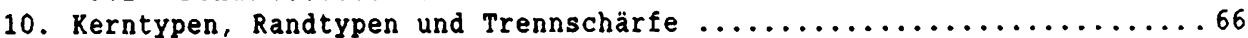

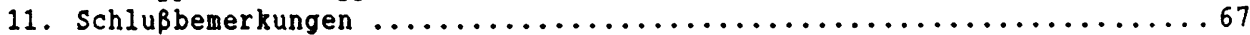

FOKUS, DEKLINATION UND WENDEPUNKT

\section{Anton Batliner}

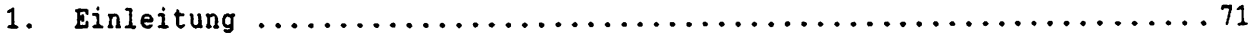

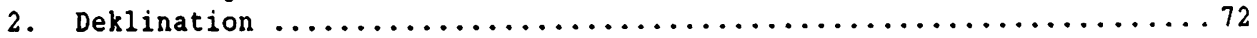

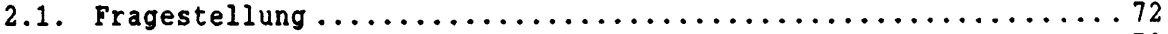

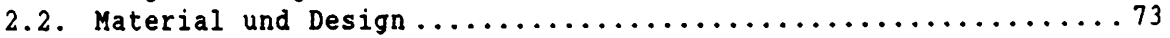

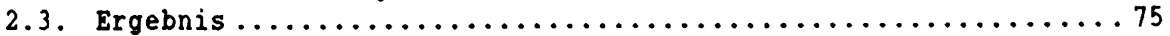

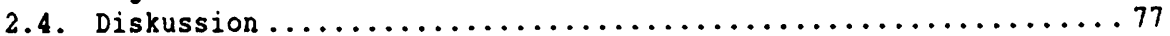

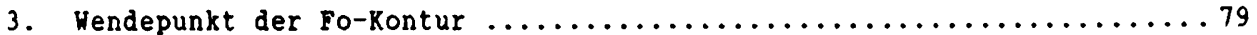

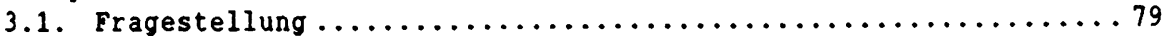

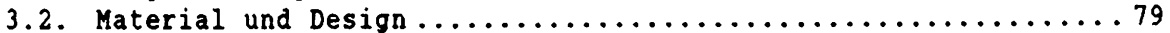

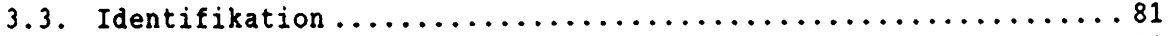

3.4. Kontextverträglichkeit ............................. 83

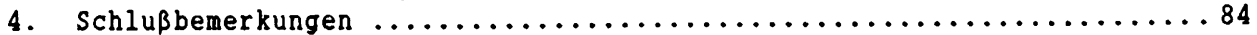

EINE FRAGE IST EINE FRAGE IST KEINE FRAGE.

\section{Anton Batliner}

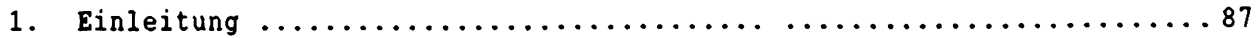

2. Im kategorialen Grenzgebiet $\ldots \ldots \ldots \ldots \ldots \ldots \ldots \ldots \ldots \ldots \ldots \ldots \ldots$

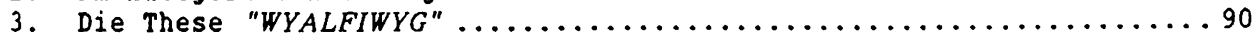

4. Auf der suche nach EINDEUTIGKEIT $\ldots \ldots \ldots \ldots \ldots \ldots \ldots \ldots \ldots \ldots \ldots \ldots$

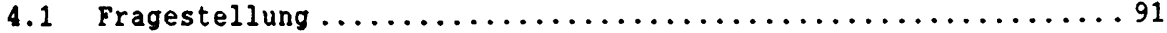

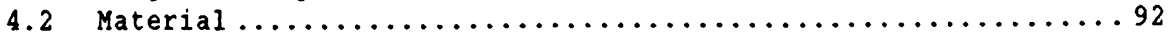

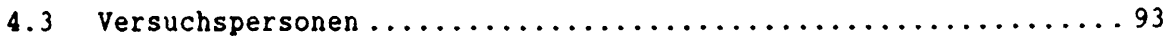

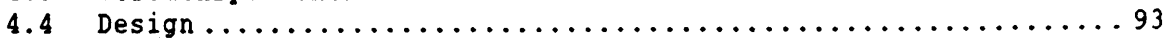

4.5 Ergebnis von Exp.1 und 2 (Akzent auf Er) :................ 93

4.6 Ergebnis von Exp. 3 und 4 (Akzent auf kommt) : ............ 94

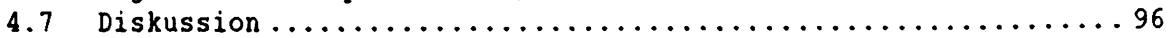

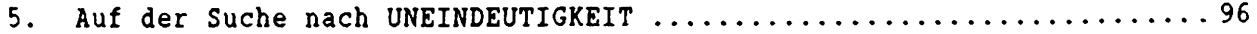

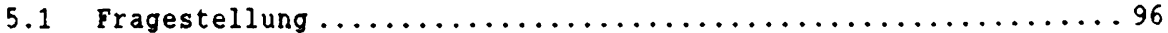

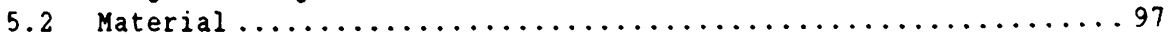

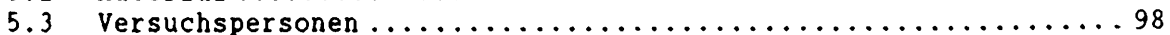

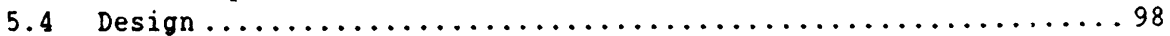

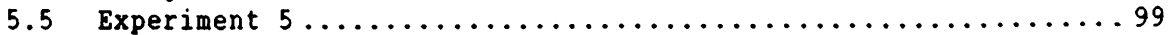

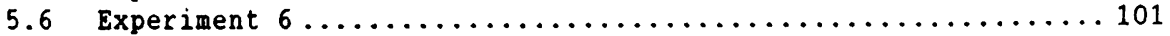

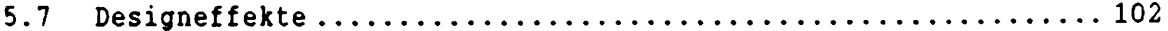

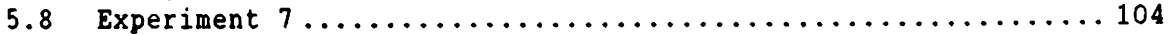

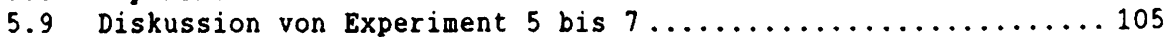




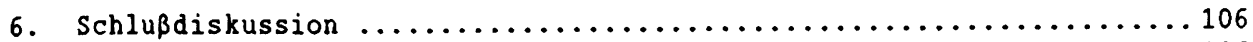

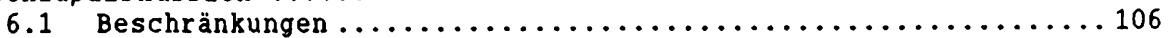

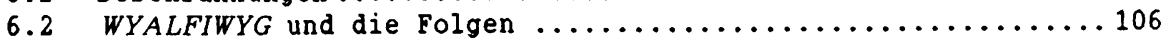

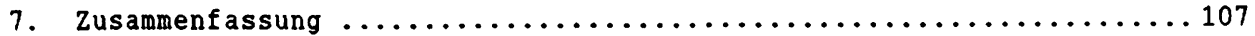

WIEVIEL HALBTONE BRAUCHT DIE FRAGE?

MERKMALE, DIMENSIONEN, KATEGORIEN.

\section{Anton Batliner}

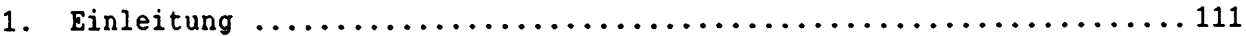

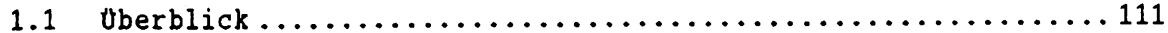

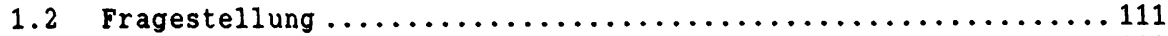

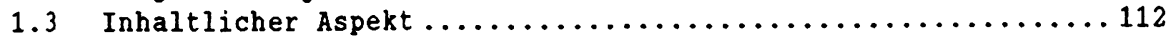

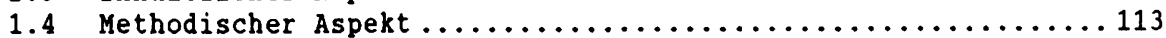

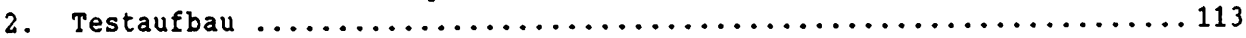

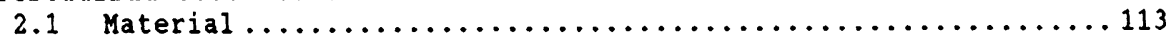

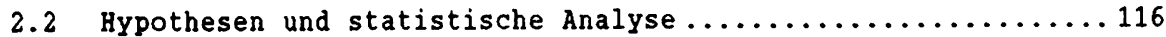

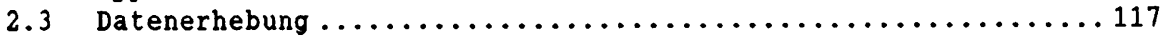

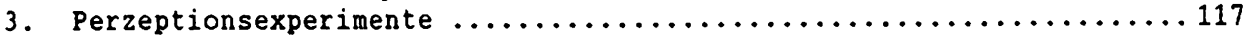

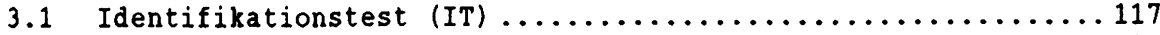

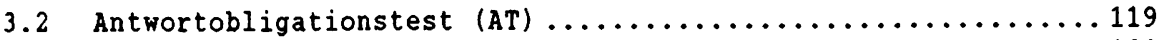

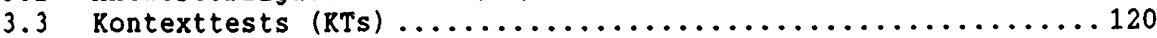

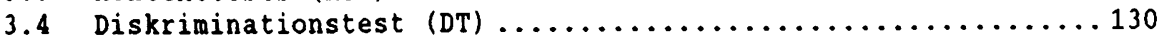

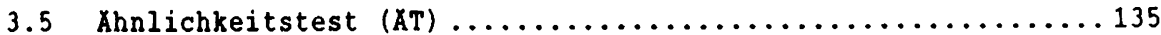

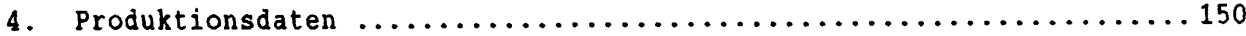

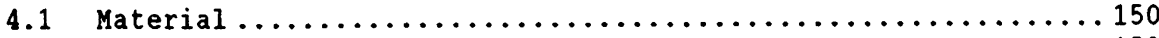

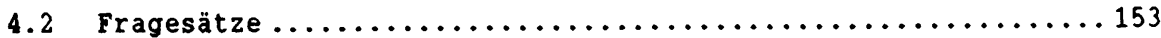

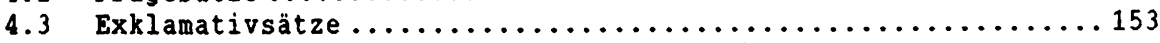

4.4 Die Relevanz der NMDS-Dimensionen für die Satz-

modi 'Verb-Erst-Frage' - und 'Verb-Erst-Exklamativsatz' . ........ 154

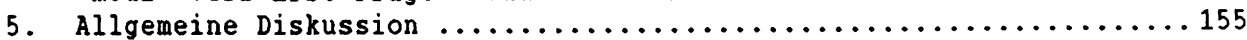

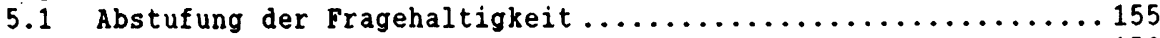

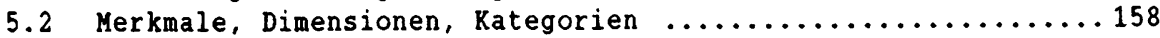

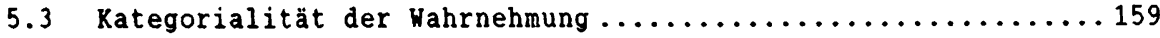

SELBSTANDIGE VERB-LETZT-SATZE: IHR PLATZ IM SATZMODUSSYSTEM UND IHRE INTONATORISCHE KENNZEICHNUNG

\section{Wilhelm Oppenrieder}

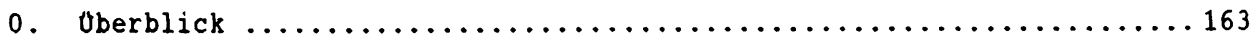

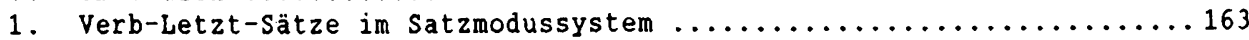

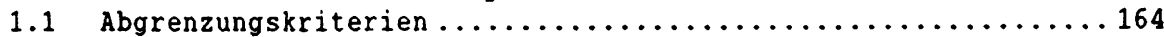

1.2 Satzeinleiter $z u$ selbständigen Verb-Letzt-Sätzen .......... 175

1.3 Typen illokutiv selbständiger Verb-Letzt-Sätze ........... 180

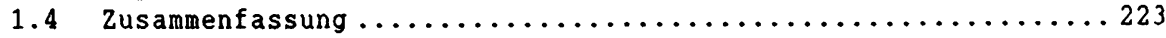

2. Experimentelle Untersuchungen zur intonatorischen Form

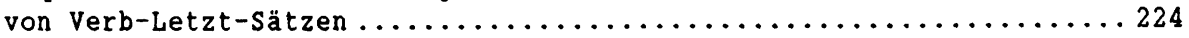

2.1 Einleitung: Minimalpaarmethode, Testsatzkonstruktion ....... 224

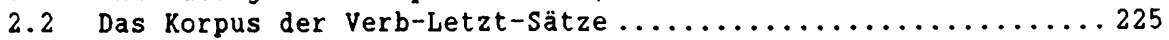

2.3 Aufnahmeprozedur, Hörtests und Parameterextraktion .........228

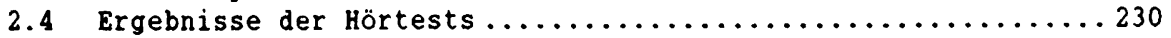

2.5 Die intonatorische Form von Verb-Letzt-Sätzen ........... 236

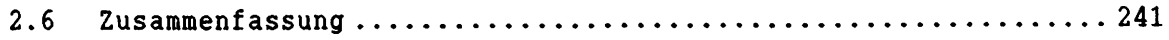




\section{Wilhelm Oppenrieder}

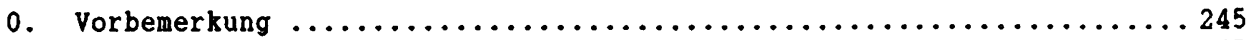

1. Das Konzept der 'Deklination' - ein kurzer uberblick ........... 245

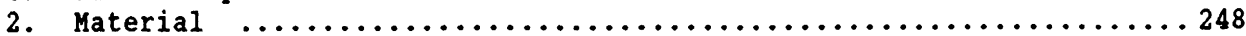

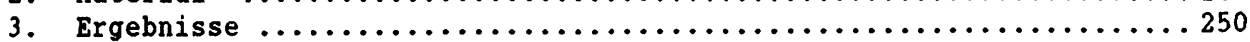

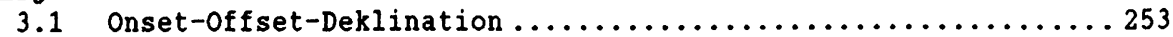

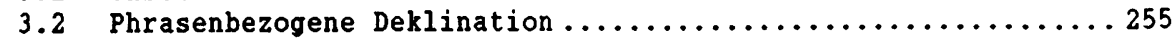

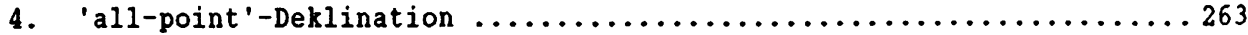

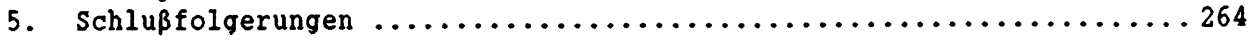

FOKUS, FOKUSPROJEKTION UND IHRE INTONATORISCHE KENNZEICHNUNG .267

\section{Wilhelm Oppenrieder}

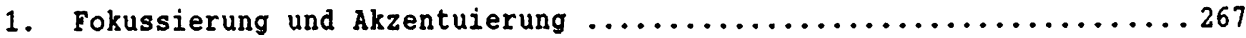

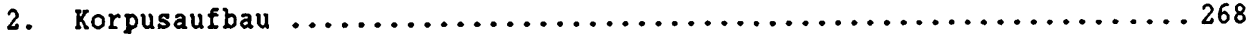

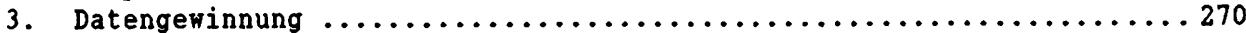

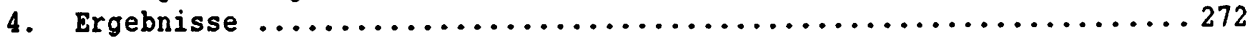

4.1 Rlassifikation und satzmodustypische

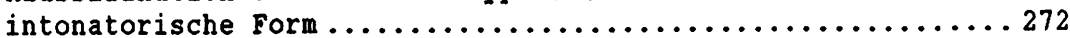

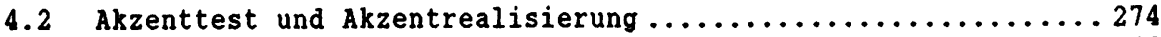

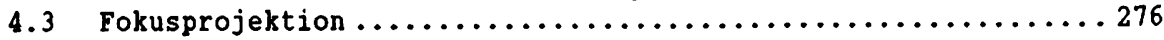

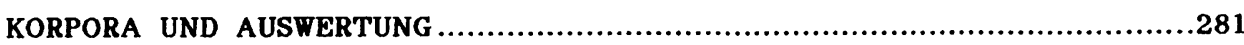

\section{Anton Batliner und Wilhelm Oppenrieder}

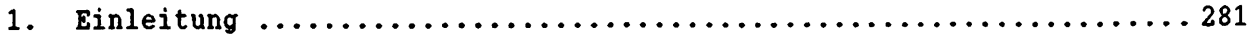

2. Die Minimalpaarmethode: Gewinnung der Testsatzkorpora $\ldots \ldots \ldots \ldots \ldots 281$

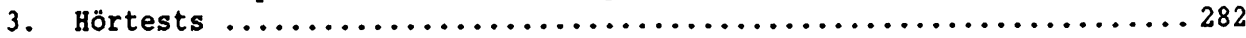

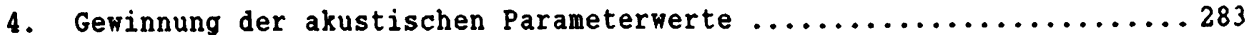

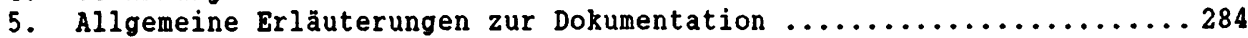

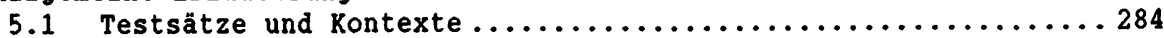

5.2 Vier Kennwerte zur Frage/Nicht-Frage-KIassifikation .........284

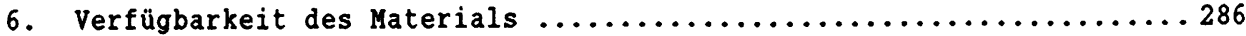

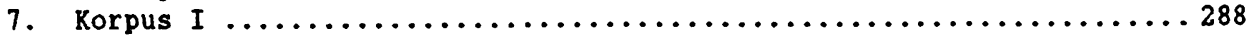

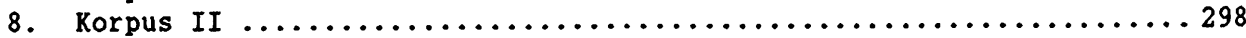

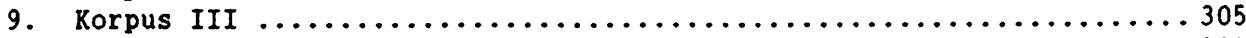

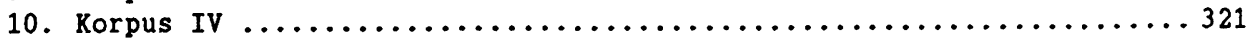


DAS PROJEKT 'MODUS-FOKUS-INTONATION'.

AUSGANGSPUNKT, KONZEPTION UND RESULTATE IM UBBERBLICK.

Hans Altmann, Anton Batliner und Wilhelm Oppenrieder

1. Begriffsklärung

Einleitend soll unser Sprachgebrauch bei den drei Begriffen 'Modus', 'Fokus' und 'Intonation' geklärt werden, die als Kurztitel das Projekt charakterisieren.

1.1 Modus

Gemeint ist hier der Begriff 'Satzmodus', wie er in Altmann (1984, 1987) verwendet wird. Satzmodi werden dort verstanden als komplexe syntaktische Strukturen (Beispiele: Aussagesatz, Entscheidungsfragesatz, Wunschsatz), denen regelhaft bestimmte abstrakte Funktionstypen (Beispiele: Aussage, Entscheidungsfrage, Wunsch) zugeordnet sind. Die jeweiligen Formtypen ergeben sich aus dem Zusammenspiel von Merkmalen aus vier verschiedenen Merkmalsmengen: a) Kategoriale Füllung (Beispiel: $w$-Ausdrücke in $w$-Fragesätzen und $w$-Exklamativsätzen) b) Stellungseigenschaften (Beispiele: Stellung des finiten Verbs an erster, zweiter oder letzter Position; Stellung des $w$-Frage-Ausdrucks in Versicherungsfragen) c) morphologische Markierung (Beispiel: Konjunktiv II in Wunschsätzen) d) intonatorische Markierung.

\section{$1.2 \quad$ Fokus}

'Fokus' und 'Fokussierung' sind semantische Begriffe, die die informationelle Gliederung von Außerungen betreffen: Vorausgesetzter Hintergrundsinformation steht fokussierte Information gegenüber, die aus einer kontextuell festgelegten Menge von Alternativen ausgewählt wird (vgl. Jacobs 1984, 1988). Gekennzeichnet wird der Fokus_durch die akzentuelle Hervorhebung eines Ausdrucks, des Fokmsexponenten (vgl. zu diesem Begriff Höhle 1982). Dieser Ausdruck trägt aber auch einen Teil der oben erwähnten intonatorischen Satzmodusmarkierung, 
so da $\beta$ jede Untersuchung die gegenseitige Beeinflussung der intonatorischen Kennzeichnung von Satzmodus und Fokus berücksichtigen muß (vgl. zu diesem Zusammenhang auch Beckman 1986 und Batliner 1989a).

\subsection{Intonation}

Der Begriff 'Intonation' wird hier in einem weiten Sinn gebraucht: Er umfaßt die nichtsegmentalen lautlichen Eigenschaften von Außerungen, also mindestens die Grundfrequenz(Fo)/Tonhöhe, die Intensität/Lautstärke und die zeitliche Strukturierung von Außerungen.

\section{Ausgangspunkt}

Genereller Ausgangspunkt für die Konzeption des Projekts war die Unzufriedenheit mit dem Stand der Intonationsforschung in Deutschland zu Beginn der 80er Jahre: In der linguistischen Intonationsforschung gab es nur wenige, häufig mit intuitiven Begriffen arbeitende Untersuchungen; der Rückstand gegenüber der internationalen Forschung (insbesondere im angelsächsischen Bereich) war auf theoretischem und empirischem Gebiet beträchtlich. In der phonetischen Intonationsforschung andererseits wurde häufig mit recht naiven Vorstellungen über die linguistische Relevanz der Intonation oder auf der Grundlage veralteter linguistischer Theorien gearbeitet. Der unbefriedigende Stand der Intonationsforschung in Deutschland war denn auch der Anlaß für die Errichtung des DFGSchwerpunktes "Formen und Funktionen der Intonation", in dessen Rahmen das hier dargestellte Projekt durchgeführt wurde:

"Die bisherige Intonationsforschung wurde durch zwei weithin unabhängige Forschungsstränge - einen linguistischen und einen phonetischen - geprägt. Dies erklärt zum Teil den nicht befriedigenden Forschungsstand [...] Besonders wünschenswert erscheint es, wenn Phonetiker und Linguisten gemeinsame Projekte oder einen Projektverbund bilden." (DFG-Schwerpunkt. FORMEN UND FUNKTIONEN DER INTONATION, S.11. Ms.).

In unserem Projekt versuchten wir, diese Interdisziplinarität konsequent durchzuhalten, so weit es beim derzeitigen stand der beiden Wissenschaften Linguistik und Phonetik möglich ist.

Spezieller Ausgangspunkt des Projekts war das in Altmann (1984, 1987) vorgestellte Satzmodusmodell, das die Zusammenarbeit von Linguistik und Phonetik an einem zentralen Teilbereich des Intonationssystems ermöglicht. Satzmodi werden als komplexe syntaktische strukturen (Formtypen) aufgefaßt, denen abstrakte Funktionstypen zugeordnet sind. $\mathrm{Zu}$ den syntaktischen Mitteln, die die 
Formtypen konstituieren, gehört unter anderem die Intonation. In Altmann (1984) wurden zunächst hypothetisch bestimmte intonatorische Merkmale (global steigende oder fallende Tonmuster, verschiedene Akzenttypen wie der 'Exklamativakzent') angesetzt. Diese selbst (wie auch die anderen syntaktischen Merkmale) haben keine eigene Bedeutung, die direkt einem Funktionstyp zugeordnet werden könnte, sondern erst der komplexe Formtyp ist ein eigenständiges sprachliches Zeichen. Nichtsdestoweniger können die verschiedenen Merkmale ein unterschiedliches Gewicht bei der Unterscheidung zweier Formtypen haben. Von besonderem Interesse sind hier diejenigen Formtyppaare, bei denen alle nichtintonatorischen Merkmale neutralisiert sind, so daß segmentgleiche Sätze gebildet werden können, bei denen allein die intonatorische Form satzmodusunterscheidend wirkt. Auf dem Konzept solcher intonatorischen Minimalpaare, wie es in Altmann (1984) detailliert erläutert wird, beruht die Konstruktion der von uns verwendeten Testsätze. Die Minimalpaarmethode erleichtert die Untersuchung der Intonation und die Auffindung der linguistisch relevanten intonatorischen Eigenschaften ganz wesentlich, indem sie einen direkten Vergleich $z$ wischen den intonatorischen Formen der Minimalpaar-Satzmodi ermöglicht, da alle anderen Faktoren (segmentelle und Akzentstruktur) konstant gehalten werden können. Zudem ist anzunehmen, daß bei einer starken funktionalen Belastung der satzmodusspezifischen intonatorischen Form diese auch besonders 'deutlich' realisiert wird, die wesentlichen Merkmale also klar erkennbar sind.

3.

Konzept

Es sollte vermieden werden, daß eine der beiden Wissenschaften reine Hilfswissenschaft der anderen bleibt. Wir suchten also eine Anbindung an die in den beiden Wissenschaften jeweils vorherrschenden Modellvorstellungen, die im Anschluß erläutert werden. Die grundsätzlich eigenständige, aber aufeinander bezogene Forschung spiegelt sich auch in der Form dieses Bandes, der Einzelbeiträge mit entweder linguistischer oder phonetisch/experimenteller Ausrichtung enthält.

Auf der linguistisch-phonologischen Seite wurde als Beschreibungsmodell der Ton-Sequenz-Ansatz in Betracht gezogen. Bei diesem Ansatz wird der Fo-Verlauf/Tonhöhenverlaup einer Außerung bzw. Intonationsphrase als das Resultat der fortlaufenden Verkettung von wenigen, lokal bestimmbaren 'Tönen' analysiert (vgl. z.B. Pierrehumbert 1980, Liberman/Pierrehumbert 1984, Beckman/Pierrehumbert 1986). Es wird also mit wenigen, einfachen und klaren Grundkategorien gearbeitet. Die tonalen Merkmale passen in dieser Hinsicht völlig zu den anderen syntaktischen Merkmalen: Neben einem morphologischen Merkmal wie 'Kon- 
junktiv II' steht ein intonatorisches Merkmal wie 'hoher rechter Grenzton' (Hochton am Außerungsende). Gegenüber solch globalen Beschreibungen wie 'steigendes Tonmuster' zeichnet sich die tonsequentielle Beschreibung zudem durch größere Flexibilität sowie durch Abbildungstreue der phonetischen Realität aus.

Auf der phonetisch/experimentellen Seite wurde das Paradigma der Kategorialen Wahrnehmung in Betracht gezogen. Das Paradigma hatte sich im segmentalen Bereich bei der Abgrenzung von Kategorien bewährt; zur intonatorischen Indizlerung der Kategorien Modus und Fokus gab es aber nur vereinzelte Arbeiten. Es war zu uberprüfen, ob sich nach den Kriterien des Paradigmas (z.B. Gipfel der Diskriminationskurve und steile Flanke der Identifikationskurve, vgl. Repp 1984 und Schiefer/Batliner 1988) auch bei intonatorisch markierten Kategorien klare Grenzen ergeben würden; das Paradigma sollte als Instrument eingesetzt werden, um damit klare und nicht klare Kategorien voneinander trennen zu können.

Tertium comparationis für linguistisches und phonetisches Vorgehen war also die Annahme von grundsätzlich distinkten Kategorien, die auch mit grundsätzlich distinguierenden Merkmalen (darunter intonatorische Merkmale) klar trennbar sind.

\section{Konkrete Planung}

Mit umfangreichen, natürllchsprachlichen Korpora einerseits und mit manipulierten Stimuli andererseits sollten das Satzmodusmodell überprift und insbesondere die hypothetisch angesetzten intonatorischen Formen empirisch abgesichert und näher spezifiziert werden; die oben skizzierten phonologischen und phonetischen Modellvorstellungen waren dabei auf ihre Leistungsfähigkeit als Analyse- und Beschreibungsinstrumente zu überprüfen: In Produktionsexperimenten wurden zwei Korpora mit jeweils 6 Sprechern aufgenommen; dle insgesamt 2074 Außerungen wurden akustisch analysiert sowie in Hörtests überprüft. Manipulierte Stimuli wurden in verschiedenen Typen von Perzeptionsexperimenten eingesetzt. Grundsätzlich begrenzten wir unsere Untersuchungen auf die Satzebene und auf makroprosodische Phänomene; diskurs- bzw. textlinguistische Phänomene und mikroprosodische Effekte mußten daher i.A. außer Betracht bleiben. 
Grundlage der Korpora waren Testsätze, die nach der oben beschriebenen Minimalpaarmethode gebildet sind; d.h. die Testsätze sind so konstruiert, daß sle je nach intonatorischer Form zu einem von zwei (oder mehr) Minimalpaar-Satzmodi gehören. Sie wurden in Produktionstests 'naiven' Versuchspersonen ohne Kenntnis des Satzmodussystems oder der intonatorischen Modelle vorgelegt, die sie satzmodusgerecht und - zur Erleichterung des Minimalpaarvergleichs - mit einer bestimmten festgelegten Akzentstruktur realisieren sollten. Die Versuchspersonen erhielten jedoch keine expliziten Anweisungen; vielmehr wurden die Testsätze in Kontexte eingebettet, die die intendierte Satzmodus- und Akzentrealisierung implizit steuern sollten.

Nach diesen Prinzipien wurden zwei große Korpora gebildet. Das erste Korpus enthält Past ausschließlich Verb-Erst- und Verb-Zweit-Sätze. Das zweite Korpus besteht aus drei Teilkorpora, die (1) (selbstständige und unselbstständige) VerbLetzt-Sätze, (ii) systematisch neben dem Satzmodus auch den Fokus variierende Sätze und (iii) kurze Verb-Erst- und Verb-Zweit-Sätze enthalten; im einzelnen vgl. Batliner/Oppenrieder (1989).

An den Produktionstests zum ersten und zweiten Korpus nahmen jeweils 6 Versuchspersonen ( 3 weiblich, 3 männlich) teil. Dabei wurden den Versuchspersonen die Testsatz-Kontext-Kombinationen auf Karten in (pseudo-) randomisierter Reihenfolge vorgelegt. Die Aufnahmen erfolgten im schallarmen Raum des Instituts für Phonetik, München.

Die Testsatzrealisationen wurden in verschiedenen Hörtests verwendet: Mit diesen sollte sowohl die Güte der Realisationen überprüft werden als auch die Frage, ob die intonatorische Form allein ausreicht, die segmental ambigen Strukturen auf einen Satzmodus festzulegen. Teilnehmer an den Hörtests waren im Durchschnitt 12 Versuchspersonen.

Die Akzentrealisierung wurde in sogenannten Akzenttests überprüft: Den Versuchspersonen wurden die Testsatzrealisationen kontextlos und gleichzeitig in schriftlicher Form dargeboten; auf dieser schriftlichen Vorlage war die am stärksten hervorgehobene Silbe zu markieren.

Mit den Kategorisierungstests sollte überprüft werden, inwieweit die intonatoriscine Form allein, ohne stützung durch einen vereindeutigenden Kontext, ausreicht, den Funktionstyp einer Außerung eindeutig festzulegen: Die Versuchspersonen mußten dementsprechend die kontextfrei dargebotenen Realisationen der Testsätze den 'Funktionstypen' Aufforderung, Frage, Aussage, Ausruf/Exklamativ und Wunsch zuordnen, die mit Hilfe einer funktionalen Beschreibung und einiger charakteristischer Beispiele elngeführt worden waren. Auf 
diese Weise lassen sich die Realisationen herausfiltern, die eindeutig dem intendierten Funktionstyp zugeordnet werden können.

Ziel der Natürlichkeitstests war es, die kontextgerechte Realisierung der Testsätze zu überprüfen: Deshalb lag den Versuchspersonen hier neben dem Testsatz auch der gesamte Kontext vor; der Testsatz war jeweils markiert. Von den Versuchspersonen sollte beurteilt werden, wie gut der Testsatz zum Kontext paßte, m.a.W. wie 'natürlich' die Testsatzrealisation war. Vorgegeben war eine Fünferskala mit 1 für 'paßt sehr gut', 2 für 'paßt gut ', 3 für 'paßt noch gut', 4 für 'paßt schlecht' und 5 für 'paßt sehr schlecht'. Jeder Testsatzrealisation kann ein (kontextbezogener) Natürlichkeitswert als arithmetisches Mittel aus den Beurteilungen zugeordnet werden.

Die Ergebnisse der Kategorisierungs- und Natürlichkeitstests wurden als Filter für die Trennung der prototypischen ('natürlichen' und 'satzmodusrichtigen') von den nicht-prototypischen ('nicht natürlichen' und/oder 'nicht satzmodusrichtigen') Realisationen genutzt. Als prototypische Realisierungen in diesem Sinn gelten Exemplare, die einen kleineren (= besseren) Natürlichkeitswert als 2.5 aufweisen und die gleichzeitig von mehr als $80 \%$ der Versuchspersonen im Kategorisierungstest so klassifiziert worden waren, wie es von uns nach der Testsatzkonstruktion intendiert war. ${ }^{1}$

Prototypisch sind also solche Exemplare, bei denen zum einen die intonatorische Form allein die Auflösung von Satzmodusambiguitäten zuverlässig gestattet und die zum anderen auch generell als akzeptable Realisationen beurteilt wurden. Aussagen über satzmodustypische intonatorische Formen sollten sich, soweit möglich, nur auf diese Realisationen stützen.

Der nächste Schritt war die akustische Auswertung der Testsatzrealisationen. $\mathrm{Zu}$ diesem $\mathrm{Zweck}$ wurden von allen 2074 Außerungen das Zeitsignal, der Fo-Verlauf (gemessen mit einem Fo-Meter) und der Intensitätsverlaup (gemessen mit einem Intensity-Meter) auf einem Mingogramm ausgegeben. Ein entscheidender Schritt ist nun, für welche Art und welches Ausmaß der Datenreduktion man sich bei der Analyse dieser akustischen Daten entscheidet. Anfangs wurde daran gedacht, die Fo-Verläufe der Testsätze mittels des phonologischen Ton-SequenzAnsatzes als eine Abfolge von 'Tönen' zu beschreiben, also sofort eine sehr abstrakte Repräsentationsform zu wählen. Wir mußten jedoch sehr schnell feststellen, daß dieser Ansatz - so geeignet er auch für eine generativ ausgerich-

1 Diese Schwellenwerte sind nicht so $z u$ verstehen, daß die exakten Werte 'begründet' sind. Sie dienten in diesem Zusammenhang ausschließlich dazu, 'gute' Produktionen von - aus welchen Gründen auch immer - weniger guten Produktionen zu trennen. Für bestimmte Fragestellungen können andere Schwellenwerte verwendet oder nur die Werte des einen oder des anderen Tests berücksichtigt werden. 
tete Beschreibung des 'tonalen Systems' einer Sprache sein mag - für die Analyse umfangreicher Korpora kein optimales Werkzeug ist (vgl. zur folgenden Kritik Oppenrieder 1988b, 181ff.).

Zunächst ist unklar, wieviele unterschiedliche Arten von Tönen sinnvollerweise verwendet werden sollten: In Pierrehumbert (1980) z.B. werden die einfachen Hoch- und Tieftöne auch $\mathrm{zu}$ verschiedenen komplexen 'Doppeltönen' verbunden. Dies hängt davon $a b$, ob eine möglichst getreue Repräsentation der verschiedenen tatsächlich vorkommenden Fo-Verläufe erwünscht ist, oder ob man - des distinktiven Charakters phonologischer Einheiten eingedenk - Verlaufsstücke mit der gleichen 'Bedeutung' auch tonal identisch repräsentiert (eine solche 'tonale Bedeutungslehre' existiert aber unseres Wissens nicht). Weiter erfolgt die Zuordnung von Tönen zu Fo-Konturstücken völlig intuitiv, eindeutige Kriterien werden nirgends in der Literatur angegeben (auch hier ist wieder von Belang, ob eine möglichst genaue Abbildung der Kontur oder eine Kennzeichnung der bedeutungsdifferenzierenden oder -tragenden struktur beabsichtigt ist). offensichtlich kann die linguistisch relevante intonatorische Beschreibung nicht auf tonale Merkmale beschränkt werden.

Wegen dieser grundsätzlichen Schwierigkeiten mit dem Ton-Sequenz-Ansatz entschlossen wir uns, unser Material nicht tonal zu beschreiben - schließlich war dieser Ansatz nur als ein mögliches Beschreibungsinstrument für unser Korpus und nicht umgekehrt das Korpus zur Bestätigung des Ton-SequenzAnsatzes konzipiert. Wir versuchten vielmehr, möglichst viele akustische Kennwerte für diejenigen Bereiche der Außerungen zu extrahieren, die für die Satzmoduskennzeichnung zentral sind: Das sind die 'Ränder' der Außerungen, im Deutschen insbesondere der rechte (d.h. das Außerungsende), und der Bereich der in den Akzenttests festgestellten Hauptakzentsilbe. Es kam uns also darauf an, zunächst behutsame, möglichst nicht-interpretative Datenreduktionen vorzunehmen; zur Extraktion der akustischen Kennwerte vgl. Batliner/Oppenrieder (1989).

\subsection{Perzeptionsexperimente}

Bei den Perzeptionsexperimenten lagen der Manipulation meist zwei Sätze mit gleicher segmentaler Struktur, aber unterschiedlicher Verbstellung zugrunde (Der Leo säuft, Säuft der Leo); mit diesen Sätzen ließen sich sehr viele intonatorische Minimalpaare bilden, bei denen allein die Intonation Modus- und Fokusunterschiede indiziert; vgl. im einzelnen Batliner (1988b,c). Diese Beschränkung war zum einen praktisch motiviert - eine pitchsynchrone Manipulation, so wie 
sie von uns durchgeführt wurde, ist sehr zeitaufwendig; die Beschränkung ermöglichte aber zum anderen eine strikte Vergleichbarkeit aller Experimente. ${ }^{2}$

Wie beim Ton-Sequenz-Ansatz mußten wir auch beim Paradigma der Kategorialen Wahrnehmung feststellen, $d a \beta$ es für die Untersuchung solcher Intonationsphänomene allenfalls bedingt geeignet ist. Einerseits läßt sich die strikte Algorithmisierung der Kriterien - die das Paradigma anfangs attraktiv machte nicht durchführen. Andererseits zeigte sich bald, daß die durch die Intonation indizierten Kategorien (-grenzen) anderer Art sind als die im Paradigma typischerweise untersuchten (vgl. zu den Kritikpunkten Schiefer/Batliner 1988). Das übliche experimentelle Design (Identifikationstests und Diskriminationstests) wurde daher ergänzt durch andere Designs, hauptsächlich durch sog. Kontexttests, bei denen eine Kontexteinbettung der Stimuli eine - in Maßen - natürlichere Bewertung ermöglichte, als dies bei direkter Befragung der Fall ist.

\section{Intonatorische Prototypen}

Bei den Produktionsdaten ist der nächste Schritt nach der Extraktion der akustischen Kennwerte für die einzelnen Außerungen die Abstraktion von den äußerungsspezifischen Besonderheiten zugunsten der satzmodustypischen und satzmodusunterscheidenden Form. Das Ergebnis sind die sogenannten 'intonatorischen Prototypen'. Den einzelnen Satzmodi sind typische Fo-Verläufe im Bereich der Hauptakzentsilbe und typische Fo-Werte an den Rändern, insbesondere am Außerungsende, sowie zum Teil auch typische Dauerstrukturen und Intensitätsverläufe (bei Exklamativsätzen) zugeordnet (vgl. Oppenrieder 1988b). Zentral für unseren Ansatz ist die Annahme, daß auch auf dieser abstrakten Ebene der intonatorischen Form berücksichtigt werden muß, daß es sich bei diesen typischen Ausprägungen nur um einen Kernbereich handelt, der Abweichungen zuläßt: Wir legen unserer Beschreibung keine klar voneinander abgegrenzten und in sich unstrukturierten 'klassischen' Kategorien zugrunde, sondern 'prototypisch' strukturierte, bei denen ein zentraler Kernbereich von typischen Realisationen in zulässige, aber weniger typische Realisierungen 'ausfranst', die sich zudem mit den Randbereichen anderer intonatorischer Prototypen überlappen können. ${ }^{3}$

2 Eine solche Vergleichbarkeit ist dann nicht gegeben, wenn auf Grund von Unterschieden im Ausgangsmaterial oder in der Art der Stimulusgenerierung nicht entschieden werden kann, ob unterschiedliche Ergebnisse nicht doch auf diese 'äußeren' Faktoren zurückzuführen sind.

3 In der 'Prototypenliteratur' (vgl. Lakoff 1987) wird die genaue struktur der Prototypen zunehmend diskutiert, so z.B. die Frage, ob es Fälle gibt, die so $z w i s c h e n$ verschiedenen Kategorien liegen, da $\beta$ sie mehreren zugleich angehören, oder ob nicht doch jeder Fall genau einer Kategorie angehört, es aber 
An Perzeptionsdaten wurden sowohl Kern- als auch Randbereiche gezielt überprüft; vgl. dazu vor allem Batliner (1988c, 1989a,d). Dabei zeigte sich durchgehend, daß die Wahl des Designs das Ergebnis wesentlich beeinflußt; so ließer. sich - je nach Design - Grenzen zwischen Satzmodus- und BetonungsKategorien und Abstufungen innerhalb von Kategorien sowie deutliche vbergangsbereiche zwischen Kategorien nachweisen. Phonetisch/experimentelles und linguistisch/phonologisches Vorgehen konvergieren also in der gleichen Art von Kategorien.

Analog zur Phonemanalyse lassen sich intonatorische Oppositionen, z.B. zwischen einer 'Frageintonation' und einer 'Nicht-Frageintonation' aufstellen. Diese Oppositionen sind der Ausgangspunkt, auf dem unsere Untersuchungen basieren: Die Minimalpaarmethode gründet in der Hypothese, daß es derartige 'funktionale' Oppositionen gibt. Doch scheint es uns im Bereich der Intonation unangemessen, lediglich bei systemphonologisch beschreibbaren oppositionen stehenzubleiben und die Variation in der phonetischen 'Substanz' auf eine für das linguistische System nicht relevante 'realisationsphonologische' Ebene abzuschieben. Wir halten es vielmehr für geboten, die 'Unschärfe' und die prototypische Strukturierung im Bereich der Intonation (und nicht nur dort) in die systematische Beschreibung der intonatorischen Form miteinzubeziehen, da sich in unseren Arbeiten deutlich die scharf voneinander abgegrenzten und 'intern' homogenen Kategorien als Fiktion erweisen.

Auch auf der Ebene der reinen Satzmodusunterscheidung mit intonatorischen Mitteln - d.h. ohne irgendwelche Feinabstufungen innerhalb der einzelnen Satzmodi zu beachten - finden wir in der phonetischen 'Substanz' keine klaren 'Oppositionen' und können dem Kennzeichnungspotential auch nicht notwendige und hinreichende (und damit eindeutige) intonatorische Eigenschaften zuordnen. Als Beispiel betrachte man die intonatorische Unterscheidung von Frage- und Nicht-Fragesätzen in den oben erwähnten Minimalpaarkonstellationen, bei denen allein die intonatorische Form für die Satzmodusdifferenzierung sorgt. Charakteristisch für die intonatorisch markierten Fragesätze ist auf und im Bereich der Hauptakzentsilbe ein ausgeprägter Anstieg der Fo, die anschließend in etwa auf diesem hohen Niveau ('hoher Offset') bleibt; charakteristisch für den Rest der Satzmodi ist auf und im Bereich der Hauptakzentsilbe ein (nicht unbedingt sehr ausgeprägter) Abfall der Fo, die anschließend aup diesem niedrigen Niveau bleibt ('tiefer offset'). Nun findet sich aup dieser abstrakten Ebene der Satzmodusoppositionen eine prototypische strukturierung der intonatorischen Satzmodus-

innerhalb der Kategorien Abstufungen gibt. Bel einem so komplexen Phänomen, wie es die Intonation darstellt, halten wir eine Entscheidung für die eine oder die andere Ansicht für verfrüht. 
kennzeichnung: Es müssen nämlich nicht beide Bestandstücke gleichermaßen realisiert sein, sondern eines davon reicht aus: entweder ein Fo-Anstieg im Bereich der Hauptakzentsilbe mit anschließendem Abfall oder aber eine steigendfallende Fo-Bewegung mit einem Ansteigen am Außerungsende. Es gibt also zwar hinreichende intonatorische Merkmale für die Konstitution einer 'Frageintonation', aber sie sind nicht notwendig.

Die einfachen Kennzeichnungsalternativen (Fo-Anstieg auf der Hauptakzentsilbe mit tiefem offset bzw. steigend-fallende Fo-Bewegung auf der Hauptakzentsilbe mit hohem offset) sind seltener und zumindest in diesem Sinn markierter als die doppelte Kennzeichnung (Fo-Anstieg auf der Hauptakzentsilbe mit hohem Offset); vgl. die Unterscheidung von Kern- und Randtypen bei Fragesätzen mit nicht-finaler Position des Hauptakzents in Batliner (1989a). Es handelt sich nicht um drei gleichwertige Möglichkeiten, sondern um eine typische, häufige und zwei seltenere. Es existiert demnach bereits auf dieser stufe eine 'Prototypizitätsskala' ('Präferenzskala'), die in eine angemessene Beschreibung eingehen muß.

Eine prototypische Strukturierung ist auf allen Beschreibungsebenen zu finden. Sie setzt bereits im Satzmodussystem selbst an: Bestimmte Struktureigenschaften dieses Systems lassen sich am besten erklären, wenn man auch hier 'zentrale' (Aussage-, Frage- und Aufforderungssätze) und 'periphere' (Exklamativ- und Wunschsätze) Satzmodi annimmt. Zunächst sind die zentralen Typen offensichtlich auch eher Kandidaten für universelle Satzmodi (vgl. zur Typologie der Satzmodi Sadock/Zwicky 1985). Bei den zentralen Typen besteherı im allgemeinen weniger grammatische Einschränkungen, z.B. im Gebrauch der Tempusund Modusformen oder auch in der lexikalischen Füllung. Die formale Abgrenzung der zentralen Typen untereinander ist im allgemeinen viel klarer als die von den Randtypen: Die Verbstellung trennt die (typischen) Aussagesätze von den Entscheidungsfragesätzen; die w-Ausdrücke markieren die Ergänzungsfragesätze; das Zusammenspiel von morphologischer Markierung und Subjektlosigkeit kennzeichnet die typischen Aufforderungssätze; dagegen sind z.B. die Exklamativsätze nur durch relativ markierungsschwache intonatorische Kennzeichnungsmittel von Aussage-, Entscheidungs- und w-Fragesätzen unterschieden. Die zentralen Formen werden schließlich weitaus häufiger gebraucht. So sind sie auch im allgemeinen 'illokutiv neutraler' als die markierten Satzmodi.

Die prototypische strukturierung findet sich auch auf allen tieferliegenden Markierungsebenen (vgl. dazu Oppenrieder 1988b, 187ff.), insbesondere den Ebenen der intonatorischen Satzmoduskennzeichnung: 
1 Zunächst spielt die intonatorische Markierung im Gesamtzusammenhang der formalen Markierungsmittel eine zentralere oder peripherere Rolle: Bei den Exklamativsätzen ist sie zentral, bei den Imperativsätzen der 2.Ps. dagegen verhältnismäßig unwichtig.

2 Wie oben erwähnt, können verschiedene Formen bei der Frageintonation nach typisch und markiert geordnet werden.

3 Innerhalb des Komplexes der intonatorischen Kennzeichnungsmittel, z.B. bei einer spezifischen Form der 'Frageintonation' ist die Fo/Tonhöhe zweifellos wichtiger als die Dauerstruktur und die Intensität/Lautstärke.

4 Schließlich gibt es auch bei den verschiedenen relevanten Einzelmerkmalen, z.B. der Fo/Tonhöhe am Außerungsende typische und markiertere Ausprägungen.

Kennzeichnend für unser 'Intonationsmodell' ist also, da $\beta$ wir nicht mit den klassischen homogenen und klar voneinander abgegrenzten Kategorien arbeiten, sondem mit prototypisch strukturierten: Ein Kernbereich mit typischen Fällen geht iber in eine Peripherie, in der es auch zu Úberlappungen mit anderen genauso strukturierten Kategorien kommen kann.

6. Modellvorstellung und empirische Grundlage

Wir haben im vorigen Abschnitt unser 'Intonationsmodell' skizziert; dieses Modell ist nicht als ausformuliertes, algorithmisiertes Modell zu verstehen, sondern eher als Modellvorstellung, innerhalb derer wir versuchen, eine breite empirische Datengrundlage $z u$ schaffen. Unsere Beschreibungsgrößen sind dabei nach Möglichkeit so gewählt, daß sie nicht nur innerhalb eines ganz bestimmten Modells Bestand haben, sondern auch in andere Modelle überführt werden können; sie sind nicht theorienah, sondern datennah oder zumindest in konkrete Werte 'rücküberführbar'. Die Grundzüge dieses Vorgehens wurden oben erläutert; wir möchten an dieser Stelle keine weiteren methodischen uberlegungen anschließen, da gerade für die Empirie gilt: "Es gibt nichts Gutes, es sei denn, man tut es." In diesem Sinne verweisen wir auf den folgenden Literaturüberblick im allgemeinen und auf die weiteren Beiträge in diesem Band im besonderen.

Dieser Literaturüberblick verzeichnet alle im Rahmen des Projekts oder im Zusammenhang mit ihm erstellten Arbeiten, die schon veröffentlicht sind oder in absehbarer Zeit veröffentlicht werden; unveröffentlichte Magisterarbeiten sind nicht aufgeführt. Die Arbeiten werden dabei thematisch den Gebieten 'Linguistik' oder 'Phonetik' zugeordnet; meist wurden aber in den unter 'Linguistik' ver- 
zeichneten Arbeiten auch phonetische Aspekte behandelt und umgekehrt. Gesamtdarstellungen des Projekts finden sich unter 'Linguistik'; Arbeiten, die Vorstufen späterer Arbeiten darstellen, sind als solche gekennzeichnet.

\subsection{Sammelband}

Altmann, H. (Hg.) (1988b): Intonationsforschungen. Tübingen.

- Sammelband mit Aufsätzen zur Intonation vor allem des Deutschen. Im einzelnen sind Beiträge enthalten zur Methodik der Intonationsforschung (Erstellung und Auswertung geeigneter Korpora; apparative und auditive Analyse von Intonation; Planung, Durchführung und statistische Auswertung phonetischer und linguistischer Experimente; Methodenkritik), zur akustischen und perzeptiven Phonetik, zur Phonologie (Art und formales Beschreibungsinventar von Akzenten und Tonmustern), zur Syntax (Prinzipien der Fokussierung; Fokus-Hintergrund-Gliederung; intonatoris che Markierung im Satzmodussystem und bei den einzelnen Satzmodi) sowie zur Pragmatik.

\subsection{Linguistik}

Altmann, H. (1984): Linguistische Aspekte der Intonation am Beispiel Satzmodus. In: Forschungsberichte des Instituts für Phonetik und Sprachliche Kommunikation der Universität München (FIPKM) 19. S.132-152.

- Klärung des Satzmodusbegriffs; Beschreibung der Formtypen im Satzmodussystem (Aussagesatz, Entscheidungsfragesatz usw.) anhand ihrer grammatischen Merkmale (kategoriale Füllung, Verbmorphologie, Verbstellung, Intonation etc.); hypothetische Ansetzung von zwei Tonmustern und mehreren Akzentarten; Entwicklung des Konzepts der intonatorischen Minimalpaare, also von Satzmodus-Ausdrücken, die nur durch ihre intonatorischen Eigenschaften mehreren Satzmodi/Formtypen zugewiesen werden können.

Altmann, H. (1987): Zur Problematik der Konstitution von Satzmodi als Formtypen. In: Meibauer, J. (Hg.) (1987): Satzmodus zwischen Grammatik und Pragmatik. Tübingen. S.22-56.

- Gegenüber Altmann (1984) ausgeweitete und verbesserte Fassung; ausführliche Darstellung des Satzmodussystems; Beschreibung der intonatorischen Merkmale von Verb-Erst- und Verb-Zweit-Sätzen anhand der Ergebnisse aus dem DFG-Projekt nach dem Konzept der intonatorischen Prototypen.

Altmann, H. (1988a): Satzmodus und Intonation. In: Modus und Modularität. Akten des Round-Table-Gesprächs ILK Berlin 87. ZISW Reihe A, Arbeitsberichte.

- Beruht auf der Darstellung der Formtypen in Altmann (1987). Liste der bei der intonatorischen Beschreibung berücksichtigten Parameter; Eigenschaften der Akzentsilben; akustische und perzeptive Eigenschaften der verschiedenen Akzentarten; Tonhöhenverlauf; Diskussion der Variabilität intonatorischer Merkmale und theoretische Schlußfolgerungen. 
Altmann H. (1989): Artikel 'Satzmodus'. In: Jacobs, J. et al. (Hgg.): Handbuch der iyntax. Berlin. (Erscheint voraussichtlich 1990)

- Detallierte Darstellung der Formtypen im Satzmodussystem nach ihren syntiktischen Eigenschaften, insbesondere der für ihre Kennzeichnung notwencigen grammatischen Merkmale; punktueller Vergleich mit den syntaktischel Eigenschaften der Satzmodi in anderen Sprachen.

Altmann H./Batliner, A./Oppenrieder,W. (1989): Das Projekt 'Modus-Fokus-Intonatiın'. Ausgangspunkt, Konzeption und Resultate im Überblick. (In diesem Banc).

- Dargettellt werden Ausgangspunkt und Konzeption des Projekts, die Erstellung und Verarbeitung der Produktionskorpora sowie das Vorgehen bei den Perz:ptionsexperimenten; eine Modellvorstellung $\mathrm{zu}$ intonatorischen Prototy?en wird entwickelt; die im Rahmen des Projekts bzw. im Zusammenhang mit ihm erstellten Arbeiten werden aufgeführt und kurz charakterisiert. Darüberhinaus enthält der Artikel einen selbstreferentiellen Bezug.

Batliner, A./Oppenrieder, W. (1989): Korpora und Auswertung. (In diesem Band)

- Die Produktionskorpora des Projekts werden im einzelnen vorgestellt und komnentiert; bei allen Korpora sind Testsätze und Kontexte dokumentiert, bel zwei Korpora zusätzlich schematisierte Fo-Verläufe aller Außerungen sowis die wichtigsten statistischen Kennwerte.

Luukko-Vinchenzo, L. (1987): Entscheidungsfragesätze im Finnischen. Mit einem Exkurs ins Deutsche. In: Meibauer, J. (Hg.) (1987): Satzmodus zwischen Grammatik und Pragmatik. Tübingen. S.125-140.

- Der Beitrag untersucht die finnischen Entscheidungsfragesätze unter formalen Aspekten in Anlehnung an die Satzmoduskonzeption von Altmann. Behandelt werden die Fragepartikel $-k o$, fragetypische Modalpartikeln, verbmorphologische Wortstellung und die intonatorische Form (diese anhand eines Korpus natürlichsprachlicher finnischer Außerungen); die formalen Merkmale der finnischen Entscheidungsfragesätze werden kurz mit denen der deutschen verglichen.

Luukko-Vinchenzo, L. (1988a): Zur Intonation finnischer Fragesätze. In: Altmann, H. (Hg.) (1988b). S.135-152.

- Untersucht werden die intonatorischen Eigenschapten von Pinnischen $m / k-$ Fragesätzen (die den deutschen $w$-Fragesätzen entsprechen) und SatzPragesätzen. Datengrundlage ist ein umfangreiches Korpus intonatorischer Minimalpaare. Als zentrale akustische Parameter erweisen sich die eng miteinander korrelierten Größen Fo und Dauer.

Luukko-Vinchenzo, L. (1988b): Formen von Fragen und Funktionen von Fragesätzen. Eine deutsch-finnische kontrastive Studie unter besonderer Berücksichtigung der Intonation. Tübingen.

- Jie Studie untersucht auf einer breiten Datengrundlage einen zentralen Bereich des deutschen und des finnischen Satzmodussystems, den der Fragen/Fragesätze; sie basiert auf dem Satzmodussystem von Altmann. Der Schwerpunkt liegt in der (kontrastiven) Herausarbeitung der formalen Merkmale der verschiedenen Fragesatztypen; dabel werden die intonatorischen Merkmale besonders ausführlich behandelt. Grundlage dafür sind detaillierte 
Analysen der deutschen Korpora des Projekts sowie eines umfangreichen finnischen Korpus intonatorischer Satzmodus-Minimalpaare.

Oppenrieder, W. (1987): Aussagesätze im Deutschen. In: Meibauer, J. (Hg.) (1987): Satzmodus zwischen Grammatik und Pragmatik. Tübingen. S.161-190.

- Untersucht werden die funktionalen und insbesondere die formalen Eigenschaften von Aussagesätzen im Rahmen des Satzmodussystems von Altmann. Der Formtyp 'Aussagesatz' wird im wesentlichen negativ durch die Abgrenzung gegenüber anderen Satzmodi auf den verschiedenen Merkmalsebenen (kategoriale Füllung; topologische, morphologische und intonatorische Merkmale) gekennzeichnet. Aup Problemfälle bei dieser Abgrenzung wird ausführlich eingegangen.

Oppenrieder, W. (1988a): Intonation und Identifikation. Kategorisierungstests zur kontextfreien Identifikation von Satzmodi. In: Altmann, H. (Hg.) (1988b): S.153-167.

- Dargestellt wird ein Hörtest ('Kategorisierungstest'), der an allen Korpora des Projekts durchgeführt wurde. Mit diesem Test sollte untersucht werden, inwieweit intonatorische Merkmale eine einwandfreie Identifikation des Satzmodus durch die Hörer auch dann ermöglichen, wenn die Außerungen in ihrer nicht-intonatorischen Struktur satzmodusambig sind. Insbesondere auf die problematischen Exklamativstrukturen wird ausführlich eingegangen.

Oppenrieder, W. (1988b): Intonatorische Kennzeichnung von Satzmodi. In: Altmann, H. (Hg.) (1988b). S.169-205.

- Ein Teilkorpus mit Verb-Erst- und Verb-Zweit-Strukturen aus dem Projekt bildet die Datengrundlage für die Aufstellung satzmodustypischer intonatorischer Prototypen. Das Prototypenkonzept im Bereich der Intonation und des Satzmodussystems generell wird ausführlich erläutert und begründet. Den Hintergrund dafür bildet eine kritische Auseinandersetzung mit anderen Methoden der Intonationsbeschreibung, insbesondere mit dem Ton-SequenzAnsatz.

Oppenrieder, W. (1989a): Selbständige Verb-Letzt-Sätze: Ihr Platz im Satzmodussystem und ihre intonatorische Kennzeichnung. (In diesem Band)

- Den ersten Teil bildet eine ausführliche Darstellung der selbstständigen Verb-Letzt-Sätze im Rahmen des Satzmodussystems von Altmann. Einem Versuch der Abgrenzung der verschiedenen Typen nicht-eingebetteter VerbLetzt-Strukturen und der generellen Charakterisierung selbstständiger VerbLetzt-Sätze folgt die formale und funktionale Beschreibung der einzelnen Typen selbstständiger Verb-Letzt-Sätze. Im zweiten Teil wird anhand eines Korpus aus dem Projekt die intonatorische Form der verschiedenen Typen von Verb-Letzt-Sätzen im Rahmen des Prototypenkonzepts vorgestellt.

Oppenrieder, W. (1989b): Deklination und Satzmodus. (In diesem Band)

- Grundlage bildet ein Korpus aus dem Projekt ('Fokus-Korpus'), das längere Außerungen enthielt, die im Satzmodus und in der Fokussierungsstruktur systematisch variiert wurden. Es wird untersucht, ob sich Satzmodi durch globale Charakteristika des Fo-Verlaups voneinander unterscheiden lassen. $\mathrm{Zu}$ diesem $\mathrm{Zweck}$ werden die durchschnittlichen Steigungen verschiedener Typen von 'Deklinationslinien' (Linien durch prominente Fo-Werte wie Minima 
oder Maxima) miteinander verglichen. (Satz-) Fragesätze lassen sich auf diese Weise deutlich von Aussage- und Imperativsätzen abgrenzen.

Oppenrieder, W. (1989c): Fokus, Fokusprojektion und ihre intonatorische Kennzeichnung. (In diesem Band)

- Es wird untersucht, in welcher Weise die intonatorische Kennzeichnung von Satzmodi bei unterschiedlichen Fokussierungsstrukturen variiert. Besonders interessiert, ob sich Fokusprojektion und enge Fokussierung in der intonatorischen Realisierung konsistent unterscheiden lassen. Datengrundlage ist ein Korpus natürlichsprachlicher Außerungen aus dem Projekt ('FokusKorpus') mit intonatorischen Satzmodus- und Fokusminimalpaaren.

Oppenrieder, W. (1989d): Struktur und Verwendung von 'irrealen Vergleichssätzen'. (In Vorbereitung)

- Untersucht werden die 'irrealen Vergleichssätze' unter den Gesichtspunkten ihrer Binnenstruktur und ihrer selbstständigen oder unselbstständigen Verwendung. Dabei wird zum einen auf die verschiedenen Typen von Einleitungsausdrücken im Zusammenhang mit Verbstellung und -modus, zum anderen auf die Stellung der selbstständigen irrealen Vergleichssätze im Satzmodussystem von Altmann eingegangen.

Scholz, U. (1987): Wunschsätze im Deutschen - formale und funktionale Beschreibung. In: Meibauer, J. (Hg.) (1987): Satzmodus zwischen Grammatik und Pragmatik. Tübingen. S.234-259.

- Gegenstand der Arbeit ist die formale und funktionale Beschreibung des VerbErst-Wunschsatzes im Rahmen des Satzmodussystems von Altmann. Im ersten Teil werden die für den Satztyp konstitutive Verbmorphologie (Konjunktiv II), die intonatorische Form sowie die typischen Modal- und Interjektionspartikeln behandelt. Der zweite Teil bringt eine funktionale Charakterisierung der Wunschsätze, die insbesondere die Abgrenzungsproblematik gegenüber den Imperativsätzen berücksichtigt.

Scholz, U. (1989): Wunschsätze im Deutschen - formale und funktionale Beschreibung. Satztypen mit Verberst- und Verbletztstellung. Ms.

- Verb-Erst- und Verb-Letzt-Wunschsätze werden im Rahmen des Satzmodussystems von Altmann untersucht. In der formalen Beschreibung werden nicht nur die kategorialen, morphologischen, topologischen und intonatorischen (auf der Grundlage des 1. Korpus aus dem Projekt) Eigenschaften von Wunschsätzen behandelt, sondern es wird auch auf die Typologie der wunschsatzähnlichen Strukturen eingegangen.

Winkler, E. (1989): Der Satzmodus "Imperativsatz" im Deutschen und Finnischen. Tübingen.

- Im Rahmen des Satzmoduskonzepts von Altmann werden deutsche Verb-Erstund Verb-Zweit-Imperativsätze sowie finnische Imperativsätze in ihren formalen und funktionalen Eigenschaften (kontrastiv) beschrieben. Im formalen Teil wird jeweils eingegangen auf die Verbmorphologie, die zulässigen Subjektsausdrücke, die Verbstellung, die Merkmale der kategorialen Füllung (insbesondere Modalpartikeln), die Sequenzierungseigenschaften und die intonatorische Form. Den Abschluß bildet eine ausführliche Funktionsbeschreibung des Imperativ-Satzmodus. 
7.3 Phonetik

Batliner, A. (1987): Der Einsatz der Diskriminanzanalyse zur Prädiktion des Satzmodus. In: Tillmann, H.G./Willée G. (Hgg.) (1987): Analyse und Synthese gesprochener Sprache. Hildesheim etc. S.125-132.

- Größtenteils Vorfassung von Batliner (1988a).

Batliner, A. (1988a): Produktion und Prädiktion. Die Rolle intonatorischer und anderer Merkmale bei der Bestimmung des Satzmodus. In: Altmann, H. (Hg.) (1988b). S.207-221.

- Einsatz eines statistischen Klassifikationsverfahren zur Bestimmung des Satzmodus anhand weniger Fo-Werte (Werte am Anfang und am Ende der Außerung sowie maximaler und minimaler Wert). Die Klassifikation wird verbessert durch (i) eine Umwandlung (Transformation) der Fo-Werte in Halbtonwerte relativ zu sprecherspezifischen oder äußerungsspezifischen werten, (ii) eine Beschränkung der Klassifikation auf die 'Prototypen', vgl. oben 4.1 (damit bestätigt sich die Relevanz der Hörtests, die der Prototypenauswahl zugrundelagen) und (iii) eine Beschränkung auf die Fälle mit hoher intonatorischer Belastung (damit bestätigt sich das Konzept der Bestimmung von Prototypen anhand intonatorischer Minimalpaare).

Batliner, A. (1988b): Modus und Fokus als Dimensionen einer Nonmetrischen Multidimensionalen Skalierung. In: Altmann, H. (Hg.) (1988b). S.223-24l.

- Aus zusammenpassenden Testsätzen und Kontextsätzen, die jeweils eine bestimmte Satzmodus- und Satzfokuskonstellation indizieren, wurden durch die Kombination jedes Testsatzes mit jedem Kontextsatz mehr oder weniger zusammenpassende Kombinationen gebildet und von Hörern bewertet. Ein statistisches Verfahren stellt die Ergebnisse anschaulich im zweidimensionalen Raum dar: Fokus ist bei Frage und Aussage relevant, beim Exklamativ irrelevant. Frage vs. Nicht-Frage sind deutlich voneinander geschieden, Aussage und Exklamativ weniger deutlich. Die Frage ist eine in sich abgestufte Kategorie.

Batliner, A. (1988c): Der Exklamativ: Mehr als Aussage oder doch nur mehr oder weniger Aussage? Experimente zur Rolle von Höhe und Position des Fo-Gipfels. In: Altmann, Hans (Hg.) (1988b). S.243-271.

- Experimente mit manipulierten Stimuli zur Unterscheidung von Aussagesatz und Exklamativsatz anhand von Höhe und Position des Fo-Gipfels. Ergebnis: Breiter Ubergangsbereich beim Fo-Gipfel mit einer Höhe von sieben Halbtönen; exklamativtypisch ist (i) die Kombination von hohem Gipfel mit Dehnung, (ii) ein fehlender Fokusakzent, (iii) ein später Fo-Gipfel.

Batliner, A. (1988d): Eine Frage ist eine Frage ist keine Frage.

Perzeptionsexperimente zum Fragemodus im Deutschen. In: Modus und Modularität. Akten des Round-Table-Gesprächs ILK Berlin 87. ZISW Reihe A, Arbeitsberichte.

- Vorfassung von Batliner (1989c). 
Batliner, A. (1989a): Fokus, Modus und die große Zahl. Zur intonatorischen Indizierung des Fokus im Deutschen. (In diesem Band)

- Anhand eines von mehreren Sprechern produzierten, kontrollierten Korpus wird die intonatorische Markierung des Satzfokus im Deutschen bei Fragen und Nicht-Fragen untersucht. Mit Hilfe eines statistischen Verfahrens (Diskriminanzanalyse) werden die für die Markierung relevanten intonatorischen Parameter und Transformationen der Pararneterwerte bestimmt. Durch Prototypenbildung 'über die große Zahl' (Mittelwertbildung) und über Hörerurteile werden 'normale', prototypische Fälle von 'nicht-normalen', aber dennoch regulären Fällen abgegrenzt sowie die sprecherübergreifende und die sprecherspezifische Relevanz der einzelnen intonatorischen Merkmale untersucht.

Batliner, A. (1989b): Fokus, Deklination und Wendepunkt. (In diesem Band)

- In Perzeptionsexperimenten wird untersucht, (i) ob ein später Fo-Gipfel akustisch weniger prominent sein muß als ein früher, um Träger des Fokusakzents sein zu können (Effekt der Deklination), und (ii) ob die Verschiebung des Fo-Wendepunkts bei einer fallend-steigenden Fo-Kontur mit einer Verschiebung des Fokusakzents einhergeht.

Batliner, A. (1989c): Eine Frage ist eine Frage ist keine Frage.

Perzeptionsexperimente zum Fragemodus im Deutschen. (In diesem Band)

- Experimente zur Abgrenzung von Frage und Aussage bzw. Frage und Exklamativ mit manipuliertem Material; zugleich Uberlegungen zur Frage, inwieweit das experimentelle Design und die ihm zugrundeliegenden Modellvorstellungen das Ergebnis beeinflussen. Es zeigt sich, daß die Wahl des Designs mitbestimmt, ob man Kategoriengrenzen oder Abstufungen zwischen Kategorien erhält.

Batliner, A. (1989d): Wieviel Halbtöne braucht die Frage? Merkmale, Dimensionen, Kategorien. (In diesem Band)

- Detaillierte Weiterführung von Batliner (1989c): Die Rolle unterschiedlicher Ausprägungen eines fallend-steigenden Tonverlaufs für die Satzmodusindizierung bei Verb-Erst-Fragesatz und Verb-Erst-Exklamativsatz wird untersucht. In Perzeptionsexperimenten mit unterschiedlichem Design werden Kategoriengrenzen und Abstufungen innerhalb der Kategorien sowie der Zusamenhang zwischen akustischen und phonetischen Merkmalen, perzeptiven Dimensionen und linguistischen Kategorien genauer bestimmt.

Batliner, A. (1989e): Ein einfaches Modell der Frageintonation und seine Folgen. (in Vorbereitung)

- Mit einem statistischen Klassifikationsverfahren, bei dem der finale Fo-Wert als Prädiktorvariable dient, und durch Hörtests werden bel 1999 Außerungen Fragen und Nicht-Fragen getrennt. In ca $20 \%$ der Fälle entscheiden entweder das statistische Verfahren oder die Hörer 'falsch'; es wird untersucht, welche Faktoren (weitere intonatorische Merkmale, nicht-intonatorische Merkmale o.ä.) für diese falschen Entscheidungen verantwortlich sind. 
Batliner, A./Oppenrieder, W. (1988): Rising Intonation: Not Passed Away But Still Alive. A Reply to R. Geluykens. In: Journal of Pragmatics 12. S.373-379.

- Kritik der Ansicht, daß bei assertiven Fragen grundsätzlich nicht die Intonation, sondern pragmatische Faktoren für die Frageinterpretation ausschlaggebend sind; zugleich Kritik eines Experimentes, mit dem diese Ansicht belegt werden soll.

Batliner, A./Nöth, E. (1989). The prediction of focus. Proceedings of the European Conference on Speech Communication and Technology, Paris, 2628 September 1989. (Im Druck)

- Darstellung der Hauptergebnisse von Batliner (1989a) unter stärkerer Berücksichtigung der Automatischen Spracherkennung.

Batliner, A./Nöth, E./Lang, R./Stallwitz, G. (1989): Zur Klassifikation von Fragen und Nicht-Fragen anhand intonatorischer Merkmale. (Erscheint in: Tagungsband der 15. Gemeinschaftstagung der Deutschen Arbeitsgemeinschaft für Akustik (DAGA), 13.-16. März 1989).

- Der Beitrag untersucht an einem großen Korpus (1999 Fälle), inwieweit der Fo-Wert am Außerungsende als Frage/Nicht-Frageindikator ausreicht, wie dieser Wert aussehen soll (Rohwert in $\mathrm{Hz}$ oder umgerechnet $\mathrm{zu}$ Bezugsgrößen), und ob es sprecherspezifische Strategien beim Einsatz dieses Parameters gibt.

Batliner, A./Schiefer, L. (1987): Stimulus Category, Reaction Time, and Order Effect - An Experiment on Pitch Discrimination. In: Proceedings XIth ICPhS, Vol. 5. S.46-49.

- Der sog. Ordnungseffekt bewirkt, daß in einem Diskriminationstest die eine Anordnung der zwei stimuli besser diskriminiert wird als die andere. Der Effekt wird bei der Wahrnehmung von Tonverläufen untersucht mit sprachlichem und nicht-sprachlichem Material sowie mit steigenden und pallenden Verläufen. Bei steigenden Verläufen ergibt sich ein größerer Ordnungseffekt als bei fallenden. Nicht-sprachliches Material wird besser diskriminiert als sprachliches, steigende Verläufe werden besser diskriminiert als fallende.

Nöth, E./ Batliner, A./ Lang, R./ Oppenrieder, W. (1987): Automatische Fo-Analyse und Satzmodusdifferenzierung. In: Tillmann, H.G./Willée G. (Hgg.) 1987): Analyse und Synthese gesprochener Sprache. Hildesheim etc. S.59-66.

- Es werden Algorithmen zur Extraktion der Fo vorgestellt und die damit extrahierten Werte eingesetzt, um mit Hilfe zweier Klassifikationsverfahren (fuzzy membership function bzw. Diskriminanzanalyse) den Satzmodus aus dem Korpus IV ('Leo-Korpus', vgl. Batliner/Oppenrieder 1989) vorherzusagen.

Schiefer, L./Batliner, A. (1988): Intonation, Ordungseffekt und das Paradigma der Kategorialen Wahrnehmung. In: Altmann, H. (Hg.) (1988b). S.273-291.

- Es werden Experimente zur Akzentzuweisung und zur Wahrnehmung von Tonverläufen beschrieben, bei denen ein 'Ordnungseffekt', d.h. ein signifikanter Unterschied bei der Bewertung von Stimulusabfolgen, vgl. Batliner/Schiefer (1987), zu beobachten ist. Mögliche Erklärungen für den Ordnungseffekt sowie seine Auswirkungen auf das Paradigma der Kategorialen 
Wahrnehmung werden diskutiert. Die Brauchbarkeit des Paradigmas bei der Untersuchung von Intonationsphänomenen wird kritisch beleuchtet.

\section{LITERATUR}

Die zitierten Arbeiten der Autoren sind in Teil 7 aufgeführt.

Beckman, M.E. (1986): Stress and Non-Stress Accent. Dordrecht.

Beckman, M.E./Pierrehumbert, J.B. (1986): Intonational Structure in Japanese and English. In: Phonology Yearbook 3. S.255-309.

Höhle, T.N. (1982): Explikationen für 'normale Betonung' und 'normale Wortstellung'. In: Abraham, W. (Hg.) (1982): Satzglieder im Deutschen. Tübingen. S.75-152.

Jacobs, J. (1984): Funktionale Satzperspektive und Illokutionssemantik. In: Linguistische Berichte 91. S.25-58.

Jacobs, J. (1988): Fokus-Hintergrund-Gliederung und Grammatik. Altmann, H. (Hg.) (1988b). S.89-134.

Lakoff, G. (1987): Women, Fire, and Dangerous Things. What Categories Reveal about the Mind. Chicago und London.

Liberman, M./Pierrehumbert, J.B. (1984): Intonational Invariance Under Changes in Pitch Range and Length. In: Aronoff, M./Oehrle, R.T. (Hgg.) (1984):

Language, Sound, Structure. Cambridge, Mass./London. S.157-233.

Pierrehumbert, J.B. (1980): The Phonology and Phonetics of English Intonation. Phil. Diss. MIT.

Repp, B.H. (1984): Categorical perception: Issues, methods, findings. In: Lass, N.J. (Hg.): Speech and Language. Orlando etc. S.243-335.

Sadock, J.M./Zwicky, A.M. (1985): Speech act distinctions in syntax. In: Shopen, T. (Hg.) (1985): Language Typology and Syntactic Description. Volume I: Clause Structure. Cambridge etc. S.155-196. 

FOKUS, MODUS UND DIE GROSSE ZAHL. ZUR INTONATORISCHEN INDIZIERUNG DES FOKUS IM DEUTSCHEN.

Anton Batliner

1. Einleitung

1.1 Uberblick

In diesem Beitrag wird anhand eines von mehreren Sprechern produzierten, kontrollierten Korpus die intonatorische Markierung des Satzfokus im Deutschen bei Fragen und Nicht-Fragen untersucht. Mit Hilfe eines statistischen Klassifikationsverfahrens (Diskriminanzanalyse) werden die für die Markierung relevanten intonatorischen Parameter und Transformationen der Parameterwerte bestimmt. Durch Prototypenbildung 'über die große Zahl' (Mittelwertbildung) und über Hörerurteile werden 'normale', prototyplsche Fälle von 'nicht-normalen', aber dennoch regulären Fällen abgegrenzt sowie die sprecherübergreifende und die sprecherspezifische Relevanz der elnzelnen intonatorischen Merkmale untersucht.

\subsection{Begriffsklärung, Stand der Forschung}

Der Begriff 'Satzmodus' wird von uns im Sinne des Satzmodusmodells von Altmann (1987) gebraucht. 'Fokus' bezeichnet den semantisch 'wichtigsten' Teil des Satzes, der durch den Fokusakzent (FA) gekennzeichnet ist. Mit phonetischen Mitteln kann der FA intonatorisch durch Grundfrequenz (Fo), Dauer und Intensität indiziert werden, nicht-intonatorisch durch damit z.T. gekoppelte Phänomene, wie spektrale Unterschiede oder Pausensetzung; auf die nicht-intonatorischen Merkmale werden wir nicht weiter eingehen. Im folgenden verzichten wir normalerweise auf eine explizite Trennung der physikalischen Größen von ihren perzeptiven Korrelaten (Fo vs. Tonhöhe, Intensität vs. Lautstärke, etc.). In dieser Arbeit sollen keine unterschiedlichen Akzentarten gekennzelchnet 
werden, wie Normalakzent, Kontrastakzent, emphatischer Akzent o.ä. Im normalen (nicht-kontrastiven) Fall kann der FA nur auf einer 'betonbaren' Silbe realisiert werden, auf der ein Wortakzent liegt. Wortakzent und FA können mit den gleichen intonatorischen Mitteln markiert werden; im einzelnen ist natürlich zu untersuchen, wie diese Mittel eingesetzt werden (vgl. dazu Bannert 1985 und Thorsen 1987). ${ }^{1}$

Normalerweise wird angenommen, daß für die Akzentmarkierung Fo am wichtigsten ist, Dauer weniger wichtig und Intensität am unwichtigsten; vgl. dazu den Literaturüberblick in Beckman (1986), die aber fürs Englische der Intensität größere Bedeutung beimißt. Die intonatorische Markierung des Akzents ist z.B. fürs Englische besser untersucht als fürs Deutsche; noch Klein (1980:32) mußte feststellen: "Es scheint mir kein gutes Zeichen für den Stand der deutschen Phonetik, daß völlige Unklarheit darüber herrscht, was das signalphonetische Gegenstück des 'Akzents' ist." Seitdem sind einige Arbeiten zur Form des Akzents im Deutschen erschienen, vgl. etwa Adriaens (1984), Bannert (1985), sowie einige Arbeiten im Sammelband von Altmann (1988). Allerdings sind die Fragestellungen meist speziell, so daß Generalisierungen nur vorsichtig gezogen werden können.

\subsection{Methodik}

Die traditionelle Intonationsforschung zog in den Augen des Forschers 'repräsentative' Fälle nicht nur zur Illustration, sondern auch zur Begründung von Regeln heran; zur Kritik an diesem Vorgehen vgl. Klein (1980, 1982). Diese 'qualitative' Vorgehensweise wurde in der neueren Forschung durch eine 'quantitative' abgelöst, bei der angegeben wird, auf der Grundlage welchen Materials Aussagen gemacht werden. Problematisch bleibt dabei noch immer der Schluß vom untersuchten Material auf die Gesamtheit (Schluß von der Stichprobe auf die Population), also etwa auf 'die intonatorische Fokusmarkierung im Deutschen'. Wir wollen nun drei in diesem Zusammenhang wichtige Punkte aufzeigen und skizzieren, welche Strategie wir jeweils einschlagen werden.

1 Wir haben in diesem Abschnitt unsere sprachregelung skizziert, ohne da $\beta$ damit impliziert sein soll, daß wir sie für 'besser' halten als andere Regelungen, wie etwa eine Einschränkung des Begriffs 'Intonation' auf Grundfrequenz/Tonverlauf, die Verwendung von 'Satzakzent' statt 'Fokusakzent' o.ä. 


\subsubsection{Sprecherauswahl}

Sprecherauswahl und damit der Umfang des Materials sind zuallererst ein ökonomisches Problem; man kann nicht versuchen, alle Sprecher des Deutschen (das es als Einheit letztlich gar nicht gibt) zu repräsentieren, sondern muß sich auf bestimmte Varietäten beschränken. Die von uns untersuchte Varietät ist die süddeutsch/bairisch gefärbte Standardsprache. Alle im folgenden gemachten Aussagen beziehen sich also nur auf diese Varietät. Es wurden sechs Sprecher (3 weibliche, 3 männliche) gewählt. Damit können schon vorsichtige Schlüsse z.B. über die Repräsentativität der Produktionen einzelner Sprecher gezogen werden. ${ }^{2}$ Die Sprecher werden nicht nur interpretativ verglichen (via Inspektion von Kurvenverläufen o.ä.), sondern mit Hilfe eines statistischen Verfahrens. Damit lassen sich objektive, in Zahlen ausdrückbare Angaben machen.

\subsubsection{Sprecherinstruktion}

Oft werden die Sprecher angewiesen, aup welcher Silbe der Akzent gesetzt werden soll, welche Emphasestufe verwendet werden soll, etc. Auf diese Weise sollen 'Störvariablen' ausgeschaltet werden. Eine solche Instruktion entspricht allerdings nicht der Forderung nach größtmöglicher Nähe zur natürlichsprachlichen Kommunikation; man begibt sich dabei auch der Möglichkeit, auf interessante Abweichungen zu stoßen: Wenn man z.B. vorgibt, daß Fragen immer mit steigendem Tonverlauf produziert werden sollen, so wird man nie herausfinden, wann und in welchem Umfang Fragen einen fallenden Tonverlauf aufweisen. Wir gaben deshalb unseren Sprechern keine expliziten Instruktionen, sondern nur 'implizite', d.h. über eine Kontextvorgabe gesteuerte; im einzelnen vgl. dazu Oppenrieder (1988b) und Batliner/Oppenrieder (1989).

\subsubsection{Konvergierende, sich stützende Strategien}

So wie man bei Perzeptionsexperimenten mit manipulierten und damit per se unnatürlichen stimuli nie sicher sein kann, ob man überhaupt das 'Richtige' oder

2 Die Forderung der theoretischen Statistik nach zufälliger und repräsentativer Auswahl der Stichprobe aus der Population wird in der Praxis so gut wie nie erfüllt. Unser Begriff der 'Repräsentativität' ist denn auch bescheidener: Bei einem Sprecher hat man eine Kasuistik, bei zwei Sprechern zwei Kasuistiken. Bel sechs Sprechern könnte man sogar mit einfachen statistischen Tests (z.B. Chi-Quadrat) signifikante Unterschiede feststellen, wenn sich ein Sprecher von allen anderen unterscheidet. Ob die Stichprobe insgesamt repräsentativ ist, bleibt ein inhaltliches Problem und kann nur durch Replikation der Experimente mit anderen und größeren Stichproben gelöst werden. 
doch nicht nur experimentelle Artefakte untersucht, so kann man bei Produktionsdaten nicht sicher sein, ob im Einzelfall 'richtig' produziert wurde, und welches perzeptive Korrelat das Gemessene hat (vgl. die Fehleranalyse in Teil 9). Alle unsere Außerungen wurden deshalb den weiter unten beschriebenen Hörtests unterzogen.

\section{$1.4 \quad$ Statistisches Modell}

Das von uns gewählte Verfahren der Diskriminanzanalyse gehört zu den in der Mustererkennung (und damit auch in der automatischen spracherkennung) üblichen Verfahren für die Klassifikation von Fällen in bekannte Gruppen anhand möglichst relevanter Merkmale (Prädiktorvariablen); es handelt sich um einen 'Klassifikator mit überwachtem Lernen' (vgl. Niemann 1974, Ruske 1988). Die Diskriminanzanalyse funktioniert ähnlich wie die multiple Regressionsanalyse: Es werden lineare Kombinationen der unabhängigen (Prädiktor-) Variablen gebildet, die möglichst optimal zwischen den Gruppen unterscheiden können. Jede Prädiktorvariable erhält einen Gewichtungskoeffizienten, der anhand der vorgegebenen Daten und Gruppenzugehörigkeit der Fälle so geschätzt wird, daß die resultierende Diskriminanzfunktion zwischen den Gruppen möglichst stark differiert; anders gesagt: die Variabilität zwischen den Gruppen soll im Verhältnis zur Variabilität innerhalb der Gruppen möglichst groß sein.

In der Mustererkennung sind für die Wahl des Verfahrens zum einen praktische Gründe (Verfügbarkeit, Zeitbedarf), zum anderen die Effizienz entscheidend, also die Güte der Klassifikation. In der Phonetik sollte man darüber hinaus das Modell wählen, das am ehesten die Prozesse im 'natürlichen sprecher/Hörer' abbildet. Letztlich dürften beide Auswahlverfahren konvergieren, also das Modell am effizientesten sein, das die menschliche Fähigkeit am besten abbildet. ${ }^{3}$ Auch wenn man keine $\mathrm{zu}$ weitreichenden Schlüsse ziehen sollte, so wird sich doch zeigen, daß die Diskriminanzanalyse sowohl unsere Produktions- als auch unsere Perzeptionsdaten sehr gut abbildet. Die folgenden Elgenschaften lassen die Diskriminanzanalyse für unsere Zwecke geeignet erscheinen (zum Verfahren im einzelnen vgl. Norusis 1986 und Klecka 1980; eine Anwendung auf die Prädiktion des Modus zeigen Batliner 1988 und Batliner et al. 1989):

3 Diese Aussage produzierte bisher jedesmal entweder Widerspruch oder den Wunsch nach eingehender Begründung und im weiteren Verlauf Diskussionen über statistische Verteilungen, Künstliche Intelligenz und das Wesen des Menschen. Eine Begründung kann hier nicht gegeben werden, einen Beweis gibt es nicht, genausowenig wie für die gegentellige Ansicht. 
1. Die Hörerurteile, die wir unserer FA-Zuweisung zugrundelegen, erfüllen nicht unbedingt die Verteilungsvoraussetzungen für regressionsanalytische Verfahren (vgl. unten Fig.1), die deshalb weniger geeignet sind als Verfahren, bei denen die Fälle bekannten Klassen zugeordnet werden.

2. Die Umwandlung von kontinulerlichen Betonungsabstufungen in 'betont/unbetont' dürfte eine wichtige Stufe in der natürlichsprachlichen Kommunikation widerspiegeln: Der Mensch kann nur zwischen einer sehr begrenzten Zahl von Akzentuierungsstufen unterscheiden, und er wird sich ziemlich bald für eine relativ grobe Repräsentation des Gehörten in 'betont=wichtig' vs. 'unbetont=unwichtig' entscheiden, um nicht zuviel Information speichern zu müssen. (Im bisher einzigen Experiment zur Frage, wieviele Akzentstufen der Mensch konsistent mit alleiniger Hilfe intonatorischer Merkmale unterscheiden kann, kommt Lieberman 1965 auf zwei Stufen: 'betont' vs. 'unbetont'. Zur Umkodierung von Außerungen bzw. Sätzen im Verstehensproze $\beta$ vgl. Hörmann 1978:460ff.)

3. Das Verfahren kann die für die Klassenzuweisung relevanten Merkmale (Prädiktorvariablen) auswählen und sie nach ihrer Relevanz einstufen. Es bietet die Möglichkeit, einzelne Variablen (univariate Analyse) und mehrere oder alle Variablen gleichzeitig (multivariate Analyse) zu testen.

4. Es können beliebige Teilstichproben (Lernstichproben) zum Trainieren der Klassenzuweisung anhand der Prädiktorvariablen sowie andere Teilstichproben zum Klassifizieren (Prüfstichproben) ausgewählt werden. Wir können systematisch einzelne Sprecher(-gruppen) zu anderen Sprechern in Beziehung setzen und Gemeinsamkeiten und Unterschiede herausarbeiten; anders gesagt: man kann Sprecherunabhängigkelt simulieren und damit das nachbilden, was der Mensch auch kann, nämlich 'richtige' Akzenturteile bei Außerungen eines ihm bis dato unbekannten Sprechers abgeben (im einzelnen vgl. Teil 4).

5. Das Verfahren liefert quantifizierte Gütemaße, insbesondere Prozentwerte für die Zahl der richtig klassifizierten Fälle.

\subsection{Das Prototypenkonzept}

Medin/Barsalou (1987) weisen auf einen entscheidenden Unterschied $z$ wischen zwei Auffassungen von Kategorien hin: Einmal sind Kategorien definiert durch Regeln, das andere Mal durch typische Vertreter, die viele bzw. charakteristische Merkmale der Kategorie aufwelsen; die Vertellung der Merkmale ist aber nicht einfach durch Regeln definlerbar: Die Kategorien sind prototypisch strukturiert. Wir nehmen analog zur zweiten Auffassung an, daß es (proto-) typische Realisierungen bestimmter Akzentstrukturen gibt, und daß eine Definition dieser Realisierungen ausschließlich durch Regeln zumindest auf Schwierigkeiten stößt.

Wir wollen zwei unterschiedliche Arten der Prototypengewinnung miteinander vergleichen: einmal die Gewinnung über 'die große Zahl', d.h. Bildung über Mittelwerte der relevanten Parameter; zum anderen die Gewinnung über 'typische' Exemplare, die bei Hörtests eine besonders gute Bewertung erhielten.4

Die Art der Gewinnung von Prototypen hat natürlich etwas mit dem zugrundegelegten Prototypenkonzept zu tun. Zu diesem Punkt machen wir keine 
Eine Gewinnung über die große Zahl setzt voraus, daß Prototypen etwas mit den häufigsten Fällen zu tun haben - im Idealfall, daß sie unimodal und normalverteilt sind. Diese Annahme ist natürlich zu einfach. Es seien anhand der Verteilung einer einzigen Variablen (eines einzigen Merkmals) drei mögliche Verteilungen diskutiert:

I

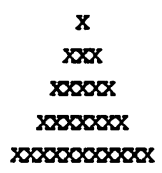

II

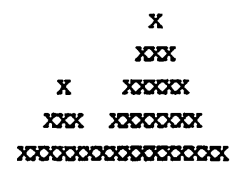

III

\begin{tabular}{|c|c|c|}
\hline$x$ & $x$ & $x$ \\
\hline$x x x$ & $x x x$ & $x x x$ \\
\hline$x \operatorname{xxx}$ & $x x x x x$ & $\operatorname{xxs} x$ \\
\hline$x \operatorname{sex} x$ & & $x \times x \times x \times x$ \\
\hline
\end{tabular}

Der 'ideale' Fall der Verteilung einer Variablen ist eine unimodale, symmetrische - die Normalverteilung; so könnte man annehmen, da $\beta$ Fragen sich immer durch einen hohen finalen Fo-Wert (Offset) auszeichnen (als 'Zielton'), der von den Sprechern angestrebt und mehr oder weniger genau getroffen wird; die Verteilung um den Mittelwert ist dann zufällig (I). Die Verteilung des offsets bei Fragen entspricht aber eher (II), vgl. Batliner et al. (1989): Der offset ist in den meisten Fällen hoch, kann aber auch tief sein. Weitere Verteilungen sind vorstellbar, von denen (III) einen möglichen Fall darstellt: eine trimodale Verteilung. Grundsätzlich kann man annehmen, daß sich bei einer genügend großen Stichprobe die Verteilung der Normalverteilung annähert; bei den Fragen muß man dann zwei Normalverteilungen, eine mit hohem, eine andere mit tiefem offset annehmen, wobei die mit dem hohen offset den häufigsten Fall repräsentiert. Man kann also über die große Zahl repräsentative prototypische Fälle ermitteln. Auf der anderen Seite ist damit nicht gesagt, daß nur solche repräsentativen Fälle prototypische Fälle sind in dem Sinn, daß sie voll akzeptabel sind. Natürlich ist es auch eine Frage der 'Klassifikationstiefe': Je nachdem wieviele Gruppen (Clusters) angesetzt werden, und wieviele Fälle in die Analyse eingehen, bekommt man ein mehr oder weniger differenziertes Bild der möglichen Merkmalsausprägungen bestimmter Klassen.

Aussagen und verweisen auf den guten ưberblick über die unterschiedlichen Richtungen und Kritikpunkte in Lakoff (1987). 
Bei den Fällen, die nicht dem häufigsten Fall, den wir als Normal-Fall bezelchnen, zuzurechnen sind, sollte unterschieden werden zwischen solchen, die bei jedem Sprecher auftreten können, und solchen, die auf sprechereigenheiten zurückzuführen sind. Eindeutige Beispiele sind auf der einen seite Fragen mit hohem vs. Fragen mit tiefem offset; jeder Sprecher kann beides äußern. Auf der anderen Seite stehen Stimmqualitäten wie behauchte Stimme oder creaky voice (Laryngalisierung), die zumindest im Deutschen sprecherspezifisch sind. Bei den intonatorischen Merkmalen, die zur Kennzeichnung des Akzents eingesetzt werden, ist noch nicht klar, was obligatorisch, was fakultativ ist, oder anders gesagt, was kennzeichnend für die untersuchte Varietät (in unserem Fall die süddeutsche/bairische Standardsprache) und was kennzeichnend für einen Idiolekt ist. In vielen Fällen dürften sich Varietät und Idiolekt nicht klar voneinander trennen lassen, sondern nur in dem Sinn, daß einzelne Sprecher gewisse Formen häufiger oder systematischer gebrauchen als andere sprecher.

\subsection{Unterschiedliche Gewinnung der Prototypen}

In einem ersten Schritt sollen die für die Fokussierung relevanten intonatorischen Parameter und ihr Stellenwert ermittelt werden (Teil 3 und 4). Die Diskriminanzanalyse hat dabei den Vorteil, daß sie exakte (wenn auch nicht immer leicht $\mathrm{zu}$ interpretierende) Maße liefert; sie hat den praktischen Nachteil, daß eine Vielzahl von solchen Maßen zwar exakt, aber doch recht unanschaulich ist. Im zweiten Schritt wird deshalb versucht, über die häufigen, repräsentativen Fälle (gewonnen über eine Mittelwertsbildung) Prototypen als Kerntypen darzustellen (Teil 7). Dabei arbeiten wir interpretativ anhand von anschaulichen Abbildungen, aber immer gestützt auf die exakten Maßzahlen des statistischen Verfahrens. Kontrastiv und ergänzend dazu sollen sprecherübergreifende und sprecherspezifische Merkmalausprägungen ermittelt werden (Teil 5). Dazu dient auch im dritten Schritt die Gewinnung von Prototypen über Hörerurteile (Teil 8) sowie der Vergleich der im zweiten (Kerntypen) und im dritten (Kerntypen und Randtypen) Schritt gewonnenen Prototypen.

\section{Material}

Tab. 1 zeigt die verwendeten Testsätze sowie die indizierten Satzmodi. Da wir uns in diesem Beitrag nur mit der Position des FA beschäptigen, wie sie von Versuchspersonen bestimmt wurde, gehen wir nicht weiter auf die Konstruktion 
der Kontextsätze und Testsätze und auf die intendierten unterschiedlichen Fokusstrukturen wie einfacher Fokus, Fokusprojektion, und geteilter Fokus ein; (vgl. dazu Oppenrieder 1989 sowie die Korpusbeschreibung in Batliner/Oppenrieder 1989).

Tab.1: Testsătze und Satzmodi:

$\begin{array}{llllll} & \text { Matrixsatz } & \text { 1.Phrase } & \text { 2. Phrase } & \text { 3. Phrase } & \\ \text { 1. } & \text { Sie IäBt } & \text { die Nina } & \text { das Leinen } & \text { weben } & \text { ?/, } \\ \text { 2. Lassen Sie } & \text { den Manni } & \text { die Bohnen } & \text { schneiden } & \text { ?/! } \\ \text { 3. } & \text { Lassen wir } & \text { den Leo } & \text { die Blumen } & \text { düngen } & \text { ?/! }\end{array}$

\section{Fragesätze}

1. Assertiver Tragesatz

2. V-1-Fragesatz

3. V-1-Fragesatz

\subsection{Produktionsexperimente}

\section{Nicht-Fragesätze}

$\begin{array}{ll}\text { vs. } & \text { Aussagesatz } \\ \text { vs. } & \text { Imperativsatz } \\ \text { vs. } & \text { Adhortativsatz }\end{array}$

Sprecher waren StudentInnen der Germanistischen Linguistik in den Anfangssemestern ohne eine genaue Kenntnis des zugrundegelegten Satzmodussystems; sie wurden für ihre Teilnahme bezahlt. Sechs Sprecher (Sp1, Sp2 und Sp3 weiblich, Sp4, Sp5 und Sp6 männlich) produzierten die Testsätze im schallarmen Raum des Instituts für Phonetik in München jeweils zwei- bis viermal; irısgesamt erhielten wir 360 Realisationen, pro Sprecher also ca. 60 (vgl. Batliner/Oppenrieder 1989). Von jeder Außerung wurde ein Mingogramm mit Fo-Verlauf, Intensitätsverlaup und Zeitsignal erstellt. Da später alle Außerungen zusätzlich in einer digitalisierten Kopie vorlagen, konnten die daran ermittelten Intensitätswerte sowie die Gesamtsteigung der Außerungen (all point regression line) mit in die endgültige Auswertung eingehen. An den Mingogrammen wurden neben den üblichen Parameterwerten (vgl. Batliner/Oppenrieder 1989) zusätzliche speziell für dieses Korpus extrahiert. Eine Beschreibung der extrahierten Parameterwerte findet sich in Batliner/Oppenrieder (1989); vgl. auch Tab.2. Hier möchten wir deshalb nur auf zwei Punkte eingehen:

1. Als zu beschreibende Einheiten wurden die Phrasen 1 bis 3 , vgl. Tab.1, festgesetzt, nicht die Nukleussilben innerhalb dieser Phrasen. Damit lie $\beta$ sich das Segmentierungsproblem z.B. beim Vokal-Nasal-Vokal-ubergang bei die Nina oder das Leinen umgehen. Dieses Vorgehen schien gerechtfertigt, da die segmentale Struktur der drei Testsätze sehr ähnlich ist. Die 1. Phrase wird im weiteren nicht mehr erwähnt; sie wurde $z$ war von 
einigen Hörern ab und an auch als akzentuiert bezeichnet, aber doch so selten, daß eine statistische Analyse darüber wenig sinnvoll wäre.

2. Beim Messen wurde zwar auf phonetisches, nicht aber auf phonologisches Wissen rekurriert; mikroprosodische Kontexteffekte (starker Fo-Abfall nach stimmlosem Plosiv etc.) wurden - als für die Perzeption irrelevant - nicht mitgemessen. Es wurden aber nicht phonologische Sequenzen hypostasiert (etwa 'Hochton, gefolgt von Tiefton') und dann die phonetische auf diese phonologische struktur abgebildet, sondern es wurde quasi-automatisch gemessen. (Man kann sich einen automatischen Algorithmus vorstellen, der starke Fo-Veränderungen nach stimmlosen Konsonanten unberücksichtigt läßt. Eine automatische Extraktion phonologischer Sequenzen scheint uns nicht möglich zu sein - zumindest kennen wir keine.) Im Zweifelsfall entschieden wir uns dafür, Bewegungen zu berücksichtigen, auch wenn unklar ist, ob sie perzeptiv auffällig sind. So zeigt sich oft bei der unbetonten Endsilbe der Verben und grundsätzlich steigendem Fo-Verlauf ein mehr oder weniger ausgeprägter Fo-Abfall am Ende. Da kein Kriterium gefunden werden konnte, wie man einen perzeptiv irrelevanten Fo-Abfall (relaxation gesture) von einem deutlich intendierten, perzeptiv auffälligen trennen kann, wurde er immer mitberücksichtigt (s. auch Teil 7).

Von den gemessenen Variablen sind in unserem Zusammenhang die folgenden relevant:

Gesamtäußerung: Die Höhe des Grundfrequenzwertes am Außerungsende (Fooffset), gemessen in $\mathrm{Hz}$; die Fo-Steigung als Regressionskoeffizient für alle ermittelten Fo-Werte; die Dauer in Centisekunden.

2. und 3. Phrase: Fo-Maximum und Fo-Minimum in $\mathrm{Hz}^{5}$; die Positionen von Phrasenanfang, Phrasenende (und damit die Phrasendauer), Fo-Maximum und FoMinimum auf der Zeitachse in Centisekunden; Intensitätsmaximum und Intensitätsmittelwert. (Da nicht absolute Intensitätswerte, sondern die Energieunterschiede zwischen verschiedenen Bereichen des Sprachsignals interessierten, wurden die berechneten Millibelwerte linear in der Weise transformiert, daß sie als 16 Bit-Werte speichergünstig darstellbar waren; zur genauen Berechriung vgl. Nöth 1989. Die Werte lagen für drei Bänder vor: $0-5000 \mathrm{~Hz}, 100-3400 \mathrm{~Hz}$ und $300-2300 \mathrm{~Hz}$.)

\section{$2.2 \quad$ Hörtests}

Es wurden vier Hörtests durchgeführt, an denen jeweils durchschnittlich 12 Hörer teilnahmen. Versuchspersonen waren Studentinnen der Phonetik oder Germanistischen Linguistik, die für ihre Teilnahme bezahlt wurden. In einem Natür-

5 Die Entscheidung Pür die Messung prominenter Punkte (Maxima und Minima) ist primär auf praktische Gründe zurückzuführen: Sie können relativ genau bestimmt werden, wogegen Fo-Verläufe und damit zusammenhängende Größen wie die Steigung Schwierigkeiten bereiten. Zur Frage der perzeptiven Relevanz vgl. auch Batliner (1989b). 
lichkeitstest wurden die Außerungen im Kontext (vgl. Batliner/Oppenrieder 1989) vorgegeben; die Hörer mußten auf einer Ratingskala von 1 bis 5 ( 1 für 'paßt sehr gut zusammen' und 5 für 'paßt überhaupt nicht zusammen') angeben, wie gut der Testsatz in den Kontext paßt. Als 'Natürlichkeitsmaß' erhalten wir den Mittelwert aus diesen Beurteilungen. In einem Kategorisierungstest wurden die Außerungen ohne Kontext dargeboten; die Versuchspersonen mußten den $\AA u ß e-$ rungstyp (Frage, Aussage, Exklamativ, Imperativ oder Wunsch) bestimmen. Wenn etwa bei einem Fragesatz von 14 Hörern 12 auf Frage und 2 auf Aussage entschieden, so ist die Trefferquote für diese Außerung $12 /(12-2)$, also 0.86 ; im einzelnen vgl. Oppenrieder (1988a).

Im 1. Akzenttest wurden die Außerungen wie beim Kategorisierungstest zusammen mit anderen Außerungen (vgl. Batliner/Oppenrieder 1989) ohne Kontext dargeboten. Die Hörer mußten sich für eine Silbe als die am stärksten betonte entscheiden. Da in einigen wenigen Fällen die Hörer den Hauptakzent auf dem Matrixsatz, aber nicht, wie intendiert, auf der 2. oder 3. Phrase hörten, wurde zusätzlich ein 2. Akzenttest durchgeführt, der nur die uns interessierenden 360 Außerungen enthielt. Aup den 1. Akzenttest werden wir nur in Teil 9.2 bei der Fehleranalyse eingehen. Der 2. Akzenttest wurde hauptsächlich in Hinblick auf andere als hier interessierende Fragestellungen durchgeführt (phonetisches Korrelat der Fokusprojektion etc; vgl Oppenrieder 1989). In unserem Zusammenhang lieferte er die Kenngröße für die Stärke des Akzents auf der 2 . und der 3. Phrase und damit für die Zuweisung des FA.

Die Versuchspersonen durften den Matrixsatz nicht als akzentuiert bewerten und mußten für die Phrasen 1 bis 3 zwei Urteile vergeben: Hauptakzent und zweitstärkster (Neben-)Akzent. Wenn also bei einer Außerung von 12 Hörern einer den Hauptakzent auf der 1. Phrase, acht auf der 2. und drei auf der 3 . hörten, so wurde daraus für die uns interessierende Beziehung der 2. zur 3. Phrase ein Kennwert gebildet; in unserem Beispiel wäre er: $(8-3) /(1+8+3)$. Die Zahl der Akzentzuweisungen auf die 3. Phrase wird also von der Zahl der Zuweisungen auf die 2. Phrase abgezogen, und das Ergebnis durch die Anzahl der Versuchspersonen dividiert. Der wert liegt zwischen +1.0 und -1.0 , er ist positiv, wenn der Hauptakzent auf der zweiten, und negativ, wenn er auf der dritten Phrase gehört wurde. In acht Fällen wurden 2. und 3. Phrase als gleich stark akzentuiert bewertet, der Kennwert war also '0.0'. Diese Fälle werden in der Auswertung nicht berücksichtigt. ${ }^{6}$

$6 \quad$ Fälle mit fehlender Klassenzugehörigkeit können von der Diskriminanzanalyse in der Lernphase nicht berücksichtigt werden, sie werden aber klassifiziert. Wie erwartet, wurden die acht Fälle je nach Konstellation und Prädiktorvariablen mal der einen und mal der anderen Gruppe zugeschlagen, mit 
Es herrscht bei weitem keine Einigkeit darüber, welche der intonatorischen Merkmale Fo, Dauer und Intensität in welcher Form (Umrechnung) die relevanten für die Akzentmarkierung sind. Wenn man nun nicht wle im Perzeptionsexperiment mit manipuliertem Material nur einen Parameter variiert und die anderen konstant läßt, also etwa $\mathrm{Hz}$ - und Halbton- ( $\mathrm{Ht}-)$ Unterschiede beurteilen läßt, so steigt der Aufwand durch die Kombinationsmöglichkeiten exponentiell an und ist nicht mehr zu bewältigen. Man muß sich daher i.a. auf ein heuristisches Verfahren beschränken, das auf plausiblen Annahmen beruht. Eine ausführliche Darstellung der von uns vorgenommenen Berechnungen würde über den Rahmen dieses Beitrags hinausgehen. Wir wollen uns deshalb auf einen ưberblick beschränken und dabei die wichtigsten Teilergebnisse anführen.

\subsection{Fo-Werte}

Wenn man Sprecher mit unterschiedlichen Stimmlagen (Frauen und Männer) analysieren möchte, so ist der $\mathrm{Hz}$-Rohwert keine optimale Skalierung. (Das mag bei großen Stichproben weniger auffällig sein, ist aber sehr relevant, wenn z.B. von einem einzigen sprecher auf andere geschlossen wird.) Eine 'gehörsadäquate' Transformation in $\mathrm{Ht}$-Werte nach der Formel $H t=17.31^{\star} L N(H z)$ zur Normierung des Stimmumfangs verbesserte die Prädiktionsgüte nicht entscheidend (vgl. dazu auch Batliner et al. 1989). Entscheidend ist eine Normierung der Stimmlage durch einen Bezug zu einem Wert, der entweder für den Sprecher oder für die Außerung 'repräsentativ' ist. Dieser Wert kann der sprecherspezifische Basiswert sein (der tiefste vom jeweiligen Sprecher erreichbare Wert) oder der Außerungsmittelwert (den man sogar grob aus wenigen Fo-Werten berechnen kann, vgl. Nöth et al. 1987, Batliner 1988a).

Die Unterschiede in der Klassifikationsgüte $\mathrm{zwischen}$ normierten $\mathrm{Hz}$ - und Ht-Werten bzw. zwischen Normierung zum Basis- oder zum Mittelwert waren nicht auffällig. Eine Entscheidung zwischen diesen Transformationen kann also fürs erste aus praktischen Gesichtspunkten erfolgen. Ein automatisches Verfahren wird dann, wenn es sprecherunabhängig arbeiten soll, natürlich den Außerungsmittelwert nehmen. Wir entschieden uns dafür, im weiteren mit der HtTransformation zum Basiswert zu arbeiten, da sie im Schnitt etwas bessere Ergebnisse lieferte bzw. bei anderen, aber ähnlichen Konstellationen geliefert hat,

einer Tendenz zur Gleichverteilung. Es handelt sich also um Grenzfälle; vgl. dazu die Fehleranalyse in Teil 9.2. 
vgl. Batliner (1988). Diese Transformation ist in der phonetischen Literatur auch üblich und deshalb eher vergleichbar.

\subsection{Deklination}

Es ist bekannt, daß die zeitliche Positionierung der akzentuierten Phrase in der Außerung eine Rolle spielt: Am Ende einer Außerung genügt eine geringere Ausprägung der Parameterwerte als zu Beginn, um den Fokus zu markieren (vgl. auch Batliner 1989a). Für die Berechnung der Dekliriationsiinle als Bezugsgröße gibt es kein anerkanntes Standardverfahren (vgl. dazu die Kontroverse zwischen Lieberman et al. 1985, 't Hart 1986, Lieberman 1986). Problematisch ist weiter, daß Untersuchungen zur Deklination normalerweise an relativ konsistenten Korpora mit Aussagesätzen durchgeführt wurden. Es ist unklar, welche Bezugswerte man für einen Fragesatz annehmen soll: den aktuellen Deklinationsverlauf der Außerung oder einen 'unmarkierten' Referenzverlauf? Man kann natürlich versuchen, die Fo-Werte zu einer Deklinationslinie in Beziehung zu setzen, um damit, ähnlich wie im nächsten Abschnitt bei den Verhältniswerten, die Perzeption des Hörers nachzubilden. Diese Deklinationslinie kann eine abstrakte Referenzlinie sein oder aus den jeweils konkret gegebenen Fo-Werten der Außerung berechnet werden. Solche Werte führten bei uns $z u$ inkonsistenten und insgesamt schlechteren Ergebnissen. Damit ist nicht nachgewiesen, daß die Deklination keine Rolle spielt - es mag einfach daran liegen, daß wir nicht die richtige Deklinationslinie als Bezugsgröße ansetzten. (Wir errechneten eine abstrakte ('neutrale') Deklinationslinie pro sprecher aus den Nicht-Fragesätzen mit 'unmarkierter' Deklination bzw. eine konkrete pro Außerung als eine all point regression line.)

Grundsätzlich ist u.E. die Deklination immer noch eher ein Untersuchungsgegenstand an sich als eine sinnvolle, normierte Bezugsgröße. Die Auswirkung der Deklination - da $\beta$ die intonatorischen Merkmale späterer Akzente weniger stark ausgeprägt sein müssen als früherer, um den Fokus zu markieren - geht sowieso in das Klassifikationsverfahren ein, da die Diskriminanzanalyse ja genau diese Verhältnisse in der Analysephase bei der Lernstichprobe vorfindet und sich 'merkt': Das Verfahren betrachtet keine absoluten Größen, sondern Verteilungen (vgl. weiter unten Fig.2).

Aus all diesen Gründen wurden die Fo-Werte nicht zu einer Deklinationslinie in Beziehung gesetzt. 
Die Positionen von Fo-Maximum und Fo-Minimum wurden nicht transformiert. Bei der Dauer der 2. und 3. Phrase brachte die folgende Divisions- und Multiplikations-Umrechnung immer bessere Ergebnisse als die Dauer-Rohwerte oder eine additive Umrechnung:

Dauer der Phrase relativ zur mittleren Dauer der Phrase pro Sprecher und zur mittleren Silbendauer der cesamtäußerung; Formel:

$\frac{\text { Dauer der Phrase }}{\text { mittl. Phrasendauer }} \star \frac{\text { Dauer der Phrase }}{\text { Außenungsdauer / Silbenzahl }}$

Dieses Ergebnis war zu erwarten, da Länge und Dauer als Maß nur relativ zur unmittelbaren Umgebung bzw. zur globalen Umgebung (Sprechtempo) sinnvoll sind. Die ebenfalls relevante intrinsische Dauer besonders der Vokale wurde nicht berücksichtigt; das schien bei unserem konsistenten Material vertretbar.

\subsection{Intensitätswerte}

Die Maxima waren grundsätzlich bessere Prädiktoren als die Mittelwerte. Von uns vorgenommene Transformationen (zum Mittelwert wie bei den $\mathrm{Hz}$-Werten, vgl. oben, oder zur intrinsischen Lautstärke anhand eines Bezugskorpus) ergaben keine systematische Verbesserung. Wir entschieden uns deshalb dafür, die Intensitätsmaxima im Bereich von 0 bis $5000 \mathrm{~Hz}$ für die endgültige Analyse zu übernehmen, die im Schnitt etwas bessere Ergebnisse brachten als die beiden anderen $\mathrm{Hz}-\mathrm{Bänder}$.

Man beachte, daß die Intensitätswerte für die ganzen Phrasen ermittelt wurden, nicht für die betonten Silben. Es gehen also auch die Intensitäten der Frikative und Plosive in die Berechnung der Intensität ein, nicht nur die der stimmhaften Passagen. Bestimmte Umrechnungen, die z.B. Beckman (1986) vorschlägt, konnten deshalb nicht durchgeführt werden. Die Bezugswerte zur intrinsischen Lautstärke der Vokale stammen von anderen Sprechern; man weiß, daß diese Werte von Sprecher zu Sprecher differieren können. Aus diesen Gründen spiegelt sich in der Unmöglichkeit, die Intensitätswerte durch Transformationen aufzuwerten, sowie in der relativ geringen Relevanz der Intensitätswerte für die Klassifikation (vgl. Teil 4) zwar die allgemeine Ansicht über die geringe Bedeutung der Intensität bei der Markierung des Akzents wider, ein Nachweis dieser geringen Bedeutung ist damit aber nicht gegeben. (Vgl. auch die Diskus- 
sion zur unterschiedlichen Rolle der Intensität bei den einzelnen Sprechern in Teil 5.)

\subsection{Verhältniswerte}

Der Hörer beurteilt natürlich bei der Akzentzuweisung die Parameterwerte nicht absolut, sondern jeweils in Relation zur Umgebung. Wir können also auch in unserer Analyse nicht die absoluten Werte der 2. und der 3. Phrase zugrundelegen, sondern z.B. nur die Differenz der Werte. Solche Verhältniswerte haben den rechentechnischen Vorteil, daß sie die Zahl der Prädiktorvariablen um die Hälfte reduzieren; gleichzeitig erhalten Extremwerte ein geringeres Gewicht, die Verteilung wird regulärer und nähert sich schneller der Verteilung der Population an. Auf der anderen Seite geht Information verloren. Grundsätzlich bleibt zu untersuchen, ob und in welcher Form solche Verhältniswerte psychophysisch adäquater sind als die Rohwerte.

Taylor/Wales (1987) haben für das australische Englisch vergleichbare Untersuchungen durchgeführt. Sie haben allerdings nur mit Verhältniswerten gearbeitet, ohne auf die Frage einzugehen, ob Rohwerte nicht doch genausogut oder besser geeignet sind. Aus den folgenden Gründen ist ihre Untersuchung nicht direkt mit der unseren vergleichbar: Ihr Material stammt von nur einer Sprecherin; das Problem der Normierung der unterschiedlichen Sprechlagen ergab sich also bei ihnen nicht. Sie gaben die Akzentposition explizit vor. Ihr Material variiert etwas mehr als unseres. Bei der Akzentbewertung gaben sie eine Ratingskala von 1 bis 5 vor, keine einfache 'betont-unbetont'-Entscheidung. Die von ihnen extrahierten Parameter sind mit unseren zwar nicht immer identisch, aber durchaus vergleichbar; Fo-Werte wurden bei ihnen nicht transformiert. Bei der Bildung der Verhältniswerte setzten sie die akzentuierte mit der unakzentuierten Phrase in Bezlehung; drei Werte wurden gebildet (Taylor/Wales 1987:239):

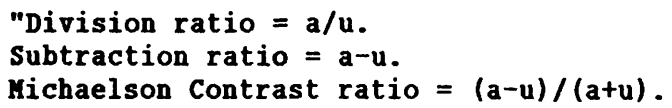

$$
\text { ( } \mathbf{a}=\text { accented, } u \text { = unaccented }) "
$$

Die Autoren erzielen mit dem Kontrast-Verhältnis (Contrast-ratio) bei den Regressionsanalysen deutlich bessere Ergebnisse als mit den beiden anderen: Durchschnittlich ist $\mathrm{R}^{2}$ als das $\mathrm{Ma \beta}$ der 'erklärten Varianz' bei Subtraktion und Division .29 bzw. .15, beim Kontrast-Verhältnis .85 . Sie folgern deshalb:

"Although contrast ratios do not appear to have been used in prosody perception, lour results show] contrast-based features to be consistently 
superior in predicting accent rating than any of the other types of combinations of ratios investigated." (Taylor/Wales 1987:243).

Wir haben für die von uns extrahierten Rohwerte und die daraus ermittelten transformierten Werte Regressionsanalysen analog $\mathrm{zu}$ Taylor/Wales (1987) sowie Diskriminanzanalysen durchgeführt. Allerdings wurde dabei nicht die akzentuierte Phrase zur unakzentuierten in Bezug gesetzt, sondern immer die zweite zur dritten. Dadurch ändern sich die Verteilungen der Werte, aber natürlich nicht die grundsätzliche Beziehung. Das kann also nicht erklären, warum wir nie einen so ausgeprägten Unterschied zugunsten des Kontrast-Verhältnisses gefunden haben. Beim Vergleich der Verhältniswerte mit den Rohwerten waren die Verhältniswerte bei kleinen Lernstichproben etwas besser als die Rohwerte, bei großen Lernstichproben war es umgekehrt; dafür dürften die o.a. rechentechnischen Unterschiede verantwortlich sein.

Man muß bedauern, daß Taylor/Wales (1987) die Rohwerte überhaupt nicht berücksichtigt haben. Bei einem Vergleich der Verhältniswerte untereinander ergaben sich für unsere Daten keine so großen Differenzen, die Subtraktion war im Schnitt etwas besser als das Kontrast-Verhältnis, und beide besser als die Division. Dies gilt für alle Werte, untransformierte und transformierte, also auch für die Verhältniswerte der Hz-Ausgangswerte. Es kann daher nicht daran liegen, daß durch Transformation und nachfolgende Verhältnisbildung eine 'Ubertransformation' stattfand.

Dieses Ergebnis bedeutet für unser weiteres Vorgehen, daß wir keine Verhältniswerte, sondern die Werte der 2. und der 3. Phrase als Prädiktoren annehmen. Für den großen Unterschied zwischen den Ergebnissen von Taylor/Wales (1987) und unseren können wir keine plausible Erklärung finden. Natürlich sind das australische Englisch und das Deutsche sowie das experimentelle Design nicht direkt miteinander vergleichbar; es ist aber unwahrscheinlich, daß sich die Psychophysik der Sprecher des australischen Englisch so sehr von der deutscher Sprecher unterscheidet. Unsere Ergebnisse lassen also die Schlußfrage von Taylor/Wales (1987:245), "[...] whether a neural analogue can be found for contrastbased acoustic-features detectors" in einem etwas dunkleren Licht erscheinen.

\section{Klassifikationsphase}

Tab.2 zeigt alle bei der endgültigen Analyse verwendeten Variablen, ihre Kurzbezeichnungen, sowie die an ihnen vorgenommenen Transformationen. In der ersten Spalte stehen die Prädiktorvariablen, die Werte aus den Hörtests sowle die zu prädizierenden Klassen. In der zweiten spalte folgen die im weiteren 
verwendeten Kurzbezeichnungen, in der dritten ist angegeben, ob die Variable für die Moduszuweisung (M) und/oder die FA-Zuweisung (F) verwendet wurde. In der letzten Spalte ist die Art der Berechnung und der Transformation angegeben.

Tab.2: Variablen der Klassifikationsphase

\begin{tabular}{|c|c|c|c|}
\hline VARIABLE & KURZB. & $\mathbf{M} / \mathbf{F}$ & BERECHNUNGEN/TRANSFORMATIONEN \\
\hline \multicolumn{4}{|c|}{ Prädiktorvariablen: } \\
\hline Steigung & $s t g$ & $\mathbb{M}$ & Ht-Werte $(17.31 * \operatorname{LN}(\mathrm{Hz}))$ \\
\hline Offset & off & $\mathbf{M}$ & $\begin{array}{l}\text { Ht-Werte, transformiert zum } \\
\text { sprecherspezifischen Basiswert }\end{array}$ \\
\hline $\begin{array}{l}\text { Fo-Maximum, } 2 \text {. } \\
\text { und } 3 \text {. Phrase } \\
\text { Fo-Minimum, } 2 \text {. } \\
\text { und } 3 . \text { Phrase }\end{array}$ & $\begin{array}{l}\operatorname{Max2} \\
\operatorname{Max} 3 \\
\operatorname{Min} 2 \\
\operatorname{Min} 3\end{array}$ & $\begin{array}{l}M / F \\
M / F \\
M / F \\
M / F\end{array}$ & $\begin{array}{l}\text { Ht-Werte, transformiert zum sprecher- } \\
\text { spezifischen Basiswert in Halbtönen }\end{array}$ \\
\hline $\begin{array}{l}\text { Rel. Position } \\
\text { von Fo-Max./Min. } \\
\text { (2.u.3.Phrase) }\end{array}$ & $\begin{array}{l}\text { Pos2 } \\
\text { Pos } 3\end{array}$ & $\mathbf{M} / \mathbf{F}$ & $\begin{array}{l}\text { Differenz der Werte von Fo-Maximum } \\
\text { und Fo-Minimum auf der Zeitachse; } \\
\text { positiver Wert, wenn Maximum vor } \\
\text { Minimum, sonst negativer Wert. }\end{array}$ \\
\hline $\begin{array}{l}\text { Dauer der } 2 . \\
\text { und } 3 \text {. Phrase }\end{array}$ & $\begin{array}{l}\text { Dau2 } \\
\text { Dau3 }\end{array}$ & $\begin{array}{l}\mathbf{F} \\
\mathbf{F}\end{array}$ & $\begin{array}{l}\text { Dauer/mittl. Phrasendauer * } \\
\text { Dauer / (Außerungsdauer/Silbenzahl) }\end{array}$ \\
\hline $\begin{array}{l}\text { Intensität ( } 2 \text {. } \\
\text { und } 3 \text {. Phrase) }\end{array}$ & $\begin{array}{l}\text { Int2 } \\
\text { Int } 3\end{array}$ & $\begin{array}{l}F \\
F\end{array}$ & $\begin{array}{l}\text { relative Millibelwerte } \\
\text { im Bereich } 0-5000 \mathrm{~Hz}\end{array}$ \\
\hline
\end{tabular}

Hörerurteile:

\begin{tabular}{lll}
\hline Moduszuweisung & MOD & $\begin{array}{l}\text { Prozent Fragekategorisierung, errech- } \\
\text { net aus den Kategorisierungstest }\end{array}$ \\
FA-Zuweisung & FOK & $\begin{array}{l}\text { errechnet aus dem } 2 \text {. Akzenttest } \\
\text { (Werte zwischen }+1.0 \text { und }-1.0 \text { ) }\end{array}$ \\
\hline
\end{tabular}

Zu prädizierende Klassen:

\begin{tabular}{lll}
\hline Satzmodus & MODUS & aus MOD errechnete Dichotomisierung: \\
& FRAGE/NICHT-FRAGE \\
Satzfokus & FOKUS & aus FOR errechnete Dichotomisierung: \\
& & 2. $/$ 3. Phrase fokussiert
\end{tabular}

Off und Stg werden nur für die Moduszuweisung, Dau2, Dau3, Int2 und Int3 nur Pür die FA-Zuweisung analysiert; alle anderen Variablen lagen sowohl der Modus- als auch der FA-Zuweisung zugrunde. Die Dauer- und die Intensitäts- 
werte ergaben bei MODUS eine sehr schlechte Prädiktion. Bei FOKUS ist es intuitiv nicht einsehbar, was z.B. ein hoher offset mit der FA-Zuweisung zu tun haben soll - es sei denn, er liegt in der Nähe des Fo-Maximums aup dieser Silbe oder ist damit identisch. Eine Reduzlerung der Prädiktorvariablen ist auch aus rechentechnischen Gründen erwünscht.

Tab.3 zeigt die Korrelation der Prädiktorvariablen für das gesamte Korpus, Tab.4 getrennt nach Fragen (untere Dreiecksmatrix) und Nicht-Fragen (obere Dreiecksmatrix). Zur einfacheren Darstellung sind die Korrelationskoeffizienten mit 100 multipliziert. Wie erwartet, sind die Werte z.T. hoch miteinander korreliert. Das kann triviale Gründe haben, etwa wenn off mit Max3 korreliert: Z.T. sind die Werte identisch, zum Teil interdependent. Dauer- und Intensitätswerte sind untereinander sowie mit den $\mathrm{Ht}$-Werten weniger stark korreliert als die $\mathrm{Ht}$ Werte untereinander. Aus Tab.4 ist ersichtlich, daß die Korrelationen bei Fragen und bei Nicht-Fragen doch sehr unterschiedlich ausfallen - ein Hinweis darauf, daß die Parameter bei Fragen und bei Nicht-Fragen unterschiedlich eingesetzt werden. Wir werden deshalb im folgenden die Prädiktion von FOKUS auf drei verschiedene Art und Weisen berechnen:
(a) Fragen und Nicht-Fragen zusammen:
(b) nur Fragen:
FOKUS
(c) nur Nicht-Fragen:
FOKUS-F
FOKUS-NF.

Tab.3: Korrelation ( $* 100)$ der Prädiktorvariablen untereinander.

\begin{tabular}{|c|c|c|c|c|c|c|c|c|c|c|c|c|}
\hline & off & Stg & $\operatorname{Max} 2$ & $\operatorname{Max} 3$ & Min2 & $\operatorname{Min} 3$ & Pas2 & Pos 3 & Dau2 & Dau3 & Int2 & Int3 \\
\hline Off & - & & & & & & & & & & & \\
\hline Stg & 68 & - & & & & & & & & & & \\
\hline $\operatorname{Max} 2$ & 39 & 44 & - & & & & & & & & & \\
\hline Max3 & 91 & 75 & 42 & - & & & & & & & & \\
\hline $\operatorname{Min} 2$ & 34 & 37 & 30 & 40 & - & & & & & & & \\
\hline $\operatorname{Min} 3$ & 70 & 80 & 41 & 67 & 38 & - & & & & & & \\
\hline Pos2 & -44 & -66 & -24 & -50 & -30 & -73 & - & & & & & \\
\hline Pos 3 & -74 & -44 & -15 & -58 & -14 & -43 & 25 & - & & & & \\
\hline Dau2 & -11 & 05 & 10 & -13 & -25 & 14 & -22 & 09 & - & & & \\
\hline Dau3 & -17 & -21 & -06 & -03 & 06 & -37 & 29 & 15 & -38 & - & & \\
\hline Int2 & 09 & -04 & 09 & 06 & -07 & 03 & 03 & 08 & 05 & -03 & - & \\
\hline Int3 & 34 & 24 & 15 & 35 & 17 & 26 & -14 & -08 & -11 & 04 & 63 & - \\
\hline
\end{tabular}


Tab.4: Rorrelation ( $(100)$ der Prädiktorvariablen untereinander. Fragen $(F)$ : untere Dreiecksmatrix; Nicht-Fragen (NF): obere Dreiecksmatrix.

\begin{tabular}{l|rrrrrrrrrrrr} 
Furf & Off & Stg & Max2 & Max3 & Min2 & Min3 & Pas2 & Pas3 & Dau2 & Dau3 & Int2 & Int3 \\
\hline Off & - & 20 & 37 & 22 & 21 & 69 & -15 & -13 & 09 & 17 & -03 & -17 \\
Stg & 11 & - & 33 & 46 & 22 & 24 & -19 & -06 & -05 & 24 & -08 & 07 \\
Max2 & -06 & 09 & - & 26 & 31 & 32 & 02 & 13 & 10 & 06 & 10 & 06 \\
Max3 & 89 & 23 & -05 & - & 39 & 34 & -22 & 23 & -16 & 56 & -07 & 13 \\
Min2 & 17 & 25 & 09 & 19 & - & 31 & -26 & 10 & -14 & 23 & -14 & 07 \\
Min3 & 29 & 73 & 15 & 25 & 26 & - & -18 & 16 & 03 & 24 & 18 & 09 \\
Pas2 & 06 & -62 & -04 & -03 & -12 & -71 & - & 08 & -17 & -06 & -07 & -07 \\
Pas3 & -84 & 00 & 13 & -65 & -01 & -17 & -17 & - & -00 & 11 & 37 & 37 \\
Dau2 & -11 & 34 & 20 & -04 & -32 & 39 & -42 & 10 & - & -42 & 07 & -17 \\
Dau3 & -04 & -36 & -00 & -00 & 02 & -57 & 44 & -05 & -41 & - & -04 & 21 \\
Int2 & 22 & -12 & 08 & 19 & -02 & -02 & 14 & 14 & 03 & 00 & - & 65 \\
Int3 & 45 & 11 & 04 & 45 & 15 & 17 & 03 & -24 & -01 & -05 & 63 & -
\end{tabular}

Fig.1 zelgt die Verteilung der Variablen MOD und FOK, also der Hörerurteile bei der Modus- und FA-Zuweisung. Die Hörer waren sich offensichtlich bei der Moduszuweisung viel sicherer als bel der FA-Zuweisung. Man kann darüber spekulieren, ob die Unterscheidung Frage/Nicht-Frage kommunikativ wichtiger und damit vom Sprecher eindeutiger signalisiert und vom Hörer eindeutiger bestimmbar ist als die FA-Zuweisung. In unserem Korpus hat sicher auch eine Rolle gespielt, daß unser Material zu einem Drittel Kontexte enthielt, die Doppelfokus indizieren; damit mag die FA-Indizierung oft weniger eindeutig ausfallen als bei einfachem Fokus. Es ist auch bekannt, daß die Akzent- bzw. FAZuweisung grundsätzlich keine leichte Aufgabe ist (vgl. Thorsen 1987:87ff).

Die Variablen MOD und FOK erfüllen nicht unbedingt die Voraussetzungen für eine parametrische Statistik, etwa für eine Korrelation mit Pearsons $\mathbf{r}$ zwischen ihnen und den Prädiktorvariablen. Es wurde deshalb Goodmans und Kruskals Gamma-Koeffizient als Assoziationsmaß berechnet; in Tab.5 sind die Werte analog zu Tab.3 und 4 mit 100 multipllziert. ${ }^{7}$ Es zeigt sich, daß MOD stärker mit den Prädiktorvariablen assozilert ist als FOK, daher auch möglicherweise stärker bzw. eindeutiger die Gruppenzuweisung indiziert.

7 Die Berechnung des Gammakoeffizienten ist ähnlich der von Kendalls $\tau$, dessen Berechnung wiederum ähnlich der Berechnung des bekannteren nichtparametrischen Korrelationskoeffizienten $\mathbf{r}$ von Spearman. Die Gammaund $\tau$-Werte fallen i.a. kleiner aus als Spearmans $\mathbf{r}$, allen liegen aber die gleichen Ordnungsrelationen zugrunde. (Vgl. im einzelnen zur unterschiedlichen Berechnung Hildebrand et al. 1977 und Norusis 1986). 
Fig.1: Verteilung der Hörerurteile MOD (Koduszuweisung: Frage/Nichtfrage) und FOK (FAZureisung: 2./3. Phrase betont).
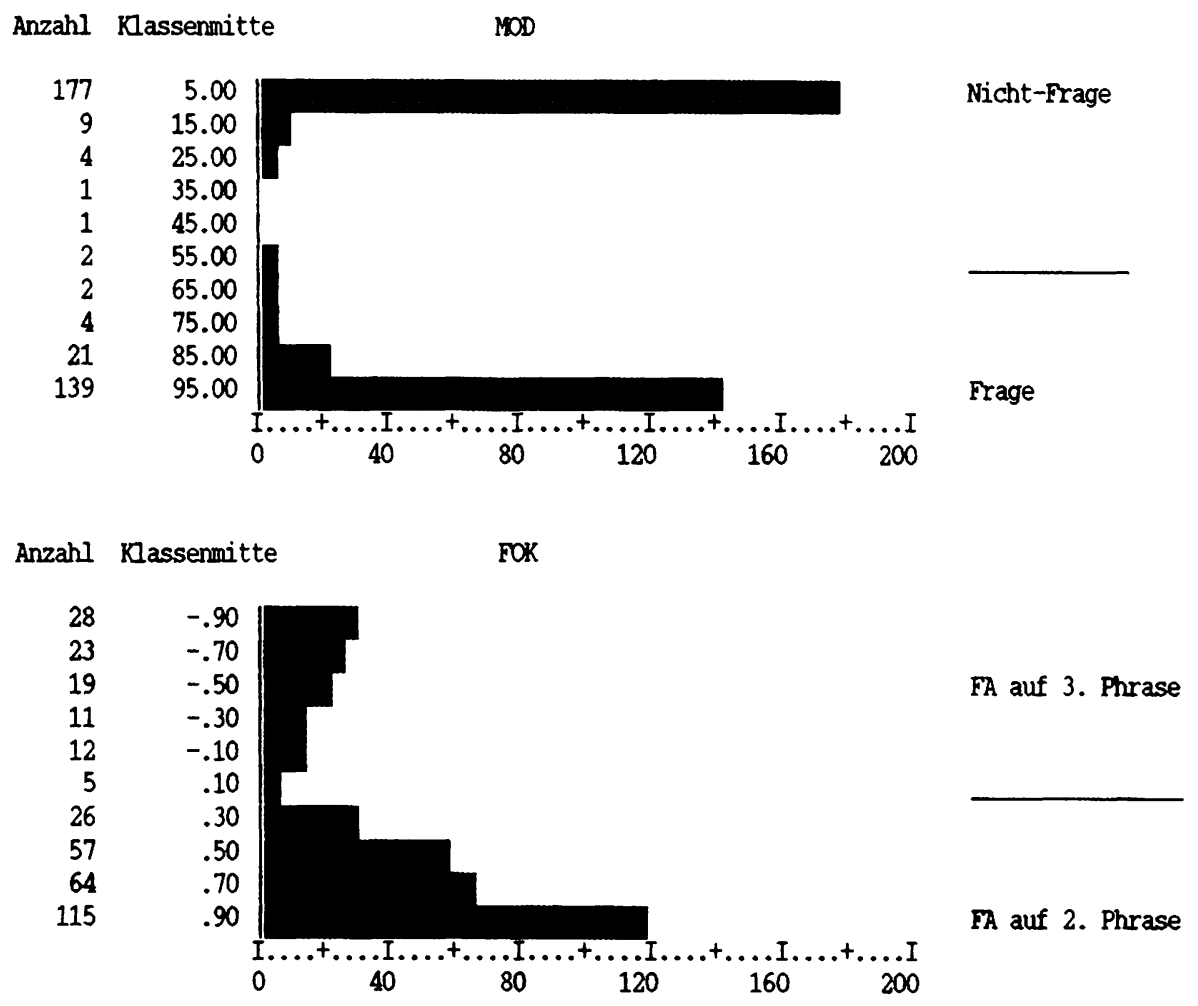

Tab.5: Assoziation $(* 100)$ der Prädiktorvariablen mit den Hörerurteilen MOD (Moduszurweisung) und FOK (FA-Zuweisung) aufgeteilt nach Fragen (FOK-F) und Nicht-Fragen (FOK-NF). Goodmans und Kruskals Garma-Koeffizient. 8

\begin{tabular}{l|rrr} 
& MOD & FOK-F & FOK - F F \\
\hline Off & 81 & & \\
Stg & $\star$ & & \\
Max2 & 39 & 36 & 14 \\
Max3 & 79 & -17 & -31 \\
Min2 & 33 & -04 & -20 \\
Min3 & 62 & 12 & 02 \\
Pos2 & -39 & -19 & 10 \\
Pas3 & -54 & 22 & -07 \\
Dau2 & & 19 & 33 \\
Dau3 & & -17 & -29 \\
Int2 & & 03 & 17 \\
Int3 & & -04 & -02
\end{tabular}

8 Die Assoziation mit Stg konnte aus rechentechnischen Gründen (mangelnder Speicherplatz) nicht ermittelt werden. 
Bei den im folgenden besprochenen Analysen, deren Ergebnisse in Tab. 6 bis 8 aufgelistet sind, gingen immer alle Fälle in die Lern- und in die Prüfstichprobe ein (vgl. unten die Konstellation (3) in Tab.9).9 Tab.6 zeigt die Ergebnisse der univariaten Analyse mit jeweils nur einer Variablen als Prädiktorvariable, der 'bivariaten' (paarweise werden Werte für die 2. und die 3. Phrase zugrundegelegt), sowie einen Vergleich mit der multivariaten Analyse. Wie man sieht, ermöglicht bei der univariaten Analyse Max3 bei MODUS und FOKUS-NF schon eine sehr gute Prädiktion; bei FOKUS-F ist die Verbesserung von unibzw. bivariater zur multivariaten Analyse am größten; die multivariate ist aber immer besser. (Dieses Ergebnis ist nicht trivial, da eine Hinzunahme von irrelevanten Prädiktorvariablen oft das Ergebnis eher verschlechtert als verbessert.)

Um den Beitrag der einzelnen Prädiktorvariablen abzuschätzen, kann man entweder die Diskriminanzfunktionskoeffizienten der Variablen oder die Korrelation zwischen Variable und Diskriminanzfunktion interpretieren; das letztere ist in unserem Fall vorzuziehen, da die Variablen z.T. miteinander korrelieren (vgl. dazu im einzelnen Klecka 1980:33f).

Tab.7 zeigt deshalb die Korrelation der Prädiktorvariablen mit der Diskriminanzfunktion; die Koeffizienten sind wieder mit 100 multipliziert. Da die Richtung der Vorzeichen pro Spalte arbiträr ist, werden hier wie auch im weiteren die Vorzeichen der Werte zur Vereinfachung immer so wiedergegeben, daß der Wert dann positiv ist, wenn ein hoher und/oder positiver Wert eine Frage bzw. einen FA auf der 2. Phrase indizieren, und vice versa ein niedriger und/oder negativer Wert eine Nichtfrage bzw. einen FA auf der 3. Phrase. Mit ' $\star^{\prime}$ gekennzeichnete Variablen passieren den Toleranztest nicht, da sie lineare Kombinationen aus anderen Variablen sind. Sie gehen deshalb nicht in die Diskriminanzfunktion ein. Verantwortlich dafür sind Korrelationen der Variablen untereinander; es ist damit aber nicht gesagt, daß gerade diese ausgeschiedenen Variablen perzeptiv irrelevant sind (zum Toleranztest vgl. Norusis 1986:B-18).

9 'Realistischer' wäre natürlich das 'leave-one-out'-Verfahren, bei dem reihum ein sprecher beim Lernen ausgelassen und dann allein klassifiziert wird. Die Werte in Tab.6 und 7 sind also alle etwas $z u$ optimistische Schätzungen (vgl. zu dieser Uberadaptation bei Identität von Lern- und Prüfstichprobe Ruske 1988 und Teil 9). Es ist aber unwahrscheinlich, daß die Relation der Werte zueinander sich stark ändern würde. Wir haben deshalb in dieser Phase davon abgesehen, Lern- und Prüfstichprobe zu trennen. 
Tab.6: Prozent richtige Klassifikation bei univariater Analyse, 'bivariater' (paarweiser) sowie multivariater Analyse: Frage/Nicht-Frage (MODUS), FA gesamt (FONUS), FA bei Frage (FOKUS-F) und FA bei Nicht-Frage (FOKUS-NF).

\begin{tabular}{|c|c|c|c|c|c|c|c|c|}
\hline & MOD & & FON & & Fơvus & & FOKNJ & $-\sqrt{F}$ \\
\hline & & & & & & & & \\
\hline oift & 93.0 & & - & & - & & - & \\
\hline Sig & 85.2 & & - & & - & & - & \\
\hline Mex2 & 72.8 & & 59.9 & & 79.6 & & 53.5 & \\
\hline Mex3 & 93.5 & 93.8 & 59.8 & & 62.9 & 80.8 & 88.4 & 91 \\
\hline Hin2 & 64.7 & 043 & 58.5 & ? & 52.7 & 712 & 61.6 & \\
\hline$M: n 3$ & 84.3 & 04.5 & 47.4 & 62.4 & 69.5 & 71.3 & 47.0 & 10. \\
\hline Ptas2 & 76.1 & & 51.1 & & 77.8 & & 69.2 & \\
\hline$P \cos 3$ & 77.5 & & 51.1 & 09.3 & 62.3 & 02.0 & 48.1 & \\
\hline Dau2 & - & & 66.1 & & 59.9 & & 69.6 & \\
\hline Dau3 & $\ldots$ & > & 71.8 & & 70.1 & & 81.5 & 81 \\
\hline Int2 & - & & 58.2 & & 52.1 & & 61.5 & \\
\hline Int3 & 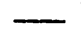 & & 54.7 & & 50.9 & & 53.3 & \\
\hline multiv. & & 97.1 & & 92.7 & & 94.6 & & 95.5 \\
\hline $\begin{array}{l}\text { Mindest- } \\
\text { verbes- } \\
\text { serung }\end{array}$ & 3.6 & 3.3 & 26.6 & 15.1 & 15.0 & 12.6 & 7.1 & 3.8 \\
\hline
\end{tabular}

Tab.7: Rorrelation ( $* 100)$ zwischen den Prädiktorvariablen und der Diskriminanzfunktion bei multivariater Analyse für Frage/Nicht-Frage (F/NF), FA gesant (FONUS), FA bei Frage (FOKUS-F) und FA bei Nicht-Frage (FOKUS-NF).

\begin{tabular}{l|cccc} 
& F/NF & FOKUS & FOKUS-F & FOKUS-NF \\
\hline Off & 82 & - & - & - \\
Stg & 55 & - & - & - \\
Max2 & 24 & 25 & 43 & 12 \\
Max3 & 78 & -28 & -24 & -61 \\
Min2 & 15 & -11 & -02 & $-15^{\star}$ \\
Min3 & 46 & 13 & $28^{\star}$ & $-\infty$ \\
Pos2 & -31 & -16 & -43 & $02^{\star}$ \\
Pos3 & -35 & 12 & $23 \star$ & $-02^{\star}$ \\
Dau2 & - & $31 \star$ & 25 & 32 \\
Dau3 & - & -37 & -26 & -41 \\
Int2 & - & 12 & 03 & 18 \\
Int3 & - & -10 & -06 & -08
\end{tabular}

* Variablen, die das Toleranzkriterium nicht passiert haben (lineare Kombinationen aus anderen Variablen). 
Die univariaten Analysen können mit solchen Perzeptionstests verglichen werden, bei denen jeweils nur ein Parameter manipuliert wird, die multivariaten mit natürlicher Sprache, bei der alle Variablen zur Modus- oder Fokusindizierung beitragen können. Genausowenig wie man direkt aus Perzeptionstests auf die Rolle des jeweiligen Parameters in der natürlichsprachlichen Kommunikation schließen kann, genausowenig ist die multivariate Analyse einfach eine Addition aus den univariaten Analysen. Wie schon erwähnt, bedeutet die Korrelation der Variablen untereinander, da $\beta$ man grundsätzlich etwas vorsichtig bei der Interpretation sein sollte. Um ein einfaches Beispiel zu nehmen: Die hohen Werte bei off und Max 3 bedeuten nicht, daß beide Variablen gleichermaßen sehr wichtig sind, da sie bei Fragen oft nahe beieinanderliegen und in manchen Fällen sogar identisch sind. Wenn man nun aber Tab.5, 6 und 7 miteinander vergleicht, also die Assoziation der Prädiktorvariablen mit den Hörerurteilen, die Klassifikationsgüte bei der univariaten Analyse sowie die Korrelation der Prädiktorvariablen mit der Diskriminanzfunktion, so stimmen die Werte gut überein. Hohe Werte in der einen Tabelle entsprechen jeweils hohen in den beiden anderen und vice versa niedrige in der einen niedrigen in den beiden anderen. Um diese Ubereinstimmung größenmäßig darzustellen, wurden die Werte der drei Tabellen miteinander korreliert, also eine Art 'Metakorrelationsanalyse' durchgeführt. (Dabei wurden beim Vergleich mit den Klassifikationsergebnissen der univariaten Analyse die Werte aus den anderen beiden Tabellen gleichgerichtet, d.h. negative in positive umgeformt.) Da der Korrelationskoeffizient eine zu optimistische Schätzung des Zusammenhangs wiedergibt, ist in Tab.8 das Quadrat des Korrelationskoeffizienten, die sog. 'erklärte Varianz' $R^{2}$ aufgeführt.

Tab.8: 'Erklärte Varianz' $\left(\mathbf{R}^{2}\right)$ aus den Korrelationen $z$ wischen den Werten in Tab.5, 6 und 7 .

\begin{tabular}{ll|lll} 
'abhängig' & 'unabhängig' & MODUS & FOKUS-F & FOKUS-N \\
\hline Hörerurteile & univariate Analyse & .94 & .66 & .69 \\
Hörerurteile & multiv. Analyse & .94 & .92 & .90 \\
multiv. An. & univ. Analyse & .94 & .92 & .77
\end{tabular}

Auch wenn die Assoziationen der Hörerurteile mit den Prädiktorvariablen univariat gewonnen wurden, so stimmen die Hörerurteile doch weitaus besser mit der multivariaten als mit den univariaten Analysen überein: Die erklärte Vari- 
anz liegt immer über .90. Es war zu erwarten, daß die univariaten Analysen nicht ganz so gut mit der multivariaten Analyse übereinstimmen. ${ }^{10}$

Tab.9 zeigt die Ergebnisse für unterschiedliche Konstellationen von Lernund Prüfstichproben. Die Reihenfolge der Behandlung ergibt sich aus dem folgenden Bild:

Lern- = Prüfstichprobe

ein Sprecher

fünf Sprecher

alle Sprecher
(3)
Lern- $\neq$ Prüfstichprabe

(5) fünf Sprecher

(4) ein Sprecher

FOKUS ohne Trennung von Fragen und Nicht-Fragen soll hier nur kurz behandelt werden, da eine Trennung der Hauptmodi grundsätzlich bessere Ergebnisse bringt. (Natürlich könnte man auch den gegensätzlichen Gesichtspunkt betonen: Es ist erstaunlich, daß die Ergebnisse trotz der fehlenden Trennung noch recht gut sind.)

(1) Reihum ist ein Sprecher Lern- und gleichzeitig Prüfstichprobe. Damit kann die Konsistenz der Sprecher in sich überprüft werden. Dieses Vorgehen ist dem häufigen Vorgehen in der Phonetik vergleichbar, bei dem Daten nur eines Sprechers interpretiert werden. Die Ergebnisse sind immer sehr gut und liegen zwischen 98.2 und 100\%. Da wir hier Lern- und Prüfstichprobe wegen der relativ geringen Zahl von Fällen nicht trennen, ist eine Uberadaptation wahrscheinlich; d.h. die Konsistenz der Sprecher in sich dürfte bei Trennung von Lern- und Prüfstichprobe ohne eine Erhöhung der Stichprobengröße nicht mehr so eindeutig sein.

(2) Reihum werden fünf Sprecher als Lern- und Prüfstichprobe genommen. Bei MODUS ist das Ergebnis unwesentlich schlechter als in (1), bei FOKUS-F und FOKUS-NF ist es um ca. $5 \%$ schlechter. ${ }^{11}$

(3) Alle sechs Sprecher sind Lern- und zugleich Prüfstichprobe. Die Hinzunahme eines weiteren Sprechers verschlechtert offensichtlich die Prädiktion nicht, da die Unterschiede unwesentlich sind.

(4) Reihum ist ein Sprecher Prüfstichprobe auf der Grundlage der anderen fünf Sprecher, s. (2). Dieses Vorgehen wird als 'leave one out-Verfahren' bezeichnet; damit läßt sich eine sprecherunabhängige automatische Spracherkennung simulieren. Zugleich stellt man das Verfahren vor eine Aufgabe, wie sie jeder Mensch lösen kann, wenn er einem unbekannten Sprecher zum ersten Mal begegnet. Bei FOKUS-NF ist das Ergebnis immer noch sehr gut, bei FOKUS-F bzw. MODUS ist es um $8 \%$ bzw. $5 \%$ schlechter.

(5) Reihum sind fünf Sprecher Prüfstichprobe auf der Grundlage des jeweils sechsten Sprechers. Damit kann die Repräsentativität eines einzelnen

10 Dieses Ergebnis zeigt die eingeschränkte Relevanz von Perzeptionsexperimenten, bei denen immer nur ein Parameter variiert wird.

11 Diese Konstellation wird beim 'leave-one-out'-Verfahren (Konstellation 4) automatisch mitberechnet. Sie wird mit aufgeführt, da man im Vergleich zu Konstellation (3) ein grobes Maß dafür erhält, welchen Einfluß die Hinzunahme eines weiteren Sprechers auf die Klassifikationsgüte hat. 
Sprechers getestet werden. Bei FOKUS-F und FOKUS-NF verschlechtert sich das Ergebnis deutlich gegenüber (4), bei MODUS wird es dagegen besser. Wir werden in Teil 7 auf dieses prima facie unerwartete Ergebnis zurückkommen.

Tab.9: Lern- vs. Prüfstichproben für Frage/Nicht-Frage (MODUS), FA gesamt (FOKUS), FA bei Frage (FOKUS-F) und FA bei Nicht-Frage (FOKUS-NF).

\begin{tabular}{|c|c|c|c|c|}
\hline & Lern $=$ Prüf & & & Lem $\neq$ Prüf \\
\hline MODUS & $\begin{array}{l}\text { ein Sprecher } \\
\text { fünf Sprecher } \\
\text { alle Sprecher }\end{array}$ & $\begin{array}{l}98.9 \\
97.2 \\
97.1\end{array}$ & $\begin{array}{l}92.0 \\
91.7\end{array}$ & $\begin{array}{l}\text { fünf Sprecher } \\
\text { ein Sprecher }\end{array}$ \\
\hline FOKUS & $\begin{array}{l}\text { ein Sprecher } \\
\text { fünf Sprecher } \\
\text { alle Sprecher }\end{array}$ & $\begin{array}{l}97.0 \\
93.1 \\
92.7\end{array}$ & $\begin{array}{l}77.5 \\
84.1\end{array}$ & $\begin{array}{l}\text { fünf Sprecher } \\
\text { ein Sprecher }\end{array}$ \\
\hline FONUS-F & $\begin{array}{l}\text { ein Sprecher } \\
\text { fünf Sprecher } \\
\text { alle Sprecher }\end{array}$ & $\begin{array}{l}98.2 \\
94.6 \\
94.6\end{array}$ & $\begin{array}{l}75.7 \\
86.2\end{array}$ & $\begin{array}{l}\text { fünf Sprecher } \\
\text { ein Sprecher }\end{array}$ \\
\hline FOKUS-NF & $\begin{array}{l}\text { ein Sprecher } \\
\text { fünf Sprecher } \\
\text { alle Sprecher }\end{array}$ & $\mid \begin{array}{r}100.0 \\
93.4 \\
95.5\end{array}$ & $\begin{array}{l}81.6 \\
93.8\end{array}$ & $\begin{array}{l}\text { fünf Sprecher } \\
\text { ein Sprecher }\end{array}$ \\
\hline
\end{tabular}

5. Relevante Merkmale: sprecherübergreifend oder sprecherspezifisch?

Wenn man nur an einem sprecher trainiert und auf die übrigen sprecher schließt, so verschlechtert sich die Prädiktion des FA deutlich. Das läßt vermuten, daß einzelne Sprecher nicht-repräsentative Eigenheiten im Einsatz der Merkmale aufweisen. Tab.10 bis 12 zeigen deshalb die Korrelationen der Prädiktorvariablen für MODUS, FOKUS-F und FOKUS-NF mit der Diskriminanzfunktion für eine Analyse, bei der jeweils ein Sprecher Lern- und Prüfstichprobe zugleich ist, also für die Konstellation (1) aus Tab.9; die Korrelationskoeffizienten sind wieder mit 100 multipliziert. Die drei rechten Spalten zeigen den Mittelwert pro Zeile $(\bar{x})$, die Standardabweichung (s) sowie die Differenz zwischen dem maximalen und dem minimalen Wert pro Zeile (Spannweite), jeweils auf die ganze Zahl gerundet. Die Mittelwerte stimmen gut mit Tab.7, also den Werten für die multivariate Diskriminanzanalyse für alle Sprecher (Konstellation (3) in Tab.9) überein. Betrachtet man nun die Standardabweichungen und die Spannweiten, so finden sich manchmal recht hohe Werte $(s>20$ und Spannweite > 50). Sucht 
man unter den sechs Sprechern nach den 'Verantwortlichen', so sieht man, daß immer dann, wenn einzelne Sprecher von den anderen abweichen, es sich um Sp1 oder Sp6 handelt: bei MODUS Sp1 bei Off und Sp6 bei Pos3; bei FOKUS-F Spl bei Pos3 und Sp6 bei Max2, Min2, Min3, Pos2 und Int3; bei FoKUS-NF Sp6 bei Pos2. Die niedrigere Korrelation von off mit der Diskriminanzfunktion bei MODUS bei Sp1 weist darauf hin, daß diese Sprecherin oft Fragen mit einem niedrigen Offset indiziert (vgl. unten Fig.5); bei Sp6 ist die Korrelation von Pos3 mit der FA-Zuweisung auf der 2. Phrase zwar auch negativ, aber viel größer. Dieser Sprecher setzt also ein übliches Merkmal für Fokus auf der 3. Phrase (Fo-Minimum vor Fo-Maximum, also ein ausgeprägter 'Tiefton' im Ton-Sequenz-Ansatz von Pierrehumbert 1980) stärker ein als die anderen Sprecher. Die anderen Abweichungen können analog interpretiert werden. Wir warten aber damit bis zur Diskussion von Fig.2 bis 10, da die Interpretation anhand der Abbildungen eingängiger sein dürfte.

Standardabweichung und Spannweite sind bei MODUS und bei FOKUS-NF geringer als bei FOKUS-F; für FOKUS-F zeigt auch Tab.9 durchgehend eine schlechtere Klassifikationsgüte als für MODUS und FOKUS-NF. Man kann also daraus schließen, daß bei Fragen eine überlagerung von modus- und von fokusspezifischen Parameterausprägungen die Klassifikation erschwert. 
Tab.10: Korrelation ( $\star 100)$ der Prädiktorvariablen mit der Diskriminanzfunktion pro Sprecher bei MODUS

\begin{tabular}{l|rrrrrr|rrr} 
& Sp1 & Sp2 & Sp3 & Sp4 & sp5 & Sp6 & $\bar{x}$ & s & Spannweite \\
\hline Off & 31 & 88 & 83 & 86 & 80 & 75 & 74 & 22 & 57 \\
Stg & 55 & 29 & 38 & 23 & 23 & 42 & 35 & 13 & 32 \\
Max2 & 26 & 02 & 23 & 06 & 12 & 36 & 18 & 13 & 34 \\
Max3 & 68 & 74 & 66 & 26 & 60 & 56 & 58 & 17 & 48 \\
Min2 & 01 & 08 & 16 & 09 & 09 & 10 & 09 & 05 & 15 \\
Min3 & 32 & 45 & 43 & 17 & 18 & 29 & 31 & 1.2 & 2.8 \\
Pos2 & -21 & -30 & -48 & -10 & -09 & -09 & -21 & 16 & 39 \\
Pos3 & -03 & -35 & -18 & -17 & -16 & -65 & -26 & 22 & 62
\end{tabular}

Tab.11: Korrelation ( ${ }^{100}$ ) der Prädiktorvariablen mit der Diskriminanzfunktion pro Sprecher bei FONUS- $F$

\begin{tabular}{l|rrrrrr|rrr} 
& $\mathrm{Sp1}$ & $\mathrm{Sp2}$ & $\mathrm{Sp} 3$ & $\mathrm{Sp4}$ & $\mathrm{Sp5}$ & $\mathrm{Sp6}$ & $\overline{\mathrm{x}}$ & $\mathrm{s}$ & Spannweite \\
\hline Max2 & 54 & 27 & 19 & 40 & 34 & 75 & 42 & 20 & 56 \\
Max3 & -16 & -12 & -07 & -12 & 01 & -33 & -13 & 11 & 34 \\
Min2 & 13 & -03 & -03 & 24 & 17 & -32 & 03 & 20 & 56 \\
Min3 & 32 & 28 & 23 & 66 & 48 & -18 & 30 & 28 & 84 \\
Pos2 & -53 & -41 & -19 & -55 & -52 & 17 & -34 & 28 & 72 \\
Pos3 & 64 & 05 & 08 & 12 & 28 & 11 & 21 & 22 & 59 \\
Dau2 & 08 & 15 & 11 & 15 & 18 & 05 & 12 & 05 & 13 \\
Dau3 & -30 & -07 & -07 & -49 & -35 & 14 & -19 & 23 & 63 \\
Int2 & -19 & 02 & 03 & -29 & -01 & 10 & -06 & 15 & 39 \\
Int3 & -06 & -18 & 05 & -02 & -04 & -47 & -12 & 19 & 52
\end{tabular}

Tab.12: Korrelation $(* 100)$ der Prädiktorvariablen mit der Diskriminanzfunktion pro Sprecher bei FOKUS-NF

\begin{tabular}{l|rrrrrr|rrr} 
& Sp1 & Sp2 & Sp3 & Sp4 & Sp5 & Sp6 & $\bar{x}$ & s & Spannweite \\
\hline Max2 & 14 & 19 & 07 & 03 & 16 & 04 & 11 & 07 & 16 \\
Max3 & -52 & -25 & -44 & -60 & -43 & -32 & -43 & 13 & 35 \\
Min2 & 07 & -15 & -20 & -14 & -10 & -14 & -11 & 09 & 27 \\
Min3 & -01 & 05 & -12 & -06 & 06 & 04 & -01 & 07 & 18 \\
Pos2 & -09 & -08 & 15 & 10 & -02 & 56 & 11 & 24 & 65 \\
Pos3 & -02 & -01 & -16 & -30 & -04 & -03 & -09 & 12 & 29 \\
Dau2 & 23 & 33 & 08 & 19 & 13 & 39 & 23 & 12 & 31 \\
Dau3 & -48 & -19 & -29 & -17 & -34 & -34 & -30 & 11 & 31 \\
Int2 & 12 & 07 & 10 & 02 & 16 & 10 & 09 & 06 & 18 \\
Int3 & 01 & -23 & -10 & -09 & -11 & -27 & -13 & 10 & 28
\end{tabular}


Tab.7 zeigt, daß z.B. Int2 und Int3 bei FOKUS-F und FOKUS-NF sowie Pos2 und Pos 3 bei FOKUS-NF praktisch nicht mit der Diskriminanzfunktion korrelieren, d.h. nur sehr wenig zur Trennung der beiden Klassen beitragen. Man könnte natürlich solche Variablen ausscheiden; das hätte rechentechnische Vorteile. Unser Ziel war aber nicht in erster Linie eine Minimierung der Prädiktorvariablen, sondern eine phonetische Analyse der sprecherübergreifenden und der sprecherspezifischen Relevanz der Variablen. Wir werden im folgenden sehen, da $\beta$ auch solche Merkmale, die insgesamt wenig zur Fokusindizierung beitragen, bei einzelnen Sprechern sehr wohl relevant sein können.

Die Ergebnisse der statistischen Analyse sind exakt, aber unanschaulich; es ist zwar möglich, aus den Zahlen repräsentative Parameterausprägungen und damit Verläufe bzw. Größenkorrelationen zu rekonstruieren - das wäre aber recht mühsam. Es soll deshalb genügen, daß man aus den Zahlen die relative wichtigkeit der einzelnen Prädiktorvariablen erschließen kann. Im weiteren wollen wir uns eine Eigenschaft unserer Daten zunutze machen, die sie vor anderen Daten auszeichnet: Eine Darstellung im zweidimensionalen Raum ist nicht nur anschaulich und möglicherweise phonetisch/kognitiv relevant, wie das etwa bei der Nonmetrischen Multidimensionalen Skalierung der Fall ist (vgl. Batliner $1989 \mathrm{~b})$; eine solche Darstellung ist außerdem sehr konkret und bei phonetischen Daten üblich. Wir wollen uns deshalb im nächsten Abschnitt anhand der Mittelwertsdarstellungen der Prädiktorvariablen ein Bild von den typischen Vertretern, eben den Prototypen machen. Diese Darstellungen gleichen denen von aktuellen Fo-Verläufen; zum Unterschied vgl. Teil 7 . Wenn wir diese Mittelwerte interpretieren, dann machen wir zwar nicht das Gleiche, aber doch etwas sehr Ahnliches wie die Diskriminanzanalyse, die ja versucht, über Verteilungsmaße die Abstände zwischen den Gruppen zu maximieren: Der Mittelwert ist ein bedeutendes, wenn auch nicht das einzig relevante Verteilungsmaß. Wenn nun die Unterschiede in Fig.2 bis 10 ins Auge fallen, dann sprechen sie für sich; wenn sie gering sind, dann können wir uns immer mit einem Blick in die Tabellen vergewissern, wie groß bzw. wie relevant dieser Unterschied ist. 
7. Gewinnung der Prototypen über die große Zahl

In Fig.2 bis 10 sind die Mittelwerte für die relevanten Parameter aufgetragen, und zwar in Fig.2 für alle Außerungen, in Fig.3 für die 'eindeutigen' Fokussierungen, in Fig.4 für die 'uneindeutigen' (vgl. $\mathrm{zu}$ den Begriffen weiter unten) und in Fig.5 bis 10 für die einzelnen Sprecher. Eine genauere Aufgliederung nach Satzmodi (z.B. bei den Nicht-Fragen nach Aussagesatz, Imperativsatz und Adhortativsatz) hat sich bei unserem Material nicht als sinnvoll erwiesen, da daîur die Zahi der Fälle pro Sprecher zu gering war. Fragen und Nicht-Fragen sowie Fokus auf der 2. und Fokus auf der 3. Phrase werden getrennt dargestellt; in Klammern ist jeweils die Zahl der Fälle angegeben ( $n$ ), über die gemittelt wurde. ${ }^{12}$ Die $x$-Achse zeigt den Zeitbereich in Centisekunden, die $\mathrm{y}$-Achse ist Ht-skaliert, wobei der sprecherspezifische Basiswert auf Null gesetzt ist.

Die Werte Max2, Min2, Max3 und Min3 werden als Volltonquadrate dargestellt. Anfang und Ende der Phrasen 2 und 3 sind durch Kreise gekennzeichnet. Da die relationalen Einheiten Dau2 und Dau3 nicht in Centisekunden angegeben werden können, sind die Mittelwerte der Positionen von Phrasenanfang und ende angegeben; die Linie zwischen Anfang und Ende kennzeichnet die mittlere Dauer der Phrasen. Um auch die Intensitätsverhältnisse in der gleichen Abbildung wiedergeben zu können, dient die y-Achse als Hilfsachse für die Differenz zwischen Int2 und Int3: Die Phrase mit der schwächeren Intensität (bis auf eine Ausnahme immer die 3. Phrase) wird bezüglich der Dauerkennzeichnung auf 1 gesetzt, die mit der höheren auf $1+($ Int2-Int3)/100. Auf der $y$-Achse läßt sich damit die linear transformierte Differenz zwischen der Intensität auf der 2. und der auf der 3. Phrase ablesen. Man beachte, daß es sich nicht um 'produzierbare' Konturen handelt! So zeigt etwa Fig.10 mit Sp6 bei FOKUS 3, FRAGEN auf der zweiten Phrase keinen abrupten Tonsprung, also kein Jodeln; man kann aber ersehen, da $\beta$ die Position der Minima um die der Maxima oszilliert, da $\beta$ also die Minima etwa zu gleichen Teilen vor und nach den Maxima liegen. Aus diesem Grund sind Max2 und Min2 bzw. Max3 und Min3 nicht durch Linien miteinander verbunden.

\footnotetext{
12 Beim Aufsummieren über die Sprecher oder über die Konstellationen können sich Unstimmigkeiten ergeben, die dadurch begründet sind, da $\beta$ für einige Außerungen nicht alle Variablen ermittelt werden konnten.
} 
Fig.2: Alle Außerungen
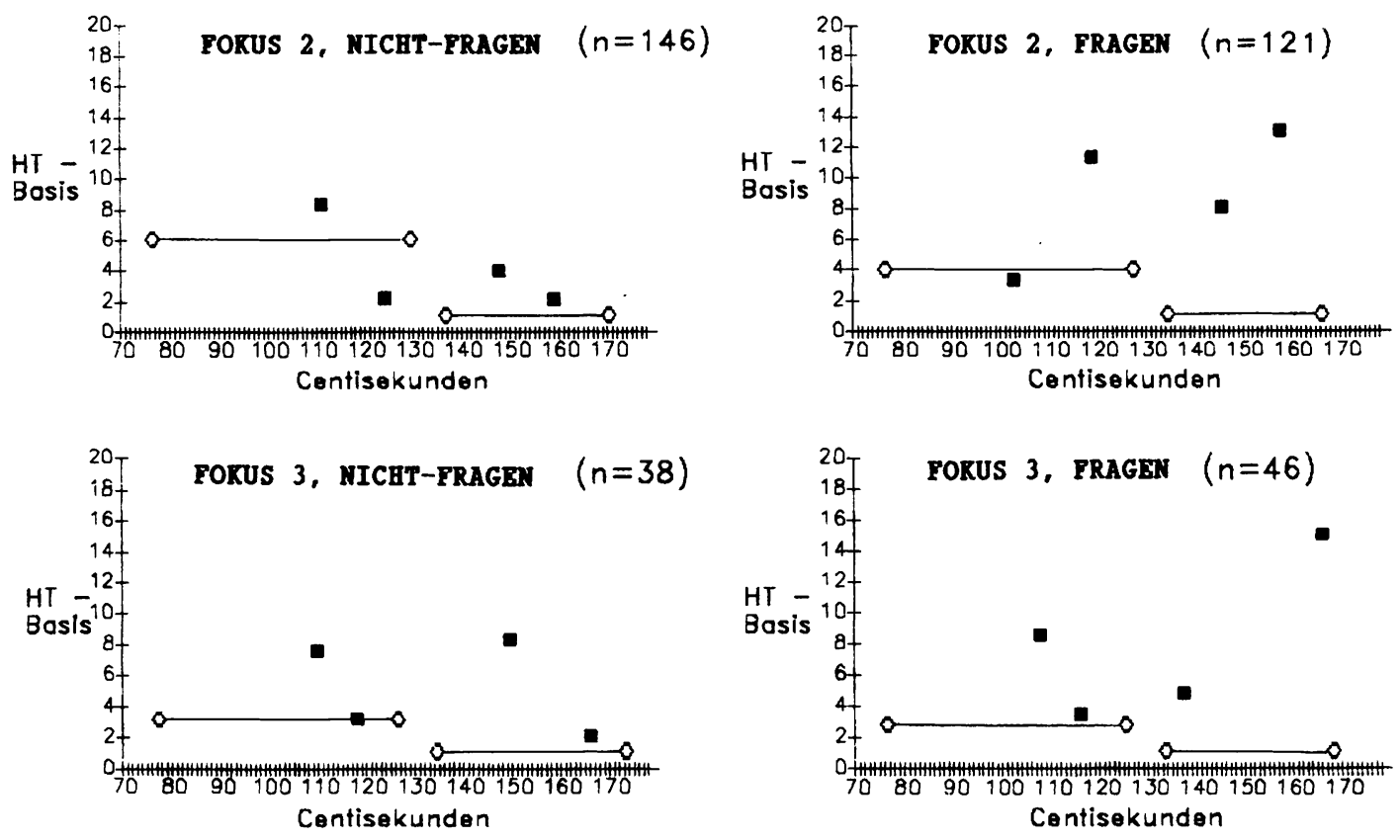

Fig.3: Eindeutige Fokussierungen
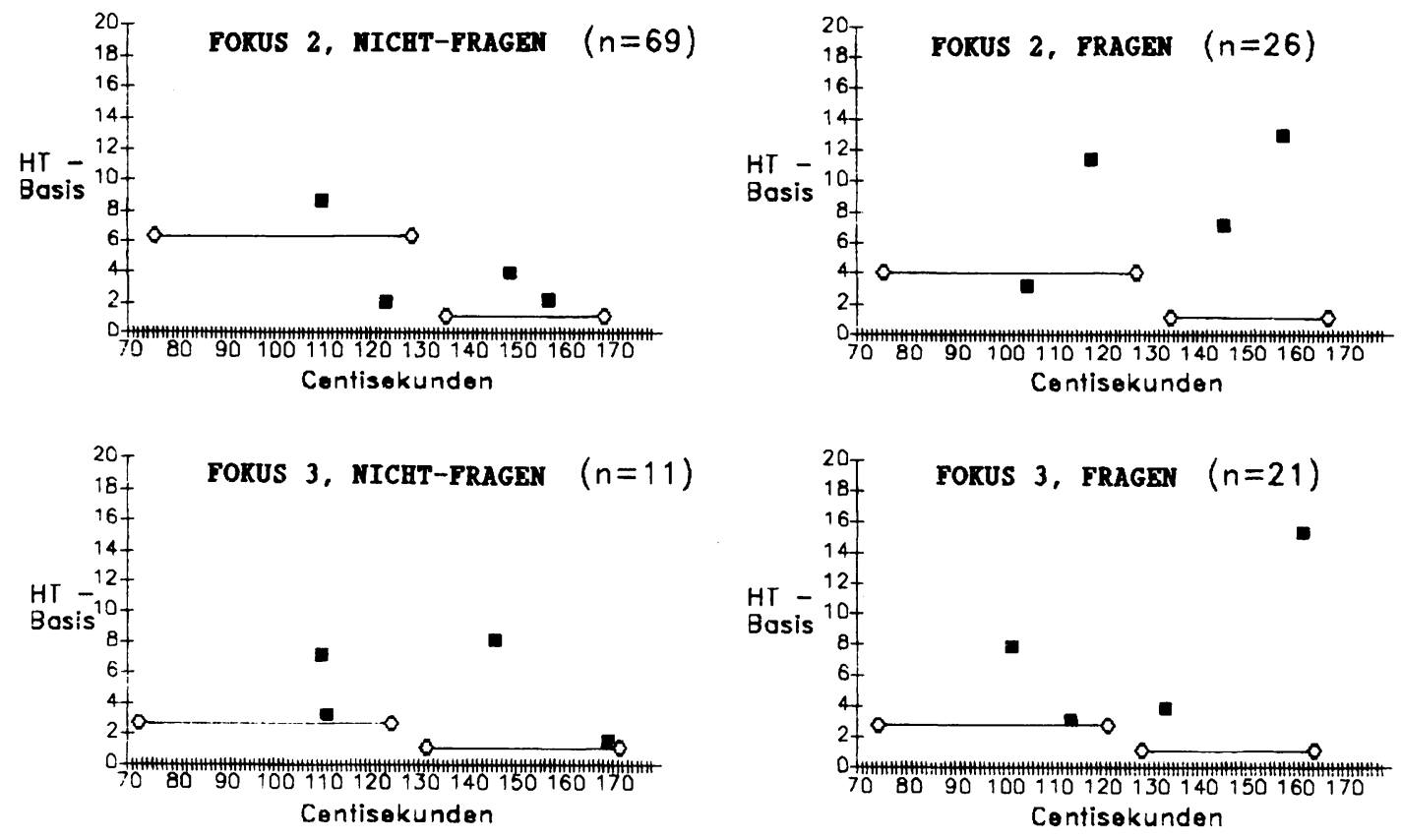
Fig.4: Uneindeutige Fokussierungen
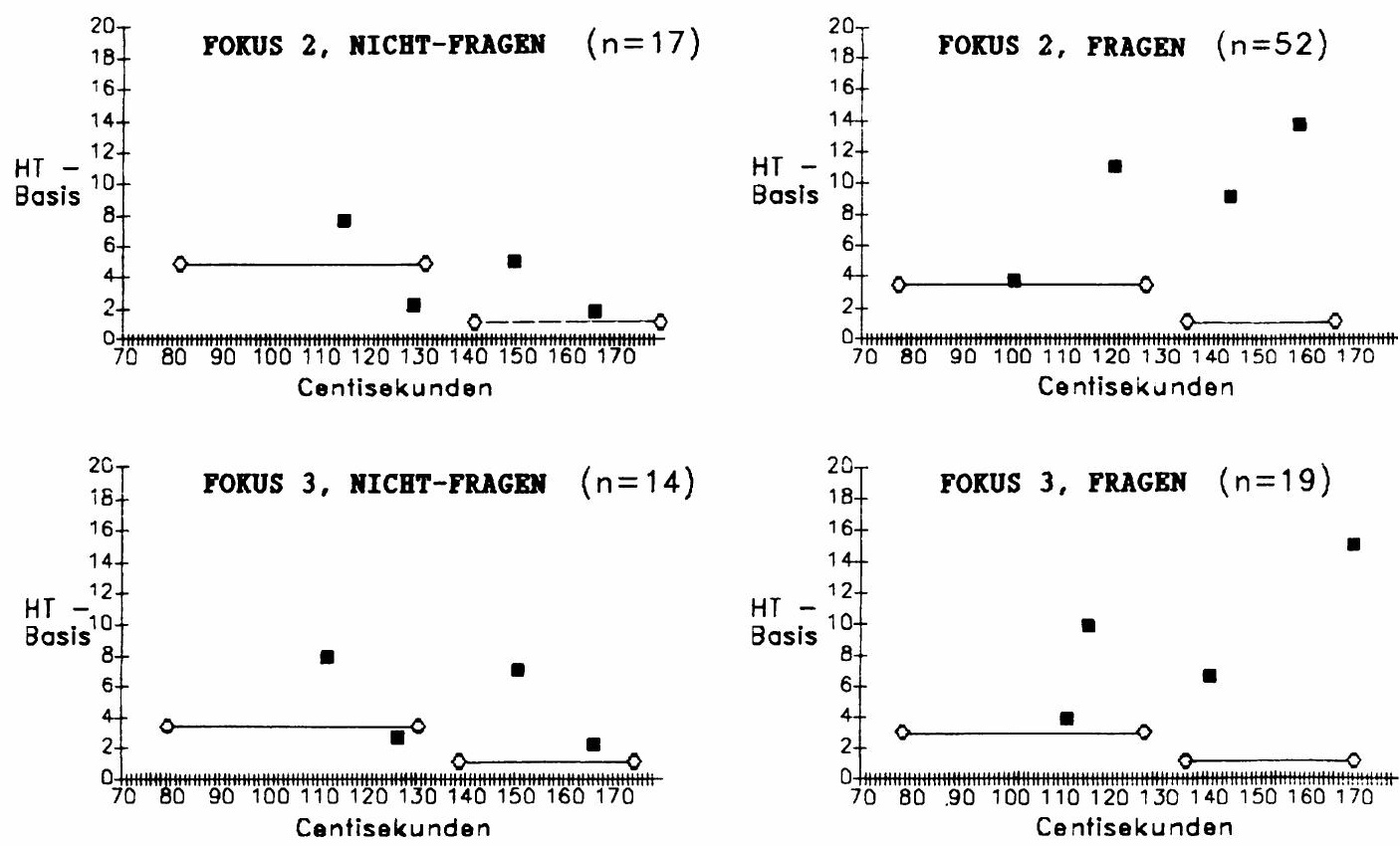

Interpretiert werden können die Unterschiede zwischen den Werten der 2. und der 3. Phrase innerhalb einer Konstellation sowie die Unterschiede in den Spalten und in den Zeilen der Figuren zwischen jeweils den Werten der 2. oder der 3. Phrase. Natürlich hängt bei einer solchen Darstellung der sichtbare Unterschied auch von der Skalierung ab; wie schon erwähnt, kann man aber immer auf die statistischen Werte in den Tabellen zurückgreifen.

Wir nehmen an, daß man einen besonders ausgezeichneten intonatorischen Prototyp, nämlich den Normalfall (Kerntyp) über die große Zahl erhalten kann. Wir müssen dabei natürlich unterstellen, daß unsere stichprobe eine gewisse Repräsentativität besitzt - nicht in dem Sinn, da $\beta$ alle möglichen Kombinationen und damit auch Randtypen enthalten sind, aber in dem Sinn, daß eine andere Stichprobe die gleichen Normalfälle zeigen würde. Beim Betrachten von Fig.2 ist diese Annahme intuitiv einsehbar: Es zeigt sich, was man erwarten würde und was wir im folgenden erläutern. 
Nicht-Fragen zeigen auf akzentuierter und unakzentuierten Phrase eine fallende Kontur ('Hochton' mit folgendem 'Tiefton' im Ton-Sequenz-Ansatz). Fokussierung wird durch einen höheren Tonumfang (Range) markiert, der hauptsächlich über die Maxima gesteuert wird. Die Prominenz der Maxima muß auch in Beziehung gesetzt werden zu ihrer Position auf der Zeitachse, da Max3 bei FOKUS 3 nur unwesentlich höher ist als Max2 im Gegensatz zum ausgeprägteren Unterschied von Max2 und Max3 bei FOKUS 2 (Einfluß der Deklinationslinie). Dauer und Intensitätsunterschiede sind nicht sehr ausgeprägt, sie markieren aber doch systematisch die fokussierte Phrase durch eine größere Ausprägung relativ zur nicht fokussierten Phrase.

Fragen zeigen auf der fokussierten Phrase eine steigende Kontur ('Tiefton' mit folgendem 'Hochton' im Ton-Sequenz-Ansatz), ebenfalls auf der nicht-fokussierten bei FOKUS 2 (bedingt durch den hohen Offset); bei FOKUS 3 hat die nicht-fokussierte zweite Phrase eine fallende Kontur. Dauer und Intensität markieren zwar auch die fokussierte Phrase durch eine größere Ausprägung, der Unterschied ist aber weniger augenfällig als bei den Nicht-Fragen. (Exakte Kennwerte dafür finden sich in Tab.9 bei der Korrelation der Prädiktorvariablen mit der Diskriminanzfunktion.)

Die Aufteilung in eindeutige und uneindeutige Fälle (Fig.3 und 4) wurde anhand von FOK, also anhand der Werte aus dem 2. Akzenttest vorgenommen. Es sollten dabei in beiden Gruppen in etwa gleich viele Fälle sein und nicht zu wenige, um Extremwerten kein zu großes Gewicht zu geben. Die Variable FOK hat einen Bereich zwischen -1.0 (alle Hörer setzten den Akzent auf die 3 . Phrase) und +1.0 (alle Hörer setzten den Akzent auf die 2. Phrase). Wir definieren als 'eindeutig' die Fälle mit dem Wert $>+.8$ oder $<-.8 \quad(n=127)$, und als 'uneindeutig' die Fälle mit Werten zwischen .5 und 0.0 bzw. -.5 und 0.0 $(n=102)$. Es zeigt sich, daß sowohl bei den eindeutigen wie bei den uneindeutigen Fällen die grundsätzlichen Relationen die gleichen sind; das kann als Argument für eine Prototypenbildung über die große Zahl genommen werden. Die Ausprägungen sind allerdings bei den uneindeutigen Fällen geringer. 
Fig.5: Sprecher 1 (Sp1)
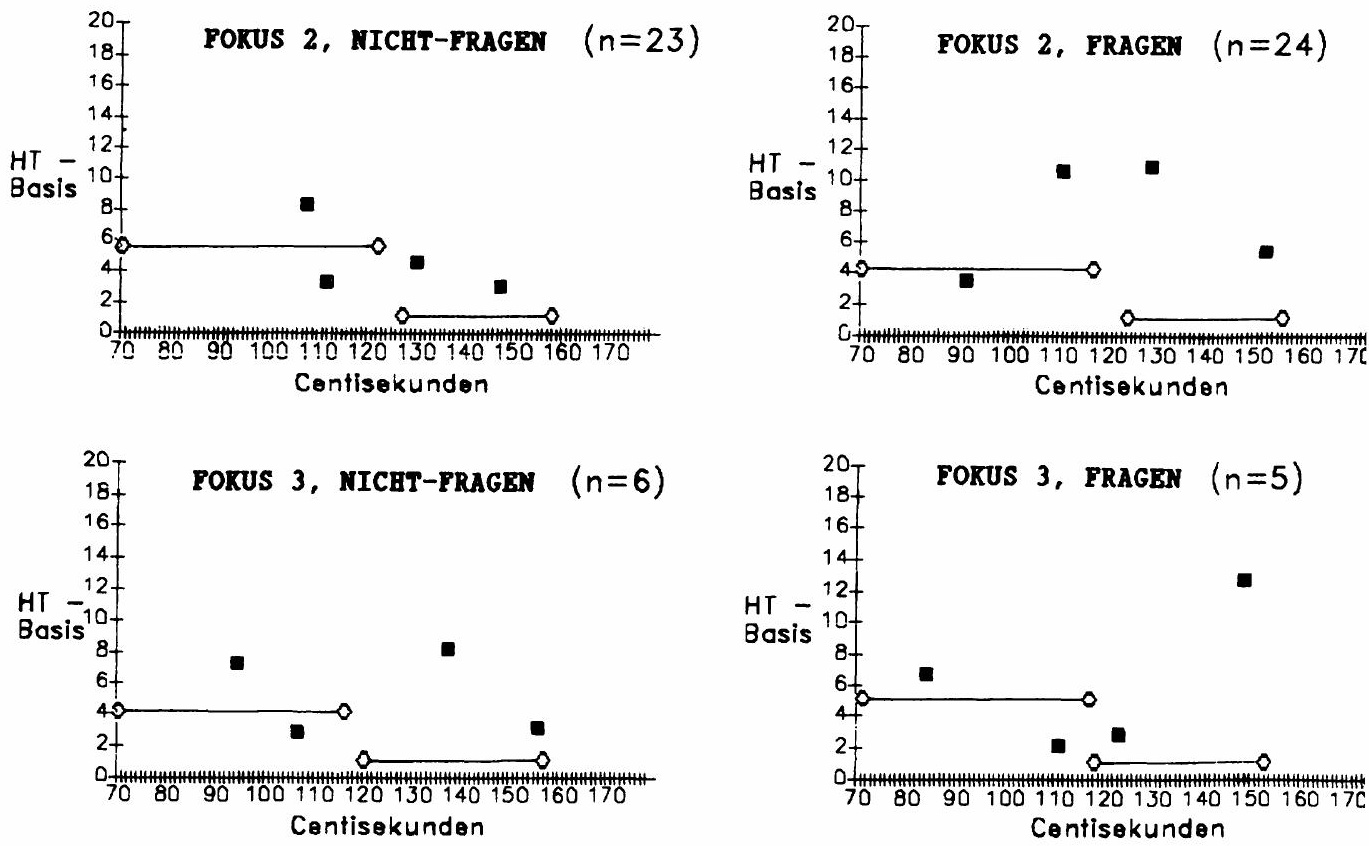

Fig.6: Sprecher 2 (Sp2)
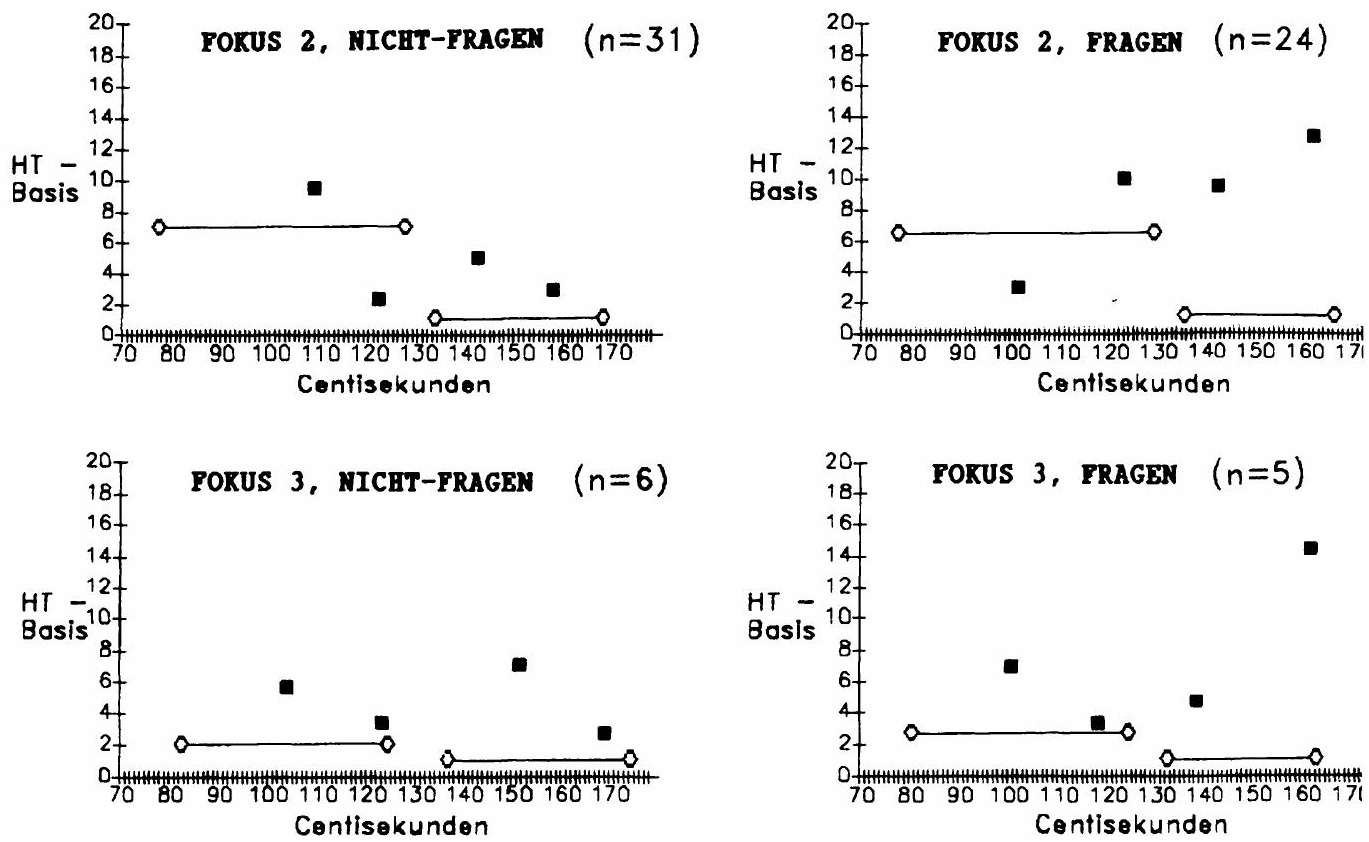
Fig.7: Sprecher 3 (Sp3)
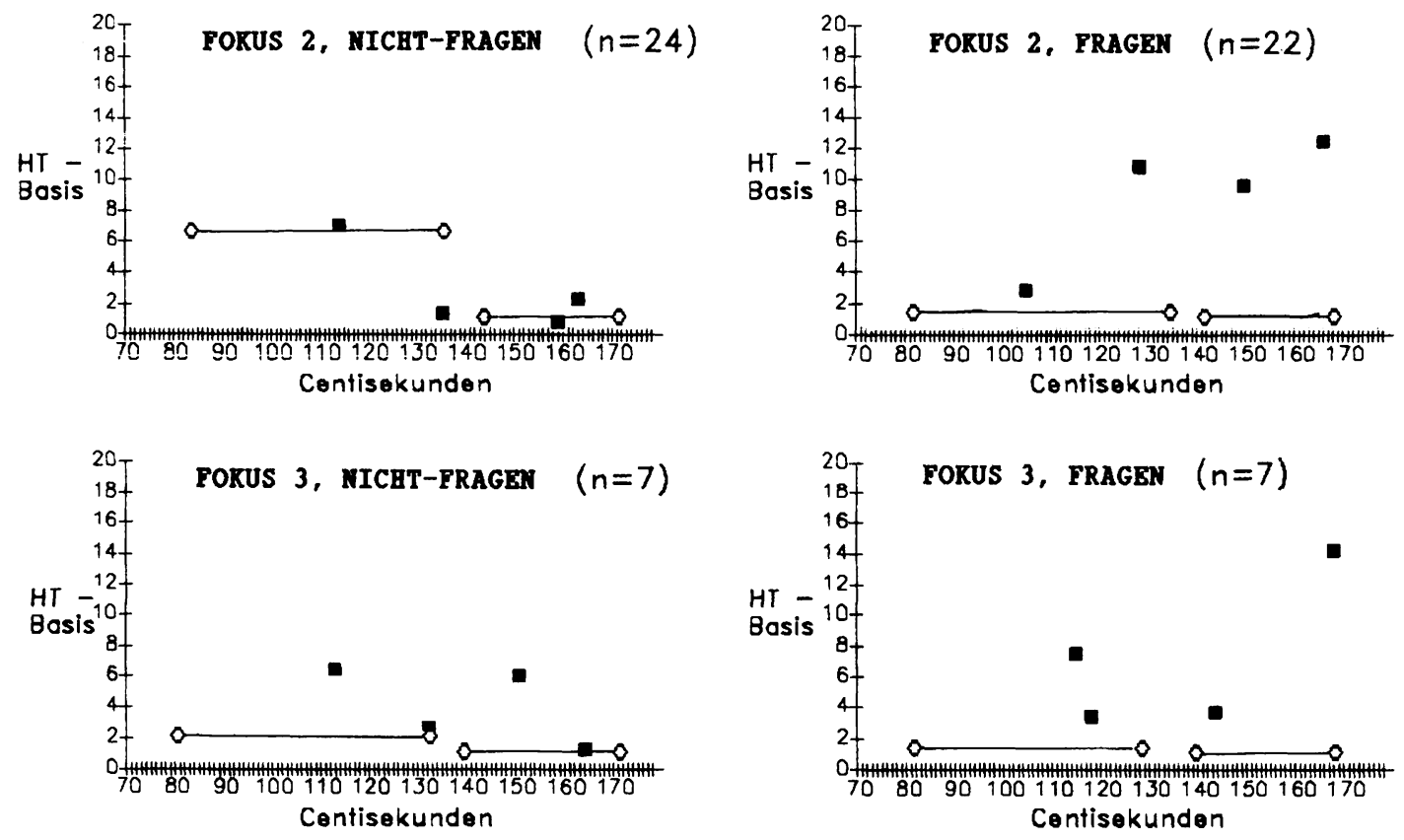

Fig.8: Sprecher 4 (Sp4)
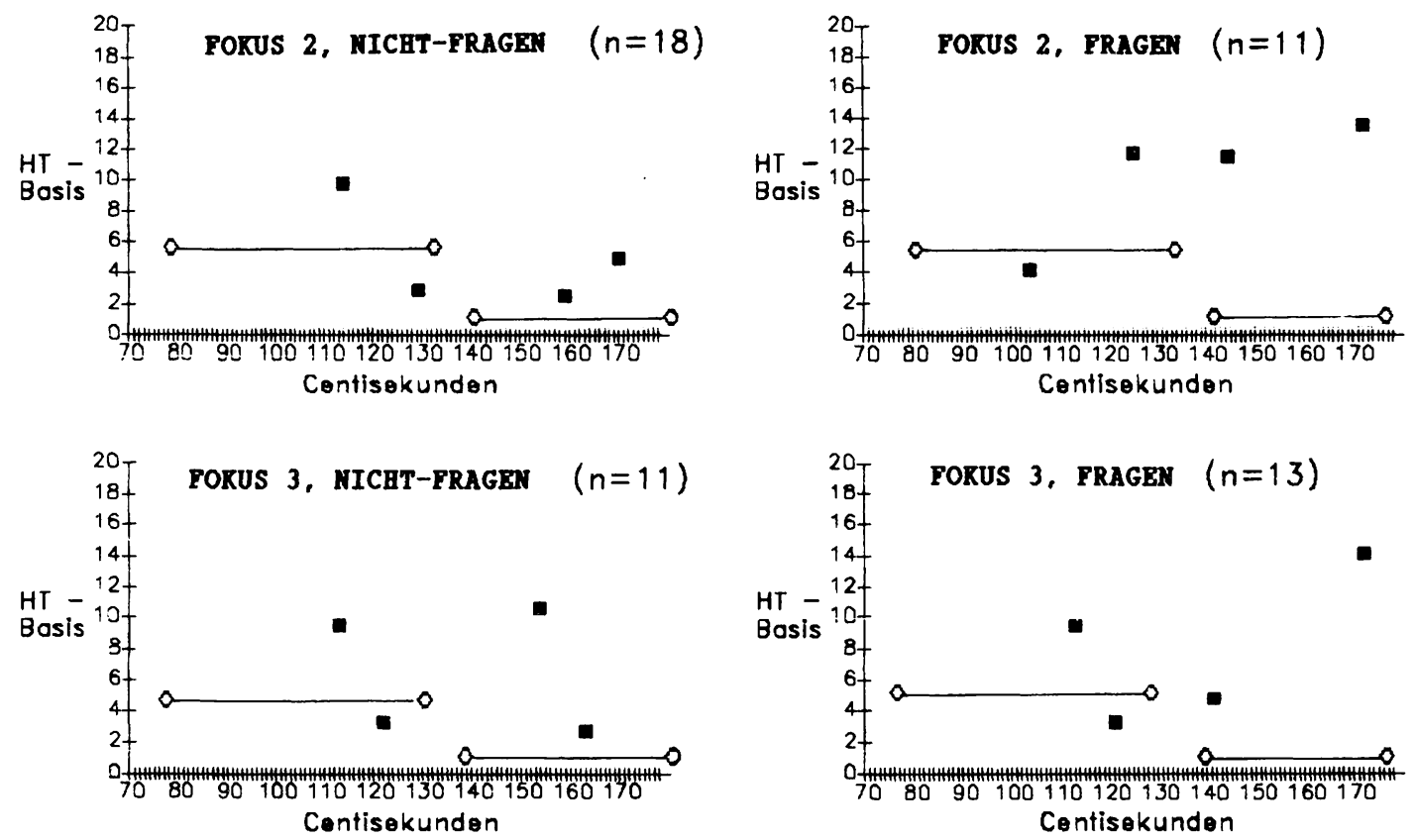
Fig.9: Sprecher 5 (Sp5)
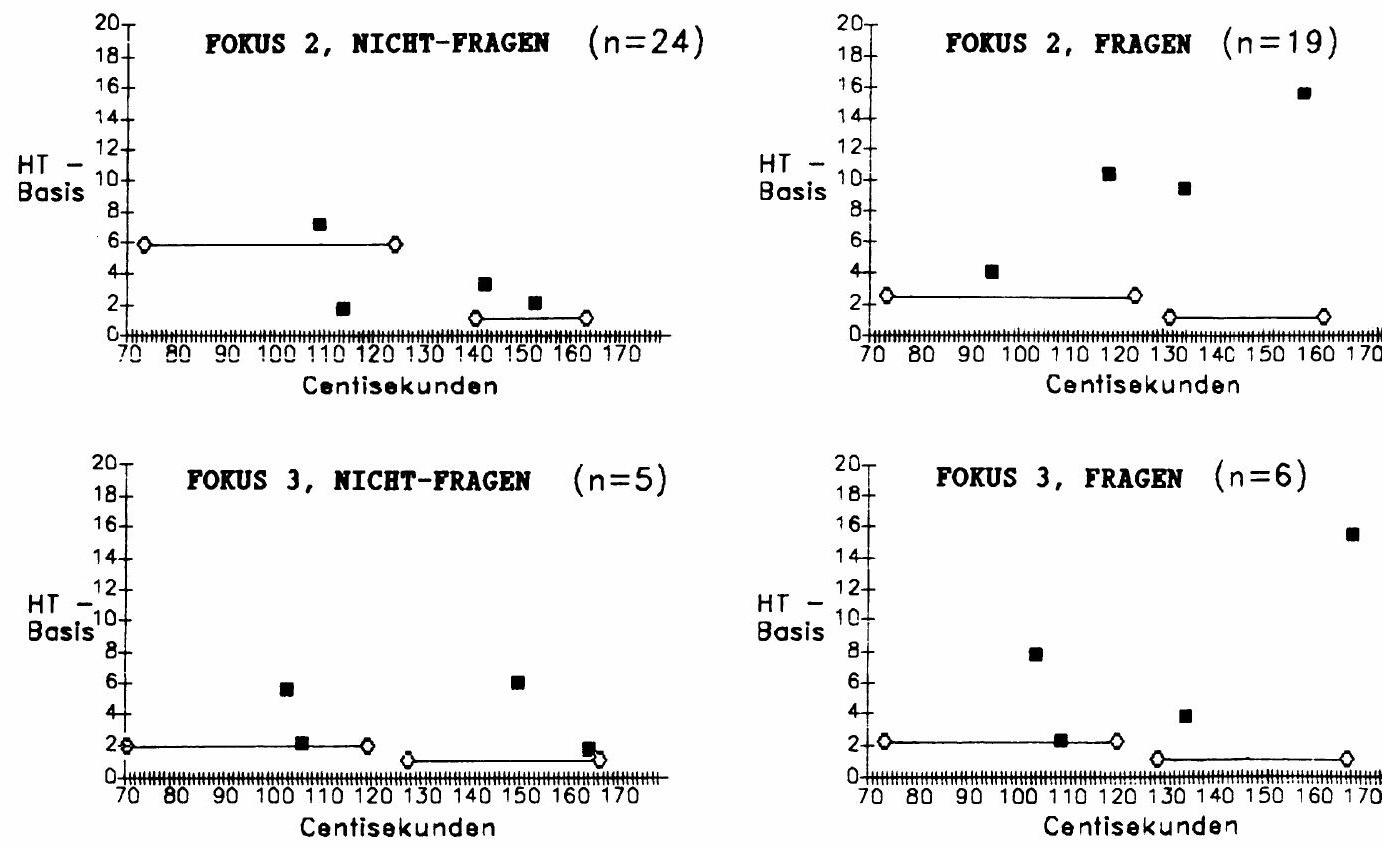

Fig.10: Sprecher 6 (Sp6)
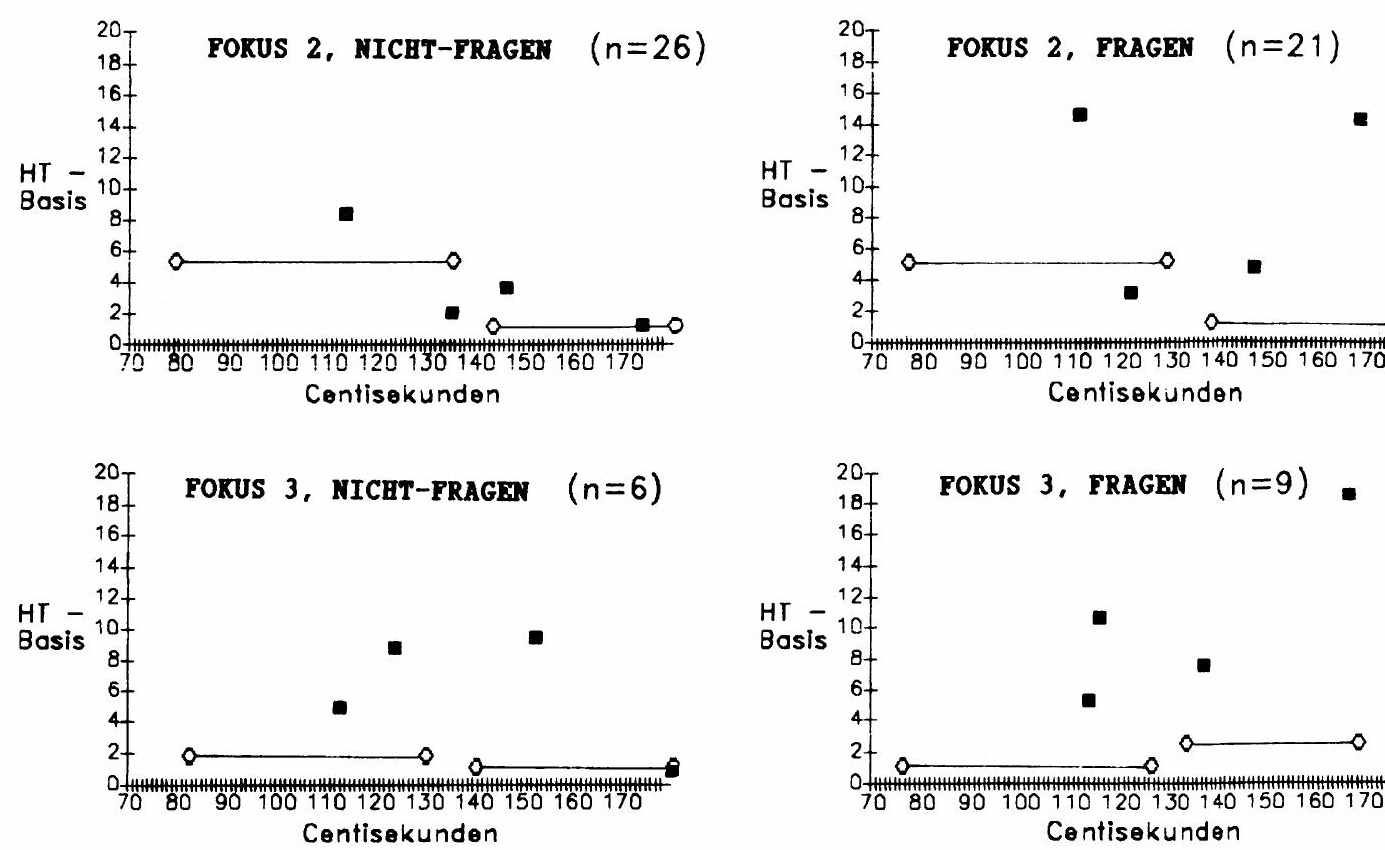
In Fig.5 bis 10 ergibt sich bei den einzelnen Sprechern grosso modo das gleiche Bild, allerdings mit einigen interessanten Abweichungen, die nun besprochen werden.

Sp1: Bei FOKUS 2, FRAGEN weist die dritte unbetonte Phrase keinen Fo-Anstieg, sondern einen Abfall von im Mittel $5 \mathrm{Htn}$ auf. Der Abfall ist je nach Ausprägung perzeptiv unterschiedlich auffällig: Mal wird eher ein ebener Verlauf wahrgenommen, mal ein leicht oder stark fallender. Immer unterscheiden sich die Außerungen aber eindeutig von Außerungen mit einem steigenden Verlauf. 20 Außerungen wurden von $\mathrm{Spl}$ auf diese Weise produziert. Wir können damit das seltsam anmutende Ergebnis in Tab.9 erklären: Bei MODUS wurden bei der Konstellation (5), also bei einer Lernstichprobe von je einem Sprecher, bessere Ergebnisse erzielt als bei der 'leave-one-out'-Methode von Konstellation (4). Im letzten Fall 'verläßt' sich das Verfahren auf die fast immer entscheidende Rolle der Offset-Höhe bei der Klassifizierung von Fragen und Nicht-Fragen auch bei der Klassifizierung von Spl; die 20 Fälle werden dabei fehlklassifiziert. Wenn das Verfahren aber von $\mathrm{Spl}$ ausgehend die anderen Sprecher klassifiziert, so 'verläßt' es sich auf die bei Spl relevanteren Variablen Stg und Max3 (vgl. Tab.10) und kann auf dieser Grundlage die anderen Sprecher gut klassifizieren.

Sp2: Diese Sprecherin setzt die Intensität relativ stark ein (vgl. Int3 in Tab.11 und 12).

Sp3: Bei Nicht-Fragen wird die Intensität deutlich eingesetzt, bei Fragen kann man keinen Unterschied feststellen.

Sp4: Dau3 wird bei Nicht-Fragen von diesem Sprecher weniger eingesetzt als etwa von Sp1. Der Unterschied zwischen Sp4 und Spl bei den entsprechenden Zeileneinträgen von Dau3 in Tab.12 ist gleich der Spannweite dieser Variable. Standardabweichung und Spannweite liegen im mittleren Wertebereich. Daraus kann man zum einen schließen, daß dieser Parameter relativ konsistent eingesetzt wird, daß aber auf der anderen seite doch sprecherspezifische Unterschiede bestehen.

Sp5: Bei Nicht-Fragen sehen wir deutliche Intensitäts- und Dauerunterschiede, bei den Fragen nur Dauer-, aber keine Intensitätsunterschiede.

Sp6: Der Tonverlaup bei FOKUS 3, NICHT-FRAGEN ist bei der 2., unbetonten Phrase ein Anstieg, kein Abfall wie bei den anderen Sprechern ('HutKontur' nach Cohen/'t Hart 1967; 'Brückenkontur' nach Wunderlich 1988). Ein solcher Verlauf ist - unterstellt man unserer Stichprobe eine gewisse Repräsentativität - zwar voll akzeptabel, aber nicht häufig und möglicherweise sprecherspezifisch.

Intensität wird sowohl bei Frage als auch bei Nicht-Frage stark eingesetzt: nur bei diesem Sprecher kehrt sich bei FOKUS 3, FRAGEN die Relation von Int2 und Int3 um. Das könnte natürlich durch den sehr großen Range auf der 3. Phrase bei diesem Sprecher, bedingt sein; Sp5 hat aber einen größeren Range, ohne daß sich das Verhältnis bei der Intensität umkehrt.

Bei FOKUS 2, FRAGE weist die fokussierte Phrase im Mittel einen starken Abfall auf, keinen Anstieg wie bei den Mittelwerten aller anderen Sprecher. Dieser Sprecher macht also eine Art 'Anleihe' im Akzentinventar der Nicht-Fragen, ohne daß die Außerung deswegen unnatürlich ist (s. Teil 8). Ein Anstieg wie in Fig.2 ist also bei dieser 
Konstellation zwar Pragetypisch, aber das Gegenteil nicht irregulär. Wir können daher schließen, da $\beta$ wir es hier mit einem Randtyp zu tun haben.

Die ins Auge fallenden und soeben aufgeführten Unterschiede zwischen den Sprechern, die man auch an den Extremwerten in Tab.11 und 12 festmachen kann, sind also auf die 'untypischen' Verläufe bei $\mathrm{Sp} 1$ und Sp6 zurückzuführen.

Wenn wir eine - vorsichtige - Gesamtinterpretation versuchen, so kann man sagen, daß bei allen Parametern eine gewisse Varianz tolerabel ist; diese Varianz ist für die normale Spannweite in Tab.11 und 12 verantwortlich. Bei den Tonverläufen gibt es aber größere Diskrepanzen, die darauf zurückzuführen sind, daß man hier auf ein unterschiedliches Markierungsinventar zurückgreifen kann. Diese Sachlage stützt ein Prototypenkonzept, das nicht mit Regeln, sondern mit typischen Merkmalbündeln arbeitet: Eine gewisse Varianz kann auf Unschärfen in der phonetischen Realisierung der Regeln zurückgeführt werden. 'Gegenläufige', also stark unterschiedliche Realisierungen, wie wir sie vorfinden, könnten aber nur mit Regeln erfaßt werden, die mal die eine, mal die andere Realisierung erlauben. Eine Klassifikation anhand typischer, aber nicht immer obligatorischer Merkmale erscheint hier einleuchtender. (Vgl. dazu Medin/Barsalou 1987:463).13

Wir müssen im Augenblick dahingestellt lassen, inwieweit die Korrelationen der Prädiktorvariablen untereinander automatisch, also produktionsbedingt sind; ein ausgeprägter Fo-Anstieg bedingt z.T. auch eine größere Dauer als ein sehr geringer Fo-Anstieg, ein höheres Fo-Maximum erhöht die Intensität, usw. Auf der anderen Seite lassen unsere Daten doch den Schluß zu, da $\beta$ die Parameter in einem gewissen Ausmaß auch willkürlich und voneinander unabhängig gesteuert werden können.

\section{Gewinnung der Prototypen über Hörerurteile}

Wir wollen nun einen anderen Weg der Gewinnung von Prototypen beschreiben, der uns zum gleichen Ziel führen wird. Man kann Prototypen als die Fälle auffassen, die in Hörexperimenten als die 'natürlichsten' bestimmt werden, wobei wir Natürlichkeit operational im sinne unseres 'Natürlichkeitstests' (vgl. Teil 2.2) definieren. ( $\mathrm{Zu}$ einem ähnlichen Vorgehen, allerdings mit anderen Schwel-

13 In einigen Fällen kann man eventuell pragmatische oder textlinguistische Verwendungsbedingungen festlegen, die unterschiedliche Realisationen möglich bzw. regulär machen; Beispiele wären die 'Fragehaltigkeit' oder die 'intonatorische Kongruenz' in Batliner $(1989 \mathrm{~b})$. Bei unserem Material wurden aber die Randbedingungen - also auch die pragmatischen Verwendungsbedingungen - bei allen sechs Sprechern konstant gehalten. 
lenwerten, vgl. Oppenrieder $1988 \mathrm{~b}$ und Batliner 1988). Wir wollen die Schwelle nun sehr hoch ansetzen und definieren als Prototypen die Fälle, die bei $|F O K|$ einen Wert von 1.0 (100\%ige Übereinstimmung), bei MOD einen Wert von $80 \%$ für Fragen bzw. 20\% für Nichtfragen (sehr gute Kategorisierung) und im Natürlichkeitstest Werte unter 2.0 (passend bis 'sehr passend') erreichen.14 Auf diese Weise erhalten wir gut $6 \%$ der Fälle (24 Außerungen). Mit fünf Ausnahmen entsprechen die Verläufe denen aus Fig.2, also den über die große Zahl gewonnenen Prototypen. Fig. 11 bis 14 sind typische Vertreter für je eine der vier Modusund Fokuskonstellationen; sie illustrieren an konkreten Außerungen die Mittelwerte von Fig.2. Von oben nach unten finden sich in diesen Abbildungen das Zeitsignal, die Intensität, der Verlauf der Ht-transformierten Fo-Werte sowie der Verlauf der Fo-Werte. ${ }^{15}$

Für drei der fünf Ausnahmen (Randtypen) steht Fig.15, also der für Sp6 typische Verlauf bei FOKUS 2, FRAGEN. Die beiden anderen sind von Sp5 produziert, der also in seinem Inventar über beide Fokussierungsarten verfügt (vgl. Fig.9, die verdeutlicht, daß für diesen sprecher die Mittelwerte bei FOKUS 2, FRAGEN dem Kerntyp entsprechen). Fig.16 zeigt den für Sp6 typischen Verlauf bei FOKUS 3, NICHT-FRAGEN (vgl. die Mittelwerte in Fig.10), Fig.17 den für Spl typischen Verlauf bei FOKUS 2, FRAGE (vgl. die Mittelwerte in Fig.5).

\footnotetext{
14 Die exakten Schwellenwerte sind natürlich arbiträr. Man könnte untersuchen, wie sich eine schrittweise Veränderung dieser Werte im einzelnen auswirkt.

15 Bei den Fo-Werten wird für je drei Frames ein Wert dargestellt, wobei ein Frame 12,5 msec umfaßt. Die Skalierung der Ht-Achse ergibt sich aus (Ht*100); zur Skalierung der Intensitätswerte vgl. Teil 2.1. Geringfügige fehlende Ubereinstimmungen zwischen Zeitsignal und Fo-Verlauf sind auf die automatische Stimmhaft-Stimmlos-Entscheidung zurückzuführen, die der FoExtraktion vorausging.
} 
Fig.11: FOKUS 2, NICHT-FRAGE (Sp5)

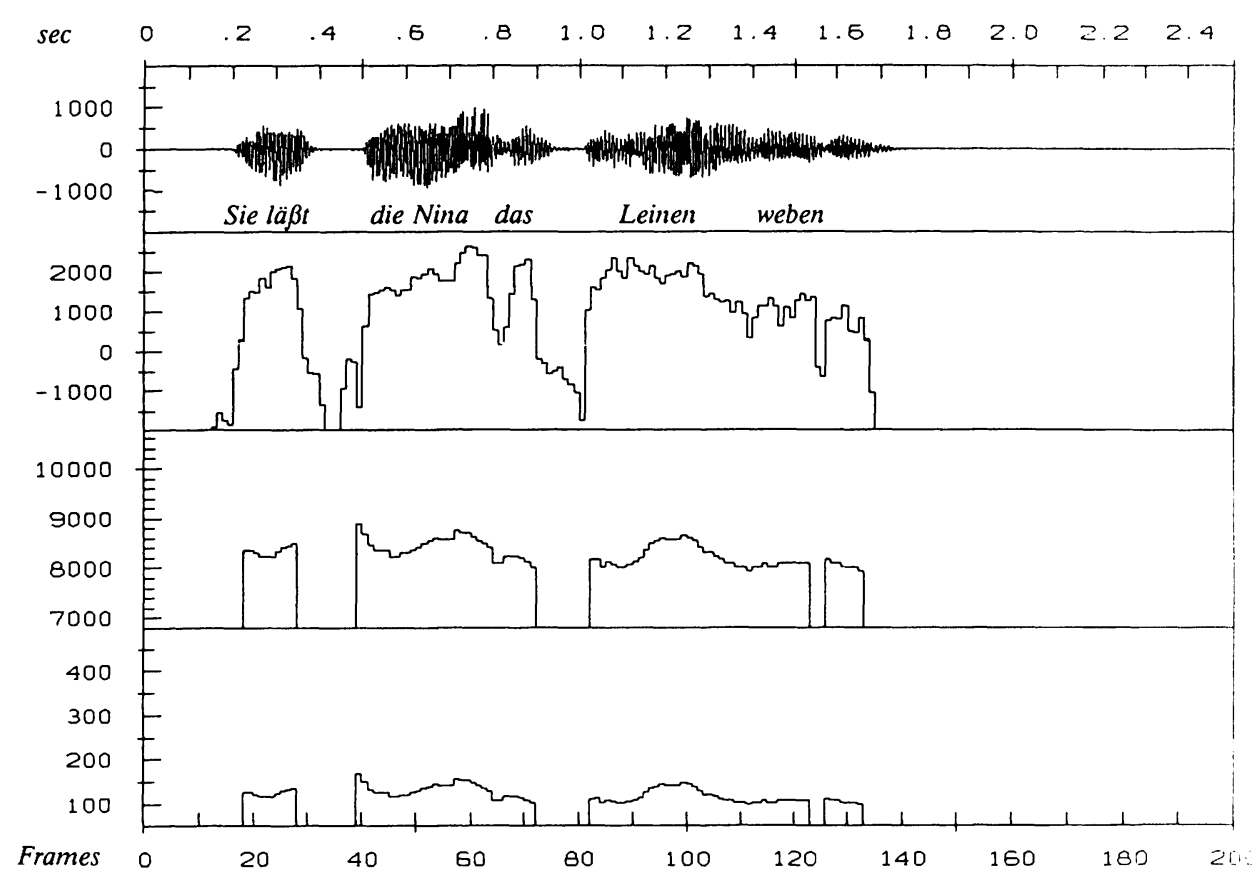

Fig.12: FOKUS 3, NICHT-PRAGE (Sp5)

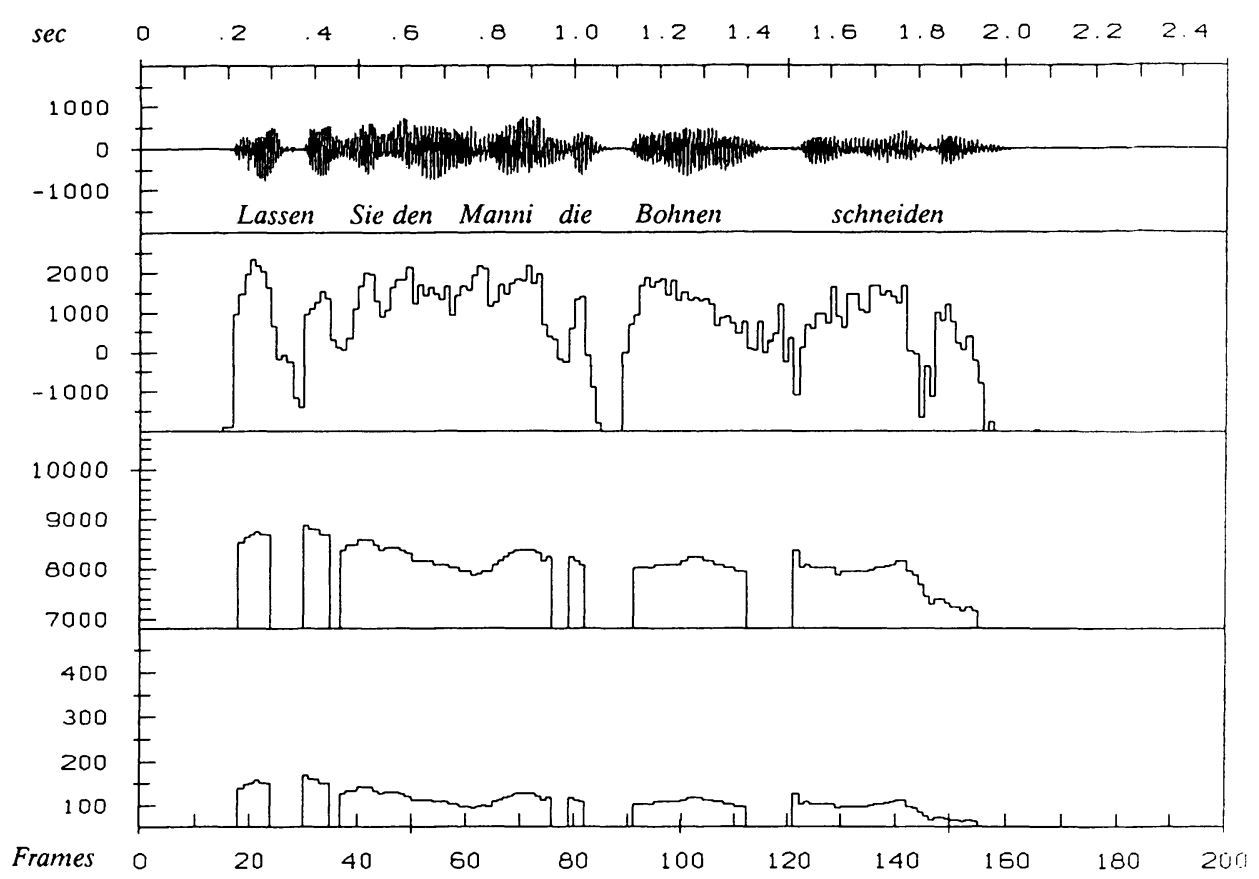


Fig.13: FOKUS 2, FRAGE (Sp5)

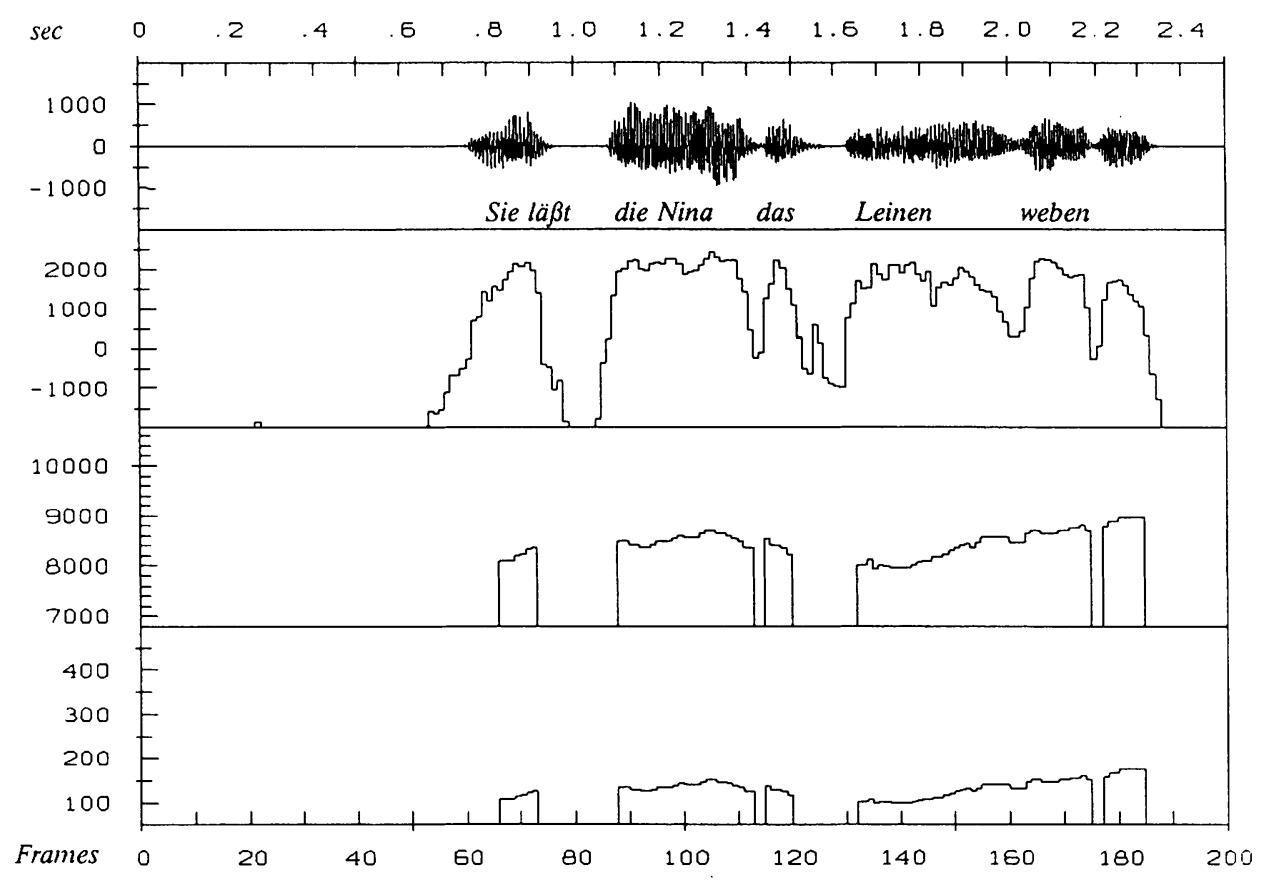

Fig.14: FOKUS 3, FRAGE (Sp1)

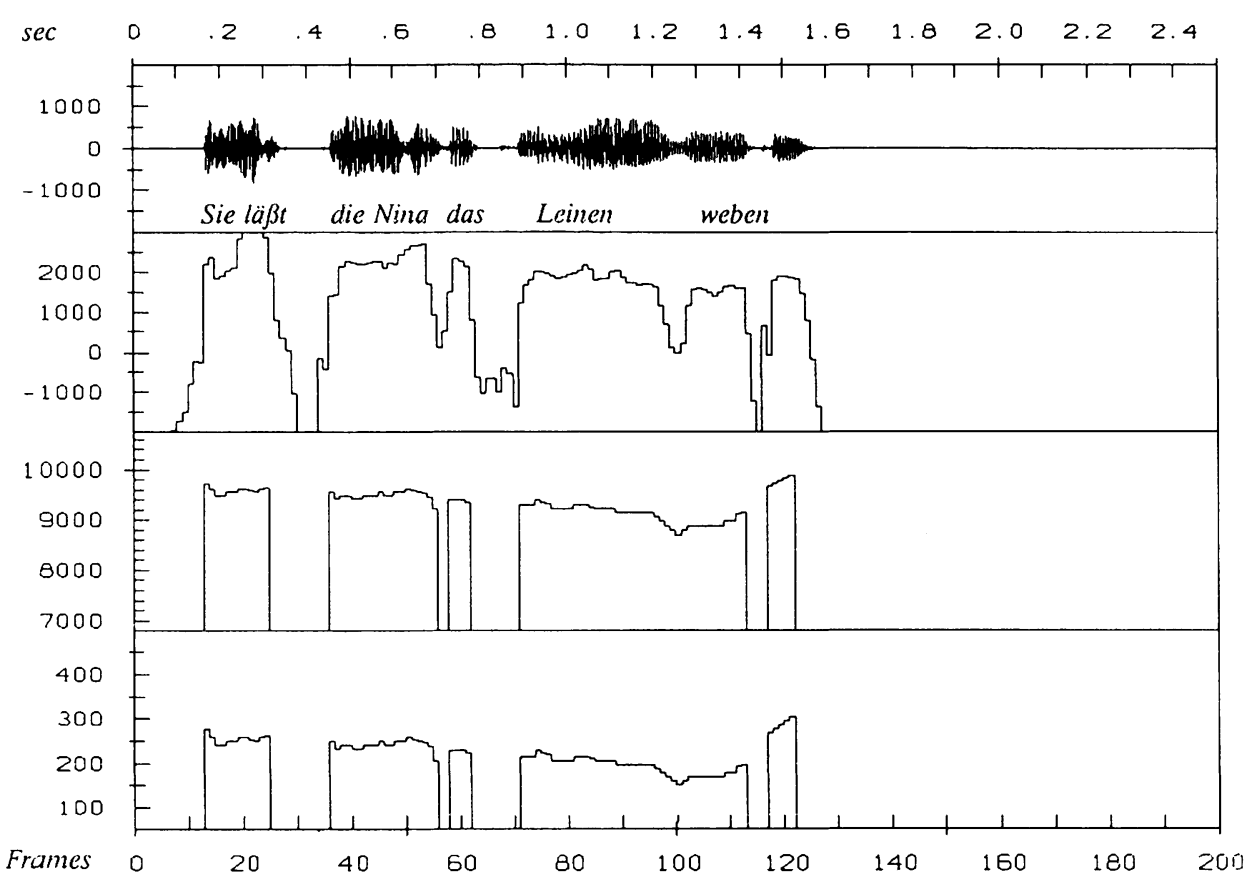


Man sieht, daß sich die beiden Methoden der Prototypengewinnung z.T. ergänzen, z.T. komplementär sind: mit beiden Methoden erhält man die repräsentativen (Kern-) Typen; Randtypen, die nicht repräsentativ, aber ebenso akzeptabel sind, erhält man dagegen nur über die Hörerurteile - wobei wir immer die Einschränkung machen müssen, daß dies alles nur gilt, wenn die sprecher (= die Stichprobe) in einem gewissen Ausmaß repräsentativ sind für die untersuchte Varietät (= die Population).

Die Abbildungen nach Fig.17 sind Batliner/Nöth (1989) entnommen; in ihnen sind die Mittelwerte der Kerntypen aus Fig.2 (stbas $=$ HT-Basis, $t=$ Centisekunden) auf die konkreten Verläufe der über die Hörerurteile gewonnenen Prototypen von Fig.11 bis 17 projiziert. Dadurch ist ein direkter Vergleich möglich. Neben den Abbildungen sind jeweils ihre Entsprechungen in Fig.2 bzw. in Fig.11 bis 17 angegeben. Die gestrichelte Linie zeigt die Grenze zwischen der 2. und der 3. Phrase für die konkrete Außerung.

Fig.15: FOKUS 2, FRAGE (Sp6)

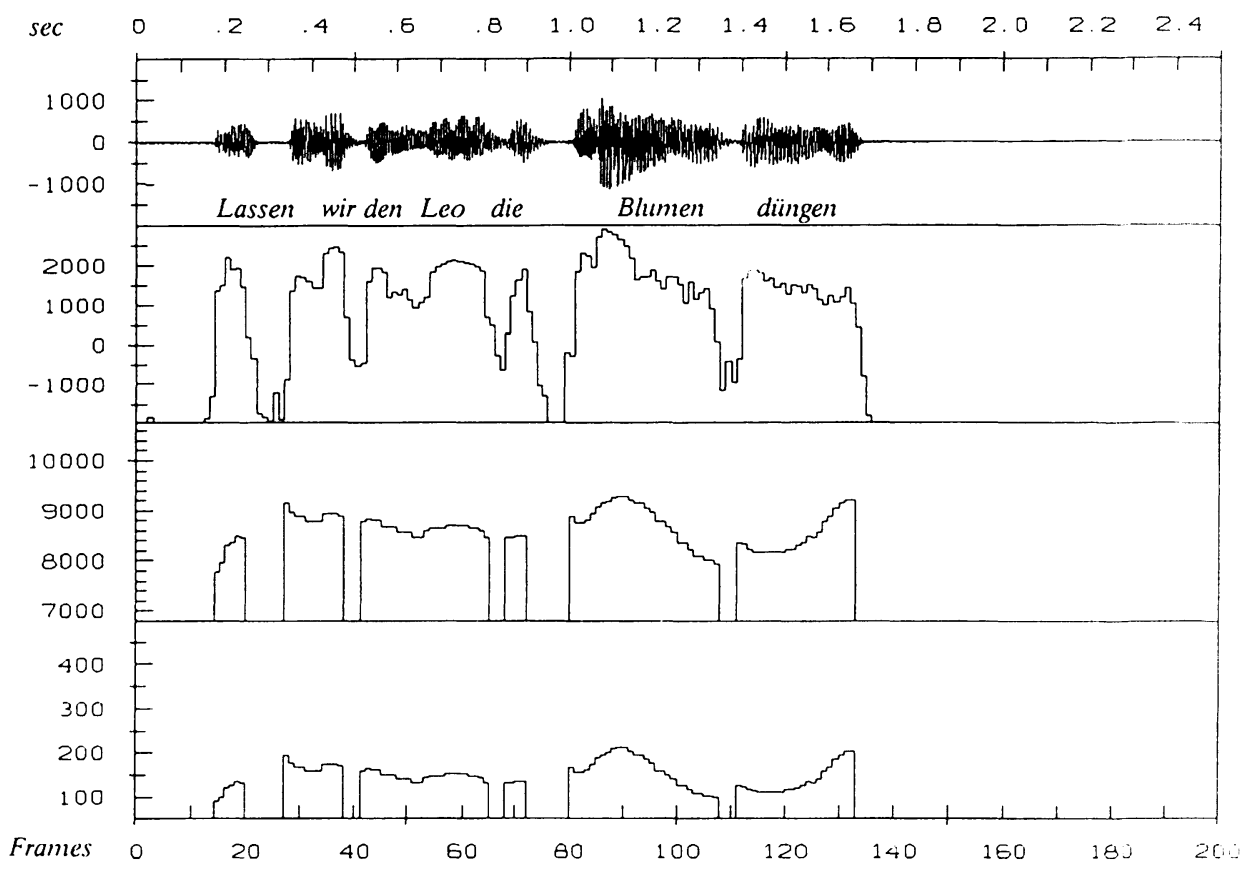


Fig.16: FOKUS 3, NICHT-FRAGE (Sp6)

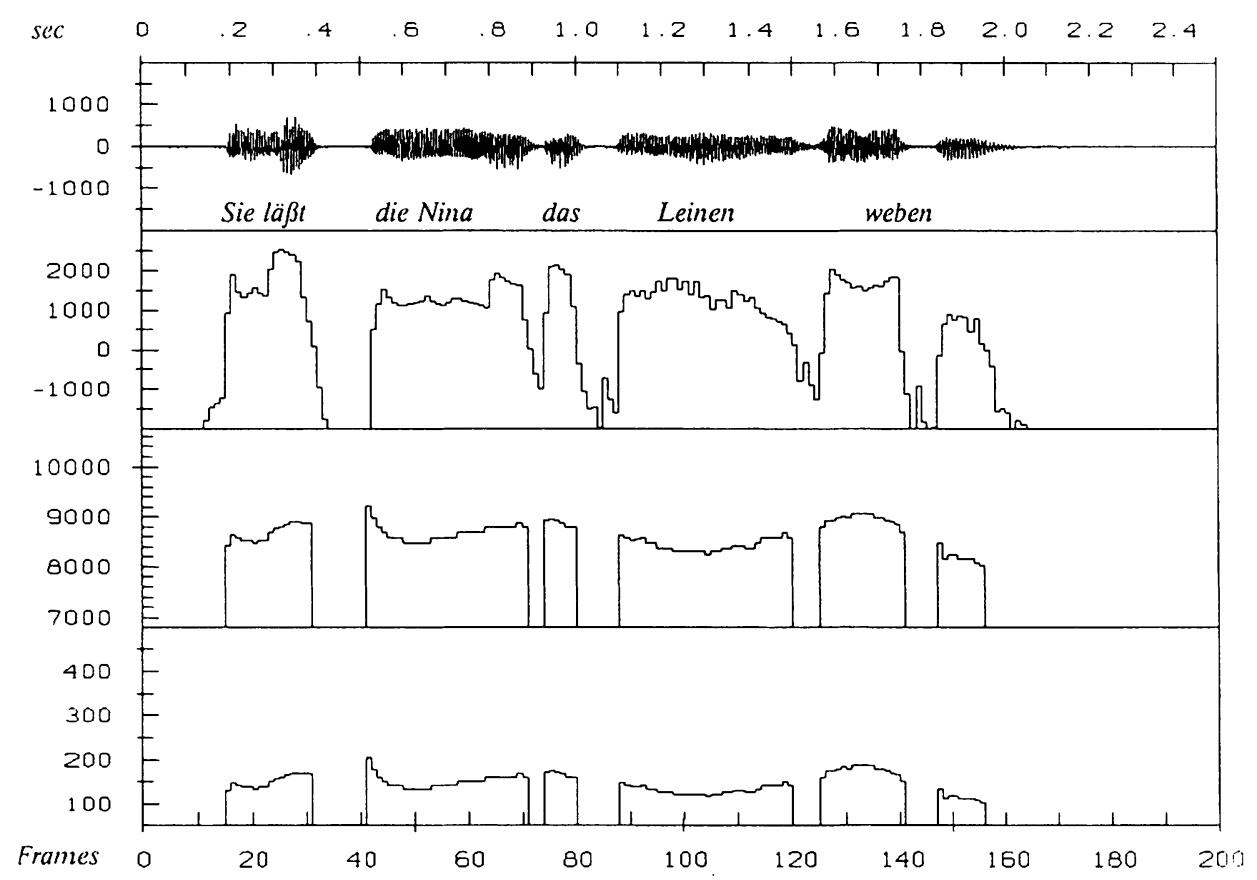

Fig.17: FOKUS 2, FRAGE (Sp1)

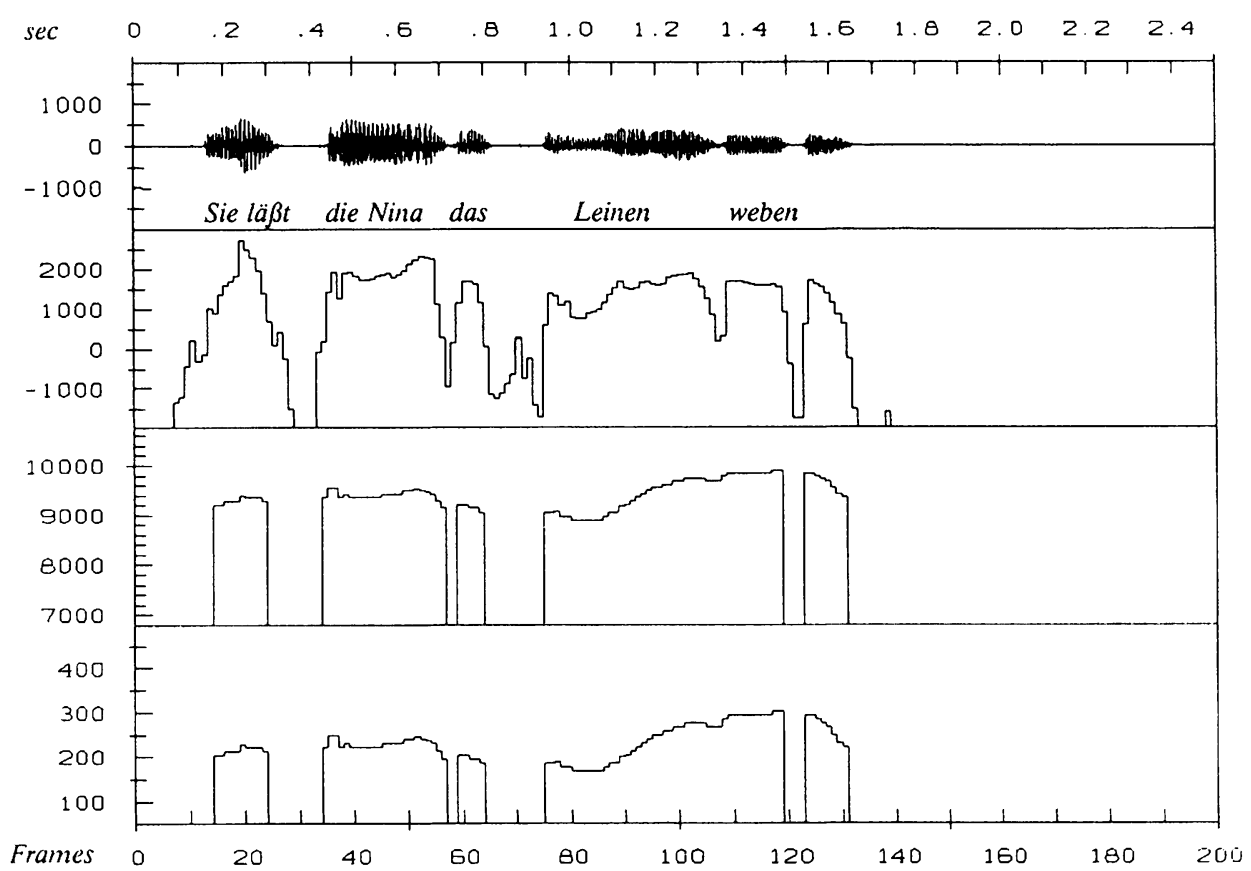


Aus Batliner/Nöth (1989):

Projestion von

Fig. 2 (oben links) auf Fig.11 st $_{\text {bas }} \quad$ Fig.1: Focus on $2^{\text {id }}$ phrase, non-question, central type

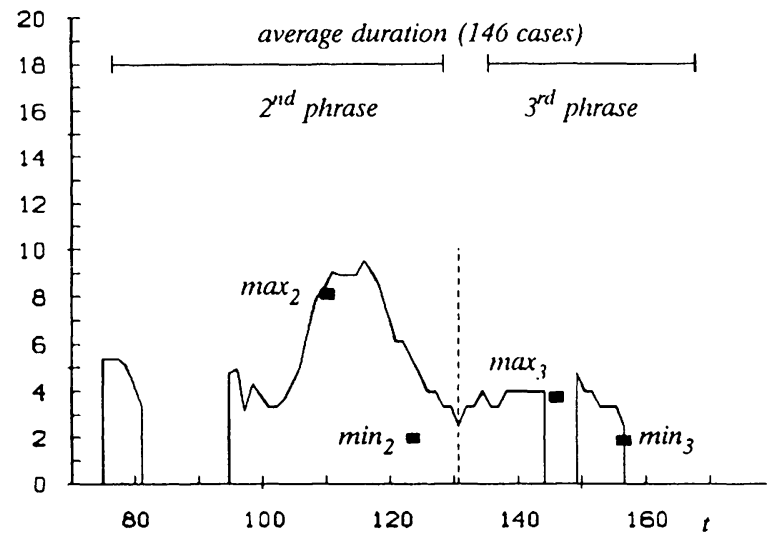

st bas $\quad$ Fig.3: Focus on $3^{\text {rd }}$ phrase, non-question, central type

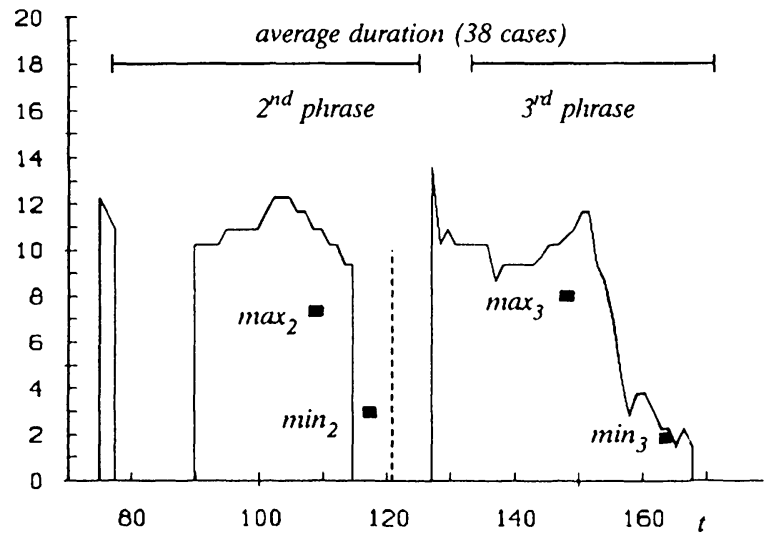

Fig.2 (unten links) auf Fig.12

Projektion von

Fig.2 (oben rechts) auf Fig.15

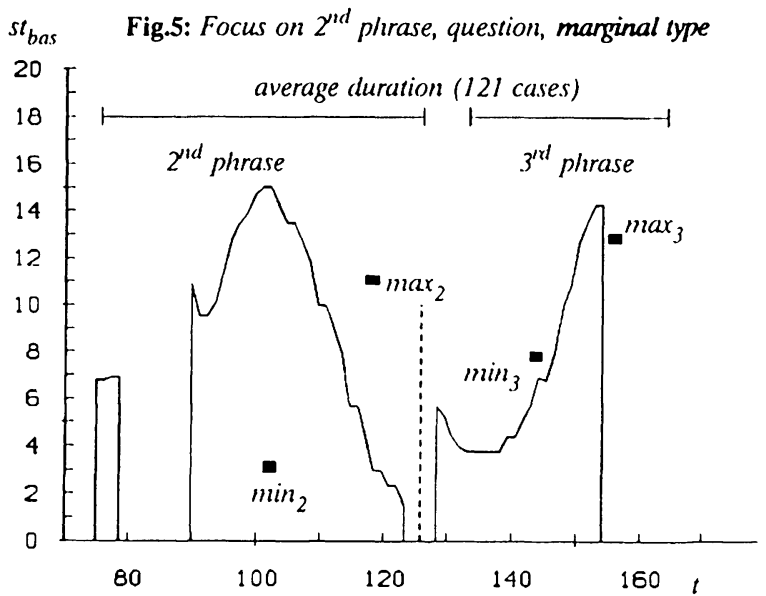




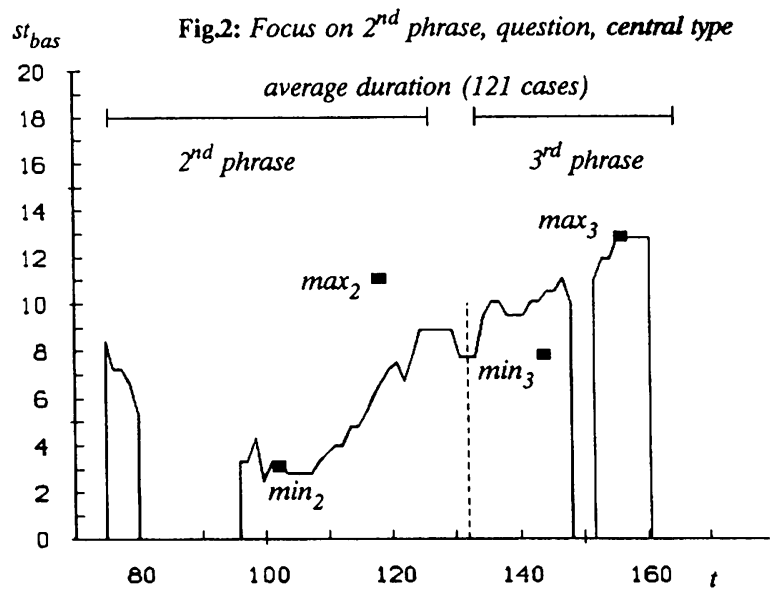

Projektion von

Fig.2 (oben rechts) auf Fig.13

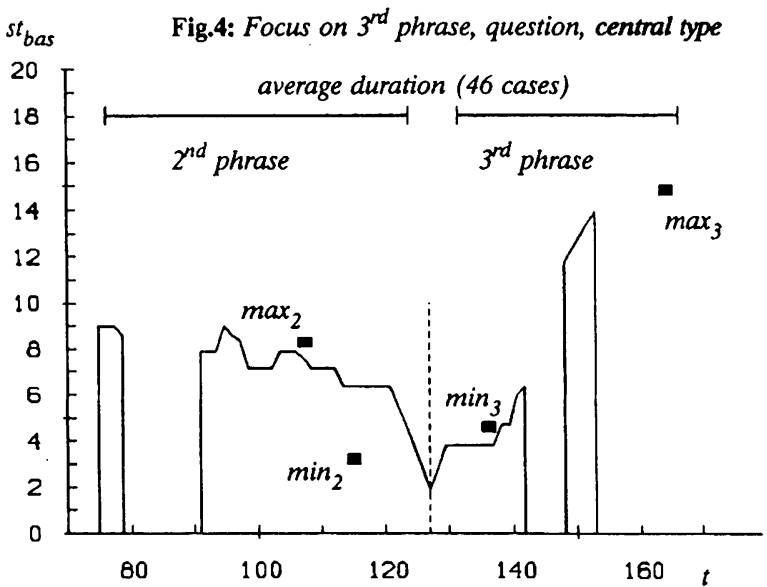

Projektion von

Fig.2 (unten rechts) auf Fig.14

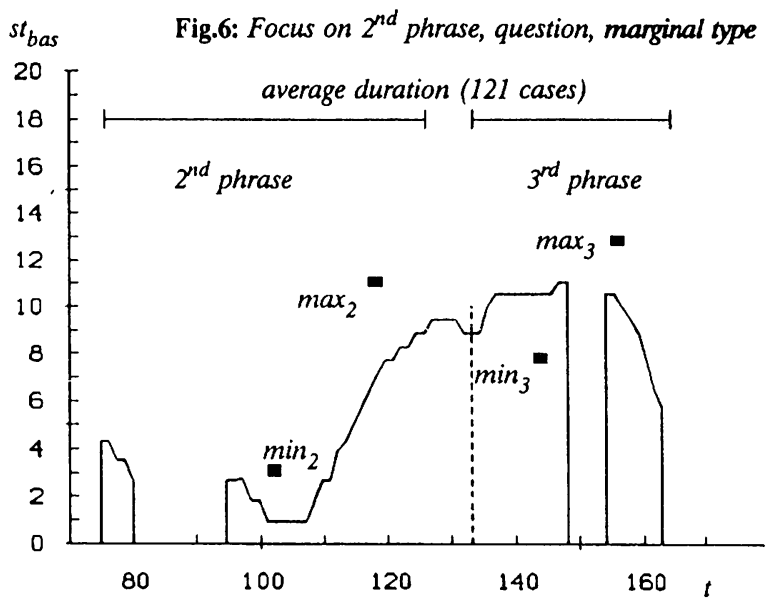

Projektion von

Fig.2 (oben rechts) auf Fig.17 


\section{Projektion von}

Fig.2 (unten links) auf Fig.16

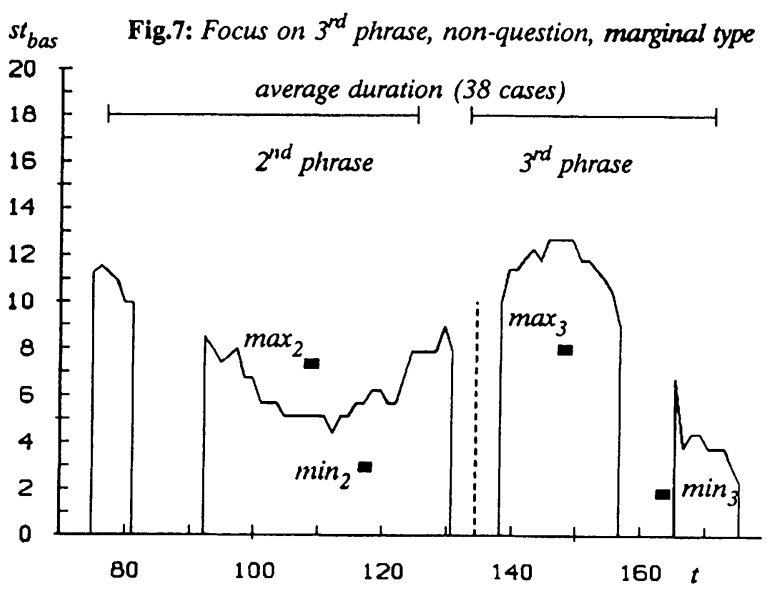

9. Fehleranalyse der Klassifikation durch die Diskriminanzanalyse

Die Fehleranalyse wird anhand der Konstellation (3) aus Tab.9, also für Lernstichprobe $=$ Prüfstichprobe, durchgeführt. Der Unterschied zwischen dieser Konstellation und Konstellation (4) ('leave-one-out') liegt 2 wischen $2 \%$ und $8 \%$. Wir legen also eine Klassifikation zugrunde, die in diesem Ausmaß auf uberadaptation beruhen dürfte (vgl. Ruske 1988:25). Auf der anderen Seite ist zu bedenken, daß die Stichprobe immer noch relativ klein ist; man kann also damit rechnen, da $\beta$ sich bei einer genügend großen Stichprobe die Prädiktion auch bei 'leave-one-out' verbessern würde (für $n \rightarrow \infty$ gilt: Die Klassifikationsgüte bei 'leave-one-out' geht gegen die Klassifikationsgüte bei Lern- = Prüfstichprobe). Wir entschieden uns für dieses Vorgehen, da bei dieser Konstellation wirklich nur die Fälle fehlklassifiziert werden, bei denen selbst eine sehr gute, überadaptierte Klassifikation versagt (das heißt nicht unbedingt, daß das statistische Modell für diese Fälle nicht adäquat ist; vgl. Teil 9.2 und 10).

Obwohl die Güte der Klassifikation sehr hoch ist, bleiben noch 27 Fehlklassifikationen, 10 bei MODUS und 19 bei FOKUS; d.h. daß zwei Fälle sowohl bei MODUS als auch bei FOKUS fehlklassifiziert wurden. Bei einer automatischen Zuweisung zu Frage/Nicht-Frage durch die Diskriminanzanalyse und darauffol- 
gender Fokusprädiktion ist das Ergebnis sogar besser, da zwei Fälle ('Fehlproduktionen', vgl. Teil 9.1) 'dank' der falschen Moduszuweisung richtig klassifiziert werden.

\section{$9.1 \quad$ Modus}

In allen 10 Fällen wurden Fragen fehlkategorisiert; In 8 der 10 Fälle ist MOD gleich Null, d.h. die Hörer konnten im kontextfreien Kategorisierungstest die Außerungen nicht als Frage erkennen. Die Natürlichkeitsbewertung war schlecht bis sehr schlecht (>3.5). Es handelt sich also bei diesen 'Fragen' mit einem tiefen offset eindeutig um Fehlproduktionen. Eine Außerung wurde zwar als Frage erkannt, erhielt aber eine sehr schlechte Natürlichkeitsbewertung. Es bleibt ein letzter und interessanter Fall: Diese Außerung wurde zwar nicht als Frage erkannt, erhielt aber eine relativ gute Natürlichkeitsbewertung. Möglicherweise handelt es sich dabei um den in unserem Korpus singulären Fall einer assertiven Frage mit tiefem offset (vgl. dazu Geluykens 1987 und Batliner/Oppenrieder 1988).

$9.2 \quad$ Fokus

Drei Beobachtungen können gemacht werden:

1. Bei den 19 Fehlklassifikationen von FOKUS ist bei 11 Fällen eine auffällige Diskrepanz (geringe Ubereinstimmung oder gar Gegenläufigkeit) $z$ wischen den beiden Akzenttests $z u$ beobachten. Zum einen mag ein systematischer Einfluß des unterschiedlichen Versuchsdesigns die Ursache sein: Es ist nicht ganz klar, ob die Versuchspersonen das gleiche machen, wenn die Fälle unterschiedlich randomisiert sind (wie das beim 1 . und bejm 2. Akzenttest der Fall ist) und einmal nur die am stärksten betonte Silbe, das andere Mal zwei Akzentstufen vergeben werden müssen. Zum anderen sind Akzenturteile grundsätzlich nicht sehr konsistent, vgl. die Diskussion in Teil 10.

2. In 12 Fällen (60\% der Fehlklassifikationen) ist die Akzentstruktur offensichtlich nicht sehr ausgeprägt, da FOK hier zwischen .4 und -.4 liegt. In diesem Bereich liegen nur 15\% aller Fälle, vgl. Fig.1.

3. In 7 Fällen zeigt sich eine 'Unsicherheit' der Diskriminanzanalyse dadurch, daß die Wahrscheinlichkeit der Gruppenzuordnung nicht ausgeprägt ist, sondern nahe der 50\%-Grenze liegt. 'Unsicherheiten' dieser Art sind sehr selten, normalerweise entscheidet sich die Diskriminanzanalyse mit hoher Wahrscheinlichkeit Pür eine der Gruppen. Bei solchen 'Grenzfällen' kann auch eine Verletzung der für die Diskriminanzanalyse erforderlichen Voraussetzungen eine Fehlklassifikation bewirken.

Wie man sieht, summieren sich die Zweifelsfälle auf mehr als 19, da in 11 Fällen mehr als eine der Beobachtungen gemacht werden konnte, also etwa eine 
gleichzeitige 'Unsicherheit' der Diskriminanzanalyse und eine geringe Ausprägung der Akzentstruktur. Zwei Fälle bleiben, die auch durch Inspektion der Daten nicht erklärt werden können.

Bei den Fehlklassifizierungen von FOKUS muß man bedenken, daß die $\mathrm{Zu}-$ weisung zu FOKUS 2 oder FOKUS 3 auf Hörerurteilen beruht, die - wie der Unterschied $z$ wischen den beiden Akzenttests und die relativ hohe Anzahl von nicht sehr entschiedenen Urteilen zeigt - selbst mit einer gewissen Unschärfe behaftet sind. Diese Unschärfe war auch beabsichtigt, da der Kontext nur in einem Drittel der Fälle einen einfachen Fokus vorgab, ansonsten aber entweder Fokusprojektion oder zweifachen Fokus. Die Annahme erscheint also plausibel, da $\beta$ das Verfahren unter ansonsten vergleichbaren Randbedingungen dann, wenn der Sprecher eindeutig fokussiert - und nur dann können die Hörer auch zu eindeutigen Urteilen kommen - praktisch fehlerfrei arbeitet.

\section{Kerntypen, Randtypen und Trennschärfe}

Ob ein Vokal kurz oder lang ist bzw. sein soll, kann man auch dem Aussprachelexikon entnehmen; ein vergleichbares FA-Lexikon gibt es nicht - und das ist kein Zufall: Ohne daß uns einschlägige Arbeiten bekannt wären, vermuten wir, da $\beta$ Vokallängen und -kürzen von nativen Sprechern konsistenter produziert und auch eindeutiger perzipiert werden, als das bei den für den FA relevanten Parametern der Fall ist (diese Vermutung gilt für im Labor elizitierte Sprache; bei spontaner sprache mag es anders aussehen). Wie auch schon Lieberman (1965) festgestellt hat, ändert sich die Transskription der Tonverläufe durch kompetente Linguisten in starkem Maße, wenn statt der natürlich produzierten Außerungen synthetisierte Aquivalente der Fo-Verläufe vorgegeben werden. Brown et al. (1980:139ff) berichten von ähnlichen Schwierigkeiten bei der Zuweisung des tonic (also des FA); vgl. auch Lickey/Waibel (1985). Bei uns ergaben sich aufällige Diskrepanzen zwischen Akzenttest 1 und Akzenttest 2; sicher hätten sich weitere Diskrepanzen ergeben, wenn wir in einem zusätzlichen Test die Außerungen in ihren modus- und fokussteuernden Kontexten zur Akzentzuweisung vorgegeben hätten. ${ }^{16}$ Erst bei einer Kontexteinbettung hätten aber die intonatorischen Merkmale den ihnen zukommenden Platz als eine - wenn auch wichtige - Gruppe fokusindizierender Mermale unter anderen grammatischen (phonetischen, syntaktischen, semantischen).

16 Der normale 'Default'-FA liegt bei unseren đußerungen auf der 2. Phrase. Ohne Kontext erwarten die Hörer diese Position, d.h. im Zweifelsfall dürften sie den FA dieser Position zuweisen. 
Die von uns vorgenommene Zuweisung des FA auf die 2. oder die 3. Phrase anhand des Akzenttests ist also zwar intersubjektiv abgestützt; da ihr aber die Beurteilung der isolierten Außerungen zugrundelag, kann nicht ausgeschlossen werden, daß die intonatorischen Merkmale im einzelnen oder der Komplex der intonatorischen Mermale im ganzen im Zusammenhang mit all den anderen grammatischen Merkmalen anders gewichtet wären. Wenn wir also innerhalb der intonatorischen Merkmale multivariate (und damit 'realistischere' als univariate) Analysen durchführen, so arbeiten wir auf einer höheren stufe trotzdem nur univariat, da wir von den relevanten grammatischen Merkmalskomplexen nur den intonatorischen berücksichtigen.

Eine gleichzeitige und systematische Berücksichtigung aller grammatischen Merkmale in elnem experimentellen Design ist auf lange Sicht wegen des damit verbundenen Aufwands nicht vorstellbar; die Lage ist aber nicht hoffnungslos, wenn man sich von den unscharfen (fuzzy) Grenzen zwischen Kategorien abund den Kernbereichen zuwendet: Eindeutig fokussierte Phrasen werden vom Menschen auch eindeutig als fokussiert wahrgenommen und vom automatischen Verfahren richtig klassifiziert. Es dürfte also sinnvoll sein, sich stärker als bisher nicht mit Kategoriengrenzen (wie im Paradigma der Kategorialen Wahrnehmung, vgl. Schiefer/Batliner 1988) zu beschäftigen, sondern mit den Kernbereichen der Kategorien, eben den Prototypen.

\section{Schlußbemerkungen}

Wir haben versucht, nicht nur einen Faktor zu variieren und alle anderen konstant $\mathrm{zu}$ halten, sondern mehrere Faktoren $\mathrm{zu}$ variieren, um dadurch eine größere Nähe zur natürlichsprachlichen Kommunikation zu erreichen: es wurden Außerungen mehrerer Sprecher untersucht, die ohne explizite Instruktionen und bei gleichzeitiger Varilerung von Modus und Fokus produziert wurden. Dagegen stehen zwei Beschränkungen, zum einen die auf eine bestimmte Varietăt des Deutschen und zum anderen die auf drei Testsätze mit syntaktisch und segmental āhnlicher Struktur. Die Ergebnisse sollten auch an spontansprachlichen Korpora überprüft werden. 
LITERATUR

Adriaens, L.M.H. (1984): A preliminary description of german intonation. In: IPO Annual Progress Report 19. S.36-41.

Altmann, H. (1987): Zur Problematik der Konstitution von Satzmodi als Formtypen. In: Meibauer, J. (Hg.) (1987): Satzmodus zwischen Grammatik und Pragmatik. Tübingen. S.22-56.

Altmann, H. (Hg.) (1988): Intonationsforschungen. Tübingen.

Bannert, R. (1985): Fokus, Kontrast und Phrasenintonation im Deutschen. In: Zeitschrift für Dialektologie und Linguistik 52. S.289-305.

Batliner, A. (1988): Produktion und Prädiktion. Die Rolle intonatorischer und anderer Merkmale bei der Bestimmung des Satzmodus. In: Altmann, H. (Hg.) (1988). S.207-221.

Batliner, A. (1989a): Fokus, Deklination und Wendepunkt. (In diesem Band)

Batliner, A. (1989b): Wieviel Halbtöne braucht die Frage? Merkmale, Dimensionen, Kategorien. (In diesem Band)

Batliner, A./Nöth, E./Lang, R./Stallwitz, G. (1989): Zur Klassifikation von Fragen und Nicht-Fragen anhand intonatorischer Merkmale. (Erscheint in: Tagungsband der 15. Gemeinschaftstagung der Deutschen Arbeitsgemeinschaft für Akustik (DAGA). 13.-16. März 1989)

Batliner, A./Oppenrieder, W. (1988): Rising intonation: Not passed away but still alive. A reply to $R$. Geluykens. In: Journal of Pragmatics 12. S.227-233.

Batliner, A./Oppenrieder, W. (1989): Korpora und Auswertung. (In diesem Band)

Batliner, A./Nöth, E. (1989). The prediction of focus. Proceedings of the European Conference on Speech Communication and Technology, Paris, 2628 September 1989. (Im Druck)

Beckman, M.E. (1986): Stress and Non-Stress Accent. Dordrecht.

Brown, G./Currie, K.L./Kenworthy, J. (1980): Questions of Intonation. London.

Cohen, A./'t Hart, J. (1967): On the anatomy of intonation. In: Lingua 19. S.177-192

Geluykens, R. (1987): Intonation and speech act type: An experimental approach to rising intonation in queclaratives. In: Journal of Pragmatics 11. S.483494.

't Hart, J. (1986): Declination has not been defeated - A reply to Lieberman et al. In: J. Acoust. Soc. Am. 80. S.1838-1840.

Hildebrand, D.K./Laing, J.D./Rosenthal, H. (1977): Analysis of Ordinal Data. Sage University Paper series on Quantitative Applications in the Social Sciences, 07-008. Beverly Hills and London.

Hörmann, H. (1978): Meinen und Verstehen. Frankfurt am Main. 
Klecka, W.R. (1980): Discriminant Analysis. Sage University Paper series on Quantitative Applications in the Social Sciences, 07-019. Beverly Hills and London.

Klein, W. (1980): Der Stand der Forschung zur deutschen Satzintonation. In: Linguistische Berichte 68. S.3-33.

Klein, W. (1982): Einige Bemerkungen zur Frageintonation. In: Deutsche Sprache (1982). S.289-310.

Lakoff, G. (1987): Women, Fire, and Dangerous Things. What Categories Reveal about the Mind. Chicago und London.

Lickey, S.A./Waibel, A. (1985): Perceptual Stress Assignements. (Anhang zu: Waibel, A. (1986): Prosody and Speech Recognition. Carnegie Mellon University, Computer Science Departement. S.192-198.

Lieberman, P. (1965): On the Acoustic Basis of the Perception of Intonation by Linguists. In: Word 21. S.40-54.

Lieberman, P. (1986): Alice in declinationland - A reply to Johan 't Hart. In: J. Acoust. Soc. Am. 80. S.1840-42.

Lieberman, P./Katz, W./Jongman, A./Zimmerman, R./Miller, M. (1985): Measures of the sentence intonation of read and spontaneous speech in American English. In: J. Acoust. Soc. Am. 77. S.649-657.

Medin, D.L./Barsalou, L.W. (1987): Categorization processes and categorical perception. In: Harnad, S. (Hg.) (1987): Categorical perception. The groundwork of cognition. Cambridge etc. S.455-490.

Niemann, H. (1974): Methoden der Mustererkennung. Frankfurt am Main.

Norusis, M.J. (1986): SPSSPC+ Advanced Statistics. Chicago.

Nöth, E. (1989): Prosodische Information in der automatischen Spracherkennung - Berechnung und Anwendung. Diss., Lehrstuhl für Informatik 5, Universität Erlangen. (in Vorbereitung)

Nöth, E./ Batliner, A./ Lang, R./ Oppenrieder, W. (1987): Automatische Grundfrequenzanalyse und Satzmodusdifferenzierung. In: Tillmann, H.G./Willée G.

(Hgg.) (1987): Analyse und Synthese gesprochener Sprache. Hildesheim etc. S.59-66.

Oppenrieder, W. (1988a): Intonation und Identifikation. Kategorisierungstests zur kontextfreien Identifikation von Satzmodi. In: Altmann, H. (Hg.) (1988). S. 153-167.

Oppenrieder, W. (1988b): Intonatorische Kennzeichnung von Satzmodi. In: Altmann, H. (Hg.) (1988). S.169-205.

Oppenrieder, W. (1989): Fokus, Fokusprojektion und ihre intonatorische Kennzeichnung. (In diesem Band)

Pierrehumbert, J.B. (1980): The Phonology and Phonetics of English Intonation. PhD Dissertation, MIT. 
Ruske, G. (1988): Automatische Spracherkennung. Methoden der Klassifikation und Merkmalsextraktion. München/Wien.

Schiefer, L./Batliner, A. (1988): Intonation, Ordnungseffekt und das Paradigma der Kategorialen Wahrnehmung. In: Altmann, H. (Hg.) (1988). S.273-291.

Taylor, S./Wales, R. (1987): Primitive mechanisms of accent perception. In: Journal of Phonetics 15. S.235-246.

Thorsen, N.G. (1987): Suprasegmental Transcription. In: Almeida, A./Braun, A. (Hgg.): Probleme der phonetischen Transkription. Stuttgart. S.79-109.

Wunderlich, D. (1988): Der Ton macht die Melodie - Zur Phonologie der Intonation des Deutschen. In: Altmann, H. (Hg.) (1988). S.1-40. 
FOKUS, DEKLINATION UND WENDEPUNKT

Anton Batliner

1. Einleitung

In Batliner (1989a) wird die intonatorische Markierung des Fokus im Deutschen an nicht manipuliertem, natürlichsprachlichen Material (elizitierten Außerungen) untersucht. Bei solchen Produktionsdaten ist es nicht möglich, den Beitrag einzelner Merkmale exakt zu bestimmen - wohl aber bei Perzeptionsexperimenten mit manipuliertem Material. Diese größere 'Exaktheit' wird aber mit zwei Nachteilen erkauft: Zum einen können durch die Manipulation Artefakte entstehen, wie z.B. eine nicht vorhersagbare Reaktion auf Stimuli, die in dieser Form zwar vom Rechner generierbar, vom Menschen aber nicht produzierbar sind; zum anderen kann man nur einzelne Merkmale durch eine systematische Variierung dieser Merkmale bei gleichzeitigem Konstanthalten der anderen Merkmale, kaum aber das Zusammenspiel aller relevanten Merkmale untersuchen.

Mit diesen Bemerkungen ist der grundsätzliche Stellenwert der im folgenden beschriebenen Perzeptionsexperimente umrissen. Wir werden an zwei Testsätzen mit gleicher segmentaler Struktur, aber unterschiedlicher Wortstellung die Rolle der Grundfrequenz- (Fo-) Deklination für den Aussagesatz (Testsatz: Der Leo säuft) und die des Fo-Wendepunkts bei einer fallend-steigenden Kontur für den Fragesatz (Testsatz: Säuft der Leo) bei der Markierung des Fokus untersuchen (zur Wahl gerade dieser Testsätze vgl. Batliner 1988a). Bei unseren kurzen Testsätzen kann der Fokusakzent (FA) im nicht-kontrastiven Normalfall zwei Positionen einnehmen: eine frühe auf der ersten oder eine späte auf der zweiten Phrase. 


\subsection{Fragestellung}

Im allgemeinen versteht man unter 'Deklination' den allmählichen Abfall des Fo-Verlaufs zum Außerungsende hin, wobei der Abfall der Fo-Minima weniger ausgeprägt ist als der der Fo-Maxima. Anhand von nicht-interrogativen Außerungen ohne besondere Hervorhebungen läßt sich eine unmarkierte Deklinationslinie gewinnen, zu der prominente Fo-Werte als potentielle Indikatoren von Akzenten in Beziehung gesetzt werden können.

Der eben unternommene Versuch einer Beschreibung mag ein intuitives Vorverständnis widerspiegeln; beim Versuch einer exakten Definition stößt man aber in praktisch allen Punkten auf ungeklärte Kontroversen (vgl. die Debatte $z$ wischen Lieberman et al. 1985, Lieberman 1986 und 't Hart 1986; sowie Pierrehumbert 1979, Umeda 1982 und Ladd 1984). Die folgende Liste führt einige der wesentlichen Punkte auf:

1. Hat die Deklination in spontaner Sprache die gleiche Relevanz wie in gelesener?

2. Wie unterscheidet sich die Deklination bei kürzeren von der bei längeren Außerungen?

3. Soll man bei Fragesätzen mit einem steigenden Fo-Verlauf die gleiche Deklinationslinie als Referenz ansetzen wie bei Nicht-Fragesätzen mit fallendem Fo-Verlauf?

4. Ist die Deklination automatisch (produktions-) bedingt oder willkürlich gesteuert?

5. Wie soll die Deklinationslinie aussehen: ist sie linear oder kurvilinear, durchgehend oder mit Brüchen?

6. Wie mißt man eigentlich die Deklination? - Kandidaten sind z.B. eine Regressionsgerade durch alle Fo-Werte oder nur durch besonders ausgezeichnete, z.B. durch die Fo-Minima oder Fo-Maxima. Die erste Berechnung erscheint objektiver, die zweite mag perzeptiv adäquater sein. Ladd (1984) hinwiederum bezweifelt die Relevanz solcher Berechnungen überhaupt und sucht die Lösung in der Phonologie. ${ }^{1}$

Als wir die Experimente durchführten, waren wir noch der Ansicht, man könnte auch bei unseren Stimuli (vgl. unten Tab.1) den Deklinationsverlauf genügend exakt messen. Diese Ansicht haben wir inzwischen modifiziert: Messen läßt er sich schon, aber auf unterschiedliche Weise, und welche Art der Messung die 'richtige' ist, läßt sich nicht ohne weiteres feststellen: Welcher Punkt eines ebenen oder fallenden Fo-Verlaufs, bei dem also kein klarer Fo-Wendepunkt vorliegt, kann z.B. als Fixpunkt für die Berechnung des Deklinationsverlaufs genommen werden? Die Entscheidung wird dann kritisch, wenn - wie das bei unseren stimuli (vgl. Tab.1) der Fall ist Stimuli mit einem finalen Fo-Abfall verglichen werden sollen mit Stimuli, die auf der letzten Phrase einen Gipfel aufweisen. Ohne Experimente, die sich speziell - analog zu Rossi 1978 - mit solchen Problemen befassen, bleibt die Entscheidung, ob etwa beim rein fallenden Verlauf die der Position des Gipfels im anderen Stimulus entsprechende Position auf der Zeitachse als 
Auch in diesem Beitrag werden wir diese Fragen nicht beantworten. Es ist 'nur' unser Ziel, für das Deutsche die Relevanz der Deklination für die Zuweisung des FA im Perzeptionsexperiment nachzuweisen. ${ }^{2}$ Die generelle Hypothese ist die folgende:

Später in der Außerung genügt eine geringer ausgeprägte Hervorhebung als früher in der Außerung, um einen FA zu indizieren.

Wir beschränken uns dabei auf den Fo-Verlauf und lassen Dauer- und Intensitätsphänomene außer acht. Es soll bei einem Quasi-Kontinuum untersucht werden, wann ein Umschlagen der Akzentuierung zu beobachten ist, und welchen Einfluß die Art des Fo-Verlaufs (fallend, steigend oder eben) auf die FAZuweisung hat.

\subsection{Material und Design}

Als Stimulus wurde die Außerung Der Leo säuft gewählt, da es sich in Pilottests herausgestellt hat, da $\beta$ die FA-Zuweisung aup dem frühen Element Leo bzw. auf dem späten Element säuft durch geeignete Kontextsätze gut zu steuern ist. $^{3}$ Kontextsätze waren der Satz Ihr fragt mich, wer säuft? (FRUHER FOKUS auf der Leo) und Was soll schon mit dem Leo sein? (SPATER FOKUS auf säuft); die Intonationskonturen der Kontextsätze finden sich in Batliner (1988a:225). Der Autor produzierte die Kontextsätze mit natürlicher Intonation (vgl. Batliner 1988b:245) und den Testsatz mit monotoner Intonation mehrmals im schallarmen Raum des Instituts für Phonetik in München (Aufnahmegerät Telefunken: M15, Geschwindigkeit: $19 \mathrm{~cm} / \mathrm{sec}$ ). Die monotone Intonation sollte dabei einen möglichst neutralen Ausgangspunkt für die Manipulation darstellen. Die besten

Referenz genommen werden soll, oder doch die Position des Fo-Endpunktes, arbiträr. - Wir sehen deshalb bei unseren Stimuli von einer Berechnung des Deklinationsverlaufs ab. Eine solche Berechnung ist für den Nachweis der Relevanz der Deklination auch nicht erforderlich.

2 Natürlich sind auch für das Deutsche die Auswirkungen der Deklination bekannt, es gibt aber noch keine Untersuchung, die die Rolle der Deklination ausdrücklich zum Gegenstand hat.

3 Eine Einbettung in einen fokussteuernden Kontext ist einem Identifikationstest, bei dem gefragt wird, ob die erste oder die zweite Phrase betont ist, aus dem folgenden Grund vorzuziehen: Die unmarkierte und damit ohne Kontextvorgabe erwartete Position des FA ist die auf der letzten - in unserem Fall auf der zweiten - Phrase. Es kann also bei unserem Testsatz die letzte Position nicht nur wegen des Einflusses der Deklination stärker gewichtet sein, sondern auch wegen der erwarteten unmarkierten Position des FA. Das würde aber bedeuten, da $\beta$ der Einfluß der Deklination nicht genau $\mathrm{zu}$ bestimmen wäre. Es ist anzunehmen, daß eine Kontextvorgabe diesen Effekt ausschaltet. (Vgl. auch Oppenrieder 1989 sowie die Diskussion in Teil 2.4.) 
Realisationen wurden auf einer PDP11/50 mit einer Abtastrate von $20 \mathrm{KHz}$ digitalisiert und mit einem Tiefpaß von $8 \mathrm{kHz}$ gefiltert. Die Kontextsätze blieben unmanipuliert, der Testsatz wurde mit einem Segmentierungsprogramm periodenweise geschnitten und diente damit als Grundlage zur pitchsynchronen Manipulation auf Halbtonbasis.

Fig.1 zeigt eine schematische Darstellung der Stimuli 1-7, Tab.1 die Unterschiede zwischen den Stimuli in Halbtönen. Für Leo und säuft ist bei einem linearen Verlauf von Fo die Differenz von Anfang und Ende in Halbtönen angegeben (' 0 ' steht für ebenen Verlauf (LEVEL) und ' 2 ' für einen fallenden Verlauf (FALL) von 2 Halbtönen); sonst steht vor dem Schrägstrich der Betrag des steigenden Verlaufs (RISE), danach der des FALL in Halbtönen. Der FoGipfel ist immer in der Mitte des vokalischen Teils, bei der Transition vom einen zum anderen Vokal. Ab dem Stimulus 5 mit gleich hohen Fo-Gipfeln auf Leo und auf säuft setzt der Fo-Verlauf von säuft auf der Anfangshöhe von Leo ein, also nicht, wie bei Stimulus 1 bis 4 , zwei Halbtöne tiefer. ${ }^{4}$ Die gleichbleibenden Teile benachbarter Stimuli sind mit einem Gleichheitszeichen verbunden. Die Stimuli 8 und 9 fallen etwas aus dem Rahmen; sie haben einen unnatürlichen, da stark monotonislerten Fo-Verlauf. Mit ihnen sollte untersucht werden, welche Bewertung solche LEVEL-Verläufe im Experiment erfahren.

Tab.1: Teststimuli

\begin{tabular}{lcccccc|ccc}
\hline Stimulus & 1 & 2 & 3 & 4 & 5 & 6 & 7 & 8 & 9 \\
Leo & $5 / 7$ & $3 / 5$ & $1 / 3=$ & $1 / 3=1 / 3$ & 0 & $=$ & 0 & 0 & $=$ \\
säuft & $2=$ & 2 & 2 & 0 & $1 / 1=1 / 1$ & $3 / 3$ & 2 & 0 \\
\end{tabular}

Zwischen den Stimuli 4 und 5 ist also ein größerer Sprung, der dadurch bedingt ist, daß wir auf säuft beim Eckstimulus einen Gipfel generieren wollten, der zumindest dem auf Leo in Stimulus 2 entspricht. Wenn wir nun, ausgehend von $80 \mathrm{~Hz}$ wie bei Stimulus 4, ein 'Gipfelkontinuum' erzeugen, so kommen wir bei der Kürze des Diphthongs bald zu unnatürlich klingenden Verläufen. Abgesehen davon wäre ein 'echtes' Kontinuum auf säuft zwischen den Verläufen von Stimulus 1 und Stimulus 7 schematisch nicht $z u$ erzeugen, sondern nur durch punktweise Interpolation wie etwa bei Experiment 1 bis 4 in Batliner $(1989 \mathrm{~b})$. Da jedoch in unserem Zusammenhang der genaue ubergang an der Kategoriengrenze nicht im Mittelpunkt steht, begnügen wir uns mit dem vorllegenden Quasikontinuum. 
Fig.1: Stimuli 1-7, schematisch dargestellt

Halbtöne

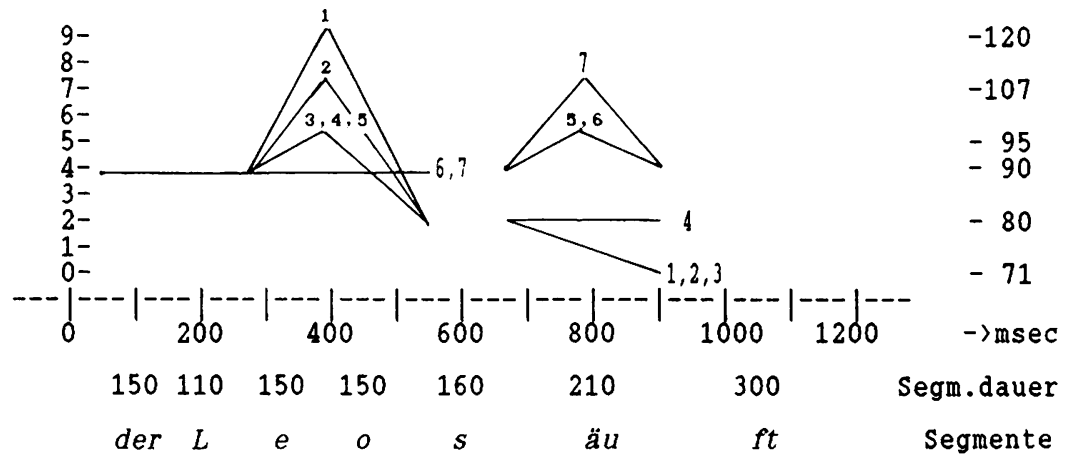

Je 6 Wiederholungen der 18 Kontext-Testsatz-Paare $(9$ Stimuli mit zwei verschiedenen Kontextsätzen, $0.5 \mathrm{sec}$ Pause $z$ wischen Kontextsatz und Testsatz, 4 sec Pause zwischen den Paaren) wurden randomisiert und auf zwei Sitzungen verteilt 17 Versuchspersonen dargeboten. Die Versuchspersonen waren Studentinnen der germanistischen Linguistik oder der Phonetik, die für ihre Teilnahme bezahlt wurden. Sie bewerteten auf einer Ratingskala, wie gut die beiden Sätze zusammenpaßten. Die Skala hatte fünf Stufen: 1 = 'sehr gut', 2 = 'gut', 3 = 'noch gut', 4 = 'schlecht', 5 = 'sehr schlecht'. Für die Auswertung wurde die Skala in eine Skala von 0-4 umgerechnet.

\subsection{Ergebnis}

In Fig.2 sind auf der $x$-Achse die Stimuli und auf der $y$-Achse die Bewertung durch die Versuchspersonen aufgetragen. Die Kurven sind für die Stimuli 1 bis 7 bei den beiden unterschiedlichen Fokuszuweisungen konsistent gegenläufig mit einem Schnittpunkt bei Stimulus 3 . Wie sich schon in Batliner (1988b) gezeigt hat, vergeben manche Versuchspersonen ungern extreme Bewertungen in einem solchen Kontexttest; damit läßt sich erklären, warum die Eckstimuli 1 und 7 nicht näher bei den Extremwerten 0 bzw. 4 liegen. 
Fig.2: FRÜHER vs. SPÄTER FOKUS

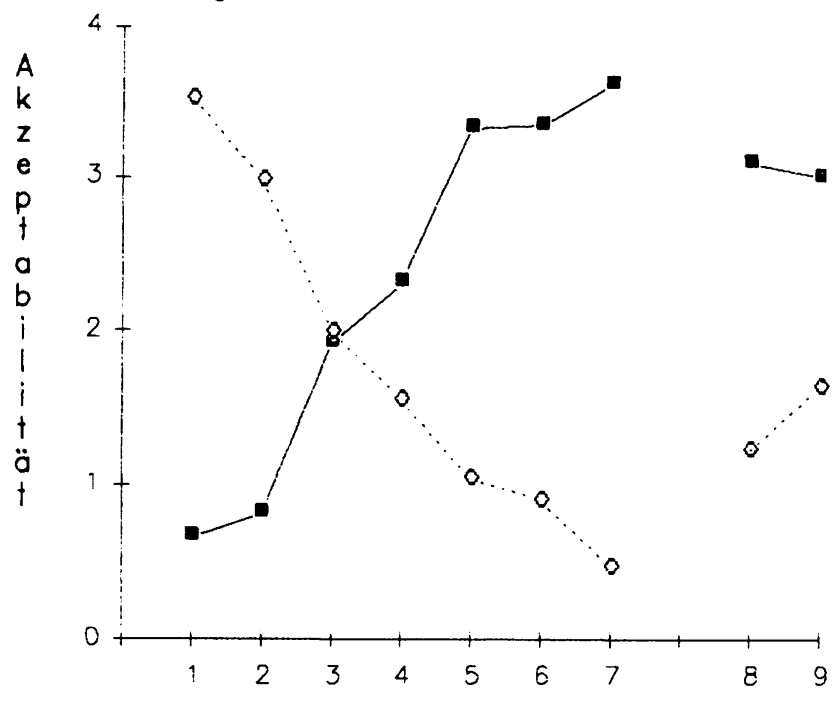

FRÜHER FOKUS

O SPÄTER FOKUS

Stimuli

Die Bewertungen für FRÜHEN und für SPATEN FOKUS bei den benachbarten Paaren $1 / 2,2 / 3, \ldots 6 / 7$, und $8 / 9$ wurden mit einem nichtparametrischen Signifikanztest für abhängige Stichproben (Wilcoxon) auf Unterschiede geprüft. Das Ergebnis ist in Tab.2 dargestellt; unter den Paaren stehen die jeweils erhaltenen $p$-Werte. Als Signifikanzniveau wurde $a=.05$ angesetzt; da über die gleiche Stichprobe vierzehnmal getestet wurde, mußte das Niveau mit der Formel $1-(1-\alpha)^{1 / c}$ abgesenkt werden, wobei $c$ für die Anzahl der durchgeführten Tests steht. Die Formel ergab ein reduziertes Signifikanzniveau von .0036; p-Werte, die unter diesem Niveau liegen, also für signifikante Unterschiede stehen, sind in Tab.2 mit einem Stern gekennzeichnet.

Tab.2: Ergebnisse der Signifikanztests (Wilcoxon, einseitig geprüft, erha1tene p-Werte)

\begin{tabular}{lllllll|l} 
stimuluspaare & $1 / 2$ & $2 / 3$ & $3 / 4$ & $4 / 5$ & $5 / 6$ & $6 / 7$ & $8 / 9$ \\
früher Fokus & .0936 & $.0001^{\star}$ & .0166 & $.0002^{\star}$ & .4860 & .0119 & .1047 \\
später Fokus & $.0003^{\star}$ & $.0002^{\star}$ & .0045 & $.0016^{\star}$ & .1912 & $.0011^{\star}$ & $.0033^{\star}$ \\
\hline
\end{tabular}




\subsection{Diskussion}

Die Stimuli 3 und 4 liegen im Übergangsbereich zwischen FRUHEM und SPATEM FOKUS. Der Stimulus 5 mit gleichhohen Fo-Maxima auf Leo und auf säuft wird schon eindeutig dem SPATEN FOKUS zugeordnet. Es zeigt sich also deutlich der vorhergesagte Einfluß der Deklination: Nicht nur die absolute FoHöhe, sondern auch die Position des entsprechenden Gipfels auf der Zeitachse ist für die Fokuszuweisung relevant. Bei gleicher Höhe ist ein später Fo-Gippel perzeptiv prominenter als ein früher. ${ }^{5}$

Für den Grad der Eindeutigkeit der Fokussierung ist nicht nur die Relation der Fo-Verläufe zwischen den Phrasen verantwortlich, sondern auch die Verhältnisse innerhalb der Phrasen; dies gilt besonders für die zweite Phrase. Die Absenkung von drei zu einem Halbton aup der ersten Phrase (Stimulus 2 zu 3) läßt zwar den Stimulus 3 in beiden Kontexten ambig erscheinen; solange aber auf der zweiten Phrase kein Gipfel erscheint, bleibt die Bewertung uneindeutig (Stimulus 4). Stimulus 5 unterscheidet sich von Stimulus 6 nur auf der ersten, nicht aber auf der zweiten Phrase. In beiden Fällen ist der Fo-Gipfel auf der zweiten Phrase für die Bewertungen verantwortlich; die Bewertungen unterscheiden sich aber nicht, obwohl auf der ersten Phrase der leichte RISEFALL-Verlauf in einen LEVEL-Verlauf übergeht. Erst wenn der Fo-Gipfel auf säuft weiter angehoben wird (Stimulus $6 \mathrm{zu} 7$ ), wird auch die Fokussierung auf der zweiten Phrase eindeutiger.

Daß sich nicht nur ein RISE-FALL-Verlauf auf der zweiten Phrase, sondern auch ein FALL in Relation zu einem LEVEL-Verlauf, m.a.w. überhaupt eine FoVeränderung, fokussierend auswirken kann, zeigt sich an den Bewertungen von Stimulus 8 im Vergleich zu Stimulus 9: Beim SPATEN FOKUS wird Stimulus 8 mit der Fo-Veränderung signifikant besser bewertet als stimulus 9 .

Bisher haben wir die Punkte kommentiert, die auf einen globalen Einfluß der Deklination zurückgeführt werden können. Betrachten wir nun den Verlauf der Bewertungsfunktionen für FRUHEN und SPATEN FOKUS, so zeigt sich, daß der Verlauf für SPATEN FOKUS stetiger ist als der für FRUHEN FOKUS: Wenn wir als 'eindeutige' Stimuli diejenigen bezeichnen, die unter '1.0' liegen, also die Bewertung 'gut' bis 'sehr gut' erhielten, sowie diejenigen, die über '3.0' liegen, also die Bewertung 'schlecht' bis 'sehr schlecht' erhielten, so gehören beim frühen Fokus die Stimuli 1,2, und 5 bis 7 eindeutig zu einer der beiden Klassen; jeweils benachbarte stimuli unterscheiden sich nicht signifikant. Beim SPATEN

Die steigung der RISE-FALL-Verläufe wurde von uns nicht kontrolliert. Es müßte also in weiteren Experimenten noch untersucht werden, inwiefern sie die FA-Zuweisung mit steuert. 
FOKUS unterscheiden sich im Gegensatz zum FRUHEN FOKUS zusätzlich die Paare $1 / 2$ und $6 / 7$ signifikant voneinander.

Man könnte also sagen, daß wir es beim FRUHEN FOKUS mit einer kategorialen Unterscheidung $z u$ tun haben, beim SPATEN FOKUS dagegen mit einer kontinuierlichen. Damit wären die Verläufe zwar benannt, aber nicht erklärt. Wir werden nun versuchen, diese Unterschiede zu erklären, indem wir einen weiteren Effekt einführen: Einstellungseffekte sind in der psychologischen Wahrnehmungsforschung seit langem bekannt (vgl. Graumann 1966:1074ff). Sie wirken sich grundsätzlich in der Art aus, da $\beta$ man das, was erwartet wird, eher wahrnimmt. Beim Kontextsatz Ihr fragt mich, wer säuft? wird ein FRUHER FOKUS, beim Kontextsatz Was soll schon mit dem Leo sein? ein SPATER FOKUS erwartet. Die fokusindizierenden intonatorischen Merkmale können dabei also je nach Erwartung stärker oder schwächer wahrgenommen werden. Der Einstellungseffekt - es wird entweder eine frühe oder eine späte Hervorhebung erwartet interagiert nun mit dem Deklinationseffekt, der spätere Hervorhebungen stärker gewichtet als frühere: Beim SPATEN FOKUS erwartet der Hörer einen möglichst niedrigen bzw. keinen Gipfel auf der ersten Phrase und einen möglichst hohen Gipfel auf der zweiten Phrase.

Der Einstellungseffekt bewirkt also beim SPATEN FOKUS, daß ein früherer Gipfel niedriger wahrgenommen wird und ein späterer höher. Der Deklinationseffekt unterstützt diesen Einstellungseffekt. Beim FRUHEN FOKUS sind dagegen der Einstellungseffekt und der Deklinationseffekt gegenläufig: Erwartet wird ein hoher Gipfel auf der ersten und ein entsprechend niedriger auf der zweiten Phrase, der Deklinationseffekt gewichtet aber einen niedrigen Gipfel auf der zweiten höher als einen gleich hohen auf der ersten Phrase.

Bei Stimulus 2 indiziert der Gipfel von 3 Halbtönen auf der ersten Phrase noch eindeutig einen FA, wenn ein FRUHER FOKUS erwartet wird (vgl. zur Kategoriengrenze im Bereich von 3 Halbtönen Batliner 1988b, 1989c, dieser Band). Die Stimuli 1 und 2 unterscheiden sich deshalb beim FRUHEM FOKUS nicht signifikant. Beim SPATEN FOKUS 'senkt' dagegen der Einstellungseffekt den Fo-Gipfel auf der ersten Phrase mit der Folge, daß der Gipfel von 3 Halbtönen schon so nahe am übergangsbereich liegt, daß er sich von Stimulus 1 mit einem Gipfel von 5 Halbtönen signifikant unterscheidet.

Bei Stimulus 6 vs. Stimulus 7 liegen beim FRUHEN FOKUS beide Stimuli schon so eindeutig im 'paßt nicht'-Bereich, daß der Unterschied nicht mehr signifikant ist; möglicherweise liegt ein ceiling-Effekt vor, d.h. beide Stimuli liegen schon so nahe am Maximum (= schlechteste Bewertung), daß etwaige Unterschiede dieser Größenordnung nicht mehr zum Tragen kommen. Beim SPATEN FOKUS 
verstärken sich der Einstellungseffekt und der Deklinationseffekt dagegen derartig, daß der Unterschied von 2 Halbtönen perzeptiv aufälliger ist als der identische Unterschied beim FRUHEN FOKUS oder auch der gleich große Unterschied auf der ersten Phrase beim FRUHEN FOKUS zwischen Stimulus 1 und Stimulus 2. Folge davon ist der signifikante Unterschied zwischen Stimulus 6 und Stimulus 7 beim SPATEN FOKUS, der beim FRUHEN FOKUS nicht vorhanden ist. 6

3. Wendepunkt der Fo-Kontur

\subsection{Fragestellung}

Es hat sich in Batliner (1989a, dieser Band) gezeigt, daß die Position des Fo-Wendepunktes einer konkaven (FALL-RISE-) Kontur bei Fragen ein relevantes Merkmal Pür die Fokusindizierung ist. Wir wollen deshalb nun untersuchen, in welchem Ausma $\beta$ bei einem globalen FALL-RISE-Verlauf eines VerbErst-Fragesatzes eine Verschiebung des Wendepunkts der Fo-Kontur von links nach rechts einhergeht mit einer Verlagerung vom frühen auf den späten FA (zu ähnlichen Untersuchungen vgl. Goldbeck/Sendlmeier 1988).

\subsection{Material und Design}

Die Stimuli wurden analog zu den eingangs beschriebenen stimuli generiert. Beim Testsatz Säuft der Leo erhielt säuft einen FALL von 5 Halbtönen; der finale Fo-Wert (Offset) lag immer 6 Halbtöne über dem Basiswert. Der Anstieg des Fo-Verlaufs lag (1) vor der Leo (FRUHER WENDEPUNKT), (2) zwischen der und Leo (MITTLERER WENDEPUNKT) bzw. (3) zwischen [e] und [0] in Leo (SPATER WENDEPUNKT). Fig.3 stellt diese drei stimuli schematisch dar.

\footnotetext{
6 Man beachte, da $\beta$ es sich um a posteriori-Erklärungen handelt, für die keine Hypothese formuliert wurde. Den technischen Anforderungen der Prüfstatistik wurde insofern Rechnung getragen, als wir alle Unterschiede zweiseitig und nicht einseitig prüften. Inhaltlich sollte die Relevanz des Einstellungseffekt nicht als 'nachgewiesen' angesehen werden. Es dürfte sich dabei aber um einen interessanten Faktor handeln, der in ähnlichen Experimenten selbst zum Untersuchungsgegenstand gemacht werden könnte.
} 
Fig.3: Fo-Verläufe der Stimuli mit Wendepunktverschiebung

Halbtöne

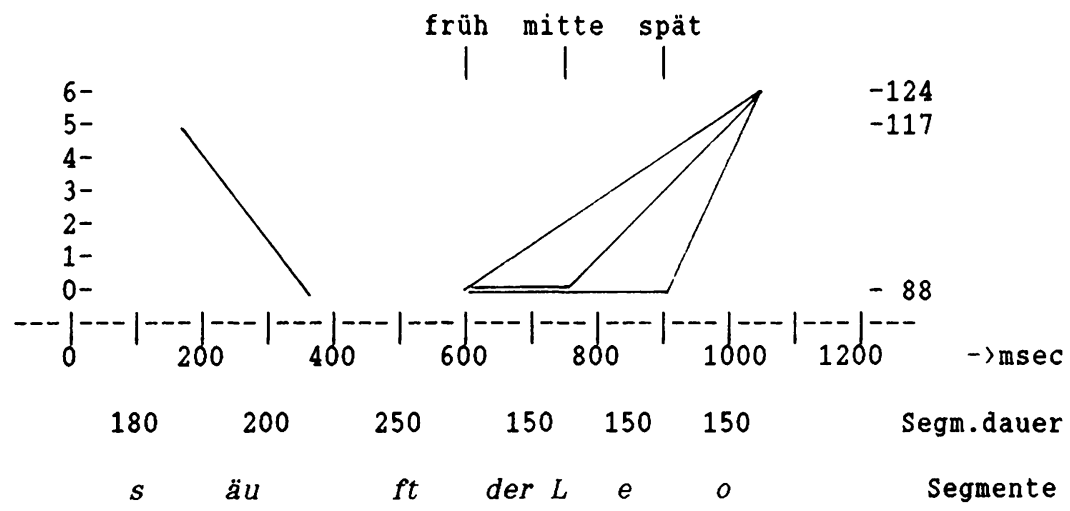

Eine überprüfung dieser drei Stimuli, bei denen die Intensität nicht manipuliert, sondern vom monoton produzierten Ausgangsstimulus übernommen wurde, ergab, daß die Unterschiede zwischen den Stimuli perzeptiv nicht sehr ausgeprägt waren. Das war einerseits beabsichtigt, da wir ja nicht schon durch große Unterschiede zwischen dem Ausmaß des initialen FALL und dem des finalen RISE die Fokussierung steuern wollten; um aber auf alle Fälle im Experiment einen Fokuswechsel zu erzielen, wurde eine zusätzliche Manipulation der Intensität vorgenommen: Bei vier weiteren Stimulusgruppen wurden entweder die Intensitäts-Werte der ersten Phrase säuft oder die der zweiten Phrase der Leo durchgehend um den Faktor 0.7 bzw. 0.5 und damit um 3 bzw. 6 dB abgesenkt. (Eine Reduzierung des Schalldruckpegels um $6 \mathrm{~dB}$ entspricht in etwa einer Halbierung des Schalldrucks.) Wir erhalten also die fünf Gruppen in Tab.3 ('1.0' steht für 'unmanipuliert'.):

Tab. 3: dB-Manipulationen

Faktor:

\begin{tabular}{lccccc} 
säuft & 0.5 & 0.7 & 1.0 & 1.0 & 1.0 \\
der Leo & 1.0 & 1.0 & 1.0 & 0.7 & 0.5 \\
\cline { 2 - 6 } Stimulusgruppe & 1 & 2 & 3 & 4 & 5
\end{tabular}

Es ist klar, daß eine solche schematische Manipulation keine sehr natürlich klingenden Stimuli zur Folge hat. Besonders bei der Absenkung um den Faktor 0.5 entsteht leicht der Eindruck, als ob ein Lautstärkeregler kurz zurückgedreht 
wird. Entscheidend ist aber die Möglichkeit, innerhalb der gleichen dBManipulation die drei verschiedenen Wendepunkte miteinander $\mathrm{zu}$ vergleichen und zugleich sicherzustellen, daß sich im Test sowohl Stimuli befinden, die auf der ersten, als auch Stimull, die auf der zweiten Phrase akzentuiert sind. Wir begnügten uns deshalb mit dieser zwar schematischen, aber sehr einfach durchzuführenden dB-Manipulation.

Die 15 Stimuli wurden in einem Identifikationstest, bei dem 15 Versuchspersonen bestimmen mußten, ob die erste oder die zweite Phrase betont war, je fünfmal randomisiert dargeboten (Inter-Stimulus-Abstand $4 \mathrm{sec}$ ). Bei einem Kontexttest bewerteten 11 Versuchspersonen die Kombinationen der 15 Teststimuli mit zwei Kontextsätzen, einmal mit dem Satz Stimmt das mit dem Leo? (Fokus aup säuft), das andere Mal mit dem Satz Wer säuft denn eigentlich? (Fokus auf Leo); die Pause zwischen Kontextsatz und Testsatz betrug $0.5 \mathrm{sec}$, die zwischen den Paaren $4 \mathrm{sec}$ (zur genaueren Instruktion vgl. oben; die FoVerläufe der Kontextsätze finden sich in Batliner 1988a:226). Versuchspersonen waren wieder StudentInnen der germanistischen Linguistik oder der Phonetik, die für ihre Teilnahme bezahlt wurden. Die Kombinationen wurden in zwei Testsitzungen insgesamt je viermal randomisiert dargeboten.

\subsection{Identifikation}

Fig.4 zeigt, daß die FA-Zuweisung aup Leo beim FRÜHEN WENDEPUNKT deutlich und durchgehend schlechter ist als beim MITTLEREN oder beim SPATEN. Der dB-Manipulation entsprechend verlaufen alle drei Kurven parallel und fallend; der Unterschied zwischen FRUHEM WENDEPUNKT auf der einen und MITTLEREM bZw. SPATEM WENDEPUNKT auf der anderen Seite beträgt ca. 20\%. Für eine eindeutigere Zuweisung des FA zur 2. Phrase war offenbar der FALL von 5 Halbtönen auf der 1. Phrase zu groß. 
Fig. 4: Identifikationstest: Zuweisungen

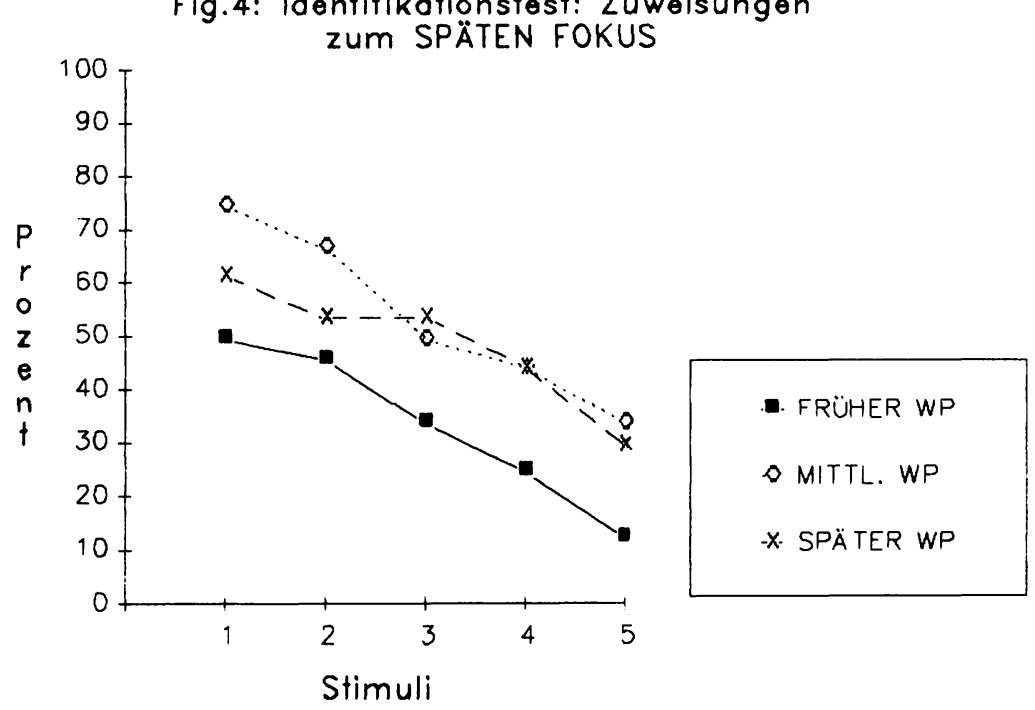

Die drei Stimulusserien wurden wieder mit einem Wilcoxon-Test paarweise miteinander verglichen. Das nach der o.a. Formel abgesenkte Signifikanzniveau a betrug .016. Tab.4 zeigt die erhaltenen p-Werte.

Tab.4: Ergebnisse der Signifikanztests (Wilcoxon, einseitig geprüf, erhaltene $\mathrm{p}$-Werte)
früher vs. mittlerer Wendepunkt
$.0065^{\star}$
früher vs. später Wendepunkt
.0345
mittlerer vs. später Wendepunkt

Die Stimuli waren nicht so unterschiedlich, um eindeutig FRUHEN und SPATEN FOKUS unterscheiden zu können. Der FRUHE WENDEPUNKT indiziert aber signifikant öfter einen FRUHEN FOKUS als der MITTLERE WENDEPUNKT. Zwischen MITTLEREM und SPATEM WENDEPUNKT ist kein Unterschied. Der 'Einbruch' beim SPATEN WENDEPUNKT bei Stimulus 1 und 2 ist dafür verantwortlich, daß er sich nicht signifikant vom FRUHEN WENDEPUNKT unterscheidet. Bei Stimulus 1 und 2 war die Intensität von säuft abgesenkt. Diese Absenkung, verbunden mit einem sehr späten Anstieg auf [o], ergab also einen perzeptiv unauffälligeren RISE und verhinderte damit eine eindeutigere FA-Zuweisung auf Leo. 


\subsection{Kontextverträglichkeit}

Die Kurvenverläufe in Fig.5 (FRUHER FOKUS) und Fig.6 (SPATER FOKUS) decken nur einen mittleren Ausschnitt aus dem möglichen Bereich von 0-4 ab. Signifikanztests, bei denen die Serien analog zum Identifikationstest miteinander verglichen wurden, ergaben keine signifikanten Unterschiede.

Fig.5: FRÜHER FOKUS

Fig.6: SPÄTER FOKUS
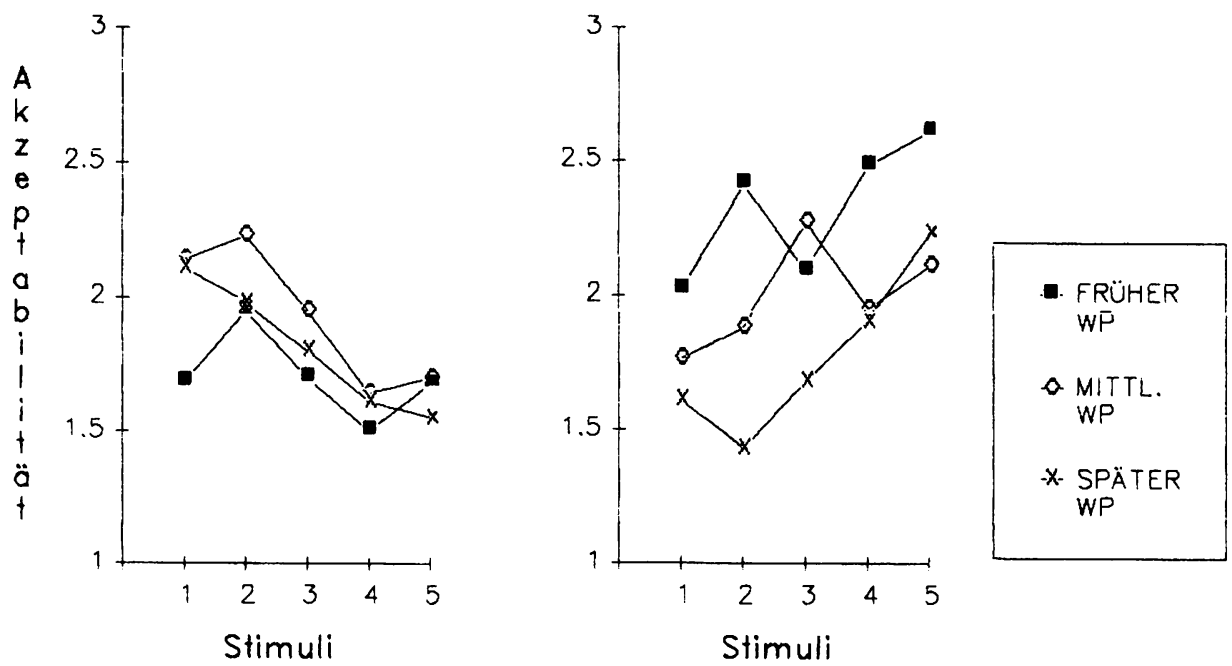

Experimente, in denen die Alternativhypothese nicht bestätigt wird, verlaufen - vordergründig - nicht 'nach Wunsch'. In unserem Fall war die Alternativhypothese, daß eine Verschiebung des Fo-Wendepunkts nach rechts in die 2. Phrase hinein mit einer signifikant besseren Verträglichkeit mit dem Kontextsatz Wer säuft denn eigentlich? einhergeht. Natürlich kann ein Einfluß der in der Einleitung angesprochenen Artefakte bei der Verwendung schematisch manipulierter Stimuli nicht ausgeschlossen werden. U.E. gibt es aber eine systematische und weiterführende Erklärung: Identifikationstests haben sich grundsätzlich als trennschärfer erwiesen als Kontexttests (cf. Batliner 1988b, 1989c). Das Resultat des Identifikationstest (cf. Fig. 4 und Tab.4) ist nun signifikant, aber nicht trennscharf: der Unterschied in der Bewertungsfunktion von FRÜHEM vs. MITTLEREM und SPATEM WENDEPUNKT beträgt etwa $20 \%$. Es gab also keine klare Trennung der Kategorien. In einem solchen Fall können sich im 
Kontexttest keine signifikanten Unterschiede ergeben. Man kann aber bei Inspektion von Fig. 5 noch übereinstimmende Tendenzen feststellen:

1. Der grundsätzlich fallende Verlauf der Bewertungsfunktionen in Fig.5 und der grundsätzlich steigende Verlauf der Bewertungsfunktionen in Fig. 6 ist auf die dB-Manipulation zurückzuführen.

2. Beim FRUHEN FOKUS haben die Stimuli mit dem FRÜHEN WENDEPUNKT, beim SPATEN die mit dem SPATEN WENDEPUNKT die beste Bewertung. Am schlechtesten schneiden die Stimuli mit den FRÜHEN WENDEPUNKT beim SPATEN FOKUS ab. Diese Tendenz entspricht den Ergebnissen des Identifikationstests.

Man kann darüber spekulieren, ob diese Tendenzen sich bei einer Erhöhung der Zahl der Versuchspersonen als signifikant erweisen würden. Das würde aber nichts an der naheliegenden Schlußfolgerung ändern: Dle Position des FoWendepunktes scheint zwar ein relevantes Merkmal für die Zuweisung des FA zu sein, sie ist aber doch nicht so relevant, daß eine Verschiebung dieser Position bei ambigen stimuli mit konstant gehaltenen anderen relevanten Merkmalen einen eindeutigen kategorialen Umschlag zur Folge hätte.

\section{Schlußbemerkungen}

Bei beiden Testserien hat sich gezeigt, daß eine deutliche Anderung der Bewertungsfunktion dann eintritt, wenn sich etwas Hörbares (und damit beim grafisch dargestellten Fo-Verlauf auch etwas Sichtbares) auf der zweiten Phrase tut; beim Aussagesatz war das der übergang von Stimulus 4 mit LEVEL auf säuft zu Stimulus 5 mit einem Gipfel von einem Halbton auf säuft, beim Fragesatz war es der Übergang vom FRUHEN zum MITTLEREN WENDEPUNKT und damit die Verlagerung des Wendepunktes in die zweite Phrase hinein. Bei der Manipulation des Fo-Gipfels erzielten wir einen kategorialen Wechsel von FRUHEM zu SPATEM FOKUS. Nach den vorliegenden Ergebnissen und vergleichbaren Arbeiten (cf. Batliner 1988b) scheint sich dagegen eine Wendepunktverschiebung weniger bzw. weniger systematisch auszuwirken als eine Anhebung des Gipfels. 


\section{LITERATUR}

Altmann, H. (Hg.) (1988): Intonationsforschungen. Tübingen.

Batliner, A. (1988a): Modus und Fokus als Dimensionen einer Nonmetrischen Multidimensionalen Skalierung. In: Altmann, H. (Hg.) (1988). S.223-241.

Batliner, A. (1988b): Der Exklamativ: Mehr als Aussage oder doch nur mehr oder weniger Aussage? Experimente zur Rolle von Höhe und Position des FoGipfels. In: Altmann, H.(Hg.) (1988). S.243-271.

Batliner, A. (1989a): Fokus, Modus und die große Zahl. Zur intonatorischen Indizierung des Fokus im Deutschen. (In diesem Band)

Batliner, A. (1989b): Eine Frage Ist eine Frage ist keine Frage. Perzeptionsexperimente zum Fragemodus im Deutschen. (In diesem Band)

Batliner, A. (1989c): Wieviel Halbtöne braucht die Frage? Merkmale, Dimensionen, Kategorien. (In diesem Band)

Goldbeck, T.P./Sendlmeier, W.F. (1988): Wechselbeziehung zwischen Satzmodalität und Akzentuierung in satzfinaler Position bei der Realisierung von Intonationskonturen. In: Altmann, H. (Hg.) (1988). S.305-321.

Graumann, C.-F. (1966): Nicht-sinnliche Bedingungen des Wahrnehmens. In: Metzger, W./Erke, H. (Hgg.): Allgemeine Psychologie. I. Der Aufbau des Erkennens. 1. Halbband: Wahrnehmung und Bewußtsein. (Handbuch der Psychologie in 12 Bänden. 1.Band.) Göttingen. S.1031-1096.

't Hart, J. (1986): Declination has not been defeated - A reply to Lieberman et al. In: J. Acoust. Soc. Am. 80. S.1838-1840.

Ladd, D.R. (1984): Declination: A review and some hypotheses. In: Ewen, J.C./Anderson, J.M (Hgg.):Phonology Yearbook 1. S.53-74.

Lieberman, P. (1986): Alice in declinationland - A reply to Johan 't Hart. In: J. Acoust. Soc. Am. 80. S.1840-42.

Lieberman, P./Katz, W./Jongman, A./Zimmerman, R./Miller, M. (1985): Measures of the sentence intonation of read and spontaneous speech in American English. In: J. Acoust. Soc. Am. 77. S.649-657.

Oppenrieder, W. (1989): Deklination und Satzmodus. (In diesem Band)

Pierrehumbert, J.B. (1979): The perception of fundamental frequency declination. In: J. Acoust. Soc. Am. 66. S.363-369.

Rossi, M. (1978): Interactions of Intensity Glides and Frequency Glissandos. In: Language and Speech 21. S.384-396.

Umeda, N. (1982): "Fo declination" is situation dependent. In: Journal of Phonetics 10. S.279-290. 

EINE FRAGE IST EINE FRAGE IST KEINE FRAGE. PERZEPTIONSEXPERIMENTE ZUM FRAGEMODUS IM DEUTSCHEN 1

Anton Batliner

1. Einleitung

In diesem Beitrag wird versucht, mit Hilfe zweier Testserien die Rolle intonatorischer Merkmale, insbesondere des Verlaufs der Tonhöhe, bei der Markierung des Fragemodus im Deutschen genauer zu bestimmen. Begleitend dazu werden wir zwei methodische Teilaspekte ansprechen: Der eine davon betrifft die Erwartungshaltung solcher Untersuchungen, die sich auch in der wahl des experimentellen Designs niederschlägt, der andere das Verhältnis der den Designs zugrundellegenden Modellvorstellung zur abgebildeten Wirklichkeit. Unserer These zu diesen beiden Aspekten geben wir die Kurzform WYALFIWYG (What you are looking for is what you get). ${ }^{2}$ Wir werden im nächsten Abschnitt zwei unterschiedliche, aber gleichermaßen einschlägige Forschungsrichtungen skizzieren und dann zeigen, daß der Fragemodus als Untersuchungsgegenstand eine gewisse Sonderstellung einnimmt. (Im folgenden sprechen wir vereinfachend von der 'Frage', verstehen darunter aber normalerweise den Fragemodus und die ihn konstituierenden Merkrnale in dem Satzmodussystem, das von Altmann 1987 beschrieben ist. Generelle Aussagen über den Status der 'Frage' können dabei auch auf andere Satzmodi verallgemeinert werden.)

1 Dieser Beitrag ist eine veränderte und (hoffentlich) verbesserte Fassung eines Beitrags, der unter dem gleichen Titel erschienen ist in: Studien zum Satzmodus II (Papers from the Round Table Sentence and Modularity at the XIVth International Congress of Linguistics, Berlin 1987) Linguistische Studien. Reihe A. Arbeitsberichte 185. Berlin 1988. S.18-38.

2 In Anlehnung an das Desiderat der Textverarbeitung WYSIWYG (What you see is what you get). Das Akronym ist neu, die These natürlich nicht, auch wenn sie u.E. bisher zu wenig beachtet wurde. Ihre Beachtung hätte den therapeutischen Effekt, voreilige Reifikationen vermeiden zu helfen. 
Medin/Barsalou (1987) haben als erste den Versuch unternommen, zwei Forschungsrichtungen $\mathrm{zu}$ vergleichen, die wenig miteinander $\mathrm{zu}$ tun haben, obwohl sie letztlich das gleiche Phänomen beschreiben: Die eine Richtung beschäftigt sich mit sensory perception categories (SP-Kategorien), die andere mit generic knowledge categories (GK-Kategorien). ${ }^{3}$ Typische SP-Kategorien sind z.B. stimmhaft vs. stimmlos (/b/ vs. /p/); hier wird untersucht, welche Korrelation zwischen physikalischen Parametern, etwa der voice onset time (VOT, i.e. Einsetzen des Stimmtons relativ zur Lösung des artikulatorischen Verschlusses), und der Klassifizierung als stimmhaft/stimmlos besteht. Typische GK-Kategorien sind z.B. Sitz- vs. Liegemöbel; hier wird untersucht, wie solche semantischen Kategorien im Gedächtnis organisiert sind. (Letztlich scheint nur eine solche Charakterisierung der beiden Kategorienarten über typische Exemplare möglich zu sein, nicht aber eine strenge Definition oder Abgrenzung; vgl. Medin/Barsalou $1987: 456 f f)$.

Die angeführten Beispiele sind sicher eindeutige SP- bzw. GK-Kategorien. Das zeigt schon allein die Tatsache, da $\beta$ es keine logisch-semantisch oder sprachphilosophisch orientierten Arbeiten über das "Stimmhaft/Stimmlos-Problem" gibt, so wie es auf der anderen seite keine psychoakustisch oder phonetisch orientierten Arbeiten über "Möbelkategorien" gibt - es sei denn, solche Bezeichnungen würden zufälligerweise ein phonetisches Minimalpaar bilden. Bei der Frage liegen die Dinge anders, wie sich leicht an der Existenz einschlägiger Arbeiten zeigen läßt: Es gibt sowohl Untersuchungen, die den Einfluß physikalischer Parameter auf perzeptive Kategorien beim Fragemodus untersuchen, z.B. Studdert-Kennedy/Hadding (1973) und Ainsworth/Lindsay (1986), als auch semantisch-pragmatische Untersuchungen über die Frage, z.B. Karttunen (1977), Sadock/Zwicky (1985) und Zaefferer (1984). Die im Polgenden skizzierten Punkte dürften dafür verantwortlich sein:

1. Der Tonverlauf einer Außerung mit seinem physikalischen Korrelat Grundfrequenz (Fo) kann wesentlich daran beteiligt sein, ob diese Außerung als Frage interpretierbar ist oder nicht. Zwei segmental und syntaktisch identische Außerungen mit unterschiedlichen Tonverläufen, die verschiedene Satzmodi indizieren, können dabei ein sog. intonatorisches Minimalpaar bilden. Für solche Minimalpaare lassen sich nun z.B. im relevanten Parameter Fo physikalisch

3 Wir belassen die Abkürzungen SP und GK, da sie auch im Deutschen zumindest mnemotechnisch einen Sinn ergeben ('sensorische Perzeption' bzw. 'generische Kenntnis'). 
äquidistante Kontinua bilden, mit denen die üblichen Identifikations- und Diskriminationstests durchgeführt werden können. (Diese Begriffe werden weiter unten erläutert.) Damit ist die Frage prädestiniert für SP-Untersuchungen, ohne allerdings auf genau derselben Ebene zu liegen wie etwa die Kategorien stimmhaft/stimmlos: Der Fo-Verlauf einer Außerung kann sich über mehr als eine Sekunde erstrecken und damit den normalerweise angenommenen Bereich des (auditorischen) Kurzzeitgedächtnisses weit überschreiten; im Gegensatz dazu liegen z.B. die für die stimmhaft/stimmlos-Unterscheidung relevanten Parameter typischerweise im Zeitbereich von weniger als $100 \mathrm{msec}$. Noch wichtiger ist ein zweiter Punkt:

2. Bei der Wahrnehmung von sprachlichen SP-Kategorien (stimmhaft/stimmlos wie /b/ vs. /p/ etc.) spielen phonetische Merkmale (i.e. die physikalischen Parameterausprägungen via ihre perzeptorischen Korrelate) eine entscheidende, andere - syntaktische, lexikalische, semantische - eine bescheidenere distinktive Rolle (vgl. Repp/Liberman 1987). Unter den phonetischen Merkmalen gibt es Hauptmerkmale, wie z.B. die VOT bei der stimmhaft/stimmlos-Unterscheidung. Eine geringere Ausprägung eines solchen Hauptmerkmals kann durch eine größere Ausprägung anderer (Neben-)Merkmale ausgeglichen werden; diese Merkmale stehen also zueinander in einer sog. trading relation (vgl. Repp/Liberman 1987:98f, Repp 1981). Eine überdeutliche positive VOT (also ein sehr spätes Einsetzen des Stimmtons) kann aber, da sie Hauptmerkmal ist, nicht mit einer eindeutigen stimmhaft-Klassifizierung einhergehen, und vice versa, eine überdeutliche negative VOT nicht mit einer eindeutigen stimmlosKlassifizierung. Anders bei der Frage: Was die phonetischen Merkmale einer intonatorisch gekennzeichneten Frage betrifft, so gibt es hier zwar - nach allem, was man bis jetzt weiß - auch ein Hauptmerkmal, den Tonverlaup, und einige Nebenmerkmale (Intensität, Dauer, etc.); normalerweise indiziert ein ansteigender Tonverlauf (RISE) eine Frage, ein abfallender (FALL) eine NichtFrage. Die phonetischen Merkmale spielen aber nicht immer die entscheidende Rolle. In bestimmten Konstellationen sind andere Merkmale entscheidend, wie (1) das Vorhandensein von Ausdrücken bestimmter syntaktischer Kategorien (z.B. ein W-Ausdruck), (2) die Stellungseigenschaften dieser Ausdrücke (z.B. die Verbstellung) und (3) die Verbmodusmarkierung (vgl. Oppenrieder 1988). Dazu kommen pragmatische und kontextuelle Faktoren (vgl. Geluykens 1987). Die für die Frage relevanten phonetischen Nebenmerkmale können zwar auch zueinander und zum Hauptmerkmal Tonverlaup in trading relation stehen. Allerdings kann ein überdeutlicher FALL sehr wohl mit einer eindeutigen Frage-Klassifizierung einhergehen, wenn die genannten anderen grammatischen Merkmale dafür 
sprechen. Im Unterschied zum Hauptmerkmal VOT bei der klaren SP-Kategorie stimmhaft/stimmlos ist also das (phonetische) Hauptmerkmal Tonverlauf auch in seinen klaren Ausprägungen nicht eindeutig der einen oder der anderen Kategorie zuzuordnen. Bei der Frage ist damit das Verhältnis phonetische/nichtphonetische Merkmale komplexer als bei eindeutigen SP-Kategorien.

Demnach steht die Frage zwischen SP- und GK-Kategorien: sie ist beides. ${ }^{4}$ Noch in einem anderen Punkt unterscheidet sie sich grundsätzlich von den meisten SP- und wohl auch von vielen GK-Kategorien; dieser Punkt wurde u.W. zwar einige Male angesprochen, aber zumindest für SP-Kategorien noch nie thematisiert oder gar experimentell untersucht: Inwiefern ist die experimentelle Fragestellung im Hinblick auf die dabei modellierten real-life-Situationen "natürlich", also nicht nur für ganz spezifische Taxonomien relevant? ob das Schachspiel zur Kategorie sport oder spiel zählt, interessiert hauptsächlich den Layouter der Sportseite. Ob ein Wal ein Fisch oder ein Säugetier ist, interessiert den Walfänger wohl am wenigsten. Ob ein /b/ oder ein /p/ geäußert wurde, interessiert zwar den Phonetiker, nicht aber den "normalen" Menschen (vgl. auch Schiefer/Batliner 1988:288ff). Die (funktionalen) Aspekte des Satz- und damit eben des Fragemodus (vermittelt auch durch phonetische Merkmale) tangieren aber die normale sprachliche Kommunikation viel direkter: Jeden, der an einem Gespräch teilnimmt, muß interessieren, ob der Redepartner eine Aussage, eine Frage oder eine Aufforderung geäußert hat. ${ }^{5}$

3. Die These "WYALFIWYG"

Untersucht man nun die phonetischen Merkmale der Frage, d.h. betrachtet man sie als SP-Kategorie, so ist klar, daß sie eine komplexere Kategorie ist als die meisten anderen SP-Kategorien ${ }^{6}$. Bei der Betrachtung eines komplexen Phänomens kann man sich nun leicht auf einen Aspekt konzentrieren, und das herausfinden, was man - heuristisch völlig plausibel - angenommen hat, wäh-

4 Medin/Barsalou (1987) zeigen auf, daß zwischen SP- und GK-Kategorien viel mehr Parallelen bestehen, als man annehmen könnte. Insofern schiene die Zwischenstellung der Frage nichts besonderes zu sein. Was sie auszeichnet, ist aber, $d a \beta$ sie eine eindeutige $Z$ wischenstellung hat, und da $\beta$ sich das schon immer, wenn auch nicht explizit, in der Literatur widergespiegelt hat.

- Diese Sonderstellung des Satz- und damit des Fragemodus können wir nicht beweisen, sondern fürs erste nur behaupten. Die Behauptung ist u.E. aber bis zum Beweis des Gegenteils plausibel.

6 GK-Kategorien können per se beliebige Komplexität annehmen, auch wenn viele der experimentell untersuchten relativ einfach sind. Daß auch SPKategorien komplexer sind, als man sich denken möchte, zeigt schon Lisker (1978). 
rend ein anderer genau zum entgegengesetzten Ergebnis kommt. Diese These, die wir WYALFIWYG (What you are looking for is what you get) nennen, wollen wir in diesem Beitrag mit zwei unterschiedlichen experimentellen Fragestellungen demonstrieren. Das eine Mal suchen wir nach Kategoriengrenzen zwischen Fragen und Nicht-Fragen und finden sie auch, das andere Mal suchen wir nicht nach Grenzen, sondern nach ubergängen und Abstufungen und finden diese ebenfalls.

4. Auf der Suche nach EINDEUTIGKEIT

\subsection{Fragestellung}

Aussagesätze und Assertive Fragesätze können bei syntaktisch und segmental gleicher Struktur Minimalpaare bilden, bei denen allein die Intonation die Satzmoduszuweisung steuert, also dementsprechend funktional hoch belastet ist. Mit der im folgenden beschriebenen Testserie wurde untersucht, ob sich bei einem intonatorischen Kontinuum $z$ wischen den beiden Satzmodi eine deutliche Kategoriengrenze ergibt. Dazu wurden die im Paradigma der Kategorialen Wahrnehmung üblichen Identifikationstests (ITs) und Diskriminationstests (DTs) durchgeführt. Im IT werden äquidistante Stimuli eines physikalischen Kontinuums, das zwei (gelegentlich auch mehrere) Kategorien enthält, in randomisierter Folge Versuchspersonen (VPn) dargeboten, die die stimuli einer der vorgegebenen Kategorien zuordnen müssen. Beim DT werden benachbarte oder weiter entfernte Stimuli auf Gleichheit beurteilt. In unserem Fall sind die Stimuli benachbart und zu Paaren zusammengestellt ( $A X-T e s t$, also AB, BA und die 'gleich'-Paare AA bzw. BB; zu den Designs im einzelnen vgl. Repp 1984). Im strikten Paradigma gibt es zwar genau definierte Kriterien für die Bestimmung einer Kategoriengrenze; sie lassen sich aber in der Praxis kaum befolgen (vgl. dazu im einzelnen Repp 1984, Harnad 1987 und Schiefer/Batliner 1988). Für unsere Zwecke nehmen wir vereinfachend eine Kategoriengrenze dann als gegeben an, wenn die IT-Kurve die 50\%-Linie dort überschreitet, wo ein eindeutiger Gipfel der DT-Kurve liegt. Wir nehmen weiter an, daß beim DT nicht nur die aus den Anordnungen $A B$ und $B A$ gemittelte Kurve, sondern auch $A B$ oder $B A$ gesondert der Interpretation zugrunde gelegt werden können; vgl. dazu ausführlich Schiefer/Batliner 1988.7

7 Wir werden aus Platzgründen auf diese und ähnliche Fragen nicht weiter eingehen und die Experimente auch nur soweit dokumentieren, als es für unsere Argumentation nötig ist. 
Der Satz Er kommt wurde vom Autor mehrmals im schallarmen Raum des Instituts für Phonetik in München mit Akzent auf $E r$ bzw. auf kommt und mit steigendem bzw. fallendem finalen Tonverlaup produziert. Die besten Realisationen der vier möglichen Konstellationen wurden auf einer PDP11/50 mit. einer Abtastrate von $20 \mathrm{KHz}$ und einem Tiefpa $\beta$ von $8 \mathrm{KHz}$ digitalisiert. In Fig. 1 sind jeweils Intensität und Fo schematisch dargestellt; das akzentuierte Element ist unterstrichen. Satz 1 und 2 bzw. Satz 3 und 4 bilden die Eckstimuli von zwei 10-stufigen Kontinua, die mit einem Programmpaket zur Interpolation von natürlich produzierten Sprachsignalen erzeugt wurden ( $\mathrm{Zu}$ dem Paket vgl. Simon 1983 und Hadersbeck 1987). Mit dem Verfahren können die Parameter FoVerlauf, Intensitätsverlauf, spektraler Energieverlauf, segmentale und damit auch suprasegmentale Zeitstruktur zweier Außerungen jeweils isoliert oder, wie in unserem Fall, gleichzeitig ineinander überführt werden. (Bei der Interpolation aller Parameter gleichzeitig ist eine möglichst natürliche Kovariation dieser Parameter gewährleistet. Allerdings läßt sich der jeweilige Beitrag der einzelnen Parameter nur ungefähr abschätzen.) Da die Intensität der resynthetisierten Eckstimuli, bedingt durch einen inzwischen behobenen Fehler im Programm, relativ $z u$ den interpolierten stimuli $z u$ hoch war, wurden sie in den Experimenten weggelassen. Es ergaben sich also zwei Kontinua mit je acht bezüglich der perzeptiv relevanten Parameter physikalisch äquidistanten Stimuli. ${ }^{8}$

Fig.1: Resynthetisierte Eckstimuli

(oben: Intensität, unten: Fo)
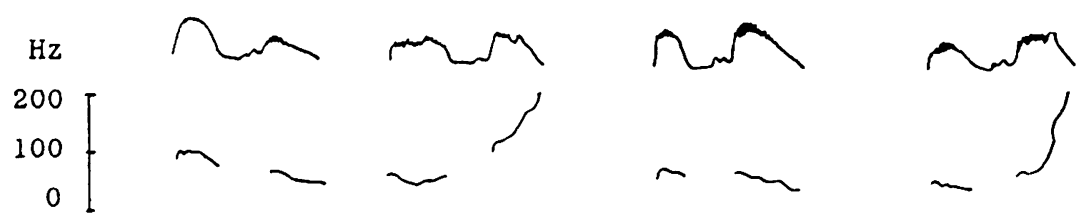
1. Er kommt
2. Er kommt
3. $\operatorname{Er} \underline{\text { kommt }}$
4. $\operatorname{Er} \underline{\text { kommt }}$

\footnotetext{
8 Fo wurde bei diesen Kontinua nicht logarithmisch (auf Halbtonbasis), sondern linear interpoliert. Wir sind inzwischen der Ansicht, daß eine logarithmische Interpolation gehörsadäquater ist. Es muß dahingestellt bleiben, ob eine Interpolation auf logarithmischer Basis zum gleichen Ergebnis geführt hätte; wir nehmen aber an, daß das Ergebnis für die Zwecke unserer Argumentation vergleichbar wäre.
} 
Versuchspersonen waren sieben (Exp.1 und 2) bzw. zehn (Exp.3 und 4) StudentInnen der Linguistik bzw. der Phonetik, die freiwillig teilnahmen.

\subsection{Design}

Es wurde ein IT (Exp.1 und 3) und darauffolgend, mit einer Woche Abstand, ein DT (Exp.2 und 4) durchgeführt. Beim IT wurden die acht Teststimuli je zehnmal randomisiert mit einem zeitlichen Abstand von $3.5 \mathrm{sec}$ zwischen den einzelnen Items dargeboten. Beim DT wurden die Items im Einerschritt $(1 / 2,2 / 3$, $\ldots 2 / 1,3 / 2, \ldots)$ gepaart und die drei unterschiedlichen Anordnungen AB, BA, AA bzw. $B B$ mit einem Abstand von $3.5 \mathrm{sec} z$ wischen den Paaren und 0.5 sec innerhalb der Paare je fünfmal randomisiert dargeboten. Die VPn saßen dabei im Sprachlabor des Instituts für Phonetik vor einem Abstimmkästchen und hörten die Stimuli über Raumlautsprecher. Beim IT lautete die Instruktion: "Bitte drücken Sie die linke Taste, wenn der Akzent Ihrer Ansicht nach auf Er, oder die rechte, wenn er auf kommt liegt." Beim DT lautete die Instruktion: "Bitte entscheiden Sie, ob die beiden Stimuli innerhalb eines Paares in allen Belangen gleich klingen oder nicht, und drücken sie dann die linke Taste für 'gleich' oder die rechte für.'verschieden'." Genau nach jedem Stimulus-(paar) wurde die Abstimmung freigegeben (angezeigt durch eine Lampe an jedem Kästchen). Die VPn hatten dann drei Sekunden Zeit, ihre Antwort zu überlegen und die entsprechende Taste $\mathrm{zu}$ drücken. Die Antworten wurden auf einer PDP11/03 gesammelt und zur weiteren Bearbeitung aufbereitet.

\subsection{Ergebnis von Exp.1 und 2 (Akzent auf Er):}

Fig.2 zeigt das Ergebnis des IT und des DT ( $A B$ und $B A$ ) auf der linken Seite; rechts finden sich schematisch Intensität und Fo-Verlaup der 'Grenzstimuli' 5, 6 und 7. Stimulus 6 liegt nahe der 50\%-Linie, er ist also nicht eindeutig zuzuordnen, wohl aber Stimulus 5 und 7 . Der Diskriminationsgipfel liegt entsprechend bei $5 / 6$ ( $A B$ ) bzw. bei 6/7 (BA) im Bereich eines psychophysischen Referenzpunktes (vgl. dazu Medin/Barsalou 1987:474 ff.), nämlich des überganges von FALL zu RISE. 
Fig.2
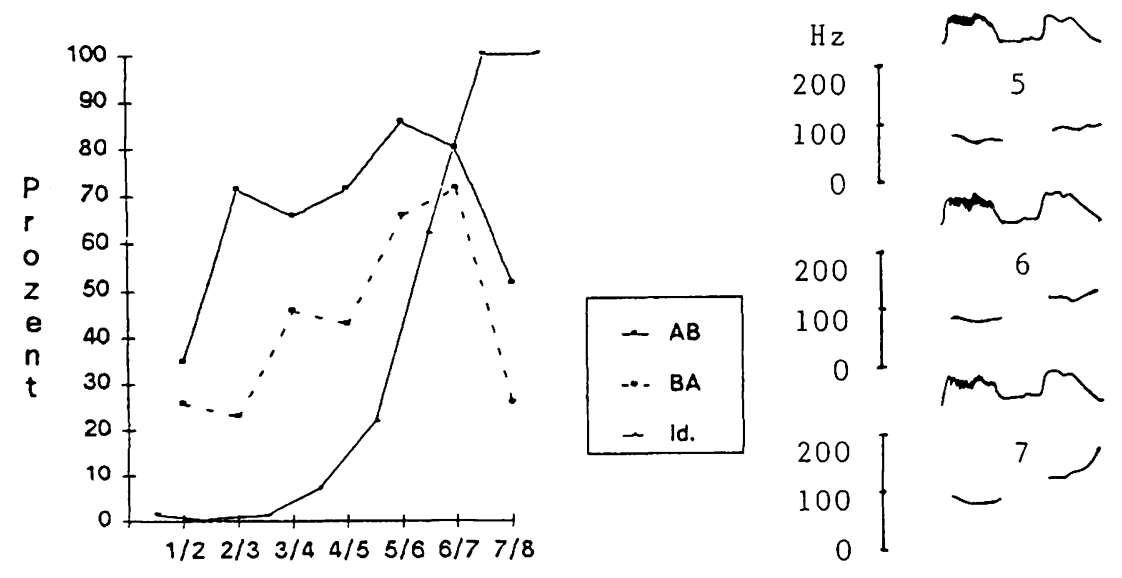

Stimuli

4.6 Ergebnis von Exp.3 und 4 (Akzent auf kommt):

Fig.3 zeigt in analoger Anordnung die Ergebnisse von Exp.3 und 4. Beim IT liegt die 50\%-Grenze zwischen Stimulus 6 und 7. Beim DT liegt der Gipfel der Kurve bei $5 / 6$ ( $A B$ ) bzw. 6/7 (BA), also ebenfalls in der Nähe der psychophysischen Grenze zwischen FALL und RISE. Daneben zeigt sich allerdings ein weiterer, kleinerer Gipfel bei 2/3. Es liegt nahe, den höheren Gipfel, der auch mit der IT-Kurve überelnstimmt, als Kategorienwechsel der Hauptmodi Aussage vs. Frage zu interpretieren, und den niedrigeren, früheren Gipfel als Kategorienwechsel innerhalb der Hauptkategorie Aussage, etwa von bestimmter, abgeschlossener Aussage $\mathrm{zu}$ unbestimmter, nicht abgeschlossener Aussage. (In der traditionellen Terminologie, vgl. v.Essen 1956, wäre das natürlich der progrediente im Gegensatz zum terminalen bzw. interrogativen Tonverlauf.)9 Fig.4 zeigt die Kurven der VPn mit einer klaren 'mittleren' Kategorie (Operationale

9 Diese Interpretation wird durch zwei Folgeexperimente unterstützt, bei denen den VPn im IT drei Kategorien zur Auswahl vorgegeben wurden, nämlich entweder 'Aussage', 'weiß nicht', 'Frage' bzw. 'Aussage', 'unvollendete Außerung', 'Frage'. In beiden Fällen ist der Verlaup der 'Frage'-Kurve ähnlich der in Exp.3, während die mittlere Kategorie den Verlaup der 'Aussage'-Kurve beeinflußt. 
Fig.3
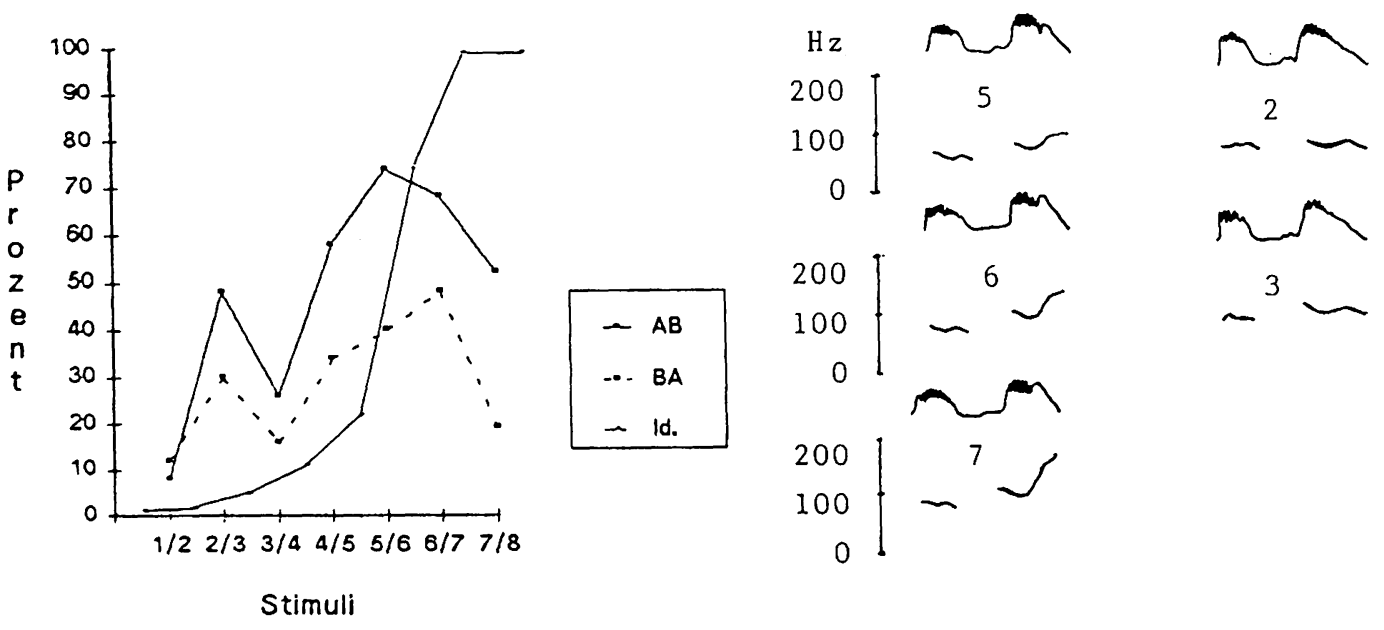

Fig.4: Drei Kategorien $(n=4)$

Fig.5: Zwei Kategorien $(n=6)$
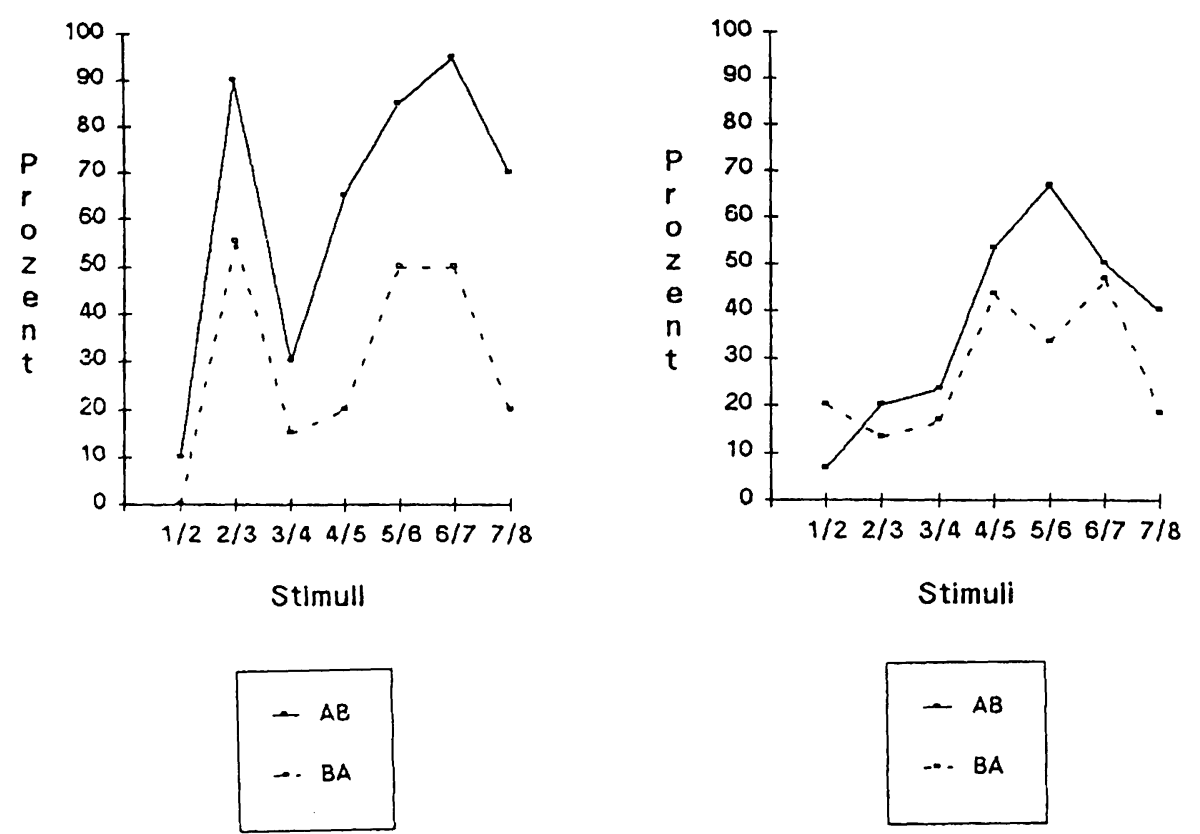
Definition: Alle VPn, bei denen der Diskriminations-Wert, also der Mittelwert aus $A B$ und $B A$, für $2 / 3$ größer als $40 \%$ ist), Fig. 5 die Kurven der restlichen VPn ohne diese dritte Kategorie. (Man kann also annehmen, da $\beta$ VPn solche ( $\mathrm{Ne-}$ ben-)Kategoriengrenzen im Experiment unterschiedlich einsetzen. Da $\beta$ sie unterschiedliche Kategoriengrenzen 'besitzen', ist ein möglicher, aber weitergehender Schluß.) Bei einer Zuordnung der Diskriminationsgipfel zum Fo-Verlauf der entsprechenden Stimuli (vgl. Fig.3) zeigt sich, daß der erste Gipfel dort auftritt, wo ein ebener Tonverlauf (LEVEL) in einen sichtbaren, aber nicht ausgeprägten FALL-RISE übergeht (Stimuli 2/3), der Hauptkategorienwechsel tritt erst dann ein, wenn ein weniger ausgeprägter FALL-RISE in einen ausgeprägten übergeht (Stimuli 5/6/7). Es ist noch nicht klar, warum sich nur beim Kontinuum mit dem Akzent auf dem finalen Element kommt zwei Kategoriengrenzen ergaben. Eventuell lenkt der Zusammenfall von prominenter Wortbetonung und (finaler) Satzintonation die Aufmerksamkeit gezielter auf dieses Element.

\subsection{Diskussion}

Das Versuchsdesign von Exp.1 bis 4 ist daraufhin angelegt, Kategoriengrenzen zu 'entdecken'. Auch wenn einige wesentliche Fragen noch offenbleiben (Relevanz der einzelnen intonatorischen Parameter, Repräsentativität der beiden Minimalpaare für die beiden Satzmodi, exakte Kriterien bei der Entscheidung für oder gegen eine kategoriale Wahrnehmung, exakte Bestimmung der Kategoriengrenzen), so kann doch festgehalten werden, da $\beta$ wir, entsprechend der These WYALFIWYG, nach Grenzen zwischen Kategorien, die eindeutig durch bestimmte Ausprägungen der relevanten physikalischen Parameter definierbar sind, gesucht und sie auch gefunden haben.

5. Auf der suche nach UNEINDEUTIGKEIT

\subsection{Fragestellung}

Auch in Exp.5 bis 7 wird versucht, die intonatorische Kennzeichnung des Fragemodus genauer zu bestimmen. Es werden aber ein anderes Material sowie ein anderes Testdesign verwendet. Grundlage ist der Testsatz Säuft der Leo, der, je nach Intonationsverlauf, ein Verb-Erst-Fragesatz oder ein Verb-ErstExklamativsatz sein kann (Zur Wahl dieses speziellen Testsatzes vgl. Batliner 1988a,b). Die übliche Auffassung kann wie folgt skizziert werden: Bei einer solchen Konstellation indiziert ein terminaler RISE eindeutig eine Frage. Ein 
RISE ist hinreichend, aber nicht notwendig für eine Frageinterpretation; diese ist auch mit finalem und sogar mit initialem FALL möglich. Pasch (1988:121) nimmt an, daß in einem solchen Fall der genaue Konturverlauf auf der Hauptakzentsilbe über die Moduszuweisung entscheidet:

"[Es zeigt sich,] da $\beta$ der einzige Intonationstyp bei Spitzenstellung des nichtimperativischen finiten Verbs, der nur nichtinterrogativ zu interpretierenden sätzen eigen ist, der ist, in dem das finite verb gelängt ist und steigend-fallende Tonhöhenbewegung in der Silbe aufweist, die im finiten Verb den Hauptakzent des Wortes trägt [...]."

Dieser Intonationsverlauf auf einem Verb-Erst-Exklamativsatz ist sicher typisch, aber nicht der einzig mögliche (vgl. die Teststimuli in Batliner 1988a): Es ist auch nur fallender Tonverlauf möglich, wobel dann das größere Ausma $\beta$ des FALL und damit verbunden eine gewisse Dehnung den Exklamativ indizieren.

Bei diesen Experimenten interessiert uns nicht nur eine Kategoriengrenze, sondern besonders auch die Frage, ob sich mehr oder weniger starke Ausprägungen des initialen FALL oder des finalen RISE auch in einer mehr oder weniger eindeutigen Moduszuweisung widerspiegeln.

\section{$5.2 \quad$ Material}

Der Autor produzierte die zwei Kontextsätze (s.u.) mit natürlicher sowie den Testsatz Säuft der Leo mit monotoner Intonation. Die weiteren Bedingungen waren die gleichen wie bei Exp.1 bis 4 (vgl. auch ausführlich zur Generierung und Manipulation solcher Teststimuli Batliner 1988b, 1989). Der Testsatz wurde mit einem segmentierungsprogramm periodenweise geschnitten und diente damit zur pitchsynchronen Manipulation auf Halbtonbasis. Es wurden die folgenden 13 Stimuli erzeugt, wobei vor dem Schrägstrich immer der Betrag des FALL in Halbtönen auf säuft und nach dem Schrägstrich der LEVEL-Verlauf (mit ' 0 ' gekennzeichnet) bzw. der Betrag des RISE in Halbtönen auf -eo verzeichnet sind; der $L$ - erhielt immer einen FALL von 2 Halbtönen. Fig.6 zeigt die schematischen Verläufe der stimuli.

Stimuli 1-10:2/10,2/8, 2/6, 2/4, 2/2, 2/0,4/0,6/0,8/0, 10/0;

Stimuli $11-13: 4 / 6,6 / 4,8 / 2$. 
Fig.6: Schematische Stimulusverläufe

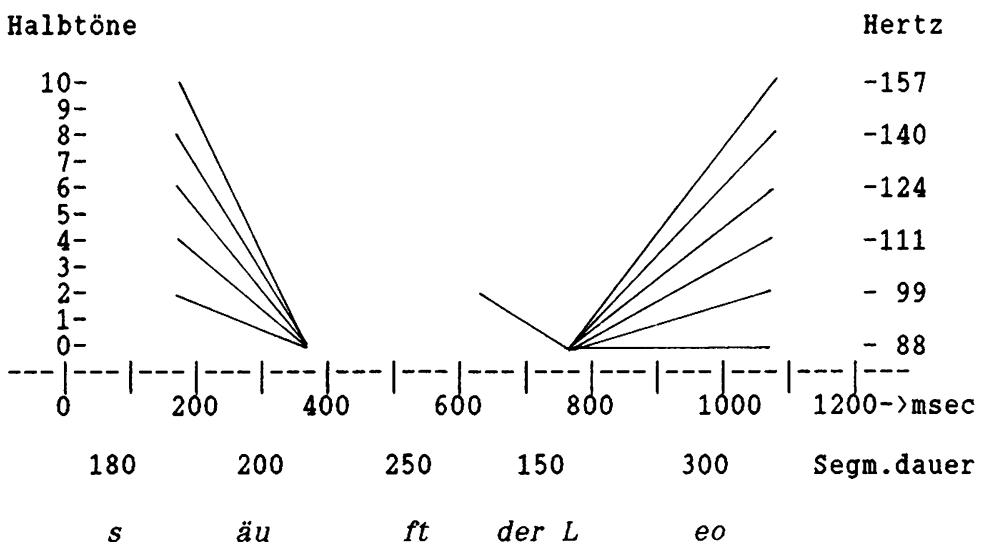

Die ersten 10 Stimuli sind eindeutig in dem Sinn, da $\beta$ entweder ein FALL auf säuft oder ein RISE auf Leo liegt. (Die zwei Halbtöne FALL auf säuft waren nötig, da dieser Teil sonst zu monoton geklungen hätte; sie sind auch wegen des zu erwartenden phonetischen Kontexteffekts - Fo-Abfall nach stimmlosem Konsonanten - motiviert.) Die letzten drei Stimuli sind uneindeutig oder hybrid, da sie aus FALL und RISE bestehen. Im Vergleich zu Exp.1 bis 4 sind alle diese Stimuli bzgl. Fo genau kontrolliert und bzgl. aller anderen Parameter konstant gehalten.

\subsection{Versuchspersonen}

Versuchspersonen waren StudentInnen der Germanistik oder Phonetik, die für ihre Teilnahme bezahlt wurden. .

\section{$5.4 \quad$ Design}

Es wurden "Kontexttests" (KTs) (Exp.5 und 6) und ein All-Step-Test (Exp.7) durchgeführt. Bei den KTs wurden die Teststimuli mit einem vorangehenden Kontextsatz kombiniert, der die Modusinterpretation festlegen sollte: Bist $d u$ sicher (mit drei verschiedenen Tonverläufen: RISE bei Exp.5, RISE, RISE-FALL und FALL bei Exp.6) für die Frage und Also sowas hätt'ich nicht gedacht für den Exklamativ. Fig.7 zeigt die Verläufe der Kontextsätze. Die Kontextsätze sind fokusneutral, d.h. sie indizieren auf dem Testsatz keinen bestimmten Fokus. Ein Prominenzwechsel von Leo bei Stimulus $1 \mathrm{zu}$ säuft bei Stimulus $10 \mathrm{kann}$ also für unsere Zwecke unberücksichtigt bleiben (vgl. aber die Diskussion zu Exp.6 in Teil 5.7). Die VPn mußten anhand einer Fünferskala (1-5) entscheiden, wie gut 
die beiden Sätze zusammenpaßten, und die entsprechende Taste auf dem Abstimmkästchen drücken. (Die Vorgaben waren: die beiden Sätze passen 'sehr gut', 'gut', 'noch gut', 'schlecht', 'sehr schlecht' zusammen.) Bei dem All-stepTest wird jedes Testitem mit jedem anderen Testitem gepaart, und die VPn müssen die Ahnlichkeit der zwei Stimuli innerhalb eines Paares ebenfalls auf einer Fünferskala beurteilen (von 'sehr ähnlich' bis 'sehr unähnlich'). Die weitere Verarbeitung geschah ähnlich wie bei Exp.1 bis 4. Dafür wurden die Skalen von $1-5$ in Skalen von 0-4 umgerechnet.

Fig.7: Schematische Fo-Verläufe der Kontextsătze

Bist du sicher?

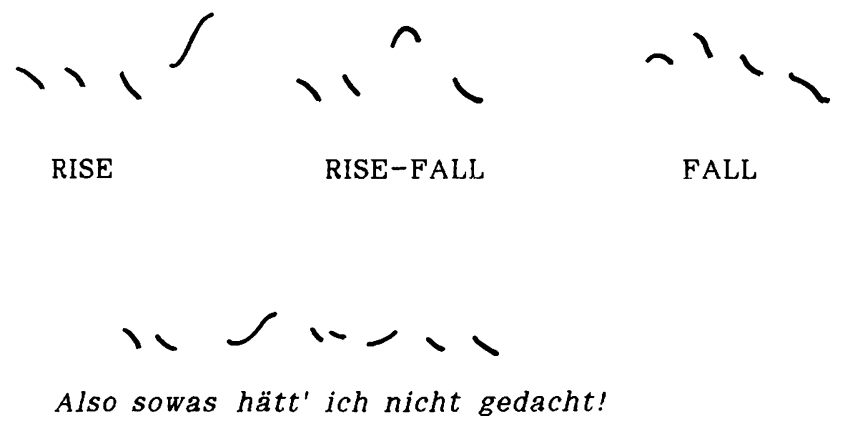

5.5 Experiment 5

Zwei Kontextsätze (Bist du sicher mit RISE und Also sowas hätt'ich nicht gedacht) wurden mit den 13 Teststimuli gepaart und je dreimal randomisiert mit einer Pause von $4 \mathrm{sec} z$ wischen den Paaren und einem minimalen Interstimulusintervall von ca. $200 \mathrm{msec}$ (Zugriffszeit des Rechners) 20 VPn dargeboten. In Fig.8 zeigen die beiden linken Kurven das Ergebnis für die eindeutigen, die beiden rechten Kurven das Ergebnis für die hybriden stimuli. Folgende Punkte lassen sich festhalten:

1. Das obere und das untere Quartil sind nicht belegt, d.h. keine der Kombinationen wurde als sehr gut bis gut, aber auch keine als sehr schlecht bis schlecht bewertet. Dafür gibt es verschiedene Erklärungsmöglichkeiten: Reine Moduszuweisungen fallen nur selten sehr schlecht aus, da es hier 'nichts gibt, was es nicht gibt'; d.h. man kann sich immer eine Situation vorstellen, bel der auch Außerungen mit eigentlich nicht zusammenpassender Intonationskontur miteinander verträglich sind. Daß wir keine sehr gut bewerteten Kombinationen 
erhielten, mag an der Koppelung eines unmanipuliert resynthetisierten, sehr gut klingenden Kontextsatzes mit einem schematisch nur in einem Parameter manipulierten Testsatz liegen. (Informelle Befragungen der VPn weisen darauf hin.) Ein weiterer Faktor ist sicher die Tendenz einiger VPn, generell keine ganz schlechten oder ganz guten 'Noten' zu vergeben.

Fig. 8:

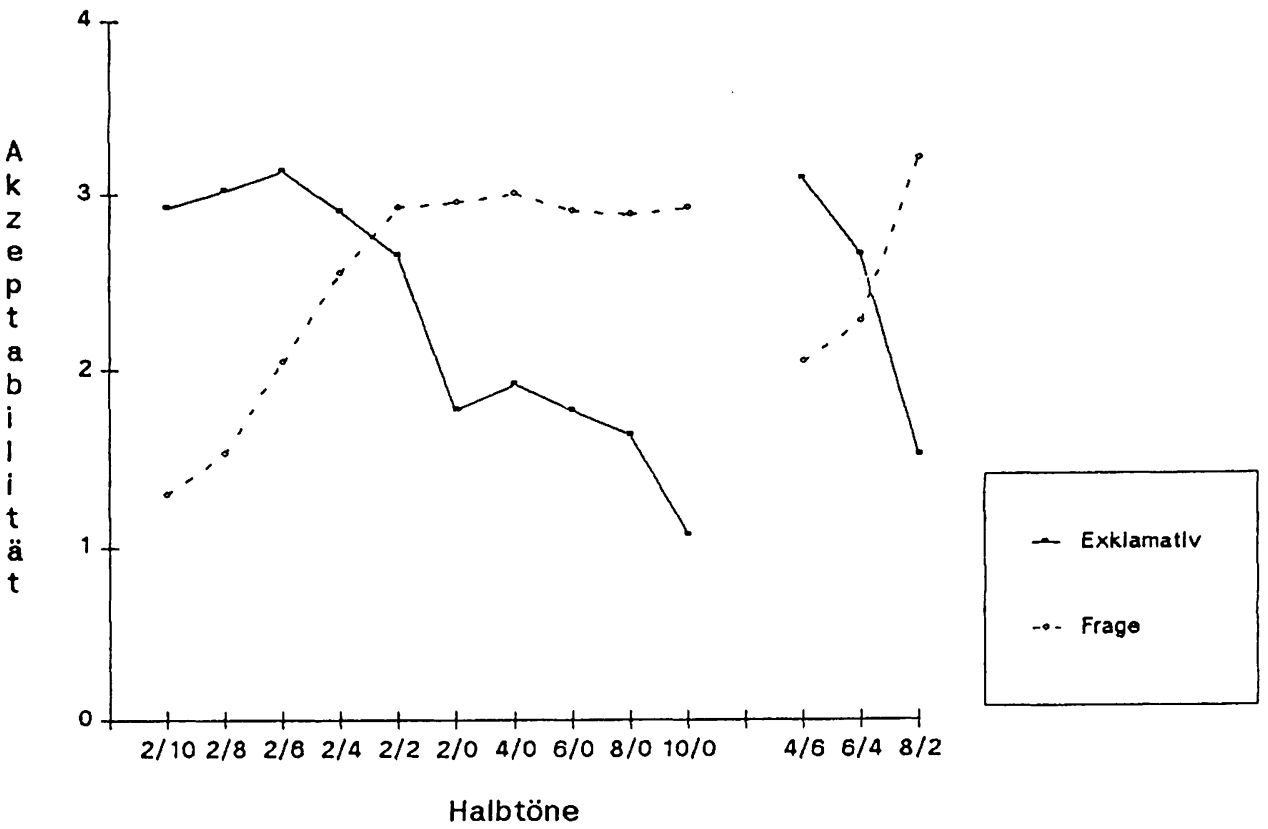

2. Der 'Schnittstimulus' der Kurven (und damit die in diesem Test gefundene Kategoriengrenze) liegt bei den eindeutigen Stimuli zwischen $2 / 4$ und $2 / 2$ und analog dazu bei den hybriden zwischen $6 / 4$ und $8 / 2$. Das stimmt mit Exp.1 bis 4 überein, wo die Kategoriengrenze nicht etwa bei LEVEL, sondern bei einem leichten RISE liegt. Die 'eigentliche' Frage beginnt also erst bei einem stärkeren RISE.

3. Der Kurvenverlauf ist unterhalb des Schnittpunktes steiler als oberhalb. Am prototypischsten in diesem Kontinuum sind jeweils die stimuli mit der größten Fo-Bewegung, also mit dem größten finalen RISE für die Frage oder mit dem größten initialen FALL für den Exklamativ. Schwächt sich dieser Parameter $a b$, so ist der Modus etwa proportional zur Abschwächung weniger ausgeprägt. Damit läßt sich der steile Verlauf unterhalb des Schnittpunktes erklären. Oberhalb des Schnittpunktes ist der Verlauf bei beiden Modi eher eben. Das könnte als ein Hinweis auf eine Kategorialität bei der Moduszuweisung aufgefaßt werden. Wir müssen aber auch mit anderen Ursachen rechnen. Es ist nämlich nicht ganz 
auszuschließen, daß nach einem exklamativindizierenden Also sowas hätt' ich nicht gedacht nicht ein Exklamativ, sondern eine "überraschte" Frage folgt: (Ist das denn wirklich wahr?) Säuft der Leo? Dadurch ließe sich erklären, da $\beta$ etwa der Stimulus $2 / 10$ nicht noch schlechter bewertet wird als der Stimulus 2/6: Die VPn bewerten den Testsatz nicht als unterschiedlich gut geglückte Realisation eines Exklamativsatzes, sondern bewerten eine etwas ungewöhnliche, aber nicht unvorstellbare Kombination von "Exklamativsatz-Kontext" und anschließender Frage.

Der Kontextsatz Bist $d u$ sicher wurde in den drei unterschiedlichen Versionen RISE, RISE-FALL und FALL mit den 13 Testsätzen gepaart und 12 VPn je zweimal randomisiert dargeboten; es sollte mit diesem Experiment untersucht werden, ob der Verlauf der Fo-Kontur auf einem frageindizierenden Kontextsatz einen Effekt auf die Bewertung der Kombinationen von Kontext- und Testsatz ausübt, m.a.w. ob es nicht nur eine allgemeine Textkohärenz gibt, sondern auch eine spezielle, die nur den Tonverlauf betrifft. Fig.9 zeigt, daß mit Ausnahme des Stimulus 2/10, der mit dem FALL-Kontext deutlich schlechter bewertet wird, die Intonation des Kontextsatzes keine Rolle spielt. Das Ergebnis - geringer Effekt des unterschiedlichen Tonverlaufs des Kontextsatzes - steht in einem gewissen widerspruch $\mathrm{zu}$ in Batliner (1989) referierten Ergebnissen, bei denen allerdings das experimentelle Design unterschiedlich war: während in Exp.6 nur frageindizierende Kontextsätze dargeboten wurden, wurden dort auch Kontextsätze dargeboten, die andere Satzmodi indizierten. Möglicherweise ist ein Designeffekt analog zu dem weiter unten beschriebenen für diese Unterschiede verantwortlich.

Deutlich ist ein Wendepunkt der drei Kurven beim Stimulus $2 / 0$ zu beobachten - ein auf den ersten Blick kontraintuitives Ergebnis: Je stärker der FALL auf säuft, m.a.W. je exklamativsatztypischer der Intonationsverlauf, desto besser wird die Bewertung für die Frage - ohne natürlich gleich so gut zu werden wie bei den Stimuli mit einem fragetypischen, deutlichen finalen RISE. Es läßt sich eine Beziehung herstellen $\mathrm{zu}$ den Ergebnissen von StuddertKennedy/Hadding (1973), die nachweisen, daß die Fo-Gipfelhöhe mit dem finalen RISE in einer trading relation steht: je höher der Gipfel, desto weniger ausgeprägt muß der finale RISE sein, um dennoch eine Frage zu indizieren. Ihr 'höherer Gipfel' ergibt aber ebenso wie unser 'stärkerer FALL' einen größeren Fo-Range (i.e. eine größere Differenz zwischen dem Fo-Maximum und dem FoMinimum der Außerung). Damit ist aber noch nicht erklärt, warum die gleichen Kombinationen von Kontext- und Testsatz in Exp.5 anders bewertet werden als in Exp.6: Die Stimuli 2/0 bis $10 / 0$ unterscheiden sich in Exp.6 in Kombination 
mit dem in beiden Experimenten verwendeten RISE-Kontext bei einem nichtparametrischen Test (Kendalls Konkordanzkoeffizient) signifikant voneinander $(p<.029)$, während sie sich in Exp.5 natürlich nicht unterscheiden $(p<.946)$.

Fig.9:

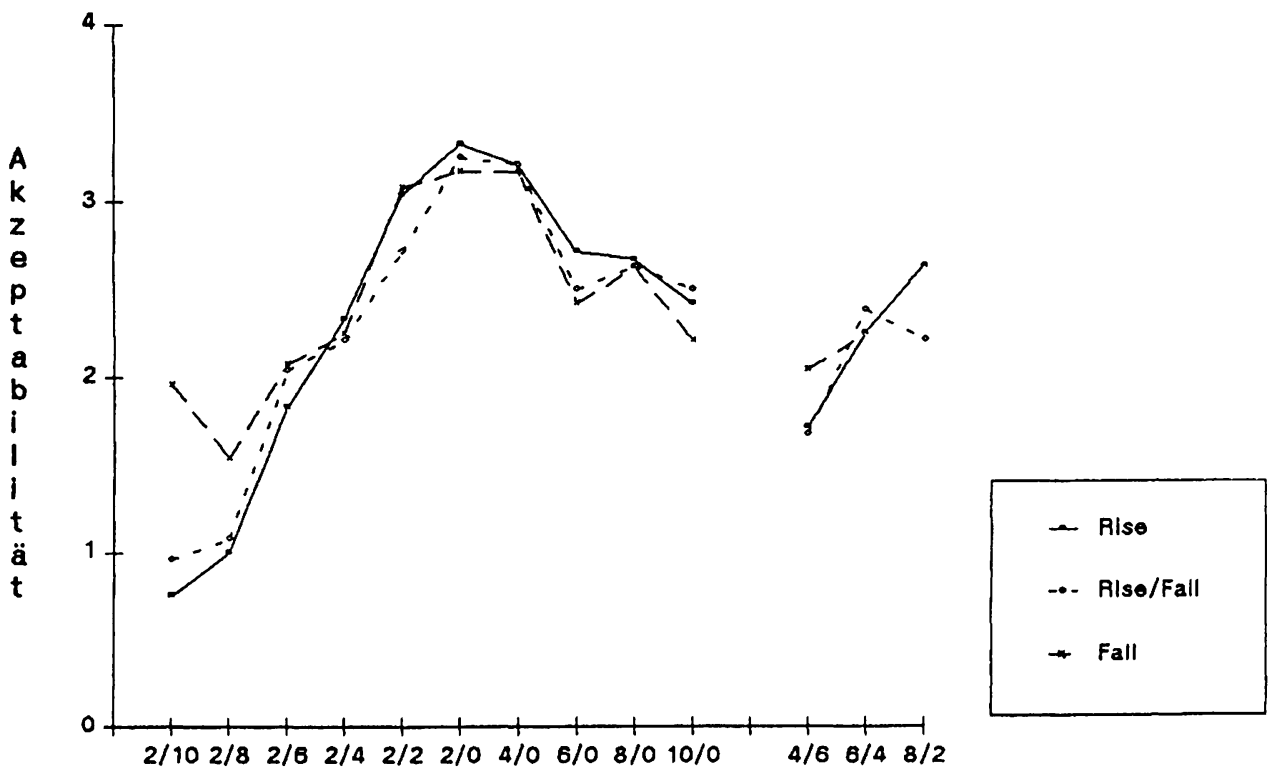

Halbtöne

\subsection{Designeffekte}

Wir wollen uns nun den möglichen Erklärungen für die unterschiedlichen Ergebnisse von Exp.5 und Exp.6 zuwenden.

\subsubsection{Situationskontext}

Den VPn wurde in der Instruktion gesagt, welche Kontextsätze vorkommen werden; sie konnten sich also von Anfang an darauf einstellen, daß in Exp.5 ein Frage- und ein Exklamativ-Kontext und in Exp.6 nur Fragekontexte vorkamen. In Exp.6 wissen die VPn also, daß sie nur innerhalb der Fragen differenzieren müssen. Nun ist bei Verb-Erst-Fragesätzen der finale RISE zwar prototypischer, 
ein (finaler oder initialer) FALL aber auch zulässig. Es sieht also so aus, als hätten die VPn hier nach zwei Regeln geurteilt:

1. Ein RISE ist fragetypischer als ein FALL.

2. Je ausgeprägter die Bewegung auf dem Testsatz, desto besser wird die Kombination bewertet.

Die Regeln wirken grob additiv, ein ausgeprägter RISE wird also bei einer Frage in etwa doppelt so gut bewertet wie ein ausgeprägter FALL. In Exp.5 dagegen wußten die VPn, daß auch Exklamative möglich sind, und kamen deshalb eher auf die Idee, daß ein FALL exklamativtypisch, ergo frageuntypisch ist. Dieser Designeffekt kann nun in einen allgemeinen situationellen (pragmatischen) Effekt umgemünzt werden, in den Effekt des Situationskontextes also im Gegensatz zum Effekt des reinen Sprachkontextes (wie er bei unseren Kombinationen aus Kontext- und Testsatz untersucht wurde): So wie man bei den Experimenten 5 und 6 von einem (Situations-)Kontext sprechen kann, der entweder eine Nicht-Frage-Interpretation grundsätzlich ermöglicht (Exp.5) oder nicht (Exp.6), so kann man in einer natürlichen Kommunikationssituation von einem Situationskontext sprechen, der eine Frageinterpretation eher wahrscheinlich oder unwahrscheinlich macht. Im ersten Fall ist dann eine Intonationskontur, die nicht fragetypisch ist, eher zulässig als im zweiten, bei dem die Intonation eine größere funktionale Belastung trägt.

\subsubsection{Sprachkontext}

Der Kontextsatz Bist $d u$ sicher? ist fokusneutral; im Kontinuum der Testsätze dürften dagegen zumindest die Randstimuli $2 / 10$ und $2 / 8$ bzw. $8 / 0$ und 10/0 auch als unterschiedlich akzentuiert perzipiert werden: Bei den ersten beiden wird man den Fokusakzent auf Leo plazieren, bei den letzten beiden auf säuft. Nun stellt der Kontextsatz Bist du sicher? keine Gesprächseinleitung dar: Er impliziert einen vorangegangenen Beitrag des Gesprächspartners, in dem dieser mitteilt, daß 'der Leo säuft'. Der Sprecher kann nun das, worüber er Gewißheit haben möchte, in der Versicherungsfrage gesondert hervorheben: entweder Säuft, oder Leo, oder die Kombination Säuft und Leo. Hervorgehoben wird durch eine größere Ausprägung des Range, bei unseren stimuli also entweder durch einen größeren initialen FALL oder durch einen größeren finalen RISE. Mit dieser Hypothese kann der Kurvenverlauf in Fig.10 erklärt werden, aber nicht der Unterschied zwischen Exp. 5 und 6. 


\subsubsection{Situationskontext und Sprachkontext}

Möglicherweise treffen beide Erklärungen zu: In Exp.5 konzentrieren sich die VPn auf den Unterschied zwischen Exklamativ und Frage; d.h. daß Erklärung 1 zutrifft. In Exp.6 können sich die VPn auf Unterschiede innerhalb der Fragen und zwischen ausgeprägten Fokusakzenten konzentrieren; d.h. daß sowohl Erklärung 1 wie Erklärung 2 zutreffen.

\subsection{Experiment 7}

Die Ergebnisse des All-Step-Tests, an dem 32 VPn teilnahmen, gingen in die Analyse einer Nonmetrischen Multidimensionalen Skalierung (NMDS) ein. Ein iterativer Algorithmus führt dabei zu einer Optimierung der Lösung, bei der die Items im $n$-dimensionalen Raum dargestellt werden, wobei ähnliche Items nahe beieinander und unähnliche weit auseinander zu liegen kommen. Aus der Lage der Items zueinander können (vorsichtige) Schlüsse auf kognitiv relevante Cluster und Dimensionen gezogen werden. Zum Verfahren im einzelnen vgl. Borg (1981) und Batliner (1988a). Um die Zahl der Stimuli und damit die Dauer des Experimentes im Rahmen $\mathrm{zu}$ halten, wurden von den 13 Stimuli nur die eindeutigen Stimuli $10 / 0,6 / 0,2 / 6$ und $2 / 10$ sowie die hybriden $8 / 2,6 / 4$ und 4/6 getestet. Mit der Statistik-Prozedur SSAl wurden ein- und zweidimensionale NMDS-Lösungen berechnet; als Gütekoeffizient erhält man bei diesem Verfahren den Alienationskoeffizienten. Eine Lösung wird üblicherweise dann als ausreichend gut bewertet, wenn dieser Koeffizient unter .15 liegt.

Fig.10 zeigt die zweidimensionale Lösung mit einem Alienationskoeffizienten von .045. Es ist keine Clusterbildung zu erkennen, wohl aber eine Anordnung im Halbkreis, der man zwei Dimensionen unterlegen kann. Die eine Dimension, die übrigens auch die eindimensionale Lösung widerspiegelt, entspricht genau der intendierten Abstufung der Modusindizierung: von eindeutiger Frage zum eindeutigen Exklamativ; sie wird indiziert durch die Ausprägungen des initialen FALL bzw. des finalen RISE. Es bietet sich an, eine zweite Dimension 'Eindeutigkeit' anzunehmen, da an dem einen Ende dieser Dimension die Stimuli mit entweder dominantem FALL oder RISE angeordnet sind, am anderen Ende die hybriden stimuli. Die VPn urteilten also nicht nur anhand der stärkeren Ausprägung von FALL oder RISE und damit entlang einer eindimensionalen Kategorialität, sondern waren in der Lage, konstistent sowohl die Ausprägung des RISE als auch die des FALL zu berücksichtigen. ${ }^{10}$

10 Es liegt natürlich der Einwand nahe, daß die VPn ihrer Bewertung keine kognitiven Dimensionen unterlegen, sondern einfach die Oberflächenähnlich- 
Fig.10:

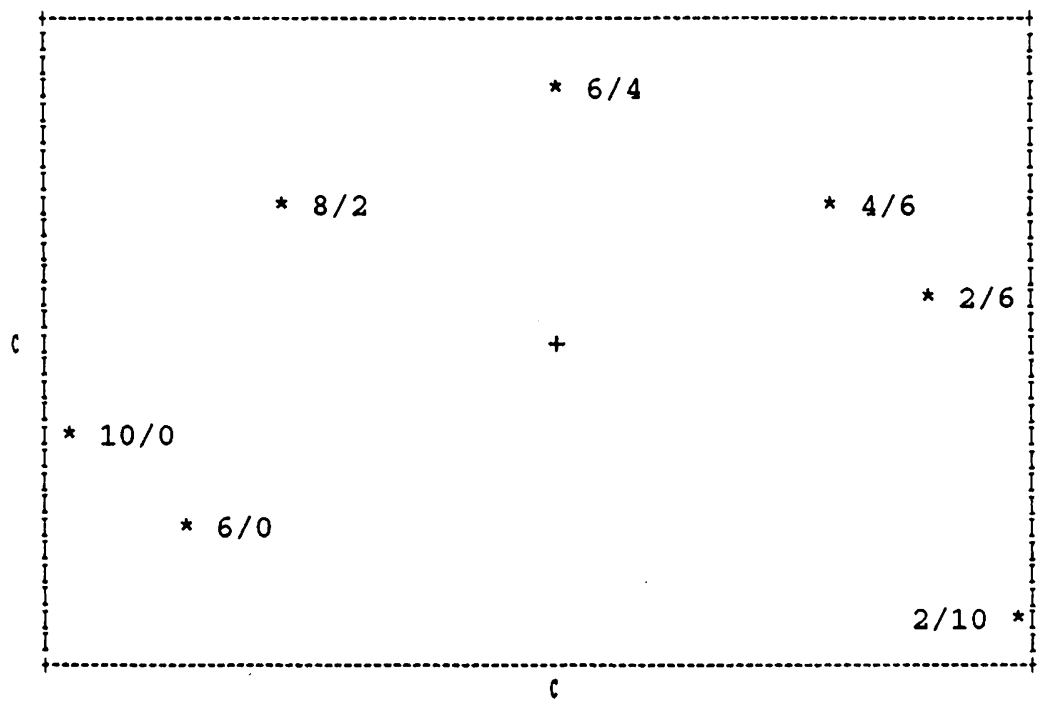

\subsection{Diskussion von Experiment 5 bis 7}

Das Versuchsdesign dieser Experimente diente dazu, Abstufungen innerhalb der Kategorien sowie Uneindeutigkeiten bei der Zuordnung von Parameterausprägungen zu Satzmodi zu entdecken. Entsprechend unserer These WYALFIWYG wurde beides gefunden.

keit. Dieser Einwand betrifft den Schluß vom experimentellen Modell auf die abgebildete 'Realität' und gilt für jede Art von Experiment. Er kann nicht endgültig, wohl aber mit kumulativer Evidenz abgelehnt werden. Zumindest die folgenden Punkte sind dabei relevant: (1) Experimentelle Replikation: das gleiche Experiment mit einer anderen Instruktion (nicht 'Oberflächen'Ahnlichkeit, sondern Bedeutungsgleichheit sollte bewertet werden) führte zum grundsätzlich gleichen Ergebnis. (2) Konvergenz mit anderen Ergebnissen: Exp.7 konvergiert in seinen Ergebnissen mit Exp.5 und 6. (3) Interpretierbarkeit der Lösung: Die (konvergierenden) Ergebnisse unserer Experimente sind allesamt sinnvoll interpretierbar (vgl. auch die weiterführenden Experimente in Batliner 1989). 
6. Schlußdiskussion

\subsection{Beschränkungen}

Grundsätzlich muß gesagt werden, daß Schlüsse aus einem begrenzten experimentellen Material auf 'die deutsche Sprache' nur vorsichtig gezogen werden können - schon allein deshalb, weil viele andere Strukturen möglich sind und sicher auch ein anderes Bild ergeben würden (vgl. die von Geluykens 1987 fürs Englische getesteten Strukturen). Eine einfache Generalisierung der Ergebnisse der einen Testserie auf die in der anderen Testserie untersuchten Konstellationen und vice versa ist natürlich nicht möglich, da die intonatorischen Parameter bei den beiden Arten der Minimalpaarkonstellation unterschiedlich belastet sind. In Exp.1 bis 4 ist die Rolle der einzelnen (immer kovariierend manipulierten) Parameter unberücksichtigt geblieben. In Exp.5 bis 7 blieb z.B. die Dehnung - ein stabiles Merkmal des Exklamativs, vgl. Batliner (1988b) - konstant. Die grundsätzliche Rolle der Intonation ist aber aup der anderen Seite durchaus vergleichbar: Sie disambiguiert bei syntaktisch und segmental ansonsten gleicher Struktur. Tertium comparationis ist also der funktionale Aspekt.

\subsection{WYALFIWYG und die Folgen}

Genauso wie ein Werkzeug, das zugleich Hammer, Beil, Schraubenzieher und Nagelfeile ist, nichts taugt, gibt es kein experimentelles Design, mit dem sich sowohl Kategoriengrenzen als auch Übergänge und Abstufungen innerhalb von Kategorien gleichermaßen gut untersuchen lassen. Anders gesagt: Unterschiedliche Testdesigns arbeiten unterschiedliche Aspekte heraus - die sich auf den ersten Blick widersprechen können. So würde sich eine einfache Generalisierung aus der ersten Testserie und eine entsprechende einfache Generalisierung aus der zweiten widersprechen. Unterschiedliche Modelle (in unserem Fall das Modell der Kategorialen Wahrnehmung bzw. das eher funktional ausgerichtete Modell, das mit den KTs in der zweiten Testserie untersucht wurde) bilden unterschiedliche Funktionen bzw. Bereiche ab. Deshalb kann es auch letztlich kein experimentum crucis geben, das zwischen zwei solchen Modellen entscheidet.

Damit kommen wir auf die in Teil 1 bis 3 diskutierten Punkte zurück: U.E. bilden die in SP-Untersuchungen üblichen ITs und DTs nur ganz bestimmte real life-Situationen ab, nämlich solche, in denen explizit Taxonomien aufgestellt 
bzw. überprüft werden (also nicht Urteile über Ereignisse, sondern Urteile über Merkmale, die diesen Ereignissen zu eigen sind, vgl. Medin/Barsalou 1987:465f). KTs dagegen bilden eher die natürlichsprachliche Kommunikation $a b$, auch wenn sie natürlich nicht mit ihr deckungsgleich sind; es handelt sich um eine Metakompetenz (Urteile über Außerungen), und nicht um die 'echte' Kompetenz (Gebrauch dieser Außerungen). Unabhängig davon, welchen (ontologischen) Status man einer im IT und DT gefundenen Kategoriengrenze zuordnet, so erscheint es doch heuristisch sinnvoller, nicht automatisch anzunehmen, daß solche Kategoriengrenzen eins-zu-eins auf real life-Situationen abgebildet werden können. U.E. bilden sie eher ein (möglicherweise normgebendes) Bezugssystem, auf das 'im Zweifelsfall' referiert wird. (Das stimmt damit überein, daß solche Kategoriengrenzen flexibel sind, vgl. Repp/Liberman 1987.) Wir sehen zwei Möglichkeiten, das Ergebnis von Experimenten realitätsnäher zu gestalten: Zum einen Perzeptions- und besonders Produktionsdaten aus real life-Situationen zu gewinnen - dabei gibt es natürlich praktische und ethische Probleme. Zum anderen eine gleichzeitige Berücksichtigung von Perzeptions- und Produktionsdaten aus Experimenten mit unterschiedlichen Designs und Modellvorstellungen, die in vergleichbaren Ergebnissen konvergieren (vgl. Batliner 1989).

\section{Zusammenfassung}

Die Experimente haben gezeigt, $d a \beta$ es bei dem für den Fragemodus relevanten phonetischen Merkmal des Tonverlaufs zu anderen Satzmodi hin eine relativ stabile Kategoriengrenze im Bereich eines psychophysischen Referenzpunktes (der Grenze zwischen RISE und FALL) gibt, sowie Abstufungen innerhalb der Kategorie und die Möglichkeit, daß der außersprachliche Kontext eine andere Bewertung der intonatorischen Merkmale verursacht. Diese Ergebnisse widersprechen sich nicht, sondern sind auf die unterschiedlichen getesteten Konstellationen, die unterschiedlichen Gesichtspunkte der zugrundeliegenden Modellvorstellungen und letztlich auf die entscheidenden Charakteristika der intonatorischen Merkmale zurückzuführen. Anders gesagt: Das Merkmal Tonverlauf hat zwar bevorzugte Interpretationen, aber keine Eigenbedeutung; es erhält Bedeutung nur im Kontext mit anderen Merkmalen. Das Ganze ist auch hier mehr als die summe der Teile. 


\section{LITERATUR}

Ainsworth, W. A./Lindsay, D. (1986): Perception of pitch movement on tonic syllables in British English. In: J. Acoust. Soc. Am. 79. S.472-480.

Altmann, H. (1987): Zur Problematik der Konstitution von Satzmodi als Formtypen. In: Meibauer, J. (Hg.) (1987): Satzmodus zwischen Grammatik und Pragmatik. Tübingen. S.22-56.

Altmann, H. (Hg.) (1988): Intonationsforschungen. Tübingen.

Batliner, A. (1988a): Modus und Fokus als Dimensionen einer Nonmetrischen Multidimensionalen Skalierung. In: Altmann, H. (1988). S.223-241.

Batliner, A. (1988b): Der Exklamativ: Mehr als Aussage oder doch nur mehr oder weniger Aussage? Experimente zur Rolle von Höhe und Position des FoGipfels. In: Altmann, H. (Hg.) (1988). S.243-271.

Batliner, A. (1989): Wieviel Halbtöne braucht die Frage? Merkmale, Dimensionen, Kategorien. (In diesem Band)

Borg, I. (1981): Anwendungsorientierte Multidimensionale Skalierung. Berlin etc.

v.Essen, O. (1956): Grundzüge der hochdeutschen Satzintonation. Ratingen/Düsseldorf.

Geluykens, R. (1987): Intonation and speech act type. An experimental approach to rising intonation in queclaratives. In: Journal of Pragmatics 11. S.483494.

Hadersbeck, M. (1987): A new program for manipulation of natural speech interpolation between two natural utterances. In: Proceedings XIth ICPhS, Vol.5. S.35-38.

Harnad, S. (Hg.) (1987): Categorical perception. The groundwork of cognition. Cambridge etc.

Karttunen, L. (1977): Syntax and semantics of questions. In: Linguistics and Philosophy 1. S.3-44.

Lisker, L. (1978): Rapid vs. rabid: A catalogue of acoustic features that may cue the distinction. In: Haskins Laboratories: Status Report on Speech Research SR-55/56. S.181-188.

Medin, D.L./Barsalou, L.W. (1987): Categorization processes and categorical perception. In: Harnad, S. (Hg.) (1987). S.455-490.

Oppenrieder, W. (1988): Intonatorische Kennzeichnung von Satzmodi. In: Altmann H. (Hg.) (1988). S.169-205.

Pasch, R. (1988): Fragen als Aufforderungen? - Kommentare zu U. Brauße: Modalpartikeln in Fragesätzen. In: Lang, E. (Hg.): Studien zum Satzmodus I. Berlin. S.114-133.

Repp, B.H. (1981): Phonetic trading relations and context effects: New experimental evidence for a speech mode of perception. In: Haskins Laboratories: Status Report on Speech Research SR-67/68. S.1-40. 
Repp, B.H. (1984): Categorical perception: Issues, methods, findings. In: Lass, N.J. (Hg.): Speech and Language. Orlando etc. S.243-335.

Repp, B.H./Liberman, A.M. (1987): Phonetic category boundaries are flexible. In: Harnad, S. (Hg.) (1987). S.89-112.

Sadock, J.M./Zwicky, A.M. (1985): Speech act distinctions in syntax. In: Shopen, T. (Hg.) (1985): Language Typology and Syntactic Description. Volume I: Clause Structure. Cambridge etc. S.155-196.

Schiefer, L./Batliner, A. (1988): Intonation, Ordnungseffekt und das Paradigma der Kategorialen Wahrnehmung. In: Altmann, H. (Hg.) (1988), S.273-291.

Simon, Th. (1983): Manipulation of natural speech signals according to the speech parameters of different speakers. In: Forschungsberichte des Instituts für Phonetik und Sprachliche Kommunikation der Universität München (FIPKM) 17. S.233-245.

Studdert-Kennedy, M./Hadding, K. (1973): Auditory and linguistic processes in the perception of intonation contours. In: Language and Speech 16. S.293313.

Zaefferer, D. (1984): Frageausdrücke und Fragen im Deutschen. Zu ihrer Syntax, Semantik und Pragmatik. München. 

WIEVIEL HALBTONE BRAUCHT DIE FRAGE?

MERKMALE, DIMENSIONEN, KATEGORIEN.

Anton Batliner

1. Einleitung

\subsection{Uberblick}

In diesem Beitrag wird die Rolle unterschiedlicher Ausprägungen eines fallend-steigenden Tonverlaufs für die Satzmodusindizierung bei Verb-ErstFragesatz und Verb-Erst-Exklamativsatz untersucht. In Perzeptionsexperimenten mit unterschiedlichem Design werden Kategoriengrenze und Abstufungen innerhalb der Kategorien sowie der Zusammenhang zwischen akustischen und phonetischen Merkmalen, perzeptiven Dimensionen und linguistischen Kategorien genauer bestimmt.

\subsection{Fragestellung}

Der Tonverlauf eines Verb-Erst-Fragesatzes kann steigend oder fallend sein. Es gibt keine gesicherten Angaben darüber, wann ein Sprecher den einen und wann er den anderen Verlauf wählt. Traditionellerweise nahm man einen steigenden 'interrogativen' Verlauf als üblich an; Klein (1982) setzt dagegen den fallenden Verlauf als normal, den stelgenden als mit besonderen Merkmalen behaftet an ('erstaunte Frage' o.ä.). Fest steht aber, daß der Tonverlauf (und insgesamt die Intonation) funktional unterschiedlich belastet ist: In vielen Fällen reichen andere Merkmale wie Verbsemantik und/oder Modalpartikeln aus, um den Fragemodus festzulegen, wie bei Gehört das (etwa) Ihnen hier? oder Stellt Ihr euch etwa an? In anderen Fällen (graduierbare Verben und/oder andere bzw. ambige Modalpartikeln) muß allerdings die Intonation zwischen einer Frage- und einer Nicht-Frage- (d.h. Exklamativ- oder Imperativ-) Interpretation unter- 
scheiden. Beispiele sind Stellt Ihr euch (vielleicht) an ?/! oder Säuft der Leo ?/!. 1

Wenn auch Verwendungsbedingungen und damit einhergehende Merkmalsausprägungen noch kontrovers sind, so dürfte wohl darüber Einigkeit bestehen, daß ein leichter initialer Abfall (FALL) der Grundfrequenz (Fo) und ein starker finaler Anstieg (RISE) der Fo auf einem Satz wie Säuft der Leo eindeutig eine Frage indizieren - sei das nun eine 'normale' Frage oder eine 'erstaunte' ('Versicherungs' bzw. 'Rück'-) Frage. Bei den im folgenden beschriebenen Perzeptionsexperimenten wurde daher eine entsprechende eindeutige Kontur für den einen 'Eckstimulus' der generierten Kontinua gewählt. Bei den weiteren Stimuli wurden der initiale FALL und der finale RISE systematisch variiert, wobei allerdings die Richtung der initialen und der finalen Bewegung nie gegenlăufig wurde, d.h. aus dem initialen FALL wurde kein RISE und aus dem finalen RISE kein FALL. Diese Beschränkung ergab sich schlicht aus der Notwendigkeit, die Anzahl der Stimuli und damit den Umfang der Experimente im Rahmen zu halten. Es soll damit nicht impliziert werden, da $\beta$ diese anderen Verläufe nicht auch mögliche oder vielleicht sogar 'bessere' Verläufe für Frageoder Exklamativ-Sätze wären; vgl. dazu die Diskussionen der einzelnen Perzeptionsexperimente in Teil 3 sowie die Produktionsdaten in Teil 4. Ebensowenig impliziert der Gebrauch der Begriffe FALL und RISE, daß wir der Fo-Bewegung eine größere perzeptive Relevanz beimessen als der Fo-Höhe - man könnte ja statt des finalen RISE die Höhe des Fo-Offset und statt des initialen FALL die Höhe des Fo-Onset als Parameter ansetzen. In diesem Punkt schließen wir uns Thorsen (1987:99) an:

"[...] it may be argued that our perception of pitch is continuous, i.e. we fill in - by interpolation - the empty spaces left by unvoiced sounds in the speech chain. On the other hand, it may also be argued that we anchor our perception of intonational phenomena on certain points in the time varying course of pitch and disregard what lies between such fix points. I do not know that anyone has yet settled this argument, or suggested an experiment which can resolve the issue."

\subsection{Inhaltlicher Aspekt}

In diesem Beitrag soll untersucht werden, wie gro $\beta$ der finale RISE sein muß, um eine Frage zu indizieren, und welche Veränderung des initialen FALL in wel-

1 In manchen Fällen kann sogar Merkmalsneutralisation vorliegen, d.h. der Kontext muß über die Satzmoduszuweisung entscheiden (vgl. Oppenrieder 1988a, Batliner 1988a und Batliner et al. 1989). 
chem Ausmaß Auswirkungen auf die Frageindizierung hat. Komplementär dazu ist die Frage, welche Ausprägungen der FALL-RISE-Bewegung nicht nur keine Frage indizieren, sondern einen - mehr oder weniger 'guten' - Exklamativ. Das zugrundegelegte Satzmodussystem ist in Altmann (1987) beschrieben.

\section{$1.4 \quad$ Methodischer Aspekt}

Wir haben in Batliner (1989) gezeigt, da $\beta$ unterschiedliche Fragestellungen und damit experimentelle Designs durchaus $\mathrm{zu}$ prima facie widersprüchlichen Ergebnissen führen können. Es soll deshalb untersucht werden, ob unterschiedliche Testanordnungen und Instruktionen der Versuchspersonen (VPn) bei gleichem bzw. vergleichbarem stimulusmaterial im Ergebnis konvergieren oder sich systematisch und interpretierbar unterscheiden. Ein Korrektiv zu den Perzeptionsdaten stellen Produktionsdaten dar, auf die wir in Teil 4 eingehen.

\section{Testaufbau}

\section{$2.1 \quad$ Material}

Als Ausgangsstimulus wurde der Satz Säuft der Leo gewählt, der auch schon in Batliner (1989) Verwendung fand. Modusindizierende 'Kontextsätze' waren für die Frage Bist du sicher? mit steigendem, steigend-fallendem und fallendem FoVerlauf sowie für den Exklamativ Also sowas hätt' ich nicht gedacht! Die FoVerläufe der Kontextsätze finden sich in Fig.9. Der Autor produzlerte die Kontextsätze mit natürlicher Intonation (vgl. Batliner 1988b:245) und den Testsatz mit monotoner intonation mehrmals im schallarmen Raum des Instituts für Phonetik in München (Aufnahmegerät: Telefunken M15, Geschwindigkeit $19 \mathrm{~cm} / \mathrm{sec}$ ). Die monotone Intonation sollte dabei einen möglichst neutralen Ausgangspunkt für die Manipulation darstellen. Die besten Realisierungen wurden auf einer PDP11/50 mit einer Abtastrate von $20 \mathrm{KHz}$ digitalisiert und mit einem Tiefpaß von $8 \mathrm{KHz}$ gefiltert. Die Kontextsätze blieben unmanipuliert, der Testsatz wurde mit einem Segmentierungsprogramm periodenweise geschnitten und diente damit als Grundlage zur pitchsynchronen Manipulation auf Halbton- (Ht-) Basis.

Die Stimuli in Batliner (1989) hatten eine Schrittgröße von zwei Htn. Dadurch wurden abrupte Ubergänge in den Bewertungsfunktionen der Tests vermieden, bei denen die exakte Kategoriengrenze nicht genau bestimmbar ist. Da in diesem Beitrag zum einen die Kategoriengrenze nicht im Mittelpunkt des Interesses steht, zum anderen die Zahl der Paare bei dem geplanten All-Step-Test 
(jeder Stimulus wird mit jedem anderen gepaart) exponentiell zur Zahl der Stimuli steigt (10 Stimuli ergeben 100, 30 schon 900 Paare), legten wir die Schrittgröße auf drei Ht fest.

Neben den zwölf Stimuli mit einer FALL-RISE-Kontur (von nun an FR-Stimuli, vgl. Fig.1 und Tab.1) wurden weitere sechs mit einem Tonsprung zwischen der ersten und der zweiten Phrase (von nun an TS-Stimuli, vgl. Fig.2 und Tab.2) erzeugt, mit denen untersucht werden sollte, inwiefern ein mehr oder weniger ausgeprägter Tonsprung frage- oder exklamativtypisch ist. Die beiden Stimulusgruppen sind in Fig.1 und Tab.1 bzw. in Fig.2 und Tab.2 dargestellt. Zur Verdeutlichung sind in Fig.2 die Kurzbezeichnungen der Stimuli eingetragen.

Bei den FR-Stimuli steht vor dem senkrechten Strich der Betrag des initialen FALL, nach dem Strich der des finalen RISE. ' $O$ ' steht für einen ebenen (LEVEL-) Verlauf. In der zweiten Phrase erhielt der $L$ immer einen FALL von 3 Htn. Stimuli mit einem LEVEL-Verlauf auf säuft oder der $L$ wurden nicht aufgenommen, da sie im Verhältnis zu den anderen stimuli sehr unnatürlich klangen.

Fig.1: FALL-RISE-Stimuli, schematisch dargestellt

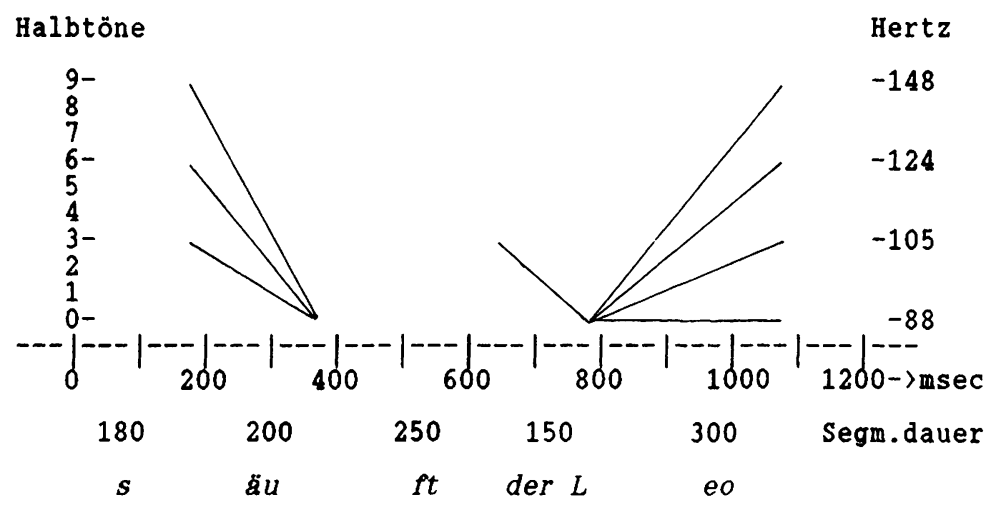


Tab.1: FALL-RISE-Stimulus-Matrix

initialer FALL in Halbtönen

\begin{tabular}{ll|ccc} 
& & 9 & 6 & 0 \\
\hline $\begin{array}{l}\text { finaler } \\
\text { RISE in }\end{array}$ & 0 & $9 / 0$ & $6 / 0$ & $3 \mid 0$ \\
Halb- & 3 & $9 \mid 3$ & $6 / 3$ & $3 / 3$ \\
tönen & 6 & $9 / 6$ & $6 / 6$ & $3 / 6$ \\
& 9 & $9 / 9$ & $6 / 9$ & $3 \mid 9$
\end{tabular}

Bei den TS-Stimuli steht am Anfang der Betrag der Bewegung auf säuft, am Ende der Betrag der Bewegung auf Leo bzw. der Leo. ' $L$ ' steht für finalen LEVEL-Verlauf auf Leo, und ' 7 ' für initialen LEVEL-Verlauf auf säuft. Bei den Stimuli in der ersten spalte von Tab.2 wurde der Fo-Verlauf auf der Leo konstant gehalten (FALL von $3 \mathrm{Htn}$ auf der und LEVEL auf Leo); der FALL auf säuft varilert derart, daß sich Tonsprünge von 0,3 und $6 \mathrm{Htn}$ ergeben. Bei den Stimuli in der zweiten Spalte von Tab.2 wurde der LEVEL-Verlaup auf säuft konstant gehalten; Anfang und Ausmaß des FALL auf der Leo variieren derart, da $\beta$ sich ebenfalls Tonsprünge von 0,3 und 6 Htn ergeben.

Fig.2: Tonsprung-stimuli

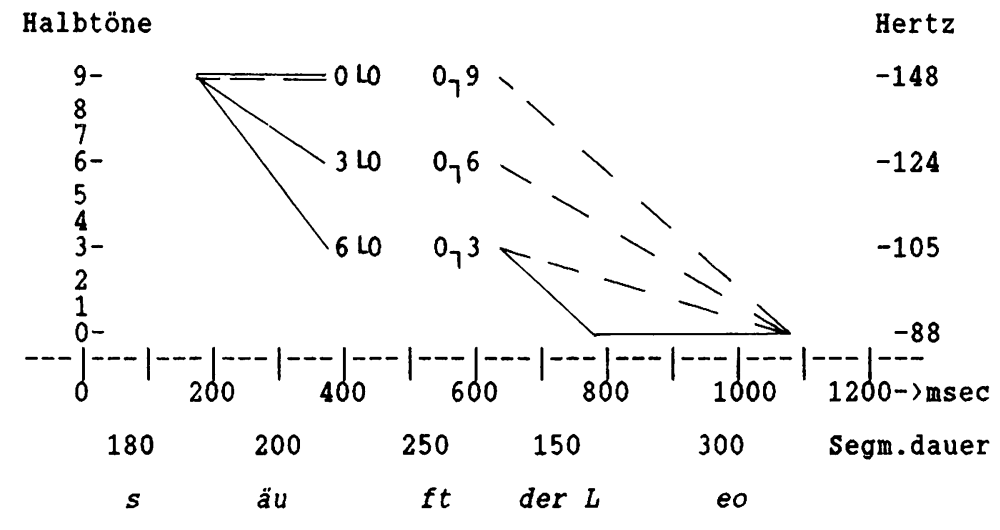

Tab.2: Tonsprung-stimulus-Matrix (Verlauf und Ausmaß des FaLl in Halbtönen)

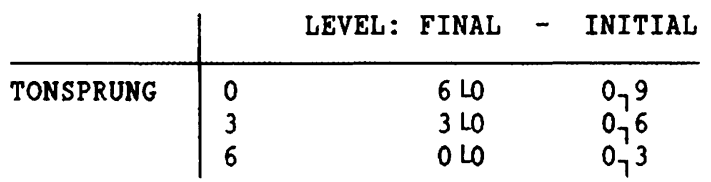


Für die FR-Stimuli nehmen wir die folgenden Hypothesen an:

- Je höher der finale RISE, desto wahrscheinlicher ist eine Frage- und desto schlechter eine Exklamativinterpretation.

- Je höher der initiale FALL, desto wahrscheinlicher ist eine Exklamativinterpretation.

In all diesen Fällen können wir bei der Prüfstatistik einseitig testen. Für die Fragen läßt sich beim initialen FALL keine Vorhersage machen, da zum einen eine bessere Exklamativinterpretation eine schlechtere Frageinterpretation bedingen könnte; zum anderen haben wir aber in Batliner (1989) gesehen, daß beim gleichen Testsatz ein höherer initialer FALL auch eine bessere Frageinterpretation bedingen kann. In diesen Fällen müssen wir also zweiseitig testen.

Die FR-Stimuli könnten im Rahmen eines varianzanalytischen Designs untersucht werden: Es gibt zwei Faktoren, den Faktor FALL mit drei Klassen (9, 6, 3 $\mathrm{Ht}$ ) und den Faktor RISE mit vier Klassen $(0,3,6$ und $9 \mathrm{Ht})$. Da die dafür benötigten Voraussetzungen der Varianzhomogenität und der Normalverteilung aber bei unseren experimentellen Daten mehr schlecht als recht erfüllt sind, nehmen wir grundsätzlich einen 'konservativen' Standpunkt ein und testen mit nicht-parametrischen Verfahren. Dabei werden die Klassen eines Faktors gemittelt und jeweils benachbarte Klassen des anderen Faktors mit einem WilcoxonTest für abhängige Stichproben auf signifikante Unterschiede geprüft. Da dabei über die gleiche Stichprobe mehrfach getestet wird, muß das gewählte Signifikanzniveau $a$ von 0.05 nach der Formel $1-(1-\alpha)^{1 / c}$ abgesenkt werden, wobei $c$ für die Zahl der durchgeführten Tests steht. Bei den elnzelnen Experimenten sind die durchgefuhrten Vergleiche und die dabei erzielten $p$-Werte sowie das abgesenkte Signifikanzniveau $\alpha$ angegeben. Signifikante Werte sind mit einem Stern gekennzeichnet; wenn einseitig getestet wurde, sind die Werte unterstrichen.

Bei den TS-Stimuli können keine Hypothesen aufgestellt werden, da ein größerer Tonsprung gleichermaßen eine bessere Frage- oder Exklamativinterpretation bewirken könnte. Zusätzliche (zweiseitige) Tests für diese Stimuli hätten darüber hinaus den Nachteil, daß das Signifikanzniveau weiter abgesenkt werden müßte. Wir begnügen uns deshalb bei diesen Stimuli mit einer explorativen, deskriptiven Statistik. 
Die Daten wurden bei allen Experimenten auf die gleiche Weise erhoben: VPn waren StudentInnen der Germanistischen Linguistik oder der Phonetik, die für ihre Tellnahme bezahlt wurden. Sie saßen im Sprachlabor des Instituts für Phonetik in München vor einem Abstimmkästchen und hörten die stimuli über Kopfhörer. Genau nach jedem Stimulus(-paar) wurde die Abstimmung freigegeben; dies wurde durch eine Lampe an jedem Kästchen angezeigt. Die VPn hatten dann vier Sekunden Zeit, ihre Antwort zu überlegen und die entsprechende Taste zu drücken. Die Antworten wurden auf einer PDP11/03 gesammelt und zur weiteren Verarbeitung aufbereitet. Die statistische Auswertung wurde mit dem Programmpaket SPSS-PC+ vorgenommen.

\section{Perzeptionsexperimente}

Es wurden die folgenden Perzeptionsexperimente durchgeführt; in Klammern stehen die im weiteren gebrauchten Abkürzungen sowie ein Hinweis auf das jeweilige Unterkapitel:

\begin{tabular}{|c|c|}
\hline Identifikationstest & $(\mathrm{IT}-3.1)$ \\
\hline Antwortobligationstest & $(\mathrm{AT}-3.2)$ \\
\hline Kontexttests & $(\mathrm{KT} 1-3.3 .1 ; \mathrm{KT} 2-3.3 .2 ; \mathrm{KT} 3-3.3 .3)$ \\
\hline Diskriminationstest & $(\mathrm{DT}-3.4)$ \\
\hline Ahnlichkeits- (All-Step-) Test & $(A T-3.5)$ \\
\hline
\end{tabular}

3.1 Identifikationstest (IT)

\subsubsection{Design}

Beim IT handelt es sich um einen sog. 'forced-choice'-Test: Die VPn erhalten zwei (manchmal auch mehrere) Möglichkeiten vorgegeben, zwischen denen sie sich entscheiden müssen. ITs werden üblicherweise zusammen mit DTs bei Untersuchungen zur kategorialen Wahrnehmung verwendet (vgl. Repp 1984; zur Kategorialität von Intonationsphänomenen vgl. Lindsay/Ainsworth 1985, Schiefer/Batliner 1988 und Batliner 1989).

Die 18 Stimuli wurden 17 VPn randomisiert je 5 mal dargeboten. Die Instruktion lautete: "Bitte entscheiden Sie, ob es sich bei den stimuli um eine Frage oder um keine Frage handelt, unủ drücken Sie die linke Taste für 'Frage' oder die rechte für 'Nicht-Frage'". 


\subsubsection{Ergebnis}

Fig.3 zeigt das Ergebnis; auf der y-Achse sind in Prozent die Nicht-FrageKlassifizierungen aufgetragen. Hier wie in den folgenden Abbildungen sind auf der $\mathrm{x}$-Achse für die FR-Stimuli die drei Klassen des initialen FALL und für die TS-Stimuli die Tonsprünge aufgetragen. Die Legende zeigt die symbole der Klassen des fínalen RISE für die FR-Stimuli bzw. der TS-Stimuli mit finalem vs. initialem LEVEL-Verlauf. Die FR-Stimuli mit LEVEL-Verlauf (0 Ht RISE) werden eindeutig als Nicht-Fragen klassifiziert, die mit 6 und 9 Ht RISE eindeutig als Fragen; die mit $3 \mathrm{Ht}$ RISE liegen im Zufallsbereich, sind also ambig. Signifikante Unterschiede zeigen sich zwischen 0 und $3 \mathrm{Ht}$ RISE und zwischen 3 und $6 \mathrm{Ht}$ RISE. Das Ergebnis stimmt mit Batliner (1989) überein, wo sich beim gleichen Testsatz, aber einer kleineren Schrittgröße von $2 \mathrm{Htn}$ eine Kategoriengrenze zwischen 2 und 4 Ht RISE ergab.

Fünf der sechs TS-Stimuli llegen lelcht über dem Zufallsbereich, der sechste, Stimulus $6 \mathrm{LO}$, wird in über 90\% als Nicht-Frage klassifiziert. Das läßt den Schluß zu, daß die TS-Stimuli grundsätzlich schlechte Fragekandidaten sind.

Fig.3: Identifikationstest

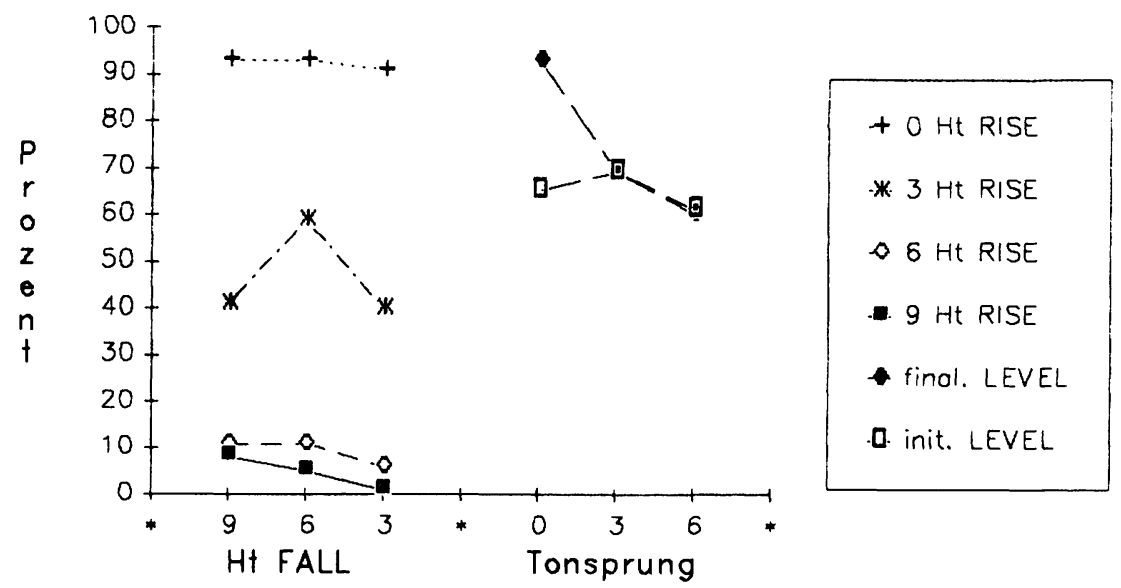

Paarweiser Vergleich der Klassen mit Wilcoxon $(\alpha=0.0102)$

$\begin{array}{ccccc}0 \mathrm{Ht} \text { RISE } & 3 \mathrm{Ht} \text { RISE } & 6 \mathrm{Ht} \text { RISE } & 9 \mathrm{Ht} \text { FALL } & 6 \mathrm{Ht} \text { FALL } \\ 3 \mathrm{Ht} \mathrm{RISE} & 6 \mathrm{Ht} \text { RISE } & 9 \mathrm{Ht} \text { RISE } & 6 \mathrm{Ht} \text { FALL } & 3 \mathrm{Ht} \mathrm{FALL} \\ \underline{0.0001^{*}} & \underline{0.0002^{*}} & \underline{0.0807} & 0.0465 & 0.0277\end{array}$




\subsubsection{Design}

Mit diesem Experiment soll untersuchen werden, ob sich bei einer abgestuften Antwortvorgabe andere Bewertungsfunktionen ergeben als bei der binären des ITs. Implizit wird mit diesem Design natürlich ein Konzept der Kategorialität, das sich mit dem Ermitteln von Kategoriengrenzen begnügt, infrage gestellt. Der AT leitet über zu den Kontexttests (KTs), da er mit dem IT die Explizitheit der Bewertung, mit den KTs aber die Abstufungen der Bewertung gemeinsam hat.

18 VPn wurden die 18 Stimuli randomisiert je $5 \mathrm{mal}$ dargeboten. Die Instruktion lautete: "Bitte geben Sie an, wie stark Sie sich durch die Außerungen zu einer Antwort gedrängt fühlen und drücken Sie dann die entsprechende Taste der Tasten 1 bis 5." Die Skala lautete: 1 'sehr stark', 2 'stark', 3 'mittel', 4 'wenig', 5 'sehr wenig'. Diese Skala wurde für die Auswertung und für die Darstellung in eine Skala von 0-4 umgerechnet. Als Maßzahl pro Item und VP erhält man also einen Mittelwert aus den fünf Einzelbeurteilungen.

\subsubsection{Ergebnis}

Fig.4 zeigt das Ergebnis. Es war zu erwarten, daß im Gegensatz zum IT die Extrembereiche bei einer solchen Instruktion nicht besetzt sind, da manche VPn die extremen Bewertungen 1 oder 5 selten bis nie vergeben. Interessant ist aber, daß die Abstufung zwischen den Klassen von RISE kontinuierlicher ist und sich alle vier Klassen vom jeweiligen Nachbarn signifikant unterscheiden, also auch $6 \mathrm{Ht}$ RISE von $9 \mathrm{Ht}$ RISE. Lenkt man demnach die Aufmerksamkeit der VPn auf eine graduelle Abstufung, so zeigt sich auch innerhalb des Fragemodus - in unserem Fall bei den Stimuli mit 6 oder 9 Ht RISE - eine Differenzierung. Eine stärkere Antwortobligation beim Hörer hat ihre Ursache in einer stärkeren 'Fragehaltigkeit' beim stimulus, und diese wiederum ist offensichtlich bedingt durch eine stärkere Ausprägung des Prageindizierenden finalen RISE. (Der Begriff 'Fragehaltigkeit' sei hier informell eingeführt, da er für sich sprechen kann; er wird unten in Teil 5 genauer erläutert und abgeleitet.)

Die TS-Stimuli liegen alle, einschließlich des Stimulus 6 LO, im mittleren, unentschiedenen Bereich. In der leicht fallenden Tendenz beim Tonsprung von 0 über 3 nach $6 \mathrm{Htn}$ deutet sich an, daß ein stärkerer Tonsprung - wenn auch in geringem Ausmaß - die Frageindizierung verbessern kann. 
Fig.4: Antwortobligationstest

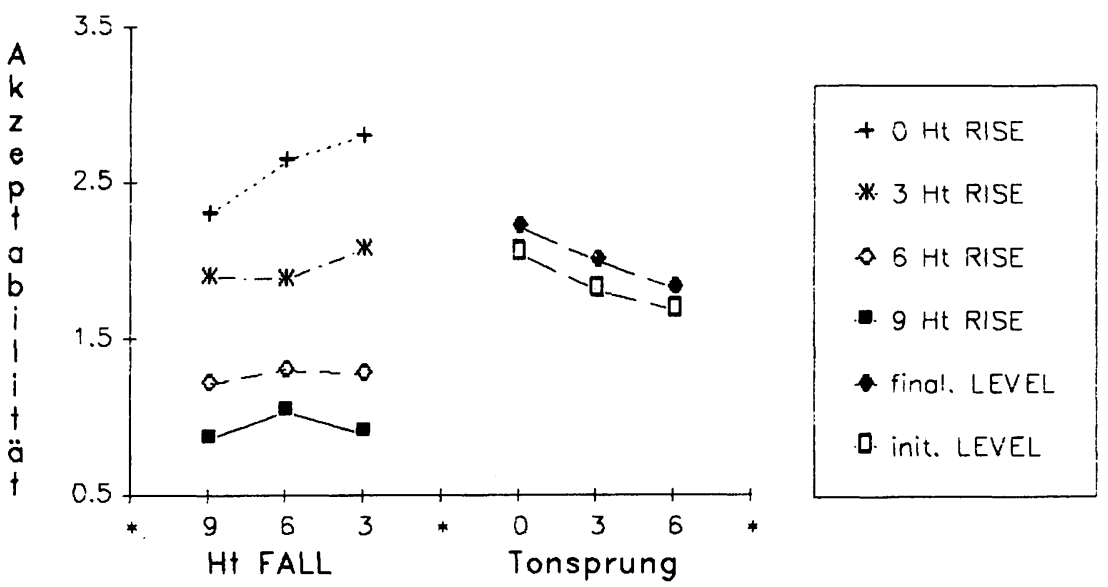

Paarweiser Vergleich der Klassen mit Wilcoxon $(\alpha=0.0102)$

$\begin{array}{ccccc}0 \text { Ht RISE } & 3 \text { Ht RISE } & 6 \text { Ht RISE } & 9 \text { Ht FALL } & 6 \text { Ht FALL } \\ 3 \text { Ht RISE } & 6 \text { Ht RISE } & 9 \text { Ht RISE } & 6 \text { Ht FALL } & 3 \text { Ht FALU } \\ \underline{0.0025^{*}} & \underline{0.0025^{*}} & \underline{0.0054^{*}} & 0.0979 & 0.8970\end{array}$

\subsection{Kontexttests (KTs)}

Die Kombination der Teststimuli mit Kontexten, die die Kategorie (in unserem Fall also den Satzmodus) indizieren, bietet die Möglichkeit einer indirekten und auch 'natürlicheren' Ermittlung der Bewertungsfunktionen. Man kann durch eine gezielte Veränderung des Kontexts unterschiedliche Einflüsse untersuchen. So varileren wir zum einen den indizierten Modus durch zwei unterschiedliche Vorgängerăußerungen, die entweder einen Fragesatz oder einen Exklamativsatz indizieren. Zum anderen variieren wir den Intonationsverlauf der fragesatzindizierenden Vorgängeräußerung, um damit zu untersuchen, inwiefern eine intonatorische Kongruenz von benachbarten (adjazenten) Teiläußerungen gefordert ist.

Als fokusneutralen Kontextsatz, der einen Fragesatz indizieren soll, wurde der Satz Bist du sicher?, als exklamativindizierender Kontextsatz der Satz Also sowas hätt' ich nicht gedacht! verwendet. Der Satz Bist du sicher? erhielt drei unterschiedliche Konturverläufe, einen steigenden (RISE), einen steigend-fallenden (RISE-FALL) und einen fallenden (FALL). In KT1 (Teil 3.3.1.) hatte Bist du sicher? den RISE-, in KT2 (Teil 3.3.2.) den FALL-Verlauf; in KT1 und KT2 wurde der gleiche exklamativindizierende Kontextsatz Also sowas hätt' ich nicht ge- 
dacht! verwendet, Testsätze waren die FR- und die TS-Stimuli; die Verläufe der Kontextsätze finden sich unten in Fig.9. KT3 unterscheidet sich von KT1 und KT2 darin, daß kein manipuliertes, sondern nur natürlichsprachliches Material verwendet wurde; im einzelnen s. Teil 3.3.3.

\subsubsection{Kontexttest 1 (KT1)}

\subsubsection{Design}

Kontextsätze waren Also sowas hätt' ich nicht gedacht! und Bist du sicher? mit RISE-Verlauf. Die 36 Paare aus Kontextsatz und Testsatz wurden 15 VPn je viermal, randomisiert und aufgeteilt auf zwei Testsitzungen, dargeboten. Das Intervall innerhalb der Stimuli betrug $0.5 \mathrm{sec}$, das zwischen den Paaren 4 sec. Die VPn gaben ihre Antwort auf einer fünstufigen Ratingskala ab. Sie entschieden damit, ob die beiden Sätze als Außerung ein und desselben sprechers 'sehr gut' $=1,{ }^{\prime}$ gut' $=2$, 'noch gut' $=3$, 'schlecht' $=4$ oder 'sehr schlecht' $=5$ zusammenpaßten. Diese Skala wurde für die Auswertung und für die Darstellung wieder in eine Skala von 0-4 umgerechnet. Als Maßzahl pro Item und VP erhält man also einen Mittelwert aus den vier Einzelbeurtellungen.

\subsubsection{Ergebnis}

Fig.5 zeigt die Ergebnisse und die Signifikanz der erhaltenen p-Werte beim Vergleich der Klassen für den Fragekontext. Die Verläufe in Fig.5 sind ähnlich denen belm $\mathrm{AT}$ in Fig.4. Wieder unterscheiden sich alle vier RISE-Klassen signifikant vom jeweiligen Nachbarn. Obwohl sich die Klassen des Faktor FALL (bedingt durch die nötige Absenkung des Signifikanzniveaus) nicht signifikant voneinander unterscheiden, kann man doch die Tendenz feststellen, daß ein ausgeprägter initlaler FALL eine Frageinterpretation begünstigt. Setzt man den finalen RISE als Hauptmerkmal, den initialen FALL als Nebenmerkmal bei der Fragemarkierung an, so läßt dieses Ergebnis den Schluß zu, daß Nebenmerkmale dann relevant werden, wenn die Aufgabenstellung 'natürlicher' ist.

Die TS-Stimuli liegen wieder im mittleren, ambigen Bereich. 
Fig.5: Kontexttest 1, Fragekontext

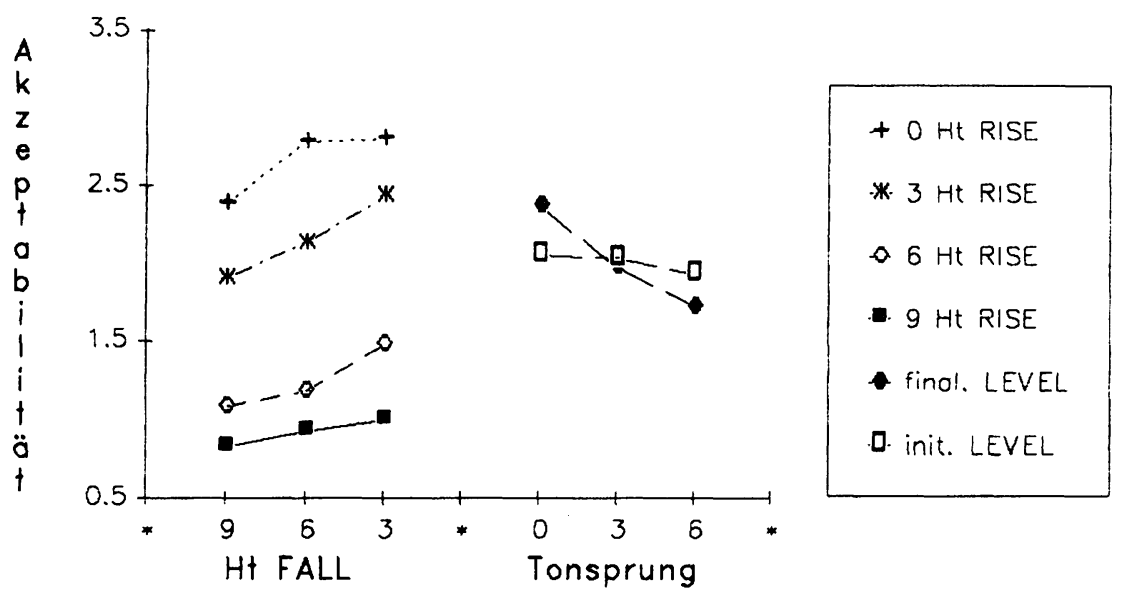

Paarweiser Vergleich der Klassen mit Wilcoxon $(\alpha=0.0051)$

\begin{tabular}{|c|c|c|c|c|}
\hline $\begin{array}{l}0 \text { Ht RISE } \\
3 \text { Ht RISE }\end{array}$ & $\begin{array}{l}3 \text { Ht RISE } \\
6 \text { Ht RISE }\end{array}$ & $\begin{array}{l}6 \mathrm{Ht} \text { RISE } \\
9 \mathrm{Ht} \text { RISE }\end{array}$ & $\begin{array}{l}9 \text { Ht FALU } \\
6 \text { Ht FALL }\end{array}$ & $\begin{array}{l}6 \text { Ht FALL } \\
3 \text { Ht FALL }\end{array}$ \\
\hline $0.0007 *$ & $0.0007 *$ & $0.0010 *$ & 0.0995 & 0.0144 \\
\hline
\end{tabular}

Fig.6 zelgt die Ergebnisse für den Exklamativkontext. 0 und $3 \mathrm{Ht}$ RISE unterscheiden sich signifikant, sowie 9 und 6 bzw. 6 und 3 Ht FALL. Damit bestätigt sich die Annahme, daß unter unseren Stimuli ein hoher initialer FALL und ein finaler LEVEL-Verlauf am exklamativtypischsten ist (Stimulus $9 / 0$ ) und daß sowohl eine Absenkung des FALL als auch eine Anhebung des RISE (von 0 auf $3 \mathrm{Ht}$ RISE) eine Verschlechterung mit sich bringen.

Beim Vergleich von Fig.5 und Fig.6 zeigt sich, daß der Stimulus $9 / 0$ in Fig.6 schlechter bewertet ist als die Pragetypischen mit 9 oder 6 Ht RISE in Fig.5. Die schematische Fo-Manipulation allein kann nicht dafür verantwortlich gemacht werden, da dann ja auch der gleiche Effekt einen Einfluß auf die fragetypischen Stimuli haben müßte. Man kann also vermuten, da $\beta$ der bei unseren Teststimuli 'exklamativtypischste' Stimulus $9 / 0$ an sich eine weniger exklamativtypische FoKontur aufweist; wir werden bel der Behandlung der Produktionsdaten in Teil 4 auf diesen Punkt zurückkommen. 
Fig.6: Kontexttest 1, Exklamativkontext

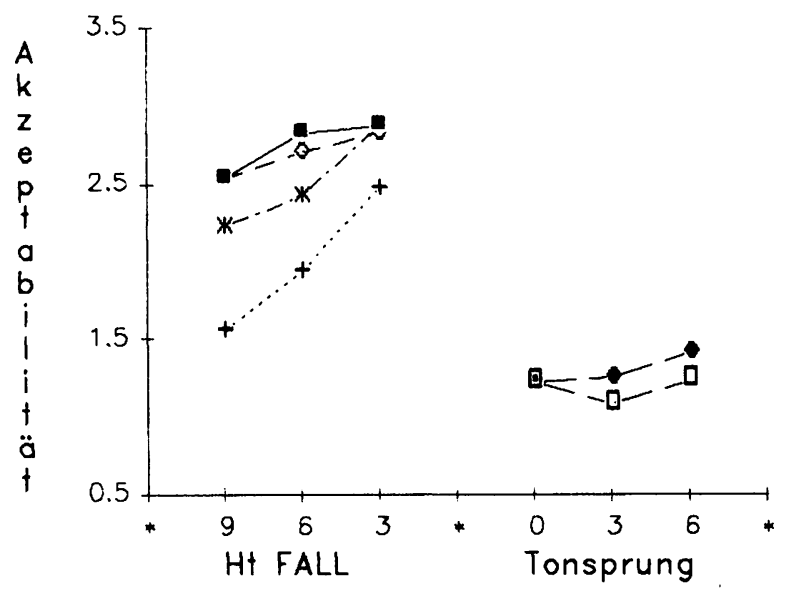

+ O Ht RISE

* 3 Ht RISE

๑ $6 \mathrm{Ht}$ RISE

- 9 Ht RISE

- final. LEVEL

]. init. LEVEL

Paarweiser Vergleich der Klassen mit Wilcoxon $(\alpha=0.0051)$

0 Ht RISE $3 \mathrm{Ht}$ RISE $6 \mathrm{Ht}$ RISE $9 \mathrm{Ht}$ FALL $6 \mathrm{Ht}$ FALL

3 Ht RISE 6 Ht RISE 9 Ht RISE 6 Ht FALL 3 Ht FALL

$\begin{array}{lllll}0.0037 * & 0.5098 & 0.2585 & \underline{0.0038^{*}} & \underline{0.0038^{*}}\end{array}$

3.3.2 Kontexttest 2 (KT2)

\subsubsection{Design}

Design und Ablauf des Experiments waren identisch mit dem in 3.3.1. geschilderten, mit einer Ausnahme: Der frageindizierende Kontextsatz wies einen FALL auf (cf. Fig.9). Es nahmen 14 VPn teil. 


\subsubsection{Ergebnis}

Fig.7 zeigt das Ergebnis und die Signifikanzen der erhaltenen p-Werte für den Fragekontext: Im Gegensatz zum eben beschriebenen Experiment unterscheiden sich 9 und 6 sowie 3 und 0 Ht RISE nicht signifikant voneinander; einzig zwischen 6 und 3 Ht RISE, also im Bereich der Kategoriengrenze, zeigt sich ein signifikanter Unterschied.

Vergleicht man Fig. 5 mit Fig.7, so sieht man, daß in Fig.7 9 und 6 Ht RISE deutlich schlechter bewertet werden als in Fig.5; dieser Unterschied muß auf den unterschiedlichen Intonationsverlauf der Kontextsätze zurückzuführen sein. Eine eingehende Diskussion stellen wir bis zum nächsten Experiment zurück.

\section{Fig.7: Kontexttest 2, Fragekontext}

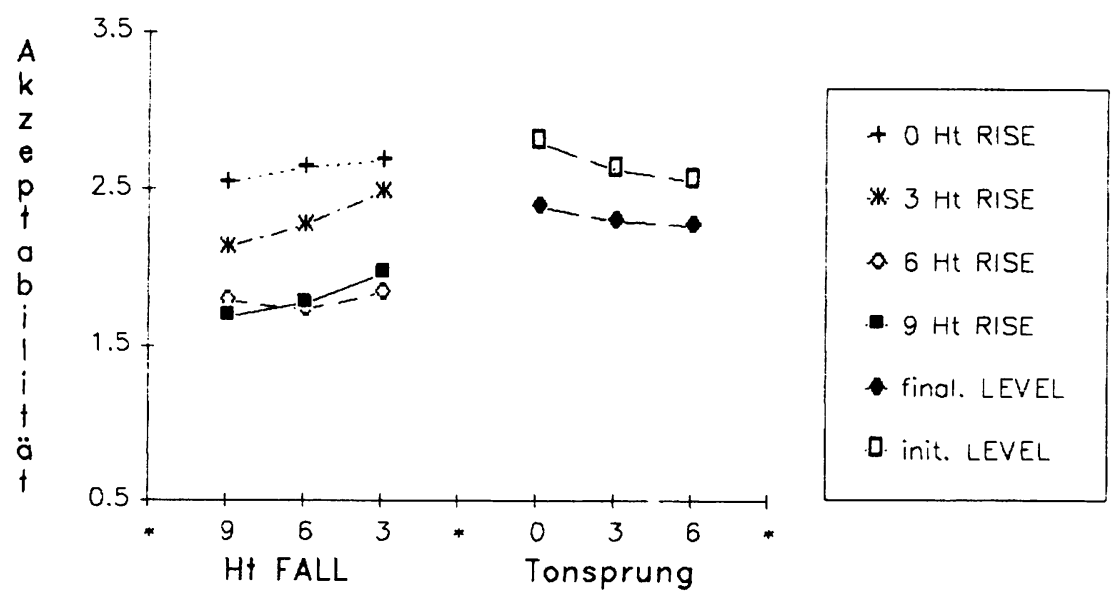

Paarweiser Vergleich der Klassen mit Wilcoxon $(a=0.0051)$

$\begin{array}{ccccc}0 \mathrm{Ht} \mathrm{RISE} & 3 \mathrm{Ht} \text { RISE } & 6 \mathrm{Ht} \text { RISE } & 9 \mathrm{Ht} \text { FALL } & 6 \mathrm{Ht} \text { FALL } \\ 3 \mathrm{Ht} \mathrm{RISE} & 6 \mathrm{Ht} \mathrm{RISE} & 9 \mathrm{Ht} \text { RISE } & 6 \mathrm{Ht} \mathrm{FALL} & 3 \mathrm{Ht} \mathrm{FALL} \\ \underline{0.0252} & \underline{0.0028^{*}} & \underline{0.4860} & 0.5302 & 0.3152\end{array}$

Fig.8 zeigt die Ergebnisse für den Exklamativkontext und die Signifikanzen der erhaltenen $\mathrm{p}$-Werte. Die Verläufe von 0 und $3 \mathrm{Ht}$ RISE entsprechenen denen in Fig.6; sie unterscheiden sich signifikant. Allerdings werden die 'schlechten' Reihen 6 und 9 Ht RISE deutlich schlechter als in Fig. 6 bewertet; daher auch der zusätzliche signifikante Unterschied zwischen 3 und $6 \mathrm{Ht}$ RISE. Dieses Ergebnis ist überraschend; wenn kein unkontrollierter VPn-Effekt vorliegt, so muß es darauf zurückgepührt werden, daß die Tatsache, daß alle Kontextsätze einen 
fallenden Fo-Verlauf aufweisen, eine Auswirkung auf die Bewertung der Testsätze mit deutlich steigenden Verläufen (6 und 9 Ht RISE) hat.

Es bleibt aber festzustellen, daß die fragetypischen Testsätze mit deutlichem RISE bei der Kombination mit einem FALL im Prageindizierenden Kontextsatz (KT2) deutlich schlechter bewertet werden als bei der Kombination mit einem RISE im frageindizierenden Kontextsatz (KT1), die exklamativtypischen Testsätze - bei Kombination mit dem gleichen exklamativindizierenden Kontextsatz wie in KT1 - dagegen in KT2 gleich gut.

Die TS-Stimuli ergeben etwa das gleiche Bild wie in KT1, d.h. eine schlechte Bewertung beim Frage- und eine gute beim Exklamativkontext.

Fig.8: Kontexttest 2, Exklamativkontext

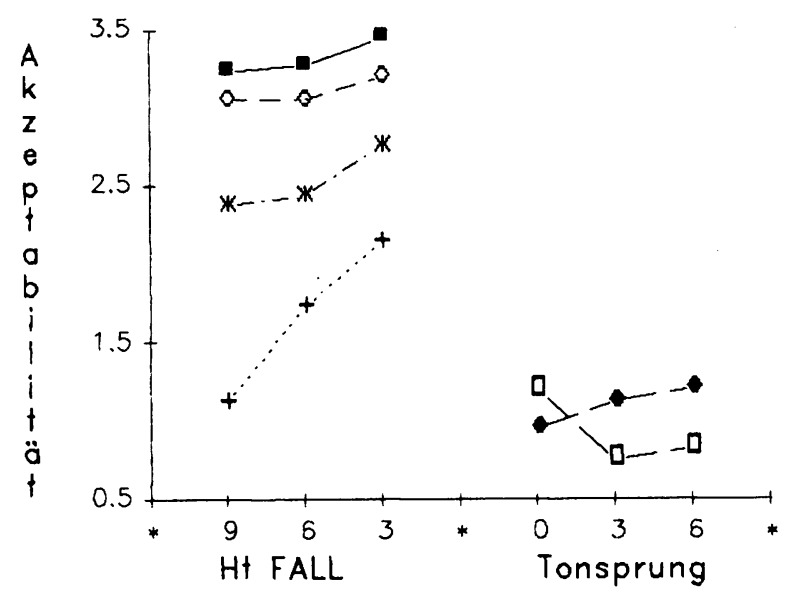

+ O HT RISE

* 3 Ht RISE

$\diamond 6$ HI RISE

- 9 Ht RISE

- final. LEVEL

o. init. LEVEL

Paarweiser Vergleich der Klassen mit Wilcoxon $(\alpha=0.0051)$

$\begin{array}{ccccc}0 \text { Ht RISE } & 3 \text { Ht RISE } & 6 \text { Ht RISE } & 9 \text { Ht FALL } & 6 \mathrm{Ht} \mathrm{FALL} \\ 3 \mathrm{Ht} \mathrm{RISE} & 6 \mathrm{Ht} \text { RISE } & 9 \mathrm{Ht} \text { RISE } & 6 \mathrm{Ht} \text { FALL } & 3 \mathrm{Ht} \text { FALL } \\ 0.0019^{\star} & 0.0019 * & 0.0229 & \underline{0.0121} & \underline{0.0039 *}\end{array}$




\subsubsection{Kontexttest 3 (KT3)}

Beim zuletzt besprochenen Experiment konnte nicht eindeutig entschieden werden, ob die beobachteten Effekte nur auf einen systematischen Faktor (Anforderung der intonatorischen Kongruenz, d.h. schlechtere Bewertung von Sätzen mit gegenläufigen Konturen) oder zusätzlich auf einen anderen Faktor zurückzuführen sind. Wir beschreiben deshalb nun als KT3 ein Teilexperiment, das zusammen mit den in Batliner (1988b) dargestellten Experimenten durchgeführt, dort aber nicht weiter erwähnt wurde.

\subsubsection{Design}

Das experimentelle Design ist ausführlich in Batliner (1988b:224ff) beschrieben; wir beschränken uns deshalb auf die wichtigsten Angaben:

Im Tonstudio wurden vom Autor jeweils mehrere Realisationen der in Fig.9 aufgeführten Kontext- und Testsätze produziert und mit $19 \mathrm{~cm} / \mathrm{sec}$ auf einer Telefunken M15 aufgenommen. Nach einer Beurteilung durch mehrere kompetente Sprecher und einer Uberprüfung der relevanten phonetischen Parameter anhand von Mingogrammen mit Zeitsignal, Fo- und Intensitätskurve wurde je ein typisches Exemplar ausgewählt. Jeder Kontextsatz wurde mit jedem Testsatz gepaart und (zusammen mit anderen Kontext-Testsatz-Kombinationen, vgl. Batliner $1988 \mathrm{~b}$ ) in randomisierter Reihenfolge im Tonstudio analog auf ein Testband kopiert; das Intervall zwischen Kontext- und Testsatz betrug ca. 300-800 msec, das zwischen den Paaren ca. 4 sec. 22 VPn nahmen teil; die Aufgabenstellung war die gleiche wie in KT1 und KT2. Abgesehen davon, daß jedes Paar nur einmal vorkam, ist also der wesentliche Unterschied zwischen KT1 und KT2 auf der einen und KT3 aup der anderen Seite, daß in KT3 nicht nur die Kontextsätze, sondern auch die Testsätze natürlich produziert waren. Zur Verdeutlichung werden bei KT3 die Exklamativ-Testsätze 'E-Testsätze' und die Frage-Testsätze 'F-Testsätze' genannt; wir beschränken uns im folgenden auf die 21 Kombinationen der sieben Testsätze aus Fig.9 mit dem frageindizierenden Kontextsatz Bist du sicher? mit den drei unterschiedlichen Fo-Verläufen aus Fig.9, da alle anderen Kombinationen schon in Batliner (1988b) behandelt wurden. ${ }^{2}$ In Fig.9 sind bei den Testsätzen die Phrasen, die den Hauptakzent tragen, unterstrichen.

2 Die beiden E-Testsätze mit Satzakzent auf säuft unterscheiden sich durch den Fo-Verlauf auf säuft, der einmal fallend, das andere Mal leicht steigend und dann fallend ist (vgl. Fig.9). Der erste Fall entspricht also dem FR-Stimulus $9 / 0$, der zweite Fall weist den exklamativtypischen RISE-FALL-Verlauf mit Dehnung auf. 
Fig.9: Schematische Fo-Verläufe der Kontext- und der Testsätze

\section{KONTEXTSATZE:}

exklamativindizierend:

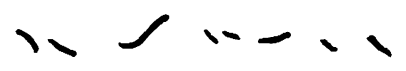

Also sowas hätt' ich nicht gedacht!

frageindizierend:

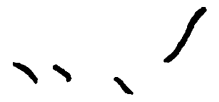

Bist du sicher? RISE

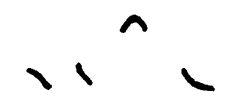

Bist du sicher? RISE-FALL

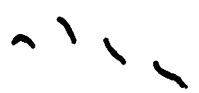

Bist du sicher? FALL

TESTSATZE, Satzakzent unterstrichen:

Exklamativtestsätze

'E-Testsätze' :

Fragetestsätze

'F-Testsätze':

FALL:

RISE :

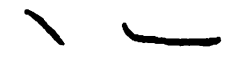

Säuft der Leo!

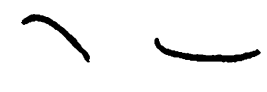

Säuft der Leo!

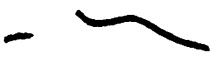

Säuft der Leo!

Säuft der Leo?

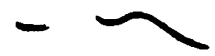

Säuft der Leo?

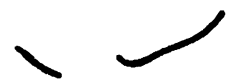

Säuft der Leo?

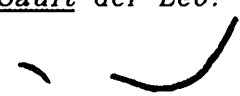

Säuft der Leo? 


\subsubsection{Ergebnis}

Die Darstellung in Fig.9 und die der Ergebnisse in Tab.3 und Fig.10 sind zur Erleichterung des Vergleichs möglichst ähnlich aufgebaut, analog zu einer Matrix mit drei Spalten (Kontextsätze) und drei Zeilen (Testsätze); die Reihenfolge ist identisch. Der Kontextsatz Bist du sicher? indiziert keinen bestimmten Satzakzent; dementsprechend sind die Unterschiede bei der Moduszuweisung $z w i s c h e n$ den Einzelwerten fur die zwei unterschiedlichen Positionen des Satzakzents nicht groß, vgl. Tab.3; die Werte können also gemittelt werden. ('S' steht für 'Satzakzent auf säuft', ' $L$ ' für 'Satzakzent auf $L e o^{\prime}$. ) Mit dieser Mittelung kann die Verteilung der Werte normalisiert und zugleich die Zahl der prüfstatistischen Vergleiche verringert werden. Die Mittelwerte aus den in Tab.3 aufgeführten Einzelwerten pro Kästchen sind in Fig.10 aufgetragen. Wieder wurde nicht-parametrisch getestet (Wilcoxon für abhängige Stichproben, zweiseitige Fragestellung, abgesenktes Signifikanzniveau $a=0.0046)$. Die erhaltenen $p$-Werte sind in Tab.3 aufgeführt und, sofern signifikant, mit einem Stern gekennzeichnet; die pWerte stehen immer $z$ wischen den verglichenen Paaren, wobei jeweils ein Faktor wechselt, der andere konstant bleibt.

Es war zu erwarten, daß die E-Testsätze bei allen drei Fragekontextsätzen am schlechtesten abschneiden. Allerdings ist der Verlauf von RISE über RISEFALL zu FALL in Fig.10 fallend, mit einem signifikanten Unterschied zwischen den letzten belden Klassen. Das gleiche gilt für die F-Testsätze mit FALL-Verlauf, dle sich aber für RISE/FALL- und FALL-Verlauf des Kontextsatzes signifikant von den E-Testsătzen unterscheiden. Die F-Testsätze mit FALL- und die mit RISE-Verlauf sind klar gegenläufig, mit zwei signifikanten Unterschieden bei RISE- und RISE/FALL-Verlauf des Kontextsatzes. In varianzanalytischer Terminologie können wir also von einer Interaktion zwischen den beiden Faktoren FoVerlauf auf dem Kontextsatz und Fo-Verlauf auf dem Testsatz sprechen. 
Fig.10: Kontexttest 3 (natürlichsprachliche Testsätze)
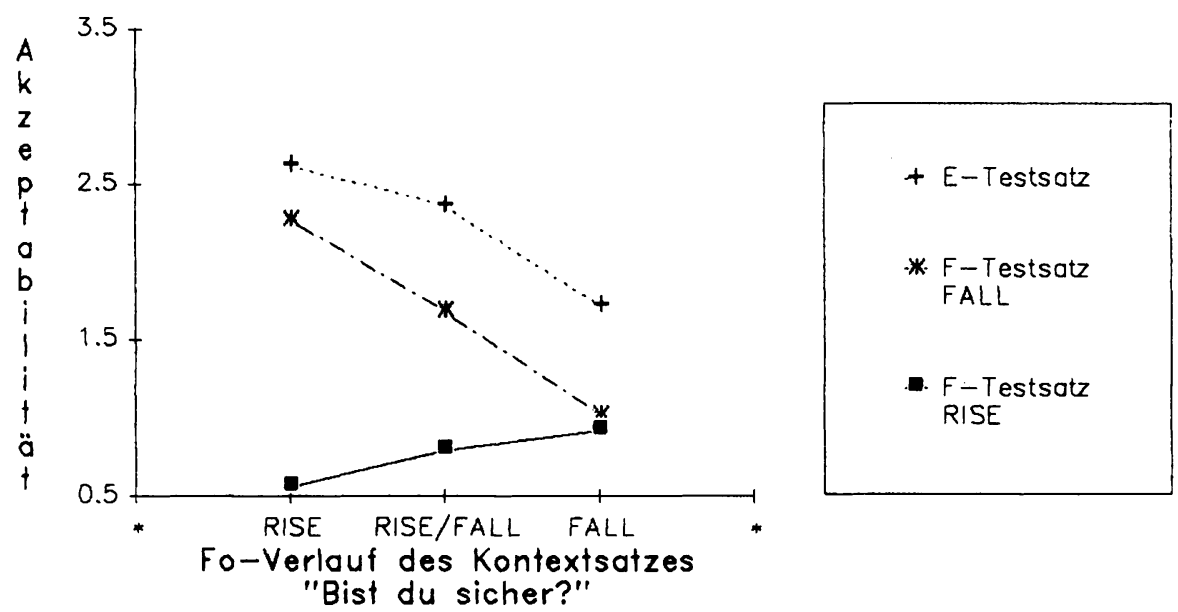

Tab.3: Paarweiser Vergleich der benachbarten Klassen (Wilcoxon, zweiseitig geprüft, abgesenktes Signifikanzniveau $a=0.0046$ )

\section{KONTEXTSATZ}

TESTSATZ

RISE RISE-FALL

FALL

\begin{tabular}{|c|c|c|c|c|c|c|}
\hline \multirow[t]{2}{*}{ EXKLAMATIV } & $\begin{array}{l}S \\
S \\
L\end{array}$ & $\begin{array}{l}4.00 \\
3.41 \\
3.45\end{array} \mid$ & $p=0.0687$ & $\left|\begin{array}{l}3.19 \\
3.57 \\
3.33\end{array}\right|$ & $p=0.0005^{*}$ & $\begin{array}{l}2.43 \\
2.82 \\
2.91\end{array} \mid$ \\
\hline & & $=0.0720$ & & $p=0.0040 *$ & & $p=0.0009$ \\
\hline \multirow[t]{2}{*}{$\begin{array}{r}\text { FRAGE } \\
\text { FALL }\end{array}$} & $\mathrm{s}$ & $\begin{array}{l}3.23 \\
3.32\end{array} \mid$ & $p=0.0033$ & $\begin{array}{l}2.45 \\
2.95\end{array} \mid$ & $p=0.0026 *$ & $\begin{array}{l}1.59 \\
2.45\end{array} \mid$ \\
\hline & & $=0.0001 \times$ & & $p=0.0046 x$ & & $p=0.615$ \\
\hline $\begin{array}{l}\text { FRAGE } \\
\text { RISE }\end{array}$ & $\mathrm{s}$ & $\left|\begin{array}{l}1.45 \\
1.68\end{array}\right|$ & $p=0.3066$ & $\left|\begin{array}{l}1.76 \\
1.82\end{array}\right|$ & $p=0.7223$ & $\left|\begin{array}{l}1.76 \\
2.09\end{array}\right|$ \\
\hline
\end{tabular}

Ein kongruenter steigender Verlauf auf Kontextsatz und Testsatz schneidet am besten ab; eine Kombination Kontextsatz/FALL + Testsatz/RISE ist nicht viel schlechter, und beide sind bedeutend besser bewertet als die Kombination Kontextsatz/RISE + Testsatz/FALL. Es kann sich nicht um eine simple Oberflächen- 
kongruenz handeln, da zum einen die Kombination der beiden gegenläufigen Konturen Kontextsatz/FALL + Testsatz/RISE gut bewertet wird, und da zum anderen die hier schlecht bewertete Abfolge Kontextsatz/RISE + Testsatz/FALL in anderem Zusammenhang, bei geschlossenen Alternativfragen, völlig normal ist. So ist die Abfolge Möchtest Du Zucker? mit steigendem und Oder möchtest Du Zitrone? mit fallendem Verlauf bei einem Fünf-Uhr-Tee dann, wenn keine Sahne oder Milch vorhanden sind, die natürliche (vgl. dazu in Batliner/Oppenrieder 1989 die geschlossenen Alternativfragen im 2. Korpus, Nr. 42 und 44).

Um den Unterschied erklären zu können, wollen wir drei Annahmen machen:

1. Ein steigender Intonationsverlauf bei einem Verb-Erst-Fragesatz (der ja regulär auch mit elnem fallenden Verlauf produziert werden könnte) indiziert ein gesteigertes Interesse des Sprechers an einer Antwort (Appellfunktion).

2. Im unmarkierten Regelfall hat ein fallender Intonationsverlauf auch beim Verb-Erst-Fragesatz eine geringe(re) Appellfunktion.

3. Was am Ende eines Redebeitrags (Turn) signalisiert wird, ist wesentlich für seine funktionale Bedeutung.

Wenn nun sowohl Kontextsatz als auch Testsatz fallenden oder steigenden Intonationsverlauf aufweisen, dann besteht auch funktional zwischen ihnen eine Kongruenz, d.h. beide Kombinationen sind akzeptabel. Hat der Kontextsatz fallenden und der Testsatz steigenden Verlauf, so entspricht die Abfolge 'wenig Appell - Appell' einem unmarkierten Redebeitrag, an dessen Ende die Aufforderung zur Gesprächsübernahme (Turn-Taking) steht. Wenn die Reihenfolge aber umgekehrt ist, so folgt einem deutlichen Appell, indiziert durch den steigenden Intonationsverlauf, eine Rücknahme des Appells, indiziert durch den fallenden Verlaup und verstärkt durch die Position am Ende des Satzes. Die 'Botschaft' des Sprechers ist damit widersprüchlich und deshalb weniger akzeptabel.

\subsection{Diskriminationstest (DT)}

Bei den bisherigen Experimenten hat es sich gezeigt, daß ein systematischer Zusammenhang zwischen der Ht-Manipulation auf der einen und der Frage/Exklamativ-Zuweisung auf der anderen Seite existiert; m.a.W. die unterschiedliche Ausprägung der Ht auf FALL und RISE ist linguistisch/phonetisch relevant. Es soll nun untersucht werden, inwiefern sie im gleichen Ausmaß auditiv/psychoakustisch relevant ist, und ob sich in einem weiteren schritt aus Ahnlichkeits - und Unähnlichkeitsrelationen zwischen unseren Stimuli Faktoren erschließen lassen, die die Bewertungsfunktionen der bisherigen Experimente 'erklären' können. Die Beziehung zwischen der Ht-Manipulation und den lingui- 
stischen Kategorien kann linear und trivial sein: ein ausgeprägter RISE indiziert eine Frage, ein fehlender RISE eine Nicht-Frage. Die Beziehung kann aber auch nicht-trivial sein, wenn sich etwa ein komplexer und nicht-linearer Zusammenhang zwischen Merkmalen und Kategorialität ergibt. Um diesen Fragen nachzugehen, wurden ein Diskriminationstest (DT, vgl. Teil 3.4) und ein Ahnlichkeitstest ( $\AA$ T, vgl. Teil 3.5) durchgeführt.

Im DT werden benachbarte oder auch entferntere stimuli eines physikalisch äquidistanten Kontinuums, die zu Paaren (AX-Test) zusammengestellt sind, auf Gleichheit beurteilt. Es werden die Stimulusabfolgen AB, BA und die 'gleich'Paare AA bzw. BB dargeboten. Im Paradigma der Kategorialen Wahrnehmung (vgl. Repp 1984) wird angenommen, daß Paare, innerhalb derer eine Kategoriengrenze liegt, deutlich öfter als 'verschieden' bewertet werden als Paare, bei denen beide Stimuli zur gleichen Kategorie gehören. In unserem Fall sind die Kategorien Frage und Exklamativ (bzw. Nicht-Frage). Das Konzept des strikten Paradigmas der Kategorialen Wahrnehmung und die dort durchgeführte Berechnung der Kategorialität wurden von uns in Schiefer/Batliner (1988) kritisiert; vgl. dazu auch die Arbeiten in Harnad (1987). Das ändert aber nichts daran, daß wir es auch hier mit Kategorien zu tun haben, und daß die grundsätzliche Fragestellung sinnvoll ist, ob solche Kategoriengrenzen, wie wir sie in den bisher besprochenen Experimenten gefunden haben, auch bei der Diskrimination sichtbar werden.

\subsubsection{Design}

Bei den TS-Stimuli ist ein Diskriminationstest nicht sinnvoll, da sie nicht eindeutig zwei verschiedenen Kategorien angehören, und da sie auch kein 'echtes' Kontinuum bilden, sondern höchstens zwei Kontinua mit je drei Stimuli. Wir beschränken uns deshalb auf die FR-Stimuli. In einem Diskriminationstest, der auf drei Sitzungen aufgeteilt war, wurden für jede Kombination benachbarter Stimuli pro Spalte und pro Zeile (vgl. Tab.1) die Anordnung AB und BA sowie die 'gleich'-Paare $A A$ und $B B$ je dreimal randomisiert dargeboten; insgesamt waren es 46 Paare. Die Instruktion lautete: "Bitte entscheiden Sie, ob die Stimuli innerhalb eines Paares in allen Belangen gleich klingen, und drücken Sie dann die linke Taste für 'gleich' oder die rechte für 'verschieden'." Es nahmen 11 VPn teil. 
Anhand der Matrix von Tab.l lassen sich zwei Kontinua bilden, wenn man von Stimulus $9 / 0$ ausgehend im Uhrzeigersinn oder im Gegenuhrzeigersinn zu Stimulus $3 / 9$ geht. Beim ersten Kontinuum wird das exklamativindizierende Merkmal initialer FALL auf 3/0 abgesenkt und dann das frageindizierende Merkmal RISE angehoben. Beim zweiten ist es umgekehrt: Das Prageindizierende Merkmal RISE wird angehoben, während der FALL auf $9 \mathrm{Htn}$ bleibt; ab 9/9 wird der FALL abgesenkt. Im zweiten Kontinuum gibt es also 'widersprüchliche' Merkmalsausprägungen: hoher FALL und hoher RISE. Es interessiert in beiden Fällen, ob und wo man einen Diskriminationsgipfel beobachten kann. Da darüberhinaus auch alle anderen möglichen Paare, also auch solche, die nicht $\mathrm{zu}$ den beiden Kontinua gehören, getestet wurden, kann grundsätzlich untersucht werden, ob sich ein $3 \mathrm{Ht}$-Unterschied bei FALL und bei RISE unterschiedlich auswirkt.

\subsubsection{Ergebnis}

In Fig. 11 sind das Ergebnis für das erste Kontinuum, in Fig.12 das Ergebnis für das zweite dargestellt. Neben den Verläufen für dle Anordnungen $A B$ und BA sind die Verläufe für die 'Gleich'-Paare und das Ergebnis des IT (vgl. Fíg.3) angegeben. Die BA-Kurve zeigt bei beiden Kontinua eher als die AB-Kurve einen Diskriminationsgipfel korrespondierend zum Zufallsbereich beim IT (Stimulus $9 \mid 3$ bzw. $3 \mid 3) .{ }^{3}$ Wir können also - wenn wir uns mit dieser introspektiven Betrachtung der Kurvenverläufe begnügen - feststellen, daß Identifikations - und Diskriminationstest die gleiche Kategoriengrenze bei beiden Kontinua ergeben: beim RISE von drei Htn.4

3 Ublicherweise werden $A B-$ und $B A-W e r t e$ gemittelt und nur diese gemittelten Werte weiter betrachtet; vgl. aber die Uberlegungen in Schiefer/Batliner (1988) dazu, daß die Stimulusabfolge einen ähnlichen Status wie die Schrittgröße hat. D.h. man kann sich für die Interpretation entweder auf $A B$ oder auf BA beschränken.

4 Interessant ist, daß in Fig.11 zwar alle 'Gleich'-Paare sehr gut als 'gleich' erkannt wurden, da $\beta$ sich aber in Fig.12 genau bei der Kategoriengrenze (Stimuli $9 \mid 3$ und $9 \mid 6$ ) ein deutlicher Gipfel zeigt; eine mögliche Erklärung für dleses Phänomen findet sich in Schiefer/Batliner (1988:279f). 
Fig. 11: Diskriminationstest $(9 / 0-3 / 0-3 / 9)$

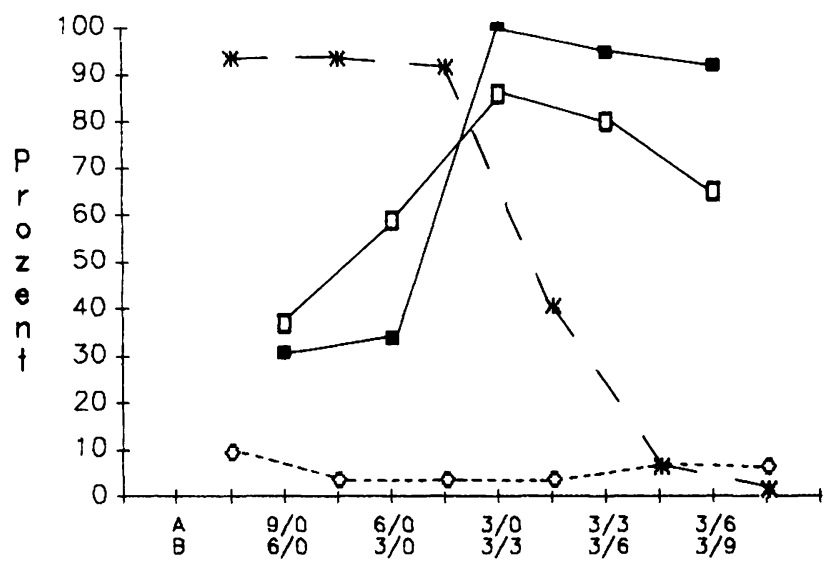

- $A B$

ㅁ. BA

'gleich'-Paare

* Identifikation

Stimuli und Stimuluspare

Fig.12: Diskriminationstest $(9 / 0-9 / 9-3 / 9)$

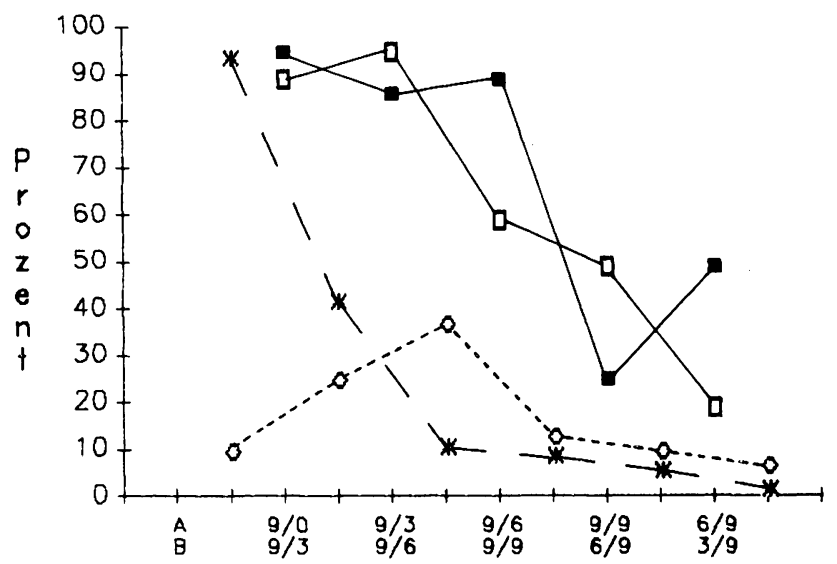

- $A B$

D. BA

- 'gleich'-Poore

* Identifikation

Stimuli und Stimuluspoare

In Tab.4 sind zum einen - in der Anordnung der Matrix wie in Tab.1 - die gemittelten 'Verschieden'-Antworten in Prozent für jeden Vergleich angefuhrt. Tabellarisch folgen die Bewertungen aufgeteilt nach $\mathrm{AB}$ und $\mathrm{BA}$. $\mathrm{Zu}$ den schon anhand von Fig.11 und 12 gemachten Feststellungen lassen sich $z$ wel weitere hinzufügen: 
1. Der finale RISE wird durchgehend besser diskriminiert als der initiale FALL. Das entspricht bekannten Ergebnissen, vgl. Rossi (1978); man muß allerdings beachten, daß bei unseren Stimuli der RISE immer länger war als der FALL, vgl. Fig. 1; der Faktor Dauer spielt sicher auch eine Rolle.

2. Der in Batliner/Schiefer (1987) und Schiefer/Batliner (1988) besprochene Ordnungseffekt tritt bei den RISE-Paaren deutlich in Erscheinung: Wenn der höhere RISE an zweiter Stelle steht, werden die Stimuli besser diskriminiert als wenn er an erster stelle steht.

Tab.4: Diskrimination zwischen benachbarten stimuli, gemittelt und aufgeschlüsselt nach $A B$ und $B A$.

1. Mittelwerte aus $A B$ und $B A$, Matrixanordnung

\begin{tabular}{rrrrr}
$9: 0$ & 338 & $6: 0$ & 468 & $3: 0$ \\
918 & & 908 & & 928 \\
$9 ! 3$ & 448 & $6: 3$ & 568 & $3: 3$ \\
908 & & 878 & & 868 \\
916 & 418 & $6: 6$ & 388 & 316 \\
738 & & 808 & & 778 \\
919 & 368 & $6: 9$ & 338 & $3: 9$ \\
\hline
\end{tabular}

2. $A B$ und $B A$ getrennt

a) Variation des initialen FALL

\begin{tabular}{|c|c|c|c|c|c|c|c|c|}
\hline & $|9| 0-6 \mid 0$ & $6|0-3| 0 ;$ & $9|3-6|^{3}$ & $\left.\left.6\right|^{3-3}\right|^{3}$ & $: 9|6-6| 6$ & $6|6-3| 6 ;$ & $9|9-6| 9$ & $6|9-3| 9$ \\
\hline AB & 308 & 338 & $58 \%$ & 768 & 368 & 488 & 248 & 488 \\
\hline$B A$ & 368 & 588 & $30 \%$ & 368 & 458 & $27 \%$ & $48 t$ & $18 \%$ \\
\hline
\end{tabular}

b) Variation des finalen RISE

\begin{tabular}{c|ccccccccc} 
& $9|0-9| 3$ & $9|3-9| 6$ & $9|6-9| 9 ; 6|0-6|^{3}$ & $\left.6\right|^{3-6} \mid 6$ & $6|6-6|^{9} ; 3|0-3| 3$ & $3|3-3| 6$ & $3|6-3| 9$ \\
\hline $\mathrm{AB}$ & 948 & 858 & 888 & 948 & 888 & 918 & 1008 & 948 & 918 \\
$\mathrm{BA}$ & 888 & 948 & 568 & 858 & 858 & 708 & 858 & 798 & 648
\end{tabular}




\subsubsection{Fragestellung und statistisches Modell}

Im DT konzentriert sich das Interesse auf das Stimuluspaar, zwischen dem die erwartete Kategoriengrenze liegt. Die 'Verschieden'-Antworten können dabei als Abstände $z$ wischen den Stimuli ein Maß für die Ahnlichkeit dieser Stimuli sein. Da allerdings immer nur benachbarte stimuli auf ihre Ahnlichkeit/Unähnlichkeit hin überprüft werden, ist das sich ergebende Bild (vgl. Tab.4) nicht hinreichend genau. Ein genaueres Bild kann man mit einem Experiment erreichen, bei dem jeder Stimulus mit jedem anderen stimulus gepaart ist, dem All-Step-Test. Dabei können auch die TS-Stimuli bewertet werden. Man erhält in unserem Fall bei 18 Stimuli eine 18x18-Matrix mit Ahnlichkeitswerten, vgl. unten Tab.5. Eine solche Matrix enthält alle nötige Information, ist aber - gerade deshalb - kaum interpretierbar, weil die Information ausgesprochen unübersichtlich dargeboten ist. Ein Verfahren zur anschaulichen, (mehr oder weniger) leicht interpretierbaren Darstellung ist die Nonmetrische Multidimensionale Skalierung (NMDS). Die Grundzüge dieses Verfahrens werden von Kruskal/Wish (1978:7) wie lolgt beschrieben:

Multidimensional scaling $[\ldots]$ refers to a class of techniques. These techniques use proximities among any kind of objects as input. A proximity is a number which indicates how similar or how different two objects are, or are perceived to be, or any measure of this kind. The chief output is a spatial representation, consisting of a geometric configuration of points, as on a map. Each point in the configuration corresponds to one of the objects. This configuration reflects the "hidden structure" in the data, and often makes the data much easier to comprehend. By reflecting the data structure we mean that the larger the dissimilarity (or the smaller the similarity) between the two objects, as shown by their proximity value, the further apart they should be in the spatial map.

Nonmetrisch ist die Skalierung dann, wenn die Abstände zwischen den Items nicht als intervallskaliert (metrisch) aufgefaßt werden, sondern als ordinalskaliert (nonmetrisch). In die Analyse gehen in einem solchen Fall nicht die $\mathrm{Ab}-$ stände, sondern die Rangplätze ein. ${ }^{5}$ Das Verfahren der NMDS ist nicht konfirmatorisch in dem Sinn, daß es den strikten Anforderungen genügt, die an ein prüfstatistisches Verfahren gestellt werden; solche Anforderungen können aller-

5 Die NMDS ist ausführlich beschrieben in Borg (1981), eine Anwendung aup Modus/Fokus zeigt Batliner (1988b); dort werden auch einige Kritikpunkte diskutiert. Eine multidimensionale Skalierung von Tonverläufen und Tönen wurde von Gandour (1978) und Gandour/Harshman (1978) durchgeführt. 
dings auch in Frage gestellt werden, cf. Henkel (1976) und Guttman (1977). Die NMDS ist ein anschauliches Verfahren, das nutzbringend explorativ eingesetzt werden kann; als solches sei es auch im folgenden verstanden.

\subsubsection{Design}

Jeder der 18 FR- und TS-Stimuli wurde mit jedem anderen und mit sich selbst gepaart (324 Paare); die Stimuli wurden pseudorandomisiert auf 6 Sitzungen mit je 54 Stimuluspaaren aufgeteilt. (Die Pseudorandomisierung sah so aus, daß jeder Stimulus dreimal an erster Stelle vorkam und die zweite Stelle nach einem Zufallsverfahren vergeben wurde; damit konnte eine, bei einer 'echten' Randomisierung immer mögliche, ausgeprägte ungleiche Verteilung zwischen den einzelnen Teilsitzungen vermieden werden.) Die Pause innerhalb der Paare betrug $0.5 \mathrm{sec}$, die zwischen den Paaren $4 \mathrm{sec}$. Die Anweisung lautete: "Bitte beurteilen Sie die Ahnlichkeit innerhalb eines jeden Paares und bewerten Sie diese Ahnlichkeit auf einer Skala von 1 bis 5 (1: 'kein Unterschied = identisch', 2: 'ähnlich', 3: 'noch ähnlich', 4: 'unähnlich', 5: 'sehr unähnlich'). Es kommen Paare mit identischen Stimuli vor, Paare mit sehr unähnlichen, und Paare mit mehr oder weniger ähnlichen Stimuli." Die sechs Testsitzungen wurden im Abstand von ein bis drei Wochen abgehalten; die Zahl der Teilnehmer schwankte $(12,16,16,18,18,19)$; sechs Teilnehmer nahmen an allen sitzungen teil, die anderen an weniger. ${ }^{6}$

Die NMDS-Lösungen (getrennt für die FR-Stimuli, die TS-Stimuli und für FR- und TS-Stimuli zusammen) wurden mit der SPSS-Prozedur ALSCAL am Leibniz-Rechenzentrum in München berechnet. Eine getrennte und eine gemeinsame Berechnung der FR- und der TS-Stimuli ermöglichte es, innerhalb der beiden Gruppen eindeutigere Dimensionen und über die Gruppen hinweg beiden gemeinsame Dimensionen zu ermitteln.

- Die relativ geringe $Z$ ahl der Teilnehmer und die nicht ganz regelmäßige Besetzung der Gruppen kann natürlich den experimentellen Fehler vergrößern; mehr Teilnehmer und ein ausbalanciertes Verhältnis unter den Gruppen hätte aber den Aufwand stark erhöht. Da die NMDS in unserem Zusammenhang als exploratives Verfahren eingesetzt wurde, schien dieses Vorgehen vertretbar. Indiz dafür, daß die erzielten Lösungen relativ stabil sind, war ein Vergleich der sechs VPn, die an allen Tests teilnahmen, mit den anderen: Bei den nledrigen Dimensionen 1 und 2 waren die Lösungen praktisch identisch. 


\subsubsection{Bestimmung der Dimensionalität}

Tab.5 zeigt die Matrix der Ahnlichkeitswerte; die Werte für die 'gleich'Paare in der Diagonalen sind fett und kursiv. Mit ALSCAL wurden ein- bis vierdimensionale Lösungen berechnet; als Abstandsma $\beta$ wurde das euklidische genommen, die Matrix wurde nicht symmetrisiert, sondern ging als asymmetrische in die Berechnungen ein. (Der beim Diskriminationstest erwähnte Ordnungseffekt ist wahrscheinlich zum großen Teil für die Asymmetrie verantwortlich; auf ihn kann aber in diesem Zusammenhang nicht weiter eingegangen werden.) Es gibt kein 'wasserdichtes' Kriterium dafür, wieviele Dimensionen man für eine NMDSLösung akzeptieren kann; zumindest die folgenden groben Kriterien können aber bei der Entscheidung behilflich sein:

1. Das Gütemaß der Lösung, bei ALSCAL der sog. 'Stress-Wert': Je kleiner dieser Wert ist, desto 'besser' ist die Lösung. (Ein Stress-Wert unter 0.10 gilt als 'gut'; eine blinde Anwendung dieses oder eines ähnlichen Kriteriums ist aber sinnlos.)

2. Ein sog. 'Ellbogen' bei einer grafischen Darstellung des Stress-Wertes vs. Zahl der Dimensionen im 'Stress-Diagramm', vgl. Fig. 13: ein Ellbogen tritt dann auf, wenn höhere Dimensionen wenig und niedrigere viel zur Verbesserung der Lösung beitragen.

3. Das Maß der 'erklärten Varianz' $\left(R^{2}\right)$. Je mehr der Wert gegen 1.0 geht, desto 'besser' wird die Varianz erklärt.

4. Die 'Eindeutigkeit' der Lösung: eine Lösung wird dann als eindeutig angenommen, wenn die Zahl der Items mindestens viermal größer ist als die Zahl der Dimensionen.

5. Die Interpretierbarkeit der Lösung. 
Tab.5: Ahnlichkeitsmatrix

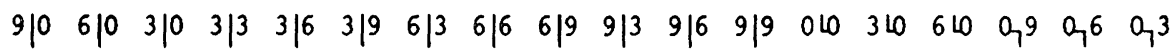

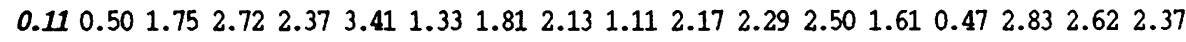

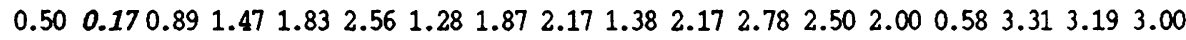

310

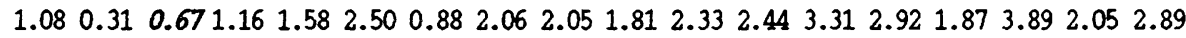

$3 \mid 3$

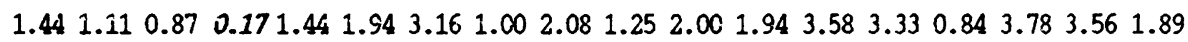

$3 \mid 6$

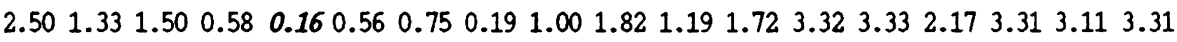

$3 / 9$

$2.251 .941 .741 .13 \quad 0.37 \quad 0.441 .501 .060 .192 .001 .211 .173 .063 .392 .723 .83 \quad 3.503 .17$

$6 / 3$

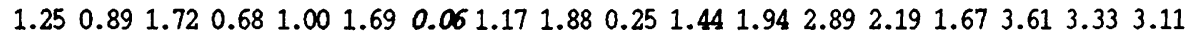

$6 \mid 6$

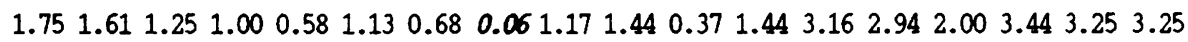

$6 / 9$

$\begin{array}{llllllllllllllllll}2.06 & 1.62 & 2.39 & 1.33 & 0.56 & 0.19 & 1.11 & 0.38 & 0.28 & 2.06 & 1.13 & 0.47 & 3.33 & 2.83 & 2.42 & 3.18 & 2.68 & 2.88\end{array}$

$9 \mid 3$

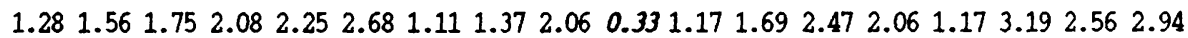

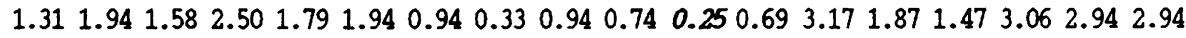

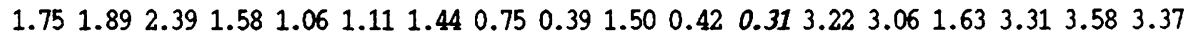

010

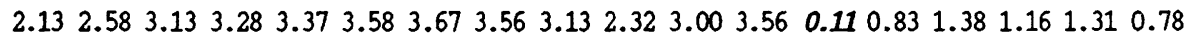

360

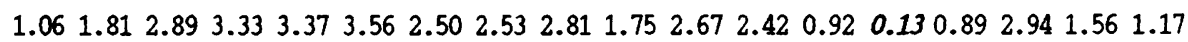

610

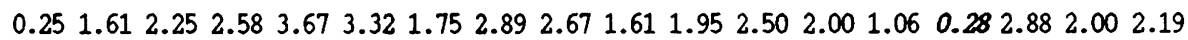

0,9

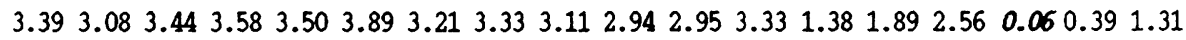

0,6

$\begin{array}{lllllllllllllllllllll}2.17 & 2.72 & 2.92 & 3.89 & 3.44 & 3.68 & 2.94 & 3.83 & 3.37 & 2.67 & 3.13 & 3.37 & 0.75 & 1.89 & 1.67 & 0.31 & 0.42 & 0.31\end{array}$

0,3

$\begin{array}{lllllllllllllllllll}2.31 & 3.11 & 3.42 & 3.62 & 3.42 & 3.78 & 2.58 & 3.56 & 3.67 & 3.33 & 3.33 & 3.05 & 0.44 & 0.73 & 1.84 & 1.19 & 0.63 & 0.45\end{array}$

Fig.13 zeigt das Stress-Diagramm, Tab. 6 die Stress-Werte, die 'erklärte Varianz' $R^{2}$, die Eindeutigkeit ('+' für 'eindeutig', '-' für 'nicht eindeutig') sowie die Interpretierbarkeit (' + ' für 'interpretierbar', ' - ' für 'nicht interpretierbar'; vgl. im einzelnen 3.5.3. - 3.5.5.) pro Dimension. 
Fig. 13: Stress-Diagramm
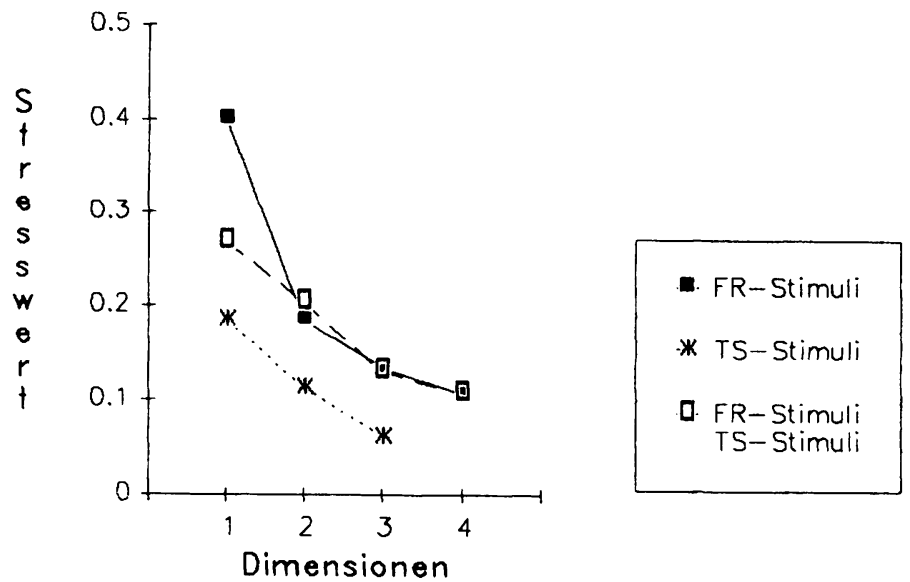

Tab.6: Kriterien zur Wahl der Dimensionalität

\begin{tabular}{|c|c|c|c|c|c|}
\hline stimuli & Dimensionen & stress & $\mathrm{R}^{2}$ & $\begin{array}{l}\text { Eindeutig- } \\
\text { keit }\end{array}$ & $\begin{array}{l}\text { Interpretier- } \\
\text { barkeit }\end{array}$ \\
\hline FALL-RISE & $\begin{array}{l}4 \\
3 \\
2 \\
1\end{array}$ & $\begin{array}{l}.108 \\
.135 \\
.185 \\
.399\end{array}$ & $\begin{array}{l}.92 \\
.89 \\
.84 \\
.59\end{array}$ & $\begin{array}{l}- \\
+ \\
+ \\
+\end{array}$ & $\begin{array}{l}+ \\
+ \\
+ \\
+\end{array}$ \\
\hline TONSPRUNG & $\begin{array}{l}3 \\
2 \\
1\end{array}$ & $\begin{array}{l}.062 \\
.113 \\
.185\end{array}$ & $\begin{array}{l}.98 \\
.95 \\
.91\end{array}$ & $\begin{array}{l}- \\
\overline{+} \\
+\end{array}$ & $\begin{array}{l}- \\
+ \\
+\end{array}$ \\
\hline $\begin{array}{l}\text { FALL-RISE } \\
\text { TONSPRUNG }\end{array}$ & $\begin{array}{l}4 \\
3 \\
2 \\
1\end{array}$ & $\begin{array}{l}.107 \\
.131 \\
.204 \\
.269\end{array}$ & $\begin{array}{l}.93 \\
.91 \\
.85 \\
.82\end{array}$ & $\begin{array}{l}+ \\
+ \\
+ \\
+\end{array}$ & $\begin{array}{l}- \\
+ \\
+ \\
+\end{array}$ \\
\hline
\end{tabular}

Man sieht, daß die Kriterien 1 bis 4 sich z.T. widersprechen: bei den FRStimuli zeigt sich z.B. ein Ellbogen bei Dimension 2, der Stress-Wert ist aber dabei noch recht hoch. Wir werden uns deshalb auf das fünfte Kriterium der 
Interpretierbarkeit der Lösungen stützen, auch wenn dabei die Lösungen noch mit einem relativ hohen Stress-Wert belastet oder uneindeutig im o.a. Sinn sein können; dieses Vorgehen ist bei einer solchen explorativen Studie sinnvoller als ein mechanisches Anwenden der anderen Kriterien.

Fig.14 bis 18 zeigen in jeweils zweidimensionaler Darstellung die interpretierbaren Dimensionen (DIM): Fig.14 DIMI und DIM2 sowie Fig.15 DIM3 und DIM4 für die FR-Stimuli, Fig.16 DIM1 und DIM2 für die TS-Stimuli und Fig.17 DIM1 und DIM2 sowie Fig.18 DIM2 und DIM3 für die FR- und TS-Stimuli. Zur besseren Vergleichbarkeit sind die FR-Stimuli an ihrer Position mit den Zeichen wiedergegeben, die ihnen auch in Fig. 3 bis Fig. 8 zugewiesen waren:

für $9 \mathrm{Ht}$ RISE, ' $O$ ' Pür $6 \mathrm{Ht}$ RISE, ' $\star$ ' für $3 \mathrm{Ht}$ RISE und '+' für $0 \mathrm{Ht}$ RISE. Die TS-Stimuli werden einheitlich mit '\#' wiedergegeben.

\subsubsection{FALL-RISE-Stimuli}

DIM1 kann als Ausprägung des finalen RISE $=$ Höhe des offset interpretiert werden, DIM2 als Ausprägung des initialen FALL $=$ Höhe des Onset, cf. Fig.14. Die Dimensionen stehen ziemlich orthogonal zum Achsenkreuz. (Man beachte, da $\beta$ das keine Bedingung ist; die Punkte können beliebig rotiert oder gespiegelt werden.) Die Stimuli mit 9 und 6 Ht RISE liegen auf DIMl nahe beieinander, ebenso die mit 3 und 0 Ht RISE; die mit 6 und 3 Ht RISE liegen weiter auseinander. Diese Positionen widerspiegeln die Kategoriengrenze zwischen Frage und Nicht-Frage bzw. Exklamativ, die bei den anderen Experimenten auch zwischen 3 und $6 \mathrm{Ht}$ RISE lag. Bei DIM2 liegt $6 / 6$ näher an $3 / 6$ als an $9 / 6$, und $6 \mid 9$ näher an $3 \mid 9$ als an $9 \mid 9$. Verantwortlich dafür ist wahrscheinlich die geringere perzeptive Relevanz des FALL im Verhältnis zum RISE; d.h. ein größeres Ausmaß des FALL ist nötig, um deutliche Unterschiede zu markieren.

Die zweidimensionale Darstellung von DIM1 und DIM2 entspricht der Matrix von Tab.l, die man erhält, wenn man die Punkte um $90^{\circ}$ gegen den Uhrzeiger rotiert und dann um die Senkrechte spiegelt. Die beiden wichtigsten Dimensionen stehen also für die von uns vorgenommenen Manipulationen bei RISE und FALL; daß RISE die wichtigere ist, zeigte sich schon an der besseren Diskriminierbarkeit der RISE-Unterschiede im Diskriminationstest. Die Eckstimuli der Matrix $3|9,3| 0,9 \mid 9$ und $9 \mid 0$ liegen näher zur Mitte hin, als das bei einer Matrixanordnung der Fall ist; sie 'erzeugen' damit die Kreisstruktur in Fig.14.?

7 Wir vermuten, daß man dieser Kreisstruktur keine 'kognitive Relevanz' zuschreiben kann, sondern daß sie sich schlicht aus dem experimentellen Design ergibt, bei dem die Ahnlichkeit nicht nur benachbarter, sondern aller Stimuli, also auch der unähnlichen, gewichtet ist. 
Fig.14:FR-Stimuli

DIM1 (HORIZONTAL): finaler RISE / Höhe des Fo-Offset

DIM2 (VERTIKAL): initialer FALL / Höhe des Fo-Onset

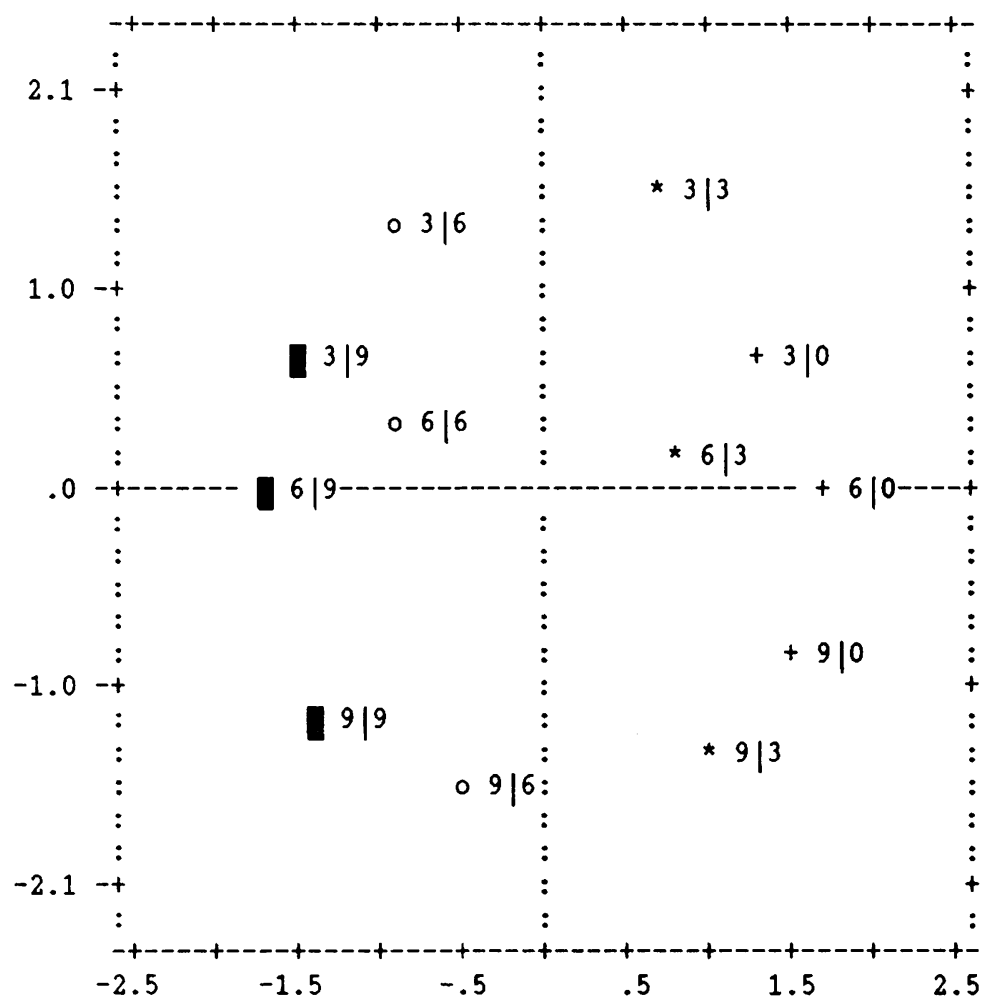

DIM3 und DIM4 tragen deutlich weniger zur erklärten Varianz bel als DIM1 und DIM2, vgl. Tab.6. Beide sind aber sinnvoll interpretierbar. Sie stehen zwar orthogonal zueinander, aber leicht rotiert im Verhältnis zum Achsenkreuz. In Fig.15 ist deshalb durch den Ursprung per Hand die Ausrichtung der Dimensionen eingetragen (durchgezogene Linien). Geht man bei DIM3, also in der Horizontalen, von links nach rechts, so kommen zuerst die stimuli mit 0 Ht RISE, dann die mit $9 \mathrm{Ht}$ RISE, dann, auf der rechten Seite, die restlichen mit 3 und 6 Ht RISE. Wir können also diese Dimension die des extremen Endpunkts nennen. (Eine solche Dimension, die nicht entlang eines Kontinuums angesiedelt ist, fanden auch Gandour 1978 und Gandour/Harshman 1978.) 
Fig.15:FR-Stimuli

DIM3 (HORIZONTAL) : Extremer Endpunkt

DIM4 (VERTIKAL): Direktionalităt der Kontur

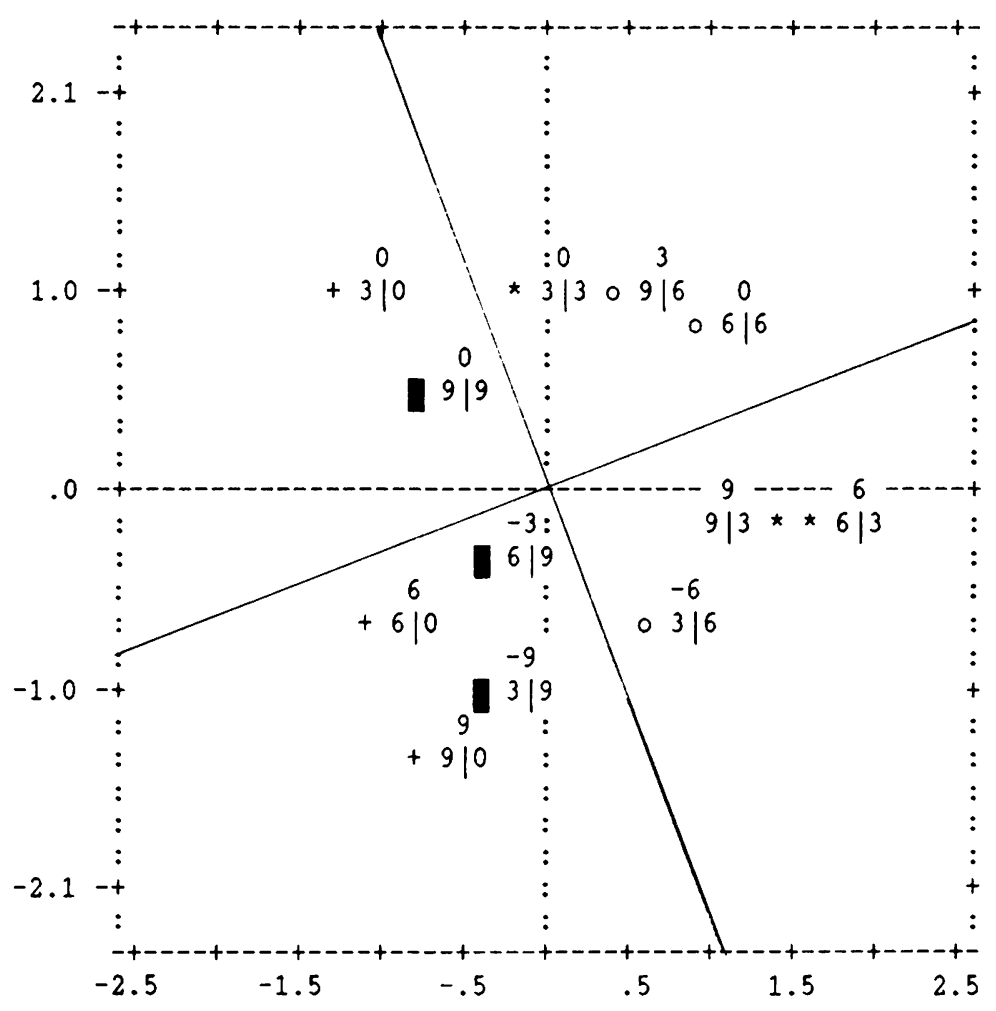

Wenn wir im folgenden die 'Existenz' einer vierten Dimension ansetzen, dann natürlich unter der Voraussetzung, daß sie replizierbar ist: die Lösung ist in unserem Fall bei vier Dimensionen nicht mehr eindeutig, vgl. Tab.6. Betrachtet man nun die Stimuli von links oben nach rechts unten, also entlang der eingezeichneten Linie, so fällt auf, daß zuerst Stimuli kommen, bei denen sich 'wenig tut', wie $3 / 0$ oder $3 / 3$, zugleich aber auch stimuli, bei denen sich 'viel tut', wie $9 / 9$ und $6 / 6$. Am anderen Ende sind Stimuli mit nur einem eindeutigen RISE ( $3 \mid 9)$ oder einem eindeutigen FALL ( $9 / 0)$ angesiedelt. Die Zuweisung zu den Positionen links und rechts von der Mitte wird eindeutig, wenn wir die stimuli mit einem primitiven Verfahren gewichten: Ein FALL von $3 \mathrm{Ht}$ ist perzeptiv unaufpăllig, da er im unmarkierten Fall wegen des Einflußes der Deklination 'erwartet' wird; wir können ihm also den 'perzeptiven Wert' ' $O$ ' zuweisen. Ein RISE von $3 \mathrm{Ht}$ ist ebenfalls perzeptiv unaupällig - nicht in dem Sinn, daß er nicht wahrgenommen wird, aber in dem Sinn, daß er zu gering ist, um eindeutig einen 
phonetisch relevanten RISE zu kennzeichnen; vgl. die Kategoriengrenze bei den weiter oben besprochenen Experimenten und die Verteilung entlang DIM1 in Fig.14. Wir können also auch diesem RISE von $3 \mathrm{Ht}$ einen perzeptiven wert von ' $O$ ' zuweisen. Wir setzen nun bei den anderen Ht-Werten den 'objektiven' Wert mit dem 'perzeptiven' gleich und ermitteln die Differenz zwischen dem FALL und dem RISE pro Stimulus; dieser Wert ist in Fig.15 bei jedem Stimulus über dem Strich angegeben. Wir erhalten dabei die folgende Aufteilung:

\section{$0+3$}

'ausgeglichene' Kontur

$$
-9-6-3
$$

entweder FALL oder RISE

unidirektional bidirektional bzw. nondirektional
$+6+9$

entweder FALL oder RISE

unidirektional

Man kann also DIM4 als die Dimension der Direktionalität bezeichnen: unidirektional vs. bi- oder nondirektional. Wir müssen es dahingestellt lassen, ob die Asymmetrie, die sich bei Stimulus 6/9 mit dem Wert ' -3 ' zeigt, objektive Gründe hat oder nicht doch auf einen experimentellen Fehler zurückzuführen ist.

\subsubsection{TONSPRUNG-Stimuli}

DIMl kann als Position des FALL: initial vs. final bzw. komplementär dazu als Position des LEVEL-Verlaufs: final - final+initial - initial bezeichnet werden. DIM!2 als Tonsprung: groß - klein. Man erhält in etwa die Darstellung von Tab.2, wenn man an der Waagrechten spiegelt. Die große Ahnlichkeit von OLO und $0_{7} 3$, vgl. Fig.2, positioniert die beiden stimuli nahe beieinander; daraus ergibt sich die Halbkreisform. Die Stimuli mit der gleichen Tonsprungdifferenz, 6 LO und 3 LO bzw. $0_{7} 9$ und $0_{7} 6$, sind unterschiedlich weit voneinander entfernt; das ist möglicherweise dadurch bedingt, daß ein steiler Abfall (Slope) perzeptiv auppälliger ist als ein flacher. 
Fig.16: TONSPRUNG--Stimuli:

DIM1 (HORIZONTAL): Position des FALL (initial vs. final)

DIM2 (VERTIKAL): Tonsprung

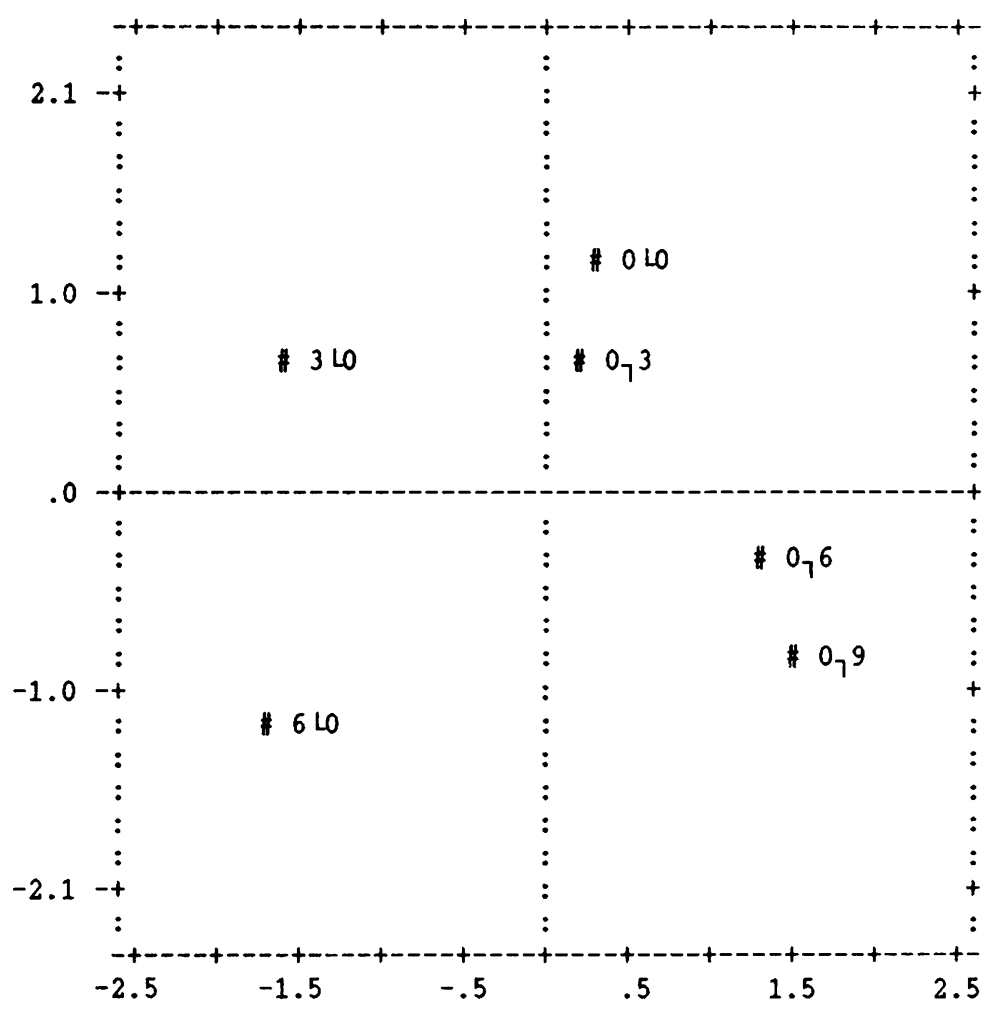

\subsubsection{FALL-RISE + TONSPRUNG - Stimuli}

In Fig.17 ist DIM1 eine Mischung aus einer Cluster-Bildung mit den TS-Stimuli auf der linken und den FR-Stimuli auf der rechten seite und einer Abfolge entlang der Dimension RISE innerhalb der FR-Stimuli. Diese Dimension RISE findet sich auch in DIM2 wieder, die man als Ausmaß der finalen Bewegung / des finalen RISE bezeichnen kann: oben ist die finale Bewegung gro $\beta$, unten gering bzw. ein LEVEL-Verlaup. Insgesamt sieht es so aus, als ob die bei der getrennten Analyse der FR- und der TS-Stimuli wichtigste Dimension (DIM1) eine 'Nahtstelle' hätte bei den ähnlichsten Stimuli 6 LO und $9 \mid 0$, und ansonsten die 
jeweilige geometrische Form, also der Kreis von Fig.14 und der Halbkreis von Fig.16, aufgebogen würden und zusammen ein Hufeisen ergeben. ${ }^{8}$

Fig.17: FALL-RISE- und TONSPRUNG-Stimuli:

DIM1 (HORIZONTAL): Cluster (TS- vs. FR-Stimuli)

DIM2 (VERTIKAL): finaler RISE

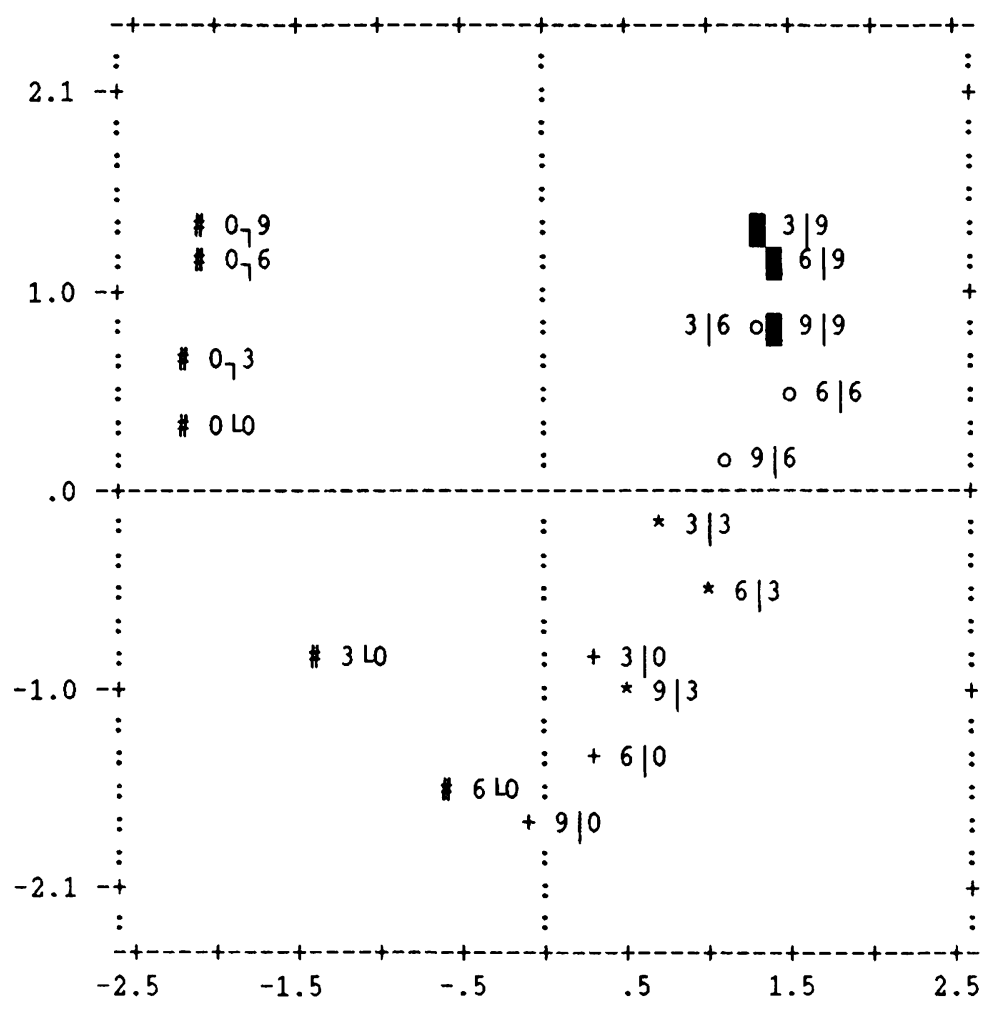

Eine solche Hufeisenform findet sich oft; sie wird manchmal als Indiz dafür genommen, daß man es eigentlich nur mit einer Dimension zu tun hat, vgl. Shepard (1974:385f). In unserem Fall dürfte aber die Kombination der beiden unterschiedlichen Stimulusgruppen dafür verantwortlich sein. 
In Fig.18 entspricht DIM2 versus DIM3 ziemlich genau DIM1 versus DIM2 bei den FR-Stimuli in Fig.14. Das weist daraup hin, daß bei einer Kombination der beiden Stimulusgruppen die erste Dimension einfach die beiden Gruppen trennt, vgl. die o.a. Cluster-Bildung, und die zweite und die dritte von der systematischeren und größeren Gruppe der FR-Stimuli geprägt ist. Es bleibt zu fragen, wo in DIM2 und DIM3 die TS-Stimuli angesiedelt sind. Wenn wir die nächsten Nachbarn betrachten, so liegen 6 LO bei $9 \mid 3,0$ LO bei $9 \mid 6,0_{7} 3$ und $0_{7} 6$ bei $6 \mid 6$ und $0_{7} 9$ bei $3 / 9$. Es geht also von einer starken Ausprägung der initialen Bewegung (FALL) zu einer starken Ausprägung der finalen Bewegung (RISE bei $3 / 9$ oder FALL bei $0_{7} 9$ ), m.a.W. zu extremen Endpunkten; vgl. DIM3 in Fig.15.

Fig.18: FALL-RISE- und TONSPRUNG-Stimuli

DIM2 (HORIZONTAL): finaler RISE (FR-Stimuli)

DIM3 (VERTIKAL): initialer FALL (FR-Stimuli)

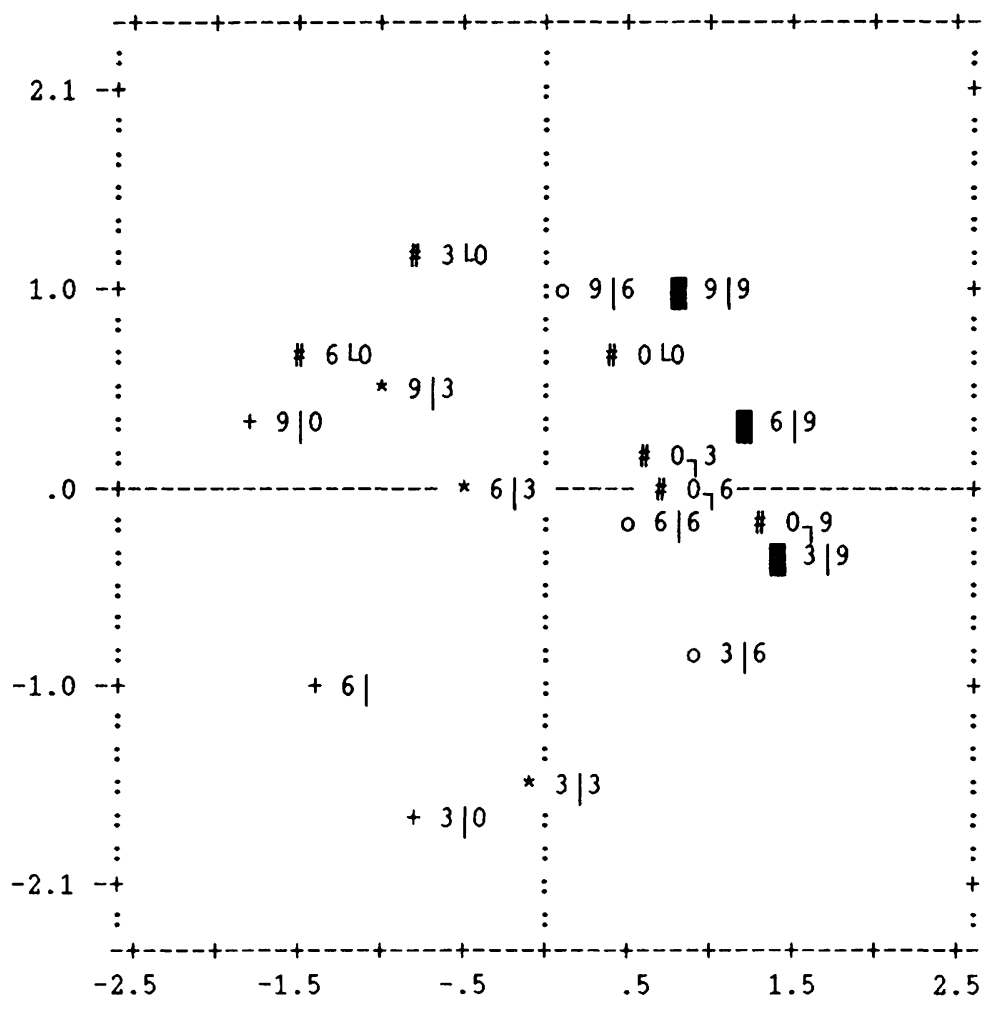




\subsubsection{Dimensionen versus linguistische Kategorien}

Welcher Zusammenhang besteht nun zwischen den Dimensionen und den Bewertungsfunktionen der anderen Experimente? Sinnvoll läßt sich diese Frage nur für die FR-Stimuli stellen, da die TS-Stimuli zu einheitlich bewertet werden: Sie sind grundsätzlich bessere Exklamativ- und schlechtere Fragesätze. Ein solcher Zusammenhang kann dadurch hergestellt werden, daß man die Dimensionen als unabhängige (Prädiktor-) und die anderen Bewertungsfunktionen als abhängige (Kriteriums-) Variablen einer Regressionsanalyse ansetzt und versucht, die 'Erklärung' der abhängigen durch die unabhängigen Variablen zu optimieren. Maßzahl dafür ist die 'erklärte Varianz' $R^{2}$. Es ist natürlich abzusehen, daß als wichtigste Dimension (DIM1) der finale RISE für die Frage/Nicht-Frage-Distinktion entscheidend ist. Interessant ist also der Beitrag der weniger wichtigen Dimensionen DIM2, DIM3 und DIM4. Da wir allerdings nur 12 Datenpunkte, nämlich die jeweiligen werte der 12 stimuli, vorhersagen können, besteht bei einer Erhöhung der Zahl der unabhängigen Variablen bald die Gefahr, daß keine systematischen Zusammenhänge entdeckt werden, sondern daß sozusagen der experimentelle Fehler 'gelernt' wird. (Wenn die Zahl der unabhängigen gegen die Zahl der abhängigen Variablen geht, wird die Vorhersage perfekt, aber sinnlos.) Die folgende Auswertung muß also immer unter dem Caveat betrachtet werden, da $\beta$ es sich um eine explorative Auswertung handelt.

In Tab.7 sind die Ergebnisse der Regressionsanalysen für alle Experimente aufgeführt. In der ersten Spalte stehen das betreffende Experiment und ein Verweis auf die Abbildung, in der zweiten Spalte die Dimensionen, in der dritten der $R^{2}$-Wert als Maß der erklärten Varianz bei einer multivariaten Regressionsanalyse mit schrittweiser Hinzufügung der unabhängigen Variablen (zuerst die wichtigste, dann die zweitwichtigste, usw.) Ein Strich zeigt an, daß diese Variable das Toleranzkriterium von 0.01 nicht passiert hat. In der vierten steht der Schritt, bei dem die Dimension in die Analyse eingefügt wurde; in der fünften ist angegeben, ob die Korrelation positiv oder negativ ist. (Man beachte, daß 'positiv' oder 'negativ' hier arbiträre Begriffe sind: 'positiv' heißt, daß ein positiver Wert auf der entsprechenden Dimension der NMDS mit einem hohen Wert, also einer schlechten Bewertung bei der Kontextverträglichkeit, korreliert!) In der letzten Spalte steht $\mathrm{R}^{2}$ für eine univariate Regressionsanalyse, bei der jeweils nur die eine Dimension einging. (Die Wurzel aus diesem Wert ergibt den 
Korrelationskoeffizienten $r$ ). ' $1-4$ ' in Spalte 2 und die entsprechenden $\mathrm{R}^{2}$-Werte in Spalte 3 stehen für Analysen, bei denen alle vier Dimensionen in die Analyse eingingen.

Wie erwartet, ist DIM1 (finaler RISE) die wichtigste Dimension, die allein schon bei den Fragen einen sehr guten Wert ergibt. DIM2 (initialer FALL) und DIM3 (extremer Endpunkt) tragen bei den Fragen auch noch bei, DIM4 (Direktionalität) ist bei den Fragen immer irrelevant. Das Bild bei den Exklamativen ist etwas widersprüchlich, es fällt aber auf, daß DIM3 nichts beiträgt, und daß beim steigenden Verlauf des Fragekontextes DIM2 und DIM4 relativ viel beitragen.

Dieses Ergebnis stimmt gut mit anderen Ergebnissen aus der Literatur, z.B. mit denen von Studdert-Kennedy/Hadding (1973), überein: Diese Autoren manipulierten bei ihren Stimuli sowohl die finale Bewegung als auch den FoVerlauf vor der finalen Bewegung. Sie stellten fest, daß ein steigender finaler Fo-Verlauf in über $90 \%$ der Fälle eine Frage (question) indizierte und vice versa ein fallender Fo-Verlaup in über $90 \%$ der Fälle eine Nicht-Frage (statement). Sie stellten aber ebenfalls fest, daß z.B. ein präfinaler hoher Fo-Gipfel eine Frageinterpretation begünstigen kann, m.a.W. daß nicht nur der finale FoVerlauf für die Frage/Nicht-Frage-Distinktion relevant ist.

Wir wollen nun im nächsten Teil die bisherigen Ergebnisse an Produktionsdaten überprüfen und danach auf die Relevanz der Dimensionen für die beiden Satzmodi zurückkommen. 
Tab.7: Regressionsanalysen mit NMDS-Dimensionen als Prädiktoren und Bewertungsfunktionen als Kriterium

\begin{tabular}{|c|c|c|c|c|}
\hline Experiment & DIM & $\begin{array}{l}\mathrm{R}^{2} \\
\text { multiv. }\end{array}$ & Schritt & Korr. \\
\hline
\end{tabular}

\begin{tabular}{|c|c|c|c|c|c|}
\hline $\begin{array}{l}\text { IT: } \\
\text { Frage-Nicht- } \\
\text { frage } \\
\text { (Fig.3) }\end{array}$ & $\begin{array}{c}1 \\
2 \\
3 \\
4 \\
1-4\end{array}$ & $\begin{array}{l}.86 \\
- \\
.94 \\
- \\
.99\end{array}$ & $\begin{array}{l}1 \\
- \\
2 \\
-\end{array}$ & $\begin{array}{l}+ \\
+ \\
- \\
-\end{array}$ & $\begin{array}{l}.86 \\
.00 \\
.13 \\
.03\end{array}$ \\
\hline (Fig.4) & $\begin{array}{c}1 \\
2 \\
3 \\
4 \\
1-4\end{array}$ & $\begin{array}{r}.91 \\
.97 \\
.94 \\
- \\
.97\end{array}$ & $\begin{array}{l}1 \\
3 \\
2 \\
-\end{array}$ & $\begin{array}{l}+ \\
+ \\
+ \\
+\end{array}$ & $\begin{array}{l}.91 \\
.03 \\
.08 \\
.03\end{array}$ \\
\hline $\begin{array}{l}\text { KTI } \\
\text { Fragekontext } \\
\text { (RISE) } \\
\text { (Fig.5) }\end{array}$ & $\begin{array}{c}1 \\
2 \\
3 \\
4 \\
1-4\end{array}$ & $\begin{array}{r}.90 \\
.98 \\
.99 \\
- \\
.99\end{array}$ & $\begin{array}{l}1 \\
2 \\
3 \\
-\end{array}$ & $\begin{array}{l}+ \\
+ \\
- \\
-\end{array}$ & $\begin{array}{l}.90 \\
.08 \\
.06 \\
.00\end{array}$ \\
\hline $\begin{array}{l}\text { KII } \\
\text { Exklamativkont. } \\
\text { (Frageksont. RISE) } \\
\text { (Fig.6) }\end{array}$ & $\begin{array}{c}1 \\
2 \\
3 \\
4 \\
1-4\end{array}$ & $\begin{array}{l}.51 \\
.75 \\
- \\
.89 \\
.93\end{array}$ & $\begin{array}{l}1 \\
2 \\
- \\
3\end{array}$ & $\begin{array}{l}- \\
+ \\
- \\
+\end{array}$ & $\begin{array}{l}.51 \\
.25 \\
.05 \\
.18\end{array}$ \\
\hline $\begin{array}{l}\text { KT2 } \\
\text { Fragekontext } \\
\text { (FALL) } \\
\text { (Fig.7) }\end{array}$ & $\begin{array}{c}1 \\
2 \\
3 \\
4 \\
1-4\end{array}$ & $\begin{array}{r}.82 \\
.96 \\
.91 \\
- \\
.97\end{array}$ & $\begin{array}{l}1 \\
3 \\
2 \\
-\end{array}$ & $\begin{array}{l}+ \\
+ \\
- \\
-\end{array}$ & $\begin{array}{l}.82 \\
.07 \\
.15 \\
.01\end{array}$ \\
\hline $\begin{array}{l}\text { KT2 } \\
\text { Exklamativkont. } \\
\text { (Fragekont. FALL) } \\
\text { (Fig.8) }\end{array}$ & $\begin{array}{c}1 \\
2 \\
3 \\
4 \\
1-4\end{array}$ & $\begin{array}{l}.81 \\
- \\
- \\
- \\
.93\end{array}$ & $\begin{array}{l}1 \\
- \\
- \\
-\end{array}$ & $\begin{array}{l}- \\
+ \\
+\end{array}$ & $\begin{array}{l}.81 \\
.03 \\
.08 \\
.09\end{array}$ \\
\hline
\end{tabular}


4. Produktionsdaten

\subsection{Material}

Es sollen nun den bisherigen Ergebnissen aus den Perzeptionsexperimenten Produktionsdaten mit den gleichen Satzmodi und gleichen oder vergleichbaren Kontext- und Testsätzen gegenübergestellt werden. Es handelt sich dabei um die Sätze Nr.80 bis 84 aus dem vierten Korpus, das ausführlich in Batliner/Oppenrieder (1989) dokumentiert ist; es wird deshalb an dieser stelle nur kurz charakterisiert: Sechs Sprecher (drei weibliche, drei männliche) produzierten die Testsätze mit dem beigefügten Kontext zwei- bis viermal im schallarmen Raum des Instituts für Phonetik. Die Kontexte indizierten die intendierte Modusund Fokus-Konstellation. In Fig. 19 sind die Verläufe für die zwei Fragesätze, in Fig. 20 die Verläufe für die drei Exklamativsätze mit dem uns in diesem $\mathrm{Zu}-$ sammenhang interessierenden Testsatz Säuft der Leo aufgeführt. Jede Außerung wurde einem 'Natürlichkeitstest' und einem 'Kategorisierungstest' unterzogen. Im ersten Test wurde die Außerung auf einer fünfstufigen Ratingskala (1-5) danach beurteilt, wie gut sie in den Kontext paßt. Bei einem Wert von $<2.5$ wird die Außerung als 'natürlich' bezeichnet. Im zweiten Test wurde die Außerung ohne Kontext dargeboten und die VPn ordneten sie einer Satztypkategorie zu. Bei einer mindestens $80 \%$ igen ubereinstimmung und richtiger Zuordnung wird die Außerung als 'richtig kategorisiert' bewertet. 'Natürliche' und zugleich 'richtig kategorisierte' Außerungen wurden als Prototypen bezeichnet, die anderen als Nicht-Prototypen; zur genaueren Darstellung und Motivation dieses Vorgehens vgl. im einzelnen Oppenrieder (1988a,b) und Batliner (1988a). Die Anordnung in Fig.19 und 20 ist wie folgt: Die $y$-Achse zeigt den Fo-Verlauf in $\mathrm{Hz}$, die $x$-Achse die Zeit. Uber der $x$-Achse stehen die Konturen der Sprecherinnen, unter der $x$-Achse die der Sprecher. Links stehen die Prototypen, rechts die Nicht-Prototypen. In den Testsätzen ist das Element, auf dem die Hauptakzentsilbe erwartet wurde, groß geschrieben.

Da die Zahl der Fälle pro Konstellation vergleichsweise gering ist, begnügen wir uns mit einer interpretativen Analyse und sehen davon ab, die Ergebnisse einer (PrüP-)Statistik zu unterziehen. 
Fig.19: Fragesätze
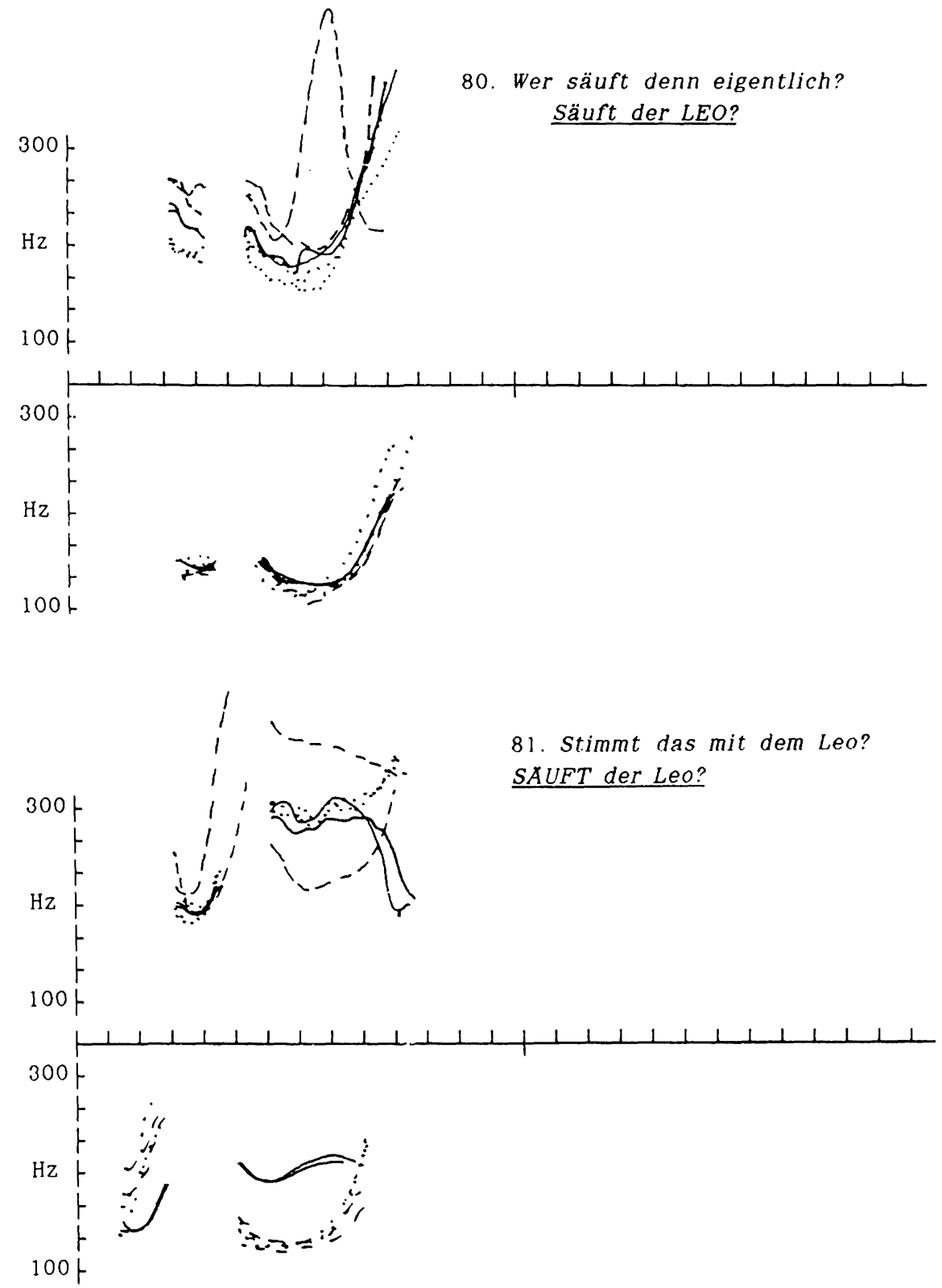
Fig.20: Exklamativsătze

82. Gestern waren wir im Wirtshaus. Mann - Säuft der LEO!

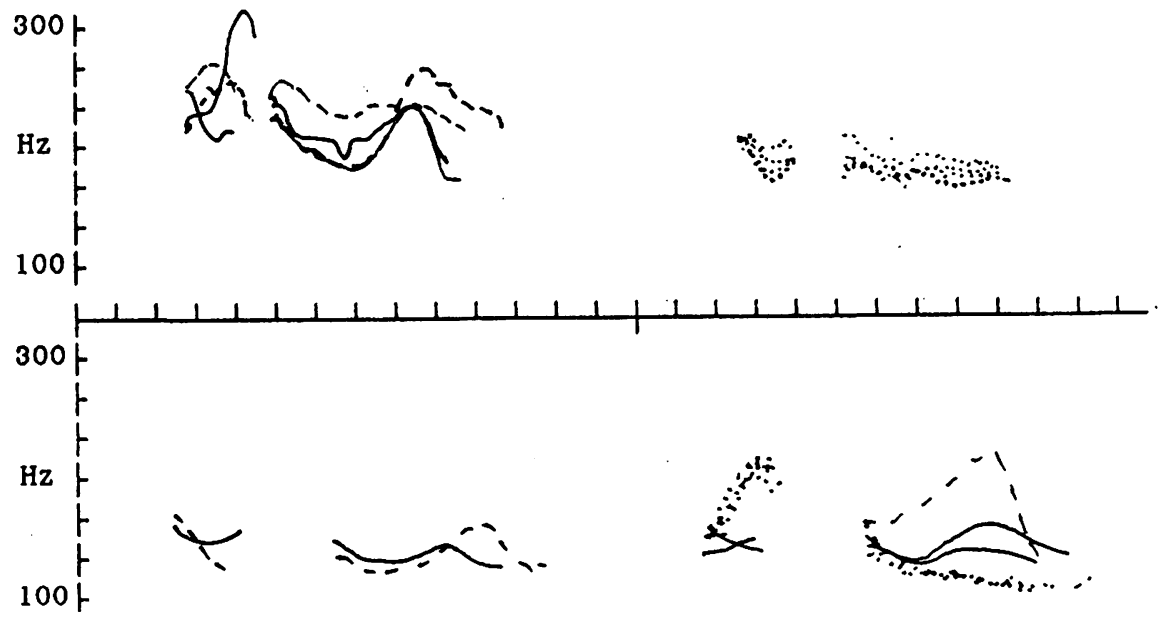

83. Gestern war ich mit dem Leo im Wirtshaus. Mann - SAUFT der Leo!

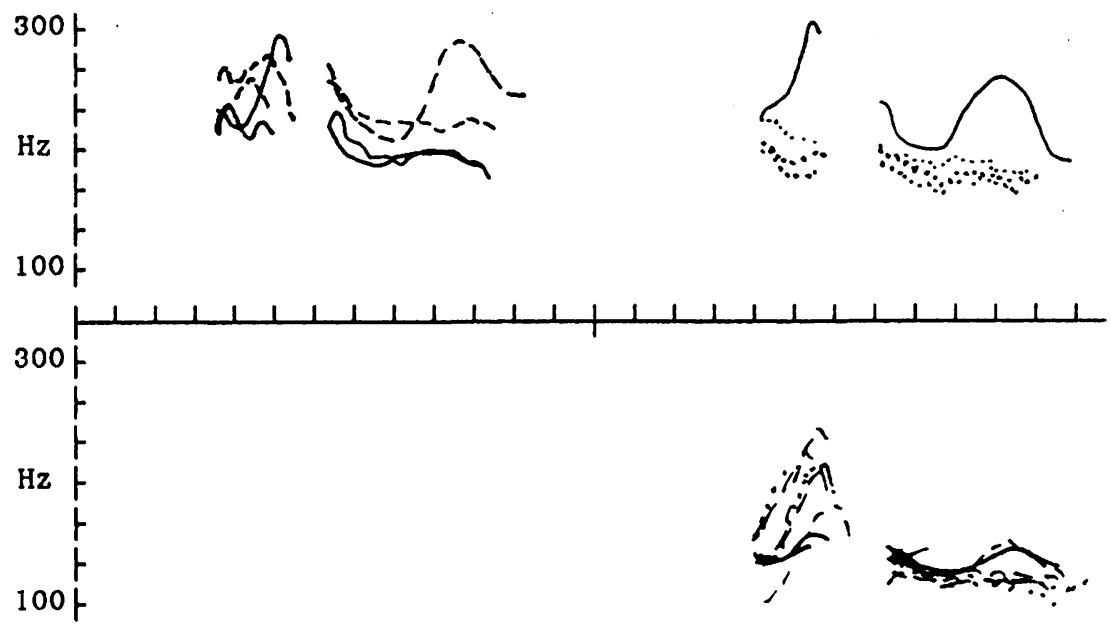


Fig.20:Exklamativsätze (Fortsetzung)

84. Also sowas hätt' ich nicht gedacht! SAUFT der Leo!

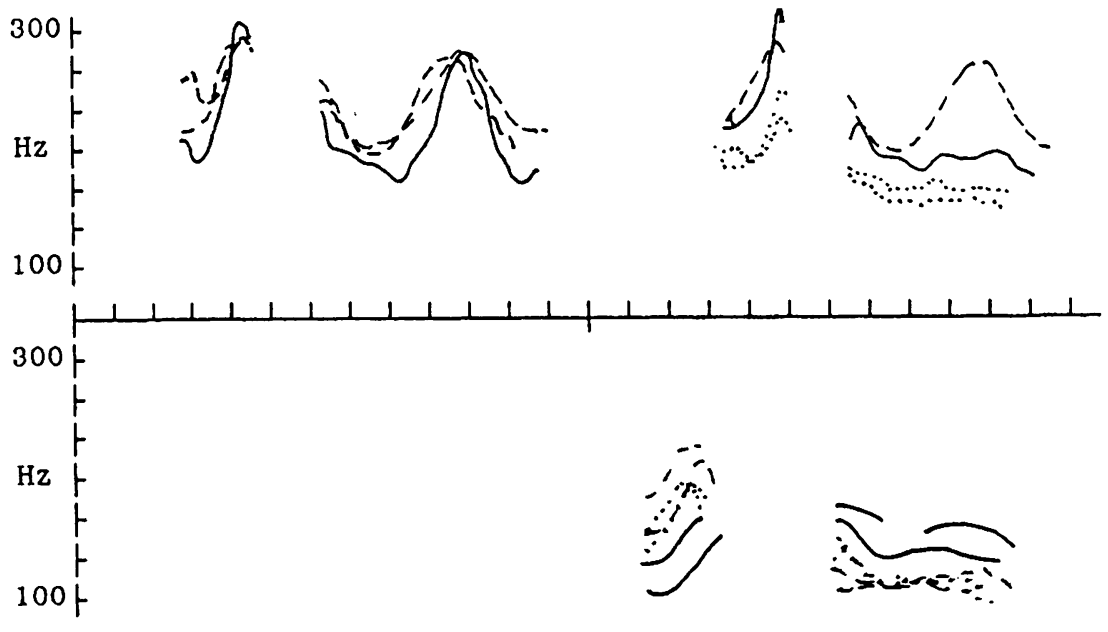

\subsection{Fragesätze}

Bei Satz 80 mit fokussiertem Leo ist der Verlauf mit einer Ausnahme immer ein schwacher FALL bzw. ein LEVEL auf säuft und ein starker RISE auf Leo. Die Außerungen sind alle natürlich und eindeutig kategorisiert. Der Verlauf entspricht unserem Stimulus $3 / 9$, der auch in den Perzeptionstests die besten Fragezuweisungen erhielt.

Einen Verlauf, der den Verläufen von Satz 81 mit Fokus auf säuft entspricht, fand sich nicht in unserem Stimulusmaterial: Man kann aber sehen, da $\beta$ bei einem nicht-finalen fokussierten Element der offset auch tief sein kann, ohne daß sich an der Natürlichkeit oder Kategorisierbarkeit etwas ändert.

\subsection{Exklamativsätze}

Bei allen drei Konstellationen fällt auf, daß sich kein Verlauf findet, der dem Stimulus $9 / 0$ vergleichbar ist. Es hat sich aber in Batliner (1988b:226ff) gezeigt, daß ein solcher Verlaup beim Exklamativsatz durchaus akzeptabel ist. Möglicherweise handelt es sich dabei um einen weniger typischen bzw. weniger oft auftretenden Verlauf. 
Bei den Exklamativsätzen zeigt sich oft eine Doppelakzentuierung (vgl. dazu Batliner 1988b,c). Bei den Prototypen von Nr.84 ist neben einem RISE auf säuft immer ein RISE-FALL auf Leo realisiert. Wenn er fehlt, so werden die Außerungen als Fragen fehlklassifiziert (die genauen Kennwerte dafür finden sich in Batliner/Oppenrieder 1989). Ahnliche Fehlklassifikationen bei entsprechenden Verläufen sind auch bei Nr.82 und Nr.83 zu beobachten. Die 'nicht-prototypischen', fast ebenen Verläufe sind zwar als natürlich bewertet, konnten aber ohne Kontext nicht ausreichend gut klassifiziert werden. Die Verläufe entsprechen dem stimulus $3 / 0$. Sie sind offensichtlich beim Exklamativsatz dann akzeptabel, wenn ihnen ein exklamativindizierendes Mann-vorausgeht, zu dem sie quasi einen intonatorischen Nachlauf bilden können. (Zur Frage, ob es sich hier überhaupt noch um Exklamativsätze handelt, vgl. Batliner 1988c:254f). Da eine Entsprechung zu Mann- in unseren Perzeptionstests fehlt, schneidet der Stimulus $3 / 0$ schlechter ab als die stimuli $6 / 0$ und $9 / 0$.

4.4 Die Relevanz der NMDS-Dimensionen für die Satzmodi 'Verb-Erst-Frage'und 'Verb-Erst-Exklamativsatz'.

Fragesätze haben typischerweise einen hohen oder einen tiefen offset; bei einem tiefen offset ist ein globaler FALL-Verlauf möglich (vgl. Oppenrieder 1988b, Batliner et al. 1989). Darin spiegelt sich die Relevanz von DIM1 (RISE), DIM2 (FALL) und DIM3 (extremer Endpunkt). DIM4 (Direktionalität) bestimmt Fragen vermutlich nicht, da Non- oder Bidirektionalität bei Fragen nicht auptritt oder dann, wenn sie auftritt, die wichtigeren Dimensionen DIM1 bis DIM3 'zuständig' sind.

Die Relevanz von DIM1 für die Exklamativsätze ist bei unseren Perzeptionsexperimenten sicher durch die Art der Stimulusmanipulation bedingt: Bei unseren Stimuli dominieren typische Frageverläufe bzw. -merkmale. ${ }^{9}$ DIM1 definiert also die Exklamativsätze 'negativ': wenn sie stark ausgeprägt ist, ist eine Exklamativinterpretation nicht möglich, wenn sie schwach ausgeprägt ist, dann ist eine solche Interpretation möglich, wenn andere Merkmale vorhanden sind. Diese anderen Merkmale sind mit bestimmt durch DIM2 (FALL) und DIM4 (Direktionalität); wir haben bei den Produktionsdaten gesehen, daß dort bei den Exklamativsatzrealisationen sowohl non-direktionale als auch bidirektionale (allerdings RISE-FALL, nicht FALL-RISE) Verläufe gehäuft auftreten. Exklamative

9 Wie sich in anderen Untersuchungen gezeigt hat, vgl. Batliner (1988b,c), ist für den Exklamativ ein hoher Gipfel bei einer RISE-FALL-Bewegung, eine Dehnung der Hauptakzentsilbe, eventuell eine späte Fo-Gipfelposition sowie eine ausgeprägte Doppelakzentuierung typisch - alles Merkmale, die bei unseren Stimuli nicht vorkamen. 
zeichnen sich also durch 'sehr viel Bewegung' aus, oder beim Vorliegen gewisser Randbedingungen ('intonatorischer Nachlauf') durch sehr geringe Bewegung. DIM3 (extremer Endpunkt) ist irrelevant: Ein ausgeprägter finaler RISE - besonders als steiler linearer Anstieg wie bei unseren Stimuli - kommt bei Exklamativsätzen praktisch nicht vor. Ein tiefer offset findet sich, aber der ist durch die Dimension (globaler) FALL abgedeckt. Typisch für den Exklamativ sind aber auch 'mittelhohe' offsetwerte. ${ }^{10}$

5. Allgemeine Diskussion

\subsection{Abstufung der Fragehaltigkeit}

Es ist eine Eigenheit der Satzmodi, da $\beta$ intonatorische Indikatoren beim gleichen Satzmodustyp strikt entgegengesetzte Ausprägungen annehmen können. So kann der Verb-Erst-Fragesatz sowohl einen hohen als auch einen tiefen offset aufweisen. Normalerweise spricht man hier von freien Varianten oder bestenfalls von paralinguistisch relevanten Nuancen - daß etwa ein steigender Intonationsverlauf auf Subordination oder Höflichkeit des Sprechers hinweist. In dieser Deutung hat also der unterschiedliche Intonationsverlauf keinen sprachlichen Zeichencharakter, sondern ist ein Anzeichen außersprachlicher Gegebenheiten. Im Gegensatz dazu nehmen wir an, daß die Variation der Intonationsverläufe innerhalb des Satzmodustyp 'Frage' mit einer Abstufung der 'Fragehaltigkeit' (und damit der Kategorialität) korreliert, also sehr wohl Zeichencharakter hat." Für diese Annahme lassen sich phonetische und linguistische Gründe ins Feld führen, wobei eine Abstufung auf der phonetischen Ebene die Voraussetzung bildet für eine Abstufung auf der Zeichenebene.

10 Vgl. dazu die Liste der intonatorischen Prototypen in Oppenrieder (1988b:203ff): die Fo-Kontur wird dort global mit drei relationalen Stufen gekennzeichnet. Bei den Exklamativsätzen überwiegt der Verlaup '132', bei dem der Fo-Offset höher liegt als der Fo-Onset, bei den damit kontrastierenden Aussage- bzw. Imperativsätzen überwiegt der Verlauf '231', bei dem der Fo-Offset tiefer liegt als der Fo-Onset.

11 Die Annahme einer Abstufung der Kategorialität o.ä. ist nicht unbedingt neu; während sie aber in der traditionellen Intonationsforschung aup Intuitionen beruht, versuchen wir, eine empirisch-experimentelle Basis zu schapfen. 


\subsubsection{Die phonetische Ebene}

Wenn man annimmt, daß Phoneme kategorial wahrgenommen werden, so läßt sich daraus nicht einfach folgern, daß die Wahrnehmung der Intonation ebenso kategorial sein muß. Die Wahrnehmung der Intonation kann kontinuierlicher sein oder zumindest mehr Zwischenstufen aufweisen als die von Segmenten: Bei den Plosivkontinua, die im Rahmen des Paradigmas der Kategorialen Wahrnehmung getestet wurden, gibt es zwischen den (Eck-)Stimuli mit einem klaren artikulatorischen Korrelat Zwischenstufen, die von natürlichen Sprechern so nicht produzierbar sind. Bei Vokalkontinua sind dagegen die Zwischenstufen produzierbar; das ist mit ein Grund dafür, daß die Wahrnehmung von Vokalen weniger kategorial ist als die von Plosiven. Solche Zwischenstufen ergeben aber distinktiv keinen Sinn, da sie immer nur einer der bedeutungsunterscheidenden Kategorien zugeschlagen werden können. Bei der Intonation liegt der Fall weniger eindeutig: Natürlich kann, wenn überhaupt, nur eine endliche und kleine Menge von Distinktionen indiziert werden, es ist aber noch nicht trivial gegeben, wieviele es wirklich sind.

\subsubsection{Die linguistische Ebene}

Der kommunikative Sinn, der sich aus der Außerung eines Fragesatzes in einer bestimmten Situation ergibt, hängt teilweise davon ab, wo die Außerung auf bestimmten fragetypischen 'pragmatischen Bedeutungsdimensionen' lokalisiert ist, z.B. auf einer Skala zunehmender Antwortdetermination oder auf einer Skala des angezeigten Interesses an einer bestimmten Antwort. Je nach der Position einer Fragesatzäußerung aup einer dieser Skalen kann die Frage als mehr oder weniger typisch, als mehr oder weniger 'fragehaltig' aufgefaßt werden. Unsere generelle These ist, daß die intonatorische Markierung des betreffenden Fragesatzes eines der Merkmale sein kann, das diese 'Fragehaltigkeit' bestimmt ein anderes Merkmal ist die Verwendung bestimmter Modalpartikeln, die für sich auch schon zeigen, daß die genannten Funktionen grammatikalisiert sein können. Voraussetzung für die intonatorische Markierung derartiger 'pragmatischer' Bedeutungen ist natürlich, da $\beta$ der betreffende intonatorische Parameter frei variieren kann und nicht bereits als festes Merkmal für die grammatische Satzmoduskennzeichnung benützt wird. Muß z.B. ein Fragesatz durch einen hohen Fooffset gekennzeichnet werden, so kann die offset-Höhe (in diesem Fall als binäres Merkmal - hoch versus tief - definiert) keine zusätzlichen pragmatischen Bedeutungen mehr vermitteln. 
Fragetypisch sind mindestens die zwei schon kurz angesprochenen und nun etwas näher $\mathrm{zu}$ charakterisierenden pragmatischen Bedeutungsdimensionen:

(1) Die erste dieser Dimensionen betrifft die Antworterwartung des Sprechers. Am einen Ende stehen hier völlig 'offene' Fragen ohne jede vom Sprecher angezeigte Antworterwartung, am anderen Ende rhetorische Fragen mit feststehender Antwort, dazwischen Fragen mit angezeigter Antworterwartung, bei denen der Sprecher aber im Prinzip auch noch mit einer nicht erwartungskonformen Antwort rechnet. So wird durch die Verwendung der unbetonten Modalpartikel nicht in Entscheidungsfragesätzen angezeigt, daß eine bejahende Antwort erwartet wird: Sind Sie nicht Herr Chomsky? Bekanntlich beeinflußt auch die Verwendung der Antwortpartikel doch die Art der Antworterwartung: Mit doch und nicht mit ja reagiert man 'negativ' auf eine Frage, auf die ein nein als Antwort erwartet wird, angezeigt durch einen betonten Negationsausdruck: A: Haben Sie KEIne Angst? B: "Ja./Doch.

Intonatorisch kann die Offsethöhe einen Hinweis auf die 'Offenheit' der Frage geben; je 'offener' die Frage, desto häufiger wird - ceteris paribus - ein hoher offset realisiert. Ist die offsethöhe bereits grammatisch festgelegt, so werden andere intonatorische Merkmale, z.B. ein erhöhter Fo-Range, für die Kennzeichnung des Grades der Antworterwartung genutzt. Als Effekt ergibt sich dann, daß die entsprechende Fragesatzäußerung einen umso 'assertiveren' und weniger fragetypischen Sinn erhält, je mehr die möglichen Antworten eingeschränkt werden.

(2) Teilweise fällt diese Dimension zusammen mit einer zweiten, die man 'angezeigtes Interesse an einer Antwort' nennen kann. Bei rhetorischen Fragen ist das Interesse des Sprechers an einer Antwort gleich Null. Am anderen Ende der Skala stehen Außerungen, mit denen der Sprecher ein großes Interesse an einer Antwort $\mathrm{zu}$ verstehen gibt. Das kann z.B. durch Verwendung der Modalpartikel eigentlich angezeigt werden: Kennst du eigentlich Herrn Chomsky? (Wenn schon durch die Situation klar ist, da $\beta$ der Angesprochene zu einer Antwort verpflichtet ist, kann die daraus folgende Antwortobligation als besonderes Antwortinteresse kaschiert werden.) Unsere These ist, daß auch hler vor allem der hohe offset ein besonderes Interesse an einer Antwort anzeigen kann. Bel festgelegter Offsethöhe wird vermutlich ein größerer Range realisiert. Eine Fragesatzäußerung ist umso typischer, je mehr der Sprecher an einer Antwort interessiert ist. Damit wird impliziert, da $\beta$ (Proto-) Typlzität funktional eingesetzt werden kann. 
Das im wesentlichen innerhalb der (strukuralistischen) Phonologie entwickelte Konzept des Merkmals beruht aup der Unterscheidung von distinktiven (d.h. bedeutungsunterscheidenden) und redundanten Merkmalen. Normalerweise wird dabei das Merkmal als binär aufgefaßt, d.h. es gibt nur zwei Ausprägungen, wie etwa [+ stimmhaft] oder [- stimmhaft] bei der Unterscheidung von [b] und [p]. Die VOT (voice onset time, das Einsetzen des Stimmtons relativ zur Verschlußlösung bei Plosiven) ist dabei als akustisch/phonetischer Parameter kontinuierlich. ${ }^{12}$ An diesem Parameter wird ein Grenzwert festgestellt, der die Kategorien - in unserem Fall stimmhafte und stimmlose Plosive - trennt. Im Paradigma der Kategorialen Wahrnehmung werden dafür ITs und DTs eingesetzt (vgl. oben Teil 3.1 und 3.4). Daß es klar trennbare Kategorien gibt, wurde nicht nur in der Phonetik und Phonologie sehr lange implizit als Faktum angenommen, vgl. Lakopp (1987:6):

From the time of Aristotle to the later work of Wittgenstein, categories were thought be [sic] well understood and unproblematic. They were assumed to be abstract containers, with things either inside or outside the category. Things were assumed to be in the same category if and only if they had certain properties in common. And the properties they had in common were taken as defining the category.

Die 'certain properties' sind bei den Plosiven also die binären Zustände des distinktiven Merkmals der Stimmhaftigkeit.

Wir können an dieser Stelle nicht allgemein aup die Kritik am traditionellen Konzept der Kategorialität eingehen; dafür sei auf Harnad (1987) und Lakopf (1987) sowie die dort zitierte Literatur verwiesen. Wenn wir uns gleich der Kategorie 'Frage' (vs. 'Nicht-Frage') zuwenden, so macht allein schon die Tatsache, da $\beta$ Fragen hohen oder tiefen Fo-offset aufweisen können, daß aber dieses Merkmal (das man natürlich auch 'Ausmaß des finalen RISE' nennen kann, vgl. oben 1.2) der einzige Kandidat Pür ein distinktives Merkmal wäre, die traditionelle Bestimmung der Kategorien, wie sie in dem Lakopp-Zitat skizziert ist, hinpällig. ${ }^{13}$ Es ist also adäquater, von der Höhe des Fo-oppsets bzw. dem Ausmaß

12 In diesem Zusammenhang sehen wir von der Frage ab, inwiefern akustisch kontinuierlich veränderbare Parameter einhergehen mit kontinuierlich veränderbaren artikulatorischen Merkmalen; so sind z.B. Vokalkontinua um einiges leichter zu produzieren als Konsonantenkontinua. - Grundsätzlich beschränken wir uns in der Diskussion aup die akustischen Merkmale und ihre perzeptiven Korrelate; aup die Frage, inwiefern eine direkte Abbildung der artikulatorischen Prozesse bzw. der Produktionsprozesse i.a. aup die Perzeption adäquater ist, können wir hier nicht weiter eingehen.

13 Man könnte nun einwenden, daß die grammatische Kategorie 'Frage' - wobei in diesem Zusammenhang irrelevant ist, ob es sich um eine Form- oder Funktionskategorie handelt - etwas prinzipiell anderes sei als die phoneti- 
des finalen RISE als (sehr relevantem) Hauptmerkmal zu sprechen, wobei andere (Neben-) Merkmale wie die Höhe des initialen FALL bei den FR-Stimuli auch zur Bestimmung der Kategorie beitragen können; die Merkmale stehen zueinander in einer trading relation, d.h. daß die geringere Ausprägung des einen Merkmals durch eine größere Ausprägung eines anderen Merkmals kompensiert werden kann; vgl. Repp (1981).

Bisher haben wir so getan, als ob eine direkte Abbildung der akustischen Merkmale auf die Kategorien möglich wäre. Dazwischen liegen aber perzeptive und kognitive Prozesse. Die Dimensionen, die sich bei den NMDS-Lösungen ergaben, sind fürs erste theoretische Konstrukte der Urteilsfindung anhand solcher perzeptiver und kognitiver Prozesse. Wenn wir ihnen nun ein 'Eigenleben' zugestehen, so sind für den relativ einfachen Fall unserer Teststimuli mit ihren klar definierbaren äquidistanten akustischen Unterschieden die Dimensionen das perzeptive und kognitive Korrelat der akustischen Struktur. Aus dem Repertoire der Dimensionen kommt das Inventar der modusindizierenden intonatorischen Merkmale. Die Abbildung der akustischen Merkmale auf die Dimensionen muß weder stetig noch linear in dem Sinn sein, daß eine Veränderung entlang des akustischen Kontinuums von einem Eckwert zum anderen immer mit einer Veränderung entlang einer Dimension korrespondiert; vgl. die Unstetigkeit der Bewertungsfunktionen an der Kategoriengrenze im AT und in den KTs und DIMl in Fig.14 sowie die nicht-lineare Abbildung der Merkmale aup DIM3 und DIM4 in Fig.15. Eine lineare Abbildung wie beim finalen RISE (DIM1) mag zwar der Regelfall sein; dafür spricht, daß DIM3 bzw. DIM4 als Prädiktoren verhältnismäßig wenig zur 'erklärten Varianz' bei IT und den KTs beitragen, vgl. Tab.7. Es mag aber auch daran liegen, da $\beta$ man immer nur nach solchen linearen Abbildungen gesucht hat: Wenn man wie bei den meisten Perzeptionsexperimenten jeweils nur ein Merkmal variiert, wird man fast immer eine lineare Abbildung erhalten. Erst bei gleichzeitiger Variation mehrerer Merkmale können nicht-lineare Abbildungen 'entdeckt' werden.

\subsection{Kategorialität der Wahrnehmung}

Die Experimente waren nicht im Hinblick daraup konzipiert, entscheidende Argumente (für oder) gegen eine kategoriale Wahrnehmung zu finden. Ein solcher Nachweis (im Sinne eines experimentum crucis) ist u.E. nur sehr schwer - wenn

sche Kategorie 'Stimmhaptigkeit'. Es mehren sich aber die Anzeichen, daß dem eben nicht so ist, vgl. z.B. die einzeinen Beiträge in Harnad (1987) sowie Batliner (1989). 
überhaupt - zu führen. Um es so $z u$ formulieren: Wenn man nach Kategorialität sucht und das experimentelle Design dementsprechend anlegt, wird man sie auch oft finden. Wenn nicht, so wird man genauso oft eine nicht-kategoriale Wahrnehmung entdecken.

Unsere Uberzeugung läßt sich wie folgt skizzieren: Natürlich muß der Mensch die erhaltene Information möglichst schnell auf ihren kategorialen Gehalt reduzieren, um nicht im ubermaß an Information $\mathrm{zu}$ ertrinken. (vgl. Hörmann 1976:460ff oder Bühlers (1934:42ff) Prinzip der 'abstraktiven Relevanz'). Diese Reduzierung kann sehr schnell vor sich gehen, wie im segmentalen Bereich (ist es ein [b] oder ein [p]?), oder langsamer, wie im suprasegmentalen Bereich (indiziert die Intonation eine Frage oder nicht?). Ebenso notwendig ist aber auch ein gewisses Nachhallen der kontinuierlichen (akustischen) Information - schon um bei eventuellen Interpretationsschwierigkeiten darauf zurückgreifen zu können. ${ }^{14}$ Kategorialität ist eher dann gegeben, wenn die phonetische Information 'einfach' und möglichst auch psychophysisch kodierbar ist (im Sinne eines 'entweder-oder', wie z.B. bei der VOT und bei der Transition des zweiten Formanten, die entweder stelgend oder fallend sein kann). Sie ist dann eher nicht gegeben, wenn die phonetische Information komplex und psychophysisch nicht kodierbar ist, und wenn es sinnvoll ist, die inhaltliche Information nicht rein kategorial zu gestalten. So ist es wohl möglich, aber nur selten sinnvoll, einen Plosiv zu produzieren, der zu zwei Drittel [b] und zu einem Drittel [p] ist; es kann aber sehr sinnvoll sein, dem Hörer eine Abstufung der Fragehaltigkeit zu signalisieren.

Vor diesem Hintergrund lassen sich die Ergebnisse in Bezug auf die Kategorialität interpretieren: Bei einer direkten Befragung und forced-choiceAufgabe wie beim IT zeigte sich eine Kategoriengrenze im Bereich einer psychophysischen Grenze (RISE versus FALL). Bei einer direkten (AT) oder indirekten (KTs) Befragung ohne forced-choice-Aufgabe zeigten sich Abstufungen innerhalb der Kategorien. Diese Abstufungen waren zweifach bedingt: Zum einen durch unterschiedliche Ausprägungen der intonatorischen Parameter (RISE und in geringerem Ausmaß FALL) innerhalb des Testsatzes, zum anderen durch die unterschiedlichen Ausprägungen der intonatorischen Parameter von Kontextsatz und Testsatz. Wir nehmen an, daß die Abstufungen zeichenhaft eingesetzt werden. Damit sind sie ein genuines Beschreibungsobjekt sowohl der Linguistik als auch der Phonetik.

14 Die Begriffe 'Reduzierung' bzw. 'Nachhallen der Information' sind hier informell verwendet; wir möchten uns jeder konkreten Aussage über die genauen Prozesse im Kurz- bzw. Langzeitgedächtnis enthalten. Es kann sich auch um verschiedene Arten des Zugriffs oder der Aufmerksamkeitsteuerung handeln. 


\section{LITERATUR}

Altmann, H. (1987): Zur Problematik der Konstitution von Satzmodi als Formtypen. In: Meibauer, J. (Hg.) (1987): Satzmodus zwischen Grammatik und Pragmatik. Tübingen. S.22-56.

Altmann, H. (Hg.) (1988): Intonationsforschungen. Tübingen.

Batliner, A. (1988a): Produktion und Prädiktion. Die Rolle intonatorischer und anderer Merkmale bei der Bestimmung des Satzmodus. In: Altmann, H. (Hg.) (1988). S.207-221.

Batliner, A. (1988b): Modus und Fokus als Dimensionen einer Nonmetrischen Multidimensionalen Skalierung. In: Altmann, H. (Hg.) (1988). S.223-241.

Batliner, A. (1988c): Der Exklamativ: Mehr als Aussage oder doch nur mehr oder weniger Aussage? Experimente zur Rolle von Höhe und Position des FoGipfels. In: Altmann, H. (Hg.) (1988). S.243-271.

Batliner, A. (1989): Eine Frage ist eine Frage ist keine Frage. Perzeptionsexperimente zum Fragemodus im Deutschen. (In diesem Band)

Batliner, A./Oppenrieder, W. (1988): Rising intonation: Not passed away but still alive. A reply to R. Geluykens. In: Journal of Pragmatics 12. S.227-233.

Batliner, A./Oppenrieder, W. (1989): Korpora und Auswertung. (In diesem Band)

Batliner, A./Nöth, E./Lang, R./Stallwitz, G. (1989): Zur Klassifikation von Fragen und Nicht-Fragen anhand intonatorischer Merkmale. (erscheint in: Tagungsband der 15. Gemeinschaftstagung der Deutschen Arbeitsgemeinschaft für Akustik (DAGA). 13.-16. März 1989)

Batliner, A./Schiefer, L. (1987): Stimulus category, reaction time, and order effect - An experiment on pitch discrimination. In: Proceedings XIth ICPhS, Vol. 5. S.46-49.

Borg, I (1981): Anwendungsorientierte Multidimensionale Skalierung. Berlin etc.

Bühler, K. (1934): Sprachtheorie. Stuttgart.

Gandour, J.T. (1978): Perceived Dimensions of 13 Tones: A Multidimensional Scaling Investigation. In: Phonetica 35. S.169-179.

Gandour, J.T./Harshman, R.A. (1978): Crosslanguage Differences in Tone Perception: A Multidimensional Scaling Investigation. In: Language and Speech 21. S.1-33.

Guttman, L. (1977): What is not What in Statistics. In: The Statistician 26. S.81-107.

Harnad, S. (Hg.) (1987): Categorical perception. The groundwork of cognition. Cambridge etc.

Henkel, R.E. (1976): Tests of Significance. Sage University Paper series on Quantitative Applications in the Social Sciences, 07-004. Beverly Hills and London. 
Hörmann, H. (1976): Meinen und Verstehen. Frankfurt a.M.

Klein, W. (1982): Einige Bemerkungen zur Frageintonation. In: Deutsche Sprache. S. $289-310$.

Kruskal, J.B./Wish, M. (1978): Multidimensional Scaling. Sage University Paper series on Quantitative Applications in the Social Sciences, 07-011. Beverly Hills and London.

Lakoff, G. (1987): Women, Fire, and Dangerous Things. What Categories Reveal about the Mind. Chicago und London.

Lindsay, D./Ainsworth, W.A. (1985): Two models of nuclear intonation. In: Journal of Phonetics 13. S.163-173.

Medin, D.L./Barsalou, L.W. (1987): Categorization processes and categorical perception. In: Harnad, S. (Hg.) (1987). S.455-490.

Oppenrieder, W. (1988a): Intonation und Identifikation. Kategorisierungstests zur kontextfreien Identifikation von Satzmodi. In: Altmann, H. (Hg.) (1988). S. 153-167.

Oppenrieder, W. (1988b): Intonatorische Kennzeichnung von Satzmodi. In: Altmann, H. (Hg.) (1988). S.169-205.

Repp, B.H. (1981): Phonetic trading relations and context effects: New experimental evidence for a speech mode of perception. In: Haskins Laboratories: Status Report on Speech Research SR-67/68. S.1-40.

Repp, B.H. (1984): Categorical perception: Issues, methods, findings. In: Lass, N.J. (Hg.): Speech and Language. Orlando etc. S.243-335.

Repp, B.H. (1986): The role of psychophysics in understanding speech perception. In: Schouten, M.E.H. (Hg.): The Psychophysics of Speech Perception. Dordrecht et al. S.3-27.

Rosch, E./Mervis, C.B. (1975): Family Resemblances: Studies in the Internal Structure of Categories. In: Cognitive Psychology 7. S.573-605.

Rossi, M. (1978): Interactions of Intensity Glides and Frequency Glissandos. In: Language and Speech 21. S.384-396.

Schiefer, L./Batliner, A. (1988): Intonation, Ordungseffekt und das Paradigma der Kategorialen Wahrnehmung. In: Altmann, H. (Hg.) (1988). S.273-291.

Shepard, R.N. (1974): Representation of Structure in Similarity Data: Problems and Prospects. In: Psychometrica 39. S.373-421.

Studdert-Kennedy, M./Hadding, K. (1973): Auditory and linguistic processes in the perception of intonation contours. In: Language and Speech 16. S.293313.

Thorsen, N.G. (1987): Suprasegmental Transcription. In: Almeida, A./Braun, A. (Hgg.): Probleme der phonetischen Transkription. Stuttgart. S.79-109. 
SELBSTANDIGE VERB-LETZT-SATZE: IHR PLATZ IM SATZMODUSSYSTEM UND IHRE INTONATORISCHE KENNZEICHNUNG'

Wilhelm Oppenrieder

0. Uberblick

Der Beitrag ist in zwei Teile gegliedert. Im ersten Teil wird die Rolle der Verb-Letzt-Sätze in einem Satzmodussystem untersucht. Zunächst wird der Frage nachgegangen, welche der nicht-eingebetteten Verb-Letzt-Sätze überhaupt als selbständig und damit als Formtypen des Satzmodussystems zu werten sind. Zweltens wird gezeigt, daß und warum adverbielle Verb-Letzt-Sätze keine Kandidaten für derartige Formtypen sind. Schließlich werden die formalen und funktionalen Eigenschaften der Verb-Letzt-Strukturen besprochen, die jeweils dem Frage-, dem Imperativ-, dem Aussage-, dem Exklamativ- und dem Wunsch-Modus zugeordnet werden können. Im zweiten Teil wird die intonatorische Form von Verb-Letzt-Sätzen untersucht. Materialgrundlage ist ein großes Korpus von Testsatzrealisationen, bei denen in der Mehrzahl allein die intonatorischen Eigenschaften die Satzmodusabgrenzung garantieren. Basierend auf diesen Realisationen werden sogenannte intonatorische Prototypen gebildet.

1. Verb-Letzt-Sätze im Satzmodussystem

Eine altbekannte Faustregel der deutschen Grammatik lautet, daß Sätze mit Erst- oder Zweitstellung des finiten Verbs 'Hauptsätze' seien und deswegen selbständig vorkommen können, während Sätze mit Endstellung des finiten Verbs 'Nebensätze' seien und deswegen nur eingebettet und nicht-selbständig vorkommen können.

1 Dank für Rat und Tat gebührt neben den Mitgliedern des Intonationsprojekts vor allem Maria Thurmair. 
Diese 'Regel' erfaßt jedoch höchstens die typischen Fälle. Denn auf der einen Seite gibt es abhängige Verb-Erst- und Verb-Zweit-Strukturen, ${ }^{2}$ während man auf der anderen Seite sehr wohl nicht-eingebettete und selbständige VerbLetzt-Strukturen findet. ${ }^{3}$

Für den ersten Fall sind die Standardbeisplele uneingeleitete Verb-ErstAntezedenssätze in Konditionalgefügen und Verb-Zweit-Sätze, die in Matrixsätze mit einem Verbum dicendi oder sentiendi eingebettet sind.

(1) Läßt du diesen los, so bist du des Kaisers Freund nicht.

(2) Johanna sagt, sie kehre niemals wieder.

Daneben gibt es weitere spezifische Typen von Matrixsätzen, die die eingebettete Verwendung von Verb-Zweit-Sătzen ermøglichen:

(3) Ich wollte/wünschte, es wäre Nacht.

(4) Es ist besser, du gehst jetzt.

Mit dem zweiten Fall, den nicht-eingebetteten Verb-Letzt-Sätzen, beschäPtigt sich dieser Aufsatz. Ihr Platz in einem Satzmodussystem im Sinn von Altmann (1984, 1987) soll bestimmt werden; d.h. es wird untersucht, welche von ihnen wie die typischen Verb-Erst- und Verb-Zweit-Sätze als illokutiv selbständig verstanden werden können und wie sie sich aufgrund ihrer formalen und funktionalen Eigenschaften in das Satzmodussystem einfügen.

\subsection{Abgrenzungskriterien}

\subsubsection{Uberblick, Terminologie}

Zur Klärung der Terminologie möge zunăchst unterschieden werden zwischen eingebetteten und nicht-eingebetteten Verb-Letzt-Sätzen, von denen die ersten den zentralen Fall der syntaktischen Verwendung von Verb-Letzt-Sätzen darstellen und Anlaß zu der Formulierung der eingangs erwähnten 'Regel' geben.

Als eingebettet $z u$ werten sind valenznotwendige Verb-Letzt-Sätze (Subjektund Objektsätze), aber auch 'frele' Adverbialsätze, sofern sie intonatorisch klar in den von ihnen modifizierten Satz integriert sind. Aber auch 'herausgestellte' (vgl. die verschiedenen in Altmann 1981 behandelten Konstruktionstypen) und

2 So werden in der Akademie-Grammatlk als Hauptsätze Verb-Erst- und VerbZweit-Sätze aufgeführt, aber eben nur solche Sătze, die "selbst nicht eingebettet sind" (Heidolph et al. 1981:786). Die Duden-Grammatik (1984:666) führt unter den Nebensätzen auch die eingebettet gebrauchten Verb-Erstund Verb-Zweit-Sätze auf.

3 In der Akademle-Grammatik (Heidolph et al. 1981:787) werden derartig verwendete Verb-Letzt-Sătze etwas mißverständlich als isolierte Nebensätze bezeichnet. 
damit intonatorisch nicht oder nicht vollständig integrierte Verb-Letzt-Sätze können in einem erweiterten Sinn als eingebettet gewertet werden, insofern sie auch formal von der Anwesenheit eines 'Trägersatzes' abhängig sind. Prinzipiell lassen sich derartig verwendete Verb-Letzt-Sätze auch enger integrieren: Sie können z.B. rechtsversetzt (vgl. (5)), als Nachtrag (vgl. (6)) oder aber als eine Art Freies Thema (vgl. (7)) verwendet werden.4

(5) Ich finde das empörend - wie Otto mit seinem Fagott umgeht.

(6) Ja, du bekommst das Eis - obwohl du es eigentlich nicht verdient hast.

(7) Wenn Sie Sorgen mit Ihrem Kanarienvogel haben - wir helfen Ihnen gern.

Die Mehrzahl der parenthetisch eingeschobenen Ausdrücke kann ebenfalls mit diesem erweiterten Begriff der Einbettung erfaßt werden.

Diesen eingebetteten Verb-Letzt-Sätzen stehen verschiedene Typen von nicht-eingebetteten Verb-Letzt-Sätzen gegenüber. Um mögliche Abgrenzungsprobleme zu Herausstellungsstrukturen $\mathrm{zu}$ vermeiden, werde ich Beispiele mit Sprecherwechsel verwenden.

Vier Typen von nicht-eingebetteten Verb-Letzt-Sätzen lassen sich unterscheiden:

Diejenigen, für die sich ein lexikalisch spezifizierter Matrixsatz aufgrund des sprachlichen Kontextes rekonstruieren läßt; dieser Typ I umfaßt die nicht-eingebetteten elliptischen Verb-Letzt-Sătze.

Diejenigen, für die sich eine Familie von 'Matrixsätzen' rekonstruieren läßt, die bestimmte Aspekte des mit der situationsspezifischen Verwendung verbundenen kommunikativen Sinns (zu diesem Begriff vgl. Bierwisch 1980) versprachlichen; beim Typ II lassen sich 'nicht-illokutive' Matrixsätze bilden, beim Typ III dagegen nur 'illokutive'.

Beim Typ IV schließlich ist jede Einbettung ausgeschlossen. Die einzelnen Typen werde ich im folgenden genauer erläutern.

Bei einer spezifischen Form von Freiem Thema ist der Verb-Letzt-Satz allerdings nicht ohne weiteres in den folgenden Satz einbettbar, nämlich dann nicht, wenn im Freien Thema eine Art Rechtfertigung für den folgenden Sprechakt gegeben wird:

(i) Weil du gerade von Rennrädern sprichst - macht Maria jetzt tatsächlich die große Pässetour?

(ii) Falls du die Salatschüssel suchst - ich habe sie gerade weggeräumt. Im ersten Fall wäre also dem Sinn nach zu ergänzen 'frage ich dich', im zweiten Fall 'sage ich dir'; eine Einbettung in den folgenden Satz ist dagegen nicht möglich. 


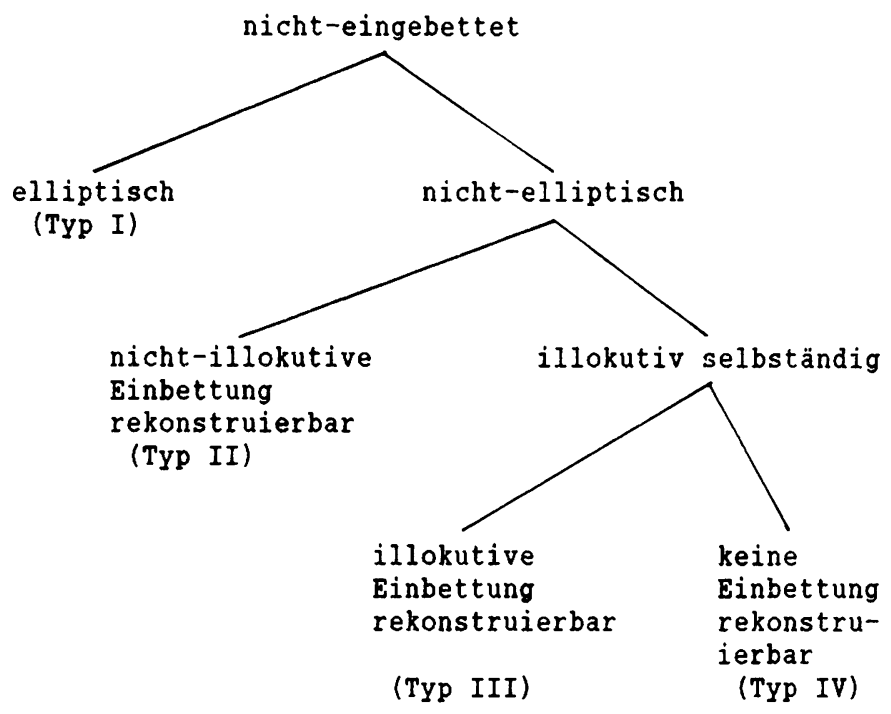

1.1.2 Typen nicht-eingebetteter Verb-Letzt-Sätze

\subsubsection{Nicht einbettbare Verb-Letzt-Sätze (Typ IV)}

Beim Typ IV ist die Einbettung prinzipiell ausgeschlossen. Derartige VerbLetzt-Sätze sind daher vom illokutiv-funktionalen standpunkt aus nicht anders einzuordnen als die selbständigen Verb-Erst- und Verb-Zweit-Sätze.

Eindeutig nicht einbettbar sind die assertiv $\mathrm{zu}$ interpretierenden $u n d o b / w^{-}$ Ausdrücke:

(8) A: Verstehen Sie überhaupt etwas von Linguistik?

$B$ : Und ob ich was davon verstehe!

Das Hauptproblem ist nicht, wie Weuster (1983:39) meint, daß und bei einer Einbettung als koordinativer Verknüpfer für den Gesamtsatz zu werten ist, sondern daß einerseits keine passende assertive Einbettung durch ich sage dir etc. möglich ist, andererseits bei einer syntaktisch passenden Einbettung durch Ausdrücke wie ich frage mich (wie bei Weuster 1983:39) gerade durch die Betonung des Satzeinleiters $o b / w$ - der assertive Sinn völlig verlorengeht.

Auch bei anderen Verb-Letzt-Strukturen liegt die fehlende Einbettbarkeit häufig an der intonatorischen Form, sowie an der Anwesenheit von Modalparti- 
keln (zu Modalpartikeln in selbständigen Verb-Letzt-Sätzen vgl. Thurmair 1989:49f.); die Blockade ist allerdings nicht in allen Fällen ganz eindeutig. So verhindert die Modalpartikelverwendung nicht in allen Fällen die Einbettung und ist damit kein eindeutiges Kriterium für die Unterscheidung von Verb-LetztSätzen des Typs IV von den restlichen Typen. Modalpartikeln lassen sich nämlich im Prinzip auch in eingebetteten Sätzen verwenden (vgl. dazu wiederum Thurmair 1989:73ff., insbesondere die Tabelle auf S. 81). Dies gilt zum einen für die indirekte Rede, zum anderen auch für die meisten Typen von Adverbialsätzen (Lokal- und Temporalsätze sind die deutlichste Ausnahme), sowie für nichtrestriktive Gliedteilsätze. Trotz formaler Einbettung sind diese verschiedenen Verb-Letzt-Sätze illokutiv dem einbettenden Satz nicht eindeutig untergeordnet, sondern können zu 'Zusatzsprechakten' verwendet werden - im allgemeinen als zusätzliche Assertionen. Die Modalpartikeln in selbständigen Verb-Letzt-Sätzen brauchen also nicht unbedingt von vornherein die Einbettbarkeit $z u$ blockieren.

Allerdings ist die Modalpartikelverwendung bei eingebetteten Verb-LetztSätzen markiert, während dies bei den meisten der selbständigen Verb-LetztSätze nicht gilt, typische Modalpartikeln zum Teil sogar obligatorisch sind.

Ein in diesem Sinn nicht ganz unstrittiges Beispiel sind die deliberativen ob/w-Verb-Letzt-Fragen mit wohl als Modalpartikel.

(9) Ob so viel Wein wohl gesund ist?

(10) Gegen wen die Bayern wohl das erste Mal verlieren?

Ein möglicher einbettender Ausdruck ist z.B. ich frage mich, der das Element der Selbstfrage deutlich macht.' $\mathrm{Zu}$ beachten ist aber, daß das Vorkommen der Modalpartikel wohl in einem tatsächlich eingebetteten ob/w-Fragesatz markiert ist, während es bei selbständigen deliberativen Verb-Letzt-Fragesätzen nahezu obligatorisch ist. Darüberhinaus paßt der hier übliche steigende Tonverlaup nicht zu dem möglichen 'Matrixsatz' ich frage mich, da sich die durch ihn angezeigte Frageeinstellung eben nicht auf den durch Einbettung entstehenden komplexen Satz bezieht, der vielmehr ein Aussagesatz sein müßte.

Ein weiteres Beispiel für die Blockierung der Einbettung durch Modalpartikeln sind die wenn-Wunschsätze, bei denen die Einfügung einer Modalpartikel nahezu obligatorisch ist: 6

Wenn du mir ??_/doch/nur glauben würdest!

vs.

5 Für Meibauer (1988:66) und Weuster (1983:38) ist der resultierende komplexe Satz trotz Modalpartikel akzeptabel, Pür Altmann (1987:27) dagegen nicht.

- Akzeptabilitätsabstufungen reichen von '?' (='nicht voll akzeptabel') über '??' (='kaum mehr akzeptabel') bis zu '.' (='inakzeptabel'). 
(12) Ich würde mich freuen/wäre zufrieden, wenn du mir_/"doch/*nur glauben würdest.

Aup die typischen Modalpartikeln wird bei der Behandlung der einzelnen Typen von selbständigen Verb-Letzt-Sätzen genauer eingegangen.

Wie an den deliberativen Fragesätzen besonders deutlich wurde, kann die intonatorische Gestalt eines Verb-Letzt-Satzes eine (sinnerhaltende) Einbettung unmöglich machen. Eine Einbettung ist aber auch dann kaum möglich, wenn der Verb-Letzt-Satz wie in den folgenden Beispielen einen besonders emphatischen Akzent enthält; aufgrund der spezifischen Ausprägung und der Tatsache, da $\beta$ der Akzent auf einen thematischen Ausdruck fällt, sollte man ihn zu den Exklamativakzenten zahlen (zum Exklamativakzent vgl. Batliner 1988; Oppenrieder 1988b:192ff.): ${ }^{7}$

(13) Daß ich DAS noch erleben durfte!

(14) Wenn ich DEN schon sehe!

(15) Als ob DAS noch eine Rolle spielen würde!

Modalpartikeln und intonatorische Form können also in zahlreichen Fällen eine Einbettung von vornherein blockieren (vgl. dagegen Weuster 1983:41, die außer bei und ob-Sätzen von der prinzipiellen Einbettbarkeit der Verb-LetztSätze ausgeht).

\subsubsection{Elliptische Verb-Letzt-Sätze (Typ I)}

Als nächstes soll der im Prinzip am leichtesten abzugrenzende Typ I besprochen werden, der sich dadurch auszeichnet, da $\beta$ nicht aufgrund der spezifischen Verwendung eine ganze Familie von möglichen Einbettungen für den nicht-elngebetteten Verb-Letzt-Satz rekonstruierbar ist, sondern aufgrund des sprachlichen Kontextes ein lexikalisch genau spezifizierter Matrixsatz. Nur in diesem Fall einer spezifischen Ergänzbarkeit, einer wörtlichen Rekonstruktion, ist es auch sinnvoll, von elliptischen Verb-Letzt-Sätzen zu sprechen.

Elliptisch sind danach zunächst jene Verb-Letzt-Sätze, die mittels einer koordinativen Konjunktion an einen komplexen Vorgängersatz mit eingebettetem Verb-Letzt-Satz angeschlossen werden. Der Satzmodus des Vorgängersatzes ist beliebig. Vgl. die folgenden Beispiele mit Sprecherwechsel (von denen sich zumindest (17), (18) und (19) an eine weitere Person oder Personengruppe C richten):

7 Hervorhebung durch Betonung werde im folgenden durch Großschreibung repräsentiert. 
(16) A: Er trinkt Jägermeister, weil schon seine Vorfahren Jägermeister getrunken haben.

B: Und weil er den Geschmack von Freiheit und Abenteuer liebt.

(17) A: Wer weiß, wie man ein Zahlenschloß knackt?

$B:$ Oder wann der nächste Wachmann kommt?

(18) A: Versuch nicht, uns übers Ohr zu haven!

$B$ : Oder gar die Polizei zu verständigen!

(19) A: Wie schade es doch ist, Tante Cilly, daß du schon gehen mußt!

$B$ : Und $d a \beta$ du uns frühestens nächstes Jahr wieder besuchen kannst!

(20) A: Hätten wir nur rechtzeitig kapiert, was die Sirenengeräusche zu bedeuten hatten!

B: Oder wieso plötzlich überall rote Lampen zu blinken anfingen!

Es besteht kein Grund hier von illokutiv selbständigen Verb-Letzt-Sätzen auszugehen. Es handelt sich einfach um Fälle von Koordinationsreduktion, bei der auch nicht-satzförmige Ausdrücke zurückbleiben können.

Eine diesen Koordinationsfällen ähnliche Konstruktion ist eine, bei der auch eine Vorgängeräußerung (zumeist ein Satz) 'erweitert' wird. Nur enthält dieser nicht einen funktionsgleichen Verb-Letzt-Satz, an den koordinativ angeschlossen werden könnte (wieder ist zumindest bei (22), (23) und (24) eine dritte Person C angesprochen):

(21) A: Zümpel wurde einer der bekanntesten Briefmarkenhändler in SachsenAnhalt.

$B$ : Obwohl er ursprünglich nur Hufschmied werden wollte.

(22) A: Hat es sehr weh getan?

B: Als man dir die Weisheitszähne zog.

(23) A: Mach bloß keine Sperenzien!

$B$ : Weil es sonst sehr ungemütlich wird.

(24) A: Wäre das aber schade!

B: Wenn du jetzt schon gehen müßtest.

(25) A: Was für einen gescheiten Eindruck Karl macht!

B: Solange er den Mund hält.

(26) A: Wäre ich doch nur ausgestiegen!

B: Solange Inspektor Clouseau noch nicht auf uns angesetzt war.

Die Beispiele gleichen den oben erwähnten Herausstellungsstrukturen, nur daß hier ein Sprecherwechsel den 'Trägersatz' und den herausgestellten Satz trennt.

Ein Sonderfall sind die sogenannten weiterführenden Relativsätze (vgl. zu diesen Holly 1988), die sowohl mit als auch ohne Sprecherwechsel auftreten können. Der relative Anschluß erfolgt entweder insgesamt an die Vorgängeräußerung(en), wenn nämlich ein sachverhaltsdenotierendes Relativpronomen vorhanden ist.

(27) A: Er ist schon immer gern gerast.

B: Was ihm schließlich den Führerschein gekostet hat./Weshalb er schließlich seinen Führerschein verloren hat. 
Oder aber das Relativum hat einen spezifischen Bezugsausdruck im Vorgängersatz.

(28) A: Otto hat sich einen funkelnagelneuen BMW gekauft.

$B$ : Den er aber leider schon wieder zu Schrott gefahren hat.

(29) A: Ich war heute den ganzen Tag im Biergarten.

$B$ : Wo du dich (ja) nicht ungern herumtreibst.

Die Umwandlung des Relativums in einen gewöhnlichen pronominalen Ausdruck - mit gleichzeitiger Veränderung der Satzstruktur zu Verb-Zweit - ist immer möglich. Andererseits gelten auch für weiterführende Relativsätze die relativumstypischen Lokalitätsbeschränkungen, die weit über die textuellen Beschränkungen für sonstige Pro-Ausdrücke hinausgehen, so daß nicht jeder pronomenhaltige Verb-Zweit-Satz beliebig in einen Relativsatz umgewandelt werden kann. Ist ein genus- und numeruskongruenter Bezugsausdruck vorhanden, dann muß er auch hier 'nah genug' im unmittelbar vorausgehenden Satz stehen; dies gilt für andere Pro-Ausdrücke nicht, vgl. die folgenden Beispiele:

(30) A: Karl hat sich wieder einen Haufen Bücher gekauft. Auch "Zettels Traum" war dabei.

B: "Der mit seinem Geld auch was besseres anfangen könnte./Er könnte mit seinem Geld auch was besseres anfangen.

VS.

(31) A: Die ganzen neuen Bücher hat übrigens Karl gekauft.

B: Der mit seinem Geld auch was besseres anfangen könnte.

Ohne Sprecherwechsel lassen sich derartige weiterführende Relativsätze wie normale appositive Relativsätze direkt im Anschluß an ihren Bezugsausdruck in den Trägersatz einschieben. Es spricht also einiges dafür, auch hier von elliptischen Ausdrücken zu sprechen. ${ }^{8}$

Aufgrund des konstitutiven Relativums kann man die entsprechenden VerbLetzt-Sätze zwar als elliptisch klassifizieren. Allerdings dürfen in weiterführenden Relativsätzen, wie auch in anderen nicht-restriktiven Relativsätzen, ohne weiteres verschiedene aussagesatztypische Modalpartikel, z.B. ja (s. (29)), verwendet werden (vgl. Thurmair 1989:81). Aufgrund der formalen Abhängigkeit von einem Vorgängertext ist es aber gerechtfertigt, die durch die Modalparti-

$8 \quad$ Neben der 'assertiven Fortsetzung' ist im Prinzip aber auch die intonatorische Kennzeichnung des Relativsatzes als Frage möglich.

(i) A: Ich habe heute nachmittag einen Mississippi-Alligator gekauft.

$B$ : Den du womöglich schon in unsere Badewanne gesetzt hast?

Bei Verwendung eines Pronomens wäre die Umwandlung in einen Verb-ErstFragesatz (oder aber in eine assertive Frage) nötig. 
kelverwendung angezeigte illokutive Selbständigkeit nicht als entscheidend für den Selbständigkeitsstatus dieser Strukturen anzusehen.

Die drei ersten elliptischen Typen (koordinative Anknüpfung, herausstellungsäquivalente Strukturen, weiterführende Relativsätze) gehören also lediglich aufgrund des (zufälligen) Sprecherwechsels zu den nicht-eingebetteten VerbLetzt-Sätzen.

Bei den folgenden Typen ist der Sprecherwechsel konstitutiv.

Erstens kann ein Verb-Letzt-Satz wie jede isolierte Phrase die durch den $w$-Ausdruck in einem Fragesatz eröffnete Leerstelle füllen, ohne da $\beta$ der Rest des vorangehenden $w$-Fragesatzes noch einmal wiederholt werden müßte. Alle Typen von Verb-Letzt-Sätzen eignen sich als derartige elliptische Antworten natürlich muß ein kategorial passender w-Ausdruck zur Verfügung stehen (bei Konzessivsätzen ist das z.B. nicht der Fall):

(32) A: Was möchtest du denn wissen?

B: Wie sich Mozart in meiner Situation verhalten hätte.

(33) A: Was hat Sie besonders beeindruckt?

$B: D a \beta$ alle hier so saubere Schuhe tragen.

(34) A: Warum hat Kain Abel erschlagen?

B: Weil Abel ihm alte jüdische Witze erzählt hat.

In den nicht-elliptischen Versionen ist der Fragesatz in einen Aussagesatz umzuformen und der $w$-Ausdruck durch den Antwortausdruck - hier den VerbLetzt-Satz - zu ersetzen. ${ }^{9}$

Ahnlich $z u$ werten wie die Antworten sind auch Korrekturen: beide Male wird die Vorgängeräußerung in einem bestimmten Aspekt spezifiziert.

(35) A: Kain hat Abel aus Neid erschlagen.

B: Nein - weil Abel alte jüdische Witze erzählt hat.

Gerade an diesen echt elliptischen Antworten läßt sich auch die unterschiedliche Modalpartikelverträglichkeit der verschiedenen Typen von nichteingebetteten Verb-Letzt-Sätzen zeigen. Die oben bei den Erläuterungen zum Typ IV als Beispiele verwendeten deliberativen Verb-Letzt-Fragesätze und wenn-Wunschsätze enthalten üblicherweise typische Modalpartikeln. In als Antworten verwendeten $o b-, w^{-}$oder wenn-Sätzen sind dieselben Modalpartikeln entweder stark markiert oder inakzeptabel:

9 Im Prinzip lassen sich die Antworten ihrerseits als elliptische Fragen formulieren:

(i) A: Warum, glaubst du, hat der Täter einen Socken zurückgelassen?

B: Weil er eine falsche Fährte legen wollte?

Der Matrixsatz ist in diesem Fall nicht als Aussagesatz, sondern als VerbErst-Entscheidungsfragesatz $z u$ rekonstruieren. Der Sprecher äußert eine Vermutung, eine Art versuchsweise Antwort, die er als Frage formuliert. 
(36) A: Was hat Otto denn wissen wollen?

$B$ : $O b$ so viel Wein (??wohl) gesund ist.

(37) A: Unter welcher Bedingung wärest du zufrieden?

B: Wenn du mir ("doch/"nur) glauben würdest.

Zweitens lassen sich offensichtlich alle Verb-Letzt-Sätze für sogenannte Fortsetzungsfragen (vgl. Luukko-Vinchenzo 1988:100ff., 180ff.) verwenden. Eine solche erweitert gewissermaßen die Vorgängeräußerung des Gesprächspartners. Der Komplex aus Verb-Letzt-Satz und kontextspezifischem Matrixsatz ist aufgrund des obligatorisch steigenden Tonverlaups als Fragesatz zu werten. ${ }^{10}$

(38) A: Ich hab's mir doch gedacht.

B: Daß Posaunen-Gustavs Alibi nicht wasserdicht ist?

(39) A: Ich Prage mich nur eines.

B: Wer die Juwelen ins Affenhaus geschmuggelt hat?

(40) A: Setzen Sie den Halunken wieder auf Preien Fuß!

$B$ : Obwohl so dringende Verdachtsmomente bestehen?

(41) A: Das ist vielleicht ein Idiot!

B: Weil er die Fingerabdrücke nicht abgenommen hat?

(42) A: Wo hatte der Kerl die Eisensäge versteckt?

B: Als wir ihn vor dem Max-Emil-Denkmal trafen?

Kennzeichnend für die elliptischen nicht-eingebetteten Verb-Letzt-Sătze ist also, daß aufgrund des sprachlichen Kontexts ein lexikalisch spezifizierter Matrixsatz rekonstruiert werden kann. Satzmodustypische propositionale Einstellungen (s. den Polgenden Abschnitt) bezlehen sich aup diesen ergänzten Komplex und nicht direkt aup die Verb-Letzt-Sätze selbst, wie dies bei den selbständigen nicht-einbettbaren Sätzen des Typs IV der Fall ist.

\subsubsection{Einbettbare Verb-Letzt-Sätze (Typ II, III)}

Die restlichen belden Typen von nlcht-eingebetteten Verb-Letzt-Sätzen, Typ II und III, unterscheiden sich von den elliptischen einerseits dadurch, daß kein lexikalisch spezifizierter Matrixsatz aufgrund des sprachlichen Kontextes rekonstruiert werden kann, von den nicht-einbettbaren andererseits dadurch, daß die Einbettung bei ihnen nicht von vornherein zu inakzeptablen Ergebnissen führt. Auch für sie scheint sich darum die Rekonstruktion eines einbettenden Matrix-

10 Die rekonstruierbaren Matrixsätze zu 'Fortsetzungsfragen' im Anschluß an Aupforderungs- und Fragesätze ähneln den Rückfragen (s.u. 1.2.3.1.2.). Mögliche Rekonstruktionen lauten also:

(i) B: Hast du dir gedacht, daß Posaunen-Gustavs Alibi nicht wasserdicht ist?

(ii) B: Ich soll den Halunken wieder auf freien Fuß setzen, obwohl so dringende Verdachtsmomente bestehen?

(iii) B: Wo der Kerl die Eisensäge versteckt hatte, als wir ihn vor dem Max-Emil-Denkmal trafen? 
satzes anzubieten. Die Tatsache, da $\beta$ ein solcher nur dem Sinn nach rekonstruiert werden kann, daß also im Prinzip eine ganze Familie von möglichen Matrixsätzen zur Verfügung steht, spricht aber zumindest dafür, sie von den echt elliptischen Verb-Letzt-Sätzen zu trennen.

Die beiden Typen ihrerseits unterscheiden sich voneinander durch die Art der rekonstruierbaren Einbettung. Im einen Fall werden durch die Einbettung bestimmte konstitutive Aspekte eines Funktions- oder Illokutionstyps (z.B. der Frage) explizit gemacht (durch Einbettungen wie ich möchte wissen, sag mir); sle kann z.B. einfach einen bestimmten Einstellungsausdruck wie fragen enthalten (Typ III). Beim anderen Typ wird dagegen kein illokutionsrelevanter einbettender Satz rekonstruiert (Typ II).

Während der Typ III deutliche Gemeinsamkeiten mit den nicht-einbettbaren, illokutiv selbständigen Verb-Letzt-Sätzen - und damit mit den illokutiv selbständigen Verb-Erst- und Verb-Zweit-Sätzen - hat, gleicht der zweite eher den elliptischen Strukturen, da erst die 'ergänzte' Fassung einem bestimmten Funktions- oder Illokutionstyp angehört. Beispiele für diesen zweiten Typ finden sich insbesondere bel den wenn-Sätzen.

(43) Und wenn er nicht kommt?

Rekonstrulert werden könnte hier je nach Kontext etwa (und) was willst du tun oder (und) was wäre. Entscheidend ist, daß sich die Frageeinstellung (angezeigt durch die steigende Intonation, hler durch das Fragezeichen) in diesem Fall nicht allein auf den wenn-Satz bezleht, sondern auf den gesamten rekonstrulerten Komplex aus Verb-Letzt-Satz und 'Matrixsatz'. Auf bestimmte andere selbständige adverbiale verb-Letzt-Sätze, die ich ebenfalls $z u$ diesem Typ rechnen möchte, werde ich weiter unten noch kurz eingehen.

Wesentlich interessanter und wohl auch häufiger sind nicht-eingebettete, aber elnbettbare Verb-Letzt-Sătze (Typ III), für die sich aufgrund des Fehlens eines spezifischen sprachlichen Kontextes nur eine Familie von 'Matrixsătzen' bilden laßßt, dle entweder einen globalen Funktionstyp, bzw. die diesem zugeordnete Einstellung, oder aber konstitutive Aspekte eines bestimmten Illokutionstyps bezelchnen.

Als Belspiel mögen noch elnmal der bereits oben angesprochene deliberative Verb-Letzt-Fragesatz und der wenn-Wunschsatz dienen.

(44) (Kaiserlicher Soldat angesichts des humpenleerenden Nusch) $O b$ so viel Wein gesund ist? 
Im Prinzip gibt es viele Einbettungen, die dem Sinn nach passen - dabei sei abgesehen von der oben erwähnten Schwierigkeit, eine intonationserhaltende Einbettung $z u$ finden. Derartig 'ergänzte' Versionen enthalten typischerweise Ausdrücke, die die ausgedrückte propositionale Grundeinstellung, bzw. den Funktionstyp bezeichnen (fragen), oder bestimmte konstitutive Aspekte eines Illokutionstyps beschreiben (wissen wollen).

(45) Ich frage mich wirklich, ob so viel Wein gesund ist.

(46) Ich möchte doch ganz gern wissen, ob so viel Wein gesund ist.

Ganz ähnlich sind die Einbettungen bei wenn-Wunschsätzen zu bewerten:

(47) Wenn du mir nur glauben würdest!

Auch hier ist die Einbettung nur unter Verlust einer eventuellen 'emphatischen' Akzentuierung möglich, ebenso muß die Modalpartikel wegfallen. Ferner kann das dem Funktionstyp angemessene Verb wünschen nicht für die Einbettung verwendet werden. Dafür stehen zahlreiche andere Prädikate zur Verfügung. die das Moment der Präferiertheit durch den Sprecher ausdrücken, z.B.:

(48) Ich wäre Proh, wenn du mir glauben würdest.

(49) Es wäre schön, wenn du mir glauben würdest.

Auppällig an allen diesen 'Vervollständigungen' ist, daß (formal) gar keine Frage- oder Wunschsätze vorzuliegen scheinen, sondern Aussagesätze, die nur kraft ihrer 'performativen', 'illokutiv relevanten' Matrixsätze als Fragen oder Wünsche interpretiert werden können (vgl. Altmann 1987:26f.).

Ausdrücke, die Funktionstypen oder bestimmte propositionale Einstellungen denotieren, spielen im Satzmodussystem in Altmann 1987 eine spezielle Rolle. Im Satzmodussystem sind den formalen Satztypen bestimmte sehr abstrakte Funktionstypen zugeordnet, die im Zusammenspiel mit lexikalischer Füllung und Kontext eine spezifische illokutive Bedeutung ergeben (vgl. das 'Akademie-Modell', wie es z.B. in Bierwisch 1980, Lang 1983 vorgestellt wird). Daß den Formtypen eine bestimmte abstrakte Grundeinstellung zugeordnet ist, soll heißen, daß sie "als Mittel zum Ausdruck einer propositionalen Grundeinstellung" (Altmann 1987:23) dienen. Die abstrakte Grundfunktion kann mit Hilfe einstellungsdenotierender Ausdrücke wie Pragen verdeutlicht werden. Die Grundfunktion illokutiv selbständiger Verb-Erst- und Verb-Zweit-Sätze läßt sich also mit Hilfe derartiger Ausdrücke umschreiben - eine formerhaltende Einbettung unter entspre- 
chende 'performative Hypersätze' ist aber (bis auf die Aussagesätze, s.u.) nicht möglich. ${ }^{11}$

Die Einbettungen mit Hilfe eben dieser einstellungsdenotierenden Ausdrücke bei bestimmten selbständigen Verb-Letzt-Sätzen haben dann aber auch keinen anderen Status als die Funktionstypumschreibungen bei Verb-Erst- und Verb- , Zweit-Sätzen: Sie explizieren die Punktionale Bedeutung, ohne eine syntaktisch relevante Ergänzung $z u$ sein. Der einzige Unterschied zwischen den Verb-Letztund den Nicht-Verb-Letzt-Satztypen besteht darin, daß im ersten Fall eine Einbettung nur selten zu ungrammatischen Ergebnissen führt. Das heißt aber natürlich noch lange nicht, daß auch tatsächlich eine (durch Ellipse unkenntlich gemachte) Einbettung vorliegt. Im Prinzip ist bei den Verb-Zweit-Aussagesätzen eine Einbettung unter einem einstellungsdenotierenden Prädikat wie mitteilen ebenfalls möglich; konsequenterweise müßte man dann auch diese als eingebettet analysieren (vgl. die 'performative Analyse' von Ross und deren Kritik aus pragmatischer Sicht in Grewendorf 1972). Es ist daher sicher sinnvoller, die syntaktische Einbettbarkeit und die Explizierung des Funktionstyps auseinanderzuhalten.

Diejenigen Verb-Letzt-Sătze, die zwar im Prinzip einbettbar sind, für die aber als einbettende Ausdrücke nur solche in Frage kommen, die einen Funktionstyp oder eine illokutiv relevante propositionale Einstellung denotieren, d.h. Verb-Letzt-Sätze des Typs III gehören also zusammen mit den nicht-einbettbaren Verb-Letzt-Sătzen des Typs IV und den nicht-eingebetteten Verb-Erst- und Verb-Zweit-Sätzen zu den illokutiv selbständigen Sätzen. Wesentliches Abgrenzungskriterium gegenüber den Typen I und II ist die Tatsache, daß nur solche Einbettungen gefunden werden können, die eine Explikation eines Funktionsoder Illokutionstyps darstellen.

\subsection{Satzeinleiter zu selbständlgen Verb-Letzt-Sätzen}

Die Entscheidung darüber, ob es sich im konkreten Fall eines nicht-eingebetteten Verb-Letzt-Satzes um eine illokutiv selbständige Verwendung handelt, hängt jedoch nicht ausschließlich vom sprachlichen Kontext und der Rekonstruierbarkeit eines bestimmten Matrixsatztyps oder dem Vorhandensein oder Fehlen von Modalpartikeln ab. Offenbar ist nämlich die Möglichkeit zur echten illokutiven Selbständigkeit nur bei bestimmten Typen von Verb-Letzt-Sätzen gegeben,

11 Zur Einbettung gehört auch die intonatorische Integration. Bei einer notwendigen intonatorischen Separierung zweier Sätze wie bei 'DoppelpunktIntonation' liegt keine Einbettung mehr vor:

(i) Ich Prage dich: Bist du bereit? 
abhängig von der Art des verwendeten Einleitungsausdrucks, der seinerseits wiederum den semantischen Typ des satzförmigen Ausdrucks bestimmt. Lediglich in diesen Fällen stellt sich die Frage 'elliptisch oder lllokutiv selbständig?'.

Zwei große Gruppen lassen sich hier unterscheiden: Aup der einen Seite stehen die adverbiellen Satzeinleiter, auf der anderen der nicht-adverbielle Rest.

\subsubsection{Adverblelle Satzeinleiter}

Die adverblellen Satzelnleiter sind relationendenotierende Ausdrücke; damit gleichen sie den (typischen) Adpositionen. Die entsprechenden Relationen bestehen zwischen dem im Matrixsatz (d.h. modifizierten Satz) ausgedrückten Sachverhalt und dem im Adverbialsatz selbst ausgedrückten Sachverhalt. Je nach Art des adverblellen Einleiters ist auch die Relation verschieden aufzuPassen, z.B. als kausal, konzessiv oder temporal.

Aus der intrinsisch relationalen Natur der Satzeinleiter und damit auch der durch sie eingeleiteten Sätze erklärt sich dann aber auch, warum derartige Sătze im allgemeinen nicht selbständig, sondern höchstens elliptisch verwendet werden können: Es müssen beide Relata vorhanden sein. (Die eine Stelle der Relation kann offensichtlich nicht durch Existenzquantifikation abgebunden werden - sie muß vielmehr ganz spezifisch gefüllt werden.)

Der Normalfall ist also die Sättigung der nicht durch den Verb-Letzt-Satz besetzten Stelle der Relation durch einen sprachlichen Ausdruck, sei dieser intonatorisch integriert oder im unmittelbaren Vortext gegeben.

Daneben gibt es aber auch noch eine markierte Verwendung, bei der die Sättigung 'kontext-deiktisch' erfolgt, nämlich durch eine spezifische Handlung, die der Sprecher durch die Außerung eines nicht-eingebetteten Adverbialsatzes begleitet. Beispiele sind:

(50) (A schiebt B einen Zettel mit seiner Telefonnummer hin.) Falls du meine Hilfe brauchst.

(51) (Vater bindet der kleinen Tochter einen Schal um.) Damit du dich nicht erkältest.

(52) (Mutter überreicht ihrer Tochter mit strengem Blick ein Eis.) Obwohl du es eigentlich nicht verdienst.

(53) (Mutter überreicht ihrer Tochter mit strahlendem Lächeln ein Eis.) Weil du bei Tante Cilly heute so brav warst.

In allen diesen Fällen ist eine nicht-spezifische Einbettung möglich, die die Handlung als das fehlende Relationsglled beschreibt: ich tue das, das geschieht, usw. Derartige Verb-Letzt-Sätze gehören also zum Typ II, nicht zu den illokutiv selbständigen Verb-Letzt-Sätzen im eigentlichen Sinn: Die assertive Einstellung 
bezieht sich nicht direkt auf den Verb-Letzt-Satz, sondern auf den rekonstruierten Komplex.

Es gilt also trotz dieser seltenen nicht-elliptischen nicht-eingebetteten Verwendungen von adverbiellen Verb-Letzt-Sätzen, daß die durch adverbielle Satzeinleiter eingeleiteteten Verb-Letzt-Sätze nicht zu den illokutiv selbständigen Verb-Letzt-Sätzen im eigentlichen Sinn zu zählen sind.

\subsubsection{Nicht-adverbielle Satzeinleiter}

Anders bei den nicht-adverbiellen Einleitern, d.h. bei daß, $\underline{o b}$ und den $w-$ Ausdrücken. Diese bezeichnen offensichtlich keine Relationen zwischen Sachverhalten. Vielmehr wird nur der 'Wahrheitsstatus' des eingeleiteten Satzes berührt: $d a \beta$ zeigt an, daß der folgende satzförmige Ausdruck als geschlossene Proposition $\mathrm{zu}$ verstehen ist, während bei ob der 'positive' oder 'negative' Abschluß der Proposition als alternative Bewertungen offenbleiben. W-Ausdrücke fallen unter die erste Charakterisierung, wenn sie exklamativ verstanden werden, unter die zweite, wenn sie interrogativ zu interpretieren sind - sie unterscheiden sich von ob nur dadurch, daß sie eine spezifische 'offene Stelle' in der Proposition charakterisieren, die bei geeigneter Füllung zu einer geschlossenen Proposition führt.

Problematisch ist für diese Erklärung die Stellung des konditionalen Satzeinleiters wenn. Wie gesehen ist es sinnvoll, bestimmte isoliert stehende wenn-Sätze als selbständig zu charakterisieren, und zwar als einen bestimmten Typ von Verb-Letzt-Wunschsätzen. Dafür sprechen alle der oben angeführten Kriterien: Zum elnen ist die Einfügung der wunschsatztypischen Modalpartikeln doch, nur, bloß - nahezu obligatorisch, zum anderen ist nicht nur aus der sprachlichen Umgebung typischerweise ein passender Matrixsatz nicht rekonstrulerbar, sondern wegen der Modalpartikeln ist es vielmehr gar nicht möglich, einen passenden einbettenden Ausdruck zu finden. Nach Eliminierung der Modalpartikeln ist zwar eine Einbettung möglich, aber nur elne Punktionstypexplizierende.

Wenn also alles dafür spricht, bestimmte nicht-eingebettete wenn-Sätze als selbständig zu betrachten, so bezeichnet doch aup der anderen Seite wenn, wie sein Gegenstück falls, eine Relation, so daß nach dem oben Gesagten elne selbständige Verwendung nicht möglich sein sollte. Insbesondere muß auch geklärt werden, wieso falls als bedeutungsähnlicher Satzeinleiter keine selbständig verwendbaren Verb-Letzt-Sätze zuläßt.

Neben den wenn-Wunschsätzen Plnden sich im Prinzip auch daß-Wunschsätze, wenngleich diese nicht mehr zu den geläufigen Satztypen zu rechnen sein dürf- 
ten. Ein eindeutig nicht-relationaler Satzeinleiter kann also durch einen anscheinend relationalen ersetzt werden. Da unklar ist, wie der nicht-relationale Satzeinleiter in diesem spezifischen Konstruktionstyp relationale Bedeutungsbestandteile enthalten können sollte, liegt es eher nahe anzunehmen, da $\beta$ der relationale Satzeinleiter wenn in dieser Verwendung seine Relationalität verliert. In der Standardverwendung, d.h. in einem Konditionalgefüge, ist es der wenn-Satz, der für die nicht-assertive Interpretation der beiden Teilsätze verantwortlich ist. Der Satzeinleiter wenn zeigt also nicht nur die Art der Verknüpfung der beiden Teilsätze an, sondern auch gleichzeitig etwas über deren 'Wahrheitsstatus'. In den selbständigen Wunschsatzverwendungen scheint gerade diese Bedeutungskomponente $z u$ dominieren, wohingegen die relationale zumindest so weit unterdrückt ist, daß sie nicht die Ergänzung durch einen passenden Konsequenz-Satz im sprachlichen Kontext notwendig macht. Diese Erklärung wird auch dadurch gestützt, daß in den wenn-Wunschsätzen wie in den $d a \beta$-Wunschsätzen Konjunktiv-II-Formen verwendet werden, die ebenfalls die Nicht-Realisiertheit der durch den Verb-Letzt-Satz ausgedrückten Proposition anzeigen.12

Wie erwähnt ist das mit wenn bedeutungsähnliche falls als Satzeinleiter für selbständige Verb-Letzt-Sätze ausgeschlossen:

(54) "Falls ich doch einmal meine Arbeiten rechtzeitig fertigbrächte!

In Analogie zur obigen Argumentation bei wenn kann man annehmen, da $\beta$ bei falls gerade nicht die Anzeige des 'Wahrheitsstatus' wesentlich ist, sondern nur die Formulierung einer Bedingung. Außerdem scheint zu gelten, daß 'irreale' Bedingungsgefüge mit falls eher ungewöhnlich sind:

(55) Wenn/?Falls Caesar den Rubicon nicht überschritten hätte, wären WürPelspiele viel weniger verbreitet.

Entsprechend der Abkunft aus dem Nomen Fall wird falls wohl hauptsächlich bel der Formulierung 'realer Bedingungen' verwendet, so da $\beta$ der für die Verwendung von selbständigen wenn-Sätzen wesentliche Bedeutungsbestandteil Pehlt. Ahnlich problematisch für die These, daß nur mit absoluten Satzeinleitern selbständig verwendbare Verb-Letzt-Sätze gebildet werden können, ist ein weiterer Typ von selbständigen Verb-Letzt-Sätzen, der aber wohl noch etwas peripherer im Satzmodussystem ist als die eben erwähnten wenn-Sätze.

12 Für die prototypischen 'irrealen' Wunschsätze ist der Konjunktiv II konstitutiv. Allerdings gibt es verschiedene 'idiomatische' wenn-Sätze, die keinen Konjunktiv II erfordern (vgl. dazu Scholz 1989). 
Es handelt sich um die durch als, bzw. wie 'eingeleiteten' sogenannten irrealen Vergleichssätze (mit der nur im Süddeutschen gebräuchlichen Kombination als wie).

(56) Als ob/Als wenn/Wie wenn/Als wie wenn ein Gemsledereinsatz schon etwas helfen würde!/Als würde ein Gemsledereinsatz schon etwas helfen!

Zunächst sollten als, bzw. wie hier nicht als Satzeinleiter fehlklassifiziert werden. Es liegt hier weder eine zweifache oder sogar dreifache Besetzung einer Satzeinleiterposition vor, noch besitzt der Satzeinleiter als die merkwürdige Eigenschaft, eine Verb-Zweit-Stellung auszulösen. Vielmehr sind auch in dieser Konstruktion, wie ja auch schon der traditionelle Name 'irreale Vergleichssätze' nahelegt, als und wie als Vergleichspartikel zu werten, die nichts mit der Binnenstruktur derjenigen Ausdrücke zu tun haben, die sie verknüpfen. Unter der Vergleichspartikel eingebettet sind durch wenn oder ob eingeleitete Verb-LetztSätze oder aber Verb-Erst-Sätze (genaueres s. 1.3.3.2).

Problematisch für die oben genannte These der zulässigen Satzeinleiter, wenn man sie nicht ganz wörtlich nehmen will, bleiben derartige strukturen aber trotzdem, da als und wie, auch wenn es sich nicht um Satzeinleiter handelt, dennoch als Vergleichspartikel ohne Zweifel relationale Ausdrücke sind. Zentral für diese Art von Vergleich ist aber offensichtlich dessen 'Irrealität' (dementsprechend ist die Verwendung von Konjunktiv-II-Formen teils der Normalfall, teils sogar obligatorisch), so daß ähnlich wie bei den wenn- und daß-Wunschsätzen die Anzeige des 'Wahrheitsstatus' dominiert. Alle selbständigen irrealen Vergleichssätze sind dabei so zu interpretieren, daß die Negation des 'irrealen Sachverhalts' gilt, es handelt sich also um eine bestimmte Art von Assertion.13

Die Aufteilung der Satzeinleiter in relationale und absolute korrespondiert also gut mit der Aufteilung der nicht-eingebetteten Verb-Letzt-Sätze in ellip-

13 Ein weiterer Randtyp, auf den aber nicht näher eingegangen werden soll, sind die durch bis eingeleiteten, als warnende Voraussagen verwendeten Verb-Letzt-Sätze:

(i) Bis du dich erkältest!

(ii) Bis ihm einer sein Fahrrad klaut!

Rekonstruierbar ist eine Familie von nicht-illokutiven 'Matrixsätzen' wie du wirst (solange) $x$-en oder es wird solange gutgehen. Insbesondere an (i) wird jedoch deutlich, daß bis intonatorisch hervorgehoben ist; dies würde bei einer Einbettung verlorengehen. Demnach lassen sich diese Sätze dem Typ IV zuordnen.

Durch bis-Ausdrücke wird der Endpunkt einer räumlichen oder zeitlichen Erstreckung gekennzeichnet; bis-Sätze bezeichnen einen bestimmten Sachverhalt, der an diesem Endpunkt besteht. Aus diesem Grund lassen sie sich als Vorhersagen verwenden. Eine angemessene Paraphrase von Sätzen wie (i) oder (ii) wäre ein Verb-Zweit-Satz, in dem bis durch am Ende o.A. ersetzt wird. 
tische (zusammen mit dem Typ II) und solche, die im Prinzip auch lllokutiv selbständig verwendbar sind. Die Relationalität der adverbiellen Satzeinleiter erfordert die sprachliche Reallsierung beider Relata. ${ }^{14}$

1.3 Typen illokutiv selbständiger Verb-Letzt-Sätze

Im folgenden werde ich die illokutiv selbständigen Verb-Letzt-Varianten der fünf Satzmodus-Gruppen Frage-, Imperativ-, Aussage-, Exklamativ- und Wunschsatzmodus vorstellen. Es werden die jeweiligen formalen und funktionalen Eigenschaften aufgeführt. Von den vier für die Satzmoduskennzeichnung relevanten Merkmalsmengen der kategorialen, topologischen, morphologischen und intonatorischen Eigenschaften (vgl. Altmann 1987) ist natürlich die kategoriale Füllung mit den verschiedenen Typen von Satzeinleitern (sowie den typischen Modalpartikeln) zentral. Die Stellung der Satzeinleiter ist auf die satzeinleitende Position (eine COMP-Position im Sinne neuerer Syntaxtheorien) und das finite Verb auf die Endposition Pestgelegt. Morphologische Verbmodus-Merkmale werden insbesondere bei den Verb-Letzt-Wunschsätzen und den als ob-Sätzen relevant und auch nur dort ausführlicher besprochen. Auf die intonatorische Form wird in diesem formal-funktionalen Uberblick nicht sehr ausführlich eingegangen. Sie ist zentraler Untersuchungsgegenstand des zweiten, 'experimentellen' Teils dieser Arbeit.

14 Nicht nur bei der Einleitung selbständiger Verb-Letzt-Sätze, sondern auch bei verschiedenen anderen syntaktischen Prozessen verhält sich der Satzeinleiter wenn eher wie $d a \beta$, ob oder eln satzeinleitender $w$-Ausdruck. Er steht daher nach Reis (1985:284) diesen 'schwachen' Komplementierern nahe und gehört nicht $\mathrm{zu}$ den 'starken' adverbiellen. In einer auf strukturbeziehungen Pußenden syntaktischen Beschreibung wie der GB-Theorie wäre das ein Indiz dafür, den beiden Typen von Komplementierern auch verschiedene Strukturpositionen zuzuweisen. Schwache Komplementierer stehen in einer spezifischen Komplementlererposition, sie sind Kopf des gesamten Verb-Letzt-Satzes, der damit als Komplementiererphrase kategorisiert wird. Wo stehen dann die starken Komplementierer? Wenn man ihren relationalen Charakter berücksichtigt, dann drängt sich die Parallele zu den ebenfalls typischerweise relationalen Präpositionen auP. Es liegt also die folgende Strukturanalogie nahe: So wie sich eine Präposition zu einer NP verhält, so verhält sich ein starker, relationaler Satzeinleiter zu einer Komplementiererphrase. Demnach würde es sich bei den sogenannten starken und schwachen Komplementierern um zwei ganz verschiedene Ausdruckstypen handeln. Um die Analogie weiterzuspinnen: Den 'echten' Komplementierern, d.h. den schwachen, könnten im Bereich der NP die Determinatoren entsprechen. Beide legen in gewisser Welse den referentiellen Status fest. Die Parallele wird noch deutlicher, wenn man, wie in neueren Arbeiten im GB-Rahmen, das, was sonst als NP bezeichnet wird, als DP, d.h. als Determinatorenphrase kategorisiert (vgl. z.B. Haider 1988). 


\subsubsection{Verb-Letzt-Fragesätze}

Sowohl zu den Entscheidungs- als auch den w-Fragesätzen existieren VerbLetzt-Typen. Sie werden durch ob oder aber wiederum durch einen $w$-Ausdruck, in diesem Fall natürlich mit anderer syntaktischer Funktion, eingeleitet.

Hier sollen nach den Sequenzierungseigenschaften zwei große Gruppen unterschieden werden, nämlich diejenigen, die nicht notwendigerweise reaktiv verwendet werden, und diejenigen, die stets gleichzeitig reaktiv und initiativ verwendet werden, insbesondere Rückfragen und Versicherungsfragen.

\subsubsection{Deliberative Verb-Letzt-Sätze}

Den ersten Typ bilden die oben bereits als Beispiel verwendeten 'deliberativen Fragesätze' (vgl. Luukko-Vinchenzo 1988:64ff., 159ff.; vgl.auch Weuster 1983:46ff.,54ff.). Zulăssige - und auch fast immer verwendete - Modalpartikeln sind wohl (und zum Teil mal: ob ich mal klopfe?) bei den ob-Sätzen, sowie bei den $w$-Verb-Letzt-Sätzen wohl, nur, bloß; die typlschsten Modalpartikeln der Verb-Erst- und w-Verb-Zweit-Fragesätze, denn und eigentlich, können dagegen nicht eingefügt werden (vgl. Thurmair 1989:54, 57). ${ }^{15}$

(57) Ob Katelbach wohl/*denn/*eigentlich kommt?

(58) Wo die Blumen wohl/nur/bloß/*denn/"eigentlich geblieben sind?

Wie oben schon ausführlich dargestellt wurde, handelt es sich eindeutig um illokutiv selbständige Verb-Letzt-Sätze. Gegenüber den Verb-Erst- und VerbZweit-Varianten unterscheiden sie sich in der eingeschränkteren Verwendbarkeit. Dies zeigt sich auch an der Unzulässigkeit der neutralsten Modalpartikel denn. Das für Fragen ansonsten zentrale Merkmal der Aupforderung an ein Gegenüber, fehlende Informationen bereitzustellen (vgl. Searles (1969:66) 'essential rule' für

15 In Thurmair (1989:57) wird auch das rhetorizitätsindizierende schon zu den zulässigen Modalpartikeln gerechnet. Anders als bei den deliberativen VerbLetzt-Fragesätzen und auch anders als bei den rhetorischen w-Verb-ZweitFragesätzen lassen sich ohne weiteres aber nur die auch für den $w$-Exklamativsatz typischen $w$-Ausdrücke, insbesondere wie, verwenden.

(i) Wie du schon schaust!

(ii) ?Wo der schon herkommt!

(iii) ??Wann der schon mal Zeit hat!

(iv) ??Wer das Zeug schon liest!

Auf die Exklamativnähe deutet auch hin, daß die implizierten 'Antworten' graduierend verstanden werden müssen: in (i) also 'sehr streng', 'sehr blöd', usw., in (ii) 'aus einem sehr merkwürdigen Ort/Haus', in (iv) 'komische Spinner', aber gerade nicht 'niemand', wie es für die Verb-Zweit-Version typisch ist. Die entsprechenden Sätze sind demnach nicht eindeutig als Fragesätze zu klassifizieren, sondern weisen sehr starke Ahnlichkeiten mit den Exklamativsätzen aup. 
Fragen) mit daraus resultierender Antwortverpflichtung, ist hier zumindest nicht wesentlich.

Zentral ist vielmehr die reine Formulierung eines Informationsmangels, dessen Behebung erwünscht ist ( $\mathrm{vgl}$. wiederum bei searle die 'preparatory rule' und 'sincerity rule'). Die typische Verwendung ist die als 'Selbstfragen'. Die Verwendung in normalen hörergerichteten Fragen ist möglich, allerdings mit dem Effekt, daß die Antwortverpflichtung für den Hörer aufgrund der 'deliberativen Problemformulierung' (wenigstens dem Anschein nach) aufgehoben ist. (Daher lassen sich die an sich schon indirekten und höflichen Aufforderungen in Fragesatzform durch die Verwendung von $o b$-Sätzen noch vorsichtiger und höflicher formulieren.)

Ein 'Deliberativitätsanzeiger' ist aber bereits die typische Modalpartikel wohl: Auch die Nicht-Verb-Letzt-Varianten der $o b / w$-Sätze lassen sich kaum durch elnen passenden Vorgängertext (in dem die 'essential rule' sozusagen explizit formuliert wird) als echte Fragen ankündigen:16

(59) Sag mal: Ist 37 denn/?wohl eine Primzahl? vs.

Sag mal: ??Ob 37 wohl eine Primzahl ist?

(60) Sag mal: Wann kommt denn/?wohl Katelbach? vs. Sag mal: ??Wann wohl Katelbach kommt?

Es ist also verständlich, wieso die deliberative Modalpartikel wohl gut in den Kontext der problemformulierenden $o b / w$-Verb-Letzt-Fragesätze paßt.

Auch für die beiden nur in den $w$-Varianten zulässigen Modalpartikeln blo $\beta$ und nur läßt sich erklären, wieso sie mit der Verb-Letzt-Form verträglich sind. Durch den Gebrauch von blo $\beta$ und nur in $w$-Fragesätzen wird nach Thurmair (1989:179) eine 'Verstärkung' der Frage angezeigt. Diese Verstärkung scheint sich aber nicht direkt auf die Antwortobligation zu richten, sondern über eine Hervorhebung des in der 'Ernsthaftigkeitsbedingung' (vgl. Searles (1969:66) 'sincerity rule') ausgedrückten Interesses an der Bereitstellung der fehlenden Information zustandezukommen. $\mathrm{Da} \beta$ gerade diese Komponente verstärkt wird, macht diese Modalpartikeln auch in $w$-Verb-Letzt-Fragen verwendbar, wo eine Verstärkung der Antwortobligation ausgeschlossen ist, vgl. wieder den Einbettungstest:

(61) Sag mal: Wann wird Katelbach nur endlich einen Schlüssel mitnehmen?/??Wann Katelbach nur endlich einen Schlüssel mitnehmen wird?

16 Allerdings ist es durchaus möglich, an diese Fragesätze Ausdrücke anzuschließen, die dem Gegenüber eine Antworthandlung nahelegen, also ein 'Pragendes' $h m$ oder auch ein was meinst du? etc. Hier handelt es sich aber um die zusätzliche Aufforderung zu einer Antworthandlung, die im Gegensatz zu der 'Vorankündigung' den Status des Fragesatzes selbst nicht berührt. 
Die Modalpartikeln müssen mit der Verb-Letzt-Form also zumindest verträglich sein. Und die charakteristische Eigenschaft von Fragesätzen dieser Form ist, daß mit ihnen keine prototypischen Fragehandlungen ausgeführt werden. Die Antworterwartung auf seiten des Fragenden und die Antwortverpflichtung für das Gegenüber sind im Vergleich zu der Verwendung von Entscheidungsfragesätzen mit Verb-Erst-Stellung und w-Fragesätzen mit Verb-Zweit-Stellung deutlich abgeschwächt.

Der Gebrauch passender Modalpartikeln ist bei den Verb-Letzt-Fragesätzen nahezu obligatorisch, insbesondere gilt das für die $w$-Versionen. Wesentlich dafür dürfte die Abgrenzung von den gleichlautenden w-Verb-Letzt-Exklamativsätzen sein. Wie bei den $w$-Verb-Zweit-Sätzen ist die intonatorische Form offenbar kein genügend sicheres Unterscheidungsmerkmal, insbesondere dann nicht, wenn kein fragesatztypischer steigender Tonverlauf realisiert wird. Wenn bei den selbständigen $w$-Sätzen die Satzmodusdifferenzierung hauptsächlich über die Verbstellung erfolgt - Verb-Zweit-Stellung bei Fragesätzen und Verb-LetztStellung bei Exklamativsätzen (s. 1.3.4) -, dann wird bei den Verb-Letzt-Fragesätzen eine zusätzliche Markierung notwendig, um die markierte Fragelesart sicherzustellen. Genau dazu dienen die Modalpartikeln.

Zur intonatorischen Form vorläufig nur soviel: Die durch ob eingeleiteten Sătze haben obligatorisch das für Fragen typische steigende Tonmuster, die deliberativen $w$-Verb-Letzt-Sätze können dagegen wie die w-Verb-Zweit-Fragesätze sowohl mit steigendem als auch mit fallendem Tonmuster (enthalten sie die Modalpartikeln nur und bloß, ist das letztere sogar üblich) versehen werden (daher die Uberschneidungsmöglichkeit mit den Exklamativsätzen).

Die (nicht sequenzgebundenen) $o b / w$-Verb-Letzt-Fragesätze werden typischerweise als eine Art 'Protlemformulierungen' gebraucht. Als Problemformulierungen sind sie wohl vor allem aufgrund ihrer formalen Verwandtschaft zu (echten) indirekten Fragesätzen ('Interrogativ-Sententialen' in der Ausdrucksweise von Zaefferer 1984) geeignet (ohne daß sie deshalb als elliptisch aufzufassen wären!): Mit solchen Interrogativ-Sententialen wird nur die Unterspezifiziertheit einer Proposition repräsentiert. Genau das ist auch für die deliberativen Fragen wesentlich.

\subsubsection{Rückfragen und Versicherungsfragen}

Rückfragen und Versicherungsfragen können ebenfalls in der Form von obund $w$-Verb-Letzt-Sätzen auftreten. Gerade in diesem Bereich ist die Unterscheidung der elliptischen und der illokutiv selbständigen Verb-Letzt-Strukturen problematísch. Das Kriterium der Modalpartikelhaltigkeit entfällt, da Rückfragen 
generell keine Modalpartikeln enthalten (Altmann 1987:48f.; Luukko-Vinchenzo 1988:71, 174; Thurmair 1989:70ff.). Auf der anderen Seite läßt sich bei VerbLetzt-Rückfragen, obwohl sie natürlich kontextgebunden verwendet werden müssen, kein spezifischer Matrixsatz aus dem Kontext rekonstruieren. Sie müssen zum Typ III der nicht-eingebetteten Verb-Letzt-Sätze gerechnet werden.

Rückfragen gehören nach Altmann (1987:48f.) zu den Mischtypen, die durch eine 'Mischung' aus den formalen Merkmalen zweier Grundtypen gekennzeichnet sind. Bei den sequenzgebundenen RückPragen werde ein "segmental identische[r] Satztyp, allerdings ohne Modalpartikel, mit stark steigendem Tonverlauf in der Nukleussilbe und hohem offset" (Altmann 1987:49) als Reaktion auf die adressatenorientierten Satztypen Aussagesatz, Fragesatz und Imperativsatz (nach Meibauer 1987 auch auf den Exklamativsatz) verwendet. Es liegt also eine Mischung aus Ursprungssatztyp und intonatorischer Fragesatzmarkierung vor. Ein typischer Verwendungszweck ist eine oft im folgenden näher ausgeführte 'Kritik' an dem Sprechakt, der mittels der replizierten Außerung vollzogen wurde.

Die Forderung nach Formidentität ist allerdings zu eng und schließt viele Strukturen aus, die aufgrund der Sequenzierung, der intonatorischen Form, der Modalpartikellosigkeit und nicht zuletzt der Funktion ganz klar unter die RückPragen einzustufen sind ( $\mathrm{vgl}$. $\mathrm{zu}$ diesen Strukturen auch Wunderlich 1986 , Meibauer 1987, Thurmair 1989:70ff.).

Das allgemeinste Charakteristikum von Rückfragen ist, daß ein Vorgängersprechakt 'zitiert' und gleichzeitig zum Gegenstand einer 'fragenden' propositionalen Einstellung gemacht wird. Daraus erklären sich die formalen Eigenschaften, die einerseits die Frageeinstellung und andererseits den Zitatcharakter verdeutlichen:

Die zusätzliche Frageeinstellung wird durch die Verwendung der 'Frageintonation', die hier obligatorisch ist, angezeigt. Die einfachste Form des 'Zitats' ist eine wörtliche Wiederholung der Vorgängeräußerung - eben bis auf die von der RückPrage überdeckte intonatorische Form (und Modalpartikeln ${ }^{17}$ ). Es geht auf der einen Seite also satzmodusrelevante Information verloren, auf der anderen Seite ist bei Rückfragen aup Fragesätze die Rückfrage selbst formal nicht eindeutig gekennzeichnet, sondern lediglich über die Sequenzierung erkennbar. Eine

17 Ein Grund für das Verbot von Modalpartikeln könnte sein, daß der 'zitierte' Satztyp die Einfügung von fragetypischen Modalpartikeln blockiert und umgekehrt der Fragecharakter das 'Zitat' nicht fragetypischer Modalpartikeln. Da diese Erklärung bei fragebezogenen Rückfragen nicht greift, ist mindestens für diesen Fall anzunehmen, daß einerseits die Einfügung einer Modalpartikel durch den RückPragenden selbst durch den Zitatcharakter, andererseits die 'Zitatübernahme' durch die Sprecherbezogenheit von Modalpartikeln blockiert wird (vgl. auch Thurmair 1989:73). 
eindeutige Trennung von 'zitiertem' und 'verwendetem' Satzmodus ist also nicht möglich. Der zitierte Satzmodus läßt sich aber durch 'Propositionalisierung' in seinem Zitatcharakter verdeutlichen. Eine extreme Möglichkeit ist die Verwendung von Einbettungen, die den zitierten Sprechakt oder die entsprechende propositionale Einstellung bezeichnen (so da $\beta$ der Form nach eine aussagesatzbezogene Rückfrage mit Verb-Zweit-Stellung und 'Frageintonation' vorliegt). Ublicherweise werden jedoch Imperativsätze in Verb-Zweit-Sätze mit einem Modalverb (typischerweise sollen) und Fragesätze in Verb-Letzt-Sätze umgewandelt, Aussagesätze erhalten dagegen tatsächlich nur eine 'Frageintonation' und werden ansonsten so weit wie nötig (Pronominalisierung von Voll-NPn) und möglich (Auslassung von Modalpartikeln) wörtlich wiederholt.

Bei den Rückfragen auf Imperativsätze ist es also zulässig und wohl auch präferiert, die Verb-Erst-Stellung eines imperativisch markierten Verbs zugunsten einer Verb-Zweit-Stellung und der Verwendung des Modalverbs sollen aufzugeben. (Auch dürfen und können sind bei Rückfragen auf Imperativsätze zulässig, die für eine Erlaubnis verwendet werden.)

(62) A: Geben Sie doch Gedankenfreiheit!

B: Geben Sie Gedankenfreiheit?/Ich soll Gedankenfreiheit geben?

(63) A: Nimm ruhig noch ein Stück Kuchen!

B: Ich darf/kann noch ein Stück Kuchen nehmen? Danke, find ich prima.

Zulässig und präferiert ist aber auch die Umwandlung von Verb-Erst- und $w$-Verb-Zweit-Fragesätzen in die entsprechenden Verb-Letzt-Varianten mit $o b$ oder einem $w$-Ausdruck als Satzeinleiter. Die 'Zitatform' für den Vorgängersprechakt 'Frage' ist die Form, die auch bei indirekten Fragesätzen, also bei der expliziten Zitatform der indirekten Rede gewählt wird.18 Auch hier ist das steigende Tonmuster der für Rückfragen konstitutiven Frageeinstellung zugeordnet.19

Die Rückfragen unterscheiden sich also in den drei präferierten Fällen auch formal von den üblicherweise 'zitierten' Vorgängeräußerungen. Bei den aussagesatzbezogenen Rückfragen ist die intonatorische Form allein unterscheidend. ${ }^{20}$

18 Dies soll natürlich nicht heißen, daß es sich bei den Rückfragen um eine Art indirekte Rede handelt: Zum einen sind keine Konjunktiv-I-Formen zulässig. Zum anderen ist die Umwandlung von Verb-Zweit-Aussagesätzen in $d a \beta$-Verb-Letzt-Sätze wie bei der indirekten Rede gerade nicht möglich (vgl. auch Thurmair 1989:73 zu Argumenten gegen eine Einstufung von Rückfragen und Versicherungsfragen als Redewiedergabe).

19 Zweifellos gibt es noch einen anderen Typ von 'zitierender' Reaktion, bei dem die Vorgängeräußerung - in diesem Fall sogar absolut segmentgleich, also auch mit Modalpartikeln - wiederholt werden kann. In diesem Fall wird die Vorgängeräußerung nachgeäfft und damit gerade nicht eine zusätzliche 'Frageintonation' über diese Außerung gelegt (vgl. Thurmair 1989:70, Fn. 90).

20 Formal nicht trennbar sind sie dagegen von den assertiven Fragen; unterscheiden kann hier nur die Sequenzierung, da bei assertiven Fragen der 
Bei den imperativsatzbezogenen Rückfragen weicht sowohl die 'modalisierte' Aussagesatzform als auch die Intonation vom Vorgänger ab. Bei den pragesatzbezogenen Rückfragen schließlich ist die Verb-Letzt-Form das unterscheidende Merkmal (es sei denn bei dem marginalen Fall der Rückfrage auf einen VerbLetzt-Fragesatz).

Diese Typen von Verb-Letzt-Rückfragen mit einer formal eindeutigen Trennung des 'zitierten' Vorgängerfrageakts und der Rückfrage selbst, sind üblicher als die echten Wiederholungsrückfragen mit dem Gebrauch des segmental identischen Ausdrucks; insbesondere gilt das für die Fälle von Pronominal/Deiktikawechsel:

(64) A: Oh pardon. Sind Sie der Graf von Luxemburg?

B: ?Sind Sie der Graf von Luxemburg?/"Bin ich der Graf von Luxemburg?/Ob ich der Graf von Luxemburg bin? Was für eine unverschämte Frage! Natürlich bin ich der gegenwärtige König von Frankreich.

(65) A: Wie schlecht haben die Bayern denn heute gespielt?

B: Wie schlecht haben die Bayern heute gespielt?/Wie schlecht die Bayern heute gespielt haben? Auf so dumme Fragen antworte ich doch gar nicht!

Auf diese Weise entstehen Verb-Letzt-Strukturen, die auf der einen Seite sequenzgebunden sind und keine Modalpartikeln enthalten, also nicht unbedingt ohne weiteres als selbständig identifizierbar sind, für die aber dennoch, genau wie für andere Rückfragen, kein spezifischer Matrixsatz aus dem Kontext rekonstruiert werden kann, sondern nur einer, der die Frageeinstellung kennzeichnet. Insofern müssen auch sie $z u$ den selbständigen Verb-Letzt-Sätzen gerechnet werden.

'Zitatcharakter' fehlt. Möglicherweise lassen sich beide Typen aber auch tatsächlich als zwei formal nicht unterscheidbare Untertypen eines übergreifenden Typs der 'Bestätigungsfrage' zusammenfassen. Bei den Rückfragen geht es darum, sich eine unmittelbar vorher vermittelte sprachliche 'Information' bestätigen zu lassen, bei den assertiven Fragen wird nach der Bestätigung einer nicht-sprachlichen, aber im situativen Kontext relevanten 'Information' verlangt. Für die nicht allzu strikte Trennung von Rückfragen und assertiven Fragen (zu einigen - erklärbaren - Unterschieden zwischen assertiven Fragen und Rückfragen vgl. Oppenrieder 1987:184, Fn. 9) spricht weiter, daß sich im Prinzip auch aufforderungs- und fragebezogene 'assertive' Fragen bilden lassen:

(i) (A macht nach einem Streit mit B demonstrativ die Tür für B auf.) $B$ : Ich soll gehen?

(ii) (A sucht auf B's Schreibtisch nach einem Stück Papier. Er hält schließlich B ein Papier vor die Nase.)

$B$ : $O b$ du das nehmen kannst?

Kennzeichen dieser formal ausgezeichneten Bestätigungsfragen ist die 'Frageintonation'. 'Bestätigungsheischend' können allerdings auch Sätze anderer Formtypen, z.B. Aussagesätze, verwendet werden, die aber aufgrund der formalen Unterschiede nicht mit Bestätigungsfragen vermischt werden sollten wie das z.B. in Geluykens (1988) geschieht. 
Typisches funktionales Charakteristikum der Rückfragen nach Altmann (1987:49) ist, daß sie die Berechtigung des replizierten Vorgängersprechaktes in Zweifel ziehen. Eine funktionale Standard-Paraphrase wäre also:

(66) Wie kannst du so etwas sagen/befehlen/fragen?

Nicht alle Verb-Letzt-Fragesätze mit steigendem Melodieverlaup, die eine Vorgängeräußerung 'zitieren', gehören aber $\mathrm{zu}$ diesem funktionalen Typ. Es scheint mir angemessener, Rückfragen allgemeiner aufzufassen und die sprechaktzurückweisenden Verwendungen als Spezialisierungen einer globaleren funktionalen Charakteristik einzuordnen: Zunächst einmal wird der Vorgängersprechakt quasi als Zitat verwendet und - angezeigt durch die Intonation - eine Pragetypische Einstellung darauf angewendet. Es handelt sich also zunächst um eine neutrale 'Nachfrage'. Die jeweilige spezialisierung kann in nachfolgenden Erläuterungen $z u$ dieser Nachfrage klargemacht werden. Im einen Extremfall kann die Berechtigung zum Vollzug des infragegestellten Sprechakts in Zweifel gezogen werden, im anderen Extremfall wird das ursprüngliche 'Ziel' des Vorgängersprechakts akzeptiert, also eine Aussage übernommen, eine Aufforderung anerkannt oder eine Frage beantwortet. Im Prinzip stehen also beide Typen von Fortsetzungen zur Verfügung:

(67) A: Dillinger ist tot.

B: Dillinger ist tot? Setzen Sie doch nicht völlig haltlose Gerüchte in die Welt./Das ist ja ganz furchtbar.

(68) A: Essen Sie Spinat!

B: Ich soll Spinat essen? Sie haben mir überhaupt keine Vorschläge zu machen./Ja, ich glaube auch, daß ich dann gesünder wäre.

(69) A: Sind Sie Bayern-Fan?

$B$ : Ob ich Bayern-Fan bin? Wie kommen Sie dazu, mir solche Fragen zu stellen./Ja, schon seit meiner Kindergartenzeit.

(70) A: Warum sitzt du hier so rum?

B: Warum ich hier so rumsitze? Auf blöde Fragen antworte ich nicht./ Weil hier mein Jägermeister steht.

Von daher gehört auch die in Luukko-Vinchenzo (1988:104ff., 187) als eigener Typ etablierte 'Frage-Ubernahme' zu den so verstandenen Rückfragen, vgl. dazu die Antwort-Versionen der beiden letzten Beispiele.

Dieselben Umwandlungen von Imperativ- und Entscheidungsfragesätzen werden auch bei einem anderen Typ von 'Rückfragen' präferiert (die obligatorische 'Frageintonation' und das Fehlen von Modalpartikeln sind eine weitere Gemeinsamkeit), nämlich bei den Versicherungsfragen im Anschluß an Imperativ- und Entscheidungsfragesätze, während bei den aussagesatzbezogenen Versicherungsfragen die Verb-Zweit-Stellung beibehalten wird ( $z u$ Versicherungsfragen, wenn 
auch nicht immer unter dieser Benennung, vgl. Wunderlich 1986, Meibauer 1987, Luukko-Vinchenzo 1988:79ff., Thurmair 1989:72f.).

(71) A: Er heißt Wrdlbrmfd.

B: Er heißt WIE?/WIE heißt er?/"WIE er heißt?

(72) A: Nennen Sie mich Abraxas!

B: ?Nennen Sie mich WIE?/"Nenne ich Sie WIE?/ICh soll Sie WIE nennen?/WIE soll ich Sie nennen?

(73) A: Ist das die S.X.F.R.T.V.?

B: ?Ist das die WAS?/Ob das die WAS ist?

(74) A: Sind Sie Phagophonologe?

$B$ : "Sind Sie WAS?/??Bin ich WAS?/Ob ich WAS bin?

(75) A: Wer hat sich denn hier auf Phenacogrammus interruptus spezialisiert?

B: ?Wer hat sich hier auf WAS spezialisiert?/Wer sich hier auf WAS spezialisiert hat?

Das teilweise 'Zitat' einer Vorgängeräußerung ist wiederum charakteristisch. Nur zielt hier, angezeigt durch die Verwendung eines obligatorisch den Satzakzent tragenden $w$-Ausdrucks, die Frageeinstellung auf die Füllung einer spezifischen Leerstelle, während bei den Rückfragen nach der Polarität gefragt wird.

Im Gegensatz zu den Rückfragen ist bei den Verb-Letzt-Versicherungsfragen eine Einbettung von vornherein wesentlich unplausibler:

(76) ??Du fragst mich, ob ich WAS bin?

Auch hier muß von illokutiv selbständigen Verb-Letzt-Sätzen gesprochen werden.

\subsubsection{Ultimative Fragen}

Ein weiterer sequenzgebundener Typ von Verb-Letzt-Fragesatz (ohne fragesatztypische Modalpartikel wie denn und eigentlich) ist die sogenannte 'ultimative Frage' (vgl. Luukko-Vinchenzo 1988:96ff., 177ff.). Sie wird verwendet, um eine unmittelbar vorher schon einmal gestellte, aber vom Gesprächspartner noch nicht beantwortete Frage noch einmal zu formulieren und damit auf einer Antwort $\mathrm{zu}$ bestehen. Im Gegensatz zur bloßen Wiederholung eines Verb-Erst- oder $w$-Verb-Zweit-Fragesatzes (in diesem Fall wohl auch ohne Modalpartikel!) wirken die Verb-Letzt-Versionen 'ultimativer', d.h. die Antwortobligation wird verstärkt:

(77) A: Hast du eigentlich deinen Aufsatz fertig?

$B$ : Stell dir vor, gestern war ich am Spitzing!

$A$ : $O b$ du ("eigentlich) deinen Aufsatz fertig hast!?

(vs.: Hast du deinen Aufsatz fertig?)

(78) A: Wann fährst du denn zum Spitzing?

B: Stell dir vor, ich hab meinen Aufsatz fertig! 
Nach den zu Anfang genannten Kriterien handelt es sich um selbständige Verb-Letzt-Sätze des Typs III, da nur unspezifische illokutive Einbettungen rekonstruiert werden können und $z$ war wiederum solche, die die einzelnen konstitutiven Aspekte einer Frage formulieren, also ich möchte wissen, du sollst mir sagen, etc. oder eben einfach ich frage dich; gerade aufgrund der Ultimativität kann auch ein imperativischer 'Matrixsatz' formuliert werden: sag mir (endlich) bzw. du sollst mir endlich sagen.

Wie bei den Rückfragen liegt auch hier eine Art 'Zitat' einer Vorgängeräußerung vor, die aber in diesem Fall als 'Selbstzitat' zu verstehen ist. ${ }^{21}$ Aufgrund des Zitatcharakters werden wieder (ob/w-)Verb-Letzt-Sätze verwendet. Vielleicht rührt daher auch die Ultimativität, die bei den Nicht-Verb-LetztVarianten fehlt, da sie zumindest in der Form von den rein initiativen 'neuen' Fragen nicht unterschieden sind.

Das Tonmuster dagegen ist obligatorisch fallend. Eine zusätzliche Frageeinstellung wie bei den Rückfragen ist nicht vorhanden, sondern es wird nur der ursprüngliche Sprechakt der Frage 'zitiert'. Während sich also bei den Rückfragen diese Frageeinstellung (auch) auf den Funktionstyp oder die Illokution der Vorgängeräußerung bezieht, bestätigt der Sprecher bei den ultimativen Fragen die Frageeinstellung seiner Vorgängeräußerung (daher das eher 'assertive' fallende Tonmuster, und daher auch scheint eine Ergänzung durch eine assertive - oder imperativische - Einbettung hier eher gerechtfertigt als z.B. bei den deliberativen Fragesätzen).

Aus dieser Bestätigung der Frageeinstellung ergibt sich auch unmittelbar ein Unterschied $\mathrm{zu}$ den Rückfragen: Bei den ultimativen w-Fragen wird tatsächlich nach einer Füllung der Leerstelle verlangt, während die auf einen w-Fragesatz bezogenen Rückfragen wie alle anderen Rückfragen auch Polaritätsfragen (Ja/Nein-Fragen) sind.

$21 \quad$ Für die enge Verwandtschaft spricht auch, daß sich parallel zu den ultimativen Fragen 'ultimative Aufforderungen' bilden lassen, in deren stärkster Version (schwächere Versionen modifizieren den imperativischen Formtyp durch Modalpartikeln wie schon und Adverbiale wie gefälligst, endlich usw.) dasselbe Mittel der Funktionstyp-Propositionalisierung benutzt wird wie bei den Rückfragen, nämlich ein mit sollen modalisierter Aussagesatz.

(i) A: Mach die Tür zu!

(Angesprochener reagiert nicht)

A: Du sollst die Tür zumachen! 
Selbständige Verb-Letzt-Fragesätze treten also in drei Varianten auf: erstens als (weder nach vorwärts noch nach rückwärts sequenzgebundene) deliberative Fragesätze (mit der typischen Modalpartikel wohl, sowie nur und blo $\beta$ bei den $w$-Fragesätzen), die als Gegenstücke zu den Verb-Erst- und $w$-VerbZweit-Fragesätze keine oder zumindest eine abgemilderte Antwortobligation auslösen; zweitens als (modalpartikellose) sequenzgebundene Rückfragen auf Vorgänger-Fragesätze, deren Frageeinstellung und propositionalen Gehalt sie 'zitieren' und - angezeigt durch das obligatorisch steigende Tonmuster - zum Gegenstand einer neuen (polaren) Frageeinstellung machen; drittens als ebenfalls sequenzgebundene ultimative Fragen (wiederum ohne Modalpartikel), die eine ungenügend beantwortete Vorgängerfrage 'zitieren' (mit fallendem Tonmuster) und benützt werden, um auf einer angemessenen Antwort zu bestehen.

\subsubsection{Fragesatz oder Imperativsatz? Ein problematischer Typ von ob-Sätzen}

Bestimmte $o b$-Verb-Letzt-Sätze sind aufgrund ihrer Funktion eindeutig unter die Aufforderungen zu rechnen:

(79) Ob du wohl den Mund hältst!

(80) Ob hier wohl gleich Ruhe herrscht!

Mindestens in ihren nicht-intonatorischen Merkmalen gleichen diese Sätze den deliberativen ob-Fragesätzen. Von der Funktion her handelt es sich aber ganz klar um Aufforderungen, sogar um sehr starke, ultimative.

Typisch, aber nicht obligatorisch für diese Konstruktionen ist daher die Einfügung eines Temporaladverbs wie gleich (seltener: sofort); allerdings scheint auch das an sich eher abschwächende mal möglich, das den Zeitpunkt der verwirklichung der Aupforderung nicht festlegt.

(81) Ob du wohl gleich/sofort/(?)mal den Mund hältst!

Die Verwendung von indikativischen Verbformen ist obligatorisch. Eindeutige Imperativformen der 2.Ps.Sg. dürfen nicht gebraucht werden (entsprechend muß ein Subjektsausdruck vorhanden sein):

(82) Ob du wohl anständig von deiner Tante 'sprich/sprichst!

Auch die zu Sie-Imperativsätzen äquivalenten Versionen müssen mit ind:kativischen Verbformen gebildet werden:

(83) Ob Sie wohl still "seien/sind! 
Mit den als Aufforderungen zu interpretierenden Aussagesätzen (Beispiel: $D u$ wäschst dir sofort die Hände!) teilen die ob-Aupforderungen die - durch den Aufforderungscharakter erklärbare - Beschränkung der Tempusformen auf Präsens und Futur I (wie bei jenen sind marginal aber auch Perfekt und Futur II verwendbar).

(84) Ob du wohl still bist/sein wirst/"warst!

Ebenfalls wie bei den 'auffordernden' Aussagesätzen (und den da $\beta$-Aufforderungssätzen) sind alle möglichen Typen von Subjektsausdrücken verwendbar, solange eine Aufforderung erschließbar ist. Es gibt also nicht wie bei den prototypischen Imperativsätzen eine Beschränkung auf Ausdrücke, die die Hörerschaft bezeichnen, obwohl diese auch hier den Großteil der Subjektsausdrücke stellen. Vgl. Beispiel (80) oben und:

(85) Ob der Fernseher wohl gleich aus ist!

Entsprechend dem Indikativ-Gebot können jedoch keine den Heischesätzen (vgl. zu diesen Oppenrieder 1987:173ff.) direkt entsprechenden Verb-Letzt-Sätze mit Konjunktiv I gebildet werden.

(86) Ob dich wohl gleich der Teufel "hole/holt!

Gerade die Restriktionen bei der Tempusverwendung zeigen deutlich den Aufforderungscharakter der entsprechenden $o b$-Sätze. Dafür, daß die ob-Sätze selbst keine Imperativsätze im eigentlichen Sinn sind, spricht jedoch - neben der Verwendung von $a b$ - die Tatsache, daß die einzige Modalpartikel, die in diesen Konstruktionen zulässig und sogar obligatorisch ist, dieselbe ist, die auch im deliberativen ob-Verb-Letzt-Fragesatz verwendet wird, nämlich wohl. Diese Modalpartikel tritt auch in einem nahezu äquivalenten speziellen VerbErst-Satz mit imperativischer Interpretation auf.

Aufforderungstypische Modalpartikeln, unter anderem die betonten ja und $b l o \beta$, sind (im Gegensatz zum $d a \beta$-Aufforderungssatz) nicht zulässig. Die Verwendung des dativus ethicus ist nicht möglich. Genausowenig kann das für starke Aufforderungen typische Gegenstück zu bitte, nämlich gefälligst, kaum eingefügt werden.

(87) Ob du wohl/"JA/*bloß/"mir/??gefälligst gleich den Mund hältst!

Es gibt $z u$ diesen ob-Aufforderungen auch eine uneingeleitete Verb-ErstVariante (vgl. Thurmair 1989:143f.), die sich in Bezug auf Partikeldistribution haargenau gleich verhält (allerdings ist die Modalpartikel wohl nicht obligato- 
risch). Auch in diesem Fall deutet von der Form her vieles auf einen Fragesatz und nicht auf einen Imperativsatz hin, von der Funktion her handelt es sich jedoch wieder um starke Aufforderungen.

(88) Hältst du (wohl) gleich den Mund!

Eine weitere Besonderheit beider Typen ist die stark verminderte Akzeptabilität syntaktischer Negationen; negative Formulierungen von Aufforderungen sind nicht erlaubt:

(89) Bleib hier!/Lauf nicht weg!

(90) Daß du ja hierbleibst!/Daß du ja nicht wegläufst! vs.

(91) Ob du wohl hierbleibst!/??Ob du wohl nicht wegläufst! oder

(92) Machen Sie keine Sperenzien!

(93) Daß Sie ja keine Sperenzien machen! vs.

(94) 'Ob Sie wohl keine Sperenzien machen!

vgl. aber die 'implizit negative' Formulierung:

(95) Ob Sie wohl gleich aufhören, Sperenzien zu machen!

Ein weiteres formales Fragemerkmal neben dem Satzeinleiter $o b$ und der Modalpartikel wohl, das nach den von uns erhobenen Produktionsdaten bei den ob-Aufforderungen vorkommt (und auch bei den Verb-Erst-Versionen zulässig ist), ist ein steigender Melodieverlauf. Allerdings weicht er in seiner Feinstruktur von der typischen 'Frageintonation' ab.

Die Abweichung kommt im wesentlichen dadurch zustande, daß ein ganz spezifisches Betonungsmuster vorliegt, das sich von dem normaler Fragesätze deutlich unterscheidet: Betont ist nämlich nicht nur der Ausdruck, auf den nach den allgemeinen Regeln der Fokus-Hintergrund-Gliederung ein Akzent fallen muß (zudem scheint sich die hier realisierte Tonbewegung von der bei den sonstigen Fragesätzen typischen Tonbewegung zu unterscheiden), sondern auch das satzeinleitende $o b$ ist akzentuell hervorgehoben. Welcher der beiden prinzipiell vorhandenen Akzente tatsächlich als der stärkere realisiert wird, ist damit noch nicht festgelegt; es kann auch zu einem 'Akzentsplitting' mit annähernd gleich starken Hervorhebungen kommen:

(96) $O B$ du wohl gleich einen DIEner machst!

(97) $O B$ du wohl gleich schläfst!

Eine vergleichbare Betonung findet sich auch bei den parallelen Verb-ErstSätzen, bei denen das finite Verb in der Spitzenposition Akzentträger ist.

(98) LASST du gleich meinen Chomsky liegen! 
Eine Möglichkeit ist, diese Hervorhebungen als emphatische oder expressive nicht-fokale Akzente $\mathrm{zu}$ beschreiben, also parallel zu den Exklamativakzenten $z u$ behandeln. Dafür könnte sprechen, daß wie in den Exklamativsätzen Akzentsplitting vorkommt.

Andererseits könnte es sich durchaus um einen Fokusakzent handeln (es läge dann natürlich Doppelfokussierung vor). Bei den Verb-Erst-Sätzen ist das finite Verb hervorgehoben, ganz gleich ob es sich um ein 'Inhaltsverb' oder ein Hilfsverb handelt: Das von der Hervorhebung betroffene Inhaltselement kann folglich nur der 'Wahrheitsstatus' des im Satz Ausgedrückten sein (vgl. Höhle 1988). Durch diese Akzentuierung wird also das tatsächliche Bestehen des im Satz beschriebenen Sachverhalts angezeigt; im Verbund mit der Fragesatzform wird nach dem tatsächlichen Bestehen allerdings nur gefragt. Bei der VerbLetzt-Variante wird mit $o b$ ein Ausdruck betont, der die Unentschiedenheit dieses Tatsachenstatus anzeigt. Beide Male ist eine fokus-explizierende Paraphrase z.B. ist es (gleich/sofort) der Fall, daß. Die Aufforderungsinterpretation ergibt sich aus der Tatsache, daß eine positive Antwort nur dann wahrheitsgemä $\beta$ sein kann, wenn der vom Satz bezeichnete Sachverhalt auch tatsächlich realisiert wird.

Eine solche Beschreibung klassifiziert die beiden Typen als die 'starken' Varianten der höflichen Aufforderungen in Fragesatzform. Die Fragesatzform wird in diesem Fall natürlich gerade nicht zur Abmilderung verwendet (daher finden sich z.B. auch keine Konjunktiv-II-Formen von Modalverben). Die besondere Stärke kommt durch die Hervorhebung desjenigen Ausdrucks zustande, der den 'Wahrheitsstatus' anzeigt: Ausschließlich die Realisierung des angesprochenen Sachverhalts ist von Interesse. Daneben wirken auch die oben angesprochenen Temporaladverbien verstärkend. ${ }^{22} \mathrm{Vgl}$. die Gegenüberstellung der verbindlichen und der ultimativen Aufforderungen:

(99) Gibst du mir (bitte) mal das Salz? vs.

(100) GIBST du mir (wohl) gleich das Salz! und

(101) Ob du mir (wohl) mal das Salz gibst? vs.

(102) $O B$ du mir wohl gleich das Salz gibst!

Die Fragesatzform erklärt wohl auch, warum negativ formulierte Aufforderungen unzulässig sind: Entscheidungsfragesätze werden nur unter speziellen Bedingungen negativ formuliert - aufgrund ihrer Polarität sind positive und

$22 \quad$ Dieselben Temporaladverbien lassen sich natürlich auch in echten Fragen verwenden. Ein Unterschied besteht aber darin, daß sie nur in diesen auch betont werden können.

(i) Kommst du denn GLEICH herüber?

(ii) *KOMMST du wohl GLEICH herüber!

Die Hervorhebung der Faktizität blockiert jede weitere (kontrastive) Hervorhebung. 
negative Versionen ja im Prinzip gleichwertig. Insbesondere das Negationsadverb nicht tritt in zwei verschiedenen Funktionen auf, erstens als unbetonte Modalpartikel (Thurmair 1989:86f., 160ff.; vgl. dagegen Meibauer 1988), die anzeigt, daß eine bestätigende Antwort erwartet wird, und zweitens als echter Negationsträger. Mit einem Modalpartikel-nicht läßt sich gar keine negative Aufforderung formulieren. Im zweiten Fall ist zwar ein echter Negationsträger vorhanden, der aber selbst betont ist und insbesondere (im Gegensatz zur Modalpartikel nicht) die Betonung des finiten Verbs mit dem damit verbundenen Effekt der Fokussierung der Faktizität blockiert. Damit entfällt die Grundvoraussetzung für die Bildung der in diesem Abschnitt untersuchten 'ultimativen Aufforderungen', seien diese ob-Sätze oder Verb-Erst-Sätze.

Die Verwendung der eigentlich eher abschwächend wirkenden Modalpartikel wohl bleibt allerdings etwas mysteriös. Vielleicht dient sie nur dazu zu signalisieren, daß keine echte Frage vorliegt. Die weitere Interpretation läuft hier natürlich anders als bei den deliberativen Fragesätzen. Zentral für die Aufforderungsinterpretation ist das Akzentmuster und damit die intonatorische Gestalt. Die Fragemerkmale dieser 'ultimativen' Aufforderungen sind aber so deutlich ausgeprägt, daß sie die Einordnung als echte Aufforderungssätze trotz der akzentuellen Eigenheiten nicht gestatten.

Immerhin reichen diese zusammen mit der Modalpartikel wohl aus, um die Einbettung bei den ob-Sätzen zu blockieren, die damit zum Typ IV gehören: Syntaktisch zulässige Ausdrücke wie ich frage (dich/euch/Sie) sind außerdem nicht 'interpretationserhaltend'.

\subsubsection{Verb-Letzt-Imperativsätze}

Aufforderungen lassen sich auch mit einem weiteren Typ von Verb-LetztSätzen vollziehen, nämlich mit daß-Sätzen (vgl. Thurmair 1989:54f.):

(103) Daß du keine Sperenzien machst!

Hier spricht einiges dafür, daß es sich um einen echten Verb-Letzt-Aufforderungssatz handelt. Allerdings ist, anders als bei den Fragesätzen mit $o b$, kein aup Imperativsätze beschränkter Satzeinleiter vorhanden, sondern nur das unspezifische nicht-interrogative daß. Eine Folge war weiter oben in 1.3.1.2 schon zu sehen: Zur Propositionalisierung des Funktionstyps Aufforderung wird bei Rückfragen nicht ein typischer Satzeinleiter, sondern ein Modalverb - im Normalfall sollen - verwendet. Die Kennzeichnung als Aufforderung kann also nicht nur über den Satzeinleiter geschehen. Andere Merkmale müssen dazukom- 
men, um $d a \beta$-Imperativsätze von den anderen selbständigen $d a \beta$-Sätzen abgrenzen zu können.

Gegenüber den daß-Exklamativsätzen wirken zum einen die spezifischen Modalpartikeln, zum anderen das Akzentmuster und dessen intonatorische Realisierung unterscheidend. Bei den Imperativsätzen kann kein Exklamativakzent verwendet werden. Wenn auch in beiden Fällen ein global fallendes Tonmuster vorkommt, so unterscheiden sich die Akzentrealisierungen (Fokusakzent vs. Exklamativakzent) doch noch hinreichend voneinander (vgl. Batliner 1988; Oppenrieder $1988 \mathrm{~b})$.

Gegenüber den - zentralen, irrealen - daß-Wunschsätzen lassen sich die daß-Imperativsätze durch die Verbmorphologie abgrenzen: Während für diese Wunschsätze Konjunktiv-II-Formen konstitutiv sind, dürfen in den Imperativsätzen (wie in den oben behandelten 'ob-Aufforderungen' und den Aussagesätzen mit Aufforderungsinterpretation) nur indikativische Formen verwendet werden.

Das bedeutet aber auch, daß eindeutige Imperativformen ausgeschlossen (und Subjektsausdrücke damit obligatorisch) sind:

(104) Daß du ja keine Schokolade "nimm/nimmst!

Auch 'Heischeformen' (imperativisch $\mathrm{zu}$ interpretierende Konjunktiv-I-Formen) sind kaum akzeptabel:

(105) Daß mir nur ja der Topf peinlich sauber ??sei/ist!

Unmarkierte Tempusform ist das Präsens. Das Futur I ist gegenüber der Verwendung in 'ob-Aufforderungen' und 'auffordernden Aussagesätzen' weitaus markierter. Zulässig ist auch das Perfekt (Futur II dagegen kaum mehr):

(106) Daß du dem Herrn Pfarrer ja die Hand gibst/?die Hand geben wirst!

(107) Daß du um 5 Uhr ja abgespült hast/??abgespült haben wirst!

Neben den normalen aktivischen können auch passivische Konstruktionen in diesem Typ von Imperativsatz verwendet werden.

(108) Daß die Türe ja fest abgesperrt wird!

Im Gegensatz zu den prototypischen Aufforderungssätzen, mit denen man sich an eine angesprochene Person oder Personengruppe richtet, die entweder vom Sprecher unterschieden ist (Imperativsätze der 2.Ps., Sie-Imperativsätze) oder diesen mit einschließt (Adhortativsätze), sind die daß-Imperativsätze flexibler, da sie alle möglichen Typen von Subjekten zulassen. Sie gleichen darin den Aussagesätzen, die als Aufforderungen verstanden werden können, müssen aber, anders als diese, schon aufgrund ihrer Form und ohne Stützung durch den 
Kontext (und eine spezifische lexikalische Füllung) 'imperativisch' interpretiert werden. Bezeichnet das Subjekt nicht den oder die Angesprochenen, so wird allerdings nicht direkt zu etwas aufgefordert, sondern es wird die Erwünschtheit eines bestimmten Sachverhalts ausgedrückt. Damit hätte man im Prinzip einen imperativischen Satztyp, der die, außer in formelhaften Wendungen, nur noch in bestimmten Textsorten (Koch- und sonstige Gebrauchsanweisungen) verwendeten Heischesätze (vgl. Oppenrieder 1987:173ff.) zu ersetzen erlaubt.

Dem steht jedoch entgegen, daß diese Verb-Letzt-Imperativsätze ein engeres Anwendungsspektrum haben als die 'klassischen' Imperativsätze mit Erst- oder Zweit-Stellung des Finitums. Mit ihnen lassen sich nämlich nur 'starke' Aufforderungen (oder Wünsche) realisieren. ${ }^{23}$

Dies zeigt sich gerade an den zulässigen Modalpartikeln. (Die Modalpartikelverwendung ist in daß-Imperativsätzen zwar nicht obligatorisch - vgl. oben (103) -, stellt aber den Normalfall dar; infolgedessen ist eine Einbettung durch einen 'Matrixsatz', der wiederum höchstens eine Paraphrase imperativischer oder imperativverträglicher Einstellungen sein könnte, auch bloß bedingt möglich.) Die mit dem daß-Imperativsatz verträglichen Modalpartikeln umfassen nämlich nur eine Teilmenge derjenigen, die in den restlichen Typen von Imperativsätzen eingefügt werden können. Dazu vergleiche man die Tabellen 5 und 7 in Thurmair (1989): In Verb-Letzt-Imperativsätzen können doch, eben, einfach, halt, mal, ruhig und schon, anders als in den typischeren Imperativsätzen, nicht auftreten: Mit ruhig wird eine Erlaubnis angezeigt; eben, einfach und halt unterstützen eine Ratschlagsinterpretation; mal mildert durch die fehlende Festlegung eines Realisierungszeitraums den Aufforderungscharakter ab; schon zeigt an, daß aup mögliche Gegengründe des Angesprochenen Bezug genommen wird; doch ist für 'schwächere' Interpretationen von Imperativsätzen, z.B. als Ratschläge, zumindest offen (vgl. die jeweiligen Bedeutungsbeschreibungen in Thurmair 1989:Kap. 2).

Die typischste Modalpartikel der $d a \beta$-Imperativsätze ist das 'emphatisch' betonte $J A .{ }^{24} \mathrm{Da}$ sie in den anderen Typen von Imperativsätzen eine besondere Stärke der Aufforderung anzeigt, paßt sie genau zu den daß-Imperativsätzen. Besondere Stärke heißt, daß eine Warnung vor den Folgen impliziert ist, die bei einer Nicht-Befolgung der Anweisung oder beim Nicht-Eintreten des erwünschten

23 Wie bei den Fragesätzen die Verb-Letzt-Form zu einer Einengung des Anwendungsbereichs auf die Formulierung 'deliberativer' Fragen führt (bei den sequenzgebundenen Typen sind die Verwendungsmöglichkeiten natürlich ebenfalls beschränkt), so kann auch hier bei den Imperativsätzen die VerbLetzt-Form für die Einschränkung der Gebrauchsmöglichkeiten verantwortlich gemacht werden (vgl. Altmann 1987:49). Dieser Effekt kann bei den Exklamativ - und Wunschsätzen allerdings nicht beobachtet werden.

24 Es handelt sich nicht um einen Fokusakzent, vgl. Oppenrieder/Thurmair (1989:33). 
Zustands drohen (nicht immer handelt es sich um eine Sanktionsandrohung); die Warnung kann durch nachgeschobene Sätze verdeutlicht werden.

(109) Daß ihr JA meinen Dackel in Ruhe laßt! Oder ihr werdet euer blaues Wunder erleben.

(110) Daß Sie JA eine Hose mit Gemsledereinsatz anziehen! Sonst werden Sie nach zwei Stunden nicht mehr sitzen können.

Das in seiner Wirkung ähnliche, 'emphatisch' betonte BLOSS wird ebenfalls, wenn auch seltener, verwendet.

\section{(111) Daß du BLOSS deinen Mund hältst!}

Das nicht betonte bloß und sein Aquivalent nur lassen sich ebenfalls verwenden, allerdings bezieht sich die 'Stärke' in diesem Fall hauptsächlich auf einen anderen Aspekt der Aufforderung - bzw. des Ausdrucks der Erwünschtheit: In diesem Fall impliziert der Sprecher ein besonderes Interesse an der Ausführung der Handlung - bzw. am Eintreten des erwünschten Zustands. Dementsprechend kann auch keine explizite Sanktionsandrohung angehängt werden.

(112) Daß du nur/bloß nicht nachgibst! ("Sonst mache ich dir Beine.)

Nur und emphatisches $J A$ lassen sich jedoch kombinieren; die beiden Bedeutungskomponenten scheinen sich einfach aufzuaddieren:

(113) Daß du nur JA deine Aufsätze rechtzeitig fertigbringst!

Die entsprechenden Modalpartikeln haben übrigens noch eine weitere Gemeinsamkeit, die sie von den restlichen prinzipiell imperativsatzverträglichen Modalpartikeln abgrenzen: Nur sie lassen sich in einem anderen Typ von NichtStandard-Aufforderungen verwenden, den verblosen, 'nominalen Aufforderungen'.

(114) Nur/bloß/BLOSS $/ J A /{ }^{*}$ doch $/{ }^{*}$ eben $/{ }^{*}$ einfach $/{ }^{*}$ halt $/{ }^{*} \mathrm{mal} /{ }^{*} \mathrm{ruhig} /{ }^{*}$ schon keine Panik/keinen Sellerie!

Positiv formulierte 'nominale Aufforderungen' sind allerdings nur mit den betonten Modalpartikeln völlig akzeptabel (vgl. aber Nur Mut!/Nur vorwärts!).

(115) ??Nur/??bloß/BLOSS/JA viel Bewegung!

Enthalten diese frei verwendeten infiniten Strukturen (vgl. dazu Fries 1983) ein nicht-finites Verb, können jedoch auch einfach und mal verwendet werden (vgl. auch Thurmair 1989:64ff.):

(116) Alle mal aufpassen/aufgepaßt!

(117) Einfach draufhalten! 
Die $d a \beta$-Aufforderungen stehen also diesen Randtypen eines formbestimmten Satzmodussystems ziemlich nahe, auch sie gehören eher zur Peripherie dieses Systems. Der gemeinsame Nenner könnte die hohe 'Nominalität' sein (eingebettete $d a \beta$-Sätze sind ja im allgemeinen alternative Strukturen zu NPn). Allerdings kommt ein bestimmter 'abtönender Ausdruck' nur in finiten Aufforderungssätzen vor (nicht in den sprecherinkludierenden Adhortativsätzen), während er bei den infiniten Typen unzulässig ist (vgl. Fries 1983:29): der ethische Dativ mir (in Thurmair 1989:39ff., 195ff. wird er zu den Modalpartikeln gezählt). ${ }^{25}$

(118) Daß du mir den Herrn Sekretär grüßt!

Auch mit der Verwendung eines mir (evtl. auch der Pluralform uns) in der Funktion des ethischen Dativs zeigt der Sprecher sein Interesse an der Ausführung der Handlung, bzw. am Eintreten des erwünschten Zustands an (vgl. Jacobs 1986:105 .), insofern eignet es sich ohne weiteres für die Verwendung in der 'starken' Verb-Letzt-Form. ${ }^{26}$

Die besondere Stärke der Verb-Letzt-Imperativsätze läßt sich auf dieselbe Weise erklären wie die besondere Stärke der 'auffordernden Aussagesätze'.27 In beiden Fällen können nur indikativische Formen verwendet werden. Der zu realisierende Sachverhalt wird ohne modale Abmilderung formuliert, d.h. so, als käme es auf jeden Fall zu seiner Verwirklichung. Dabei sind die daß-Aufforde-

25 Ebenfalls nicht in infiniten Strukturen, wohl aber in $d a \beta$-Imperativsätzen kommt auch vor. Seine Verwendung scheint mir ein gewisses Autoritätsgefälle vorauszusetzen, insofern ist das Auftreten in den 'starken' da $\beta$-Imperativsätzen erklärlich:

(i) Daß Sie sich auch anständig benehmen!

Auch das süddeutsche $f e i$ ist in infiniten Strukturen zumindest nicht sehr akzeptabel.

(ii) ?Fei gut aufpassen!

In den $d a \beta$-Imperativsätzen hat es wohl eine ähnliche interesseanzeigende Funktion wie nur. Es ist allerdings nicht auf negative Formulierungen eingeschränkt:

(iii) $D a \beta$ du dich fei warm anziehst!

26 Die Blockade in infiniten Strukturen könnte darauf zurückzuführen sein, daß derartig formulierte Aufforderungen eher als allgemeingültig (keine durch Finitheit angezeigte temporale Verankerung), jedenfalls nicht allein im Interesse des Auffordernden liegend, verstanden werden (daher die Verwendung in Kommandos). Der ethische Dativ zeigt aber gerade an, da $\beta$ die Erfüllung zugunsten und im Interesse des Auffordernden erfolgt.

27 Aussagesätze mit permissivitätsanzeigenden Ausdrücken, z.B. mit dem Modalverb dürfen, können selbstverständlich nicht in starken imperativischen Sprechakten gebraucht werden. Besonders nachdrückliche Aufforderungen lassen sich dagegen realisieren, wenn Futurformen verwendet werden. Immer bleibt jedoch die imperativische Interpretation von der lexikalischen Füllung (z.B. Subjektsausdrücken, die den oder die Angesprochenen bezeichnen; Verben mit einer 'Handlungsbedeutung') und dem Verwendungskontext abhängig. Dies trifft auf daß-Imperativsätze gerade nicht $z u$. 
rungen ausnahmslos stark, da hier auch keine Modalverben verwendet werden dürfen (daß Futurformen nicht rein temporal, sondern eben auch mit einem deutlichen modalen Bedeutungsanteil verwendet werden, könnte der Grund dafür sein, daß sie in den $d a \beta$-Imperativsätzen kaum akzeptabel sind). Vgl.:

(119) Du läßt den Hund aus dem Sack! vs.

(120) Daß du den Hund aus dem Sack läßt! mit

(121) Du kannst den Hund aus dem Sack lassen. vs.

(122) 'Daß du den Hund aus dem Sack lassen kannst!

((122) ist - mit anderer intonatorischer Form - in der Exklamativlesart akzeptabel.)

Derjenige, der solche indikativischen Aufforderungen äußert, muß natürlich auch eine entsprechende Position innehaben, um die Realisation durchsetzen zu können (ansonsten verstieße er gegen die Gricesche Maxime der Qualität, wenn er jede modale Abmilderung unterläßt). Daher werden gerade die da $\beta$-Aufforderungen als implizit sanktionsandrohend verstanden.

Dies gilt allerdings dann nicht, wenn sich die Stärke nur aus dem besonderen Interesse des Sprechers an der Realisierung des erwünschten Sachverhalts ergibt. Derartige Verwendungen von $d a \beta$-Imperativsätzen sind jedoch seltener als die in sanktionsandrohenden, bzw. vor negativen Folgen warnenden Aufforderungen. Beispiele mit den (unbetonten) Modalpartikeln nur und blo $\beta$ werden, wie oben erwähnt wurde, in diesem Sinn verstanden (vgl. aber wiederum die Kombination nur $J A$, in der durch das $J A$ zusätzlich eine Warnung vor den Folgen bei einer Nichtrealisierung impliziert ist). Eine Parallele finden sie in Verb-ErstImperativsätzen, die aufgrund der lexikalischen Füllung bevorzugt als 'Wünsche' interpretiert werden, vgl.:

(123) Erkälte dich nicht!/Erkälten Sie sich nicht!

(124) Daß du dich nur nicht erkältest!

Aber auch hier legt nicht unbedingt schon die lexikalische Füllung die 'Wunsch'-Interpretation fest:

(125) Erkälte dich JA nicht!/Daß du dich JA nicht erkältest!

Eine Besonderheit dieser als 'Wünsche' zu interpretierenden daß-Sätze ist, da $\beta$ sie im Normalfall negativ formuliert werden, vgl.:

(126) Daß du nur nicht krank wirst! vs.

(127) ?Daß du nur gesund bleibst!

Für die positiv formulierten Wünsche wird $d a \beta$ als Satzeinleiter im Normalfall durch wenn ersetzt: Bis aup den Indikativ gleichen diese Sätze dann den typi- 
schen 'irrealen' Wunschsätzen; auch ist nur eine wunschsatztypische Modalpartikel.

\section{(128) Wenn du nur gesund bleibst!}

Man hat an dieser Stelle des Satzmodussystems also ein eindeutiges Beispiel für dessen im Prinzip 'prototypische Strukturierung' (vgl. dazu Oppenrieder 1988b:186ff.): Es gibt keine klar voneinander abgegrenzten, in sich homogenen Kategorien, sondern zentrale, prototypische Fälle in den Kernbereichen der einzelnen Kategorien, die in eine Peripherie weniger typischer Vertreter der entsprechenden Kategorie übergehen.

Auf den Bereich der selbständigen Verb-Letzt-Sätze bezogen heißt das, daß es zentrale Fälle von Verb-Letzt-Imperativsätzen gibt, nämlich sanktionsimplizierende, die die 'emphatisch' betonten Modalpartikeln JA oder BLOSS enthalten. Daneben finden sich $d a \beta$-Imperativsätze, die lediglich die Erwünschtheit eines Zustands ausdrücken; typischerweise werden hier die unbetonten Modalpartikeln nur und blo $\beta$ verwendet. Dies sind Modalpartikeln, die ihrerseits in den 'irrealen' Wunschsätzen, auch im durch wenn eingeleiteten Verb-Letzt-Typ, zulässig sind. Bel den wenn-Wunschsätzen gibt es ebenfalls eine periphere Variante, nämlich eine mit indikativischen Verbformen (Typ $B$ in der Klassifikation der 'idiomatischen wenn-Verb-Letzt-Sätze' bei Scholz; vgl. Scholz 1989:46ff.).28 Diese Variante ähnelt, wie gesehen (vgl. Beispiel (128)), den als Wunsch zu interpretierenden daß-Imperativsätzen sehr stark. Ein einfacher Austausch der Satzeinleiter hat (zumindest bei einer negativen Formulierung) keinen besonders großen Effekt. Möglicherweise liegt aber auch eine vollständige Uberlappung der peripheren Bereiche der Verb-Letzt-Imperativ- und Wunschsätze vor, denn schließlich gibt es auch noch $d a \beta$-Wunschsätze ( $D a \beta$ ich doch ein Bäcker wäre!). Diese

28 Der Typ B scheint mir nicht sehr gut in die Liste der übrigen von Scholz angenommenen idiomatischen wenn-Sätze zu passen. Sie zählt noch drei weitere Typen auf, die hier durch je ein Beispiel angedeutet werden sollen (vgl. Scholz 1989:39ff.):

(i) Wenn Sie bitte mal einen Augenblick aufpassen könnten?

(ii) Wenn er auch immer Zigarren schnorren muß!

(iii) Prototypizität! - Wenn ich sowas schon höre!

Lediglich der durch (i) repräsentierte Typ hat noch eine gewisse funktionale Verwandtschaft mit den prototypischen Wunschsätzen. Die Erwünschtheit der Realisierung eines Sachverhalts wird in den beiden restlichen Fällen gerade nicht ausgedrückt. Bei den 'indikativischen Wunschsätzen' besteht hingegen eine doch sehr weitgehende Ahnlichkeit mit den zentralen Wunschsatztypen. Nicht zuletzt zeigt sich das an der Verwendung der wunschsatztypischen Modalpartikeln nur und bloß. Daß die 'kontrafaktische' Modalpartikel doch nicht verwendbar ist, läßt sich damit erklären, daß mit diesen indikativischen Strukturen eben keine kontrafaktischen, irrealen Wünsche ausgedrückt werden können. 
sind zwar im Gegenwartsdeutschen nicht mehr gebräuchlich, aber wenn es zu den wenn-Wunschsätzen indikativische Varianten gibt, dann spricht im Prinzip nichts dagegen, auch für die $d a \beta$-Wunschsätze eine solche (noch gebräuchliche) Variante anzunehmen, die von den daß-Imperativsätzen nicht mehr unterscheidbar ist (auch in Thurmair 1989:55 werden derartige Strukturen als "Sonderfall der daß-Wunschsätze" charakterisiert).29

Eine Voraussetzung für diese Überlappung ist die funktionale Verwandtschaft von Imperativsätzen und Wunschsätzen. Beide Male wird ausgedrückt, daß der repräsentierte Sachverhalt in einer 'möglichen Welt' realisiert ist; die einzelnen Typen von Imperativsätzen und Wunschsätzen unterscheiden sich aber durch die verschiedene Zugänglichkeit dieser Welt von der Welt des Sprechzeitpunktes aus (vgl. dazu Oppenrieder 1987:186, Fn. 23).

Die 'irrealen' Wünsche, die durch die prototypischen Wunschsätze mit Konjunktiv-II-Markierung des Verbs ausgedrückt werden, sind dadurch ausgezeichnet, da $\beta$ der Sprecher einerseits eine Realisierung (das Erreichen der 'Wunschwelt' von der 'Sprechwelt' aus) präferiert, andererseits weiß, daß er eine solche Realisierung nicht selbst herbeiführen kann. Ist die zweite Bedeutungskomponente abgeschwächt (eine Realisierung ist im Prinzip möglich), dann erhält man Wünsche, wie sie durch indikativische wunschsätze ausgedrückt werden können.

Auch bei Aufforderungen wird im Regelfall die Verwirklichung des repräsentierten Sachverhalts vom Sprecher präferiert werden. Insofern unterscheiden sich 'indikativische' wünsche und derartige Aufforderungen nicht wesentlich voneinander. Als Ergebnis kann vom einen zum anderen Formtyp ohne eine gravierende Anderung der Punktionalen Grundbedeutung übergewechselt werden. ${ }^{30}$

$\overline{29}$ Daß nicht automatisch alle nur-Versionen als wunschsätze klassifiziert werden können, sieht man an der zulässigen Modalpartikelkombination nur $J A$, bei der das $J A$ eindeutig dem imperativischen Satzmodus zugeordnet ist.

30 Als Grenzfall bei der allgemeinen Charakterisierung von Wunsch- und Aufforderungseinstellungen ist denkbar, da $\beta$ die 'Wunschwelt' bereits ein Teil der 'Sprecherwelt' ist, daß also die ausgedrückte Einstellung nicht 'ich wäre froh' (oder etwas Annliches), sondern 'ich bin Proh' ist. Das ist eine mögliche Beschreibung von Sätzen wie

(i) Daß du nur wieder da bist!

(Hier sind positive 'Wunschformulierungen' also ohne weiteres zulässig.) Gegen eine - alternative - Beschreibung als Exklamativ sprechen erstens

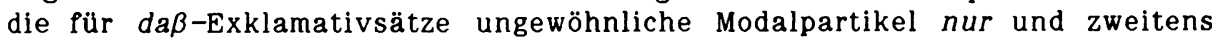
die exklamativuntypische Akzentrealisation auf $d a$; daneben paßt auch die ausgedrückte Einstellung, obwohl expressiv, nicht gut zu dieser Beschreibung. Für eine solche Einordnung könnte jedoch eine merkwürdige 'kausale' Variante sprechen, die auch bei den $d a \beta$-Exklamativsätzen möglich ist.

(ii) Weil du nur wieder da bist! 


\subsubsection{Verb-Letzt-Aussagesätze}

Den dritten 'klassischen' Satzmodus neben dem Frage- und dem ImperativModus bildet der Aussage-Modus.

Uberraschenderweise wird man jedoch bei der Suche nach einer Verb-LetztVariante des Aussagesatzes nicht fündig: "Ein V-L-Aussagesatz existiert nicht." (Altmann 1987:49)

Zumindest beim naheliegendsten Strukturtyp, dem daß-Satz, stößt man zwar auf Imperativsätze, Exklamativsätze und Wunschsätze, aber eben nicht auf solche daß-Sätze, die sich aufgrund des Verhaltens von satzmodussensitiven Ausdrücken (insbesondere Modalpartikeln, aber auch Satzadverbialien) und aufgrund der Verwendung zweifelsfrei als Verb-Letzt-Varianten der Verb-Zweit-Aussagesätze klassifizieren lassen. Ein Indiz dafür ist auch die Tatsache, daß in Rückfragen auf Aussagesätze die assertive Grundfunktion nicht durch den Gebrauch des Satzeinleiters $d a \beta$ propositionalisiert werden kann (vgl. oben 1.3.1.2).

Eine radikale Lösung, die die $d a \beta$-Sätze mit Aufforderungs-, Exklamativund Wunschinterpretation nicht als eigenständige Satztypen, sondern als Spezialisierungen einer ganz abstrakten assertiven Grundfunktion beschreibt, scheidet jedenfalls aus: Für Wunschsätze und Imperativsätze ist die Nicht-Assertivität (und nicht nur eine modifizierte Assertivität) wesentlich (vgl. die Anmerkungen zum Funktionstyp oben in 1.3.2 und Oppenrieder 1987:186, Fn. 23). Dementsprechend können auch bestimmte imperativ- und wunschsatztypische Modalpartikeln nicht in Aussagesätze eingefügt werden (vgl. die Tabellen in Thurmair 1989:49 und 62). Am ehesten ließen sich noch die daß-Sätze mit Exklamativinterpretation als illokutive Spezialisierungen einer abstrakten assertiven Sprechereinstellung analysieren. Die formale, nämlich intonatorische Verwandtschaft mit anderen Exklamativsätzen (Stichwort 'Exklamativakzent') spricht allerdings auch gegen eine solche Beschreibung.

Wenn sich unter den daß-Sätzen kein Kandidat für einen Verb-Letzt-Aussagesatz findet, dann bleibt nur übrig, sich unter anderen Typen von VerbLetzt-Sätzen umzuschauen, ob sich dort nicht welche finden, die als spezifische Typen von Assertionen verwendet werden. ${ }^{31}$

Kandidaten sind wo doch-Sätze, als ob-Sätze, und $o b / w$-Ausdrücke sowie die $w$-Schlagzeilen.

31 Nach Altmann (1987:49) gilt für selbständige Verb-Letzt-Sätze, daß "ein Sprecher mit ihnen eine propositionale Einstellung ausdrückt, die meist eine Spezialisierung der propositionalen Einstellung des entsprechenden Grundtyps ist". 


\subsubsection{1 wo doch-Sätze}

Ein möglicher Kandidat für selbständige Verb-Letzt-Aussagesätze sind bestimmte durch nicht-lokales wo eingeleitete Sätze mit global fallendem Tonverlauf, die fast immer die Modalpartikel doch enthalten (vgl. Thurmair 1989:58; Oppenrieder 1987:187, Fn. 28).

Die beiden typischen Verwendungen demonstrieren die folgenden Beispiele:

(129) Du wirst dich noch erkälten. Wo du doch nur ein dünnes Hemd anhast.

(130) Das ist vielleicht ein Knallkopf! Wo doch alles genau in der Gebrauchsanweisung steht.

(131) Warum werkelst du denn so lange an diesem blöden Verb-Letzt-Aufsatz herum? Wo ihn doch eh keiner liest.

(132) A: Max ist jetzt endgültig ans Bett gefesselt.

B: Wo er doch immer so gesund war.

In den ersten drei Beispielen wird durch den wo doch-Satz eine Begründung gegeben (vgl. zu dieser Verwendung auch Heidolph et al. 1981:801). Ob sich die Begründung auf die vorausgegangene sprachliche Handlung oder (auch) auf den propositionalen Gehalt bezieht, ist nicht immer klar. In (130) geht es z.B. primär um eine Rechtfertigung der 'exklamativen Beschimpfung', in (131) um die Erklärung für eine 'vorwurfsvolle Frage': Dementsprechend ist die Ersetzung durch einen weil-Satz (anders als bei (129)) auf jeden Fall ausgeschlossen, da mit diesem nur eine begründende Relation zwischen propositionalen Gehalten hergestellt werden kann.

Im Beispiel (132) wird dagegen eine konzessive Relation erschlossen, die zwischen zwei Sachverhalten besteht. Die Ersetzung des wo doch-Satzes durch einen Satz mit einem expliziten Anzeiger der Konzessivitätsrelation - z.B. obwohl - ist hier möglich.

Die begründend zu interpretierenden wo doch-Sätze müssen sich nicht immer auf sprachliche Handlungen beziehen:

(133) (A kauft am Obststand fünf Kilo Erdbeeren; zu B:) Wo sie doch so billig sind.

Das nicht-lokale wo in diesem Konstruktionstyp scheint also eine ziemlich unspezifizierte Relation $\mathrm{zu}$ bezeichnen. Die kausale Interpretation liegt am nächsten. $\mathrm{Zu}$ diesen Strukturen gibt es ein (im Gegenwartsdeutschen jedoch viel seltener gebrauchtes) Gegenstück: einen sehr peripheren Verb-Erst-Satz ebenfalls typischerweise mit doch als Modalpartikel.

(134) Du wirst dich noch erkälten. Hast du doch nur ein dünnes Hemd an. 
In beiden Fällen liegt eine spezifische begründende Variante des assertiven Modus vor. Eine expressive Bedeutungskomponente mag in manchen Fällen (weitaus deutlicher bei den wo doch-Sätzen) gegeben sein, sie rechtfertigt aber keinesfalls eine Einordnung der beiden Konstruktionen unter die Exklamativsätze. Zudem darf kein Exklamativakzent realisiert werden. Die Verb-Erst-Variante muß dabei unterschieden werden von einem spezifischen Typ von Exklamativsatz (oder aber emphatischem Aussagesatz ohne Vorfeldbesetzung; vgl. dazu Oppenrieder 1987:180):

(135) Hast du doch glatt nur ein dünnes Hemd an!

Die Expressivität ist wohl insbesondere ein Effekt der Modalpartikel doch (vgl. die Bedeutungsbeschreibung von doch bei Thurmair 1989:110ff.). Unklar ist, wieso hier ein offensichtlich nicht-lokal zu interpretierendes wo als Satzeinleiter verwendet werden kann. Ahnliches findet sich zum einen bei wo-Sätzen mit konditionaler Interpretation (mit ebenfalls sachverhaltsverknüpfendem wo), die jedoch bis auf ein paar Wendungen wie z.B. wo nicht (für wenn nicht) und als Bestandteil z.B. in wofern völlig verschwunden ist. Zum zweiten gibt es den süddeutschen 'Relativverstärker' wo, der bekanntlich unter bestimmtem Bedingungen auch alleine stehen kann (z.B. jeder, wo mich kennt). Es könnte sein, daß auch das hier interessierende satzeinleitende wo ein unspezifischer Relativsatzeinleiter ist, d.h. daß die wo doch-Sätze eine bestimmte Art von weiterführenden Relativsätzen sind. Allerdings sind sie, wie gesehen, auf eine im weitesten Sinn kausale Interpretation festgelegt.

Wenn sie den weiterführenden Relativsätzen zugeschlagen werden, dann dürften sie (nach den Ausführungen in 1.1.2.2) nicht $z u$ den selbständigen Verb-Letzt-Sätzen gezählt werden. Anders als die eindeutigen weiterführenden Relativsätze können sie jedoch dazu verwendet werden, eine nicht-sprachliche Handlung $\mathrm{zu}$ begründen (vgl. Beispiel (133)). Außerdem lassen sich nicht alle in den nicht-restriktiven Relativsätzen zulässigen Modalpartikeln verwenden, z.B. nicht ja; vgl.

(136) Du wirst dich noch erkälten. - Wo du ("ja) doch nur ein dünnes Hemd anhast. mit

(137) Du wirst dich noch erkälten. Was du ja/doch sicher nicht willst.

Selbst wenn die wo doch-Sätze auch den weiterführenden Relativsätzen ähneln, werden sie doch am geeignetsten als ein eigenständiger Typ von selbständigen Verb-Letzt-Sätzen mit einem spezifischen Satzeinleiter und einer, nahezu obligatorischen, spezifischen Modalpartikel (sowie einer Verb-Erst-Vari- 
ante) beschrieben, denen eine bestimmte Einschränkung der Assertion als Funktionstyp zugeordnet ist.

\subsubsection{Als ob-Sätze (Rhetorische Vergleiche)}

Bereits im Abschnitt über die verschiedenen Typen von Einleitungselementen (1.2) wurde angesprochen, daß auch die irrealen Vergleichssätze (vgl. zu deren Semantik bei nicht-selbständiger Verwendung Kasper 1987:134ff.), die durch die Vergleichspartikel als oder wie 'eingeleitet' werden, selbständig verwendet werden können. Sie werden in diesem Fall bei Weuster (1983:42ff.) und Thurmair (1989:59f.) zu den Aussagesätzen gezählt. Als echte Satzeinleiter finden sich hier $o b$ und wenn, daneben gibt es aber auch nicht-eingeleitete Verb-ErstSätze. Dabei sind nur nach als alle drei Typen von Satzstrukturen zulässig, während sich wie nur zusammen mit wenn-Sätzen verwenden läßt; die Doppelverwendung der beiden Vergleichspartikeln ist (mindestens im süddeutschen Bereich) möglich, und zwar in der Reihenfolge als wie, so da $\beta$ in diesem Fall aufgrund der wie-Dominanz nur ein wenn-Satz folgen kann. Vergleichspartikel und Satzstrukturen verteilen sich, bei Einbettung, also folgendermaßen:

(138) Du tust so, als ob/als wenn/*wie ob/wie wenn/*als wie ob/als wie wenn ich etwas dafür könnte.

Du tust so, als/*wie könnte ich etwas dafür.

Charakteristisch ist die Verwendung von Konjunktiv-II-Formen. Während für die eingebetteten Verwendungen der irrealen Vergleichssätze im Prinzip auch Konjunktiv-I-Formen und zum Teil sogar Indikativformen zulässig sind, ist deren Verwendung in den selbständigen Typen kaum mehr möglich, vgl.

(139) Frau Kümmel tut, als ob das eine Affäre wäre/sei/?ist.

(140) Als ob das eine Affäre wäre/"sei/??ist!

Bei der selbständigen Verwendung wird wohl überwiegend auf die als obVariante zurückgegriffen; die als wie wenn-Variante scheint mir ziemlich ausgeschlossen und die Variante mit als und folgendem Verb-Erst-Satz ist zumindest stark markiert. Der zentrale Fall der selbständigen Verwendung der irrealen Vergleichssätze ist also der mit als ob-Einleitung und Konjunktiv II.

Wesentlich für die selbständig verwendeten als ob-Sätze ist, da $\beta$ bei der Interpretation die Polarität vertauscht werden muß,32 es handelt sich gewisser-

32 Eine mir unerklärliche Ausnahme ist, daß

(i) Als ob ich es geAHNT hätte.

anscheinend dasselbe bedeuten kann wie die negative Version (mit regulärer Polaritätsumkehrung)

(ii) Als ob ich es nicht geAHNT hätte. 
maßen um 'rhetorische Vergleiche': Gültig ist die in ihrer Polarität umgedrehte Proposition, vgl.:

(141) Als ob ich hier was zu sagen hätte!

(142) Als ob ich nicht bereits genug gesagt hätte!

Gemeint ist in (141), daß der Sprecher nichts $z u$ sagen hat, in (142) dagegen, daß er bereits genug gesagt hat. Für die eingebetteten irrealen Vergleichssätze gilt diese automatische Polaritätsumkehrung nicht. Vielmehr kann dort der im Vergleich genannte Sachverhalt tatsächlich zutreffen:

(143) Anna geht, als ob sie einen Stein im Schuh hätte.

Hier läßt der Sprecher offen, ob Anna tatsächlich einen Stein im Schuh hat; lediglich die Charakterisierung ihrer Gehweise ist relevant. Das Nicht-Bestehen des Vergleichssachverhalts kann höchstens in manchen Fällen aufgrund des Weltwissens erschlossen werden:

(144) Dieter geht, als ob er einen Besen verschluckt hätte.

In den selbständigen rhetorischen Vergleichen kommt es dagegen gerade nicht auf die Charakterisierung eines übergeordneten Sachverhalts an.

Insofern ist auch bereits die Frage nach der Selbständigkeit, die sich bei allen nicht-eingebetteten Verb-Letzt-Sätzen stellt, entschieden: Offensichtlich ist eine Einbettung schon deshalb nicht möglich, weil dann die Polaritätsvertauschung nicht mehr garantiert wäre. ${ }^{33}$

Aber auch formal ist eine Einbettung in vielen Fällen nicht möglich. Die naheliegendste Ausbuchstabierung ist in fast allen Fällen, daß eine aus dem Kontext zu bestimmende Person oder Personengruppe 'so tut als ob':

(145) Du tust/Sie tun/Der Innenminister tut/... (so), als ob das eine Affäre wäre.

Bei genauerem Hinsehen wird jedoch klar, daß diese Rekonstruktionsmöglichkeit häufig entfällt. In (140) kann das einen 'emphatischen' Akzent tragen (der auf dem global fallenden Melodieverlauf sitzt); genau diese Akzentuierung ist jedoch bei einer echten Einbettung nicht möglich.

Bei markierter Akzentposition allerdings

(iii) Als ob ICH es geahnt hätte.

ist wieder nur die zu erwartende Interpretation möglich.

33 Die unterschiedlichen Interpretationsmöglichkeiten der eingebetteten und selbständigen irrealen Vergleichssätze werden auch von weuster festgestellt; wieso sie dann allerdings doch funktionsexplizierende Einbettungen für die rhetorischen Vergleiche zuläßt (Weuster 1983:44f.), ist mir nicht klar. 
In manchen Fällen - offensichtlich insbesondere dann, wenn ein derartiger emphatischer Akzent vorhanden ist - kann die Modalpartikel schon verwendet werden. ${ }^{34}$

Als ob DICH schon jemand fragen würde.

Auch in diesem Fall ist eine Einbettung nicht möglich, wie dies ja meistens beim Vorhandensein einer Modalpartikel gilt. Um was für eine Art von Akzent es sich handelt, ist nicht ganz klar. Er könnte im Prinzip zwei etablierten Akzenttypen zugeschlagen werden - dem Kontrastakzent (also einem speziellen Fokusakzent) oder dem Exklamativakzent (einem nicht-fokussierenden Akzent; vgl. dazu z.B. Oppenrieder 1988b:188ff.; Batliner 1988).

Bei der ersten Analyse müßte man von einer impliziten Kontrastierung ausgehen. Dies ist zumindest in manchen Fällen ganz offensichtlich die falsche Analyse, nämlich dann, wenn der Akzent auf einen Ausdruck fällt, der aufgrund der für rhetorische Vergleiche typischen Polaritätsumkehrung selbst wie sein 'polares Gegenstück' interpretiert werden muß:

Als ob IRgendeiner etwas gewußt hätte.

(147) ist so $z u$ verstehen, daß keiner etwas gewußt hat, und läßt demnach gerade nicht den zusätzlichen Schluß zu, daß eine bestimmte Person etwas gewußt hat.3s

Auch die intonatorische Form der Realisierungen scheint mir nicht der für den Kontrastakzent typischen zu entsprechen, sondern der des Exklamativakzents zu ähneln. Sollten die rhetorischen Vergleiche dann also eher als Exklamativsätze denn als Aussagesätze klassifiziert werden? Dann müßte auch eine auf den propositionalen Gehalt gerichtete exklamativtypische 'Verwunderriseinstellung' vorhanden sein, die sich entweder auf die besondere Ausprägung einer Eigenschaft oder auf das Bestehen eines Sachverhalts bezieht. Eine graduierbare Eigenschaft braucht jedoch nicht immer involviert zu sein, vgl.:

Als ob VIER eine Primzahl wäre.

34 Die genauen Bedingungen für das Auftreten der Modalpartikel schon sind mir nicht klar. In Thurmair (1989:60) wird darauf hingewiesen, daß schon ja auch in w-Fragesätzen ein Rhetorizitätsindikator ist.

35 Dies wäre allenfalls dann möglich, wenn sich die Polaritätsvertauschung auf das Objekt bezöge: 'irgendeiner hat nichts gewußt' ist aber keine mögliche Interpretation von (147). Dies gilt auch, wenn eine andere Akzentstruktur vorliegt:

(i) Als ob irgendeiner etwas geWUSST hätte.

(ii) Als ob IRgendeiner IRgendetwas gewußt hätte. 
Eine sachverhaltsbezogene Exklamativeinstellung andererseits kann weder auf die ausgedrückte Polarität (die gerade nicht gültig ist) noch auf die implizierte Polarität (die mindestens für den Sprecher nicht sehr überraschend, sondern eher trivial ist) gerichtet sein. Wenn etwas für den Sprecher überraschend ist, dann höchstens die Tatsache, daß die 'falsche' Polarität überhaupt zur Sprache gebracht wurde. Dies gilt zumindest für die unmarkierte Verwendung, bei der mit Hilfe der als ob-Außerung eine Vorgängeräußerung, bzw. eine Folgerung oder Implikatur aus dieser nicht nur einfach zurückgewiesen, sondern als geradezu absurd (eine irreale Welt wird für die wirkliche ausgegeben) charakterisiert wird, vgl.:

(149) A: Sie hätten Ferdinand aufhalten müssen.

$B$ : Als ob das noch etwas geholfen hätte.

(150) A: Der König will deinen Jodler noch einmal hören.

$B$ : Als ob er nicht selbst singen könnte.

(151) A: Fährt Anna heuer wieder ans Nordkap?

$B$ : Als ob sie sich das noch leisten könnte.

Diese emphatische Zurückweisung von erschließbaren Annahmen eines Gesprächspartners ist der Grund, daß rhetorische Vergleiche exklamativähnlich erscheinen.

Neben dieser zentralen partnerbezogenen Verwendung gibt es auch eine, bei der ein Ereignis (mit Mißbillung) kommentiert wird:

(152) (Nach dem dreizehnten Glockenschlag:) Als ob zwölf nicht schon genug wären.

\subsubsection{3 und $o b / w-A u s d r u ̈ c k e$}

Ein weiterer spezifischer Typ von selbständigem Verb-Letzt-Satz (zur Selbständigkeit vgl. oben 1.1.2.1), der aufgrund seiner Funktion zur Gruppe der assertiven Strukturen gezählt werden kann, ist gekennzeichnet durch betontes ob oder einen betonten $w$-Ausdruck als Satzeinleiter, denen ein obligatorisches anknüpfendes und vorausgeht (vgl. Weuster 1983:45f., 48f.; Luukko-Vinchenzo 1988:118ff.; Thurmair 1989:60f.). Typischerweise werden sie in Antworten auf Polaritätsfragen (Entscheidungsfragen und assertive Fragen) verwendet (daher bei Luukko-Vinchenzo die Bezeichnung 'w-Antwort'); der propositionale Gehalt der Vorgängerfrage kann wiederholt werden, oder aber der Verb-Letzt-Satz wird bis auf das anknüpfende und und den betonten Satzeinleiter gekürzt (der Tonverlauf ist beide Male global fallend):

(153) A: Lieben Sie Brahms?

$B$ : Und $O B$ (ich ihn liebe)! bzw.

$B$ : Und WIE (ich ihn liebe)! 
(154) A: Haben Sie ein Terrarium?

$B$ : Und WAS für eines (ich habe)!/Und WAS für ein tolles ich habe!

Ein negatives Merkmal ist, da $\beta$ keine Modalpartikeln auftreten können, insbesondere keine aussagesatztypischen wie z.B. ja. In den Beispielen (153) und (154) sind die Satzeinleiter verwendet, die für diese Art von selbständigem Verb-Letzt-Satz charakteristisch sind, nämlich ob, wie und was für ein-; dabei ist was für ein-das im nominalen Bereich verwendete Gegenstück zu wie.

Insbesondere das Auftreten der letzten beiden Typen von Satzeinleitern läßt eine Verwandtschaft mit den 'graduierenden' Exklamativsätzen - vor allem den $w$-Verb-Zweit- und $w$-Verb-Letzt-Varianten - vermuten. Ahnlich wie bei Exklamativsätzen zeigt die Verwendung dieser Satzeinleiter an, daß eine graduierbare Eigenschaft, die aufgrund des Vorgängertextes zur Debatte steht, in einem beachtenswerten Ausmaß realisiert ist: In der zweiten Antwortvariante von (153) ist es die Liebe zur Brahmsschen Musik, in (154) irgendeine kontextuell zu erschließende Eigenschaft von Terrarien (z.B. die Größe oder die Ausstattung).

Strukturen mit und $o b$ ließen sich dementsprechend als eine Art Gegenstück zu den 'Satz-Exklamativsätzen' (z.B. den daß-Exklamativsätzen) auffassen:

(155) A: Ist 43 überhaupt eine Primzahl?

$B$ : Und $O B$ (es eine ist)!

Offensichtlich macht es in diesem Beispiel wenig Sinn, irgendeine graduierbare Eigenschaft, z.B. die der 'typischen Primzahlhaftigkeit', anzunehmen. Eine und ob-Antwort läßt aber im Prinzip auch eine graduierende Interpretation zu. Diese kann durch einen explizierenden Zusatz verdeutlicht werden:

(156) A: Lieben sie Brahms?

$B$ : Und $O B$ (ich ihn liebe)! Sogar sehr.

Falls die und ob-Antwort (oder die nicht-verkürzte Version) einen Ausdruck enthält, der eine graduierbare Eigenschaft bezeichnet, ist die graduierende Interpretation sogar obligatorisch, vgl.:

(157) A: Bist du müde?

B: Und OB! "Aber nicht sehr. vs. Ja. Aber nicht sehr.

Die Graduierung kann sich auch auf eine Quantitätsskala beziehen:

(158) A: Hast du Geld?

$B$ : Und $O B$ (ich Geld habe)!

Die und ob-Konstruktion ist also auch eine Art Gegenstück zu denjenigen $w$-Exklamativsätzen, die einen Allquantor enthalten. Die diesen Strukturen di- 
rekt entsprechenden und w-Ausdrücke (die nicht nur wie oder was für einenthalten) gehören nicht mehr zum Kernbereich der hier untersuchten Konstruktion; insbesondere ist eine Ellipse oft kaum möglich. Vgl. die Antwort in (158) mit:

(159) B: ?Und WIEviel (ich habe)!

(160) A: Hat er denn überhaupt etwas gesehen?

$B$ : Und $O B$ (er etwas gesehen hat)!

vs.

B: ?Und WAS er alles gesehen hat!/??Und WAS alles!"Und WAS!

Während sich bestimmte Parallelen zwischen Exklamativsätzen und den hier interessierenden Strukturen finden lassen, gibt es doch auch auffällige Unterschiede:

Zum einen ist bei den und ob/w-Ausdrücken der Akzent auf den $w$-'Kopf' des $w$-Ausdrucks, bzw. auf $o b$, fixiert. Bei echten Exklamativsätzen ist demgegenüber eine derartige Akzentpositionierung ausgeschlossen (vgl. auch Zaefferer 1988:157).

(161) *WIE gut sie riecht! vs. Wie gut sie RIECHT!

(162) "WIE schön! vs. Wie SCHON!

Zum anderen unterscheiden sich die Verwendungen von Exklamativsätzen und den und $o b / w$-Strukturen eindeutig. Die ersteren sind keine zulässigen Antworten auf Fragen.

(163) A: Freust du dich denn über die neue Hose?

$B:$ *(Und) wie ich mich über die neue Hose FREUE!

Die letzeren sind immer sequenzgebunden. Wie die obligatorische Verwendung von und anzeigt, müssen sie stets an nicht-erster Position in einer 'Texteinheit' auftreten. Im Standardfall werden sie als Antworten auf Polaritätsfragen gebraucht.

Eine derartige sequentielle Fixierung ist für Exklamativsätze nicht möglich. Zwar gibt es durchaus auch Exklamativsätze, die zu einer Textsequenz gehören, also anscheinend nicht einen quasi davon abgelösten Kommentar darstellen. Solche Exklamativsätze können auch durch ein und an die Vorgängeräußerung 'angeknüpft' werden:

(164) A: Das ist ja ein sagenhaftes Rad.

$A / B$ : Und wie LEICHT es ist!/Und wie leicht die SCHALtung geht!

In diesem Fall muß eine graduierende Beurteilung auf einer anderen, den 'Gesprächsgegenstand' betreffenden Dimension unmittelbar vorausgegangen sein. Verknüpft werden die extremen Ausprägungen der verschiedenen Eigenschaften; 
die Exklamativeinstellung selbst ist dagegen nur eine Art Sprecherkommentar dazu, der nicht zur Aufzählung gehört. ${ }^{36}$ Eine Texteinbindung von Exklamativsätzen ist also zwar möglich, aber auf keinen Fall obligatorisch und betrifft zudem nicht die Exklamativeinstellung selbst.

Die und $o b / w$-Strukturen teilen zwar mit den Exklamativsätzen die Expressivität, sind aber selbst kein exklamativischer Subtyp. $\mathrm{Zu}$ den genannten Unterschieden kommt noch, daß der Sprecher mit ihnen häufig überhaupt keine 'Verwunderung' über den mitgeteilten Sachverhalt ausdrückt (insbesondere bei den und ob-Ausdrücken, vgl. oben Beispiel (155)).

Der Standardgebrauch der und $o b / w$-Strukturen ist der als Antworten auf Polaritätsfragen. Ihre Verwendung gilt als positive Entscheidung der Frage (sie sind allerdings häufig überinformativ, dann nämlich wenn ein hoher Grad einer Eigenschaft impliziert wird). Mit der und $o b$-Version, zum Teil auch mit den und w-Versionen, deutet der Antwortende zudem an, daß ein Erwägen einer negativen Antwort, wie es durch das Stellen einer Frage zum Ausdruck kommt, nicht gerechtfertigt war. Daher kommt es wohl auch, daß eine Frage mit 'negativer Tendenz' viel angemessener auf diese Weise beantwortet werden kann als eine neutrale Frage, vgl.:

(165) A: Ist 47 überhaupt eine Primzahl?/?Ist 47 eine Primzahl?

$B$ : Und $O B$ !

Dazu paßt auch, daß sehr häufig ein 'widersprechendes' na (zu den sehr verschiedenen Funktionen von na vgl. Ehlich 1986:93ff.; Willkop 1988:193ff.) als Auftakt gewählt wird.

Bei Vorgängerfragen, die eine propositionale Verneinung enthalten, wird üblicherweise zuerst die Verneinung durch das reguläre doch zurückgewiesen;

(166) Ist 47 keine Primzahl?

Doch, und $O B ! / ?$ ?Und $O B$ !

Auch ein widersprechendes na kann gebraucht werden.

Mit der Verwendung als Antwort auf Polaritätsfragesätze steht die Verwendung als 'Bestätigung' einer positiven Aussage in enger Beziehung. Dabei scheint allerdings zu gelten, daß diese resümierenden Charakter haben muß.

(167) A: Anna hat sich (also) über das Geschenk gefreut.

$B$ : Und $O B /$ Und WIE (sie sich gefreut hat)!

36 Die Anknüpfung von nur 'implizit graduierenden' Exklamativsätzen ist daher auch ausgeschlossen:

(i) Das ist ja ein sagenhaftes Rad! *Und DAS ist aber leicht! 
Ein und $o b / w$-Ausdruck kann zudem als expliziter widerspruch auf eine 'negative' Behauptung, also positiv bestreitend verwendet werden (anders als im eben angeführten Fall muß die Behauptung nicht resümierend sein):

(168) A: Du hast dich nicht einmal gefreut über mein Geschenk.

$B$ : Doch. $-/ N a$, und $O B /$ und WIE (ich mich gefreut habe)!

Hier gilt in etwa dasselbe wie für die negativen Fragen, d.h. üblicherweise geht ein explizit bestreitender Ausdruck voraus. ${ }^{37}$

Die und $o b / w$-Ausdrücke sind also als positive, zum Teil graduierende Entscheidungen von 'Fragen', 38 die durch die Vorgängeräußerung ins Spiel gebracht werden, aufzufassen. Wie bei Antworten üblich werden zumeist nur die entscheidungsrelevanten Ausdrücke realisiert, hier also die betonten Satzeinleiter; dazu kommt obligatorisch das anknüpfende und. Aussagetypische (und auch exklamativtypische) Modalpartikeln sind unzulässig. Das mag zum einen damit zusammenhängen, daß in Antworten Modalpartikel sowieso nicht ohne weiteres auftreten können (zu ja vgl. Jacobs 1986:104). Vor allem aber entsprechen die und $o b / w$-Ausdrücke in ihrer Form nicht den typischen Aussagesätzen, so da $\beta$ die von der Bedeutung her passenden Modalpartikeln schon aus diesem Grund kaum auftreten können ( $z u$ Indizien für und gegen eine Formtypabhängigkeit von Modalpartikeln vgl. Thurmair 1989:201f.).

37 Bei einer weiteren Verwendung fehlt der für die und $o b / w-A u s d r u ̈ c k e$ ansonsten konstitutive Sprecherwechsel. Voran geht jeweils eine positive Aussage (d.h. eine positiv entschiedene Frage?), die weiter qualifiziert wird; vgl. das folgende Beispiel aus Thurmair (1989:60):

(i) Ich betete zu meiner Privatgöttin Santa Claude um Fliegeralarm. Und er kam. Und wie!

Da eine positive Aussage weiter qualifiziert wird, sind und ob-Strukturen, bei denen die graduierende Interpretation nicht die einzig mögliche ist, kaum akzeptabel.

(ii) Keiner von denen hat gewußt, ob 47 eine Primzahl ist.

Natürlich ist es eine. ?Und ob!

Vielleicht werden in diesem Zusammenhang deshalb und $w$-Ausdrücke verwendet, weil sie einerseits expressiv sind, andererseits aber nicht die exklamativtypische 'Verwundernseinstellung' ausgedrückt wird: Etwas (z.B. das Ausma $\beta$ der Fliegeralarmfolgen) ist $z$ war beachtlich, aber deswegen nicht unbedingt verwunderlich oder erstaunlich. Hingewiesen werden soll auch noch auf eine weitere Verwendung, die zeigt, daß die Abgrenzung gegenüber dem $w$-Exklamativsatz im Randbereich nicht eindeutig ist:

(iii) A: Schau mal, da drüben läuft der Heinz-Rüdiger.

B: Und WIE er wieder daherstolziert!

Der Akzent kann hier - ohne gravierende Veränderung des kommunikativen Sinns - auf das Prädikat verschoben werden, so daß eine eindeutige Exklamativstruktur entsteht. Offensichtlich wird auch nicht einfach eine Aussage bestätigt, sondern es wird ein neuer Aspekt ins Spiel gebracht.

38 Im Sinn von Zaefferer (1988:149), der Propositionen, also den 'Inhalt' von assertiven Sprechakten als "positive Entscheidungen von Fragen" bezeichnet. 
Die für den Formtyp relevanten Satzeinleiter sollten der assertiven Funktion eigentlich völlig widersprechen. Dies gilt insbesondere für $o b$, während man für assertiv interpretierte $w$-Konstruktionen immerhin eine gewisse Parallele bei den Exklamativsätzen findet. Und gerade diese unpassenden Satzeinleiter sind auch noch die Träger der 'positiven Entscheidung' einer Polaritätsfrage. Allerdings sind sie es nur im Zusammenhang mit dem in dieser Konstruktion obligatorischen und. Zudem müssen sie einen starken Akzent tragen. Bereits weiter oben wurde aufgrund der Stellungsfestigkeit dieses Akzents gegen eine Klassifizierung als (nicht-fokussierenden) Exklamativakzent argumentiert. Die alternative Einordnung als Fokusakzent mit enger Fokussierung, d.h. als Kontrastakzent liegt schon deshalb nahe, weil die Satzeinleiter als einzige Ausdrücke nicht zum Hintergrund gehören und damit in den gekürzten Versionen nicht verschwinden können, sondern erhalten bleiben müssen. Die zweifellos vorhandene 'Expressivität' der Akzentrealisierung ist bei einer solch stark markierten Fokusstruktur zu erwarten. Inhaltlich scheint jedoch eine derartige Fokussierung überhaupt keinen Sinn $z u$ machen, denn offenbar wird durch die Art der fokussierten Ausdrücke ( $o b$ oder $w^{-}$) gerade die Offenheit und Unentschiedenheit der Vorgängerfrage hervorgehoben, nicht aber deren positive Entschiedenheit. Man kann annehmen, daß hier etwas Ahnliches geschieht wie bei den rhetorischen Fragen, bei denen ebenfalls ein fragesatzförmiger Ausdruck assertiv interpretiert werden muß (für $w$-Verb-Zweit-Fragesätze, die die Modalpartikel schon enthalten, ist diese Interpretation ja sogar obligatorisch).

Es wäre also ein ähnliches Räsonnement (nach Gricescher Art) wie bei den rhetorischen Fragen anzunehmen: Offensichtlich erscheinen die und ob/w-Ausdrücke (mindestens im Standardfall) in einem Textzusammenhang, in dem vom Sprecher die Entscheidung einer vorangegangenen Frage erwartet wird; wenn dieser seinerseits einen fragesatzförmigen Ausdruck gebraucht, dann hält er sich entweder nicht an Konversationsmaximen (was wir doch nicht hoffen wollen), oder aber seine Reaktion muß als eine Entscheidung der Frage verstanden werden, wobei er durch die Verwendung eines Ausdrucks mit unpassender Form bestimmte Implikaturen auslöst (z.B. die, daß er das Stellen der Frage für überflüssig hält). Die assertive Interpretation ist bei den und $o b / w-A u s d r u ̈ c k e n$ allerdings eine von vornherein festgelegte Bedeutung und braucht nicht mehr vom Hörer im Einzelfall erschlossen $z u$ werden. Anders als bei den rhetorischen Fragen (auch den $w$-Fragesätzen mit schon) ist bei ihnen die (absichtliche oder unabsichtliche) Fehlinterpretation als echte Frage trotz ihrer Fragesatzform 
ausgeschlossen. Daher können sie zu den Verb-Letzt-Aussagesätzen gezählt werden. ${ }^{39}$

\subsubsection{4 $w$-Schlagzeile}

Die drei bisher behandelten Typen von Verb-Letzt-Aussagesätzen sind zum einen dadurch gekennzeichnet, daß sie mehr oder weniger deutlich 'expressiv' verwendet werden, also den Exklamativsätzen ähneln. Zum anderen sind sie als Begründung, Zurückweisung oder Frageentscheidung an eine Vorgängeräußerung (oder -handlung) gebunden.

Beide Charakterisierungen treffen auf den letzten Typ aus der Familie der Verb-Letzt-Aussagesätze nicht zu. Es handelt sich um Strukturen wie die folgenden:

(169) Wo Bayerns Millionäre wohnen

(170) Warum in Bayern die Uhren anders gehen

(171) Was Schneewittchen bei den sieben Zwergen erlebte

(172) Wie die Alten den Tod gebildet (haben)

Dieser Verb-Letzt-Satz möge, seiner zentralen Funktion entsprechend, ' $w$ Schlagzeile' heißen (zu diesem Typ vgl. Weuster 1983:53f.; Altmann 1987:28; Luukko-Vinchenzo 1988:117f.; Thurmair 1989:56).

Der $w$-Ausdruck als Satzeinleiter ist charakteristisch für diesen Verb-LetztSatz.

Bei der mündlichen Wiedergabe der $w$-Schlagzeile ist ein fallender Tonverlauf obligatorisch. Modalpartikeln sind, wie die Bespiele in Thurmair (1989:56, Fn. 70) zeigen, im Prinzip zulässig (z.B. 'imperativische' Modalpartikeln wie ruhig oder $J A$, für die durch die Einfügung von Modalverben wie können oder müssen eine passende Umgebung geschaffen werden kann).

Aus dem Kontext ist kein Matrixsatz zu konstruieren. Vom Sinn her passende Paraphrasen sind assertiv, wie z.B. hier erfahren Sie, wir zeigen/sagen Ihnen, hier können Sie erfahren/sehen/lesen (mit der imperativischen Variante Sehen/Lesen Sie).

39 Der in Weuster (1983:58f.) erwähnte weitere Typ von 'und- Ausdruck', nämlich

(i) Und wenn (schon)!

unterscheidet sich von den und $o b / w$-Ausdrücken dadurch, daß die nichtverkürzte Version, d.h. der vollständige Verb-Letzt-Satz, kaum akzeptabel ist. Außerdem wird normalerweise eine Modalpartikel, nämlich schon, eingefügt. Die Funktion ist die Zurückweisung der Relevanz von im weitesten Sinn negativ bewerteten Aussagen (z.B. Warnungen); daher paßt auch die Modalpartikel schon (zu deren Bedeutung vgl. Thurmair 1989:146ff.) 
Es handelt sich immer um funktionskennzeichnende Paraphrasen, d.h. die wSchlagzeilen gehören zum Typ III der nicht-eingebetteten Verb-Letzt-Sätze. Die Funktion ist jeweils die Ankündigung einer Information, die den durch den Typ des $w$-Ausdrucks gekennzeichneten, inhaltlich nicht spezifizierten Teil des propositionalen Gehalts der Schlagzeile zu spezifizieren gestattet. Typischerweise wird diese Information - z.B. über die Wohnorte von Bayerns Millionären durch einen mehr oder weniger langen Text vermittelt (aber auch $w$-Schlagzeilen zu Bildern kommen vor).

Die $w$-Schlagzeile hat also eine spezialisierte assertive Funktion, nämlich die der Ankündigung; die w-Ausdrücke zeigen lediglich an, was für eine Art von Information geliefert wird. Obwohl dieser spezifische Satztyp aufgrund seiner fragesatzähnlichen Form einerseits (nur ähnlich, da ein steigender Tonverlauf als die typische Frageintonation unzulässig ist) und seiner Ankündigungsfunktion andererseits in keine der beiden satzmodus-Kategorien problemlos paßt, scheint mir die Einordnung unter den Aussage-Modus noch am ehesten gerechtfertigt. ${ }^{40}$

\subsubsection{Verb-Letzt-Exklamativsätze}

Bisher wurden Verb-Letzt-Varianten der 'klassischen' (d.h. in allen grammatischen Beschreibungen als eigenständige Typen anerkannten) Satzmodi untersucht. Charakteristisch für diese Verb-Letzt-Strukturen ist, da $\beta$ sie unter den verschiedenen Subtypen ihrer jeweiligen Modi eine ziemlich periphere Rolle spielen; zentral sind (bei Frage, Imperativ und Aussage) die Verb-Erst- oder Verb-Zweit-Strukturen. Das ändert sich bei den beiden 'nicht-klassischen' Satzmodi des Deutschen, dem Exklamativ- und dem Wunsch-Modus. ('Nicht-klassisch' sind sie insofern, als sie im Satzmodussystem eine relativ periphere stellung einnehmen, vgl. Oppenrieder 1988b:186f., und daher auch nicht immer als eigenständige Satzmodi erkannt und anerkannt werden.)

Während die zentralen Verb-Erst- bzw. Verb-Zweit-Typen der drei klassischen Satzmodi formal deutlich voneinander abgegrenzt sind, ${ }^{41}$ überlappen die

40 Im Prinzip gibt es auch eine 'polare' Variante der fragesatzähnlichen VerbLetzt-Schlagzeilen, etwa nach dem Muster der scholastischen Quaestiones ('Utrum deus sit'). Bestimmte Abschnitte dieses Aufsatzes könnten z.B. zusammengefaßt werden unter der Überschrift

(i) Ob Verb-Letzt-Sätze selbständig verwendet werden können

41 Aussagesätze haben Verb-Zweit-Stellung, Fragesätze haben Verb-Erst-Stellung oder aber einen $w$-Ausdruck im Vorfeld, Imperativsätze - zumindest die prototypischen Imperativsätze der 2.Ps. - sind durch das Zusammenspiel von Verbmorphologie, Verbstellung und Fehlen des Subjektsausdrucks formal hinreichend deutlich von den anderen beiden Satzmodi geschieden. 
Verb-Erst- und Verb-Zweit-Formtypen des Exklamativ- und Wunsch-Modus weitgehend - nämlich bis auf die häufig nicht sehr indizierungsstarken intonatorischen Merkmale und Modalpartikeln - mit jenen klassischen Formtypen.

Als Ausweg bietet sich an, die Markiertheitsverhältnisse bei VerbErst/Zweit-Strukturen und Verb-Letzt-Strukturen umzukehren. Wenn nämlich die Verb-Letzt-Strukturen zu den zentralen Formtypen der peripheren Satzmodi gemacht werden, dann ist die Gefahr der Uberlappung mit irgendwelchen Formtypen der klassischen Satzmodi weit weniger gegeben, da bei diesen die Verb-LetztVersionen stark markiert und damit auch selten sind. Als Ergebnis erhält man Satzmodi, die zumindest in den zentralen und häufigen Formtypen gut unterschieden sind: Die klassischen, zentralen Satzmodi sind außer durch die intonatorischen Merkmale und Merkmale der kategorialen Füllung vor allem durch Verbstellung unterscheidbar; die periphereren Satzmodi sind von jenen (unter anderem) durch die Verb-Letzt-Stellung und untereinander durch die Art des Satzeinleiters abgrenzbar.

Durch diese Umstrukturierung im Satzmodussystem gewinnen die Satzmodi zumindest in den zentralen Formtypen eine größere Merkmalsdistanz voneinander: Zur intonatorischen Differenzierung (die z.B. bei den Exklamativakzenten gegenüber expressiven/emphatisch realisierten Fokusakzenten notorisch schwach ausgeprägt ist) kommt noch die Differenzierung über die Verbstellung und über die Satzeinleiter hinzu. Das Ergebnis ist ein deutlicher gegliedertes system der Formtypen. Beim Exklamativ- und beim Wunsch-Modus sind also die Verb-LetztVersionen mindestens genauso häufig, mindestens genausowenig markiert und haben ein genauso weites Anwendungsspektrum wie die Verb-Erst- und VerbZweit-Varianten. Zum Teil kehrt sich die Markiertheit sogar eindeutig um: Bei den w-Exklamativsätzen sind im Gegenwartsdeutschen die Verb-Letzt-Versionen sicherlich häufiger und auch weniger markiert als die Verb-Zweit-Versionen; die wenn-Wunschsätze sind zumindest häufiger als die Verb-Erst-Wunschsätze.

\subsubsection{1 daß-Exklamativsätze}

Beisplele für diesen Typ von Exklamativsatz (vgl. die kurzen Bemerkungen in Näf 1987:156 sowie Thurmair 1989:55f.; Weuster 1983:49f. zählt sie zu den Aussagesätzen, macht aber durch entsprechende Paraphrasen das spezifische Verwendungspotential deutlich) sind die folgenden:

(173) Daß du mit dem Kerl überhaupt noch redest!

(174) Daß Sie daran gedacht haben!

(175) Daß du (aber auch) so empfindlich bist!

(176) $D a \beta$ du (aber auch) immer recht behalten mußt! 
Wie an den Beispielen zu sehen ist, gibt es zwei unterschiedliche Versionen von daß-Exklamativsätzen, nämlich eine, die das graduierend $\mathrm{zu}$ interpretierende so oder aber einen quantifizierenden Ausdruck (wie immer) enthält (175 und 176), und eine ohne einen dieser spezifischen Typen von Ausdrücken (173 und 174). Die Unterscheidung der beiden Versionen ist insofern relevant, als nur bei der graduierenden oder quantifizierenden Variante die Modalpartikel auch bzw. die Kombination aber auch vollständig akzeptabel zu sein scheint (das gilt wohl auch für die Modalpartikel doch, die allerdings noch zusätzlichen Restriktionen unterliegt):

(177) ??Daß die Frau Mittermeier aber auch nachtragend ist!

(178) Daß die Frau Mittermeier aber auch so nachtragend ist!

vs.

In der nicht-graduierenden Variante (177) wird eindeutig eine 'polare' Interpretation erzwungen (vgl. die Unterteilung in Polaritäts- und Skalaritätsexklamative bei Zaefferer 1988:152ff.); d.h. die exklamativtypische propositionale Einstellung bezieht sich auf das Bestehen eines bestimmten Sachverhalts. ${ }^{42}$

Die Frage, ob es einen 'polar' zu interpretierenden exklamativischen Formtyp mit Verb-Erst-oder Verb-Zweit-Stellung gibt, ist schwierig zu beantworten. (Von der Struktur des Satzmodussystems her wäre zu erwarten, da $\beta$ es zu jedem Verb-Erst/Zweit-Formtyp eines Satzmodus einen Verb-Letzt-Typ gibt und umgekehrt. Eine Lücke bei polar zu interpretierenden Verb-Erst/ZweitSätzen stört die Symmetrie des Systems.) Zaefferer (1988) z.B. scheint zwar 'emphatische' Verb-Zweit-Strukturen wie

(i) Der spielt (glatt) die Schell-Sau!

lieber als Aussagesatzvarianten klassifizieren $\mathrm{zu}$ wollen, rechnet aber entsprechend 'emphatische' Verb-Erst-Sätze wie (ii) als einen spezifischen Formtyp zu den Exklamativsätzen.

(ii) Spielt der (doch glatt/tatsächlich) die Schell-Sau!

Aber auch hier im zweiten Fall scheint mir nur eine emphatische Variante des Aussagesatzes vorzuliegen, bei der lediglich das Vorfeld nicht besetzt ist, vgl. die folgende Abänderung von (ii):

(iii) Jetzt spielt der doch glatt die Schellsau!

Zumindest sehr ungewöhnlich für Exklamativsätze ist auch, daß die VerbErst-Strukturen ohne weiteres in die normale Textprogression einbezogen werden können, vgl.:

(iv) Ich fahre gerade in Eurasburg den Berg hinunter. Springt mir doch glatt eine Katze ins Vorderrad!/*Daß mir eine Katze ins Vorderrad springt!

Ein weiterer nicht sehr vielversprechender Kandidat für ein Nicht-VerbLetzt-Gegenstück zu den polaren daß-Exklamativsätzen ist der 'emphatische' Aussagesatz mit ja:

(v) Da IST ja das Schreiben!

Anders als bei den 'Skalaritätsexklamativen' mit Verb-Erst- und VerbZweit-Stellung weicht hier (und bei der in (ii) exemplifizierten Variante) die Interpretation, bis auf die Expressivität, nicht von der von Aussagesätzen ab. Auch das spricht dagegen, hier einen eigenen Exklamativ-Satztyp anzunehmen.

Das bedeutet dann aber, daß im Bereich der polaren Exklamativsätze die Pur den Exklamativ-Modus typische Umkehrung des Markiertheitsverhältnisses 
Bei der graduierend interpretierbaren Variante (die weniger markiert und häufiger ist als der polare Typ) wird die Graduierung nicht durch den Satztyp ausgedrückt, sondern durch propositionale Mittel, nämlich den Ausdruck so bewirkt. Sie kann auch durch ein zusätzliches sehr expliziert werden:

(179) Daß er so sehr an seinen Formulierungen hängt!

Aufgrund dieser spezifischen Art der Graduierung sind daß-Exklamativsätze auch nicht überall mit den implizit graduieren den (Verb-Erst/Verb-Zweit-)Exklamativsätzen austauschbar; vgl.:

(180) War DAS aber schön! vs.

(181) Daß das so schön war!

Offensichtlich gibt ein Sprecher, der (181) verwendet, zu verstehen, da $\beta$ er eigentlich mit dem Gegenteil gerechnet hat, daß es nämlich nicht schön sein würde (bei der Verwendung von (180) kann das nicht automatisch geschlossen werden). Das zeigt, daß auch bei denjenigen daß-Exklamativsätzen, die ein graduierendes so enthalten, eine polare Interpretation zugrundeliegt, die lediglich über den propositionalen Gehalt durch eine graduierende Bedeutung erweitert wird: Wenn sich nämlich die Exklamativeinstellung auf die Polarität bezieht, also ausgedrückt wird, da $\beta$ das Bestehen eines bestimmten Sachverhalts überraschend oder erstaunlich ist, dann wird damit auch $z u$ verstehen gegeben, daß eher das Bestehen eines komplementären Sachverhalts (die andere Polarität) erwartet wurde. Bei impliziter Graduierung wird dagegen nur das Erstaunen über die extreme Ausprägung einer Eigenschaft ausgedrückt, ohne daß notwendigerweise auf negative Vorannahmen Bezug genommen wird.

Verb-Letzt-daß-Exklamativsätze werden also immer polar interpretiert, können aber durch die Einfügung eines so an die typischeren graduierenden Exklamativsätze angenähert werden. ${ }^{43}$

von Verb-Letzt- und Verb-Erst/Zweit-Strukturen bis ins Extrem getrieben ist.

43 Nur von den graduierenden bzw. quantifizierenden daß-Exklamativsätzen gibt es übrigens auch die merkwürdigen Varianten, bei denen der Satzeinleiter daß durch wenn (vgl. Fn 30) oder durch weil ersetzt wird, vgl.

(i) Daß/Wenn/Weil du aber auch so leichtsinnig bist!

(11) Daß/Wenn/Weil du aber auch immer Zigarren schnorrst!

(iil) Daß/"Wenn/'Weil du Arno Schmidt magst!

Die beiden anderen Satzeinleiter verleihen dem Exklamativ sozusagen einen konditionalen oder kausalen 'Beigeschmack'. Ubrigens ist bei diesen beiden untypischen Satzeinleitern die Verwendung des exklamativindizierenden (aber) auch obligatorisch. (Dies könnte dann auch der Grund für den Ausschluß der rein polaren Versionen sein, die - wie gesehen - Modalpartikeln kaum zulassen.) 
$\mathrm{Zu}$ Beginn von 1.3.3 habe ich schon darauf hingewiesen, daß die daß-Sätze, die für die Einordnung als Aussagesätze noch am ehesten in Frage kommen könnten, unter den daß-Exklamativsätzen $z u$ suchen wären. Die rein polaren (d.h. nicht graduierenden und nicht quantifizierenden) daß-Exklamativsätze kommen den Aussagesätzen in ihrer Funktion wohl noch am nächsten, aber auch bei ihnen liegt noch eine exklamativtypische Einstellung vor, die z.B. verhindert, daß die entsprechenden strukturen wie neutrale oder emphatische Aussagesätze im Textzusammenhang gebraucht werden können (vgl. Beispiel (iv) in Fußnote 42).

Noch ein Wort zur intonatorischen Form: Auch bei den daß-Exklamativsätzen kann ein typischer Exklamativakzent (überlange Dauer der Akzentsilbe und 'später Fo-Gipfel'; vgl. Oppenrieder 1988b und Batliner 1988) realisiert werden. Dies ist aber nicht notwendig. Unsere Produktionstests haben gezeigt, daß dlese Exklamativsätze überraschenderweise sehr häufig eine steigende Fo-Kontur mit einem hohen Ende aufweisen (vgl. unten 2.4 und 2.5); dies ist eigentlich eine intonatorische Form, die man eher bei Fragesätzen erwarten würde.

\subsubsection{2 w-Verb-Letzt-Exklamativsätze}

Verb-Letzt-Exklamativsätze mit einem w-Ausdruck als Satzeinleiter (vgl.

z.B. Weuster 1983:57f.; NäP 1987; Luukko-Vinchenzo 1988:116P.; Zaepperer 1988:155ff.; Thurmair 1989:57f.) sind nach meinen Beobachtungen im Gegenwartsdeutschen weitaus häufiger und auch unmarkierter als ihre Verb-ZweitGegenstücke (vgl. dagegen Näf 1987:150, der diese Ansicht nicht teilt), vgl. die folgenden Paare:

(182) Wie dreckig die Isar heuer wieder ist! vs.

(183) Wie dreckig ist die Isar (doch) heuer wieder!

(184) Was für hervorragende Salate du machen kannst! vs.

(185) Was kannst du (aber auch) für hervorragende Salate machen!

(186) Wo der überall im Aufsichtsrat sitzt! vs.

(187) Wo sitzt der (nicht) überall im Aufsichtsrat!

Den unterschiedlichen Status erkennt man z.B. daran, daß in den VerbZweit-Versionen zur Verdeutlichung der Exklamativinterpretation eine Modalpartikel eingefügt werden muß (die wohl meistens auch eingefügt wird). Bei den Verb-Letzt-Versionen ist dies hingegen nicht unbedingt nötig. Bei den klassischen Satzmodi ist das Verhältnis genau umgekehrt: Deliberative Verb-LetztFragesätze in beiderlei Gestalt enthalten nahezu obligatorisch die Modalpartlkel wohl (bzw. nur oder bloß bei den $w$-Versionen); für daß-Imperativsätze gilt gleichermaßen, daß sie fast immer eine Modalpartikel enthalten (vor allem JA, 
bloß, nur); Verb-Letzt-Aussagesätze treten sowieso nur in spezifischen markierten Konstruktionstypen auf.

Die zulässigen Modalpartikeln sind die schon in den graduierend-quantifizierenden da $\beta$-Exklamativsätzen auftretenden doch und (aber) auch, sowie in den (all)quantifizierenden Versionen (möglicherweise auch bei was für ein-Versionen) ein als Modalpartikel fungierendes nicht (vgl. Thurmair 1989:89). W-Exklamativsätze kommen in den beiden Spielarten vor, die auch schon oben bei den nicht rein polar zu interpretierenden daß-Exklamativsätzen anzutreffen waren, nämlich mit einem graduierenden bzw. skalierenden Satzeinleiter wie oder was (für ein) (vgl. die Tabelle in Näf 1987:143) oder mit einem (all)quantifizierenden Ausdruck (vgl. die Beispiele (186/187)). Der graduierende Satzeinleiter wie kann wie sein 'interrogatives Gegenstück' mit einem betonten (graduierbaren) Adjektiv verknüpft werden. In den Verb-Letzt-Strukturen sind wie und Adjektiv normalerweise adjazent:

(188) Wie reizend sich das muntere Bächlein durch die saftiggrünen Wiesen windet! vs.

(189) Wie sich das muntere Bächlein reizend durch die saftiggrünen Wiesen windet!

Bei den Verb-Zweit-Strukturen ist die Trennung von wie ('quantopere-wie' bei NäP 1987:145) und (betontem) Adjektiv dagegen ein Abgrenzungsmerkmal gegenüber $w$-Verb-Zweit-Fragesätzen (mit 'quomodo-wie') und daher eine übliche Variante:

(190) Wie ALT ist er geworden! vs.

(191) Wie ist er ALT geworden!

Die graduierbare (und $\mathrm{zu}$ einem extremen Grad ausgeprägte) Eigenschaft, auf die sich bei den wie-Versionen die Exklamativeinstellung bezieht, kann auch nur erschlossen sein:

\section{(192) Wie du dich wieder anstellst!}

Das Gegenstück $\mathrm{zu}$ wie im nominalen Bereich ist was für ein. Auch hier ist die Trennung des $w$-Ausdrucks was vom Restausdruck für ein-NP bei VerbLetzt-Strukturen die markiertere Version:

(193) Was für tolle Radtouren er in seiner Arbeitszeit macht! vs.

(194) Was er in seiner Arbeitszeit für tolle Radtouren macht!

Die durch ein 'quantopere-was' (vgl. Näf 1987:147f.) eingeleiteten Exklamativsätze sind im Gegenwartsdeutschen gegenüber den wie-Versionen sehr mar- 
kiert und kaum mehr gebräuchlich, vgl. mit (191) und (192) (ein Gegenstück zur adjazenten Version (190) existiert nicht):

(195) ?Was ist er ALT geworden!

(196) ?Was du dich wieder anstellst!

Diese Strukturen mit 'quantopere-was' müssen allerdings unterschieden werden von den noch gebräuchlichen Strukturen mit 'impliziter Allquantifizierung' (kenntlich vor allem an dem dann einfügbaren Modalpartikel-nicht):

(197) Was er nicht (alles) gesehen haben will!

(198) Was du nicht (alles) sagst!

In den Fällen, in denen eine explizite oder implizite (All-)Quantifizierung vorliegt, können auch andere $w$-Ausdrücke als die bisher besprochenen als Satzeinleiter in Exklamativsätzen verwendet werden (vgl. auch Beispiel (186)):

(199) Wo Dante überall gewesen ist!

(200) Wen der (alles) über den Styx gesetzt hat!

(201) Worüber der schon (alles) einen Aufsatz geschrieben hat!

Die Trennung von $w$-Ausdruck und Quantor (beim Plektierbaren all- nur in der Form alles) ist hier obligatorisch:

(202) "Wen alles/alle der über den Styx gesetzt hat!

(203) "Worüber alles der schon einen Aufsatz geschrieben hat!

Diese quantifizierten $w$-Verb-Letzt-Exklamativsätze lassen $z$ war neben der quantifizierenden auch eine graduierende Interpretation $z u$, diese verdrängt aber die quantifizierende Interpretation keineswegs. Wenn also eine Sprecherin z.B. (199) verwendet, dann wird sie für gewöhnlich damit auch zu verstehen geben wollen, daß die Orte, an denen sich Dante aufgehalten hat, auch häufig irgendwelche bemerkenswerte Eigenschaften aufweisen (oder aber sie bezieht sich nur auf die bemerkenswerten Stätten); sie kann (199) aber nicht dazu verwenden auszudrücken, daß sich Dante an einem einzigen bemerkenswerten Ort, z.B. in der Hölle, aufgehalten hat, d.h. Dante muß eine erkleckliche Zahl von bemerkenswerten orten besucht haben.

Wie an den Beispielen $z u$ sehen ist, sind die Verb-Letzt-Stellung und die spezifische Füllung der Satzeinleiterposition sowie auch häufig die übrige lexikallsche Füllung im Prinzip ausreichend, um die Unterscheidung der $w$-VerbLetzt-Exklamativsätze von den seltenen und zudem meist durch spezifische Modalpartikeln markierten $w$-Verb-Letzt-Fragesätzen zu ermöglichen.

Die intonatorische Form ist also funktional nicht sehr stark belastet. Spezifische Merkmale in diesem Markierungsbereich sind der global fallende Tonhö- 
henverlauf und das Auftreten von mehr oder weniger deutlich ausgeprägten Exklamativakzenten, die typischerweise auf den graduierenden Ausdruck oder auf das Prädikat fallen. Wenn ein definiter thematischer Ausdruck vorhanden ist, wie z.B. in (200), kann auch dieser den Exklamativakzent tragen; eine solche Akzentpositionierung ist auch in Verb-Erst- und Verb-Zweit-Exklamativsätzen zu beobachten; wie bei diesen kann auch Mehrfachakzentuierung auftreten (vgl. Oppenrieder 1988b:176).

\subsubsection{Verb-Letzt-Wunschsätze}

Zum Abschluß des linguistisch orientierten Teils dieser Untersuchung sei noch kurz auf die Verb-Letzt-Wunschsätze eingegangen.

Die durch wenn eingeleiteten Verb-Letzt-Varianten der Wunschsätze (eine sehr ausführliche Analyse findet sich in Scholz 1989) sind im Gegenwartsdeutschen häufiger anzutreffen als die entsprechenden Verb-Erst-Versionen, Markiertheitsunterschiede lassen sich aber wohl nicht feststellen (eventuell gehören die Verb-Erst-Versionen zu einer höheren Stilebene):

(204) Wenn es doch nur zu Ende wäre! vs.

(205) Wäre es doch nur zu Ende!

Außer durch den Satzeinleiter wenn und die durch diesen erzwungene VerbLetzt-Stellung unterscheidet sich der wenn-Wunschsatz ${ }^{44}$ auch in seinen formtyprelevanten Merkmalen kaum vom Verb-Erst-Gegenstück: Das finite Verb steht im Konjunktiv II; als (obligatorische) Modalpartikeln werden doch, nur und blo $\beta$ sowie die Kombinationen doch nur und doch bloß verwendet; der Tonhöhenverlaup ist global fallend.

Ein Unterschied zwischen den beiden Typen besteht allerdings darin, da $\beta$ durch den Satzeinleiter wenn die Wunschinterpretation schon sehr stark nahegelegt wird, während das bei Verb-Erst-Stellung des Finitums natürlich nicht gilt. Als Folge davon kann die Konjunktiv-II-Markierung bei den wenn-Sätzen durch eine indikativische Markierung ersetzt werden: Der Effekt ist, daß nicht mehr von 'irrealen' Wünschen gesprochen werden kann, d.h. der für die Verb-

44 Die wenn-Wunschsätze scheinen übrigens die im süddeutschen prinzipiell mögliche 'emphatische Topikalisierung' von Satzgliedern kaum zuzulassen, $\mathrm{vgl.:}$

(i) Den Karl wenn ich treffe, sind immer gleich zwei Stunden weg vs.

(ii) ??Den Karl wenn ich doch treffen würde!

Offenbar erlaubt also die durch den Satztyp ausgedrückte expressive Wunscheinstellung nicht, eine zusätzliche und davon unterschiedene emphatische oder expressive Hervorhebung zu realisieren. 
Erst-Sätze allein mögliche Interpretationsbereich wird verlassen. Da $\beta$ die indikativischen wenn-Sätzen trotzdem als ein Wunschsatztyp klassifiziert werden sollten, wurde bereits oben in 1.3.2 angesprochen. Als Folge der nicht vollständig festliegenden Modusmarkierung des Verbs haben die wenn-Sätze also einen größeren Anwendungsbereich als die Verb-Erst-Wunschsätze. Wahrscheinlich aus diesem Grund verdrängen sie diese auch aus dem zentralen Anwendungsbereich, nämlich dem Ausdruck von 'irrealen' Wünschen.

Neben dem wenn-Wunschsatz findet sich als heute kaum mehr gebräuchlicher Formtyp der daß-Wunschsatz:

(206) Daß ich doch tausend Finger hätte!

(207) Ach, daß ich doch einen Leberkäs hätte!

Auch hier ist die Konjunktiv-II-Markierung ein zentrales Merkmal; es sichert die Unterscheidung gegenüber den anderen selbständigen daß-Verb-Letzt-Typen. Als Modalpartikel kann nur doch verwendet werden. Der Tonverlauf ist fallend. Auf die mögliche Uberlappung von indikativischen Varianten mit den daß-Imperativsätzen wurde bereits in 1.3 .2 hingewiesen.

\subsection{Zusammenfassung}

Selbständigen Verb-Letzt-Sätzen muß ein fester Platz in einem Satzmodussystem eingeräumt werden. Sie können nicht als elliptische strukturen auf die Haupt-Formtypen mit Verb-Erst- bzw. Verb-Zweit-Stellung zurückgeführt und damit wegerklärt werden. In vielen Fällen ist eine Einbettung schon aus syntaktischen Gründen ausgeschlossen (Modalpartikeln, intonatorische Form); in anderen ist zwar eine Einbettung möglich, diese entpuppt sich jedoch immer als eine Funktionsparaphrase, die syntaktisch nicht relevant ist. Als selbständige Formtypen kommen nur nicht-adverbielle Verb-Letzt-Sätze in Frage, da nur diese, als nicht-relationale Ausdrücke, 'absolut' verwendet werden können. Bei den klassischen Haupt-Satzmodi Frage, Imperativ und Aussage sind Verb-LetztFormtypen markierte und periphere Strukturen. Das gilt insbesondere für die formal sehr uneinheitlichen Verb-Letzt-Aussagesätze. Bei den peripheren Satzmodi Exklamativ und Wunsch dagegen kehrt sich das Markiertheitsverhältnis von Verb-Letzt- und Verb-Erst/Zweit-Formtypen nahezu um: Die Verb-Letzt-Strukturen sind die zentralen Realisierungstypen. Auf diese Weise erhält man für die unmarkierten Formtypen der 5 Satzmodi des Deutschen eine größere Merkmalsdistanz und damit eine bessere formale Unterscheidbarkeit. 
2. Experimentelle Untersuchungen zur intonatorischen Form von VerbLetzt-Sätzen

\subsection{Einleitung: Minimalpaarmethode, Testsatzkonstruktion}

Im Münchner DFG-Projekt 'Modus-Fokus-Intonation' wurde die intonatorische Realisierung fast aller der im ersten Teil behandelten Typen von selbständigen Verb-Letzt-Sätzen untersucht, nämlich der durch $o b$, einen $w$-Ausdruck (im speziellen Fall wie), daß oder wenn eingeleiteten.

Genauso wie bei den im Satzmodussystem zentralen Verb-Erst- und VerbZweit-Strukturen sollten die zunächst hypothetisch angesetzten intonatorischen Formen mit Hilfe der Methode der intonatorischen Minimalpaare überprüft werden (vgl. Altmann 1984): Bei bestimmten Formtypen im Satzmodussystem sind die nicht-intonatorischen Merkmale neutralisiert, so da $\beta$ allein die intonatorische Form satzmodusunterscheidend wirkt. Untersucht man nun segmentgleiche (d.h. wort- und wortfolgengleiche) Sätze, die jeweils einem der beiden Minimalpaarsatzmodi angehören, so kann man die intonatorischen Merkmale direkt miteinander vergleichen.

Dementsprechend wurden von uns geeignete Testsätze konstruiert. Wenn segmentelle Gleichheit angestrebt wird, muß bei den Verb-Letzt-Sätzen die Art des Satzeinleiters das Kriterium für die Ordnung der Minimalpaare abgeben. Die von uns zugrundegelegten Strukturen ((S1) bis (S4)), die den vier Typen von Satzeinleitern für selbständige Verb-Letzt-Sätze zugeordnet sind, lauten:

(S1) $O b$ er kommt

(S2) Wie die blüh'n

(S3) Daß du brav bist

(S4) Wenn der Willi doch käme

Die völlig segmentale Gleichheit ist jedoch bei den selbständigen VerbLetzt-Sätzen nicht durchzuhalten: Im ersten Teil dieser Arbeit wurde gezeigt, daß sehr häufig die Einfügung spezifischer Modalpartikeln wenn nicht obligatorisch, so doch der Normalfall ist (z.B. bei den deliberativen Fragesätzen oder den $d a \beta$-Imperativsätzen). In manchen Fällen muß die Kern-Struktur erweitert (durch so bei den graduierend $z u$ interpretierenden daß-Exklamativsätzen) oder abgeändert werden ( $d u$ statt er als Subjektsausdruck bei den ob-Aufforderungen), um die angemessene Interpretation sicherzustellen. In diesen Fällen liegen also keine echten Minimalpaare vor. Kein Satzmodus-Minimalpaar läßt sich bei den wenn-Wunschsätzen bilden. Allerdings wurden hier, wie in den anderen 
Fällen, eingebettete und elliptische Versionen der entsprechenden Strukturen zum Vergleich herangezogen. ${ }^{45}$

Die Testsätze sind in Kontexte eingefügt, die jeweils einen bestimmten Satzmodus (und eine Akzentstruktur) festlegen.

Im folgenden sind die von uns getesteten Testsatz-Kontext-Kombinationen aus dem Korpus ((K1) bis (K33)) aufgeführt. Allerdings ist hier nicht immer der gesamte Kontext angegeben. Für eine vollständige Version der Kontexte verweise ich auf Batliner/Oppenrieder (1989); die in Klammern angegebenen Bezeichnungen für spezielle Verb-Letzt-Typen beziehen sich auf die dort angegebenen $\mathrm{Be}-$ zeichnungen, die zum Teil etwas von den hier eingeführten abweichen.

\subsection{Das Korpus der Verb-Letzt-Sätze}

\section{$O b-$ Sãtze:}

- Deliberativer Fragesatz:

(K1) Ob er wohl kommt?

- Aussagesatz mit extraponiertem Interrogativsentential ('Gegenstück' zum deliberativen Fragesatz):

(K2) Ich frag' mich, ob er kommt.

- Versicherungsfrage auf Entscheidungsfragesatz:

(K3) A: Kommt der Peter?

$B$ : Ob WER kommt?!?

- elliptische Rückfrage auf Entscheidungsfragesatz:

(K4) A: Weißt du, ob er kommt?

$B:$ Ob er kommt?

- ultimative Frage:

(K5) A: Kommt der Peter heute?

B: Er geht heute schwimmen, besucht seine Großmutter, und er will in die Bibliothek gehen.

A: Red doch nicht herum! ob er kommt?!?

- Aussagesatz mit extraponiertem Interrogativsentential ('Gegenstück' zur ultimativen Frage):

(K6) Red doch nicht herum! Ich will wissen, ob er kommt.

45 Die Gleichheit wird auch durch satzmodusspezifische Akzentmuster (z.B. die Betonung der Satzeinleiter in den und $o b / w$-Ausdrücken) durchbrochen. 
- elliptische Antwort ('ob-Fortsetzungsassertion'):

(K7) A: Wie bitte? Was hast du mich gefragt?

$B: O b$ er kommt.

- Aussagesatz mit extraponiertem Interrogativsentential (nicht-elliptische Antwort):

(K8) A: Wie bitte? Was hast du mich gefragt?

B: Ich habe dich gefragt, ob er kommt.

- Und ob-Satz ('ob-Antwort-Satz'):

(K9) A: Was ist jetzt? Kommt er?

$B$ : Und ob er kommt!

- ob-Aufforderung:

(K10) Ob du wohl kommst!

- elliptische Rückfrage auf Entscheidungsfragesatz ('Frage-Übernahme'):

(K11) A: Weißt du, ob er kommt?

B: Ob er kommt? Das weiß ich auch nicht.

- (elliptische) 'ob-Fortsetzungsfrage':

(K12) A: Anna hat mich ein paar Sachen über Peter gefragt.

$B$ : Auch, ob er kommt?

- Entscheidungsfragesatz mit extraponiertem Interrogativsentential ('Gegenstück' zur 'ob-Fortsetzungsfrage'):

(K13) Hat sie auch gefragt, ob er kommt?

$d a \beta$-Sätze:

- daß-Imperativsatz:

(K14) Daß du JA brav bist!

- daß-Exklamativsatz mit graduierender Interpretation:

(K15) Daß du so brav bist!

- Aussagesatz mit einem die Sprechereinstellung bezeichnenden Matrixsatz ('Gegenstück' zum daß-Exklamativsatz mit graduierender Interpretation)

- daß-Satz im Vorfeld:

(K16) Daß du so brav bist, wundert mich schon.

- extraponierter daß-Satz:

(K17) Es wundert mich schon, daß du so brav bist. 
- daß-Exklamativsatz mit polarer Interpretation ('da $\beta$-Satz-Exklamativsatz'):

(K18) Daß du brav bist!

- elliptische Antwort:

(K19) A: Das wundert mich aber schon.

B: Was denn?

A: $D a \beta$ du (so) brav bist.

- (elliptische) 'daß-Fortsetzungsfrage':

(K20) Kind: Der Nikolaus hat dem Opa nur Gutes über mich erzählt. Mutter: Auch, daß du brav bist?

- elliptische Rückfrage auf Aussagesatz:

(K21) Kind: Der Onkel hat gesagt, daß ich brav bin. Mutter: Da $\beta$ du brav bist?

- daß-Wunschsatz:

(K22) Ach, daß du doch so brav wärst wie dein Bruder.

wenn-Sätze:

- wenn-Wunschsatz:

(K23) Wenn der Willi doch käme!

- Aussagesatz mit wenn-Satz im Vorfeld ('Gegenstück' zum wenn-Wunschsatz):

(K24) Wenn der Willi käme, könnte er uns bestimmt helfen.

w-Verb-Letzt-Sätze:

- w-Verb-Letzt-Exklamativsatz:

(K25) Schau dir doch mal diese wunderschönen Kakteen an. Wie die blüh'n!

(K26) Schau dir doch mal die hübsche Form von diesen Kakteen an. Und wie die blüh'n!

- und wie-Satz ('wie-Antwort-Satz'):

(K27) A: Blühen diese Kakteen eigentlich?

$B$ : Und wie die blüh'n!

- elliptische Antwort ('Fortsetzungsassertion'):

(K28) A: Ich weiß jetzt alles, was ich schon immer über diese Kakteen wissen wollte.

B: Wo die wachsen?

A: Nein, wie die blüh'n. 
- (elliptische) 'w-Fortsetzungsfrage':

(K29) A: Ich hab gestern etwas über diese Kakteen gelesen und weiß jetzt endlich, was ich schon immer wissen wollte.

B: Wie die blüh'n?

- elliptische Rückfrage auf Entscheidungsfragesatz (Spezialverwendung 'Frageübernahme'):

(K30) A: Weißt du, wie diese Kakteen blühen?

$B$ : Laß mich mal überlegen! - Wie die blüh'n? Weiß und orange.

- elliptische Rückfrage auf Entscheidungsfragesatz:

(K31) A: Weißt du, wie diese Kakteen blühen?

$B$ : Wie die blüh'n? Das weiß doch jedes Kind!

- deliberativer $w$-Verb-Letzt-Fragesatz:

(K32) Wie die wohl blüh'n?

- ultimative Frage:

(K33) A: Wie blühen diese Blumen eigentlich?

$B$ : Das sind Kakteen.

A: Nein, wie die blüh'n?!?

2.3 Aufnahmeprozedur, Hörtests und Parameterextraktion

\subsubsection{Aufnahmeprozedur}

Die Testsätze mitsamt ihrem Kontext wurden 6 Versuchspersonen (VPen) in Produktionstests vorgelegt; die dabei erhaltenen Realisationen wurden zum einen in Hörtests überprüft und zum anderen akustisch analysiert (die Test- und Analyseprozeduren entsprechen den in Oppenrieder (1988b) geschilderten bei den Verb-Erst- und Verb-Zweit-Sătzen).

Das Verb-Letzt-Korpus bildete einen Teil eines umfangreicheren Korpus, das noch zwel weitere Teilkorpora enthielt (vgl. Batliner/Oppenrieder 1989). Die Testsatz-Kontext-Kombinationen dieses Großkorpus wurden jeweils auf Kärtchen geschrieben und den 6 VPen ( 3 weiblich, drei männlich) in (pseudo-)randomisierter Relhenfolge zur Realisation vorgelegt (Aufnahme auf Telefunken M15Tonbandgerät im schallarmen Raum des Instituts für Phonetik der Universität München). Normalerweise wurde jeder VP jede Testsatz-Kontext-Kombination zweimal vorgelegt, da aber am Ende des Durchgangs die nach Meinung des Versuchsleiters schlecht realisierten Testsätze wiederholt wurden, gibt es pro Testsatz und Sprecher bis zu vier Realisationen. 
Insgesamt erhielten wir für das Verb-Letzt-Korpus 476 Realisationen. ${ }^{46}$ Diese wurden auf zweifache Weise weiterverwendet: Zum einen wurden sie in verschiedenen Hörtests überprüft, zum anderen wurden charakteristische akustische Parameterwerte extrahiert.

\subsubsection{Hörtests}

In den Hörtests sollten vor allem die Realisierungsgüte und die Relevanz der intonatorischen Form für die Satzmoduserkennung überprüft werden. An den entsprechenden Tests nahmen durchschnittlich ein Dutzend VPen teil. Dargeboten wurde immer das gesamte Großkorpus; die Randomisierung aus den Produktionstests wurde beibehalten.

(a) Im Akzenttest sollte die Position der am stärksten betonten Silbe herausgefunden werden. Dazu wurden den VPen die Testsatzrealisationen kontextlos (eingebettete Verb-Letzt-Sätze wurden aus ihrem Matrixsatz herausgeschnitten) vorgespielt; gleichzeitig lag ihnen eine schriftliche Fassung dieser Testsätze vor, auf der sie die entsprechende Silbe markieren sollten. Als Ergebnis erhält man ein (hörerbezogenes) Betontheitsmaß für die verschiedenen Silben. Aup der am stärksten betonten Silbe (A1-Silbe) wird ein Teil der satzmoduskennzeichnenden intonatorischen Form realisiert.

(b) Kontextlos (und matrixsatzlos) wurden die Realisationen auch im Kategorisierungstest vorgespielt. Die Anweisung lautete hier, die einzelnen Realisationen den durch eine funktionale Beschreibung und einige typische Beispiele eingeführten Gruppen Aufforderung, Frage, Aussage, Ausruf/Exklamativ und Wunsch zuzuordnen (per Knopfdruck an einem Abstimmkästchen). Die Ergebnisse dieses Tests geben Auskunft darüber, ob die intonatorischen Eigenschaften ausreichen, ansonsten 'satzmodusambige' Strukturen eindeutig (und so wie bei der Testsatz-Konstruktion beabsichtigt) identifizierbar zu machen.

(c) Beim Natürlichkeitstest lagen den VPen die vollständigen Testsatz-Kontext-Kombinationen schriftlich vor; die Testsätze waren markiert. Aufgabe war es, zu beurteilen, wie gut jede vorgespielte Testsatzrealisation zu ihrem Kontext paßt. Die Einschätzung erfolgte auf einer 5-stufigen Ratingskala: 1 für 'paßt sehr gut', 2 für 'paßt gut', 3 für 'paßt noch gut', 4 für 'paßt schlecht' und 5 für 'paßt sehr schlecht' (wieder per Knopfdruck an einem Abstimmkästchen).

46 Im folgenden werden nur 440 Realisationen untersucht; es fehlen die 'idiomatischen wenn-Sätze', vgl. die Auflistung in Batliner/Oppenrieder (1989). 
Jeder Testsatzrealisation kann damit ein (kontextbezogener) Natürlichkeitswert als arithmetisches Mittel aus den einzelnen Natürlichkeitsbewertungen der VPen zugeordnet werden.

Als prototypische Realisationen bezeichnen wir diejenigen, die einen Natürlichkeitswert kleiner als 2.5 (also einen Wert, der zwischen 'gut' und 'noch gut' liegt) haben und gleichzeitig von mehr als $80 \%$ der VPen im Kategorisierungstest (kontextfrei) dem von uns intendierten Funktionstyp zugeordnet wurden. Für die Bestimmung der intonatorischen Form werden nur diese prototypischen Realisationen herangezogen.

\subsubsection{Parameterextraktion}

Von jeder Testsatzrealisation wurden Mingogramme mit Zeitsignal, Grundfrequenzverlauf und Intensitätsverlauf erstellt. Anhand dieser Mingogramme wurden systematisch für alle 476 Realisationen die folgenden Werte extrahiert (vgl. Oppenrieder 1988b:177ff.):

(a) Der Hz-Wert am Beginn (='Onsetwert') und am Ende (='Offsetwert') der Außerung

(b) Die $\mathrm{Hz}$-Werte der beiden höchsten (lokalen) Fo-Maxima und der beiden tiefsten Fo-Minima (falls die Außerung tatsächlich zwei oder mehr derartige Extremwerte enthielt

(c) Die $\mathrm{Hz}$-Werte der 'Eckpunkte' der beiden (sofern vorhanden) im Umfang größten 'Fo-Bewegungen'; als solche zählen der einfache Anstieg und Fall, sowie komplexere Konturen mit einer deutlichen Veränderung der Bewegungsrichtung

(d) Die beiden Silben mit den höchsten dB-Werten

(e) Die Dauer (des stimmhaften Teils) der Hauptakzentsilbe/A1-Silbe

(f) Die Dauer der Gesamtäußerung (vom Onset bis zum offset).

Die Werte bilden die Grundlage für die Bildung der 'intonatorischen Prototypen', d.h. der für einen Formtyp im Satzmodussystem charakteristischen intonatorischen Form.

\subsection{Ergebnisse der Hörtests}

Von den Ergebnissen der in 2.3.2 beschriebenen Tests sind insbesondere die der Kategorisierungstests interessant. ${ }^{47}$

\footnotetext{
47 Auf die Ergebnisse der anderen beiden Test werde ich dagegen nur vereinzelt eingehen.
} 


\subsubsection{Ergebnisse der Kategorisierungstests}

Die folgende Tabelle gibt für die Realisationen der oben angeführten Satztypen an, wieviel Prozent der Urteile in den Kategorisierungstests jeweils auf die vorgegebenen funktionalen Kategorien Aufforderung (AUF), Frage (FRA), Aussage (AUS), Ausruf/Exklamativ (EXK) und Wunsch (WUN) entfielen. Hinter dem Satztyp steht in Klammern zunächst, auf welche der oben angegebenen Korpus-Sätze sich die Urteile beziehen; die Zahl nach dem Schrägstrich gibt die Anzahl der Realisationen der jeweiligen Testsätze an.

AUF FRA AUS EXK WUN

\section{VERB-LETZT-FRAGESATZE}

(1)

$\begin{array}{lllllll}\text { deliberativer ob-Fragesatz (K1/12) } & 0 & 94.1 & 0 & 0.7 & 5.2 \\ \text { deliberativer W-Fragesatz (K32/12) } & 0.8 & 83.2 & 0 & 14.5 & 1.5 \\ \text { eingebetteter ob-Satz (K2/14) } & 0 & 70 & 20.3 & 7.7 & 2 \\ & & & & & & \\ \text { (2) } & & & 99.3 & 0 & 0 & 0.7 \\ \text { (elliptische) ob-Rückfragen (K4/14) } & 0 & 74.2 & 3 & 22.1 & 0.7 \\ \text { (elliptische) wie-Rückfragen (K31/12) } & 0 & 74.7 & 32.7 & 5.4 \\ \text { (elliptische) daß-Rückfragen (K21/13) } & 13 & 45.6 & 3.3 & 32.7 \\ \text { (ell.) ob-Rückfr./'Ubernahme' (K11/12) } & 0 & 97.8 & 0 & 0.7 & 1.5 \\ \text { (ell.) W-Rückfr./'Ubernahme' (K30/12) } & 0 & 43.3 & 10.4 & 45.5 & 0.8\end{array}$

(3)

$\begin{array}{lllllll}o b \text {-Versicherungsfrage } & (\mathrm{K} 3 / 14) & 0 & 98 & 0 & 1.3 & 0.7\end{array}$

(4)

(el1.) 'ob-Fortsetzungsfrage' (K12/12) $00 \begin{array}{lllll}91.8 & 3 & 0.7 & 4.5\end{array}$

$\begin{array}{lllllll}\text { (el1.) 'w-Fortsetzungsfrage' (K29/12) } & 0.8 & 73.6 & 3.9 & 21.7 & 0\end{array}$

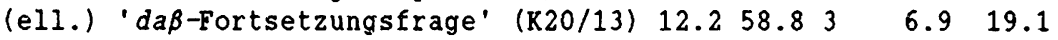

$\begin{array}{lllllll}\text { eingebetteter ob-Satz } & (\mathrm{K} 13 / 12) & 0 & 94 & 3 & 2 & 2\end{array}$

(5)

ultimative ob-Frage $(K 5 / 12)$

ultimative $w$-Frage $(\mathrm{K} 33 / 12)$

eingebetteter ob-Satz $(K 6 / 14)$

$\begin{array}{llllll}0 & 74.2 & 6.5 & 13.7 & 5.6\end{array}$

$\begin{array}{lllll}0 & 4.5 & 2.2 & 93.3 & 0\end{array}$

$\begin{array}{lllll}0.7 & 62.4 & 10.7 & 18.1 & 8.1\end{array}$

(6)

ob-Aufforderung (K10/12)

$54.930 .8 \quad 0 \quad 8.3 \quad 6$

\section{VERB-LETZT-IMPERATIVSATZ}

(7)

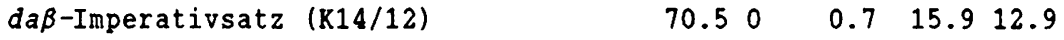


AUF FRA AUS EXK WUN

VERB-LETZT-AUSSAGESATZE

(8)

und ob-Satz (K9/12)

und $w$-Satz $(\mathrm{K} 27 / 12)$

$\begin{array}{lllll}0 & 0 & 15.7 & 83.6 & 0.7 \\ 0 & 0 & 0 & 100 & 0\end{array}$

(9)

(elliptische) ob-Antwort (K7/11)

(elliptische) w-Antwort (K28/12)

$\begin{array}{lllll}0 & 75 & 7.3 & 6.4 & 11.3\end{array}$

(ell.) daß-Antwort (K19/14)

(ell.) daß-Antwort + so (K19/12)

$\begin{array}{llllll}0.7 & 3.1 & 7.7 & 88.5 & 0\end{array}$

eingebetteter ob-Satz $(K 8 / 12)$

$\begin{array}{llllll}23.7 & 13.5 & 20.6 & 32.1 & 10.1\end{array}$

$\begin{array}{lllll}0 & 14.2 & 6.7 & 76.1 & 3\end{array}$

$\begin{array}{llllll}0.8 & 64.4 & 14.4 & 12.1 & 8.3\end{array}$

\section{VERB-LETZT-EXKLAMATIVSATZE}

(10)

w-Exklamativsatz (K25, 26/24)

(polarer) daß-Exklamativsatz (K18/14)

(grad.) daß-Exklamativsatz (K15/31)

$\begin{array}{lllllll}\text { (extrapon.) eingeb. daß-Satz (K17/12) } & 0.9 & 4.8 & 40.3 & 50 & 4 \\ \text { (im Vorf.) eingeb. daß-Satz (K16/12) } & 0 & 11.1 & 18.3 & 68.3 & 2.3\end{array}$

$\begin{array}{llllll}0.3 & 0 & 4.2 & 94.7 & 0.8\end{array}$

$\begin{array}{llllll}8.6 & 25.2 & 10.6 & 49 & 6.6\end{array}$

$\begin{array}{lllll}0 & 6.2 & 5 & 86.8 & 2\end{array}$

\section{VERB-LETZT-WUNSCHSATZE}

(11)

daß-Wunschsätze (K22/12)

wenn-Wunschsätze (K23/12)

eingebetteter wenn-Satz (K24/12)

$\begin{array}{lllll}2.9 & 0 & 0 & 10.4 & 86.7 \\ 0 & 0.7 & 0 & 4.5 & 94.8 \\ 0.8 & 6.4 & 14.4 & 6.4 & 72\end{array}$

2.4.2. Erläuterungen $\mathrm{zu}$ den Kategorisierungsergebnissen

In diesem Abschnitt werden insbesondere die vom Satzmodussystem her unerwarteten Kategorisierungen genauer erläutert. Auf klare Fälle und auch auf die elngebetteten Sätze wird nur kurz eingegangen.

Bei den Ergebnissen ist grundsätzlich zu berücksichtigen, daß die VPen eine funktionale Klassifizierung vornahmen, von der aus keine direkten Rückschlüsse auf den Formtyp möglich sind. So werden beispielsweise Außerungen, die zu einem 'expressiven' Satztyp gehören (z.B. Rückfragen) oder auch nur zufälligerweise expressiver realisiert wurden, häufig von den VPen als Exklamative klassifiziert ( $v g l$. $z u$ diesen Problemen Oppenrieder 1988a). Bei den eingebetteten Sätzen muß beachtet werden, daß sie aus ihrem Matrixsatz entfernt wurden, so daß den VPen wichtige Information für die Klassifikation nicht zur Verfügung stand. 
Generell kommt den Satzeinleitern eine zentrale Rolle bei der Klassifizierung zu. Dies zeigt sich bei den Verb-Letzt-Fragesätzen ganz klar an der unterschiedlichen Verteilung der Exklamativurteile, je nachdem, ob als Satzeinleiter $o b$ oder wie verwendet wird. Schließlich ist wie im Gegensatz $\mathrm{zu}$ ob ein Satzeinleiter, der auch in Exklamativsätzen auftritt, für w-Exklamativsätze sogar typisch ist. Wahrscheinlich liegt daher beim typischen exklamativischen $w-$ Ausdruck wie eine interrogative Interpretation von vornherein weniger nahe als bei anderen $w$-Ausdrücken. Außerdem könnte eine Rolle spielen, daß in den Kategorisierungstests ja auch echte wie-Exklamativsätze vorkamen, die bei den VPen die Bereitschaft förderten, von der struktur her exklamativverdächtige Außerungen auch als solche zu klassifizieren.

Zu (1): Der verhältnismäßig hohe Anteil an Exklamativurteilen (14.5\%) fällt insbesondere bei den deliberativen $w$-Verb-Letzt-Fragesätzen (K32) auf, da erstens alle Realisationen mit dem fragesatztypischen hohen offset enden, zweitens die exklamativunverträgliche Modalpartikel wohl vorhanden ist und drittens auch keine besonders expressiven Realisationen vorliegen (zumindest sind sie nicht expressiver als die parallelen Realisationen von deliberativen ob-Fragesätzen).

Zu (2): Bei der Gruppe der Rückfragen sind die Exklamativurteile sowohl für die Realisationen mit $w$-Satzeinleiter $(22.1 \%$ und $45.5 \%)$ als auch für die daßRückfragen sehr häufig (32.7\%); auch bei den letzteren dürfte die Ahnlichkeit mit Exklamativsätzen von Einfluß sein. Gerade bei den Rückfragen (K4), (K31) und (K21) ist schon vom Kontext her eine eher expressive Realisierung erforderlich. Hier auftretende Exklamativurteile könnten auch durch diese Expressivität bedingt sein. Es ist aber bemerkenswert, da $\beta$ der Satzeinleiter $o b$ auch bei den 'expressiven' Rückfragen (K4) ausschließlich die Frageinterpretation nahelegt (99.3\%; nur ein einziges 'Wunsch'-Urteil als Irrläufer). Die als 'Frage-ưbernahmen' (vgl. Luukko-Vinchenzo 1988:104ff.) bezeichneten w-Rückfragen (K30) werden demgegenüber sogar häufiger als Exklamative denn als Fragen beurteilt (obwohl hier ein expressivitätsfördernder Kontext fehlt); auch die Einordnung als Aussage ist hier einigermaßen häufig (zur Klassifizierung von Exklamativsätzen als Aussagen vgl. Oppenrieder 1988a); d.h. die im weitesten Sinn exklamativischen Beurteilungen überwiegen eindeutig (55.9\% $\mathrm{zu} 43.3 \%)$. Ein Teil der Realisationen hat hier auch nicht die für Rückfragen ansonsten typische intonatorische Form, sondern endet mit einem 'Tiefton'; gerade in diesem Fall liegt eine nicht-interrogative Interpretation des wie-Satzes nahe.

Zu (5): Nicht weiter überraschend ist danach, daß die Realisationen der ultimativen $w$-Fragen (K33) $\mathrm{zu} 93.3 \%$ als Exklamative klassifiziert werden. Ulti- 
mative Fragen haben nicht das eindeutige Fragemerkmal des steigenden Tonverlaufs, so daß insbesondere die wie-Varianten lediglich durch die (den VPen nicht zugängliche) Sequenzierung von den gleich strukturierten Exklamativsätzen unterschieden sind. Zudem handelt es sich bei ultimativen Fragen durchwegs um sehr expressive Außerungen.

Infolge dieser Expressivität fallen sogar auf die durch ihren fragespezifischen Satzeinleiter eigentlich klar gekennzeichneten ultimativen ob-Fragen (K5) mehr Exklamativurteile (13.7\%) als auf jeden anderen ob-Typ (mit Ausnahme der durch die Konjunktion und zusätzlich markierten und damit als Nicht-Fragesätze gekennzeichneten und ob-Sätze).

Zu (6): Eine Sonderstellung nehmen die 'ob-Aufforderungen' ein (zu deren problematischem Status s.o. 1.3.1.4). Neben den 54.9\% Aufforderungsurteilen, zu denen im Prinzip auch die $6 \%$ Wunschurteile zu zählen sind, finden sich immerhin aber noch $30.8 \%$ Frageurteile (die $8.3 \%$ Exklamativurteile verdanken sich wohl der Expressivität der Außerungen). Die Fragesatzform schlägt bei der Klassifizierung also überraschend stark durch, obwohl die lexikalische Füllung ( $d u$ als Subjekt, im Gegensatz zu allen anderen im Test verwendeten ob-Strukturen, die ein neutrales er-Subjekt enthalten) und die 'Expressivität' eigentlich eine rein funktionale Klassifizierung begünstigen sollten.

Diese Form manifestiert sich hier aber nicht nur im Satzeinleiter $o b$, sondern vor allem auch in dem fragesatztypischen steigenden Tonverlauf. Allerdings erhalten die 12 Realisationen der 'ob-Aufforderungen' auch ziemlich schlechte Natürlichkeitsbewertungen. Die Werte von 5 Exemplaren liegen auf oder über dem von uns angesetzten Schwellenwert von 2.5 (vgl. oben 2.3.2); der Rest liegt bis auf 2 Ausnahmen nur knapp darunter; der durchschnittliche Natürlichkeitswert für alle 12 Realisationen ist 2.57, also ebenfalls über dem Schwellenwert. $\mathrm{Da}$ beim Natürlichkeitstest die Außerungsgüte relativ zum Kontext bestimmt wurde, der seinerseits hier die Aufforderungsinterpretation erzwang, könnten die schlechten Natürlichkeitsbewertungen dafür sprechen, da $\beta$ wir in den Produktionsexperimenten einfach größtenteils unangemessene Realisationen erhielten, die demzufolge im Kategorisierungstest auch nicht konsistent erkannt werden konnten. Eine andere Erklärung ist die, daß 'ob-Aufforderungen' so selten und markiert sind, daß ihre Verwendung anstelle der üblichen 'Aufforderungs-Satztypen' an sich schon unnatürlich ist (allerdings werden sie im Kategorisierungstest ja immer noch ziemlich gut erkannt). Interessanterweise ist die Klassifizierung als Aufforderung bei denjenigen Realisationen am höchsten, die nach Ausweis der Akzenturteile auf dem Satzeinleiter $o b$ den relativ stärksten Akzent tragen; dessen Hervorhebung scheint also, wie bereits oben in 1.3.1.4 ausgeführt wurde, 
zu den formtyprelevanten Merkmalen zu gehören. (Bei fast allen Exemplaren liegt Akzentsplitting vor, d.h. die Al-Urteile der Akzenttests verteilen sich, wenn auch nicht gleichgewichtig, auf das Finitum als Träger des Fokusakzents und den Satzeinleiter mit seinem satzmodustypischen 'emphatischen' Akzent.)

Zu (7): Die Realisationen des $d a \beta$-Imperativsatzes (K14) werden viel häufiger als Aufforderung klassifiziert (70.5\%) als die der 'ob-Aufforderung'. Zudem sind auch die 'Fehlklassifizierungen' als Wünsche und Exklamative erklärbar: Aufforderungen lassen sich im Prinzip auch als wünsche nach der Ausführung einer Handlung verstehen; die Exklamativurteile können darauf zurückgeführt werden, daß daß-Imperativsätze mit der emphatisch betonten Modalpartikel $J A$ für starke Aufforderungen verwendet werden, also als 'expressiv' klassifiziert werden können.

Zu (8): Ebenfalls aufgrund ihrer Expressivität werden die und $o b / w$-Sätze (K9) und (K27) ganz überwiegend als Exklamative eingestuft. Typischerweise findet sich die Klassifizierung als Aussage nur bei den und ob-Realisationen.

$\mathrm{Zu}$ (9): Bei den elliptischen Antworten ist $\mathrm{zu}$ erwarten, daß sich die VPen bei der Klassifizierung vor allem nach der Art der Satzeinleiter richten; ohne den passenden Kontext kann einfach nicht erkannt werden, daß es sich um elliptische, als Antworten verwendete Aussagesätze handelt. Dementsprechend überwiegen bei der ob-Antwort (K7) die Frageurteile (75\%), bei der wie-Antwort (K28) die Exklamativurteile (88.5\%). Bei der daß-Antwort ist die Unsicherheit der VPen ganz deutlich: Da $d a \beta$ als Satzeinleiter bei sehr vielen Typen von Verb-Letzt-Sätzen verwendet wird, gehen die Klassifizierungen quer durch die vorgegebenen Kategorien. Enthält der $d a \beta-S a t z$ jedoch wie in der einen Version von (K19) einen klaren Exklamativitätsindikator wie so, dann schnellt die Anzahl der Exklamativurteile in die Höhe $(76.1 \%$ vs. $32.1 \%$ bei der Version ohne so). Die Fragekategorisierungen bei (K19) betreffen fast ausschließlich Antwortversionen mit hohem (oder wenigstens mittlerem) Fo-Offset, d.h. Antwortäußerungen mit einer an Fragesätze angenäherten intonatorischen Form (die entsprechenden Außerungen wurden übrigens im Natürlichkeitstest als akzeptabel beurteilt).

Zu (10): Bei der Gruppe der Exklamativsätze ist erwartungsgemä $\beta$ die Klassifizierung der wie-Versionen (K25) als Exklamative problemlos möglich.

Die polaren daß-Exklamativsätze (K18) werden hingegen überraschenderweise nicht nur als Exklamative (49\%) oder Aussagen (10.6\%), sondern häufig auch als Fragen (25.2\%) eingeordnet. Tatsächlich kommt ihre intonatorische Form der der Fragesätze ziemlich nahe, denn bei fast allen Realisationen steigt der Fo-Verlauf auf der Al-Silbe und der Fo-Offset ist hoch. Allerdings haben die meisten 
der 14 Exemplare sehr schlechte Natürlichkeitswerte: Nur 5 liegen unter dem Schwellenwert 2.5, der Durchschnittswert beträgt 2.71. Wie bei den 'ob-Aufforderungen' stellt sich die Frage, ob nur zufälligerweise so viele unnatürliche Außerungen produziert wurden oder ob nicht der Satztyp selbst bereits markiert und ungewöhnlich ist. Viel eindeutiger als Exklamative klassifiziert wurden die graduierenden daß-Exklamativsätze (K15), nämlich zu 86.8\%. Sie enthalten allerdings auch ein kennzeichnendes so. Auch bei ihnen kommt, wenngleich weniger häufig als beim polaren Typ, die fragesatzähnliche intonatorische Form vor, ohne daß sich dies auf die Natürlichkeitswerte negativ auswirkt. Es könnte also sein, daß beim polaren Typ nicht so sehr wegen der unangemessenen intonatorischen Form der meisten Realisierungen, sondern wegen der Markiertheit der Struktur an sich so schlechte Natürlichkeitswerte vergeben werden.

Zu (11): Die Wunschsätze schließlich werden allesamt den Erwartungen gemä $\beta$ klassifiziert, die wenn-Strukturen (K23) etwas besser als die sehr viel periphereren $d a \beta$-Strukturen (K22).

\subsection{Die intonatorische Form von Verb-Letzt-Sätzen}

Die aus den Mingogrammen der einzelnen Realisationen extrahierten akustischen Parameterwerte (s.o. 2.3.3) werden benutzt, um sogenannte intonatorische Prototypen $z u$ bilden (detaillierte Auflistungen der Parameterwerte eines Teils der hier behandelten Verb-Letzt-Typen finden sich in Luukko-Vinchenzo 1988 und Scholz 1989). Die Aufstellung dieser Prototypen erfolgt analog zu den in Oppenrieder (1988b) für das Korpus der Verb-Erst- und Verb-Zweit-Sätze vorgestellten prototypischen intonatorischen Formen. Von den extrahierten akustischen Parameterwerten gehen im folgenden nur die aus dem Grundfrequenzverlauf gewonnenen Werte in die Prototypenbildung ein (also nicht die Dauerwerte und die Positionen der intensitätsstärksten Silben), da für die intonatorische Satzmoduskennzeichnung im wesentlichen charakteristische Fo-Verläufe verantwortlich sind.

Insbesondere zwei Parameter sind von Wichtigkeit, zum einen die Höhe des Fo-offset, also der letzte Fo-Wert einer Außerung, zum anderen die Form des Fo-Verlaufs auf und in der Umgebung der Hauptakzentsilbe (der Al-Silbe aus dem Akzenttest). Sie werden in der folgenden Tabelle unter 'Offsethöhe' und 'Konturtyp' aufgeführt.

Bei der Offsethöhe wird allerdings nicht der $\mathrm{Hz}$-Wert selbst angegeben, sondern der globale Bereich, in den dieser Wert fällt. Zu diesem Zweck wird der gesamte in der Außerung ausgeschöpfte Bereich der Fo-Variation (der Fo-Range, berechnet aus den Fo-Maxima und -Minima, sowie den Fo-Werten am Anfang 
und Ende der Außerung) halbiert; fällt der Offsetwert in den oberen Bereich, so sprechen wir von einem hohen offset (oder auch Hochton, in Anlehnung an die Sprechweise des heute gängigsten phonologischen Intonationsmodells, des Tonsequenz-Ansatzes), abgekürzt ' $H$ ', ansonsten von einem tiefen offset (Tiefton), abgekürzt ' $\mathrm{T}$ '. Bei einer genauen Analyse der Verteilung der konkreten $\mathrm{Hz}-$ Werte zeigt sich, daß sie fast nie in den unscharfen Grenzbereich in der Mitte des jeweiligen Fo-Range fallen, sondern typischerweise entweder selbst die obere oder untere Grenze des Range markieren oder doch sehr nahe an dieser Grenze liegen.

Der Konturtyp wird aus den extrahierten 'auffälligen' Fo-Verläufen gebildet (s.o. (c) in 2.3.3). Vier Typen von Fo-Verläufen im Bereich der Hauptakzentsilbe (A1-Silbe) werden unterschieden: fallend, steigend, sowie die komplexen Typen konvex (d.h. steigend-fallend) und konkav (d.h. fallend-steigend).

offsethöhe und Konturtyp gehen wesentlich ein in die Bestimmung der für einen Satztyp charakteristischen intonatorischen Form. Zugrundegelegt werden dabei die bei den 'guten' Realisationen am häufigsten vorkommenden Ausprägungen der Offsethöhe und des Konturtyps. Offensichtlich sind davon abweichende Realisationen in vielen Fällen nicht ausgeschlossen: Sie werden in den Hörtests als natürlich bewertet und wie erwartet kategorisiert. Daher sind die angegebenen Ausprägungen von offsethöhe und Konturtyp als Prototypen aufzufassen, d.h. als die häufigste und unmarkierte und damit zentrale intonatorische Realisation. Zulässige Abweichungen von dieser Form werden als peripherere und damit auch markiertere Realisationen eingeordnet (zum Prototypenkonzept im Bereich der Satzmoduskennzeichnung vgl. Oppenrieder 1988b:184ff.).

Als weiteres Merkmal wird in der folgenden Tabelle der durchschnittliche Fo-Range, d.h. der in den Außerungen eines Satztyps ausgeschöpfte Fo-Umfang, in Halbtönen (HT) angegeben. Der Range ist insofern interessant, ais sich an ihm zumindest teilweise die 'Expressivität' eines Satztyps ablesen läßt. 
2.5.1 Tabelle der intonatorischen Prototypen

$\begin{array}{lll}\text { Range } & \text { offset- } & \text { Kontur- } \\ \text { (in HT) höhe } & \text { typ }\end{array}$

VERB-LETZT-FRAGESATZE

(1)

(a) deliberativer ob-Fragesatz (K1) $10.5 \quad \mathrm{H}$

$10.6 \quad \mathrm{H}$

(b) deliberativer $w-F r a g e s a t z$ (K32)

$6.7 \quad \mathrm{~T}$

steigend, konkav

(c) eingebetteter ob-Satz (K2) konkav, konvex fallend

(2)

(d) (elliptische) ob/wie/daß-Rückfragen 14.1 H (K4, K31, 21)

$12.0 \quad \mathrm{H}$

steigend, konkav

(e) (elliptische) ob/w-Rückfragen/ 'Ubernahmen' (K11, K30)

steigend, konkav

(3)

(f) ob-Versicherungsfrage (K3)

$14.7 \quad \mathrm{H}$

steigend, konkav

(4)

(g) (elliptische) ob/W-/daß-Fortsetzungsfragen (K12, K29, K20)

(h) eingebetteter ob-satz (K.13)

$12.2 \mathrm{H}$

steigend, konkav

(5)

(i) ultimative ob/w-Fragen (K5, K33)

$11.6 \quad \mathrm{H}$

steigend, konkav

(j) eingebetteter ob-Satz (K6)

$11.9 \mathrm{~T}$

konvex

10.6 T konvex

(6)

(k) ob-Aufforderung (K10)

$12.3 \quad \mathrm{H}$

steigend

VERB-LETZT-IMPERATIVSATZ

(7)

(1) daß-Imperativsatz (K14)

$10.8 \quad \mathrm{~T}$

konvex

VERB-LETZT-AUSSAGESATZE

(8)

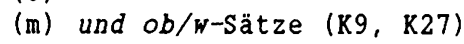
$11.5 \mathrm{~T}$
konvex
(9)
(n) (elliptische) ob/w-Antwort (K7, K28)
$9.0 \mathrm{~T} \quad$ konvex
(o) (elliptische) daß-Antwort (K19)
(p) eingebetteter ob-Satz (K8)
$10.0 \mathrm{~T}, \mathrm{H}$
$9.0 \mathrm{~T}$
konvex, konkav konvex 


$\begin{array}{lll}\text { Range Offset- } & \text { Kontur- } \\ \text { (in HT) höhe } & \text { typ }\end{array}$

VERB-LETZT-EXKLAMATIVSATZE

(10)

(q) $w$-Exklamativsatz (K25, K26)

(r) daß-Exklamativsatz (K15, K18)

(s) (extrapon.) eingeb. daß-Satz (K17)

$9.7 \mathrm{~T}$

9.5 T, H

$6.7 \mathrm{~T}$

(t) (im Vorf.) eingeb. daß-Satz (K16) konvex

konvex, konkav

konvex

konvex

\section{VERB-LETZT-WUNSCHSATZE}

(11)

(u) daß-Wunschsatz (K22)

(v) wenn-Wunschsatz (K23)

(w) eingebetteter wenn-Satz (K24)

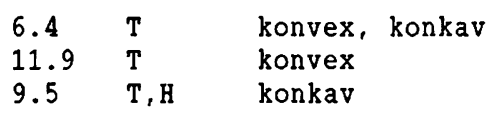

\subsubsection{Erläuterungen $z u$ den intonatorischen Prototypen}

Wie bei den Erläuterungen $\mathrm{zu}$ den Kategorisierungstests (2.4.2) wird auch hier nur auf unerwartete Fälle eingegangen.

Zu (b): Bei den deliberativen w-Verb-Letzt-Fragesätzen (Wie die wohl blüh'n?) wird in knapp der Hälfte der Fälle eine bei der 'Frageintonation' markierte konvexe Kontur auf der Al-Silbe realisiert. Sie tritt genau dann auf, wenn die Al-Silbe mit dem früh im Satz auftretenden Demonstrativum die zusammenfällt (die Sprecher realisieren diesen frühen Akzent, die Sprecherinnen, bis auf eine Ausnahme, einen späten Akzent auf blüh'n). Beide Akzentuierungen werden als natürlich beurteilt. Die markierte Akzentrealisierung hängt wohl damit zusammen, da $\beta$ hier mit der Hervorhebung des Subjekts auch eine markierte Fokusstruktur zugrundeliegt: Sie ist kontrastiv zu interpretieren ('diese da, im Gegensatz zu anderen Pflanzen'). Der hohe offset bleibt aber in allen Fällen erhalten.

Zu (c): Daß der eingebettete ob-Satz auf einen Tiefton endet und eine fallende Kontur aufweist, ist zu erwarten, da er Teil eines Aussagesatzes ist (Ich frag' mich, ob er kommt.). Der gegenüber den selbständigen deliberativen Verb-Letzt-Sätzen geringere Range erklärt sich aus dem Fehlen des normalerweise rangeerweiternd wirkenden hohen offsets.

Zu (e): Als Charakteristikum der Rückfragen wurde in 1.3.1.2 die 'Frageintonation' genannt, die anzeigt, daß das 'Zitat' des Vorgängerausdrucks im Bereich einer zusätzlichen Frageeinstellung liegt. Dies sollte auch für die Rück- 
fragen gelten, die zur Einleitung einer eigenen Antwort verwendet werden ('Frage-Übernahmen'). Bei einem Drittel der $w$-Versionen fehlt jedoch die typische Frageintonation: Der offset ist tief; in zwei Fällen ist zudem eine konvexe Kontur vorhanden. Die entsprechenden Realisationen wurden als Exklamative klassifiziert, im Natürlichkeitstest aber als kontextgerecht bewertet. Es scheint also, da $\beta$ bei den nicht-expressiven $w$-Rückfragen (man beachte die Rangeunterschiede zwischen (d) und (e)) die intonatorische Kennzeichnung als Frage nicht obligatorisch ist, zumindest dann nicht, wenn der Sprecher die gestellte Frage sofort selbst beantwortet, also gar keine typische Fragehandlung vorliegt.

$\mathrm{Zu} \mathrm{(g):} \mathrm{Auch} \mathrm{bei} \mathrm{den} \mathrm{elliptischen} \mathrm{'w-Fortsetzungsfragen'} \mathrm{haben} \mathrm{zwei} \mathrm{Exem-}$ plare einen frageuntypischen tiefen offset und eine fallende Kontur. In diesem Fall wird zwar der Hörer tatsächlich zu einer Antwort aufgefordert, anders als bei den Rückfragen wird hier aber auch nicht ein Vorgängerausdruck zitiert, so da $\beta$ die Frageeinstellung schon durch den verwendeten Satzeinleiter angezeigt wird. Die prototypische intonatorische Form ist trotzdem die fragetypische mit hohem offset und einer steigenden oder konkaven Kontur im Bereich der Hauptakzentsilbe.

Zu (i): Für die ultimativen Fragen ist wieder die Sequenzierung wichtig. Anders als die Rückfragen stellen sie aber gewissermaßen 'Selbstzitate' dar; sie führen also keine zusätzliche Frageeinstellung ein. Die fragesatztypische Form ist unzulässig, vielmehr sind ein Tiefton am Außerungsende und eine konvexe Kontur kennzeichnend.

Zu (k): Auf die 'ob-Aufforderungen' ( $O b$ du wohl kommst!) wurde im Zusammenhang mit den Kategorisierungstests schon ausführlicher eingegangen. Sie wurden fast ausschließlich mit der für Fragesätze typischen intonatorischen Form realisiert. Die einzige Ausnahme ist eine Sprecherin, die beide Male einen tiefen Offset produziert (Natürlichkeitswerte: 2.2 und 3.4). Die Anzahl der Aufforderungskategorisierungen ist bei diesen Exemplaren ziemlich hoch $(78 \%)$; eine Rolle spielt hier sicher auch, daß die Akzenturteile überwiegend auf den Satzeinleiter $o b$ fallen, also die typische Akzentstruktur vorliegt.

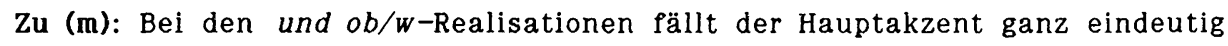
auf die beiden Satzeinleiter $o b$ bzw. wie. Der Range ist für einen Typ mit obligatorisch tiefem offset und konvexer Kontur sehr umfangreich (man vergleiche die Ranges der Exklamativsätze ( $q$ ) und ( $r$ )); die Expressivität des Satztyps ist also nicht nur eine Folge der ungewöhnlichen Akzentstruktur, sondern wird auch zusätzlich durch den großen Umfang der Fo-Bewegung aup dem akzentuierten Satzeinleiter erzielt. 
Zu (o): Bereits weiter oben bei der Besprechung der Kategorisierungsergebnisse wurde darauf hingewiesen, daß die elliptischen $d a \beta$-Antworten, also assertiv zu interpretierende Außerungen, zum Teil eine fragesatztypische intonatorische Form aufweisen. Vielleicht erlaubt die Sequenzierung eine gewisse Freiheit bei der Realisierung der Antwortäußerung, so daß die Verwendung einer an sich unpassenden intonatorischen Form zur Erzielung besonderer pragmatischer Effekte (z.B. 'Emphatisierung' der Antwort) ermöglicht wird.

Zu (r): Auch auf die nicht-prototypischen Realisierungen der daß-Exklamativsätze wurde bereits verwiesen. Sie treten insbesondere bei den polar zu interpretierenden Außerungen auf, werden dort aber als ziemlich unnatürlich bewertet. Bei den graduierend $\mathrm{zu}$ interpretierenden Außerungen sind sie seltener; dort überwiegen aber Exemplare mit einem 'halbhohen' offset, der ansonsten in den Daten kaum auftaucht.

$\mathrm{Zu}$ (s) und (t): Die Unterschiede in der Realisierung der beiden eingebetteten da $\beta$-Sätze ergeben sich aus ihrer unterschiedlichen Position im Matrixsatz: Bei der Vorfeldversion tritt häufig ein hoher oder halbhoher offset auf, d.h. es wird ein 'weiterführendes Tonmuster' realisiert. Die Unterschiede im Range lassen sich wahrscheinlich darauf zurückführen, daß bei längeren 'assertiven' Außerungen, d.h. solchen ohne hohen Offset, der Fo-Range gegen Ende zu kleiner wird.

\subsection{Zusammenfassung}

Auch bei den Verb-Letzt-Formtypen des Satzmodussystems gehört die intonatorische Form $\mathrm{zu}$ den satzmoduskennzeichnenden Merkmalen. Allerdings sind hier mit dem Satzeinleiter ( $d a \beta, o b, w$ - oder wenn) und in vielen Fällen mit den nahezu obligatorischen Modalpartikeln sehr starke nicht-intonatorische Satztypindikatoren vorhanden, die eine weniger starke funktionale Belastung der Intonation zur Folge haben. Trotzdem variiert auch bei den Verb-Letzt-Sätzen die intonatorische Form nicht beliebig, sondern es lassen sich prototypische, unmarkierte Ausprägungen finden. 


\section{LITERATUR}

Altmann, H. (1981): Formen der "Herausstellung" im Deutschen. Rechtsversetzung, Linksversetzung, Freies Thema und verwandte Konstruktionen. Tübingen.

Altmann, H. (1984): Linguistische Aspekte der Intonation am Beispiel Satzmodus. In: Forschungsberichte des Instituts für Phonetik und sprachliche Kommunikation München 19. S.132-152.

Altmann, H. (1987): Zur Problematik der Konstitution von Satzmodi als Formtypen. In: Meibauer, J. (Hg.) (1987): Satzmodus zwischen Grammatik und Pragmatik. Tübingen. S.22-56.

Batliner, A. (1988): Der Exklamativ: Mehr als Aussage oder doch nur mehr oder weniger Aussage? Experimente zur Rolle von Höhe und Position des Fo-Gipfels. In: Altmann, H. (Hg.) (1988): Intonationsforschungen. Tübingen. S.243271.

Bierwisch, M. (1980): Semantic Structure and Illocutionary Force. In: Searle, J.R./ Kiefer, F./Bierwisch, M. (Hgg.) (1980): Speech Act Theory and Pragmatics. Dordrecht. S.1-35.

Duden-Grammatik (1984): Duden. Grammatik der deutschen Gegenwartssprache. 4.Aufl. Herausgegeben und bearbeitet von Günter Drosdowski. Mannheim/ Wien/Zürich.

Ehlich, K. (1986): Interjektionen. Tübingen.

Fries, N. (1983): Syntaktische und semantische Studien zum frei verwendeten Infinitiv. Tübingen.

Geluykens, R. (1988): On the Myth of Rising Intonation in Polar Questions. In: Journal of Pragmatics 12. S.467-485.

Grewendorf, G. (1972): Sprache ohne Kontext. Zur Kritik der performativen Analyse. In: Wunderlich, D. (Hg.) (1972): Linguistische Pragmatik. Wiesbaden. S. 144-182.

Haider, H. (1988): Die Struktur der deutschen Nominalphrase. In: Zeitschrift für Sprachwissenschaft 7 . S.32-59.

Heidolph, K.E./Flämig, W./Motsch, W. (1981): Grundzüge einer deutschen Grammatik. Berlin.

Höhle, T. (1988): VERUM-Fokus. In: Sprache und Pragmatik. Arbeitsberichte 5. Lund. S.1-7.

Holly, W. (1988): Weiterführende Nebensätze in sprachgeschichtlicher Perspektive. In: Zeitschrift für germanistische Linguistik 16. S.310-322.

Jacobs, J. (1986): Abtönungsmittel als Illokutionstypmodifikatoren. In: GAGL 27. S. $100-111$. 
Kasper, W. (1987): Semantik des Konjunktivs II in Deklarativsätzen des Deutschen. Tübingen.

Lang, E. (1983): Einstellungsausdrücke und ausgedrückte Einstellungen. In: Růžiłka, R./Motsch, W. (Hgg.) (1983): Untersuchungen zur Semantik. Studia grammatica XXII. Berlin. S.305-341.

Luukko-Vinchenzo, L. (1988): Formen von Fragen und Funktionen von Fragesätzen. Eine deutsch-finnische kontrastive Studie unter besonderer Berücksichtigung der Intonation. Tübingen.

Meibauer, J. (1987): Zur Form und Funktion von Echofragen. In: Rosengren, I. (Hg.) (1987): Sprache und Pragmatik. Lunder Symposium 1986. Stockholm. S.335-356.

Meibauer, J. (1988): Satzmodus, kategoriale Füllung und nicht-propositionales nicht. In: Studien zum Satzmodus II. LS/ZISW/A 185. Berlin. S.63-76.

Näf, A. (1987): Gibt es Exklamativsätze? In: Meibauer, J. (Hg.) (1987): Satzmodus zwischen Grammatik und Pragmatik. Tübingen. S.140-160.

Oppenrieder, W. (1987): Aussagesätze im Deutschen. In: Meibauer, J. (Hg.) (1987): Satzmodus zwischen Grammatik und Pragmatik. Tübingen. S.161-189.

Oppenrieder, W. (1988a): Intonation und Identifikation. Kategorisierungstests zur kontextfreien Identifikation von Satzmodi. In: Altmann, H. (Hg.) (1988): Intonationsforschungen. Tübingen. S.153-167.

Oppenrieder, W. (1988b): Intonatorische Kennzeichnung von Satzmodi. In: Altmann, H. (Hg.) (1988): Intonationsforschungen. Tübingen. S.169-205.

Oppenrieder, W./Thurmair, M. (1989): Kategorie und Funktion einer Partikel. Oder: Was ist eigentlich 'eigentlich' EIGENTLICH? Eine Replik auf M. Kohrt. In: Deutsche Sprache 17. S.26-39.

Reis, M. (1985): Satzeinleitende Strukturen im Deutschen. Uber COMP, Hauptund Nebensätze, $w$-Bewegung und die Doppelkopfanalyse. In: Abraham, W. (Hg.) (1985): Erklärende Syntax des Deutschen. Tübingen. S.271-311.

Scholz, U. (1989): Wunschsätze im Deutschen - formale und funktionale Beschreibung. Ms.

Searle, J.R. (1969): Speech Acts. An Essay in the Philosophy of Language. Cambridge.

Thurmair, M. (1989): Modalpartikeln und ihre Kombinationen. Tübingen.

Weuster, E. (1983): Nicht-eingebettete Satztypen mit Verb-Endstellung im Deutschen. In: Olszok, K./Weuster, E. (1983): Zur Wortstellungsproblematik im Deutschen. Tübingen. S.7-87.

Willkop, E.-M. (1988): Gliederungspartikeln im Dialog. München. 
Wunderlich, D. (1986): Echofragen. In: Studium Linguistik 20. S.44-62.

Zaefferer, D. (1984): Frageausdrücke und Fragen im Deutschen. Zu ihrer Syntax, Semantik und Pragmatik. München.

Zaefferer, D. (1988): Satzmodi als Satzformkategorien. Zur Analyse grammatischer Faktoren in der Illokutionstypbestimmung. In: Studien zum Satzmodus II. LS/ZISW/A 185. Berlin. S.139-163. 


\section{DEKLINATION UND SATZMODUS}

Wilhelm Oppenrieder

0. Vorbemerkung

In diesem Beitrag wird auf der Grundlage eines umfangreichen Korpus untersucht, inwieweit in einer Außerung globalere Grundfrequenzveränderungen, die sogenannte Grundfrequenz-Deklination, satzmodustypisch ausgeprägt sind und so eine Differenzierung der Satzmodi ermöglichen.

1. Das Konzept der 'Deklination' - ein kurzer Überblick

Das Phänomen der Grundfrequenz-(Fo-)Deklination ist in der phonetisch orientierten Intonationsforschung schon lange ein wichtiger Untersuchungsgegenstand (vgl. den Uberblick in Ladd 1984). Den Anstoß gab die Beobachtung. da $\beta$ bei den üblicherweise in Intonationsuntersuchungen verwendeten gelesenen Realisationen von aussagesatzförmigen strukturen aufeinanderfolgende Fo-Gipfel (bzw. weniger ausgeprägt auch Fo-Täler) im typischen Fall immer niedriger werden, daß also eine durch die Fo-Gipfel (bzw. Fo-Täler) gezogene Linie durch eine global fallende Tendenz gekennzeichnet ist (zu Unterschieden von spontan geäußerten und gelesenen Aussagesätzen vgl. Umeda 1982 und Lieberman et al. 1985).

Dieses Phänomen spielt auch in der Wahrnehmung eine Rolle, und zwar bei der Tonhöhenskalierung von aufeinanderfolgenden Fo-Gipfeln: Der Hörer erwartet den Deklinationseffekt und kompensiert ihn bel der Gewichtung von Tonhöhengipfeln. In Pierrehumbert (1979) wird gezeigt, daß zwei aufeinanderfolgende Akzentgipfel als gleich prominent beurteilt werden, wenn dem späteren ein FoGipfel entspricht, der gegenüber dem früheren um einen bestimmten $\mathrm{Hz}-$ Wert abgesenkt wurde.1

1 Die zweigipfligen Muster, mit denen in den Experimenten (z.B. in den Perzeptionsexperimenten in Plerrehumbert 1979 oder den Produktions- und Perzeptionsexperimenten in Gussenhoven/Rietveld 1988) gearbeitet wird, sind 
Der Deklinationseffekt spielt also sowohl bei der Produktion als auch bei der Perzeption eine Rolle.

Im Laufe der Forschungen zur Fo-Deklination hat sich allerdings gezeigt, da $\beta$ der ursprünglich als einheitliches Phänomen gesehene Effekt möglicherweise in verschiedene 'Sub-Effekte' aufgespalten werden muß. In Pierrehumbert (1980) wird unterschieden zwischen einer - wohl durch physiologische Mechanismen erklärbaren - invarianten 'baseline', die als Referenzlinie für die Skalierung von Fo-Gipfeln und -Tälern dient und für den normalen und unmarkierten Deklinationseffekt in Aussagesätzen verantwortlich ist, und dem 'downstep' (bzw. 'catathesis' in Beckman/Pierrehumbert 1986), der Außerungen kennzeichnet, bei denen die Fo-Gipfel auf einer sehr viel steiler abfallenden Gipfellinie liegen.

In Liberman/Plerrehumbert (1984) wird angenommen, da $\beta$ auch lokalere Phänomene wie z.B. ein 'final lowering' am Ende einer Außerung ${ }^{2}$ in den allgemein abfallenden Trend des Fo-Verlaufs eingehen, der undifferenziert als Deklination bezeichnet wird. Auf diese Aufspaltung des Deklinationseffekts soll im folgenden aber nicht weiter eingegangen werden.

Die Untersuchungen zum Deklinationseffekt gingen üblicherweise von (gelesenen) Aussagesätzen aus, bei denen sich die global fallende Tendenz des FoVerlaufs zeigte. Es fragt sich nun, ob bei Realisationen von Strukturen anderer Satzmodi ein ähnlicher Effekt festzustellen ist: $Z w a r$ muß in Fragesätzen ein finaler Fo-Anstieg ausgeklammert werden (dieses 'steigende Tonmuster' unterscheidet sie gerade vom 'fallenden Tonmuster' der Aussagesätze), aber zumindest der 'Vorlauf' vor diesem Anstieg könnte Deklination aufweisen.

Daß solche konkreten Deklinationsverläufe bei der Satzmodusdifferenzierung eine Rolle spielen können, hat für das Dänische Nina Thorsen gezeigt. Sie nimmt

allerdings nicht unbedingt das beste Material für derartige Untersuchungen. Es ist nåmlich nicht von vornherein auszuschließen, da $\beta$ die Absenkung des zweiten Gipfels in der Produktion, bzw. seine Heraufstufung in der Perzeption, auf den Einfluß des 'Systems der Nukleusakzentuierung' zurückgeführt werden kann, das in Sprachen wie dem Deutschen, Englischen oder Holländischen die Akzente innerhalb einer Außerung in zwei Klassen teilt: in nichtletzte vs. den letzten. Der letzte Akzent zählt als Nukleus ('Hauptakzent'), als der Akzent, der den Fokusexponenten ( $z u$ diesem semantisch-syntaktischen Begriff vgl. Höhle 1982) hervorhebt und auf dem normalerweise eine satzmodustypische Fo-Kontur realisiert wird. Der letzte Tonhöhengipfel hat also schon vom Akzentuierungssystem her gegenüber den restlichen eine Sonderstellung. Es ist denkbar, da $\beta$ er allein aus diesem Grund mit verhältnismäßig niedrigerer Fo realisiert werden kann (Experimente mit zumindest dreigipfligen Mustern können den Einfluß dieses Faktors leicht überprüfbar machen). Es mag z.B. sein, daß das 'final lowering', dessen Interaktion mit der 'normalen' Deklination in Gussenhoven/Rietveld (1988) untersucht wird, ein Effekt des 'Systems der Nukleusakzentuierung' ist.

2 Beckman/Pierrehumbert (1986:303ff.) vermuten, daß bestimmte dieser Effekte nicht äußerungs-, sondern diskursbezogen sind. 
an, daß eine durch die betonten Silben einer (kurzen) Außerung gelegte Regressionslinie je nach Satzmodus einen charakteristischen Verlauf aufweist:

Terminal declarative sentences have the most steeply falling (unmarked) contours at one extreme, syntactically unmarked interrogative sentences have horizontal contours at the other extreme. In between are found other question types and non-final sentences. (Thorsen 1983a:192)

Von besonderer Bedeutung ist dabei die letzte betonte Silbe: "the vital information about the intonation contour, i.e. sentence type, is contained in the last stressed syllable" (Thorsen 1979:75).

Nach Vaissière spielt der Deklinationsverlauf auch in anderen Sprachen bei der Satzmodusanzeige eine gewisse Rolle: Das Vorhandensein gegenüber dem Fehlen eines Abfalls des Deklinationsverlaufs "contributes to the contrast between declarative and interrogative sentences" (Vaissière 1983:57). Allerdings schätzt sie den Beitrag des Deklinationsverlaufs für die Satzmodusmarkierung insbesondere in spontanen Außerungen - eher als gering ein.

Eine Gegenposition dazu nimmt u.a. Ladd ein, der diesem auf konkrete Außerungen bezogenen "regression-line view" (Ladd 1983:46) den Ansatz einer weitgehend invarianten 'Basislinie' (wie in Pierrehumbert 1980, s.o.) entgegensetzt:

The zero-line view of declination involves the stronger and more abstract hypothesis that declination is more or less invariant, a gradual (probably physiologically determined) lowering of an abstract zero value from which accent peaks are skaled. (Ladd 1983:46)

In den konkret in einer Außerung feststellbaren Deklinationsverlauf gehen dagegen neben dem Verlauf der Basislinie auch die verschiedenen Emphasestufen der entlang dieser Linie aufgereihten Akzenttöne ein. Der Einfluß der Basislinie zeigt sich also gerade in den nicht durch Akzenttöne überdeckten, unbetonten Teilen des einer Außerung zugeordneten Fo-Verlaufs.

Mit drei Beispielen spontan geäußerter deutscher Ergänzungs- und Versicherungsfragesätze versucht Ladd diese Behauptung $\mathrm{zu}$ untermauern: Nach den akzentbedingten Anstiegen der Grundfrequenz erfolge jeweils in den unbetonten Partien ein der Basislinie entsprechender leichter Abfall der Grundfrequenz. Der nicht durch Akzenttöne überdeckte Deklinationsverlauf in Fragesätzen entspricht also dem in Aussagesätzen. Auf das Phänomen, daß die betonten Silben in dänischen assertiven Fragen zumindest der Tendenz nach auf einer nichtfallenden Linie zu liegen scheinen, geht Ladd allerdings nicht näher ein. Im Basislinien-Modell sollte damit eigentlich eine zum Außerungsende hin zunehmende Prominenz der Akzentsilben einhergehen: Daß das fürs Dänische nicht 
zutrifft, könnte - so ließe sich spekulieren - damit zusammenhängen, daß es dort in 'neutralen' nicht-kontrastiven Außerungen anscheinend keinen besonders hervorgehobenen Satzakzent gibt (vgl. Thorsen 1983b:29), so daß die in anderen Sprachen für Hervorhebungszwecke genutzte Distanz der Akzenttöne zur Basislinie hier zur Satzmodusanzeige dienen kann.

\section{Material}

Geeignetes Material, um die Frage der Satzmodusabhängigkeit der Fo-Deklination untersuchen zu können, bietet das sogenannte Fokuskorpus, das im DFGProjekt 'Modus-Fokus-Intonation' konzipiert wurde, um die Interdependenz von intonatorischer Satzmodus- und Fokusmarkierung untersuchen $\mathrm{zu}$ können (vgl. Oppenrieder 1989 und zur genaueren Beschreibung des Korpus Batliner/Oppenrieder 1989). Es ist für eine solche Untersuchung deshalb besonders geeignet, weil nur verhältnismäßig lange, in ihrem Satzmodus und in ihrer Fokusstruktur zudem kontrollierte Außerungen vorkommen, bei denen die globaleren Fo-Veränderungen zuverlässig von den lokalen Hervorhebungen getrennt werden können. Bei diesem Korpus wurden für die Untersuchung der lokalen Hervorhebungen zeitlich genau lokalisierte Fo-Werte extrahiert (s.u.), die eben auch für die Untersuchung der globalen Fo-Charakteristika der Realisationen genutzt werden können.

Dem Fokuskorpus liegen drei weitgehend parallel gebaute Wortfolgen zugrunde:

(1) Sie läßt die Nina das Leinen weben

(2) Lassen Sie den Manni die Bohnen schneiden

(3) Lassen wir den Leo die Blumen düngen

Je nach intonatorischer Markierung liegt in (1) ein Aussagesatz oder eine assertive Frage, in (2) ein Sie-Imperativsatz oder ein Entscheidungsfragesatz und in (3) ein Adhortativsatz oder ein Entscheidungsfragesatz vor. Jedem Testsatz sind vier verschiedene Fokusstrukturen zugeordnet: zwei enge Foki mit a) Fokussierung des Objekts (das Leinen, die Bohnen, die Blumen) oder b) des (infiniten) Verbs (weben, schneiden, düngen) des eingebetteten 'A.c.I.-Satzes', c) Fokusprojektion vom objekt auf das Verb (das Leinen weben, die Bohnen schneiden, die Blumen düngen) und d) Doppelfokussierung von objekt und Verb (das Leinen/weben, die Bohnen/schneiden, die Blumen/düngen). Es ergeben sich also $6 \times 4=24$ in Satzmodus und/oder Fokus sich unterscheidende Strukturen.

In den Produktionsexperimenten wurden die 'Satzmodus-Lesart' und der intendierte Fokus nicht explizit vorgegeben, sondern durch die Einbettung der Sätze in einen geeigneten Kontext gesteuert. Es nahmen drei weibliche (im fol- 
genden Spl - Sp3) und drei männliche Versuchspersonen (Sp4 - Sp6) teil, die jede der Strukturen mindestens zweimal produzierten. Wiederholungen nicht angemessen produzierter strukturen dazugerechnet liegen 360 Testsatzrealisationen vor. Vom Zeitsignal, Fo- und Intensitätsverlauf dieser Exemplare wurden Mingogramme erstellt.

Für die genauere Untersuchung der intonatorischen Realisierung von unterschiedlichen Fokusstrukturen wurden anhand dieser Mingogramme für das eingebettete A.c.I.-'Subjekt' (die Nina, den Manni, den Leo), das eingebettete Objekt (das Leinen, die Bohnen, die Blumen) und das infinite Verb (weben, schneiden, düngen) jeweils das Fo-Minimum und -Maximum und deren zeitliche Lokalisierung gemessen, so daß neben dem ebenfalls extrahierten Fo-Onset- und Offset-Wert (Fo-Werte am Beginn und Ende der jeweiligen Außerung) sechs genau lokalisierte Fo-Werte für jede einzelne Testsatzrealisation vorliegen. ${ }^{3}$

Durch alle diese Punkte können Verbindungslinien gelegt werden, die genau berechenbare 'Deklinationen' aufweisen. Diese verschiedenen Deklinationsverläufe lassen sich nicht nur in Beziehung setzen zu den Akzentuiertheitsurteilen aus dem Akzenttest (in dem - ohne Kontext - die am stärksten betonte Silbe jeder Realisation markiert werden sollte; vgl. dazu Batliner 1989), sondern es läßt sich auch untersuchen, ob ihre Neigungsrichtung und ihre steigung mit bestimmten Satzmodi korrelieren.

Aus den konkreten Deklinationsverläufen lassen sich durch Mittelwertbildung Deklinationslinien gewinnen, die eine Menge von Realisationen kennzeichnen und einen 'prototypischen Deklinationsverlauf' repräsentieren. Im folgenden wurden zur Berechnung solcher Deklinationslinien bestimmte Arten von Verläufen (z.B. die durch onset und offset gelegten Verbindungslinien) in definierten Teilmengen (z.B. in Außerungen mit dem Merkmal 'Aussagesatzrealisationen der Sprecherin $\left.X^{\prime}\right)$ gemittelt.

Als Berechnungsgrundlage für die jeweiligen Deklinationsverläufe dienen die Polgenden 8, zeitlich genau lokalisierten Fo-Werte: der Wert am Beginn der Außerung (=onsetwert) und an ihrem Ende (=offsetwert), der maximale und der minimale Wert im Bereich des eingebetteten Subjektsausdrucks (die Nina, den Manni, den Leo; = Phrase 1), des Objektsausdrucks (das Leinen, die Bohnen, die Blumen; = Phrase 2) und des infiniten Verbs (weben, schneiden, düngen; = Phrase 3).

Für jede Verbindung zweier dieser Punkte läßt sich die Steigung berechnen; Als Steigungsmaß der Deklinationslinie berechneten wir das arithmetische Mittel

$3 \quad$ Der Offset kann auch mit dem Maximum oder dem Minimum zusammenfallen. 
aus den Steigungen der einander entsprechenden Verbindungslinien mehrerer Außerungen. ${ }^{4}$

Anders als Ladd (1983) gehen wir von einer viel umfangreicheren und kontrollierteren Datenbasis aus. Wie bei Thorsen (1983a) enthält unser Material Aussagesätze und assertive Fragen, d.h. Fragen, die sich nur durch intonatorische Merkmale von Aussagesätzen unterscheiden. Zusätzlich sind die im Korpus enthaltenen Realisationen von Entscheidungsfragesätzen aufgrund der Wortfolge nicht von Imperativsätzen $z u$ unterscheiden, so da $\beta$ wiederum allein die intonatorische Form für die satzmodusmarkierung verantwortlich ist (sogenannte intonatorische Minimalpaare, vgl. Altmann 1984). Es sollte sich also wenigstens ein genereller 'Deklinations'-Unterschied zwischen Fragen und NichtFragen feststellen lassen.'

Natürlich muß berücksichtigt werden, daß im Deutschen bereits die Höhe des Fo-Offset und die spezlelle 'Kontur' des Fo-Verlaufs im Bereich der Hauptakzentsilbe dominante intonatorische Merkmale für die Satzmoduskennzeichnung sind (vgl. Oppenrieder 1988). Selbst wenn die Neigungsrichtung und der Neigungsgrad der durch Minima oder Maxima gezogenen Deklinationslinien satzmodustypisch sind, so ist doch anzunehmen, daß sie gegenüber den auch perzeptiv auffälligeren Merkmalen Offsethöhe und 'Kontur' als sekundäre Merkmale einzustufen sind. Da es sich nicht um zentrale Merkmale handelt, wäre es andererseits auch nicht ungewöhnlich, wenn sich solche satzmodustypischen Deklinationslinien nicht in jedem konkreten Deklinationsverlauf manifestieren: Das Konzept der 'prototypischen intonatorischen Form' von Außerungen läßt ohne weiteres $z u, d a \beta$ im einzelnen Fall weniger zentrale Merkmale fehlen.

\section{Ergebnisse}

Im folgenden erhalten die drei oben genannten Teilphrasen des eingebetteten A.c.I.-Ausdrucks die Nummern 1 (='Subjekt'), 2 (=Objekt) und 3 (=Infinitiv). Verglichen werden die Deklinationslinien, die sich durch die Minima und Maxima von Phrase 1 und $3(1 / 3)$, bzw. von 2 und $3(2 / 3)$ legen lassen, sowie die Linie $z$ wischen On- und Offset. Die Deklinationslinien $1 / 3$ werden gewählt, weil sie neben der Onset-offset-Linie am 'globalsten' sind, also am ehesten von den

\footnotetext{
4 Der Begriff 'Deklination' wird also nicht unbedingt im klassischen Sinn verwendet, wo er sich immer auf Gesamtäußerungen bezieht.

- Von Thorsen unterscheiden wir uns zudem durch die Auswahl der 'Ankerpunkte' für die Deklinationsverläufe. Während bei ihr deren Berechnung direkt auf den betonten Silben beruht, legen wir die drei kompletten Phrasen zugrunde, deren maximaler und minimaler Grundfrequenzwert jeweils in die Berechnung der 'Deklinationen' eingeht.
} 
lokaleren Verschiebungen durch die fokusanzeigende Hervorhebung einer Phrase unberührt bleiben sollten.

Von der durch den Kontext vorgegebenen Fokussierung ist die Phrase 1 (das eingebettete Subjekt) stets ausgeschlossen; mit Hilfe des erwähnten Akzenttests läßt sich zudem leicht feststellen, ob die Phrase 1 tatsächlich im Vergleich zu den folgenden Ausdrücken nicht hervorgehoben ist, so daß die für sie notierten Minima- und Maxima-Werte 'neutral' sind und keine besondere Akzentuierung widerspiegeln.

Die Phrase 3 (das infinite Verb) sollte dagegen bei zwei der vier Fokusbedingungen eine stark hervorgehobene Silbe enthalten - beim engen Fokus auf dem Verb und bei der Doppelfokussierung auf dem objekt und dem Verb. Wiederum kann über die Akzenttests nachgeprüft werden, ob eine derartige Hervorhebung tatsächlich vorhanden ist. In diesem Fall ist eine mehr oder weniger starke Beeinflussung des Deklinationsverlaufs $1 / 3$ durch die Akzentuierung zu erwarten, so daß ein satzmodustypischer Verlauf möglicherweise nicht mehr zu erkennen ist; bei der Berechnung der Deklinationslinien sind diese Fälle eventuell auszuscheiden. Die Ergebnisse des Akzenttests zeigen allerdings, daß bei der beabsichtigten Doppelfokussierung nur jeweils eine der beiden fokussierten Phrasen stärker hervorgehoben ist - bei 5 Sprechern das objekt (wie bei der Fokusprojektion und beim engen Fokus auf dem objekt), bei Sp4 das infinite Verb. Auch die Fo- und Dauerwerte der Doppelfokus-Realisationen ähneln bei den 5 Sprechern denen der Realisationen mit objektfokus oder Fokusprojektion; die Werte für das infinite Verb unterscheiden sich jedenfalls deutlich von denen bei enger Fokussierung dieses Verbs und zeigen keine auffällige lokale Hervorhebung. 6

Generell ist anzunehmen, daß die Minima weniger stark durch die Hervorhebung einzelner Silben beeinflußt werden als die Maxima. Die 'lokaleren' Deklinationsverläufe $2 / 3$ sind auf jeden Fall stärker von der jeweils realisierten Hervorhebung abhängig, zumal die Fokussierung innerhalb dieser beiden Phrasen, Objekt und Verb, variieren sollte. Die 2/3-Verläufe sollten also weniger ausgeprägt mit den jeweiligen Satzmodi korrelieren.

Die Einzelergebnisse $z u$ den ermittelten Deklinationslinien (Onset-OpfsetDeklination, Maxima-Deklination 1/3, Minima-Deklination 1/3, Maxima-Deklination 2/3, Minima-Deklination 2/3) finden sich in den folgenden Tabellen. Je

6 Das unterscheidet unsere Realisationen der Doppelfokus-Strukturen deutlich von denen, die Eady et al. (1986) erhielten: Dort entsprechen bei Doppelfokussierung die Fo- und Dauerwerte beider fokussierter Phrasen weitgehend denen bei der jeweiligen engen Fokussierung; zu einer möglichen Erklärung für dieses Phänomen vgl. Oppenrieder (1989). 
Sprecher und Satzmodus wurde das arithmetische Mittel aus den Steigungen (in Hertz/Centisekunden) der einzelnen Verläufe gebildet; ein positiver Wert bedeutet, daß die entsprechende Deklinationslinie fällt, ein negativer, daß sie steigt. Hinter diesem Wert findet sich in Klammern die Standardabweichung.

Die in manchen Fällen zusätzlich aufgeführten Werte ergeben sich, wenn bestimmte Realisationen bei der Berechnung ausgeschieden werden. Dabei beziehen sich die hinter dem Kürzel $\mathrm{N} / \mathrm{K}$ stehenden Werte auf durchschnittliche Steigungen, die sich ergeben, wenn als unnatürlich beurteilte und nicht wie erwartet kategorisierte Realisationen nicht berücksichtigt werden. Im Natürlichkeitstest wurde überprüft, wie gut die Testsatzrealisationen in den vorgegebenen Kontext passen; im Kategorisierungstest mußte von den Testpersonen kontextfrei der Funktionstyp der Realisationen festgestellt werden.

Auf diese Weise können Realisationen ausgesondert werden, die nicht der Kontextvorgabe entsprechen, von denen daher angenommen werden kann, daß ein gegenüber den 'guten' Realisationen deutlich abweichender Deklinationsverlauf auf eine 'Fehlrealisierung' zurückzupühren ist.

Problematisch sind in diesem Zusammenhang die Adhortativsatzrealisationen, die bei 4 Sprechern im Durchschnitt nur $\mathrm{zu} 50 \%$, bei den restlichen beiden Sprechern zu 70\% (Sp5), bzw. 80\% (Sp1) wie angezielt klassifiziert wurden (vgl. dazu Oppenrieder 1989).

$\mathrm{Zu}$ beachten ist, da $\beta$ sich bis auf einen Sprecher (Sp4) die Natürlichkeitsbewertungen für die Adhortativsatzrealisationen im Rahmen der für den jeweiligen Sprecher typischen Benotungen bewegen. Die vereinzelten unerwarteten Kategorisierungen bei den restlichen satzmodi sind dagegen mit schlechten Natürlichkeitswerten verknüpft, so daß davon ausgegangen werden kann, da $\beta$ die vorgegebenen Strukturen nicht kontextgerecht realisiert wurden.

Eventuell vorhandene zusätzliche Zahlen in den Tabellen beziehen sich auf Mittelwerte, bei deren Berechnung die Realisationen bestimmter Fokusstrukturen nicht berücksichtigt werden, da die globalen Deklinationscharakteristika durch die lokale Hervorhebung überdeckt werden. Insbesondere sind davon die Strukturen mit (engem) Verbfokus betropfen.

Generell sind bei den meisten Sprechern durch alle diese verschiedenen Deklinationslinien die Frage- und die Nicht-Frage-Modi deutlich unterschieden, da die entsprechenden Verbindungslinien im ersten Fall ansteigen, im zweiten dagegen abfallen. 
Für die Verbindungslinie von Onset und offset (vgl. Tabelle 1) ist eine deutliche Trennung von Frage- und Nicht-Frage-Modi nicht überraschend, da in sie der offset-Wert eingeht, der bekanntlich ein ziemlich zuverlässiges und im Gesamtkomplex der satzmodusmarkierenden intonatorischen Merkmale dominierendes Merkmal ist (vgl. Oppenrieder 1988, Batliner et al. 1989).

Tabelle 1: Onset-offset-Deklinationslinien

\begin{tabular}{|c|c|c|c|c|c|}
\hline & $\begin{array}{l}\text { Entscheidungs- } \\
\text { fragesatz } \\
\text { Sie Vir }\end{array}$ & Assertive Frage & Aussagesatz & $\begin{array}{l}\text { Sie-Impera- } \\
\text { tivsatz }\end{array}$ & $\begin{array}{l}\text { Adhortativ- } \\
\text { satz }\end{array}$ \\
\hline Sp1 & $-.05(.34)$ & $.21(.31)$ & $.5 \quad(.24)$ & $.5 \quad(.15)$ & $.46(.11)$ \\
\hline Sp2 & $-.77(.28)$ & 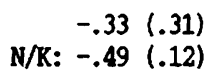 & $.57(.23)$ & $.64(.27)$ & $.65(.31)$ \\
\hline Sp3 & 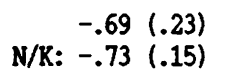 & $-.66(.18)$ & $.35(.12)$ & $.16(.07)$ & $.18(.12)$ \\
\hline Sp4 & $-.43(.12)$ & $-.41(.08)$ & $.1(.04)$ & $.11(.08)$ & $.14(.09)$ \\
\hline Sp5 & $-.59(.09)$ & $-.56(.13)$ & $.13(.04)$ & $.15(.05)$ & $.16(.09)$ \\
\hline Sp6 & $\mathrm{N} / \mathrm{K}: \begin{array}{ll}-.57 & (.29) \\
-.65 & (.17)\end{array}$ & $\mathrm{N} / \mathrm{K}: \begin{array}{ll}-.51 & (.27) \\
-.58 & (.16)\end{array}$ & $.24(.04)$ & $.14(.09)$ & $.16(.1)$ \\
\hline
\end{tabular}

Während der Onset bei allen Realisationen eine gewisse Höhe nicht überschreitet und nur geringe Variation aufweist, sind die Fragesatz-Realisationen aller SprecherInnen (mit Ausnahme von Sp1) durch einen hohen offset gekennzeichnet - im Gegensatz zu den Realisationen der restlichen Satzmodi: Bei den letzteren fält die durch on- und offset gelegte Linie ab, während sie bei den Fragesatz-Realisationen ansteigt.

Sowohl die Realisationen der Fragesätze (Entscheidungsfragesätze und assertive Fragen) als auch die der restlichen Satztypen (Aussagesätze, SieImperativsätze und Adhortativsätze) bilden nicht nur bezüglich der Neigungsrichtung, sondern auch bezüglich des durchschnittlichen Neigungswinkels bei jeder einzelnen Versuchsperson erstaunlich homogene Gruppen. Lediglich bei einer Sprecherin (Sp3) und einem Sprecher (Sp6) ist die 'Aussagesatz- Deklina- 
tion' deutlich steiler als die 'Deklination' für die beiden Imperativsatztypen (Sp3: .35 zu .16 und .18; Sp6: .24 zu .14 und .16); bei einer anderen sprecherin (Sp2) ist die Deklination der Entscheidungsfragesätze $(-.77)$ doppelt so steil wie die der assertiven Fragen $(-.33)$. $^{\text {? }}$

Einzelne besonders stark streuende Werte lassen sich auf den Einfluß von Realisierungen zurückführen, die als unnatürlich bewertet und nicht wie erwartet kategorisiert wurden; werden diese Realisationen nicht berücksichtigt, so erhält man eine deutlich geringere Streuung (vgl. Sp3: Entscheidungsfrage und assertive Frage; Sp2: assertive Frage; Sp6: Entscheidungsfrage und assertive Frage).

Die insbesondere bel den Nicht-Fragen zwischen den männlichen (Sp4 - Sp6) und zwei der weiblichen Versuchspersonen ( $\mathrm{Sp1}, \mathrm{Sp} 2$ ) auftretenden Differenzen in der Steilheit der Onset-Offset-Deklination fallen bei einer Berechnung auf Halbtongrundlage weniger deutlich aus.

Ganz allgemein kann also über diese die offset-Höhe einbeziehende Verbindungslinie eine gute Unterscheidung zwischen Fragesätzen und dem Rest getroffen werden, eine feinere Differenzierung ist jedoch nur vereinzelt bei manchen Versuchspersonen zu beobachten. Wahrscheinlich spielt in diesen Fällen auch die Onset-Höhe eine gewisse satzmodusunterscheidende Rolle. Wo Fragesatz- und Nicht-Fragesatz-Realisationen durch die Neigungsrichtung der Onset-Offset-Linie unterschieden sind, dort ist auch fast durchgängig der Neigungswinkel beim fragesatztypischen Anstieg wesentlich größer als bei der abfallenden Onset-offset-Linie der übrigen Satztypen.

Wie bereits angedeutet, fällt bei den Fragesatzrealisationen eine der sprecherinnen ( $\mathrm{Sp} 1$ ) aus dem Rahmen, da sie fast alle Fragesätze mit einem tiefen offset realisiert, so daß sich bei den assertiven Fragen im Durchschnitt eine abfallende Onset-Offset-Verbindungslinie ergibt (.21), bei den Entscheidungsfragesätzen eine nur ganz schwach ansteigende (-.05). Dem Regelfall entsprechen hingegen die Realisationen der Entscheidungsfragesätze, bei denen der satzfinale Infinitiv den Fokus bildet $(-.51)$ : Ein später akzentrelevanter FoAnstieg begünstigt hier also das eigentlich fragesatztypische Verweilen auf der erreichten Tonhöhe. Die beiden Realisationen der assertiven Frage, bei denen ein solches Muster ebenfalls zu erwarten wäre, zeigen eine schwach ansteigende $(-.28)$ Onset-offset-Verbindungslinie, bzw. eine völlig gleichbleibende.

7 Die drei Fälle zeigen also ein Verhältnis der Neigungswinkel, das man nicht unbedingt erwarten würde: Bei Sp2 ist gerade nicht die assertive Frage, sondern die 'neutralere' Entscheidungsfrage durch den steileren Abfall der Deklinationslinie gekennzeichnet, bei Sp3 und Sp6 der 'neutralere' Aussagesatz und nicht einer der beiden Imperativsatztypen. 
Uberraschenderweise werden bis auf drei Fälle alle diese Exemplare mit fallender Onset-Offset-Linie im Kategorisierungstest richtig als Fragen identifiziert - und nur diese drei Fälle werden als ziemlich unnatürlich im vorgegebenen Kontext beurteilt. Die nicht auf den Offset bezogenen Deklinationslinien $1 / 3$ und $2 / 3$ weisen auch bei den Fragesatzrealisationen dieser Sprecherin fast alle einen Pragesatztypischen Anstieg auf (weitere Ausnahme: Minima-Deklinationslinie $1 / 3$, s.u.). Die meisten intonatorischen Merkmale haben aber die für assertive Fragen erwartbare Ausprägung, z.B. einen fallend-steigenden Fo-Verlauf im Bereich der Hauptakzentsilbe. Bei der (kontextfreien) Kategorisierung können sich die Versuchspersonen also auf diese Merkmale stützen (wobei die Akzentkontur sicher ein wichtigeres Merkmal ist als die verschiedenen Deklinationslinien). Auf jeden Fall handelt es sich zwar um keine prototypischen, aber offensichtlich um zulässige Realisierungen.

\subsection{Phrasenbezogene Deklination}

\subsubsection{Maxima-Deklination $1 / 3$}

In die Maxima-Deklination 1/3 (vgl. Tabelle 2), die sich aus der Verbindung zwischen den Fo-Maxima des A.c.I.-'Subjekts' und des Infinitivs ergibt, geht bei den Fragesatz-Realisationen noch teilweise der offsetwert ein, und zwar dann, wenn dieser dem maximalen Fo-Wert aup dem Verb entspricht. Daß bei hohem offset eine ansteigende Verbindungslinie resultiert, auch wenn das infinite Verb nicht aufgrund der Fokusstruktur besonders hervorgehoben wird, verwundert also in solchen Fällen nicht, zumal der eingebettete 'Subjekts'-Ausdruck niemals fokussiert wird.

Interessant sind hier insbesondere die Ergebnisse für die oben schon erwähnte Sprecherin (Spl), die die Fragesätze, insbesondere auch die assertiven Fragen, größtenteils mit tiefem offset realisierte. Die Maxima-Deklinationslinie 1/3 steigt - im Gegensatz zu der im Durchschnitt eher abfallenden Onset-Offset-Verbindungslinie. Nach Abzug der als unnatürlich beurteilten und nicht wie erwartet kategorisierten Realisationen ist das Verhältnis der Onset-offsetDeklination zur Maxima-Deklination $1 / 3$ bei den Entscheidungsfragesätzen -.08 $\mathrm{zu}-1.05$, bei den assertiven Fragen $.14 \mathrm{zu}-.73$.

Die zwei Entscheidungsfragesätze mit einem Abfall der Maxima-Deklination 1/3 (.79 und .68) schnitten dagegen im Natürlichkeitstest sehr schlecht ab und wurden auch im Kategorisierungstest entsprechend dem anderen Glied des Minimalpaars, nämlich der Aufforderung, 'fehlklassifiziert'. 
Damit entspricht auch im Fall von Spl die Maxima-Deklination $1 / 3$ der fragesatztypischen Maxima-Deklination bei den anderen Versuchspersonen, ohne daß, wie bei diesen ziemlich häufig, in die Berechnung der offset-Wert als maximaler Fo-Wert auf dem Infinitiv eingeht.

Tabelle 2: Maxima-Deklinationslinien $1 / 3$

\begin{tabular}{|c|c|c|c|c|c|}
\hline & $\begin{array}{l}\text { Entscheidungs- } \\
\text { fragesatz } \\
\text { Sie Wir }\end{array}$ & Assertive Frage & Aussagesatz & $\begin{array}{l}\text { Sie-Impera- } \\
\text { tivsatz }\end{array}$ & $\begin{array}{l}\text { Adhortativ- } \\
\text { satz }\end{array}$ \\
\hline Sp1 & $\begin{array}{r}-.87(.59) \\
N / K=-1.05(.2)\end{array}$ & $-.7 \quad(.18)$ & $\begin{array}{c}.44(.6) \\
\text { ohne V-Folkus: } \\
.74(.26)\end{array}$ & $.51(.17)$ & $.47(.35)$ \\
\hline $\mathrm{Sp} 2$ & $-1.3 \quad(.39)$ & $\begin{array}{rll} & -.72 & (.53) \\
\mathrm{N} / \mathrm{K}: & -.94 & (.2)\end{array}$ & $.53(.29)$ & $.17(.21)$ & $.19(.4)$ \\
\hline $\mathrm{Sp} 3$ & $\mathrm{~N} / \mathrm{K}=\begin{array}{ll}-.91 & (.37) \\
-.99 & (.22)\end{array}$ & $-.7 \quad(.24)$ & $.77(.36)$ & $.42(.23)$ & $.43(.25)$ \\
\hline $\mathrm{Sp} 4$ & $-.46(.19)$ & $-.27(.09)$ & $\begin{array}{l}.21(.33) \\
\text { ohne V- und } \\
\text { Doppelfokus: } \\
.48(.12)\end{array}$ & $\begin{array}{c}.15(.12) \\
\mathrm{N} / \mathrm{K}: \\
.2 \quad(.1)\end{array}$ & $.07(.43)$ \\
\hline $\mathrm{Sp} 5$ & $-.7 \quad(.11)$ & $-.5 \quad(.18)$ & $\begin{array}{lll} & .35 & (.27) \\
\mathrm{N} / \mathrm{K}: & .47 & (.24)\end{array}$ & $.24(.09)$ & $.35(.17)$ \\
\hline Sp6 & $\mathrm{N} / \mathrm{K}=\begin{array}{ll}-.59 & (.53) \\
-.73 & (.3)\end{array}$ & $\mathrm{N} / \mathrm{K}: \begin{array}{ll}-.41 & (.47) \\
-.54 & (.21)\end{array}$ & $\begin{array}{c}.51(.46) \\
\text { ohne V-Fokus: } \\
.74(.14)\end{array}$ & $.54(.25)$ & $\begin{array}{c}.39(.46) \\
\mathrm{N} / \mathrm{K}: \\
.5 \quad(.28)\end{array}$ \\
\hline
\end{tabular}

Für alle Versuchspersonen gilt, daß die Maxima-Deklination $1 / 3$ bei den Fragen durch einen Anstieg gekennzeichnet ist. Wie zu erwarten, ist die Steigung bei den männlichen Versuchspersonen kleiner, da die Berechnung nicht auf Halbtonbasis, sondern mit den Hertz-Werten erfolgte. Bei allen sechs Versuchspersonen ist die Steigung bei den Entscheidungsfragesätzen größer als bei den assertiven Fragen.

Bei den Aussagesätzen und Imperativsätzen fällt die Deklinationslinie meist. Deutliche - und erklärliche - Ausnahmen hierzu bilden einige der Exemplare, bei denen nach der Kontextvorgabe das infinite Verb den Fokus bildet, also hervorgehoben werden sollte. Die akzentuelle Hervorhebung kann durch einen ausgeprägten Fo-Gipfel erfolgen. Entsprechend erhält man eine steilere Deklinationslinie und eine geringere Streuung, sobald man diese Realisationen bei der Mittelwertbildung nicht berücksichtigt; bis auf einen Sprecher (Sp4) blieb die Kontextanweisung der Doppelfokussierung ohne größere Auswirkung auf den Fo- 
den Fo-Range des Verbs, so daß nur bei diesem neben den Verbfokus- auch die Doppelfokus-Realisationen von der Mittelwertberechnung ausgeschlossen wurden. In vielen Fällen bleibt aber auch bei einer Fokussierung des Verbs die typische, fallende Deklination erhalten: Die Hervorhebung erfolgt dann nicht durch einen besonders hohen Fo-Wert oder -Range, sondern durch die Dehnung der Hauptakzentsilbe; möglicherweise liegt in diesen Fällen ein 'Downstep-Muster' vor, d.h. ein Muster, bei dem in einer Folge prominenzanzeigender Fo-Gipfel die Veränderungen immer geringer ausfallen, ohne daß damit eine geringer werdende Prominenz der hervorgehobenen Silben bzw. Phrasen einhergeht (vgl. die in Teil 1 erwähnte Literatur).

Die Steilheit der Maxima-Deklination $1 / 3$ bei den Nicht-Fragen scheint versuchspersonenspezifisch zu sein; möglicherweise wird von manchen Versuchspersonen das Downstep-Muster bevorzugt: Bei der Sprecherin Sp2, für die sich bei den Fragen die steilsten Deklinationslinien ergeben, entsprechen die Werte für die Nicht-Fragen eher denen der beiden Sprecher Sp4 und Sp5, während sich bei Sp6 wie bei den restlichen beiden Sprecherinnen $\mathrm{Sp} 1$ und $\mathrm{Sp} 3$ ein steilerer Abfall der Deklinationslinien ergibt.

Wie die Zahlenwerte der Tabelle 2 zeigen, sind innerhalb der Nicht-Fragen die Aussagesätze gegenüber den Imperativsätzen durch einen steileren Abfall der Maxima-Deklination $1 / 3$ ausgezeichnet. Das könnte ein Hinweis darauf sein, daß Aussagesätze bessere Kandidaten für das Downstep-Muster sind als Imperativsätze.

\subsubsection{Minima-Deklination $1 / 3$}

Die Ergebnisse bei den Minima-Deklinationslinien 1/3 (vgl. Tabelle 3) sind im Bereich der Fragesätze nicht ganz so eindeutig. Zwar steigen auch hier fast in jedem Fall die jeweiligen Deklinationslinien an. Einige Ausnahmen und Besonderheiten sind jedoch $\mathrm{zu}$ beachten. 
Tabelle 3: Minima-Deklinationslinien $1 / 3$

\begin{tabular}{|c|c|c|c|c|c|}
\hline & $\begin{array}{l}\text { Entscheidungs- } \\
\text { fragesatz } \\
\text { Sie Wir }\end{array}$ & Assertive Frage & Aussagesatz & $\begin{array}{l}\text { Sie-Impera- } \\
\text { tivsatz }\end{array}$ & $\begin{array}{l}\text { Adhortativ- } \\
\text { satz }\end{array}$ \\
\hline Sp1 & $\begin{array}{c}-.27(.24) \\
\mathrm{N} / \mathrm{K}:-.31(.22) \\
\mathrm{N} / \mathrm{K}+0 . \mathrm{V}-\text { Fokus: } \\
-.38(.19)\end{array}$ & $\begin{array}{c}.1 \quad(.28) \\
\mathrm{N} / \mathrm{K}+\text { ohne V-Fokus: } \\
-.06(.13)\end{array}$ & $.24(.2)$ & $.18(.11)$ & $.06(.14)$ \\
\hline Sp2 & $\begin{array}{l}-1 . \quad(.78) \\
\text { ohne V-Fokus: } \\
-1.42(.46)\end{array}$ & 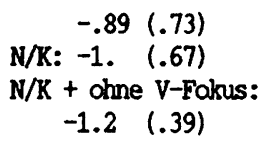 & $.36(.24)$ & $.13(.16)$ & $.23(.14)$ \\
\hline Sp3 & $\begin{aligned} & -.55(.43) \\
\mathrm{N} / \mathrm{K}: & -.59(.39) \\
\mathrm{N} / \mathrm{K} & +0 . \mathrm{V}-\mathrm{Folkus}: \\
& -.79(.21)\end{aligned}$ & $\begin{array}{l}-.84(.62) \\
\text { ohne V-Folkus: } \\
-1.1 \quad(.34)\end{array}$ & $.54(.16)$ & $.26(.09)$ & $.33(.17)$ \\
\hline Sp4 & 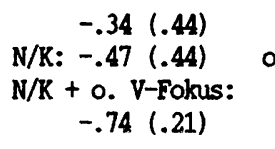 & $\begin{array}{c}-.08(.41) \\
\text { o. v-u.Doppelfokus: } \\
-.47(.05)\end{array}$ & $.27(.07)$ & $.12(.06)$ & $.13(.07)$ \\
\hline Sp5 & 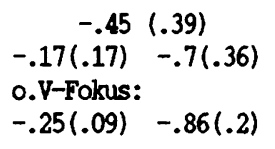 & $\begin{array}{l}-.23(.34) \\
\text { ohne V-Fokus: } \\
-.39(.18)\end{array}$ & $.15(.08)$ & $.07(.11)$ & $.08(.08)$ \\
\hline Sp6 & 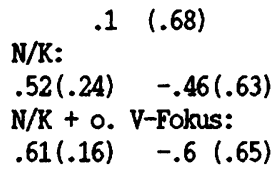 & $.19(.5)$ & $.35(.07)$ & $.24(.07)$ & $.33(.3)$ \\
\hline
\end{tabular}

Zum einen müssen bei zwei Sprechern (Sp5, Sp6) die zwei Versionen der Entscheidungsfragesätze getrennt werden. Eine - wie erwartet - steigende Deklinationslinie findet sich in den Versionen der Entscheidungsfragesätze, die die gleiche Struktur wie der Adhortativsatz aufweisen (Sp5: -.7; Sp6: -.46). Bei der zum Sie-Imperativsatz parallelen Struktur ist dagegen der Anstieg entweder weniger ausgeprägt (Sp5: -.17) oder aber es findet sich ein Abfall (Sp6: .52). Die lexikalische Füllung kann nicht der Grund für diese Unterschiede sein, da die beiden Sätze in diesem Punkt einander zu ähnlich sind. Eher könnte der einbettende Kontext bei den zum Sie-Imperativsatz parallelen Exemplaren eindeutiger 'frageindizierend' sein, so daß eine weniger prototypische Realisierung des Fragesatzes gewählt werden kann. (Allerdings ist in allen Fällen ein hoher Offset, also ein dominantes Merkmal zur Kennzeichnung von Fragesätzen, vorhanden; entsprechend steigen die Onset-offset- und die 1/3-Maxima-Deklina- 
tionslinien an.) Vielleicht ist aber auch der unterschiedliche Status des jeweils zugeordneten 'Minimalpaar-Satzmodus', also Sie-Imperativ- oder Adhortativsatz, von Belang. Eine echte Ausnahme zu den bei Entscheidungsfragesätzen typischerweise ansteigenden Deklinationslinien findet sich nur bei den 'Sie-Fragesätzen' des Sprechers Sp6. Dieser Sprecher taucht auch in der zweiten Ausnahmegruppe auf, die (untypische) nicht ansteigende Deklinationslinien bei den assertiven Fragen umfaßt. Die prototypische Realisierung eines intonatorisch markierten Fragesatzes ist durch einen Anstieg der Minima-Deklination gekennzeichnet. Bei der Sprecherin Sp1, deren untypische Realisation der assertiven Fragen im Zusammenhang der Onset-Offset-Deklination bereits angesprochen wurde, steigt die entsprechende Minima-Deklinationslinie $1 / 3$ ebenfalls nicht an. offensichtlich ist also eine Variation bei dem für die Satzmodus-Anzeige nichtdominanten Merkmal der Deklinationsrichtung durchaus möglich, allerdings nicht typisch: Die fallenden Deklinationslinien bei den Fragesätzen des Korpus sind Ausnahmen, die möglicherweise einen persönlichen Sprechstil kennzeichnen.

Die Nicht-Fragen sind demgegenüber ausnahmslos durch abfallende MinimaDeklinationslinien $1 / 3$ gekennzeichnet.

$\mathrm{Da}$ anders als bei der Maxima- und der Onset-offset-Deklination ein fragesatztypischer hoher offset auf keinen Fall in die Berechnung eingehen kann, ist der Unterschied in der Neigungsrichtung der Minima-Deklinationslinien auch nicht lediglich auf den Einfluß der offsethöhe zurückzuführen, sondern ein von dieser unabhängiges Merkmal.

Natürlich bleiben lokale Fo-Veränderungen, die eine besonders starke Hervorhebung - hier des infiniten Verbs, also der Phrase 3 - anzeigen, wiederum nicht ohne Einwirkung auf die globale Minima-Deklinationslinie 1/3. Im Gegensatz zur Maxima-Deklinationslinie $1 / 3$ sind jedoch in diesem Fall die Fragen betroffen. Dies erklärt sich aus den unterschiedlichen 'Hervorhebungsstrategien': Während bei den Realisationen der Nicht-Fragesätze die Fokussierung des infiniten Verbs durch einen aufälligen Fo-Gipfel angezeigt wird, geschieht dies bei denen der Fragesätze durch ein besonders ausgeprägtes Fo-Tal. ${ }^{8}$

Die lokale Hervorhebung überlagert die globaleren Deklinationslinien, bei den Maxima also deren Abfall und bei den Minima entsprechend deren Anstieg. Die Nichtberücksichtigung der Exemplare mit Fokussierung des infiniten Verbs bei

8 In einer tonalen Theorie der Akzentuierung muß von einer Hervorhebung durch einen Hochton, bzw. durch einen Tiefton gesprochen werden. Vgl. auch Batliner (1989), wo sich als Ergebnis diskriminanzanalytischer Untersuchungen am Fokuskorpus ergibt, da $\beta$ für die Prädiktion betonter Phrasen die Minima bei den Fragen relevanter sind als bei den Nicht-Fragen. 
der Berechnung der Deklinationslinien führt dementsprechend $\mathrm{zu}$ steileren Neigungswinkeln und zu einer geringeren Standardabweichung.

Innerhalb der Fragen steigt die Minima-Deklinationslinie $1 / 3$ (ohne die Verbfokus-Exemplare) mit Ausnahme von Sprecherin $\mathrm{Sp} 3$ bei den Entscheidungsfragen steiler an als bei den assertiven Fragen. (Auf die Ausnahmen bei Spl und Sp6 wurde bereits hingewiesen.) Bei den Nicht-Fragen zeigen die Aussagesätze den steilsten Abfall; eine mögliche Erklärung ist wieder die größere 'Anfälligkeit' dieses Satztyps für das Downstep-Muster, die sich auch auf den Verlauf der Minima-Deklination auswirkt.

Bei einem Vergleich der Minima- und Maxima-Deklinationslinien $1 / 3$ zeigt sich bei den Nicht-Fragen der bekannte Effekt, da $\beta$ die 'obere' Deklinationslinie steiler abfällt als die 'untere', so daß sich die beiden zunehmend einander nähern; nach Cohen et al. (1982:264) verschwindet dieser Annäherungs-Effekt weitgehend, sobald die Berechnung auf der 'gehörsadäquateren' Halbtonbasis erfolgt. Entsprechend dürfte auch der entgegengesetzte Spreiz-Effekt kleiner werden, der sich bei den Realisationen der Fragesätze zeigt: dort steigt die 'obere' Deklinationslinie steiler an als die 'untere'. In beiden Fällen werden die Deklinationslinien nach der Umrechnung also in etwa parallel verlaufen.

Das würde bedeuten, daß im prototypischen Fall innerhalb einer Außerung (oder vielleicht auch nur innerhalb einer intonatorischen Phrase) der Normalrange in etwa gleich bleibt - ein Normalrange, der sich aus dem Abstand der Deklinationslinien ergibt, in deren Berechnung nur Minima und Maxima der Phrasen mit in etwa gleicher 'Emphase-Stufe' eingehen. Die geringsten Standardabweichungen ergeben sich, wenn Realisationen mit einer markierten, engen Fokussierung (wie die des Verbs) nicht berücksichtigt werden. Fokussierung kann dann durch einen Fo-Gipfel - oder ein entsprechendes Tal angezelgt werden, der über die vorgegebene 'obere' Deklinationslinie hinausragt - bzw. die 'untere' Deklinationslinie durchbricht. (Vgl. dazu auch die beiden Typen von oberer und unterer Deklinationslinie im 'Lunder Intonationsmodell', Gårding 1983:14f.)

Die Neigungsrichtung der parallelen Deklinationslinien ist dabei satzmodusabhängig: In den (intonatorisch markierten) Realisationen von Fragesätzen steigen sie typischerweise an, ansonsten fallen sie mehr oder weniger stark ab, eventuell abhängig von einem graduierbaren 'Downstep-Faktor'. Demnach ist die globale Neigungsrichtung der Deklinationslinien (vielleicht auch deren steilheit) im Deutschen mit bestimmten Satzmodi korreliert, wenn auch anzunehmen ist, daß sie gegenüber dominanteren Merkmalen wie der Offsethöhe und dem Fo-Ver- 
lauf im Bereich der Hauptakzentsilbe im Normalfall eine untergeordnete Rolle spielt.

\subsubsection{Lokale Deklinationslinien}

Die Maxima-Deklinationslinie 2/3 (vgl. Tabelle 4) entspricht in der Neigungsrichtung der Maxima-Deklinationslinie 1/3; Unterschiede ergeben sich aber dadurch, daß aufgrund der größeren Nähe der miteinander verbundenen Punkte die Neigungswinkel größer werden. Die Tatsache, daß von den zwei Phrasen, die bei der Berechnung berücksichtigt werden, fast durchgängig nur eine besonders hervorgehoben ist, so daß der Neigungswinkel der Deklinationsverläufe stark schwankt, führt $z u$ den im Vergleich zu den 1/3-Maxima-Deklinationslinien größeren Standardabweichungen. Es ist zu erwarten, da $\beta$ sich der Grad der relativen Hervorgehobenheit der beiden Phrasen vor allem auch mit der Steilheit dieses Deklinationsverlaufs in Beziehung setzen läßt.

Tabelle 4: Maxima-Deklinationslinien $2 / 3$

\begin{tabular}{|c|c|c|c|c|c|}
\hline & $\begin{array}{l}\text { Entscheidungs- } \\
\text { fragesatz } \\
\text { Sie Wir }\end{array}$ & Assertive Frage & Aussagesatz & $\begin{array}{l}\text { Sie-Impera- } \\
\text { tivsatz }\end{array}$ & $\begin{array}{l}\text { Adhortativ- } \\
\text { satz }\end{array}$ \\
\hline Sp1 & $\begin{array}{lll} & -.34 & (2 .) \\
\mathrm{N} / \mathrm{K}: & -.97 & (.68)\end{array}$ & $\begin{array}{rr}-1.4 & (.93) \\
\mathrm{N} / \mathrm{K}:-1.5 & (.82)\end{array}$ & $1.73(2.58)$ & $1.35(.88)$ & $2.12(1.26)$ \\
\hline Sp2 & $-2.4(1.5))$ & $\begin{array}{r}-.61 \quad(3.2) \\
N / K=-1.85 \quad(.8)\end{array}$ & $3.8(1.7)$ & $.58(1.5)$ & $2.87(3.4)$ \\
\hline Sp3 & $\begin{array}{c}-.78(1.26) \\
\mathrm{N} / \mathrm{K}:-1 . \quad(.7) \\
\mathrm{N} / \mathrm{K}+0 . \mathrm{V}-\text { Folsus: } \\
-.73(.46)\end{array}$ & $-1.5 \quad(.83)$ & $1.26(.86)$ & $.71(.43)$ & $1.02(.6)$ \\
\hline Sp4 & $-.64(.32)$ & $-.66(.42)$ & $\begin{array}{l}.07(.88) \\
\text { O. v-u.Doppelfokus: } \\
.85(.1)\end{array}$ & $.32(.17)$ & $.67(1.4)$ \\
\hline Sp5 & $-1.3(.33)$ & $-1.5(.54)$ & $\begin{array}{c}.75(.9) \\
\mathrm{N} / \mathrm{K}: \\
1.3(.71)\end{array}$ & $\begin{array}{c}.76(.7) \\
\text { ohne V-Fokus: } \\
.9(.7)\end{array}$ & $.64(.43)$ \\
\hline Sp6 & $\begin{aligned} & -.41(1.3) \\
\mathrm{N} / \mathrm{K}: & -.82(.84) \\
\mathrm{N} / \mathrm{K}+ & 0 . \mathrm{V}-\text { Folkus: } \\
& -.38(.48)\end{aligned}$ & $\begin{array}{c}-.7 \quad(1.5) \\
\mathrm{N} / \mathrm{K}:-1 . \quad(1.2) \\
\mathrm{N} / \mathrm{K}+0 . \mathrm{V}-\text { Folus: } \\
-.61 \quad(.94)\end{array}$ & $\begin{array}{l}1.3(1.9) \\
\text { ohne v-Fokus: } \\
2.2(.67)\end{array}$ & $.82(.39)$ & $\begin{array}{c}.29(1.1) \\
\mathrm{N} / \mathrm{K}: \\
.6 \quad(.44)\end{array}$ \\
\hline
\end{tabular}

Die Fokussierungseigenschaften der jewelligen Außerungen wirken sich auf die Minima-Deklinationsverläufe 2/3 (vgl. Tabelle 5) noch stärker aus. Das gilt 
insbesondere für die Nicht-Fragen, bei denen die Minima-Deklinationslinie 1/3, wie erwähnt, durchgängig fällt. Die 2/3-Deklinationslinie steigt dagegen häufig an. Hier wirkt sich aus, daß die Phrase 2, das objekt, häufig nicht nur durch einen auffälligen Fo-Gipfel, sondern durch eine Vergrößerung des Range insgesamt und damit auch ein verhältnismäßig tiefes Minimum hervorgehoben wird, das nicht auf der globalen 'unteren' Deklinationslinie liegt.

Tabelle 5: Minima-Deklinationslinien $2 / 3$

\begin{tabular}{|c|c|c|c|c|c|}
\hline & $\begin{array}{l}\text { Entscheidungs- } \\
\text { fragesatz } \\
\text { Sie Wir }\end{array}$ & Assertive Frage & Aussagesatz & $\begin{array}{l}\text { Sie-Impera- } \\
\text { tivsatz }\end{array}$ & $\begin{array}{l}\text { Adhortativ- } \\
\text { satz }\end{array}$ \\
\hline Sp1 & $\begin{array}{rll} & -.54 & (.95) \\
\mathrm{N} / \mathrm{K}: & -.68 & (.9)\end{array}$ & $\begin{array}{l}-.38(.25) \\
\mathrm{N} / \mathrm{K}+\text { ohne V-Folsus: } \\
-.49(.15)\end{array}$ & $-.16(.54)$ & $.11(.2)$ & $-.03(.25)$ \\
\hline Sp2 & $-2.3(1.3)$ & 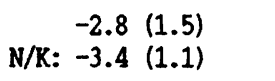 & $-.37(.77)$ & $-.03(.26)$ & $-.22(.42)$ \\
\hline Sp3 & $\begin{array}{c}-1.3(.93) \\
\mathrm{N} / \mathrm{K}+0 . \mathrm{V}-\text { Fokus: } \\
-1.6(.46)\end{array}$ & $\begin{array}{c}-2.3(1.2) \\
\text { ohne V-Fokus: } \\
-2.7(.68)\end{array}$ & $.36(.42)$ & $.77(.46)$ & $.08(.31)$ \\
\hline Sp4 & $\begin{aligned} & -1.2(1 .) \\
\mathrm{N} / \mathrm{K}: & -1.3 \\
\mathrm{~N} / \mathrm{K}+0 . \mathrm{V} & (.93) \\
-1.7 & (.3)\end{aligned}$ & $\begin{array}{c}-.94(1 .) \\
\text { o. v-u.Doppelfokus: } \\
-1.8(.32)\end{array}$ & $-.05(.2)$ & $.23(.3)$ & $.08(.22)$ \\
\hline $\mathrm{Sp} 5$ & $\begin{array}{c}-.91(.51) \\
-.49(.34)-1.3(.3) \\
\text { ohne V-Fokus: } \\
-.62(.28)-1.3(.12)\end{array}$ & $\begin{array}{c}-1 . \quad(.55) \\
\text { ohne V-Folkus: } \\
-1.3(.32)\end{array}$ & $-.06(.1)$ & $.03(.15)$ & $.01(.31)$ \\
\hline Sp6 & $\begin{array}{c}-.71(1.3) \\
N / \mathrm{K}: \\
.28(.3) \quad-2 .(.86)\end{array}$ & $\begin{aligned}-.83 & (.82) \\
\mathrm{N} / \mathrm{K}+0 . & \mathrm{V}-\text { Folkus: } \\
-1.1 & (.8)\end{aligned}$ & $.7(1.5)$ & $.13(.13)$ & $\begin{array}{l}.06(.3) \\
\text { ohne V-Fok: } \\
-.04(.13)\end{array}$ \\
\hline
\end{tabular}

Der Minima-Deklinationsverlauf $2 / 3$ ist also wahrscheinlich ebenfalls ein guter Indikator für die relative Hervorgehobenheit der Phrasen 2 oder 3 . Ein Unterschied der beiden Satzmodi-Gruppen läßt sich an ihm trotzdem durchaus noch feststellen, da die Fragesatzrealisationen durch einen deutlich steileren Anstieg gekennzeichnet sind. 
4. 'all-point'-Deklination

Die Fragesätze und die Aussage- und Imperativsätze unterscheiden sich auch deutlich voneinander, wenn pro Außerung eine auf dem gesamten Fo-Verlauf und nicht nur auf den Extrempunkten beruhende Regressionslinie (vgl. zu derartig bestimmten Deklinationslinien Lieberman et al. 1985) berechnet wird.9

Die durchschnittlichen steigungen ohne die Fälle mit Verb-Fokus sind (in Hz/Centisekunden):

$\begin{array}{llr}\text { Entscheidungsfrage (Sie) } & -.14 \\ \text { Entscheidungsfrage } & \text { (wir) } & -.29 \\ \text { assertive Frage } & -.22 \\ \text { Aussagesatz } & .29 \\ \text { Sie-Imperativsatz } & .35 \\ \text { Adhortativsatz } & .37\end{array}$

Die beiden Gruppen von Satztypen unterscheiden sich eindeutig in der Richtung dieser 'all-point'-Deklinationslinien. Allerdings ist $\mathrm{zu}$ beachten, daß Neigungsrichtung und Steilheit derartig berechneter Deklinationslinien bei den Fragesatzrealisationen stärker von der Position der Hauptakzentsilbe abhängig sind als die Neigungsrichtung und Steilheit der Linien, die aus den Extrempunkten berechnet werden. Bei einer Akzentuierung der Phrase 2 bleibt die Grundfrequenz nach dem Anstieg hoch (selbst bei Spl fällt sie höchstens ganz am Ende wieder ab). Da dieser Anstieg relativ früh liegt, gehen in die Berechnung viele spät liegende, hohe Fo-Werte ein. Anders dagegen bei den Verbfokus-Realisationen der Fragesätze: Da dort der Anstieg spät liegt, also den tieferen Fo-Werten zu Beginn nur eine geringere Zahl an hohen Fo-Werten gegenüberstehen, ist nicht immer ein Anstieg der 'all-point'-Linie festzustellen, vgl. die Werte für Verbfokus:

$\begin{array}{llr}\text { Entscheidungsfragesatz } & \text { ( Sie) } & .07 \\ \text { Entscheidungsfragesatz } & \text { (wir) } & -.11 \\ \text { assertive Frage } & & .02 \\ \text { Aussagesatz } & .19 \\ \text { Sie-Imperativsatz } & .32 \\ \text { Adhortativsatz } & .28\end{array}$

Weiter muß bei diesen beiden Tabellen beachtet werden, daß auch der einbettende Teil des A.c.I.-Satzes bei der Berechnung berücksichtigt wird. Da sich die Aussagesatzrealisationen und die Imperativsatzrealisationen anders als bel der Maxima-Deklination $1 / 3$ verhalten (s.o.), die Imperativexemplare nämlich eine steiler abfallende Deklinationslinie haben, kann nur ein höherer (unbeton-

9 Die Berechnungen erfolgten durch Elmar Nöth am Lehrstuhl für Mustererkennung der Universität Erlangen. 
ter!) Vorlauf vor der Phrase 1 der Grund für den deutlichen Unterschied sein. Diese Vorlaufhöhe könnte also ein weiteres globales Merkmal sein, das innerhalb der Nicht-Fragesätze eine Differenzierung in Aussagesätze und Imperativsätze (d.h. Typen mit 'Appellfunktion') vorzunehmen gestattet.

Auffällig sind die Unterschiede in den beiden Versionen des Entscheidungsfragesatzes, auf die schon weiter oben bei den Kommentaren zu den Minima-Deklinationslinien $1 / 3$ hingewiesen wurde: Die mit dem Adhortativsatz wort- und wortfolgengleichen Entscheidungsfragen werden mit einem steileren globalen Fo-Anstieg realisiert als die Sie-Versionen.

\section{Schlußfolgerungen}

Die globaleren Deklinationslinien, die die Fo-Maxima und -Minima der in etwa gleich stark hervorgehobenen Phrasen 1 und 3 verbinden, sind im vorliegenden Korpus ein ziemlich stabiles Merkmal zur Unterscheidung der beiden Satzmodi-Gruppen Frage und Nicht-Frage. (Die 'lokaleren' Deklinationslinien zwischen den Maxima und Minima der Phrasen 2 und 3 spiegeln dagegen sehr viel mehr die relative Hervorhebung dieser beiden Phrasen wider.)

Die Neigungsrichtung der globalen Deklinationslinien kann also auch im Deutschen durchaus als satzmodusdifferenzierendes Merkmal angesehen werden. Allerdings wird der Beitrag zur intonatorischen Satzmoduskennzeichnung geringer sein als der der dominanten Merkmale Fo-Offsethöhe und Fo-Verlauf im Bereich der Hauptakzentsilbe. Eine im Rahmen des Prototypenkonzepts (vgl. Oppenrieder 1988) erfolgende Beschreibung der Rolle der Intonation bei der Satzmoduskennzeichnung läßt außerdem $z u, d a \beta$ ein solches periphereres Merkmal im Komplex der intonatorischen Merkmale auch weniger prototypisch realisiert werden kann als ein zentraleres Merkmal.10

Wenn also bei Fragesatzäußerungen die verschiedenen, auf Extremwerte bezogenen Deklinationslinien typischerweise ansteigen, dann muß das Konzept einer invarianten, bei allen Außerungen automatisch abfallenden 'Basislinie' zumindest erweitert werden: Offenbar kann ein Sprecher dieses Abfallen gezielt überdecken. Dies kommt jedoch bei Aussagesätzen, den bevorzugten strukturen gerade bei Deklinationsuntersuchungen, nicht vor; daher konnte der Eindruck entstehen, als seien stets nur global abfallende Deklinationslinien anzutreffen. Allerdings deutet die Tatsache, daß die globalen, nicht durch Akzentuierung beeinflußten, Deklinationslinien zwar bei den (intonatorisch markierten) Frage-

10 Daher ist zu erwarten, daß der Zwang zur satzmodusgerechten Realisierung der Deklinationslinien im Dänischen stärker ist als im Deutschen. 
sätzen auch fallen, nicht aber bei den Nicht-Fragesätzen ansteigen können, darauf hin, daß fallende Deklinationslinien den unmarkierten Normalfall darstellen.

\section{LITERATUR}

Altmann, H. (1984): Linguistische Aspekte der Intonation am Beispiel Satzmodus. In: Forschungsberichte des Instituts für Phonetik und Sprachliche Kommunikation München 19. S.132-152.

Batliner, A. (1989): Fokus, Modus und die große Zahl. Zur intonatorischen Indizierung des Fokus im Deutschen. (In diesem Band)

Batliner, A./Nöth, E./Lang, R./Stallwitz, G. (1989): Zur Klassifikation von Fragen und Nicht-Fragen anhand intonatorischer Merkmale. (Erscheint in: Tagungsband der 15. Gemeinschaftstagung der Deutschen Arbeitsgemeinschaft für Akustik (DAGA). 13.-16. März 1989)

Beckman, M.E./Pierrehumbert, J.B. (1986): Intonational Structure in Japanese and English. In: Phonology Yearbook 3. S.255-309.

Cohen, A./Collier, R./'t Hart, J. (1982): Declination: Construct or Intrinsic Feature of Speech Pitch? In: Phonetica 39. S.254-273.

Eady, S.J./Cooper, W.E./Klouda, G.V./Mueller, P.R./Lotts, D.W. (1986): Acoustical Characteristics of Sentential Focus: Narrow vs. Broad and Single vs. Dual Focus Environments. In: Language and Speech 29. S.233-251.

Höhle, T.N. (1982): Explikationen für 'normale Betonung' und 'normale Wortstellung'. In: Abraham, W. (Hg.) (1982): Satzglieder im Deutschen. Tübingen. S.75-152.

Gårding, E. (1983): A Generative Model of Intonation. In: Cutler, A./Ladd, D.R. (Hgg.) (1983): Prosody: Models and Measurements. Berlin etc. S.11-25.

Gussenhoven, C./Rietveld, A.C.M. (1988): Fundamental Frequency Declination in Dutch: Testing Three Hypotheses. In: Journal of Phonetics 16. S.355-369.

Ladd, D.R. (1983): Peak Features and Overall Slope. In: Cutler, A./Ladd, D.R. (Hgg.) (1983): Prosody: Models and Measurements. Berlin etc. S.39-52.

Ladd, D.R. (1984): Declination: A Review and Some Hypotheses. In: Phonology Yearbook 1. S.53-74.

Liberman, M./Pierrehumbert, J.B. (1984): Intonational Invariance under Changes in Pitch Range and Length. In: Aronoff, M./Oehrle, R.T. (Hgg.) (1984): Language, Sound, Structure. Cambridge, Mass./London. S.157-233.

Lieberman, Ph./Katz, W./Jongman, A./Zimmerman, R./Miller, M. (1985): Measures of the Sentence Intonation of Read and Spontaneous Speech in American English. In: Journal of the Acoustical Society of America 77. S.649-657. 
Oppenrieder, W. (1988): Intonatorische Kennzeichnung von Satzmodi. In: Altmann H. (Hg.) (1988): Intonationsforschungen. Tübingen. S.169-205.

Oppenrieder, W. (1989): Fokus, Fokusprojektion und ihre intonatorische Kennzeichnung. (In diesem Band)

Pierrehumbert, J.B. (1979): The Perception of Fundamental Frequency Declination. In: Journal of the Acoustical Society of America 66. S.363-369.

Pierrehumbert, J.B. (1980): The Phonology and Phonetics of English Intonation. Ph.D. Diss. MIT.

Thorsen, N. (1979): Interpreting Raw Fundamental-Frequency Tracings of Danish. In: Phonetica 36. S.57-78.

Thorsen, N. (1983a): Standard Danish Sentence Intonation - Phonetic Data and Their Representation. In: Folia Linguistica 17. S.187-220.

Thorsen, N. (1983b): Two Issues in the Prosody of Standard Danish. In: Cutler, A./Ladd, D.R. (Hgg.) (1983): Prosody: Models and Measurements. Berlin etc. S.27-38.

Umeda, N. (1982): "Fo-declination" is situation dependent. In: Journal of Phonetics 10. S.279-290.

Vaissière, J. (1983). Language-Independent Prosodic Features. In: Cutler, A./Ladd, D.R. (Hgg.) (1983): Prosody: Models and Measurements. Berlin etc. S.53-66. 
FOKUS, FOKUSPROJEKTION UND IHRE INTONATORISCHE KENNZEICHNUNG

Wilhelm Oppenrieder

\section{Fokussierung und Akzentuierung}

'Fokussierung' bezeichnet einen Aspekt der semantischen Gliederung eines Satzes. Einem nicht-hervorgehobenen Hintergrund steht ein hervorgehobener Fokus gegenüber (zu den Begriffen vgl. Jacobs 1984, 1988). Hervorgehobene Teile sind in ihrem informationellen status besonders ausgezeichnet; oft handelt es sich um 'neue Information', gegenüber der 'bekannten Information' des Hintergrundes. Eine allgemeinere Charakterisierung dieser informationellen Gliederung findet sich in Jacobs (1988:91): Funktion der Fokus-Hintergrund-Gliederung ist die "Herstellung eines Bezugs zu Alternativen" in dem Sinn, daß von den möglichen alternativen Besetzungen der Fokusstelle, die in einem spezifischen Außerungskontext zur Debatte stehen, nur eine ausgewählt wird. Der Hintergrund ist dagegen für Alternativen nicht offen. Eine klar definierte Abgrenzung von Hintergrund und Fokus für Aussagesätze erhält man insbesondere in zwei Typen von Dialog-Sequenzen: In solchen, die aus w-Fragesätzen und (vollständigen) Antwort-Aussagesätzen bestehen und in Korrektursequenzen.

(1) A: Wer ist mit dem Einkaufen dran?

$B$ : Max ist mit dem Einkaufen dran.

(2) A: Gulda spielt Geige.

$B$ : Nein! Gulda spielt Klavier.

(Nicht-)Hervorgehobenheit ist jedoch auch ein für die Ausdrucksseite relevanter Begriff, da fokussierte Ausdrücke durch Akzentuierung gekennzeichnet werden. Allerdings sind nicht alle Ausdrücke, die zum Fokusbereich gehören, auch tatsächlich akzentuiert. Vgl. die folgenden Sequenzen:

(3) A: Was macht eigentlich Gulda?

$B$ : Gulda spielt Klavier.

(4) A: Was ist denn hier los?

B: Gulda spielt Klavier. 
Typischerweise ist in beiden Fällen das Verb nicht akzentuiert, obwohl es zum Fokus gehört. Einer der Fokusausdrücke muß aber auf jeden Fall akzentuiert werden, nämlich der sogenannte/Fokusexponent/ Die Wahl des Fokusexponenten erfolgt nach bestimmten Regeln, die wesentlich auf die syntaktische Struktur des Satzes, insbesondere auf die syntaktischen und rollensemantischen Funktionen seiner Konstituenten, Bezug nehmen (vgl. Uhmann 1987:212ff.). Ein akzentuierter Ausdruck kann in einer gegebenen syntaktischen Struktur Fokusexponent von verschieden umfangreichen Foki sein (vgl. (2) bis (4)). Abhängig von dieser struktur sind verschieden umfangreiche /'Fokusprojektionen' möglich, die eine je unterschiedliche Kontexteinbettung ermöglichen (vgl. Höhle 1982). Andererseits ist immer auch enge Fokussierung möglich, die nur die akzentuierte Konstituente selbst betrifft. In manchen Fällen ist enge Fokussierung sogar die syntaktisch einzig mögliche Fokussierung:

(5) ICh fahre immer JAPANISCHE Autos.

In anderen Fällen ist enge Fokussierung nur eine unter mehreren möglichen Fokusstrukturen, die in einem geeigneten Kontext ausgewählt wird, z.B. in der Korrektursequenz (2).

Die akzentuelle Hervorhebung des Fokus(exponenten) hängt im Deutschen eng mit der intonatorischen Satzmodusmarkierung zusammen, denn auf der Fokusakzentsilbe wird ein wesentlicher Teil der satzmoduskennzeichnenden intonatorischen Form realisiert (vgl. z.B. Oppenrieder 1988). Grundfrequenz(Fo-)Anderungen auf und im Bereich der Fokussilbe dienen zur Hervorhebung dieser Silbe; je nach dem Satzmodus der Außerung bilden diese Anderungen unterschiedliche und charakteristische Verlaufsmuster.

\section{Korpusaufbau}

Sollen die zur Fokusmarkierung eingesetzten Mittel in voller Breite untersucht werden, muß auch der Satzmodus systematisch variiert werden. Eine solche systematische Variation von Fokus und Satzmodus wurde im DFG-Projekt 'ModusFokus-Intonation' vorgenommen. Grundlage der Untersuchung war das in Altmann (1984, 1987) vorgestellte Satzmodussystem. Satzmodi werden dort als sprachliche Zeichen mit einer charakteristischen Form und Funktion aufgefaßt. Die Form wird durch das Zusammenwirken bestimmter Typen von Merkmalen konstituiert, $\mathrm{zu}$ denen auch intonatorische Merkmale gehören. Zur Untersuchung dieser satzmodustypischen intonatorischen Form bieten sich sogenannte intonatorische Minimalpaare an (vgl. Altmann 1984); das sind wort- und wortfolgengleiche Sätze 
mit unterschiedlichem Satzmodus, bei denen allein durch ihre unterschiedlichen intonatorischen Eigenschaften die Satzmodusdifferenzierung gewährleistet ist.

Das Konzept der intonatorischen Minimalpaare läßt sich in erweiterter Form auch für die Untersuchung der Fokuskennzeichnung nutzen: Die Variation betrifft in diesem Fall nicht nur den Satzmodus, sondern auch die Fokusstruktur.

$\mathrm{Zu}$ diesem $\mathrm{Zweck}$ wurden im Projekt Testsätze gebildet, die im Satzmodus und in ihrer Fokusstruktur systematisch variierten. Die Satzmodus-Minimalpaare, die der Untersuchung zugrundelagen, waren:

Minimalpaar 1: Aussagesatz vs. assertive Frage

Minimalpaar 2: Sie-Imperativsatz vs. Entscheidungsfragesatz

Minimalpaar 3: Adhortativsatz vs. Entscheidungsfragesatz

Dreimal steht also ein bestimmter Typ von Frage einer Nicht-Frage gegenüber. Da sich die Fragesätze und Nicht-Fragesätze dieser Minimalpaare in den globalen intonatorischen Eigenschaften deutlich unterscheiden ('steigender' vs. 'fallender' Fo- bzw. Tonhöhenverlauf), sollten auch die intonatorischen Mittel zur Fokuskennzeichnung, insbesondere der Fo-Verlauf, unterschiedlich eingesetzt werden.

Als Fokusstrukturen werden enger Fokus, weiter Fokus (d.h. Fokusprojektion) und Doppelfokussierung untersucht. Geeignete Fokus-Minimalpaare lassen sich mit dem Verb und seinem direkten objekt bilden, da in diesem Fall neben der engen Fokussierung des Obiekts oder des Verbs und der Doppelfokussierung als einer 'Aufaddierung' dieser einfachen Foki auch ohne weiteres eine Fokusprojektion vom Objekt auf den Komplex aus Verb und objekt (aup eine Verb-Projektion in der Sprechweise der $\mathrm{X}$-Bar-Syntax) möglich ist.

Wir wollten uns bei der Untersuchung der Fokusprojektion allerdings aup den einfachsten Fall des kontinuierlichen Fokus beschränken; der komplexere Fall eines aufgespaltenen Fokus bei einer Zweit-Position des mitfokussierten finiten Verbs sollte vermieden werden. Infolgedessen kamen nur eingebettete Infinitivphrasen als Bereich der Fokusvariation in Frage. Die Wahl der Ausgangsstrukturen wurde weiter eingeschränkt durch die Überlegung, daß die Extraposition (oder auch die Vorfeldstellung) einer Infinitivphrase möglicherweise die Realisation der Fokusstruktur in spezifischer Weise beeinflußt. Um alle diese nicht erwünschten Nebeneffekte $z u$ vermeiden, wurden als Ausgangsstrukturen A.c.I.Konstruktionen herangezogen, da bei diesen von einer weitgehenden syntaktischen (und damit auch intonatorischen) Integration des 'eingebetteten Satzes' in die Gesamtkonstruktion ausgegangen werden kann: Es liegt ein einfacher Satz vor, der aber eine kontinuierliche Abfolge von objekt und Verb bei der Fokusprojektion ermöglicht. 
Ausgangsstrukturen ((S1) bis (S3)) waren A.c.I.-Konstruktionen mit lassen, die aus einem kurzen 'Matrixsatz' mit einem pronominalen Subjekt und dem für die Fokussierung relevanten 'eingebetteten A.c.I.-Satz' bestanden, der seinerseits zwei Voll-NPn, nämlich ein (akkusativisch markiertes) 'Subjekt' und ein Akkusativobjekt, sowie einen nackten Infinitiv enthielt:

(S1) Sie läßt die Nina das Leinen weben

(S2) Lassen Sie den Manni die Bohnen schneiden

(S3) Lassen wir den Leo die Blumen düngen

(S1) bildet den Ausgangspunkt für das Satzmodus-Minimalpaar Aussagesatz vs, assertive Frage, bei den beiden anderen Strukturen stehen sich ein Entscheidungsfragesatz und ein Sie-Imperativsatz (S2) oder ein Adhortativsatz (S3) gegenüber. ${ }^{1}$

Die vier Fokusbedingungen in diesen drei Strukturen sind:

(i) (enger) Fokus auf dem Objekt (das Leinen, die Bohnen, die Blumen)

(ii) (enger) Fokus auf dem infiniten Verb (weben, schneiden, düngen)

(iii) (weiter) Fokus auf objekt und Verb, d.h. Fokusprojektion

(iv) Doppelfokussierung auf objekt und Verb; d.h. zwei enge Foki

Die Testsätze wurden in geeignete Kontexte eingebettet, die Satzmodus und Fokusstruktur steuern. Insgesamt waren es 26 Testsatz-Kontext-Kombinationen. ${ }^{2}$

Die Steuerung der Fokusstruktur erfolgte zum überwiegenden Teil durch wFragesätze. Zweimal wurden auch Korrektursequenzen gewählt.

\section{Datengewinnung}

Die Testsatz-Kontext-Kombinationen wurden 6 Versuchspersonen in Produktionstests vorgelegt; die so erhaltenen Realisationen wurden zum einen in Hörtests überprüft und zum anderen akustisch analysiert. Das sogenannte FokusKorpus war Teil eines umfangreicheren Gesamtkorpus (vgl. Batliner/Oppenrieder 1989).

Bei den Produktionstests wurden den Versuchspersonen (weiblich: Spl bis Sp3, männlich: Sp4 bis Sp6) die Testsatz-Kontext-Kombinationen des Gesamtkorpus in (pseudo-)randomisierter Reihenfolge präsentiert. Normalerweise kam

1 Die in der Standardsprache inakzeptable Form des Eigennamens mit Artikel wurde gewählt, weil wir für Produktionstests Sprecher/innen mit einer süddeutsch gefärbten Umgangssprache wählen wollten, in der nur diese Form des Eigennamens akzeptabel ist. Bei der Auswahl der lexikalischen Füllung wurde darauf geachtet, die Zahl der stimmlosen Laute klein zu halten, um Unterbrechungen des Fo-Verlaufs so weit wie möglich zu vermeiden.

2 Die vollständige Liste der Testsatz-Kontext-Kombinationen findet sich in Batliner/Oppenrieder (1989). 
jede Testsatz-Kontext-Kombination zweimal vor; die nach Meinung des Versuchsleiters schlecht realisierten Testsätze wurden am Ende noch einmal vorgelegt, d.h. pro Testsatz und Sprecher gab es maximal 4 Realisationen. Insgesamt erhielten wir 360 Realisationen.

In verschiedenen Hörtests wurden die Plazierung des Hauptakzents, die Realisierungsgüte und die Relevanz der intonatorischen Form für die Satzmoduserkennung überprüft. An den entsprechenden Tests nahmen durchschnittlich 12 Versuchspersonen teil. Sie erhielten stets alle Realisationen des Gesamtkorpus vorgesplelt, und zwar in der Reihenfolge der Produktionstests.

(a) Im Akzenttest sollten die Versuchspersonen die ihrer Meinung nach am stärksten betonte Silbe jeder Realisation auf der vor ihnen liegenden schriftlichen Fassung der Testsätze markieren. Die Testsatzrealisationen wurden kontextlos vorgespielt. Mit Hilfe dieser Tests läßt sich überprüfen, ob die Wahl der Fokussilbe(n) kontextgemä $\beta$ erfolgte: Bei Doppelfokussierung ist zu erwarten, da $\beta$ sich die Akzenturteile auf beide Fokussilben verteilen; bei einfacher enger Fokussierung und bei Fokusprojektion sollten die Akzenturteile ausschließlich auf die Wortakzentsilben des Objektsnomens fallen.

(b) Auch im Kategorisierungstest wurden die Realisationen kontextlos vorgespielt. Die Anweisung lautete hier, die einzelnen Außerungen den durch eine funktionale Beschreibung und einige typische Beispiele eingeführten Gruppen Aufforderung, Frage, Aussage, Ausruf/Exklamativ und Wunsch zuzuordnen. Die Ergebnisse dieser Tests geben Auskunft darüber, ob die intonatorischen Eigenschaften ausreichen, ansonsten 'satzmodusambige' Strukturen eindeutig identifizierbar zu machen.

(c) Im Natũrlichkeitstest lagen den Versuchspersonen die vollständigen Testsatz-Kontext-Kombinationen schriftlich vor; die Testsätze waren markiert. Aufgabe war es, zu beurteilen, wie gut eine vorgespielte Realisation zu ihrem Kontext paßt (Einschätzung auf einer 5-stufigen Ratingskala mit 1 für 'paßt sehr gut', 2 für 'paßt gut', 3 für 'paßt noch gut', 4 für 'paßt schlecht' und 5 für 'paßt sehr schlecht'). Jeder Testsatzrealisation ist ein kontextbezogener Natürlichkeitswert als arithmetisches Mittel aus den einzelnen Natürlichkeitsbewertungen der Versuchspersonen zugeordnet.

Hauptursachen für eine schlechte Bewertung sind die kontextunangemessene Satzmodus- oder die kontextunangemessene Fokusrealisierung. Die Ergebnisse aus diesem Test stützen im Idealfall die Ergebnisse der anderen beiden Tests: Wenn man im Akzenttest eine unerwartete Akzentverteilung erhält, sollte auch der Natürlichkeitswert schlecht sein; wenn im Kategorisierungstest eine $\mathrm{Auße-}$ rung nicht wie erwartet klassifiziert wurde, dann liegt bei einer schlechten $\mathrm{Na}-$ 
türlichkeitsbeurteilung (und einer 'guten' Akzentbeurteilung) der Schluß nahe, da $\beta$ ein unpassender Satzmodus-Formtyp und damit eine unpassende intonatorische Form realisiert wurde.

Von jeder Testsatzrealisation wurden Mingogramme mit Zeitsignal, Fo-Verlauf und Intensitätsverlaup erstellt. Anhand dieser Mingogramme wurden systematisch für alle 360 Realisationen folgende Parameterwerte extrahiert: Zum einen auf die Gesamtåußerung bezogene Werte (vgl. Oppenrieder 1988:177ff.):

- die Hz-Werte am Beginn (Onsetwert) und am Ende (Offsetwert) der Außerung,

- die $\mathrm{Hz}$-Werte der beiden höchsten (lokalen) Fo-Maxima und der beiden tiefsten Fo-Minima,

- die Hz-Werte der 'Eckpunkte' der beiden im Umfang größten 'Fo-Bewegungen',

- die Dauer der Gesamtäußerung.

Diese Werte bilden die Grundlage für die Bildung der 'intonatorischen Prototypen', d.h. der für einen Formtyp im Satzmodussystem charakteristischen intonatorischen Form.

Zusätzlich wurden in diesem Fokuskorpus 'lokale', phrasenbezogene Werte extrahiert, die für eine intonatorische Kennzeichnung der Fokusstruktur relevant sind. Für den eingebetteten Subjektsausdruck (=Phrase 1), das objekt (=Phrase 2) und das infinite Verb (=Phrase 3) wurden jeweils Fo-Minimum und -Maximum gemessen und zeitlich lokalisiert, sowie die zeitlichen Positionen von Anfang und Ende (des stimmhaften Teils) notiert. Schließlich wurde für die Hauptakzentsilbe der $\mathrm{Hz}$-Wert am Beginn und Ende sowie an einem (eventuell vorhandenen) Umschlagspunkt der Kontur bestimmt.

4. Ergebnisse

4.1 Klassifikation und satzmodustypische intonatorische Form

Eine kontextgerechte Satzmodusrealisierung liegt in fast allen Fällen vor. Dies zeigen die Ergebnisse des Kategorisierungstests; einzelne extreme Fälle von 'Fehlkategorisierungen' sind fast immer mit schlechten Natürlichkeitsbewertungen verknüpft: 
Satzmodus

Aussagesatz

Sie-Imperativsatz

Adhortativsatz

assertive Frage

Entscheidungsfragesatz
Urteile (in der VPen)

97\% Aussage

$88 \%$ Imperativ

8\% Aussage

56\% Imperativ

28\% Aussage

898 Frage

958 Frage

Die Adhortativsatzrealisationen werden überraschend häufig als Aussagen klassifiziert. Bei einer Erklärung ist zu berücksichtigen, daß Adhortativsätze generell seltene und daher markierte Strukturen sind; zudem könnte die Länge für einen Imperativsatz eher untypisch sein. Daß gerade so häufig AussageKlassifikationen vorkommen, könnte damit zusammenhängen, da $\beta$ eine oberflächliche Ahnlichkeit mit einem spezifischen Typ von Aussagesatz mit unbesetztem Vorfeld (wie z.B. Läßt der Leo unsere Blümchen verdursten) besteht, wenn als Subjektsausdruck ein wir vorhanden ist. Da den Versuchspersonen im Kategorisierungstest der Außerungskontext ja nicht vorlag, könnten sie die Adhortativstrukturen als diesen spezifischen Typ von Aussagesatz mißdeutet haben. Bis auf diese erklärliche Ausnahme haben die Realisationen offensichtlich die satzmodustypische intonatorische Form.

Im übrigen ist für die Adhortativsatzrealisationen auch die Natürlichkeitsbewertung generell schlechter als für die Realisationen der anderen Satztypen. Auch dies deutet darauf hin, daß es sich bei den Adhortativsätzen um markierte (und in Aufforderungskontexten nicht unbedingt völlig natürliche) Strukturen handelt.

Mit Hilfe der extrahierten intonatorischen Parameterwerte kann für jeden der fünf Satztypen eine prototypische intonatorische Form gebildet werden. Besonders relevant ist zum einen die auf den Fo-Range bezogene offsethöhe: Liegt sie in der oberen Hälfte des in einer Außerung ausgeschöpften Fo-Umfangs (FoRange), sprechen wir von einem hohen offset, ansonsten von einem tiefen offset. Als zweites ist die Form des Fo-Verlaufs im Bereich der Hauptakzentsilbe, der Konturtyp, ein wichtiges Merkmal bei der Satzmoduskennzeichnung. Die häufigste offsethöhe und der häufigste Konturtyp gehen als zentrale Merkmale in die prototypische intonatorische Form ein (vgl. Oppenrieder 1988). Ein anderes, periphereres Merkmal ist z.B. die Fo-Deklination einer Außerung (vgl. Oppenrieder 1989). Offsethöhe und Konturtyp entsprechen bei den Realisierungen des Fokuskorpus weitgehend dem Prototyp aus Oppenrieder (1988): Fragesatzrealisationen sind fast durchgängig durch einen hohen offset und einen steigenden 
oder fallend-steigenden (konkaven) Fo-Verlauf auf der Hauptakzentsilbe gekennzeichnet. Demgegenüber ist bei den Realisationen der Aussage- und der Imperativsätze der offset tief und die Kontur fallend oder steigend-fallend (konvex). ${ }^{3}$

Interessanterweise weichen eine Sprecherin (Sp1) und ein Sprecher (Sp6) bei den Fragesätzen von der prototypischen Realisierungsform ab, ohne da $\beta$ sich dies auf die (kontextfreie) Kategorisierung und die Natürlichkeitsbewertung der entsprechenden Exemplare auswirkt: Bei Spl ist kein hoher offset vorhanden, wohl aber eine steigende oder konkave Fo-Kontur im Bereich der Hauptakzentsilbe; dies gilt jedoch nicht für die Sätze mit spätem Fokus auf dem Verb. Bei Sp6 ist demgegenüber zwar ein hoher offset vorhanden, aber die Kontur der Hauptakzentsilbe ist konvex; wiederum gilt das nur dann, wenn das Verb nicht betont ist. Offensichtlich ist bei einem späten Hauptakzent eine (normal klingende) 'widersprüchliche' Kombination aus steigender Kontur und tiefem offset, bzw. fallender Kontur und hohem offset in der Kürze der zur Verfügung stehenden Zeit nicht zu realisieren. Die zwei Ausnahmen zeigen, daß neben den prototypischen Realisationen, die beide fragesatztypischen Bestandteile (hoher offset und konkave Kontur) enthalten, auch weniger typische Realisationen zulässig sind, die nur durch eines dieser beiden Merkmale gekennzeichnet sind.

\subsection{Akzenttest und Akzentrealisierung}

Die Ergebnisse des Akzenttests zeigen, daß bei drei der vier Fokusbedingungen die Sprecher/innen den Hauptakzent wie erwartet setzten, nämlich bei den beiden Fällen mit einfachem engen Fokus und beim weiten Fokus (Fokusprojektion), daß sie aber anscheinend keine Doppelakzentuierung realisierten. Ein Blick auf die extrahierten Parameterwerte bestätigt diese Vermutung: Sowohl in den Dauer- als auch in den Fo-Werten der Phrase 3 (dem Verb als zweitem der beiden engen Foki) unterscheiden sich die als Doppelfokussierung intendier-

$3 \quad$ Als weiteres Merkmal, das möglicherweise für die Satzmodusdifferenzierung genützt wird, findet sich beim Fokuskorpus die Onsethöhe. Sie liegt bei den Nicht-Fragesätzen höher als bei den Fragesätzen, nämlich etwa in der Mitte des Fo-Range, bei den Aussagesatzrealisationen der Sprecherinnen sogar im oberen Viertel. Der Unterschied zwischen den beiden Satztyp-Gruppen dürfte jedoch kleiner werden, wenn man nicht die rangebezogenen Onsetwerte betrachtet, sondern sie in Bezug setzt zu den jeweils satzmodustypischen Deklinationslinien (vgl. Oppenrieder 1989): Sowohl die durch Fo-Maxima gelegten, als auch die durch Fo-Minima gelegten Deklinationslinien fallen bei den Nicht-Fragesätzen ab, während sie bei den Fragesätzen ansteigen. Berechnet man die Onsethöhe relativ zur Minimadeklinationslinie am Beginn der Außerung, dann ist der Abstand eines mittelhohen Onsets von einer hoch ansetzenden Linie nicht sehr viel größer als der eines tiefen Onsets von einer tief ansetzenden Linie. 
ten Fälle nicht von den Fällen mit engem Objektfokus oder von denen mit Fokusprojektion. Die Realisationen mit Verbfokus heben sich demgegenüber von den restlichen Exemplaren klar ab. Die Exemplare mit vom Kontext nahegelegter Doppelfokussierung zeigen sich also weder in ihren intonatorischen Eigenschaften noch im Akzenttest als Realisationen mit einer 'Aufaddierung' zweier enger Foki. Sie werden aber nicht nur 'unifokal' realisiert, sondern auch im Natürlichkeitstest als durchschnittlich gut bewertet, während ansonsten Exemplare mit kontextunpassender Akzentstruktur auch schlechte Natürlichkeitsbewertungen erhalten.4

Die Doppelfokussierung scheint also, obwohl sie der Kontext nahelegt, nicht durch intonatorische Mittel gekennzeichnet $\mathrm{zu}$ werden. Ein möglicher Grund könnte sein, daß die Kontextvorgaben für die Doppelfokussierung $\mathrm{zu}$ kompliziert waren und sowohl die Sprecher/innen als auch die Versuchspersonen im Akzentund Natürlichkeitstest überforderten. Eine andere Erklärungsmöglichkeit ist, daß ein Doppelfokus nicht immer durch hörbare Doppelakzentuierung realisiert werden muß: Der zweite Fokus könnte, wenn er im Projektionsbereich des ersten liegt, selbst gar keine eigene intonatorische Markierung mehr benötigen (zu einer Erklärung in dieser Richtung vgl. Rochemont 1986). In den von uns zugrunde gelegten Strukturen liegt das Verb bei der Doppelfokusbedingung im Projektionsbereich des objekts (nicht aber umgekehrt, daher die schlechte Natürlichkeitsbewertung bei Sp4, vgl. Fn.4) und kann daher durch diese Art 'Pseudo-Fokusprojektion' auf einfachere Weise als Fokus ausgezeichnet werden als durch eine explizite Hervorhebung. Doppelakzente könnten nach dieser Erklärung also nur erzwungen werden, wenn keiner der beiden Foki den anderen in seinem Projektionsbereich einschließt (und das zerstört gleichzeitig die Vorbedingung für die Bildung von Fokus-Minimalpaaren).

Was die Hauptakzentverteilung betrifft, lassen sich bei unserem Material also nur zwei große Gruppen bilden: Zur ersten Gruppe gehören die Realisationen mit Hauptakzent auf der Objektkonstituente; das sind solche mit engem Fokus auf dem Objekt, sowie diejenigen mit Projektion und 'Pseudo-Projektion' auf das Verb. Auf der anderen Seite stehen die Realisationen mit engem Verbfokus. Beide Gruppen unterscheiden sich deutlich voneinander in der verteilung der

Ausnahmen zur bloßen Objektbetonung bei der Doppelfokussierung sind Sprecher Sp 4, der in diesem Fall häufig nur das Verb stark akzentuiert (die entsprechenden Realisationen werden als unnatürlich bewertet), und der Sprecher Sp 6, bei dem zwar zum Teil bei der Doppelfokus-Bedingung im Signal eine 'Aufaddierung' zweier für einfache Foki kennzeichnenden Werte $\mathrm{zu}$ sehen ist, der diese Strategie aber auch bei den anderen Fokusbedingungen anwendet. 
akzentuierungsrelevanten intonatorischen Parameterwerte auf die beiden Phrasen 2 (Objekt) und 3 (Verb).

Die von uns extrahierten akzentuierungsrelevanten Parameterwerte der beiden Phrasen sind das Fo-Maximum, das Fo-Minimum und die Dauer; daraus lassen sich weitere Größen ableiten, wie z.B. der Fo-Range und die Richtung der größten Fo-Bewegung auf der Phrase oder die relative Dauer der beiden Phrasen. Das Gewicht dieser Werte für die Fokuskennzeichnung ist jedoch unterschiedlich groß, abhängig von der Position des Fokusausdrucks im Satz, vom Satzmodus und auch vom jeweiligen sprecher/der jeweiligen Sprecherin.

Bei den Fragesătzen läßt sich eine typische Akzentuierungsstrategie erkennen, die von derjenigen bei den Aussagesätzen und den Imperativsätzen unterschieden ist: Auf der fokussierten Phrase erfolgt ein ausgeprägter Fo-Anstieg, der von einem verhältnismäßig tiefen Niveau ausgeht. (Entsprechend spielen die Fo-Minimawerte eine weitaus größere Rolle bei einer diskrimanzanalytischen Bestimmung der akzentuierten Phrase als bei den Nicht-Fragen.) Unterschiede in der Phrasendauer fallen gegenüber der auch perzeptiv sehr auffälligen Fo-Anderung kaum ins Gewicht. Die Ausnahme zu der Pragesatztypischen Hervorhebungsstrategie bildet, wie schon erwähnt, der sprecher $\mathrm{Sp} 6$, bei dem der Fokusausdruck in Fragesätzen auch durch einen Fo-Abfall hervorgehoben werden kann; allerdings geschieht dies nur, wenn das objekt als Fokus oder Fokusexponent hervorgehoben werden soll.

Bei den Nicht-Fragesätzen ist demgegenüber neben den Fo-Veränderungen auch die Dauer für die Fokuskennzeichnung relevant. Objekt- und Verbakzent unterscheiden sich in der relativen Gewichtung dieser Kennzeichnungsmittel: Beim (späten) Verbfokus spielt die Dauer eine wesentlich größere Rolle als beim (frühen) Objektfokus; dies gilt insbesondere für die Imperativsatztypen (genauere Kennwerte finden sich in Batliner 1989).

\subsection{Fokusprojektion}

Ob sich nicht nur die Hervorhebung des Fokusexponenten, sondern auch die umfangreichere Fokusprojektion auf die intonatorische Form auswirkt, läßt sich anhand eines Vergleiches des Fokustyps (i) (Objekt-Fokus) und des Fokustyps (iii) (Fokusprojektion) feststellen. Allerdings kann mit unseren Hörtests nicht überprüft werden, ob die Sprecher/innen tatsächlich diese beiden in der Akzentsetzung identischen Fokusstrukturen relativ zum Kontext richtig realisierten. Der Kategorisierungstest ist irrelevant. Der Akzenttest ist ungeeignet, da hier nur nach dem Hauptakzent gefragt wurde und der eng fokussierte Ausdruck im einen Fall und der Fokusexponent im anderen eben gerade zusammen- 
fallen sollten. Der Natürlichkeitstest ist zwar im Prinzip genau für die Aufgabe konzipiert, die kontextgerechte Realisierung zu überprüfen. Es kann jedoch nicht davon ausgegangen werden, daß sich intonatorische Unterschiede, wie sle bel Projektion gegenüber Nicht-Projektion möglicherweise vorllegen, auf die Beurteilung wesentlich auswirken, da es sich im Vergleich zur Akzentplazierung höchstens um recht subtile Unterschiede handeln kann: Paßt die Akzentposition in den Kontext, dann dürften derartig feine Differenzierungen die Natürlichkeitsbewertung kaum negativ oder positiv beeinflussen. Ob tatsächlich eine Fokusprojektionsstruktur realisiert wurde, kann also nicht überprüft werden. Man muß stattdessen voraussetzen, daß die unterschiedlichen Kontextvorgaben den Sprecher/inne/n mindestens bei diesem Fokus-Minimalpaar hinreichend klar waren.

Beim Minimalpaarvergleich sollte tatsächlich nur eine unterschiedliche Projektivität vorliegen. Der enge Fokus darf also kein Kontrastfokus (im Sinne einer expliziten Korrektur einer 'falschen' Besetzung der Objektstelle) sein, da in diesem Fall (zumindest bei Nicht-Fragesätzen) üblicherweise eine spezifische Kontrastakzentuierung gewählt wird (vgl. Bannert 1985, Batliner 1988, Oppenrieder 1988), so da $\beta$ sich enger Fokus und (nicht-kontrastiver) Fokusexponent schon deswegen unterscheiden. Die Nicht-Kontrastivität des engen Fokus ist im Korpus jedoch durch die Kontextkonstruktion gesichert.

Wie Fokusprojektion durch intonatorische Mittel angezeigt werden könnte, ist keineswegs von vornherein offenkundig. Die beiden folgenden Möglichkeiten scheinen aber zunächst einmal am plausibelsten:

(a) Der im Projektionsbereich liegende Teil wird durch einen vergrößerten Fo-Range (ein tieferes Fo-Minimum und/oder -Maximum) oder eine längere Dauer (möglicherweise auch durch eine größere Intensität bzw. ein größeres Energieintegral, vgl. Beckman 1986) gegenüber Hintergrundsausdrücken hervorgehoben: Da er nicht selbst den Hauptakzent trägt, darf die 'Extensivierung' der auf den Fokusexponenten folgenden Projektionsausdrücke ein bestimmtes Maß nicht überschreiten (als Haupt- oder Nukleusakzent gilt immer der letzte Akzent, vgl. auch Uhmann 1987).

(b) Der im Projektionsbereich liegende Teil wird enger an den Fokusexponenten gebunden, indem er in den satzmodustypischen Fo-Verlaup der Hauptakzentsilbe einbezogen wird, z.B. in der Form, daß der für Nicht-Fragesätze obligatorische Fo-Abfall auf die Projektionsausdrücke ausgedehnt wird, die dem Fokusexponenten folgen. Insbesondere diese Art der intonatorischen Kennzeichnung des Fokusprojektionsbereichs sollte demnach bei unseren Testsatzrealisationen zu erwarten sein (das objekt als Fokusexponent geht dem infiniten Verb 
voraus). Ein Einsatz der anderen Mittel ist aber ebenfalls nicht ausgeschlossen. Als möglicherweise für die Projektionskennzeichnung relevante intonatorische Markierungsmittel wurden also die Ausprägungen der Parameter Fo-Range und (relative) Dauer auf den beiden Phrasen 2 (Objekt) und 3 (infinites Verb), sowie die Steilheit und Dauer der satzmodusrelevanten finalen Fo-Veränderung auf der Hauptakzentsilbe untersucht:

(1) phrasenbezogener Fo-Range

Da keine Kontrastierung vorlag, hatte Phrase 2 beim engen Fokus (der Aussagesatz- und Imperativsatzrealisationen) keinen überhohen Fo-Range. Sowohl für den Fo-Range auf Phrase 2 als auch für den auf Phrase 3 lassen sich keine für alle Sprecher/innen gültigen, konsistenten Unterschiede zwischen den beiden Fokusbedingungen feststellen.

(2) phrasenbezogene (relative) Dauer

Auch die Unterschiede in den relativen Dauern ${ }^{5}$ der Phrasen 2 und 3 in den beiden Fokusbedingungen lassen nicht den Schlu $\beta \mathrm{zu}$, daß die Dauer von den Sprecher/inne/n konsistent zur Kennzeichnung einer Fokusprojektionsstruktur eingesetzt wird. Immerhin ist bei den Sprechern Sp4 bis Sp6 zumindest in den Aussagesätzen die Phrase 2 bei der engen Fokussierung teilweise länger, als wenn sie als Fokusexponent einer Projektionsstruktur fungiert. Sprecher 5 'längt' zudem bei Aussage- und Adhortativsätzen die im Projektionsbereich liegende Phrase 3. Wenn nun aber schon die Sprecher die Längung nicht konsequent einsetzen, so widersprechen die wenigen einigermaßen klaren Fälle von Dauerunterschieden bei den Sprecherinnen sogar dieser 'Regel': Sprecherin 2 kürzt bei den Adhortativsätzen die Phrase 3 im Projektionsbereich gegenüber der Bedingung, wo sie zum Hintergrund gehört. Auch die Dauer wird also von den 6 Sprecher/inne/n nicht konsequent und konsistent eingesetzt, um Fokusprojektion und engen (nicht-kontrastiven) Fokus zu unterscheiden.

(3) Integration in die Fo-Kontur

Für die Hauptakzentsilben wurde, falls vorhanden, die Position einer Richtungsänderung des Fo-Verlaufs, eines Fo-Umschlagpunkts festgestellt, sowie die durchschnittliche Steigung des Fo-Verlaufs in dieser Silbe berechnet. Weder für die relative Positionierung eines Umschlagpunkts in der Silbe noch für die

5 Als 'relative Dauer' wurde das Produkt aus der durchschnittlichen Dauer der Phrase relativ zur Gesamtäußerung und aus der durchschnittlichen Dauer relativ $\mathrm{zu}$ den anderen Realisationen der Phrase durch einen Sprecher errechnet (vgl. Batliner 1989). 
Steigung läßt sich ein Zusammenhang mit den beiden Fokusbedingungen ausmachen. Lediglich bei Sprecher 6 ist zum Teil der Abfall der Fo in der Hauptakzentsilbe bei der Fokusprojektions-Bedingung weniger steil und der Fo-Abfall setzt sich (anders als beim engen Objektfokus) nach der Hauptakzentsilbe weiter fort; interessanterweise ist das nur bei den markierten Fragesatzrealisationen mit 'konvexer' Kontur zu beobachten, während sich für die Nicht-Fragesätze keine derartige Regularität ausmachen läßt.

Die durchschnittliche Steigung wurde auch für längere Fo-Abschnitte berechnet, die von der Hauptakzentsilbe bis zur nächsten klaren Richtungsänderung des Fo-Verlaufs reichen. Je flacher und länger die Steigung ist, um so weiter setzt sie sich in die Phrase 3 hinein fort. Ein Zusammenhang zwischen der Steilheit und Länge eines Fo-Abfalls oder -Anstiegs und den beiden Bedingungen der engen Fokussierung und der Fokusprojektion ist nicht festzustellen.

Zumindest in unserem Material und mit den von uns extrahierten Daten läßt sich ein Zusammenhang zwischen den intonatorischen Eigenschaften einer nichtakzentuierten Phrase und ihrem Status als Hintergrunds- oder (im Projektionsbereich eines Fokusexponenten liegender) fokussierter Ausdruck nicht nachweisen. Die beiden Fokusbedingungen objektfokus und Fokusprojektion unterscheiden sich in ihrer intonatorischen Realisierung nicht.

\section{LITERATUR}

Altmann, H. (1984): Linguistische Aspekte der intonation am Beispiel Satzmodus. In: Forschungsberichte des Instituts für Phonetik und sprachliche Kommunikation München 19. S.132-152.

Altmann, H. (1987): Zur Problematik der Konstitution von Satzmodi als Formtypen. In: Meibauer, J. (Hg.) (1987): Satzmodus zwischen Grammatik und Pragmatik. Tübingen. S.22-56.

Bannert, R. (1985): Fokus, Kontrast und Phrasenintonation im Deutschen. In: Zeitschrift für Dialektologie und Linguistik 52. S.289-305.

Batliner, A. (1988): Der Exklamativ: Mehr als Aussage oder doch nur mehr oder weniger Aussage? Experimente zur Rolle von Höhe und Position des Fo-Gipfels. In: Altmann, H. (Hg.) (1988): Intonationsforschungen. Tübingen. S.243271.

Batliner, A. (1989): Fokus, Modus und die große Zahl. Zur intonatorischen Indizierung des Fokus im Deutschen. (In diesem Band)

Batliner, A./Oppenrieder, W. (1989): Korpora und Auswertung. (In diesem Band) 
Beckman M.E. (1986): Stress and Non-Stress Accent. Dordrecht.

Höhle, T.N. (1982): Explikationen für 'normale Betonung' und 'normale Wortstellung'. In: Abraham, W. (Hg.) (1982): Satzglieder im Deutschen. Tübingen. S.75-152.

Jacobs, J. (1984): Funktionale Satzperspektive und Illokutionssemantik. In: Linguistische Berichte 91. S.25-58.

Jacobs, J. (1988): Fokus-Hintergrund-Gliederung und Grammatik. In: Altmann, H. (Hg.) (1988): Intonationsforschungen. Tübingen. S.89-134.

Oppenrieder, W. (1988): Intonatorische Kennzeichnung von Satzmodi. In: Altmann, H. (Hg.) (1988): Intonationsforschungen. Tübingen. S.169-205.

Oppenrieder, W. (1989): Deklination und Satzmodus. (In diesem Band)

Rochemont, M.S. (1986): Focus in Generative Grammar. Amsterdam.

Uhmann, S. (1987): Fokussierung und Intonation. Eine Untersuchung zum Deutschen an Frage/Antwort-Sequenzen in experimentellen Dialogen. Masch. Phil. Diss. Konstanz. 
KORPORA UND AUSWERTUNG

Anton Batliner und Wilhelm Oppenrieder

\section{Einleitung}

In diesem Beitrag werden die Korpora im einzelnen vorgestellt und kommentiert, die im Projekt 'Modus-Fokus-Intonation' Untersuchungsgrundlage waren. Bei allen Korpora sind Testsätze und Kontexte dokumentiert, bei zwei Korpora zusätzlich schematisierte Grundfrequenz-(Fo-) Verläufe aller Außerungen sowie die wichtigsten statistischen Kennwerte. ${ }^{1}$

2. Die Minimalpaarmethode: Gewinnung der Testsatzkorpora

Die Korpuskonstruktion basiert auf dem von Altmann (1984, 1987) entwickelten Satzmodussystem. Danach werden Satzmodi formal durch bestimmte kategoriale, morphologische, topologische und eben auch intonatorische Merkmale gekennzíichnet. Häufig können zwei Satzmodi formal nur durch eines dieser Merkmale untorschieden werden, während die anderen gewissermaßen neutralisiert sind. Ist bei gleicher segmentaler struktur zweier Sätze lediglich die intonatorische Form satzmodusdifferenzierend, dann sprechen wir von intonatorischen Minimalpaaren. Auf dem Konzept dieser intonatorischen Minimalpare, wie es in Altmann (1984) detailliert erläutert wird, gründet die Konstruktion der Testsätze für unsere Korpora, da angenommen werden kann, daß in diesen Fällen die Intonation maximal belastet und damit besonders ausgeprägt ist. Ein weiterer Vorteil ist die Erleichterung des Vergleichs zwischen Minimalpaar-Satzmodi, wenn segmentgleiche, aber intonationsverschiedene Außerungen verglichen werden. Die Testsätze sind also nach Möglichkeit so konstruiert, daß sie je nach intonatorischer Form zu einem von zwei oder drei Minimalpaar-Satzmodi gehören.

1 Die Dokumentation der Korpora wurde zu großen Teilen von Marion Bludszuweit, Ute Hofmann und Jörg Schorradt erstellt. 
Die entsprechenden Sätze wurden jeweils 6 Versuchspersonen (3 weiblich, 3 männlich) vorgelegt, die sie satzmodusgerecht und - zur Erleichterung des Minimalpaarvergleichs - mit einer bestimmten festgelegten Akzentstruktur realisieren sollten. Dazu wurden jedoch keine expliziten Anweisungen gegeben. Vielmehr waren die Testsätze in Kontexte eingebettet, die die intendierte Satzmodus- und Akzentrealisierung implizit steuern sollten. Die Aufnahmen erfolgten im schallarmen Raum des Instituts für Phonetik, München. In randomisierter Folge wurden die Sätze je zweimal den Sprechern zur Produktion vorgelegt; am Ende der Sitzung ließ der Versuchsleiter Sätze, die seiner Ansicht nach falsch produziert waren oder bei denen andere Probleme aufgetaucht waren, wiederholen. Jeder Satz wurde also von jedem Sprecher mindestens zweimal und höchstens viermal produziert. Weitere Einzelheiten finden sich in oppenrieder (1988b:173).

Nach diesen Prinzipien wurden zwei 'Großkorpora' gebildet. ${ }^{2}$ Das erste Großkorpus enthält fast ausschließlich Verb-Erst- und Verb-Zweit-Sätze (Korpus 1). Das zweite Großkorpus besteht aus drei Teilkorpora mit (i) Verb-Letzt-Sätzen ('Verb-Letzt-Korpus' = Korpus II), (ii) systematisch neben dem Satzmodus auch den Fokus variierenden Sätzen ('Fokuskorpus' = Korpus III) und (iii) kurzen Verb-Erst- und Verb-Zweit-Sätzen ('Leo-Korpus' = Korpus IV).

\section{Hörtests}

Die Testsatzrealisationen wurden in verschiedenen Hörtests verwendet. An den einzelnen Tests nahmen im Durchschnitt 12 Versuchspersonen teil. Aufgabe bei den Kategorisierungstests war es, die kontextfrei dargebotenen Realisationen der Testsätze den durch eine funktionale Beschreibung und durch einige charakteristische Beispiele eingeführten 'Funktionstypen' Aufforderung, Frage, Aussage, Ausruf/Exklamativ und Wunsch zuzuordnen. Ziel war es, herauszufinden, ob die jeweiligen intonatorischen Charakteristika der Testsatzrealisationen ausgeprägt und charakteristisch genug sind, um auch ohne stützenden Kontext eine eindeutige Zuordnung $\mathrm{zu}$ dem intendierten Funktionstyp zu gestatten. Auf diese Weise lassen sich die eindeutigen Realisationen herausfiltern; genaueres zu diesen Tests und $\mathrm{zu}$ den mit ihnen verbundenen Schwierigkeiten findet sich in Oppenrieder (1988a).

Bei den Natürlichkeitstests lag den Versuchspersonen neben dem Testsatz auch die gesamte Kontextbeschreibung vor; der Testsatz war jeweils markiert.

2 Jedes der beiden Großkorpora wurde einer anderen Sprechergruppe vorgelegt; d.h. insgesamt gab es 12 Sprecher. Die beiden Großkorpora sind jeweils intern durchnumeriert. 
Aufgabe war es, zu beurteilen, wie gut die Testsatzrealisation zum vorgegebenen Kontext paßte. Vorgegeben war eine Ratingskala mit fünf Stufen: 1 für 'paßt sehr gut', 2 für 'paßt gut', 3 für 'paßt noch gut', 4 für 'paßt schlecht' und 5 für 'paßt sehr schlecht'. Jede Testsatzrealisation erhielt einen (kontextbezogenen) Natürlichkeitswert als arithmetisches Mittel aus den Beurteilungen.

Die Ergebnisse der Kategorisierungs- und Natürlichkeitstests bildeten einen Filter für die Trennung der prototypischen von den nicht-prototypischen Realisationen. Als Prototypen gelten Exemplare, die einen kleineren Natürlichkeitswert als 2.5 aufweisen und die gleichzeitig von mehr als $80 \%$ der Versuchspersonen im Kategorisierungstest wie von uns nach der Testsatz- und Kontextkonstruktion intendiert klassifiziert worden waren.

Bei den Akzenttests bestimmten die Hörer die Hauptakzentsilbe jeder Testsatzrealisation.

4. Gewinnung der akustischen Parameterwerte

Von jeder Außerung wurden Mingogramme erstellt mit Zeitsignal, Grundfrequenzverlauf (gemessen mit einem Frøkjær-Jensen Pitchmeter) und Intensitätsverlauf (gemessen mit einem Frøkjær-Jensen Intensity-Meter). An diesen Mingogrammen wurden die folgenden Werte extrahiert (Weitere Einzelheiten finden sich in Oppenrieder 1988b:177ff):

1. Der Hz-Wert am Beginn (Onset) und am Ende der Außerung (OPfset).

2. Der $\mathrm{Hz}$-Wert der beiden höchsten (lokalen) Fo-Maxima und der beiden tiefsten Fo-Minima (sofern tatsächlich in der Außerung zwei oder mehr dieser beiden Arten von Extremwerten vorkommen).

3. Die $\mathrm{Hz}$-Werte der 'Eckpunkte' (Anfang, Ende bzw. prominenter Umkehrpunkt) der beiden im Umfang größten Fo-Bewegungen (sofern zweí oder mehr vorhanden sind): Als solche Bewegungen zählen neben einem einfachen Anstieg und Abfall (Eckpunkte A und B) auch komplexe Konfigurationen mit drei Eckpunkten, wobei im mittleren Punkt $B$ eine deutliche Anderung in der Richtung des Fo-Verlaups auftritt (bis auf wenige Ausnahmen ein Umschlag von Anstieg in Abfall oder umgekehrt); über stimmlose Abschnitte, die zwischen den Eckpunkten liegen, wurde interpoliert. Die Ablesegenauigkeit für alle diese Fo-Werte betrug $5 \mathrm{~Hz}$; für jeden Wert wurde festgehalten, auf die wievielte Silbe der Außerung er fällt.

4. Die beiden Silben mit den höchsten dB-Werten.

5. Die Dauer (des stimmhaften Teils) der in den Akzenttests festgestellten Hauptakzentsilbe.

6. Die Dauer der Gesamtäußerung (vom Fo-Onset bis zum Fo-Offset). 
Im folgenden werden für alle Korpora die einzelnen Testsätze zusammen mit dem modus- und fokussteuernden Kontext aufgeführt, d.h. in der Form, in der sie auch den Versuchspersonen bei den Aupnahmen und in den Natürlichkeitstests vorlagen. In den beiden Großkorpora sind die Testsatz-Kontext-Kombinationen jeweils gesondert durchnumeriert. Die (Teil-)Korpora sind grob gegliedert nach übergreifenden satzmodusrelevanten Merkmalen wie Verbstellung oder Art des Satzeinleiters. Die Feingliederung erfolgt nach den zusammengehörigen Minimalpaaren und -tripeln. Innerhalb dieser Gruppen wird der Satztyp jedes einzelnen Testsatzes durch weitere $z$ wischenüberschriften kenntlich gemacht. ${ }^{3}$

In den einzelnen Testsatz-Kontext-Kombinationen ist wie üblich objektsprachliches, also der zu sprechende Text durch Kursivschreibung von der Situationsbeschreibung unterschieden. Der relevante Testsatz selbst ist in diesem Uberblick jeweils unterstrichen, die 'erwartete', d.h. die durch die Kontextvorgabe intendierte Hauptakzentsilbe ist beim Korpus III und beim Korpus IV groß geschrieben. ${ }^{4}$ (Den Testpersonen für die Aufnahmen lagen diese Markierungen selbstverständlich nicht vor.)

\subsection{Vier Kennwerte zur Frage/Nicht-Frage-Klassifikation}

Bei jedem Testsatz sind am Ende in Klammer vier Positionen vorgegeben, die entweder leer sind (markiert durch '-') oder in denen eine Zahl steht, die maximal so groß ist wie die Zahl der Außerungen pro Testsatz. Die Summe aller Zahlen ist meist gleich der Anzahl der Außerungen pro Testsatz; 75 Fälle, bei denen der Fo-Offset wegen Laryngalisierung am Mingogramm nicht abgelesen werden konnte, mußten unberücksichtigt bleiben. Die Bedeutung dieser Zahlen soll nun erklärt werden:

3 Die Benennungen entsprechen nicht immer den in Altmann (1987) verwendeten, z.B. statt 'V-1-Fragesatz' heißt es hier 'Satzfragesatz'; die Tatsache, $\mathrm{da} \beta$ es sich um einen Verb-Erst-Satz handelt, kann aber daraus entnommen werden, daß die Entscheidungsfragesätze im Block der Verb-Erst-Sätze aufgeführt werden. Die Benennung der 'Mischtypen' - wie der assertiven Fragen - ohne den formtypbezeichnenden Zusatz '-satz' folgt dem Gebrauch in Altmann $(1987,48 \%$.)

4 Die Angabe der Hauptakzentsilbe fehlt bei den beiden anderen Korpora, da hier oft mehrere Positionen möglich sind. 
In Batliner et al. (1989) wurden mit einer Diskriminanzanalyse (zum Verfahren vgl. Batliner 1989a) die Außerungen aller vier Korpora als Frage bzw. Nicht-Frage klassifiziert. Prädiktorvariable war die halbtontransformierte Höhe des Fo-Offsets, die zum sprecherspezifischen Basiswert transformiert war; d.h. vom Offsetwert wurde der tiefste vom jeweiligen Sprecher erreichbare Wert abgezogen. Die vorgegebene Frage/Nicht-Frage-Einteilung entspricht der in diesem Anhang; Zweifelsfälle bei den Verb-Letzt-Sätzen werden in Oppenrieder (1989a) besprochen. Die Diskriminanzanalyse weist jedem Fall eine Wahrscheinlichkeit der Gruppenzugehörigkeit zu. Bei zwei Gruppen wird der einzelne Fall der Gruppe zugewiesen, für die sich ein Wert von $>0.5$ ergibt. Die Zuweisung kann richtig oder falsch sein.

Die 'Trefferquoten' aus den Kategorisierungstests wurden nun derart umkodiert, daß sich für jeden Fall ein Wert zwischen 0 und 1 für die richtige Klassifizierung als Frage/Nicht-Frage ergibt: Je größer er ist, desto besser konnte die Außerung klassifiziert werden. Analog zur richtigen oder falschen Entscheidung bei der Diskriminanzanalyse anhand der Wahrscheinlichkeit der Gruppenzugehörigkeit wurden nun die Werte unter 0.5 umkodiert in 'falsche' Entscheidung, und die über 0.5 in 'richtige' Entscheidung. Wir erhalten also für die 1999 Außerungen die in der folgenden Tabelle enthaltene Kreuzklassifizierung in die Gruppen I-IV aus den richtigen und den falschen Entscheidungen des statistischen Verfahrens und der Hörerurteile bzgl. der intendierten Frage/Nicht-FrageKlassifikation; angegeben ist die Zahl der Fälle pro Gruppe und die entsprechende Prozentzahl bzgl. aller Fälle.

Tabelle: Klassifikation durch Hörer- und Diskriminanzanalyse

$$
\text { richtig falsch }
$$

\begin{tabular}{|c|c|c|c|c|}
\hline $\begin{array}{l}\text { richtig } \\
\text { falsch }\end{array}$ & $\begin{array}{rr}\text { I } & 1625 \\
\text { III } & 105\end{array}$ & $\begin{array}{r}(81.38) \\
(5.38)\end{array}$ & $\begin{array}{l}\text { II } \\
\text { IV }\end{array}$ & $\begin{array}{r}221 \\
47\end{array}$ \\
\hline
\end{tabular}

Die vier Positionen, die in Klammer nach jedem Testsatz angegeben sind, entsprechen diesen vier Gruppen in der Reihenfolge I-IV; sie werden in Batliner (1989c) genauer analysiert; vgl. auch Batliner et al. (1989). Die Gruppen seien hier kurz kommentiert:

Gruppe I: Vier Fünftel der Außerungen konnten allein anhand der Fo-OffsetHöhe richtig als Frage oder Nicht-Frage klassifiziert werden; diese Außerungen wurden auch von den Hörern richtig bestimmt. 
Gruppe II: Die richtige Klassifikation durch die Hörer erfolgte aufgrund anderer Merkmale als die Höhe des Fo-Offsets (nicht-intonatorische Merkmale wie Verb-Stellung und Verb-Semantik oder andere intonatorische wie Position des Fo-Wendepunkts auf der fokussierten Phrase, vgl. Batliner 1989a).

Gruppe III: Die meisten der Fälle stammen aus dem Verb-Letzt-Korpus (Korpus II; vgl. dazu im einzelnen Oppenrieder (1989a). Ein weiteres, typisches Beispiel sind die 8 Fälle bei der unhöflichen/ungeduldigen Frage Nr. 41 in Korpus $\mathrm{I}$, die von den Hörern funktional als Aufforderung und damit als Nicht-Frage klassifiziert wurden.

Gruppe IV: Hier handelt es sich entweder um Fehlproduktionen, vgl. Batliner (1989a), oder um Außerungen, bei denen offensichtlich allein der Kontext disambiguieren kann, wie bei drei Fällen von Nr.1 in Korpus 1 (Schlafen Sie? mit tiefem Fo-Offset).

\section{Verfügbarkeit des Materials}

Fo-Verlauf, Intensitätsverlauf und Zeitsignal aller Testsätze sind auf Mingogrammen aufgezeichnet und nach Korpus, Sprecher und Randomisierungsreihenfolge geordnet. Die Testsätze von Korpus III und IV liegen zusätzlich als digitalisierte Kopien vor (Binärdaten, $16 \mathrm{KHz}$ Abtastfrequenz, 12 Bit Auflösung, ILS-Header im ersten Block).

Die statistischen Kennwerte, d.h. die extrahierten Parameterwerte, die Ergebnisse der Hörtests sowie alle daraus erzeugten Werte sind in SPSS-PC-Systemdateien (Binärdateien) gespeichert. Inventarnummer und Testsatznummer ermöglichen eine eindeutige Identifizierung. Aup die Werte kann auf drei verschiedene Weisen zugegriffen werden:

1. Die Werte können alle oder in Auswahl einfach in der gespeicherten Reihenfolge ausgegeben werden (SPSS-PC-Prozedur LIST).

2. Nach einem Sortierdurchgang können ausgewählte Werte nach bestimmten Kriterien (z.B. pro sprecher und Satzmodus) zusammengefaßt und sowohl Mittelwert, Standardabweichung etc. der Gruppen als auch die Einzelwerte auf einer ASCII-Datei ausgegeben werden (SPSS-PC-Prozedur REPORT).

3. Die Werte können mit der SPSS-PC-Prozedur WRITE auf eine ASCII-Datei geschrieben werden, die im Gegensatz zu den beiden anderen Ausgabemöglichkeiten keine weiteren Meldungen, sondern nur die Werte enthält. In diesem Format können die Werte von allen Programmen verarbeitet werden, die spaltenund zeilenweises Einlesen von ASCII-Dateien vorsehen.

Alle extrahierten und berechneten Werte können grundsätzlich anderen Interessenten zur Verfügung gestellt werden. Einschränkend müssen wir allerdings bemerken, daß nicht alle Daten gleichermaßen einfach und schnell abrufbar sind: 
Die SPSS-PC-Beschränkung auf 200 Variablen pro Fall sowie der nur begrenzt zur Verfügung stehende Speicherplatz hatten zur Folge, daß wenig benötigte Variablen ausgelagert wurden und nur mit einem gewissen Zeitaufwand zugänglich gemacht werden können. 
7. Korpus I

Das erste Korpus besteht fast ausschließlich aus Verb-Erst- und VerbZweit-Strukturen. $\mathrm{Zu}$ den Benennungen der Satztypen vergleiche man $\mathrm{z}$.B. Altmann (1987). In den meisten Fällen wurden hier nicht nur die wort- und wortfolgengleichen echten Minimalpaare gebildet, sondern jeweils auch Beispiele, in die für einen bestimmten Satzmodus typische - und diesen damit zusätzlich verdeutlichende - Modalpartikeln eingefügt wurden, um überprüfen zu können, ob und inwieweit die intonatorischen Realisierungen bei modalpartikelhaltigen und modalpartikellosen versionen voneinander abweichen.

Zunächst werden die mit Verb-Erst-Sätzen gebildeten Minimalpaare und -tripel aufgeführt (Nr. 1-43). Die Benennung 'V-1-Ausruf' erscheint in Anführungszeichen, da nicht klar ist, ob es sich tatsächlich um eine eigenständige Verb-Erst-Exklamativsatzvariante handelt, bei der sich die exklamativtypische propositionale Grundeinstellung nicht auf den Grad der Ausprägung einer Eigenschaft, sondern auf das Bestehen eines Sachverhalts bezieht; alternativ können derartige ' $\mathrm{V}-1$-Ausrufe' als emphatische Aussagesätze mit nicht besetztem Vorfeld klassifiziert werden. Am Ende dieser Verb-Erst-Gruppe steht unter IX. ein 'Minimalpaar', bei dem nicht Satzmodi einander gegenübergestellt werden, sondern innerhalb des Satzmodus Satzfragesatz eine höfliche und eine unhöfliche Ausprägung.

Es folgen Minimalpaare mit Verb-Zweit-Sätzen ohne $w$-Ausdruck in Spitzenstellung (Nr. 44-55). Den Abschluß bilden 'W-Sätze', Sätze mit einem $W$-Ausdruck in spitzenstellung, bei denen es sich zum größten Teil auch um VerbZweit-Strukturen handelt (Nr. 56-71). Die Minimalpaare aus Ergänzungsfragesatz und $W$-Verb-Zweit-Exklamativsatz sind in zwei Gruppen eingeteilt: In der ersten Gruppe steht das graduierbare Adjektiv (reich) in Distanz zum $w$-Ausdruck wie. Die komplexe 'exklamativische' $w$-Phrase ist also diskontinuierlich realisiert. Die Ergänzungsfragesätze sind in diesem Fall nur einfache wie-Fragen (Distanzstellung ist bei den Ergänzungsfragesätzen verboten; gefragt wird nicht nach dem Ausmaß des Reichtums, sondern nach der Art und Weise des Reichwerdens). In der zweiten Gruppe steht sowohl bei den Exklamativ- wie auch bei den Fragesätzen eine komplexe w-Phrase (wie laut, wie alt) im Vorfeld. Am Ende werden einige $W$-Verb-Letzt-Satztypen einander gegenübergestellt.

Untersuchungsergebnisse $\mathrm{zu}$ diesem Korpus finden sich in Oppenrieder (1988a,b), Batliner (1988), Luukko-Vinchenzo (1988) und speziell zu den Wunschsätzen in Scholz (1989). 
A. V-1-SATZE

I. Satzfragesatz vs. Sie-Imperativsatz

\section{Satzfragesatz}

1. Situation: Sprecher und ein anderer.

Sprecher: He! Ich rede mit Ihnen. Schlafen Sie?

\section{Sie-Imperativsatz}

2. Situation:

Sprecher und ein anderer.

Sprecher: Ruhen Sie sich erst einmal aus! Schlafen Sie!

II. Satzfragesatz vs. Ihr-Imperativsatz vs. V-1-Exklamativsatz

\section{Satzfragesatz}

3. Situation:

Der Sprecher und seine Freunde sehen eine lange Schlange vor der Kinokasse.

Sprecher: Das dauert mir viel zu lange. Was mich betrifft - ich stelle mich nicht an. Stellt ihr euch an?

4. Situation:

Der Sprecher und seine Freunde sehen eine lange Schlange vor der Kinokasse.

Sprecher: Das dauert mir viel zu lange. Was mich betrifft - ich stelle mich nicht an. Stellt ihr euch etwa an?

\section{Ihr-Imperativsatz}

5. Situation:

Der Sprecher und seine Freunde sehen eine lange Schlange vor der Kinokasse.

Sprecher: Also diesmal seid ihr dran. Ich stelle mich nicht wieder so lange an. Los! Stellt ihr euch an!

6. Situation: Der Sprecher und seine Freunde sehen eine lange Schlange vor der Kinokasse.

Sprecher: Also diesmal seid ihr dran. Ich stelle mich heute nicht wieder so lange an. Los! Stellt ihr euch doch an!

\section{V-1-Exklamativisatz}

7. Situation:

Der Sprecher beobachtet zwei Ulmer, wie sie vergeblich

Sprecher: versuchen, einen Balken durch das Stadttor zu tragen. Stellt ihr euch an! Laßt mich mal machen! 
8. Situation: Der Sprecher beobachtet zwei Ulmer, wie sie vergeblich versuchen, einen Balken durch das Stadttor zu tragen.

Sprecher: $\quad$ Stellt ihr euch vielleicht an! Laßt mich mal machen!

III. Satzfragesatz vs. Adhortativsatz

\section{Satzfragesatz}

9. Situation: Sprecher und andere

Sprecher: Was machen wir jetzt? Gehen wir?

10. Situation:

Sprecher und andere

Sprecher:

Was machen wir denn jetzt? Gehen wir denn jetzt?

\section{Adhortativsatz}

11. Situation: Sprecher:

12. Situation: Sprecher:
Sprecher und andere

Hier haben wir nichts mehr verloren. Gehen wir!

$(11---)$

Sprecher und andere

Hier haben wir nichts mehr verloren. Gehen wir doch jetzt!

IV. Satzfragesatz vs. V-1-Exklamativsatz

\section{Satzfragesatz}

13. Situation:

Sprecher und ein anderer

Sprecher:

Du hast also nicht geflucht? Und der hitzköpfige Max? Hat der geflucht?

14. Situation:

Sprecher und ein anderer

Sprecher:

Du hast also nicht geflucht? Und der hitzköpfige Max? Hat der denn geflucht?

\section{V-1-Exklamativsatz}

15. Situation: Sprecher:

16. Situation: Sprecher:

Sprecher und ein anderer

Karl ist gestern das Fahrrad geklaut worden. Mann! Hat der geflucht!

Sprecher und ein anderer

Karl ist gestern das Fahrrad geklaut worden. Mann! Hat der vielleicht geflucht!

V. Satzfragesatz vs. 'V-1-Ausruf'

\section{Satzfragesatz}

17. Situation:

Sprecher:
Sprecher und ein anderer

Mein Sohn schläft nie ein, wenn ich ihm Märchen erzähle. Wie ist es denn bei deinem? Schläft der ein? 
18. Situation: Sprecher:

\section{'V-1-Ausruf'}

19. Situation: Sprecher:

20. Situation: Sprecher:
Sprecher und ein anderer

Mein Sohn schläft nie ein, wenn ich ihm Märchen erzähle. Wie ist es denn bei deinem? Schläft der denn ein?

$$
\left(\begin{array}{lll}
11 & 1 & -
\end{array}\right)
$$

Sprecher und ein anderer

Da versuche ich ihm nun mühsam zu erklären, was er tun soll

- Und was passiert? Schläft der ein!

$$
(13---)
$$

Sprecher und ein anderer

Da versuche ich ihm nun mühsam zu erklären, was er tun soll

- Und was passiert? Schläft der einfach ein!

$$
(12---)
$$

VI. Satzfragesatz vs. V-1-Exklamativsatz vs. 'V-1-Ausruf'

\section{Satzfragesatz}

21. Situation:

Sprecher:

22. Situation:

Sprecher:
Der Hörer blickt zum Fenster hinaus. Der Sprecher möchte wissen, was ein dritter macht.

Was macht er denn gerade? Rennt der wieder?

$$
(11--1)
$$

Der Hörer blickt zum Fenster hinaus. Der Sprecher möchte wissen, was ein dritter macht.

Was macht er denn gerade? Rennt der etwa wieder?

\section{V-1-Exklamativsatz}

23. Situation:

Sprecher:

24. Situation:

Sprecher:

' $\mathrm{-1}$ - Ausruf'

25. Situation:

Sprecher:

26. Situation:

Sprecher:
Sprecher und Hörer sehen einen gemeinsamen Freund über die Straße laufen.

Mensch, schau dir mal den an! Rennt der wieder!

$(141--)$

Sprecher und Hörer sehen einen gemeinsamen Freund über die Straße laufen.

Mensch, schau dir mal den an! Rennt der vielleicht wieder!

Sprecher und ein anderer

Da hab ich ihm tausendmal gesagt, er soll immer schön langsam gehen.- Und was passiert? - Rennt der wieder!

$$
(16---)
$$

Sprecher und ein anderer Da hab ich ihm tausendmal gesagt, er soll immer schön langsam gehen. - Und was passiert? - Rennt der einfach wieder! 


\section{Satzfragesatz}

27. Situation: Sprecher und ein anderer

Sprecher: Kannst du mir mal sagen, wie der Kojak seinen Kopf so glatt bekommt? Rasiert der den glatt?

$(13-1-)$

\section{V-1-Exklamativsatz}

28. Situation:

Sprecher:

'V-1-Ausruf'

29. Situation: Sprecher:
Sprecher und Hörer schauen bei einem Geschicklichkeitswettbewerb für Barbiere zu.

Mensch, schau dir den mal an! Rasiert der den glatt! So würde ich das nie zustande bringen!

$$
(13---)
$$

Sprecher erzählt einem anderen eine Geschichte. ... Und dann hab ich ihn mit seinem Teddybären allein gelassen. Und was passiert? - Rasiert der den glatt!

VII. Satzfragesatz vs. V-1-Wunschsatz

\section{Satzfragesatz}

30. Situation: Sprecher und ein anderer

Sprecher:

Und was wäre gewesen, wenn der Oberförster seine Brille nicht vergessen hätte? Hätte er getroffen?

$(14---)$

31. Situation: Sprecher und ein anderer

Sprecher: Und was wäre gewesen, wenn der Oberförster seine Brille nicht vergessen hätte? Hätte er wohl getroffen?

$$
(12---)
$$

\section{V-1-Wunschsatz}

32. Situation:

Der Sprecher und sein Freund jammern über eine knappe Niederlage der Bayern.

Sprecher: Mein Gott! Steht dieser Rummenigge allein vor dem Torwart und bringt den Ball nicht ins Tor. Ach! Hätte er getroffen!

$$
(14-1-)
$$

33. Situation: Der Sprecher und sein Freund jammern uber eine knappe Niederlage der Bayern.

Sprecher: Mein Gott! Steht dieser Rummenigge allein vor dem Torwart und bringt den Ball nicht ins Tor. Ach! Hätte er doch getroffen!

$(13---)$

VIII. Satzfragesatz vs. V-1-Wunschsatz vs. V-1-Exklamativsatz

\section{Satzfragesatz}

34. Situation: Sprecher:

Sprecher und ein anderer

Kein Wunder, daß er nicht glücklich ist. Wie ginge es mir in seiner Lage? Wäre ich glücklich? 
35. Situation: Sprecher:
Sprecher und ein anderer

Kein Wunder, daß er nicht glücklich ist. Wie ginge es mir in seiner Lage? Wäre ich denn glücklich?

$(121--)$

\section{V-1-Wunschsatz}

36. Situation: Sprecher:

37. Situation: Sprecher:

\section{Der Sprecher}

Ach! Wenn doch nicht immer nur die anderen glücklich wären! Wäre ich glücklich!

$$
(15---)
$$

Der Sprecher

Ach! Wenn doch nicht immer nur die anderen glücklich wären! Wäre ich doch glücklich!

\section{V-1-Exklamativ}

38. Situation: Sprecher:
Sprecher und ein anderer

Was meinst $d u$, wie ich mich über einen dicken Lottogewinn freuen würde. Wäre ich glücklich!

$\left(\begin{array}{lll}10 & 1 & --\end{array}\right)$

Sprecher und ein anderer

Was meinst $d u$, wie ich mich über einen dicken Lottogewinn freuen würde. Wäre ich vielleicht glücklich!

IX. Neutrale/höfliche vs. unhöfliche/ungeduldige Frage

Neutrale/höfliche Frage

40. Situation:

Der Sprecher will vom Hörer wissen, ob er mit dessen

Sprecher: Begleitung rechnen kann: Gehst du bald nach Hause?

\section{unhõfliche/ungeduldige Frage}

41. Situation:

Sprecher:
Der Sprecher fragt den Hörer, ob dieser bald nach Hause geht. Nachdem der Hörer lediglich mit ironischem Lachen reagiert, wiederholt der Sprecher seine Frage ganz böse: Gehst du nun bald nach Hause?

\section{Neutrale/höfliche Frage}

42. Situation:

Sprecher:
Sprecher und ein anderer

Entschuldigen Sie bitte vielmals! Ich habe nur eine Frage: Gehört das Ihnen hier?

unhöfliche/ungeduldige Frage

43. Situation:

Sprecher:
Der Sprecher fühlt sich durch das Gelump des Hörers äußerst gestört und möchte diesen dazu bringen, da $\beta$ er es entfernt. Böse zum Hörer: Gehört das Ihnen hier? 
B. V-2-Sätze

\section{Aussagesatz vs. V-2-Exklamativsatz}

\section{Aussagesatz}

44. Situation: Sprecher:

Sprecher und ein anderer Warum schauen Sie mich so böse an und nicht den dort drüben? Ich habe doch nicht geschimpft. Der hat geschimpft.

45. Situation:

Zwel Sprecher unterhalten sich

Sprecher 1:

Da hat doch gerade einer geschimpft.

Sprecher 2:

Also ich war's nicht. Aber frag mal den Karl. Der hat vielleicht geschimpft.

$(102--)$

\section{V-2-Exklamativsatz}

46. Situation:

Sprecher:

47. Situation: Sprecher:

\section{Aussagesatz}

48. Situation: Sprecher:

49. Situation: Sprecher:
Sprecher und ein anderer Karl ist gestern das Fahrrad geklaut worden. Mann! Der hat geschimpft!

$(104--)$

Sprecher und ein anderer Karl ist gestern das Fahrrad geklaut worden. Mann! Der hat vielleicht geschimpft!

$$
(74--)
$$

Sprecher und ein anderer

Nein, die Maria ist nicht naiv. Ich meine die Brigitte. Die ist $\underline{\text { naiv. }}$

Sprecher und ein anderer Ich glaube eigentlich nicht, daß du bei Maria Glück hast, wenn du eine Naive suchst. Aber vielleicht versuchst du's mal bei Brigitte. Die ist vielleicht naiv.

\section{V-2-Exklamativsatz}

50. Situation: Sprecher:

51. Situation: Sprecher:
Sprecher und ein anderer Also diese Maria! Die ist naiv! Die glaubt immer noch an den Weihnachtsmann!

Sprecher und ein anderer Also diese Maria! Die ist vielleicht naiv! Die glaubt immer noch an den Weihnachtsmann! 
II. Aussagesatz vs. Versicherungsfrage

\section{Aussagesatz}

52. Situation: Sprecher:
Sprecher und ein anderer

Nein, nein, er ist nicht blind. Er sieht was.

\section{Versicherungsfrage}

53. Situation:

Fluglotse A: Fluglotse $B$ :
Zwei Fluglotsen unterhalten sich. Fluglotse A über einen Piloten, mit dem er gerade Funkverbindung hat: Der Kerl behauptet, er sieht ein UFO.

Er sieht was?

\section{Aussagesatz vs. assertive Frage}

\section{Aussagesatz}

54. Situation: Sprecher:

$$
\begin{aligned}
& \text { Sprecher und ein anderer } \\
& \text { Keine Widerrede. Du kommst. }
\end{aligned}
$$

\section{assertive Frage}

55. Situation: Sprecher:

Sprecher und ein anderer Ist das denn tatsächlich wahr? Du kommst?

C. W-SATZE

I. Ergänzungsfragesatz vs. Versicherungsfrage

\section{Ergånzungsfragesatz}

56. Situation:

Sprecher und ein anderer Sprecher:

Soso, im alten Stadion wird also wieder einmal gespielt. - Und wer spielt?

\section{Versicherungsfrage}

57. Situation:

Zwei Sprecher unterhalten sich.

Sprecher 1:

Dieses Mal spielt der Club-Präsident in der Sturmmitte.

Sprecher 2:

Das gibt's doch gar nicht! Wer spielt?

$(12---)$

II. Ergănzungsfragesatz vs. W-V-2-Exklamativsatz (mit Adjektiv in Distanzstellung)

\section{Ergänzungsfragesatz}

58. Situation:

Sprecher und Hörer über eine dritte Person A.

Sprecher:

Du hast gerade gesagt, da $A$ reich geworden ist. Was ich aber gerne wissen möchte: Wie ist der reich geworden? Wie um alles in der Welt konnte der das schaffen? 
59. Situation: Sprecher und Hörer über eine dritte Person A.

Sprecher: Du hast gerade gesagt, daß A reich geworden ist. Was ich aber gerne wissen möchte: Wie ist der denn reich geworden? Wie um alles in der Welt konnte der das schaffen?

$(19--)$

W-V-2-Exklamativsatz

60. Situation: Sprecher und Hörer uber eine dritte Person A.

Sprecher: Du kennst doch A. Vor ein paar Jahren noch ein armer

Schlucker. Und jetzt? Wie ist der reich geworden! So viel Geld wie der möchte ich auch einmal haben!

$(12---)$

61. Situation: Sprecher und Hörer über eine dritte Person A.

Sprecher: Du kennst doch A. Vor ein paar Jahren noch ein armer

Schlucker. Und jetzt? Wie ist der doch reich geworden! So viel

Geld wie der möchte ich auch mal haben!

$(10---)$

III. Ergänzungsfragesatz vs. W-V-2-Exklamativsatz

\section{Ergänzungsfragesatz}

62. Situation: Der Sprecher zum Vermieter, der ihm eine zu vermietende Wohnung gezeigt hat:

Sprecher: Die Wohnung hier sagt mir wirklich sehr zu. Aber es gibt da noch einen wichtigen Punkt. Wie laut ist es hier?

$(28-3)$

\section{W-V-2-Exklamativsatz}

63. Situation: Der Sprecher tut so, als müßte er sich die Ohren zuhalten.

Sprecher: $\quad H i e r$ könnte ich mit meiner Lärmempfindlichkeit wahrhaftig nicht wohnen! Wie laut ist es hier!

\section{Ergänzungsfragesatz}

64. Situation: Der Sprecher zum Hörer, nachdem ihm dieser von einem gewissen Max erzählt hat:

Sprecher: Uber Max bin ich jetzt sehr gut informiert. Nur eines möchte ich noch gern von dir wissen. Wie alt ist er geworden?

W-V-2-Exklamativsatz

65. Situation: Der Sprecher über einen alten Freund

Sprecher: Es ist doch unglaublich, was für Falten er jetzt hat und wie gebückt er dahergeschlurft kommt! Mein Gott! Wie alt ist er geworden! 
IV. Wortfrage-Obernahme vs. W-V-L-Exklamativsatz vs. deliberativer V-L-Fragesatz

\section{Wortfrage-Obernahme}

66. Situation: Zwei Sprecher unterhalten sich.

Sprecher 1: Weißt du wie groß der Peter ist?

Sprecher 2: Wie groß der ist?

\section{$\mathrm{W}-\mathrm{V}-\mathrm{L}-$ Exklamativsatz}

67. Situation: Sprecher und ein anderer

Sprecher: Schau dir mal den Rüssel von dem Elefanten an! Wie groß der ist!

$\left(\begin{array}{lll}8 & 2 & -\end{array}\right)$

\section{Wortfrage-Obernahme}

68. Situation: Zwei Sprecher unterhalten sich

Sprecher 1: Du kennst dich doch mit Rechnern aus. Kannst du mir vielleicht sagen, wie der hier läuft?

Sprecher 2: Wie bitte? Wie der läuft?

$$
(10-2-)
$$

W-V-L-Exklamativsatz

69. Situation: Sprecher und Hörer beobachten einen Dritten.

Sprecher: Schau mal, der Typ da drüben auf der anderen Straßenseite!

Wie der läuft!

$(69-1)$

70. Situation: Sprecher und ein anderer

Sprecher: Schau mal, der Typ da drüben, was der für einen komischen

Hut hat. Und eine Stricknadel hat er auch im Ohr. Und da!

Schau mal! Wie der erst läuft!

$(96--)$

\section{Deliberativer V-L-Fragesatz}

71. Situation:

Sprecher:
Sprecher und ein anderer

Schau mal, ich hab da einen neuen Rechner, bei dem ich mich überhaupt nicht auskenne. Wie der wohl läuft? 
8. Korpus II

Das zweite Großkorpus besteht aus drei Teilkorpora, dem 'Verb-Letz-Korpus' (Korpus II), dem 'Fokuskorpus' (Korpus III) und dem 'Leo-Korpus' (Korpus IV). Das Verb-Letzt-Korpus enthält vor allem selbständige und elliptische VerbLetzt-Sätze mit $o b, d a \beta$, wenn und dem $w$-Ausdruck wie als einleitendem Ausdruck. In diesem Teilkorpus werden elliptische Strukturen durch Einfügungen in eckigen Klammern ergänzt. (Diese Ergänzungen lagen den Versuchspersonen bei den Aufnahmen und in den Natürlichkeitstests nicht vor).

Die Testsätze sind hier in größeren Gruppen, je nach dem Einleitungsausdruck, angeordnet, es handelt sich also um 'Minimal-n-Tupel', die allerdings aufgrund der Besonderheiten mancher Verb-Letzt-Konstruktionen nicht ausschließlich völlig segmentgleiche Testsätze enthalten können. Auf Sätze mit $o b$ als Satzeinleiter (Nr. 1-13) folgen solche mit daß (Nr. 14-24) und mit wenn (Nr. 25-29) als Satzeinleiter. Bei den wenn-Sätzen ist es wegen der Fixiertheit des Satzeinleiters auf wunschsätze oder damit verwandte Strukturen nicht möglich, Satzmodus-Minimalpaare zu bilden. Stattdessen wurden neben echten VerbLetzt-Wunschsätzen auch bestimmte 'idiomatische' wenn-Sätze mit aufgenommen. Die Verb-Letzt-Sätze werden abgeschlossen durch solche mit einem $w$-Ausdruck, hier wie, als Satzeinleiter (Nr. 30-38).

Am Ende folgen ein Minimaltripel von $W$-Verb-Zweit-Sätzen (Nr. 39-41) und einige Alternativfragesätze (Nr. 42-45). Bei diesen war insbesondere auch der Unterschied von offenen und geschlossenen Alternativfragen von Interesse. Die Minimalpaarbildung erfolgte also zwischen dem 'Kombinationstyp' des eigentlichen (geschlossenen) Alternativfragesatzes (Altmann 1987: 48) und der (offenen) disjunktiven oder-Verknüpfung von Satzfragesätzen Untersuchungsergebnisse zu diesem Korpus finden sich in Oppenrieder (1989a), Luukko-Vinchenzo (1988) und speziell zu den Wunschsätzen in Scholz (1989). 
$O B-$ SATZE

Deliberativer Fragesatz

1. Situation: Sprecher wartet auf seinen Freund und ist sich nicht sicher, ob dieser kommt.

Sprecher: $\quad$ ob er wohl kommt?

$\left(\begin{array}{lll}11 & 1 & -\end{array}\right)$

Aussagesatz + Interrogativ-Sentential

2. Situation: Sprecher und ein anderer, auf einen gemeinsamen Freund

Sprecher: $\quad$ wartend 1 Ich 1 mich, ob er kommt.

$(1-121)$

\section{Versicherungsfrage}

3. Situation:

Sprecher - ein guter Bekannter von Peter - und ein anderer, der von ihm noch nie etwas gehört hat.

Sprecher 1:

Kommt der Peter?

Sprecher 2:

ob wer kommt?!?

$(121--)$

\section{Rückfrage}

4. Situation:

Sprecher und ein anderer, auf einen gemeinsamen Freund wartend

Sprecher 1:

Weißt $d u, o b$ er kommt?

Sprecher 2:

[Du fragst mich,J ob er kommt? Wie kommst du überhaupt dazu, mich das zu Pragen! Natürlich kommt er!

$(14---)$

\section{Ultimative Frage}

5. Situation:

Sprecher 1:

Sprecher und ein anderer.

Sprecher 2:

Kommt der Peter heute?

Er geht heute schwimmen, besucht seine Großmutter, und er will in die Bibliothek gehen.

Sprecher 1:

Red doch nicht herum! [Ich will wissen,l ob er kommt?!?

6. Situation:

Sprecher und ein anderer

Sprecher 1:

Kommt der Peter heute?

Sprecher 2:

Er geht heute schwimmen, besucht seine Großmutter, und er will in die Bibliothek gehen.

Sprecher 1:

Red doch nicht herum! Ich will wissen, ob er kommt.

\section{Ob-Fortsetzungsassertion}

7. Situation:

Sprecher 1:

Sprecher 2:

Sprecher 1:
Sprecher und ein anderer, auf einen gemeinsamen Freund wartend

Hast du eine Ahnung, ob er kommt?

Wie bitte? Was hast du mich gefragt?

[Ich habe dich gefragt.] ob er kommt. 
8. Situation: Sprecher und ein anderer, auf einen gemeinsamen Freund wartend

Sprecher 1: Hast du eine Ahnung, ob er kommt?

Sprecher 2: Wie bitte? Was hast du mich gefragt?

Sprecher 1: $\quad$ Ich habe dich gefragt, ob er kommt.

Und-ob-Antwort

9. Situation: Sprecher und ein anderer

Sprecher 1: Was ist jetzt? Kommt er?

Sprecher 2: $\quad$ Und ob er kommt!

$(12---)$

\section{Ob-Aufforderungssatz}

10. Situation: Die Mutter hat ihren Sohn bereits mehrmals ohne Erfolg aufgefordert, zu ihr zu kommen. Schließlich wird sie ungeduldig.

Mutter: ob du wohl kommst!

$(26-4)$

\section{Frage-Obernahme}

11. Situation:

Sprecher und ein anderer, auf einen gemeinsamen Freund wartend

Sprecher 1: Weißt du, ob er kommt?

Sprecher 2: [Du fragst mich, / Fragst du mich,l ob er kommt? Das weiß ich auch nicht.

$(12---)$

\section{$O b$-Fortsetzungsfrage}

12. Situation: Sprecher und ein anderer

Sprecher 1: Anna hat mich ein paar Sachen über Peter gefragt.

Sprecher 2: [Hat siel Auch lgefragt), ob er kommt?

13. Situation: Sprecher und ein anderer

Sprecher 1: Anna hat mich ein paar Sachen über Peter gefragt.

Sprecher 2: $\quad$ Hat sie auch gefragt, ob er kommt?

$(11---)$

\section{DASS-SATZE}

\section{Daß-Aufforderungssatz}

14. Situation: Mutter zum Kind

Mutter: $\quad$ Und mach der Oma keinen Arger! Hörst du? Daß du ja brav

$\underline{\text { bist! }}$

$(12---)$

\section{Daß-Grad-Exklamativsatz}

15. Situation: Oma zur Enkelin

Oma:

Daß du so brav bist! Ich hab noch nie ein Mädchen gesehen, das so brav war. 
16. Situation: Oma zur Enkelin, die seit zwei Stunden den Mund nicht mehr aufgemacht hat

Oma:

Daß du so brav bist! Deine Eltern haben mir zwar schon erzählt, daß du sehr brav sein kannst, aber das hätte ich nicht erwartet.

$(85--1)$

Aussagesatz mit einem die Sprechereinstellung bezeichnenden Matrixsatz

17. Situation: Oma zur Enkelin

Oma: $\quad$ Daß du so brav bist, wundert mich schon.

$\left(\begin{array}{lll}6 & 6 & -\end{array}\right)$

18. Situation: Oma zur Enkelin

Oma: $\quad$ Es wundert mich schon, daß du so brav bist.

$\left(\begin{array}{lll}10 & 1 & -\end{array}\right)$

Daß-Satz-Exklamativsatz

19. Situation: Oma wundert sich über den Enkel, der still in der Ecke sitzt

Oma:

Daß du brav bist! Alle haben mir gesagt, daß du ein ungezogener und böser Bub wärst.

Da $\beta$-Antwort

20. Situation:

Oma:

Enkel:

Oma und Enkel

Das wundert mich aber schon.

Was denn?

Oma:

Daß du so brav bist. (, wundert mich.]

21. Situation:

Oma und Enkel

Oma:

Das wundert mich aber schon.

Enkel:

Was denn?

Oma:

Daß du brav bist. [, wundert mich.]

$\left(\begin{array}{lll}11 & 1 & -2\end{array}\right)$

\section{Da $\beta$-Fortsetzungsfrage}

22. Situation:

Kind:

Mutter:

Kind zu Mutter

Der Nikolaus hat dem Opa nur Gutes über mich erzählt.

[Hat er ihm] Auch [erzählt], daß du brav bist?

$$
(10-3-)
$$

\section{Rückfrage}

23. Situation:

Kind zu Mutter

Kind:

Der Onkel hat gesagt, da $\beta$ ich brav bin.

Mutter:

Kind:

Daß du brav bist? l, hat der Onkel gesagt?]

Ja, das hat er gesagt. 
Daß-Wunschsatz

24. Situation: Mutter jammert über böse Tochter

Mutter:

\section{WENN-SATZE}

V-L-Wunschsatz

25. Situation:

Sprecher und ein anderer

Sprecher:

Ob die anderen kommen, ist eigentlich ziemlich egal. Wenn der Willi doch käme! Der könnte uns bestimmt helfen.

$(12---)$

26. Situation: Sprecher:

Sprecher und ein anderer

Wenn der Willi käme, könnte er uns bestimmt helfen.

$(210--)$

Diverses, 'idiomatische' wenn-Sätze

27. Situation: Sprecher:

28. Situation: Sprecher 1: Sprecher 2:

29. Situation: Sprecher:
Sprecher und ein anderer Daß die Christa kommt, ist ja schon schlimm genug. Aber wenn der Willi erst kommt! Der würde euch vielleicht was erzählen.

$(12---)$

Sprecher und ein anderer Weißt du, wie das Wetter morgen wird?

Ach, wenn ich das wüßte!

$(12---)$

Sprecher und ein anderer

Wenn das mal gut geht!

W-V-L-SATZE

W-V-L-Exklamativsatz

30. Situation: Sprecher:

31. Situation: Sprecher:

Und-wie-Antwort

32. Situation:

Sprecher 1: Sprecher 2:
Sprecher und ein anderer Schau dir doch mal diese wunderschönen Kakteen an. Wie die $\underline{\text { blüh'n! }}$

$(12---)$

Sprecher und ein anderer

Schau dir doch mal die hübsche form von diesen Kakteen an. Und wie die (erst) blüh'n!

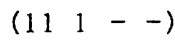

Sprecher und ein anderer vor ein paar unscheinbaren Kakteen stehend Blühen diese Kakteen eigentlich?

Und wie die blüh'n! 


\section{Fortsetzungsassertion}

33. Situation: Sprecher und ein anderer vor ein paar Kakteen stehend Sprecher 1: Ich weiß jetzt alles, was ich schon immer über diese Kakteen wissen wollte.

Sprecher 2: Wo die wachsen?

Sprecher 1: Nein, wie die blüh'n. I, weiß ich jetzt.J

\section{Fortsetzungsfrage}

34. Situation: Sprecher und ein anderer vor ein paar Kakteen stehend Sprecher 1: Ich hab gestern etwas über diese Kakteen gelesen und weiß jetzt endlich, was ich schon immer wissen wollte.

Sprecher 2: [Weißt du nun,] Wie die blüh'n?

Sprecher 1: Nein, wo die wachsen.

\section{Frageũbernahme}

35. Situation: Sprecher 1:

Sprecher und ein anderer

Weißt du, wie diese Kakteen blühen?

Sprecher 2: Laß mich mal überlegen! - [Du fragst,/Fragst du mich.] Wie die blüh'n? Weiß und orange.

\section{Rückfrage}

36. Situation: Sprecher 1:

Sprecher und ein anderer

Sprecher 2:

Weißt du, wie diese Kakteen blühen?

IDu fragst mich, I Wie die blüh'n? Das weiß doch jedes Kind!

\section{Deliberativer $W-V-L-F r a g e s a t z$}

37. Situation:

Sprecher:

Sprecher steht stirnrunzelnd vor blütenlosen Kakteen Wie die wohl blüh'n?

\section{Ultimative Frage}

38. Situation:

Sprecher 1:

Sprecher 2:

Sprecher 1:
Sprecher und ein anderer

Wie blühen diese Blumen eigentlich?

Das sind Kakteen.

Nein, lich habe gefragt,J wie die blüh'n?!? 
W-V-2-SATZE

W-Exklamativsatz

39. Situation:

Sprecher und ein anderer

Sprecher:

Willi fährt morgen für ein ganzes Jahr nach Agypten. Was wird der dann schon alles gesehen haben, wenn er wiederkommt!

$(16---)$

\section{Rhetorischer W-Fragesatz}

40. Situation: Sprecher und ein anderer

Sprecher 1: Willi hat Urlaub in Gelsenkirchen gemacht.

Sprecher 2: Und? Was hat er denn alles gesehen?

Sprecher 1: Na ja! Was wird er denn schon alles gesehen haben? Er hat

sich halt den Friedhof und ein paar Kohlengruben angeschaut.

$(-8-5)$

\section{Ergänzungsfragesatz}

41. Situation: Sprecher und ein anderer

Sprecher 1: In einem Jahr wird Willi schon durch halb Agypten gereist sein.

Sprecher 2: Und was wird er dann schon alles gesehen haben? Glaubst du, da $\beta$ er die Pyramiden dann schon kennt?

$(54-6)$

\section{ALTERNATIVFRAGESATZE}

\section{Geschlossener Alternativfragesatz}

42. Situation: Der Gastgeber hat zwei Sorten Kuchen zur Auswahl und bietet diese an.

Gastgeber: $\quad$ Möchten Sie Mohn oder Streusel?

$$
(-12--)
$$

\section{Offener Alternativfragesatz}

43. Situation: Der Gastgeber hat mehrere Sorten Kuchen zur Auswahl und bietet diese an.

Gastgeber: Möchten Sie Mohn? Oder Imöchten SieL Streusel?

Gast: $\quad$ Ich möchte einen Obstkuchen.

$(77--)$

\section{Geschlossener Alternativfragesatz}

44. Situation: Der Gastgeber hat drei Sorten Kuchen zur Auswahl und bietet diese an.

Gastgeber: Was möchten Sie von den drei Kuchen haben? Möchten Sie Sahne, Mohn oder Streusel?

$$
(-13--)
$$

\section{Offener Alternativfragesatz}

45. Situation: Der Gastgeber hat mehrere Sorten Kuchen zur Auswahl und bietet diese an.

Gastgeber: Was möchten Sie von den Kuchen haben? Möchten Sie lzum Beispiell Sahne, Mohn oder Streusel?

Gast:

Ich möchte einen Käsekuchen. 
9. Korpus III

Das zweite Teilkorpus (Nr. 46-71) ist das sogenannte Fokuskorpus. In diesem wird nicht nur der Satzmodus, sondern auch die Fokusstruktur systematisch variiert. Sowohl Modus- als auch Fokusrealisierung werden durch passende Kontexteinbettungen gesteuert. Da in allen Satzmodi sowohl enge Fokussierung als auch Fokusprojektion und Doppelfokussierung untersucht werden sollten, andererseits aber Probleme mit einem diskontinuierlichen Fokus oder möglichen spezifischen Fokussierungseigenschaften von Subjekten vermieden werden sollten, wurde die Fokussierung in A.c.I.-Sätzen mit eingebettetem hochtransitivem Verb untersucht. Dieses (infinite) Verb und sein Objekt sind die möglichen Fokusträger. Die für die jeweiligen Testsätze intendierte Fokusstruktur ist in den Zwischenüberschriften angegeben.

Ergebnisse zu diesem Korpus finden sich in Batliner (1989a), Batliner/Nöth (1989), Oppenrieder (1989b) sowie Oppenrieder (1989c).

Zusätzlich zu den für alle Korpora ermittelten Parameterwerten wurden für jede der drei Phrasen (eingebettetes Subjekt des infiniten Satzes, objekt des infiniten Verbs und das infinite Verb selbst) folgende Werte gemessen (genauere Angaben finden sich in Batliner 1989a):

1. Der Beginn der Phrase

2. Die Dauer der Phrase

3. Der höchste Fo-Wert der Phrase

4. Der niedrigste Fo-Wert der Phrase

Gemessen wurden die stimmhaften Passagen. Die folgende Abbildung zeigt eine Fo-Kontur sowie, mit Pfeilen angedeutet, die gemessenen Punkte. ${ }^{1}$

Natürlich gab es auch einige $Z$ weifelsfälle, bei denen die Punkte nicht so eindeutig gemessen werden konnten. Die zwei Hauptstrategien, die für solche Fälle auch bei den in Teil 4 beschriebenen Messungen angewandt wurden, seien kurz erwähnt: (i) Wenn die Differenz zwischen Fo-Maximum und FoMinimum einen Schwellenwert, z.B. einen Halbton, unterschritt, wurde ein ebener Fo-Verlauf angesetzt. (ii) Mit einer gewissen Willkür behaftete $\mathrm{Zu}-$ schreibungen können bei der Betrachtung des Einzelfalls als solche gekennzeichnet werden; bei der Betrachtung der statistischen Verteilungen 'mitteln sie sich aus', fallen also nicht ins Gewicht. 


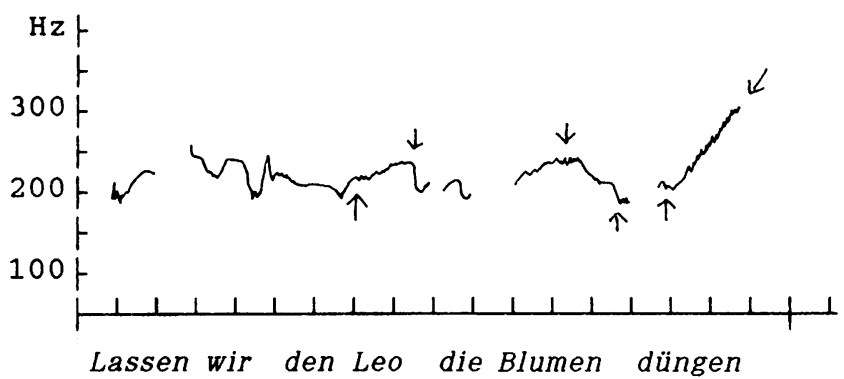

Bei den in diesem Band auch in den Einzelbeiträgen behandelten Korpora III und IV sind zusätzlich schematisierte Fo-Verläufe und einige statistische Kennwerte angegeben. Die $\mathrm{y}$-Achse ist in $\mathrm{Hz}$ skaliert, die $\mathrm{x}$-Achse gibt die Zeit wieder, wobei ein Teilstrich für $100 \mathrm{msec}$ steht. Die Kurven geben den Verlauf der Fo-Kurven auf den Mingogrammen wieder. Um die Vergleichbarkeit zu erleichtern, wurden die Kurven derart normiert, daß auf der Zeitachse der Anfang stimmhafter Passagen auf den gleichen Punkt gesetzt wird. Die Dauer der stimmlosen Passagen und damit auch die Gesamtdauer der einzelnen Außerungen kann also anhand der Abbildungen nicht ermittelt werden. Die Dauer der jeweiligen stimmhaften Passagen wurde nicht normiert und entspricht damit den Originalen.

Uber der $\mathrm{x}$-Achse stehen die Fo-Konturen der Sprecherinnen, unter der $\mathrm{x}$-Achse die der Sprecher; links stehen die 'Prototypen', rechts die 'NichtPrototypen' (vgl. dazu oben Teil 3 ).

Bei den statistischen Kennwerten stehen in jeder Zeile die Angaben für alle Außerungen pro Sprecher(in), und zwar in den Spalten von links nach rechts:

1. Linienart pro Sprecher(in): durchgezogen, gestrichelt bzw. gepunktet.

2. SprecherInnen: 1-3 weiblich, 4-6 männlich.

3. Kennzeichnung für Prototyp/Nicht-Prototyp: ' $+1 /{ }^{\prime}-$ '.

4. Position der Hauptakzentsilbe (Silbenzahl bis inkl. Hauptakzentsilbe).

5. Natürlichkeitsbewertung (Werte zwischen 1 und 5).

6. Kategorisierung (Werte zwischen 0 und 1.0).

7. Der Wert wurde ermittelt anhand von Hörtests, die speziell für dieses Korpus durchgeführt wurden; Einzelheiten finden sich in Batliner (1989a). Er gibt an, ob die zweite Phrase (positiver Wert) oder die dritte Phrase (negativer Wert) den Fokusakzent trägt. Die Werte liegen $z$ wischen 0.0 und $+/-1.0$. Je größer der absolute Betrag, desto 'sicherer' sind sich die Hörer bei der Fokusakzentzuweisung.

8. Dieser Wert steht für die Richtigkeit der Frage/NichtFrageklassifizierung und ergibt sich dadurch, da $\beta$ die Zuweisungen $z u$ den vier Nicht-Frage-Modi zusammengefaßt werden. Die Werte liegen zwischen 0.0 und 1.0; je größer der Wert, desto richtiger ist die Klassifizierung; vgl. Teil 5.2. 
9. Ein Stern bildet das Trennungszeichen zwischen den einzelnen Außerungen pro Zeile.

Aus diversen Gründen fehlende Werte sind durch einen Punkt gekennzeichnet. Um anzudeuten, daß die Werte in der 7. und in der 8. Spalte 'sekundär', d.h. durch Umrechnung ermittelt wurden, sind sie kursiv wiedergegeben. Die Spaltenbelegung sei nun an einem Beispiel erläutert. Die Werte für den ersten Fall von Nr. 46 lesen sich wie folgt:

$1+71.91 .0 .861 .00$

Die Fo-Konturen der Sprecherin 1 (2. Spalte) sind durchgezogen (1. Spalte). Der Hauptakzent liegt auf der 7. Silbe (3. Spalte), also auf Lei-, wie durch den Kontext indiziert, vgl. den Testsatz mit groß geschriebenem LEI: Sie läßt die Nina das LEInen weben. Der Natürlichkeitswert (5. Spalte) ist 1.9, die 'Treffer-

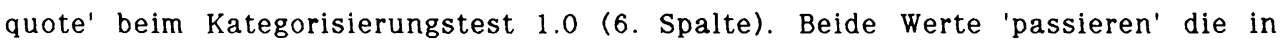
Teil 3 beschriebene Schwelle; es handelt sich also um einen Prototyp; das ist in der 3. Spalte durch ' + ' gekennzeichnet. Der Wert in der 7. Spalte ist positiv, die 2. Phrase (das Leinen) wurde demnach als akzentuiert eingestuft. Ein Wert von 0.86 ist relativ hoch, die Hörer waren sich also sicher in dieser Zuweisung. Die Klassifizierung in Frage/Nicht-Frage ist $100 \%$ richtig, wie der Wert von 1.0 in der 8. Spalte zeigt. ${ }^{2}$

Die zwei bis maximal vier Fo-Kurven pro Sprecher(in) können dann, wenn es sich gleichermaßen um Proto- oder Nicht-Prototypen handelt, nicht immer unterschieden werden; damit ist auch eine eindeutige Zuordnung der statistischen Kennzahlen zum dazugehörigen Fo-Verlauf nicht möglich. Diesen Nachteil mußten wir in Kauf nehmen, da eine unterschiedliche Strichlierung pro Außerung zwar theoretisch eine Identifizierung ermöglichen würde, in der Praxis aber auf den Abbildungen ein Chaos verursacht hätte. In den meisten Fällen unterscheiden sich die Fo-Kurven sowieso nur geringfügig, d.h. der allgemeine Verlauf ist der gleiche.

2 Wenn die 'Trefferquote' in Spalte 61.0 ist, wie in diesem Fall, ist natürlich auch der Wert in der 8. Spalte 1.0. Die Werte müssen sich aber nicht entsprechen: Wenn z.B. ein Exklamativ als Aussage klassifiziert wurde, so ist die Trefferquote niedrig. Da aber davon die Frage/Nicht-Frage-Klassifikation nicht berührt ist, kann der Wert in der 8. Spalte trotzdem sehr hoch sein. 
AUSSAGESATZ, FOKUS: OBJEKT

46. Situation: In einem Textllbetrieb; eine Mutter erkundigt sich bei einer Angestellten nach den handwerklichen Fortschritten ihrer Tochter. Mutter: Was läßt dle Meisterin meine Nina denn gerade weben? Angestellte: Sie läßt die Nina das LEInen weben.

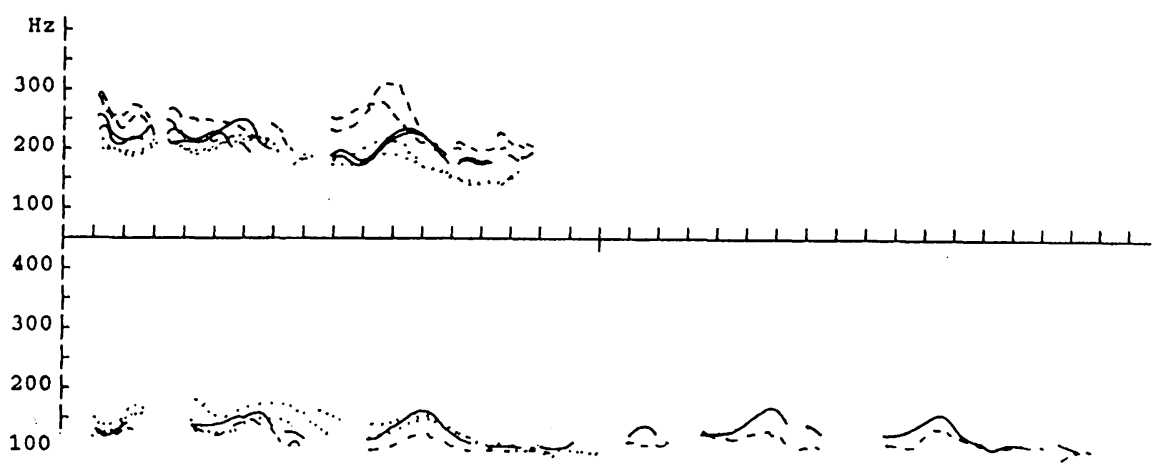

$1+71.91 .0 .861 .00 *+71.81 .0 .931 .00$

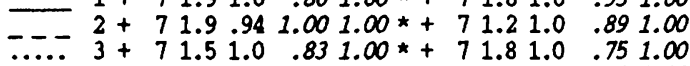

$4+72.41 .0 .801 .00 *-72.81 .0 \quad .801 .00$

$5+71.61 .01 .001 .00 *-72.61 .01 .001 .00$

$\ldots .6+71.51 .0 .801 .00 *+71.11 .0 .801 .00$

\section{AUSSAGESATZ, FOKUS: VERB}

47. Situation: In elnem Textilbetrieb; Anna erkundigt sich bei Rosa, womit ihre

gemelnsame Freundin Nina gerade beschäftigt ist. Anna: Heute ist sie also mit dem Leinen beschäftigt. Und was genau läßt die Meisterin die Nina damit machen? Rosa: Sie läßt die Nina das Lelnen WEben.

$$
(13-1-)
$$
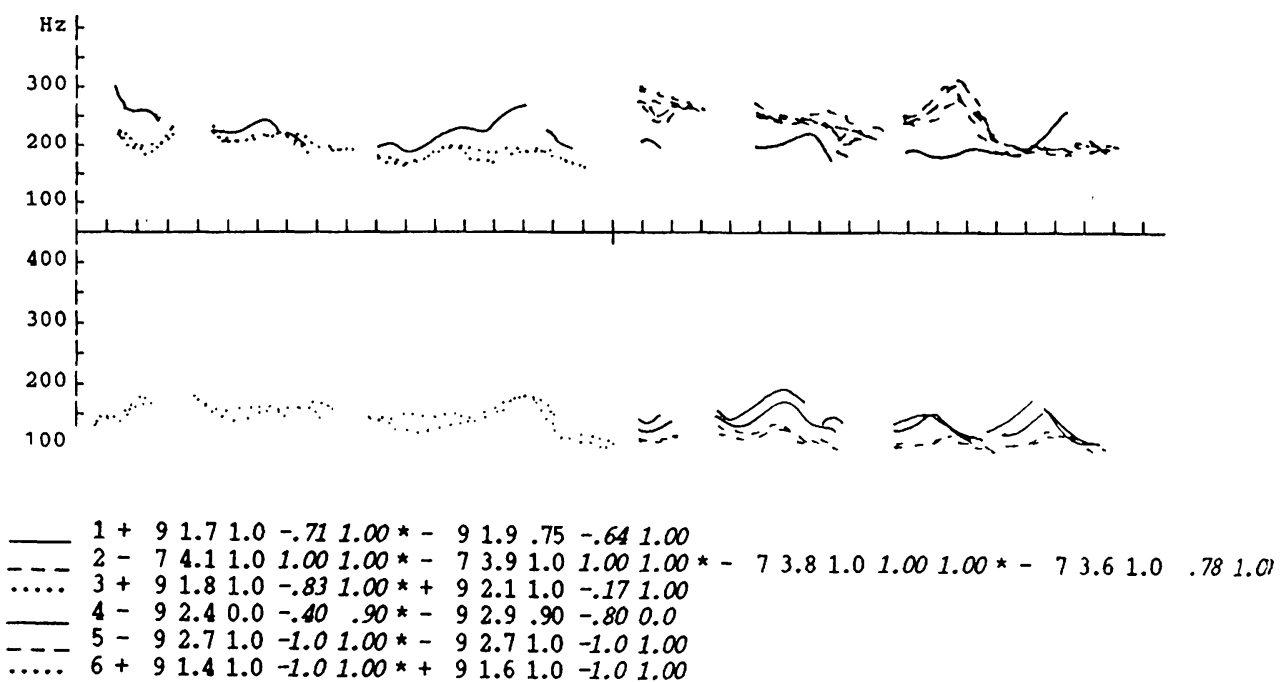
AUSSAGESATZ, FOKUS: OBJEKT + VERB

48. Situation: In einem Textllbetrieb; zwel Angestellte unterhalten sich über eine gemeinsame Freundin. Anna: Was läßt die Meisterin die Nina denn gerade machen? Rosa: Sie läßt die Nina das LEInen weben.

$$
(12---)
$$

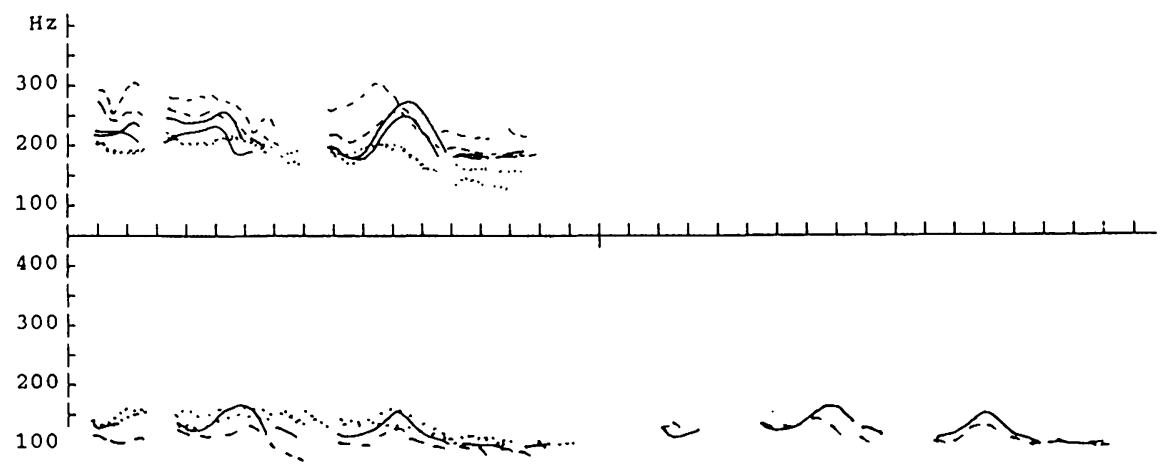

$1+71.81 .01 .001 .00 *+71.21 .01 .001 .00$

$2+71.3 .92 .781 .00 *+71.71 .0 .891 .00$

$\ldots . .-3+71.51 .0 .831 .00 *+71.91 .0 .751 .00$

$4+71.91 .0 .701 .00 *-72.51 .0 .901 .00$

$-5+72.11 .0 .671 .00 *-72.81 .01 .001 .00$

$\ldots . .6+71.7 .90 .701 .00 *+71.61 .0 .801 .00$

AUSSAGESATZ, ZWEITEILIGER FOKUS: OBJEKT, VERB

49. Situation: In einem Textilbetrieb; zwel Angestellte unterhalten sich über eine gemeinsame Freundin. Anna: Was läßt die Meisterin die Nina denn gerade mit welchem Stoff machen? Rosa: Sie läßt die Nina das LEInen WEben.

$(17---)$

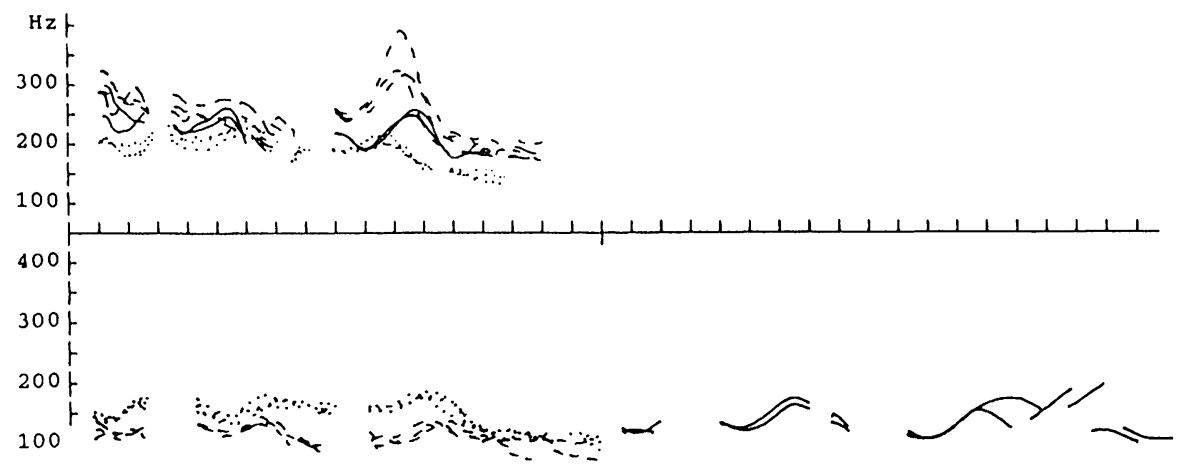

$1+72.0 .89 .691 .00 *+71.61 .0 .931 .00$

$2+71.6 .85 .891 .00 *+72.21 .01 .001 .00 *+72.21 .0 .891 .00$

$\ldots . .3+72.01 .0 .671 .00 *+72.01 .0 .921 .00 *+72.11 .0 .831 .00$

$4-94.1 .90-.30 .90 *-93.9 .92-.601 .00$

$-5+72.31 .0 .501 .00 *+72.31 .01 .001 .00 *+71.41 .0 .901 .00 *$

$\ldots .6+71.81 .0 .601 .00 *+71.51 .0 .701 .00 *+71.41 .0 \quad .801 .00$ 
AUSSAGESATZ, ZWEITEILIGER FOKUS: OBJEKT, VERB

50. Situation: In elnem Textllbetrleb; zwel Angestellte unterhalten sich über eine gemeinsame Freundin. Anna: Läßt die Meisterin die Nina denn heute mit den Baumwollstopfen arbeiten? Soll sle dle färben? Rosa: Nein, nein. - Sle läßt die Nina das LEInen WEben.

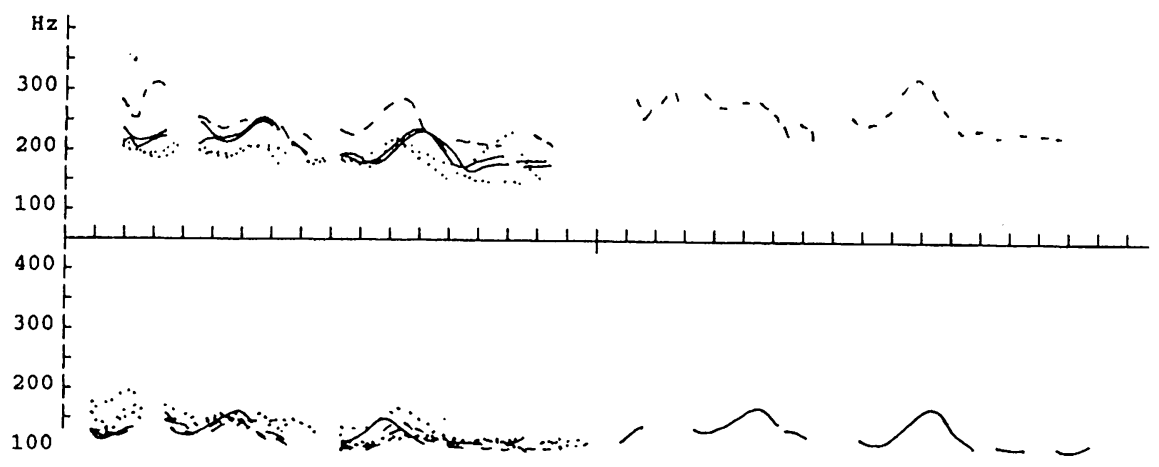

$1+71.5 .89 .711 .00 *+71.61 .0 .861 .00$

$--2+71.71 .01 .001 .00 *+72 \%-72.7 .941 .001 .00$

$\ldots \ldots .+3+92.0 .90-.831 .00 *+72.01 .0 .751 .00$

$4+71.91 .0 .801 .00 *-72.5 .92 .801 .00$

$-\ldots 5+71.41 .01 .001 .00 *+72.01 .01 .001 .00$

$\ldots . .6+71.8 .90 .601 .00 *+71.81 .0 .901 .00 *+71.61 .0 .701 .00$

\section{ASSERTIVE FRAGE, FOKUS: OBJEKT}

51. Situation: Eine Mutter besucht in einem Textilbetrieb ihre Tochter, um deren handwerkliche Fortschritte $\mathrm{zu}$ begutachten. Zu einer Angestellten: Mutter: Was läßt die Meisterin meine Nina denn gerade weben? [Sie entdeckt jetzt erst ihre Tochter vor einem Webstuhl.l Sle läß dle Nina das LEInen weben? Warum muß sle denn immer dasselbe machen?
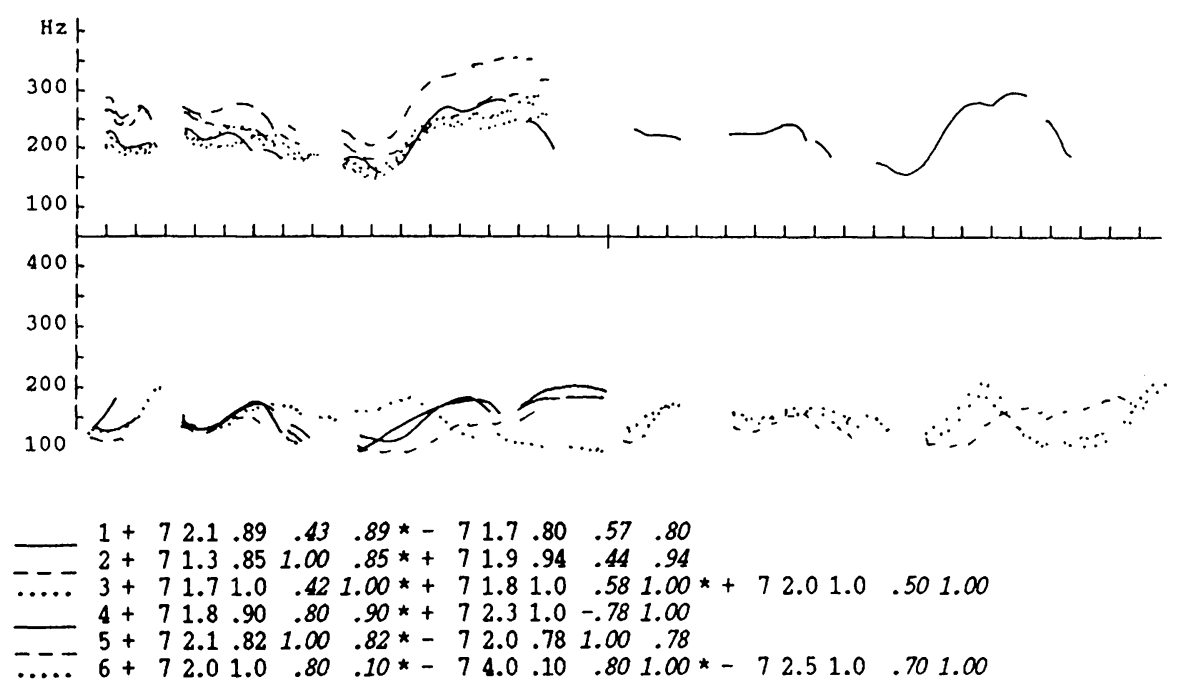
ASSERTIVE PRAGE, FOKUS: VERB

52. Situation: In elnem Textllbetrleb; elne Mutter erkundigt sich bel elner Angestellten, mlt welchen Arbelten lhre Tochter gerade beschäftigt Ist. Mutter: Aha, mit Leinen also? Was läßt die Melsterin melne Nina denn damit machen? [Sle sleht Ihre Tochter an elnem Webstuhl sitzen.] Sie läßt die Nina das Leinen WEben? Das macht sle doch dauernd. Ich habe gedacht, ihr wird jetzt endllch einmal das Färben belgebracht.

$(141--)$

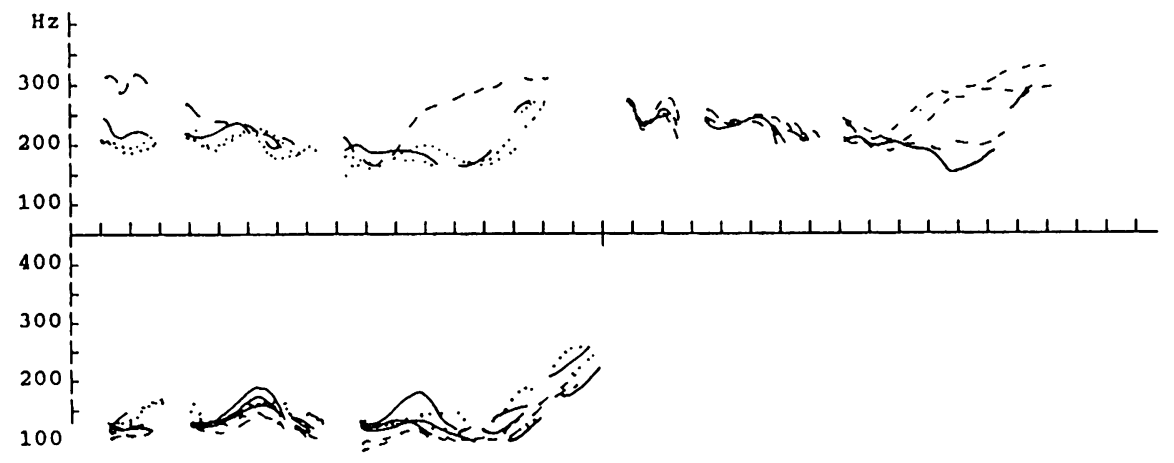

$1+92.01 .0-1.0 .78 *-91.6 .78-1.01 .00$

$-\ldots 2+91.61 .0-.781 .00 *-73.1 .78 .56 .83 \star-73.6 .83 .56 .78 *-72.91 .0 .561 .00$

$\ldots .-3+92.21 .0-.671 .00 *+92.21 .0-.251 .00$

$+4+92.1 .83-.40 .83 *+91.81 .0-1.01 .00 \star+92.41 .0-.801 .00$

$-\ldots+92.31 .0-1.01 .00 *+92.31 .0-1.01 .00$

$\ldots .-6+92.11 .0-.901 .00 *+91.01 .0-.801 .00$

\section{ASSERTIVE FRAGE, FOKUS: OBJEKT + VERB}

53. Situation: In elner Weberel; zwel Kolleginnen unterhalten sich uber elne dritte. Anna: Was läß die Meisterin dle Nina denn jetzt machen? ISle sehen Nina an elnem Webstuhl sitzen.l Rosa: Sle läßt dle NIna das LEInen weben? Und uns läßt sle putzen und dle ganze Drecksarbejt machen!

$(112--)$

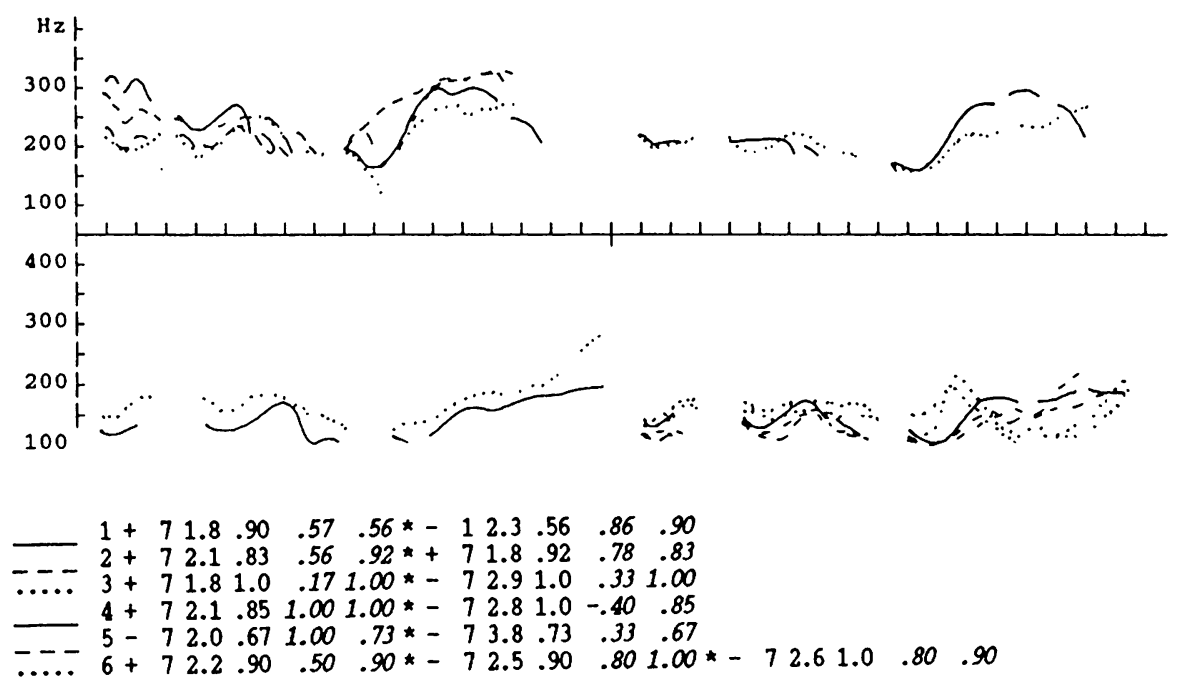


ASSERTIVE FRAGE, ZWEITEILIGER FOKUS: OBJEKT, VERB

64. Situation: In elnem Textllbetrleb; Anna sleht, wle die Melsterin ihrer Freundin eln paar Anwelsungen gibt. Zu elner Kollegin: Anna: Ich bin gespannt, was sie die Nina mit welchem Stoff machen läßt. (Nina setzt sich an den Webstuhl.I Sie läßt die Nins das LEInen WEben? Mit Leinen hat sle doch schon genug gearbeltet. Und statt dem dauernden Weben konnte sie die Nina ruhig auch mal was anderes machen lassen, färben oder so.

$(123-1)$

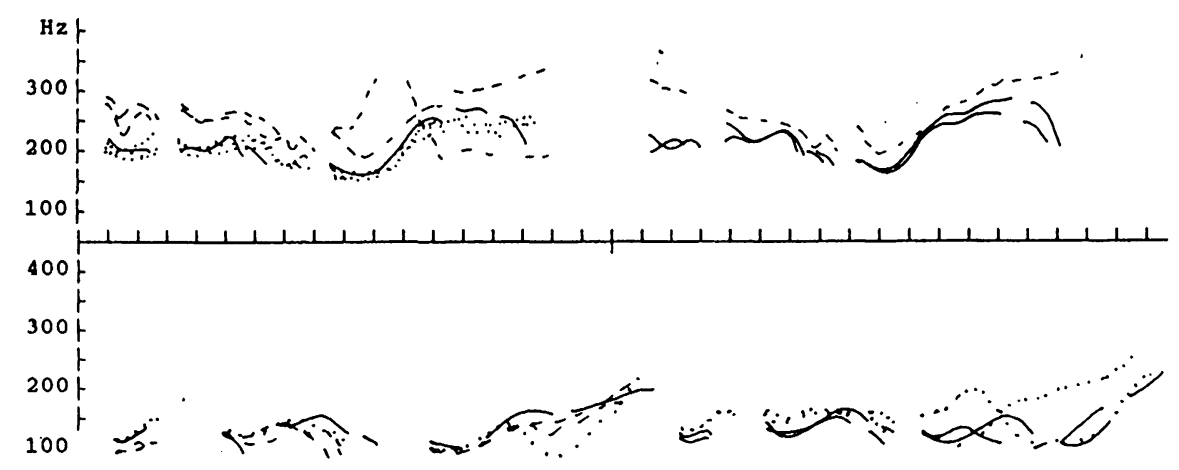

$1+72.11 .0 .641 .00 *-71.2 .801 .00 .80 *-71.9 .80 \quad .36 .80$

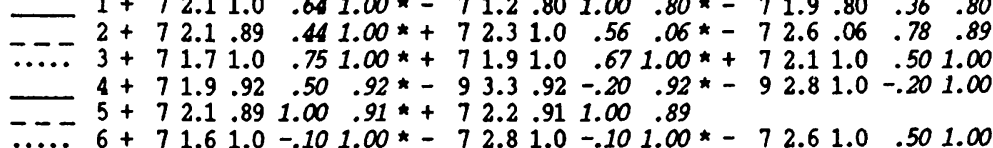

SIE-IMPERATIVSATZ, FOKUS: OBJEKT

55. Situation: In der Hotelkuche lst elne etwas ungeschickte Aushllfskraft elngestellt worden. Ein Koch zum Chefkoch: Koch: Dieser Manni kann ja kaum mit dem Messer umgehen! Was soll Ich ihn denn schnelden lassen? Chefkoch: Lassen Sle den Mann! dle BOHnen schnelden!

$(12---)$

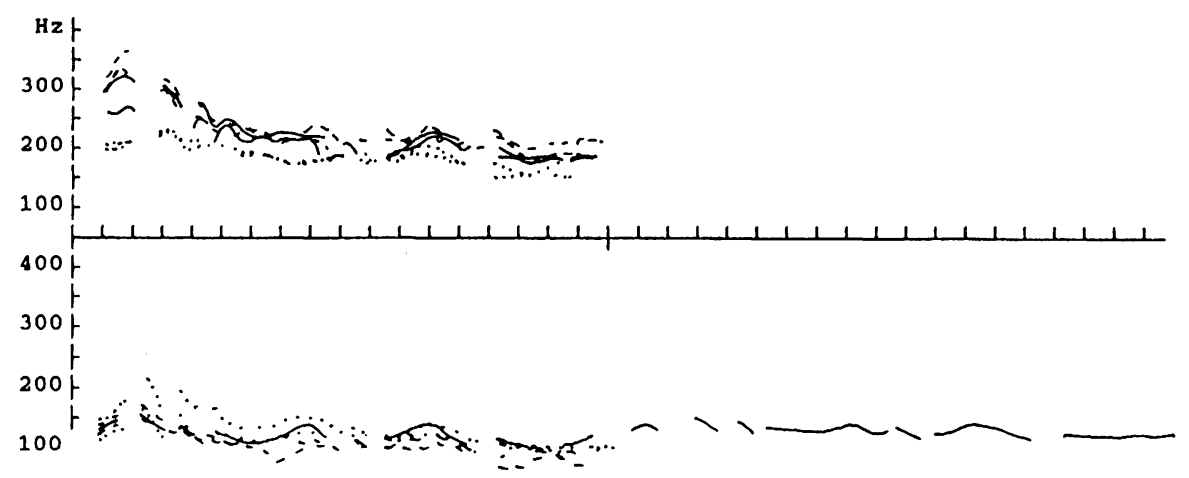

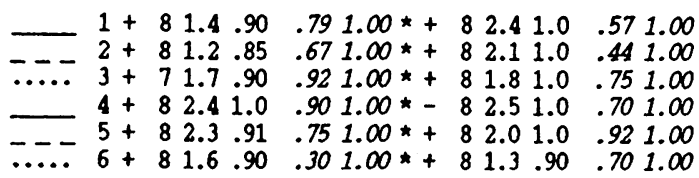


SIE-IMPERATIVSATZ, FOKUS: VERB

56. Situation: In der Hotelkuche ist elne etwas ungeschickte Aushllpskraft elngestellt worden. Ein Koch zum Chefkoch: Koch: Elgentlich könnte dieser Mannl Ja den Bohnensalat machen, aber ich traue seinen Fähigkelten nicht so recht. Was soll ich thn denn mit den Bohnen machen lassen? Chefkoch: Lassen Sle den Mannl die Bohnen SCHNEIden!

$(13---)$

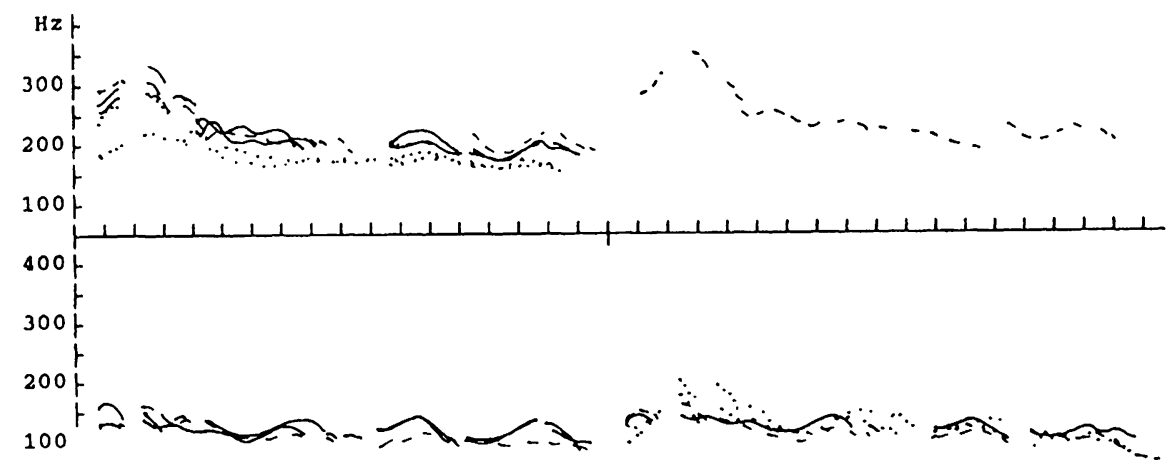

$1+101.81 .0-.291 .00 *+101.61 .0-.571 .00$

$-\begin{aligned} & 1 \\ & 2\end{aligned}+101.9 .82-.561 .00 *-101.2 .77-.671 .00$

$-\ldots . .3+102.0 .90-.081 .00 *+101.9 .90-.751 .00$

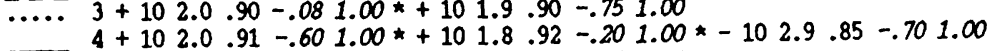

$-5+102.01 .0-1.01 .00 *-102.7 .82-1.01 .00$

$\ldots .-6-101.8 .50-.901 .00 * 101.6 .80-.701 .00$

SIE-IMPERATIVSATZ, FOKUS: OBJEKT + VERB

67. Situation: In der Hotelkuche ist elne neue Aushllfe elngestellt worden. Eln Koch zum Chepkoch: Koch: Was soll Ich den Mannl denn machen lassen? Chefkoch: Lassen Sle den Mannl die BOHnen schnelden!

$$
(11---)
$$

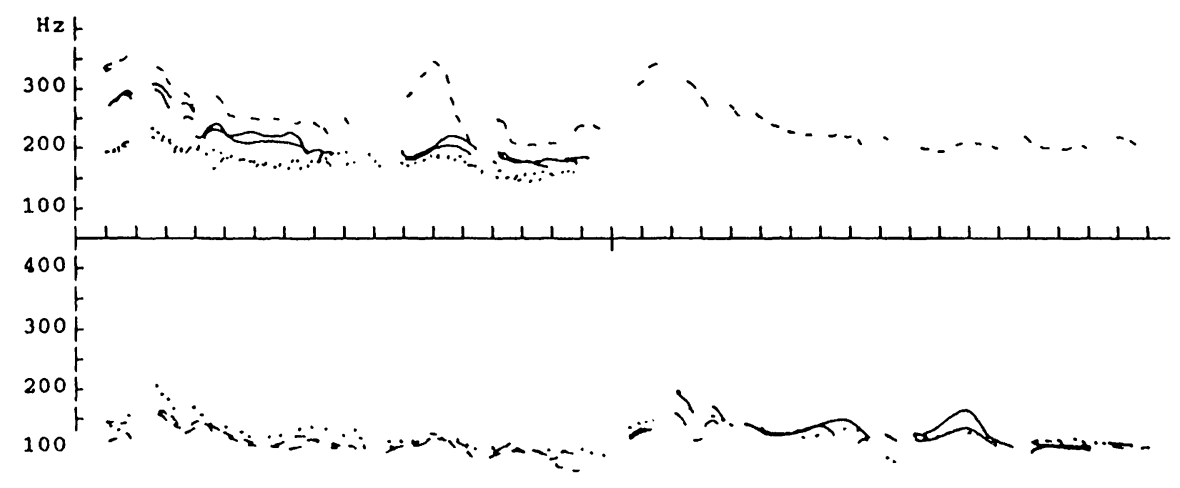

$1+81.5 .90 .711 .00 \star+81.71 .0 .711 .00$

$2+82.2 .83 .78 .62 \star-82.7 .541 .001 .00$

$\ldots . .3+81.71 .0 .671 .00 *+82.01 .0 .831 .00$

$\begin{array}{r}4-82.6 .85 .90 .92 \star-82.8 .92 .90 .92 \\ \hline\end{array}$

$\ldots-5+81.71 .0 .921 .00 *+51.81 .0 \quad .671 .00$

$\ldots . .6+81.3 .90 .671 .00 *-81.8 .80 .401 .00$ 
SIE-IMPERATIVSATZ, ZWEITEILIGER FOKUS: OBJEKT, VERB

58. Situation: In der Hotelkuche Ist elne etwas ungeschickte Aushllfskrapt eingestellt worden. Ein Koch zum Chefkoch: Koch: Der Mannl scheint mir nicht sehr geschickt. - Belm Gemüse kann wohl am wenigsten danebengehen. Was soll ich ihn denn mit welchem Gemüse machen lassen? Chefkoch: Lassen Sle den Manni die BOHnen SCHNEIden!

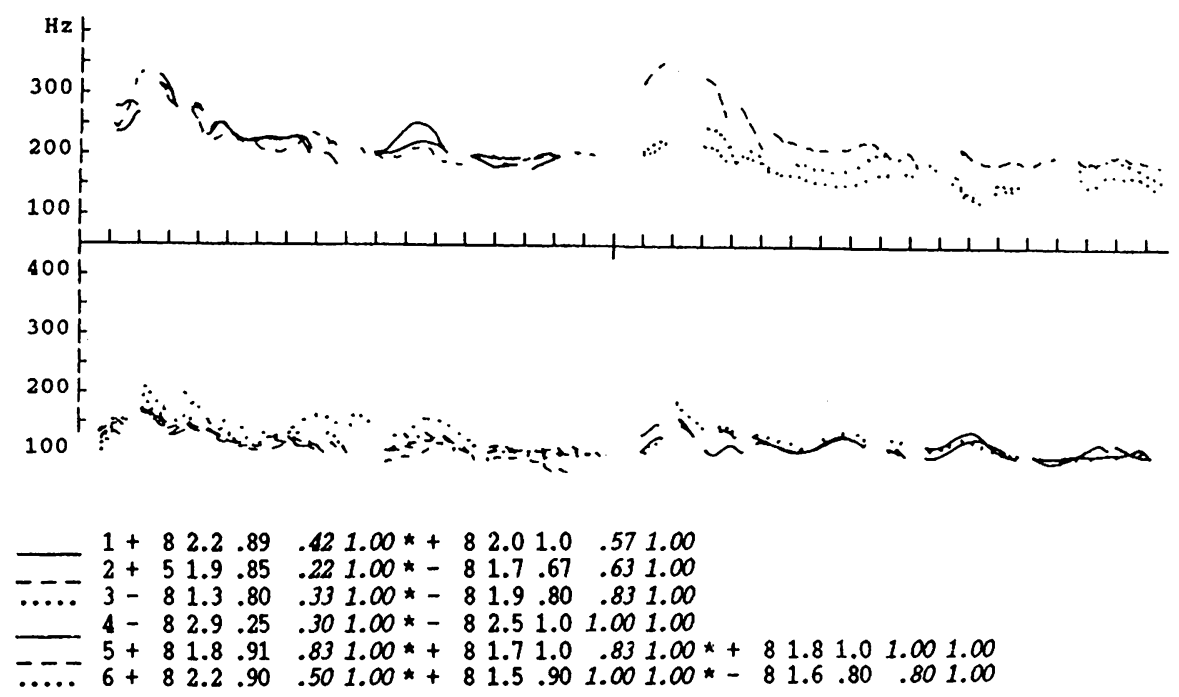

ENTSCHEIDUNGSFRAGESATZ, FOKUS: OBJEKT

59. Situation: Der Hotelbesitzer erkundigt sich in der Kuche belm Koch nach der Beschätlgung elner neuen Aushllfskrapt. Besitzer: Was lassen Sle unseren etwas ungeschlckten Jungen Freund Mannt denn schnelden, damit thm und uns am wenigsten passiert? Lassen Sle den Mannl die BOHnen schneiden? [Der Koch nickt.]
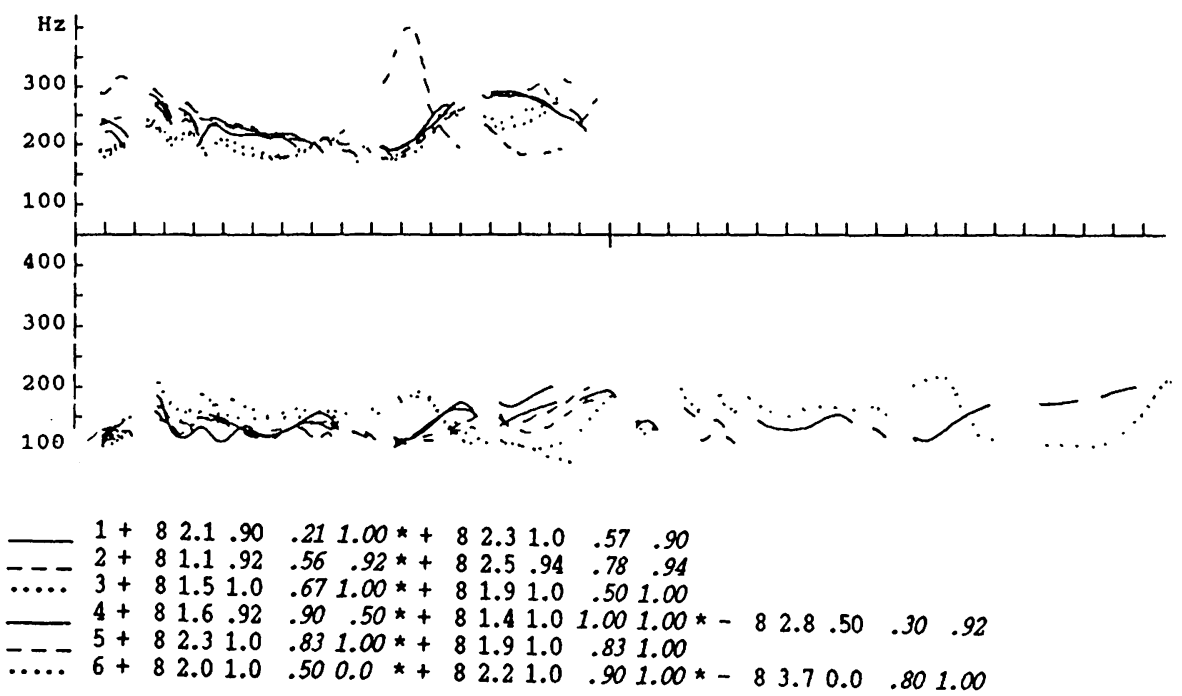
ENTSCHEIDUNGSFRAGESATZ, POKUS: VERB

60. Situation: Der Hotelbesitzer erkundigt sich in der Küche beim Koch nach der Beschäftigung einer neuen Aushilfskraft. Besitzer: Aha! Um den Bohnensalat soll sich der Manni ein wenig kümmern. Aber ich hoffe, er macht nur die vorbereitenden Arbeiten. Was lassen Sie ihn denn mit den Bohnen machen? Lassen Sie den Manni dle Bohnen SCHNEIden? (Der Koch nickt.)

$$
(12---)
$$

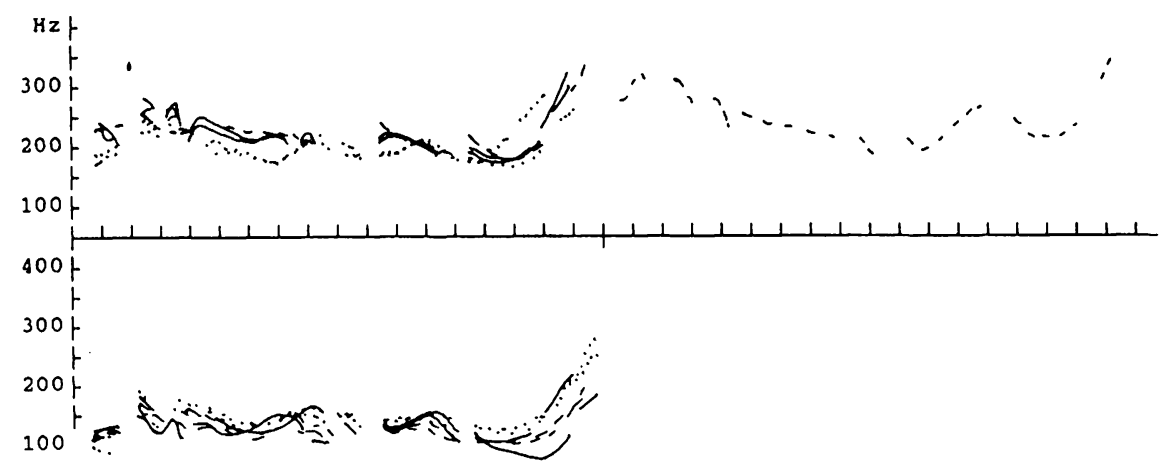

$1+101.51 .0-.861 .00 *+101.51 .0-.861 .00$

$2+101.11 .0-1.01 .00 *-103.51 .0-.331 .00$

$3+101.21 .0-.831 .00 *+102.11 .0-.751 .00$

$4+102.31 .0-.501 .00 *+101.81 .00 .001 .00$

$5+101.81 .0-1.01 .00 *+101.71 .0-1.01 .00$

$\ldots .-6+101.41 .0-1.01 .00 *+101.21 .0-.501 .00$

ENTSCHEIDUNGSPRAGESATZ, FOKUS: OBJEKT + VERB

61. Situation: In elner Hotelkuche schaut sich eine Mutter nach ihrem Sohn um, der vor kurzem als Lehrling zu arbelten angefangen hatte. Zum Koch: Mutter: Ich wollte nur mal nach meinem Sohn, dem Mannl, schauen. Was lassen Sie Ihn denn gerade machen? Lassen Sle den Manni dle BOHnen schneiden? IDer Koch nickt.]

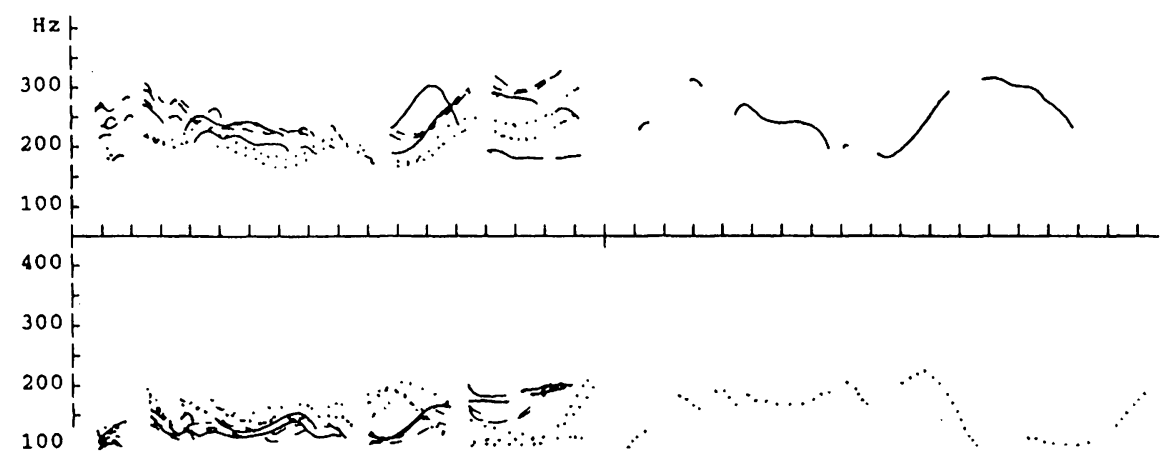

$$
\begin{array}{lllllllll}
1+8 & 1.7 .90 & .570 .0 *+82.0 .90 & .43 & .90 *-83.70 .0 & .85 & .90
\end{array}
$$$$
[-\ldots-3+81.8 .90 .501 .00 *+81.21 .0 .92 .90
$$$$
4+81.8 .92 .60 .92 *+81.61 .0 .801 .00
$$

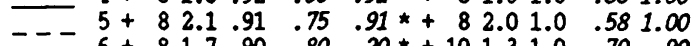

$\ldots . .6+81.7 .90 .80 .20 \star+101.31 .0 .70 .90 *+81.51 .0 .501 .00 \star-83.7 .20 .801 .00$ 
ENTSCHEIDUNGSFRAGESATZ, ZWEITEILIGER FOKUS: OBJEKT, VERB

62. Situation: Der Hotelbesitzer erkundigt sich in der Küche belm Koch nach der Beschätlgung elner neuen Aushllfskraft. Besitzer: Ich wollte nur mal nach unserem Neuen, dem Manni, schauen. Er sollte ja wohl für das Gemüse zuständig sein. Was lassen Sle ihn denn gerade mit welchem Gemüse machen? Lassen Sle den Mannl dle BOHnen SCHNEIden? (Der Koch nickt.)

$(123--)$

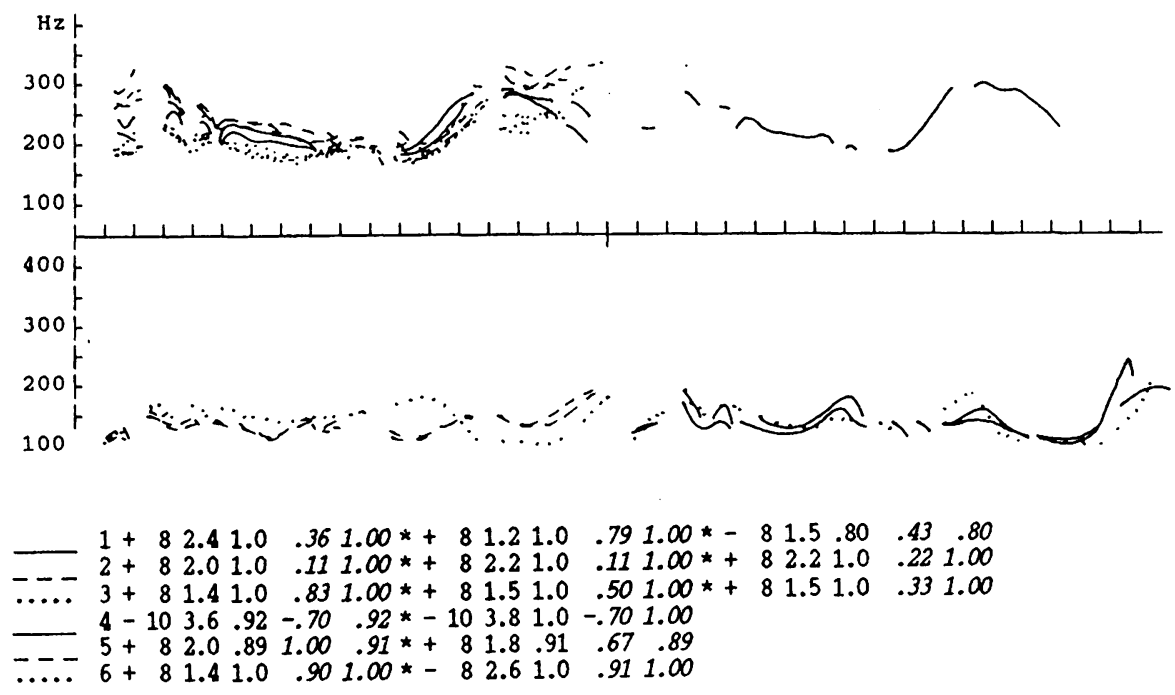

ADHORTATIVSATZ, FOKUS: OBJEKT

63. Situation: EIn Gartnermelster bespricht mit selnem Gehllfen, was sie den neuen Lehrling Leo arbelten lassen sollen. Meister: Der Leo könnte sich heute efgentlich mal mit dem Düngen beschaftigen. Was sollen wir thn denn düngen lassen? Gehilfe: Lassen wir den Leo die BLUmen düngen!

$$
(11-1-)
$$

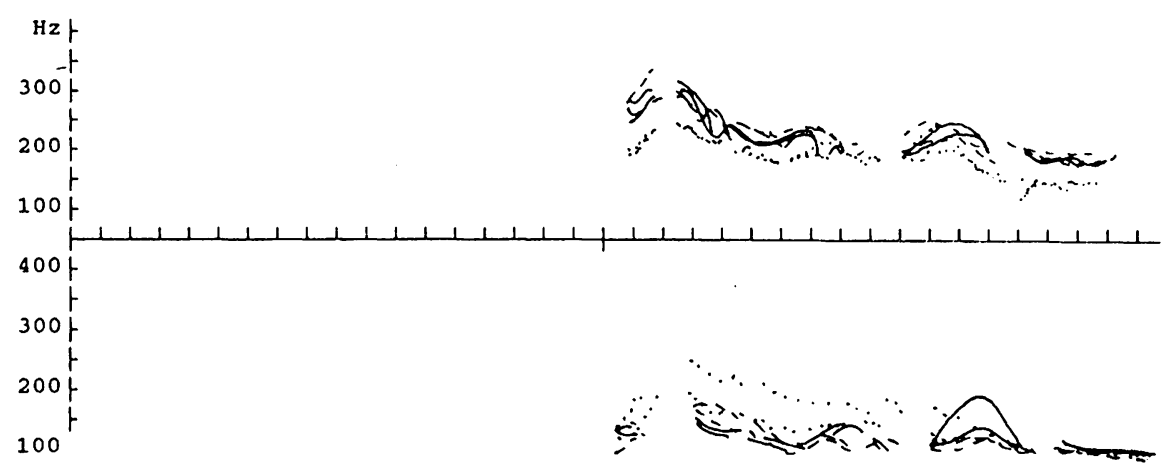

$1-81.6 .60 \quad .711 .00 *-11.6 .78 \quad .641 .00$

$-2-81.2 .27 .561 .00 *-81.7 .41 .891 .00$

$\ldots . .3-81.6 .40 .921 .00 *-81.8 .50 .921 .00$

$\begin{array}{r}4-83.7 .331 .00 .33 *-82.5 .42 .701 .00 \\ \hline\end{array}$

- $5-81.6 .451 .001 .00 *-81.6 .75 .83 .87$

$\ldots .6-81.8 .40 .70 .90 *-81.7 .50 .901 .00$ 
ADHORTATIVSATZ, POKUS: VERB

64. Situation: Ein Gärtnermeister bespricht mit seinem Gehilfen, was sie den neuen Lehrling Leo arbeiten lassen sollen. Melster: Bisher haben wir den Leo ja fast nur beim Gemüse beschätigt. Ich finde, er sollte auch einmal zu den Blumen. Was sollen wir den Leo denn mit den Blumen machen lassen? Gehilfe: Lassen wir den Leo die Blumen DuNgen!

$$
(14---)
$$

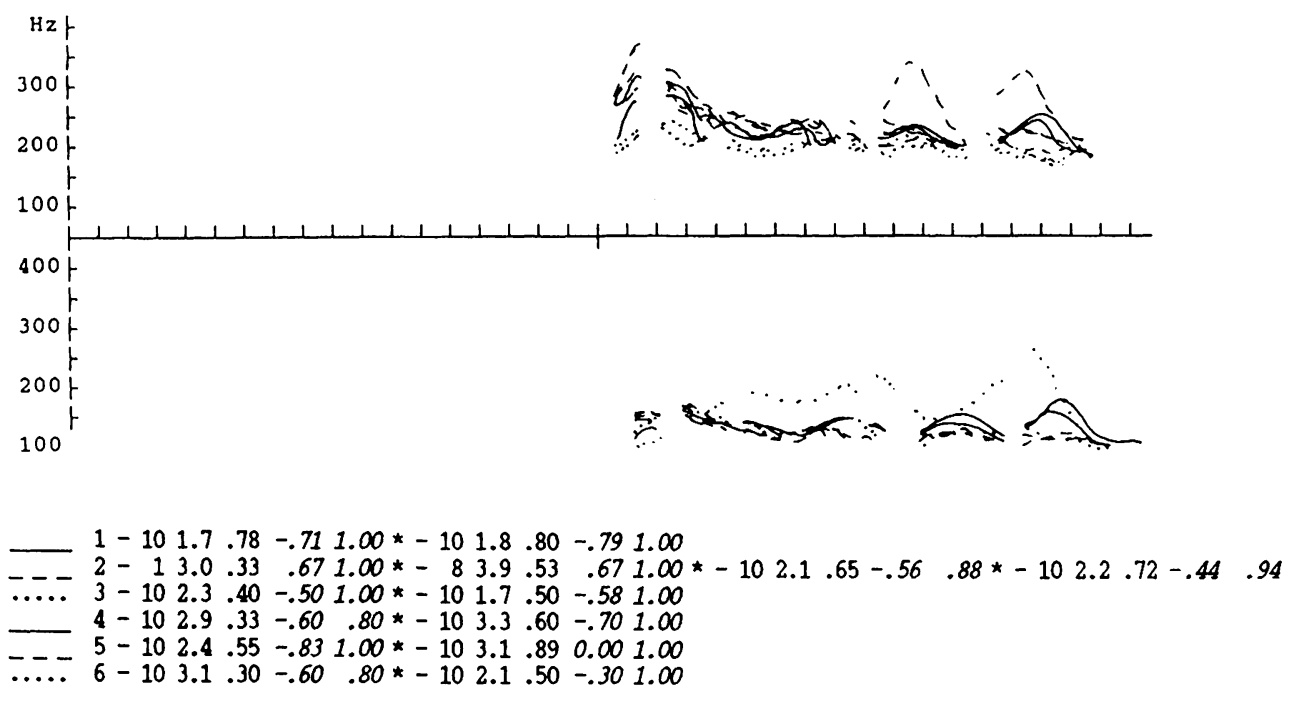

ADHORTATIVSATZ, FOKUS: OBJEKT + VERB

65. Situation: Vater zu Mutter über den Sohn. Vater: Die Ferien dauern viel zu lange. Unser Fillus beginnt sich schon zu langweilen. Was sollen wir den Leo denn machen lassen? Mutter: Lassen wir den Leo dle BLUmen düngen!

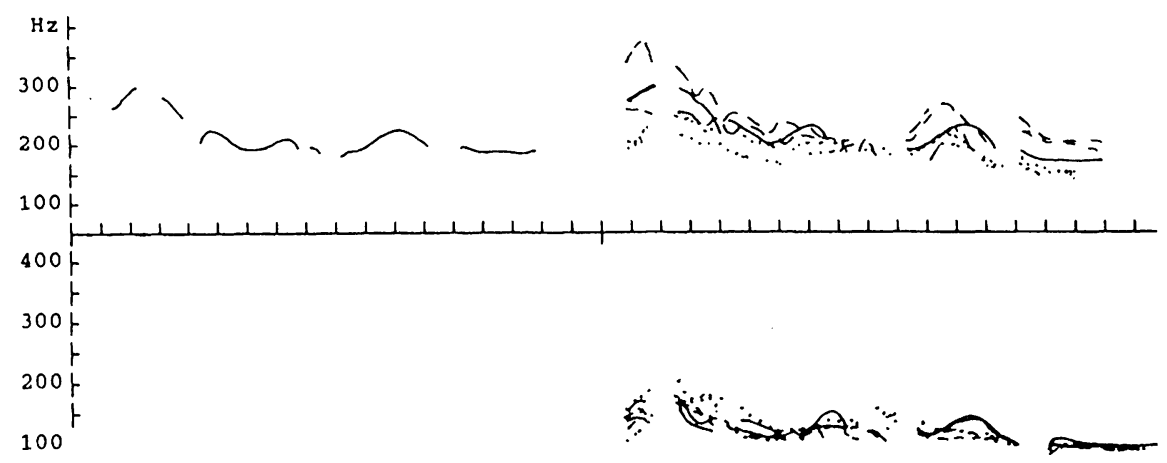

$1+82.01 .0 .641 .00 *-11.6 .70 .641 .00$

$2-82.1 .25 \quad .89 .85 \star-82.7 .621 .001 .00$

$3-82.6 .44 .831 .00 *-82.3 .44 .831 .00$

$4-83.1 .36 .601 .00 *-82.5 .58 .901 .00$

$5-82.1 .561 .001 .00 *-82.6 .82 .581 .00$

$\ldots .6-82.0 .25 .901 .00 *-82.2 .501 .001 .00$ 
ADHORTATIVSATZ, ZWEITEILIGER FOKUS: OBJEKT, VERB

66. Situation: Ein Gärtnermeister bespricht mit seinem Gehilfen, was sie den neuen Lehrling Leo arbelten lassen sollen. Meister: Was sollen wir den Leo mit welchen Pflanzen machen lassen? Gehilfe: Lassen wir den leo die BL,I/men DúNgen!

$(18---)$
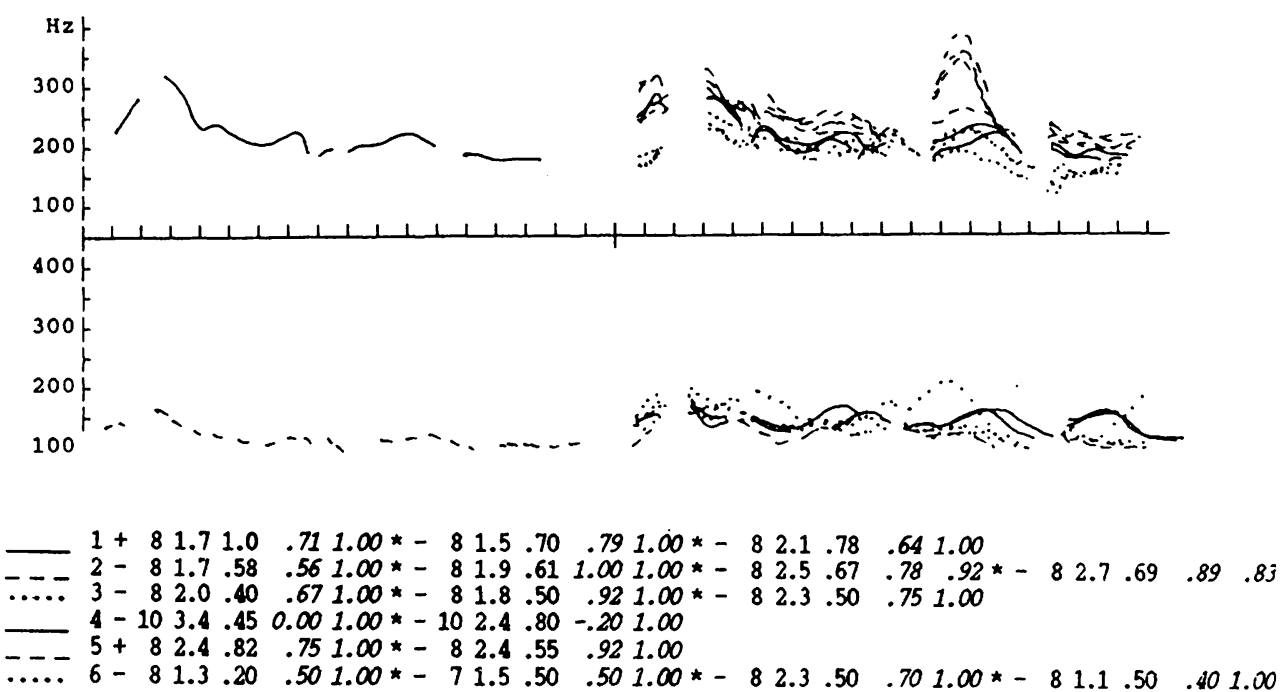

ADHORTATIVSATZ, ZWEITEILIGER FOKUS: OBJEKT, VERB

67. Situation: Eln Gärtnergehllfe $\mathrm{zu}$ selnem Meister über den Lehrling Leo: Gehilfe: Sollen wir den Leo die Bäume wässern lassen? Meister: Nein, nicht die Baume und auch nicht bloß gießen. Lassen wir den Leo die BLUmen DUNgen!

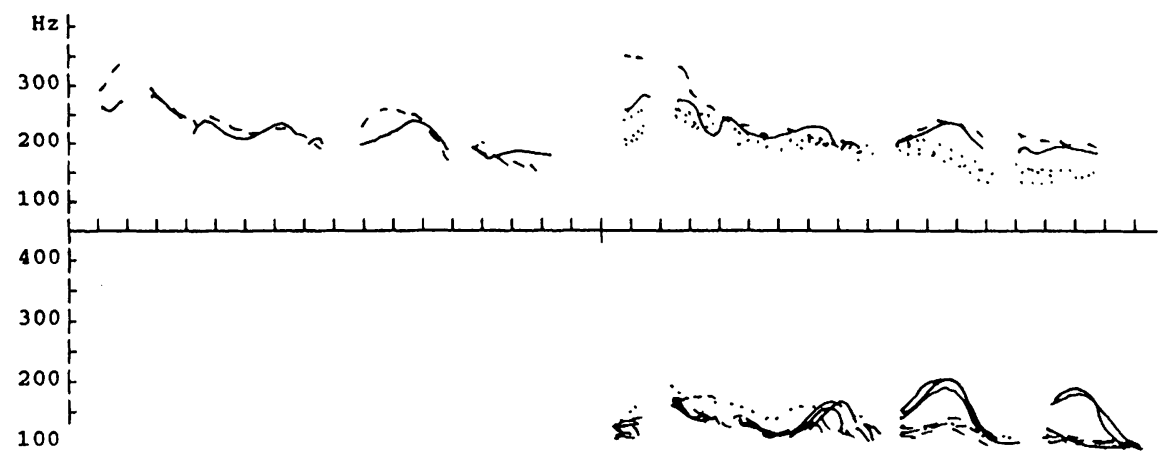

$1+82.4 .89 .691 .00 *-82.5 .90 .711 .00$

$--2+82.0 .85 .221 .00 *-82.4 .69 .891 .00$

$\ldots .3-81.7 .40 .831 .00 *-82.0 .60 .671 .00 *-82.1 .70 .671 .00$

$-4-83.60 .0 .40 .17 *-104.1 .31-.40 .54 *-82.7 .33 .90 .92$

- $-5-82.1 .781 .001 .00 *-82.0 .780 .001 .00 *-82.6 .80 .921 .00$

$-\ldots-6-81.7 .601 .001 .00$ 
ENTSCHEIDUNGSFRAGESATZ, FOKUS: OBJEKT

68. Situation: In einer Gärtnerel; ein Gärtner erkundigt sich beim Besitzer, wie sie den neu elngestellten Lehrling beschäftigen sollen. Gärtner: Gut, mit dem Düngen soll sich der Leo beschäftlgen. Aber was sollen wir thn düngen lassen? Lassen wir den Leo die BLUmen düngen? [Der Besitzer nickt.]

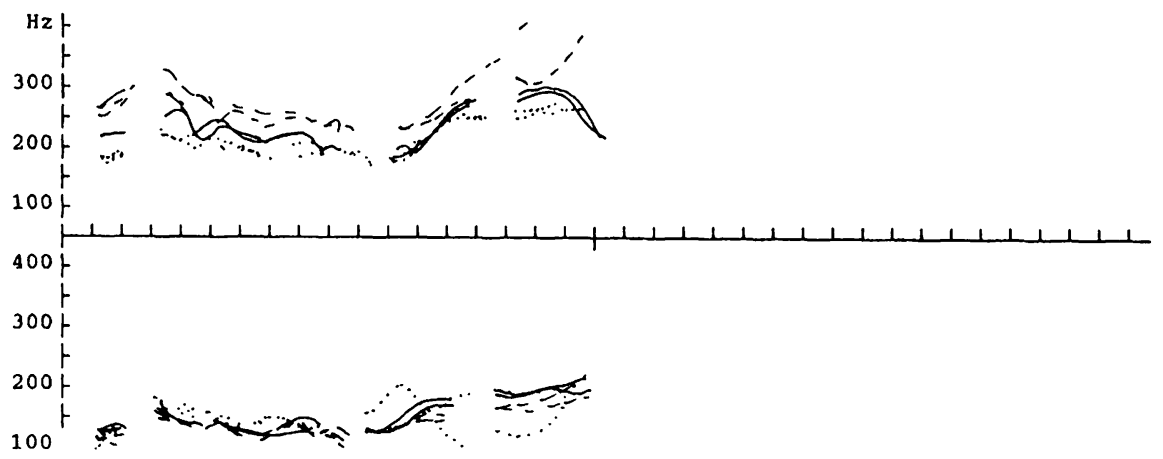

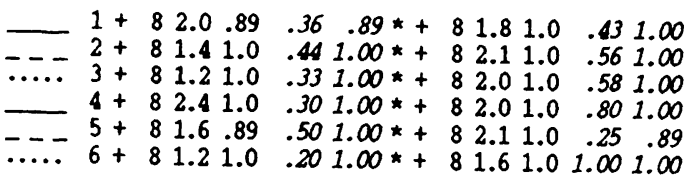

ENTSCHEIDUNGSPRAGESATZ, FOKUS: VERB

69. Situation: In elner Gärtnerel; eln Gärtner erkundigt slch beim Besitzer, was sle dem neuen Lehrling Leo für Aufträge geben sollen. Gärtner: Wir haben dem Leo jetzt die Blumen zum Umtopfen gegeben. Was sollen wir ihn denn noch damit machen lassen? Lassen wir den Leo die Blumen DUNgen? Besitzer: Ja, das soll er auch noch machen.

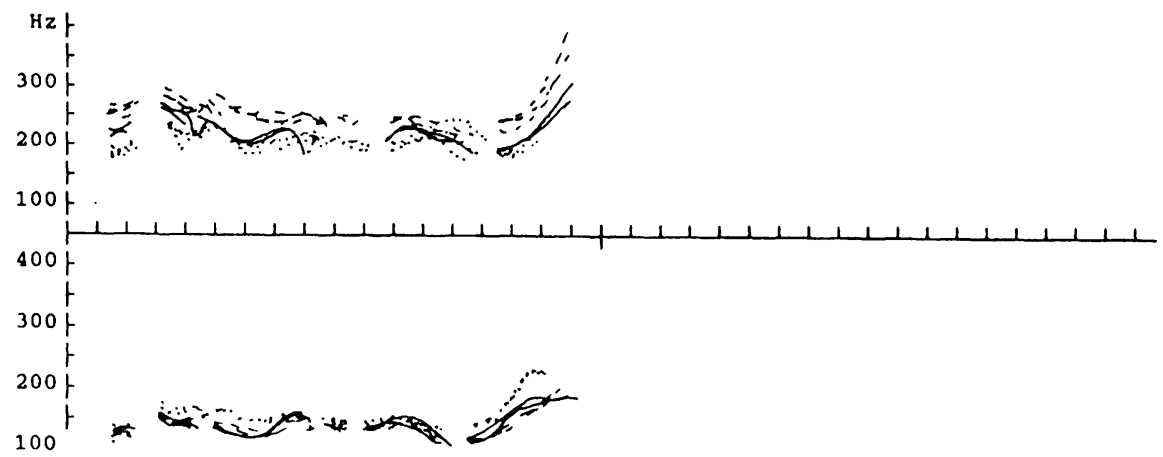

$1+102.11 .0 .641 .00 *+101.61 .0-.861 .00$

$2+101.31 .0-.501 .00 *+102.01 .0-.781 .00 *+101.81 .0-.781 .00$

$\ldots-.-3+101.61 .0-.501 .00 \star+101.81 .0-.671 .00 *+101.91 .0-1.01 .00$

$4+102.31 .0-.301 .00 *+102.01 .0-.601 .00$

$5+101.81 .0-.831 .00 *+101.71 .0-1.01 .00$

$\ldots . .6+101.31 .0-.801 .00 *+101.51 .0-.901 .00$ 
ENTSCHEIDUNGSFRAGESATZ, FOKUS: OBJEKT + VERB

70. Situation: Ein Gärtnergehilfe zu seinem Meister: Gehilfe: Die Arbeit für den Nachmittag müßte noch verteilt werden. Was lassen wir denn den Leo machen? Lassen wir den Leo die BLUmen düngen? (Der Meister nickt.)

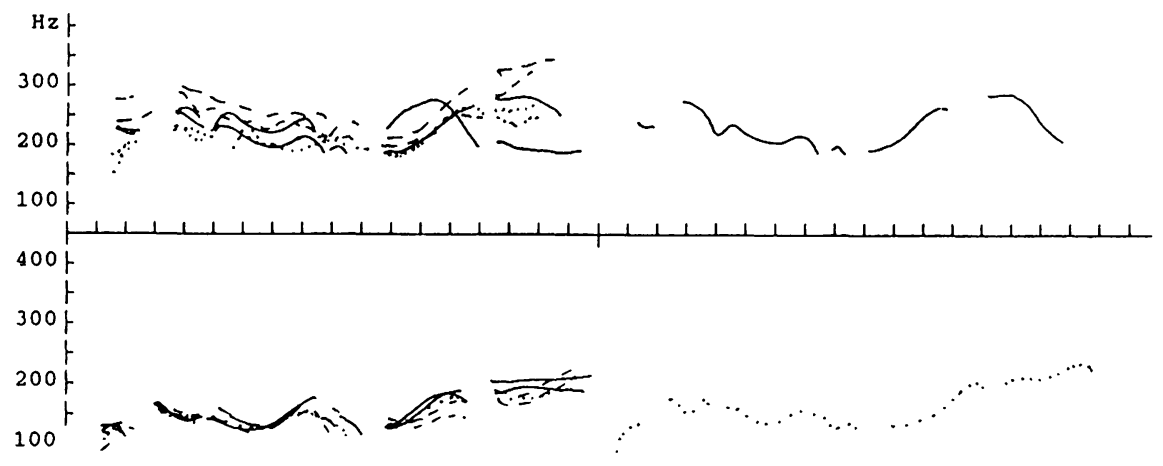

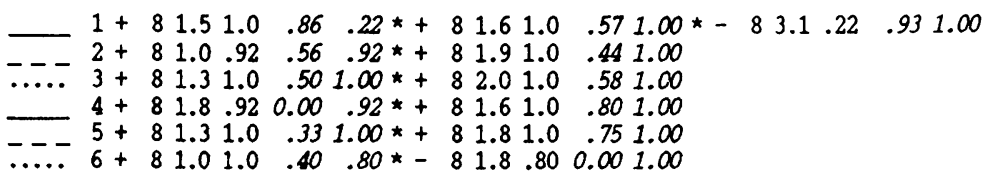

\section{ENTSCHEIDUNGSFRAGESATZ, ZWEITEILIGER FOKUS: OBJEKT, VERB}

71. Situation: Eln Gärtner erkundigt sich beim Besitzer der Gärtnerei, was der neue Lehrling Leo am nächsten Tag arbelten soll. Gärtner: Beim Gemüse kennt der Leo alle notwendigen Arbeiten und auch mit den obstbäumen kennt er sich schon aus. Was sollen wir ihn denn morgen mit welchen Pflanzen machen lassen? Lassen wir den Leo die BLUmen DÜNgen? IDer Besitzer nickt.]

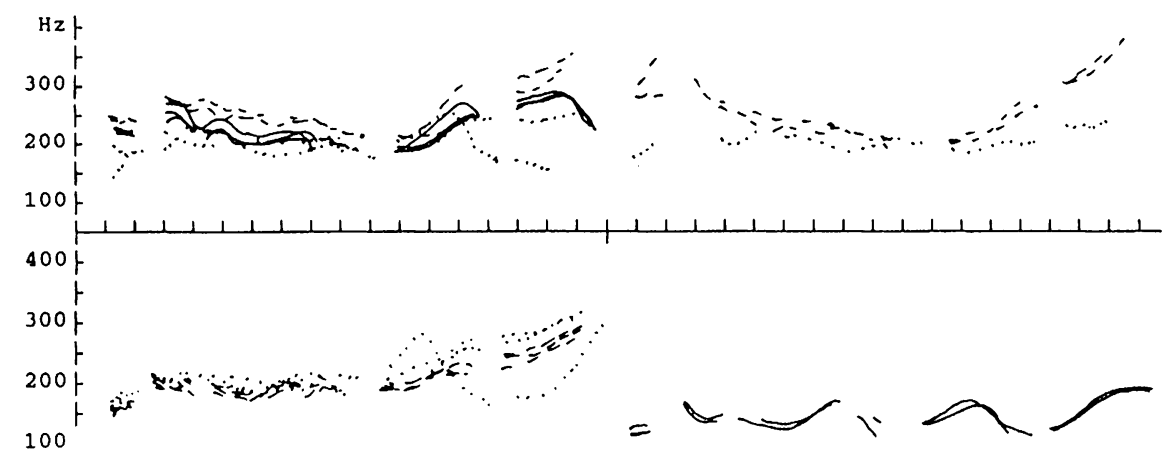

$1+82.3 .89 .641 .00 *+82.11 .0 .431 .00 *+81.51 .0 .86 \quad .89$

$\ldots-2+81.21 .0 .671 .00 *+82.41 .0 .331 .00 *-82.5 .920 .00 .92 *-82.51 .0 .221 .00$

$\ldots . .3+81.61 .0 .42 .10 \star+81.71 .0 .251 .00 \star-83.2 .101 .001 .00$ $4-104.11 .0-.701 .00 *-103.01 .00 .001 .00$

$5+81.9 .82 .751 .00 *+82.0 .89 .50 .89 *+82.01 .01 .00 \quad .82$

$\ldots \ldots 6+81.41 .0-.301 .00 *+81.31 .0 .401 .00 *+81.91 .0 .601 .00$ 
10. Korpus IV

Das dritte Teilkorpus (Nr. 72-84) ist das sogenannte Leo-Korpus. Beim Verb-Erst-Satz Säuft der Leo und beim Verb-Zweit-Satz Der Leo säuft wurden systematisch Satzmodus und Hauptakzent variiert. Absicht dieses Teilkorpus war es, natürliche Produktionsdaten als Kontrolle zu manipulierten Stimuli derselben segmentalen Struktur zu bekommen, die für eine ganze Reihe von Perzeptionstests generiert wurden (vgl. Nöth et al. 1987, Batliner 1989b).

Die Darstellung in den Abbildungen ist analog $z u$ der bei Korpus III, mit einer Ausnahme: Da der dem Wert in Spalte 7 bei Korpus III entsprechende Wert nicht erhoben wurde, steht in Spalte 7 die Richtigkeit der Frage/Nicht-Frageklassifizierung, und in Spalte 8 der Stern als Trennzeichen. 
72. Ihr fragt mich, wer säuft? Der LEO säuft. $(12---)$

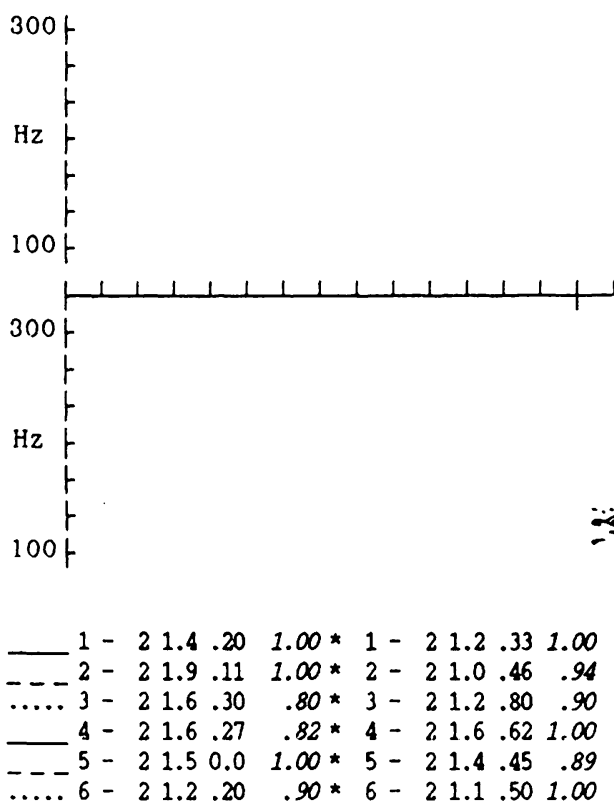

73. Was soll schon mit dem Leo sein? Der Leo SAIJFT. $(104--)$
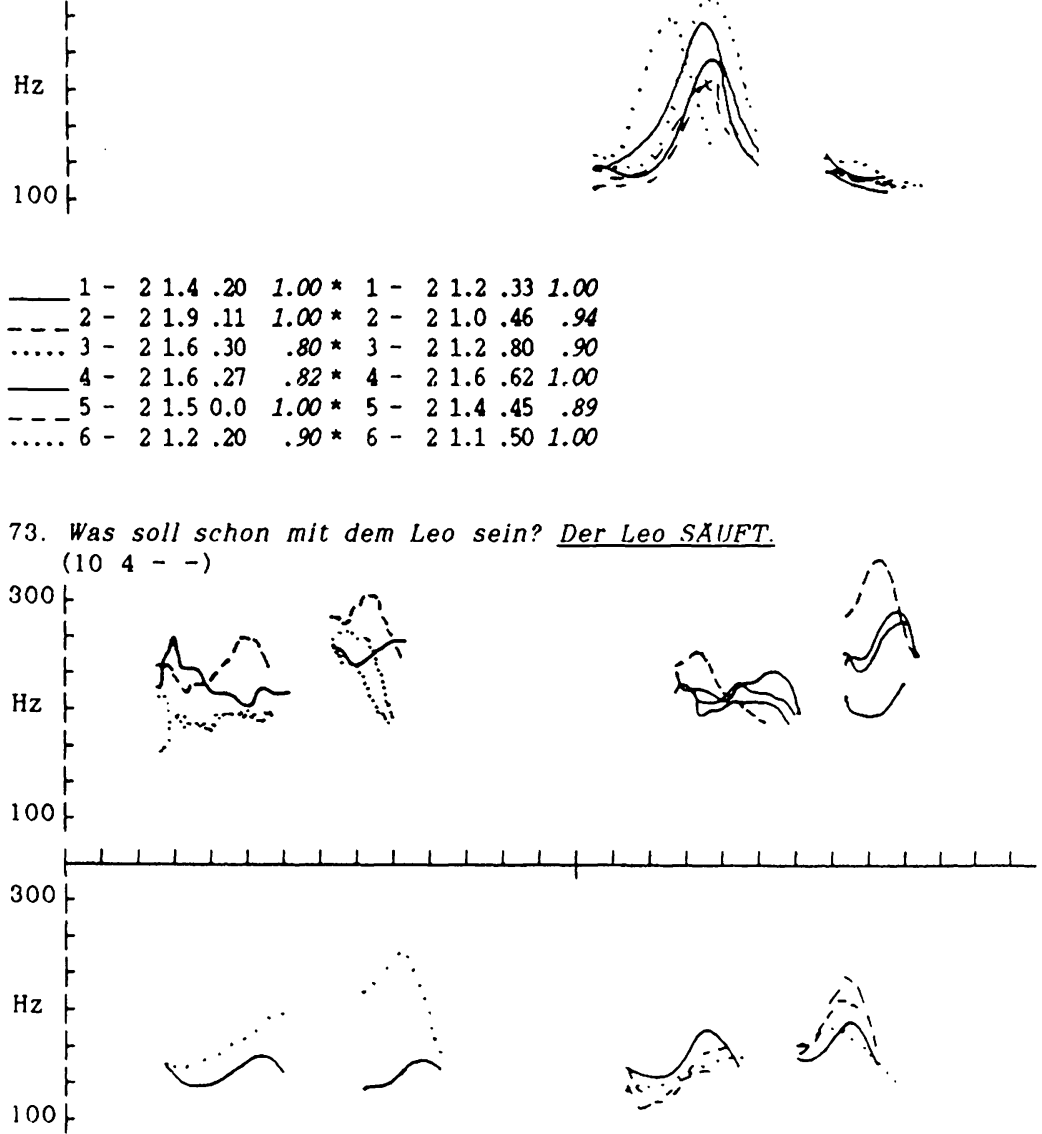

$1+41.91 .01 .00 *-41.7 .331 .00 *-43.0 .671 .00 *-41.8 .801 .00$

$2+41.4 .921 .00 *-42.5 .781 .00$

$\ldots . .3+42.0 .901 .00 *+41.31 .0 .90$

$4+42.41 .01 .00 *-42.5 .77 .92$

$5-41.50 .0 .73 *-41.8 .45 .67$

$\ldots .-5+41.4 .90 .90 *-41.4 .801 .00$ 


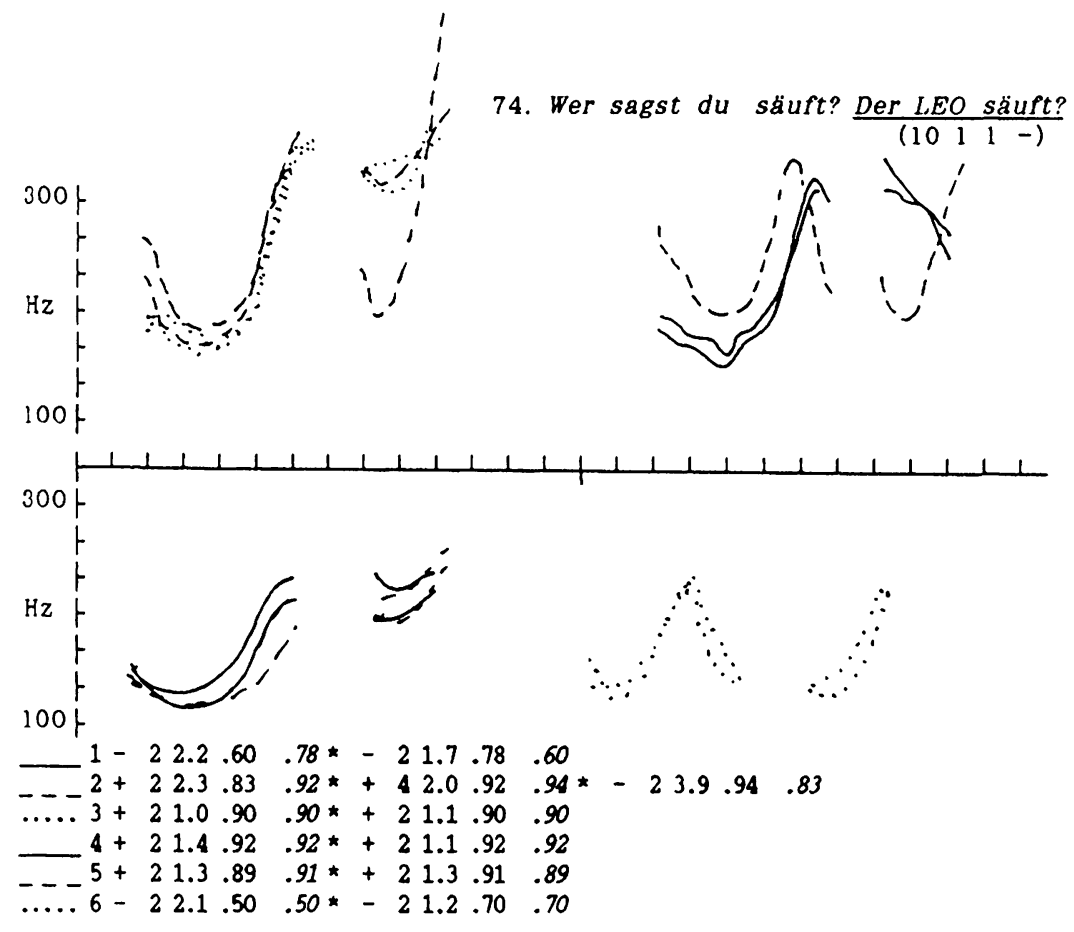

75. Stimmt das mit dem Leo? Der LeO SAUFT?

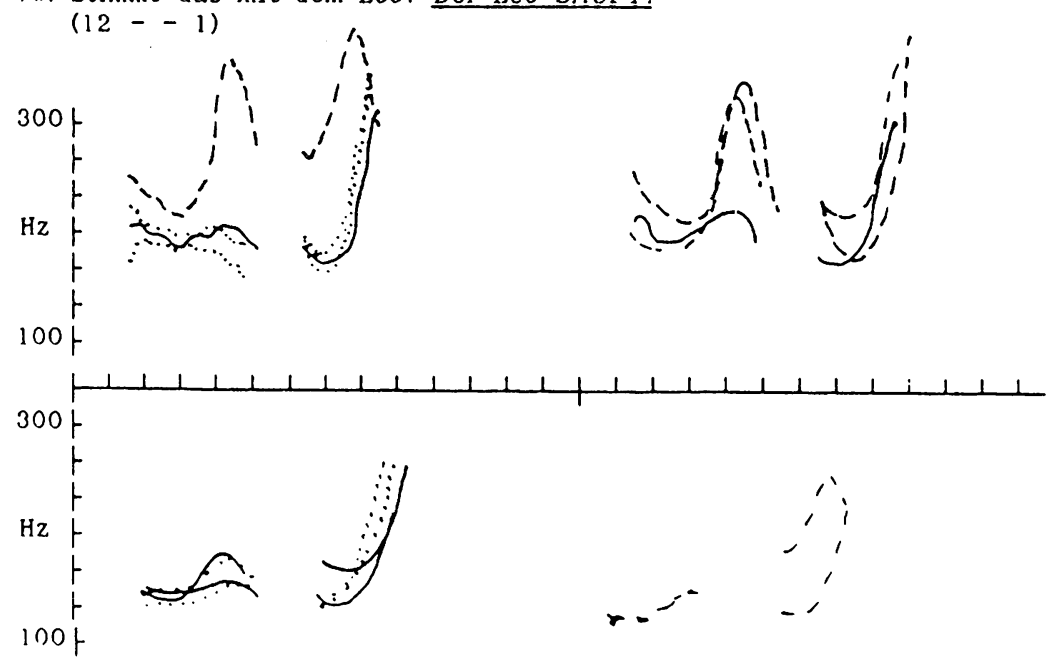

$1+41.51 .01 .00 *-41.0 .80 .80$

$--2+42.0 .89 .23 *-42.8 .23 .67 *-42.0 .67 .89$

$\ldots . .3+41.4 .901 .00 *+41.41 .0 .90$

$4+41.31 .01 .00 *+41.91 .01 .00$

$-5-42.0 .671 .00 *-42.51 .0 \quad .67$

$\ldots .6+41.11 .01 .00 *+41.11 .01 .00$ 
76. Der Theo säuft nicht, der LEO säuft! (10 - - -)

$\mathrm{Hz}$
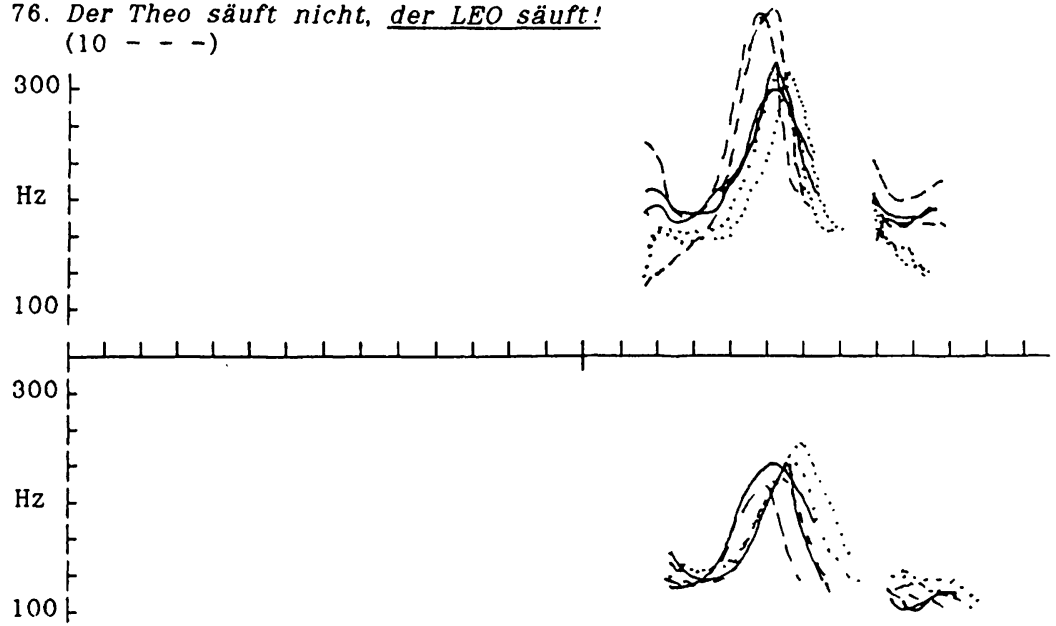

$1-21.20 .01 .00$ * -21.5 .301 .00$

$-\ldots 2-21.9 .351 .00 *-22.2 .621 .00$

$\ldots . .3-21.6 .201 .00 *-21.0 .401 .00$

$4-21.6 .621 .00 *-21.8 .801 .00$

$-5-21.3 .551 .00 *-22.1 .571 .00$

$\ldots .66-21.3 .501 .00 *-21.2 .601 .00$

77. Der Leo trinkt nicht, der Leo SAUFT!

$$
\text { (10 - - ) }
$$

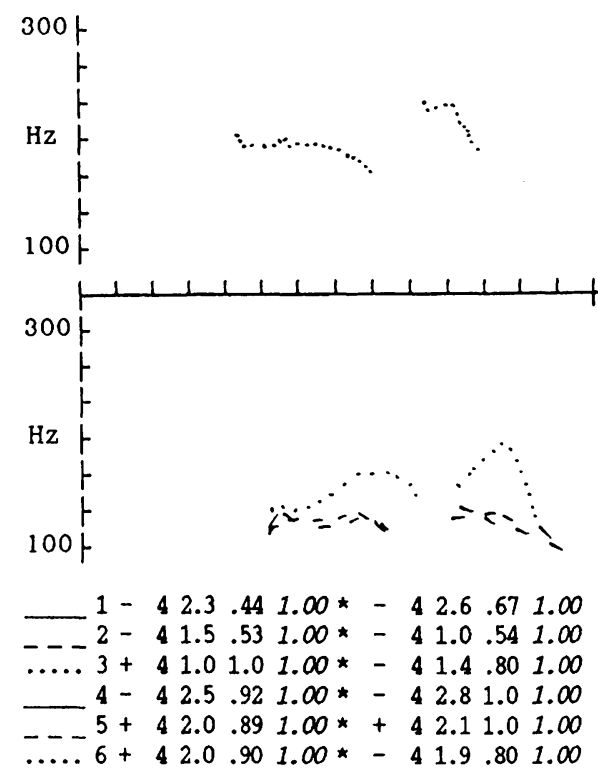


78. Gestern waren wir im Wirtshaus. Mann - der Leo SAUFT! $\left(\begin{array}{lll}14 & 1 & -\end{array}\right)$

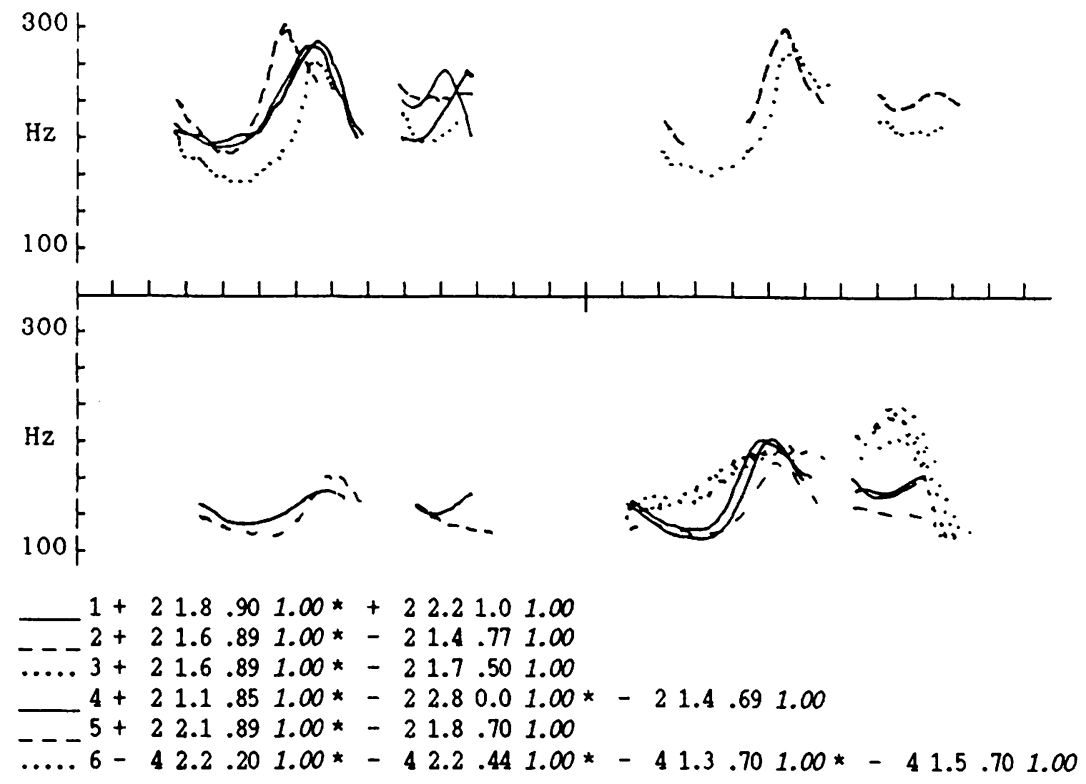

79. Gestern war ich mit dem Leo im Wirtshaus. Mann - der Leo SAUFT!

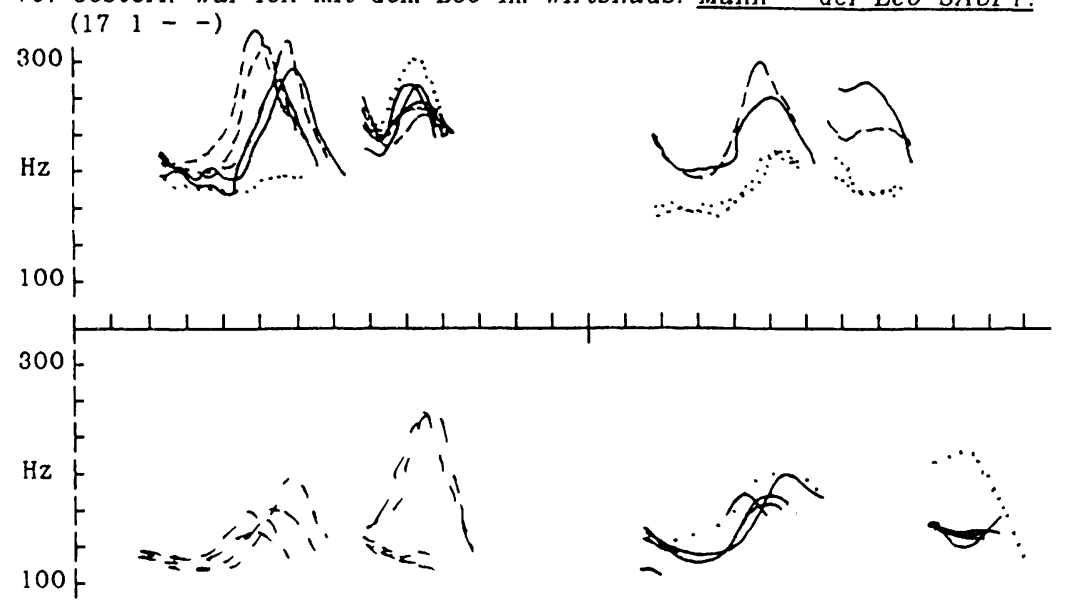

$1+22.3 .901 .00 *+42.31 .01 .00 *-43.1 .671 .00$

$-\ldots+22.1 .831 .00 \star+21.9 .941 .00 \star+22.11 .01 .00 *-22.1 .771 .00$

$\ldots . .3+41.91 .01 .00 *-22.5 .201 .00 *-22.6 .301 .00$

$4-2 . .0 *-21.9 .461 .00 *-21.8 .671 .00 *-21.9 .691 .00$

$-{ }_{-}+21.3 .891 .00 \star+41.7 .911 .00 *+41.91 .01 .00 \star+41.91 .01 .00$

$\ldots .6-41.4 .701 .00$ 

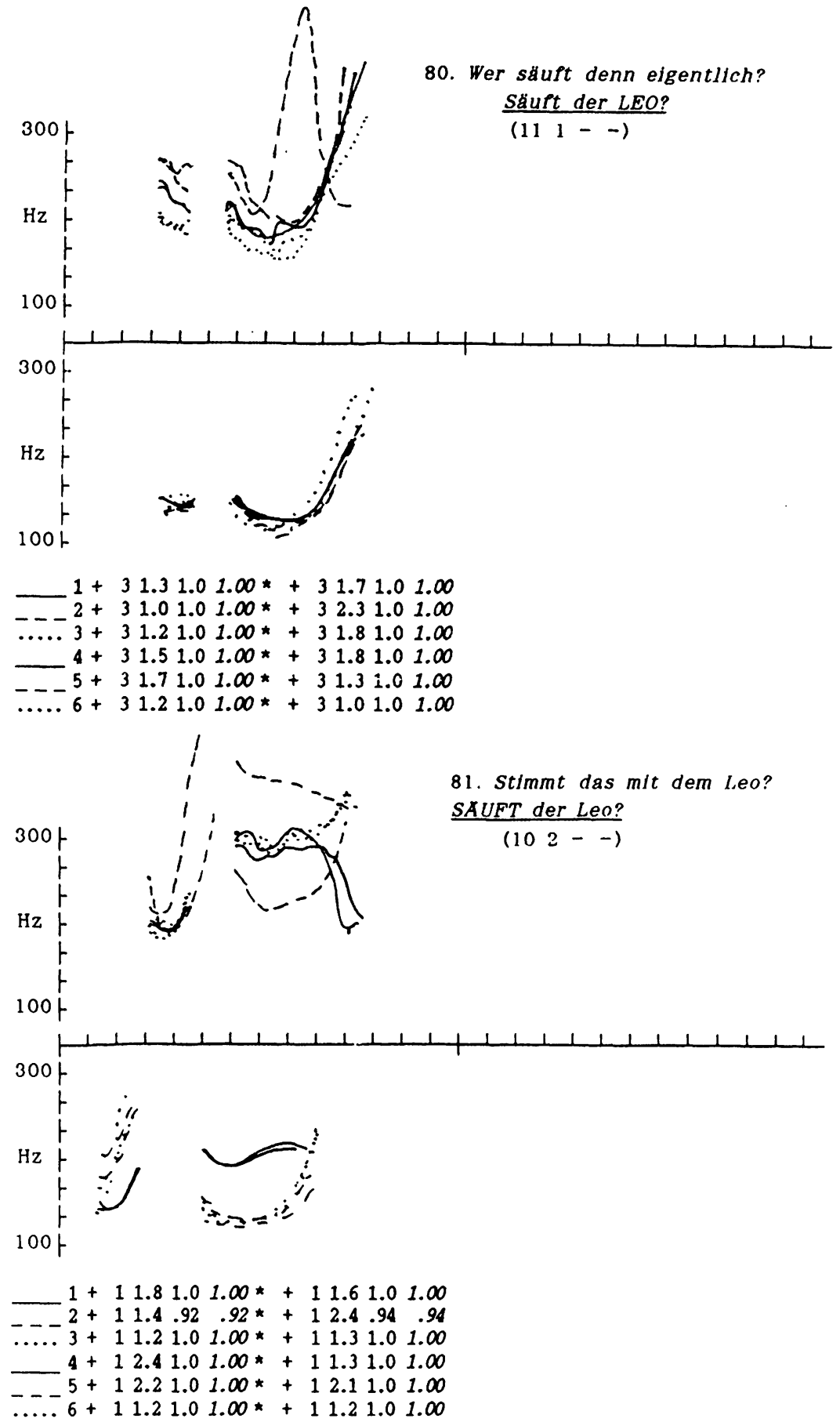
82. Gestern waren wir Im Wirtshaus. Mann - Säuft der LEO! $(16-1-)$

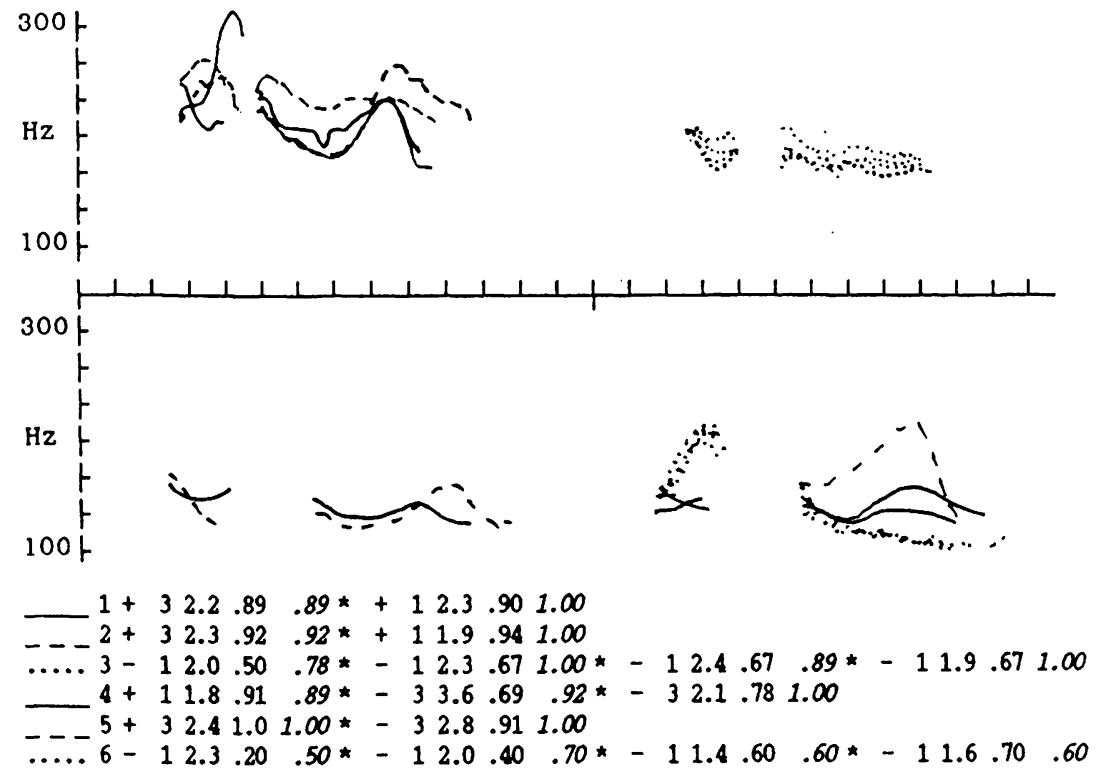

83. Gestern war ich mit dem Leo im Wirtshaus. Mann - SAUFT der Leo! $(14-2-)$

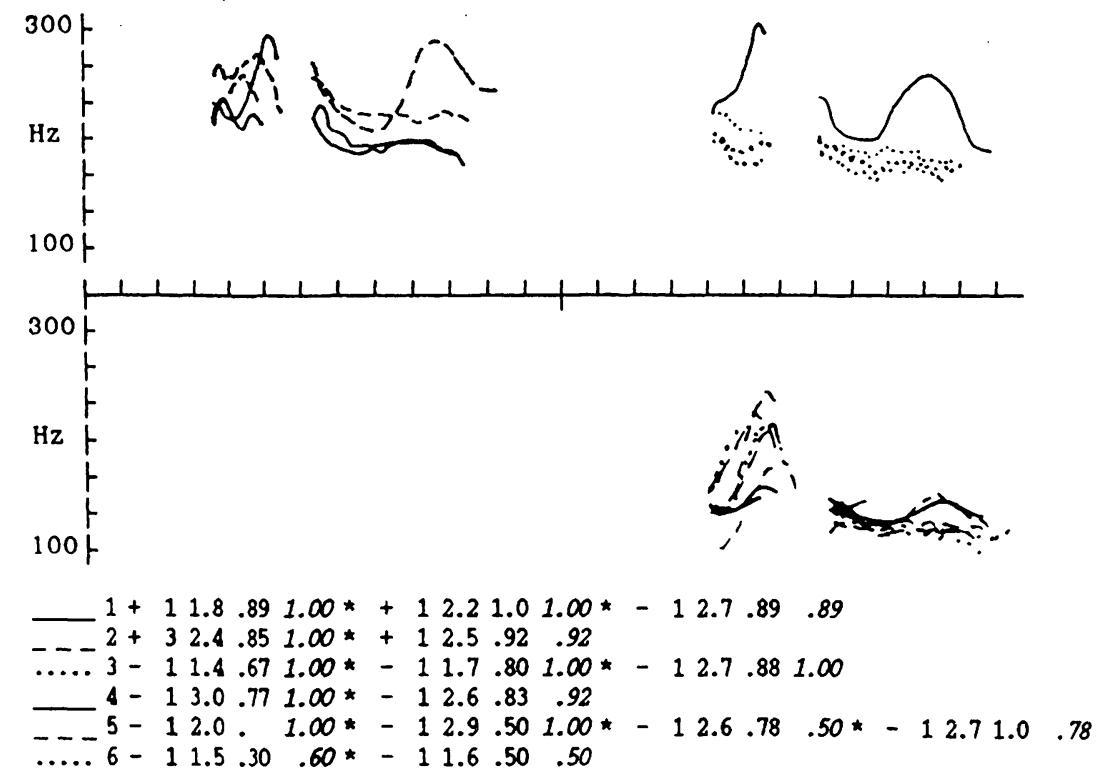


84. Also sowas hätt' ich nicht gedacht! SAUFT der Leo! $(8-5-)$

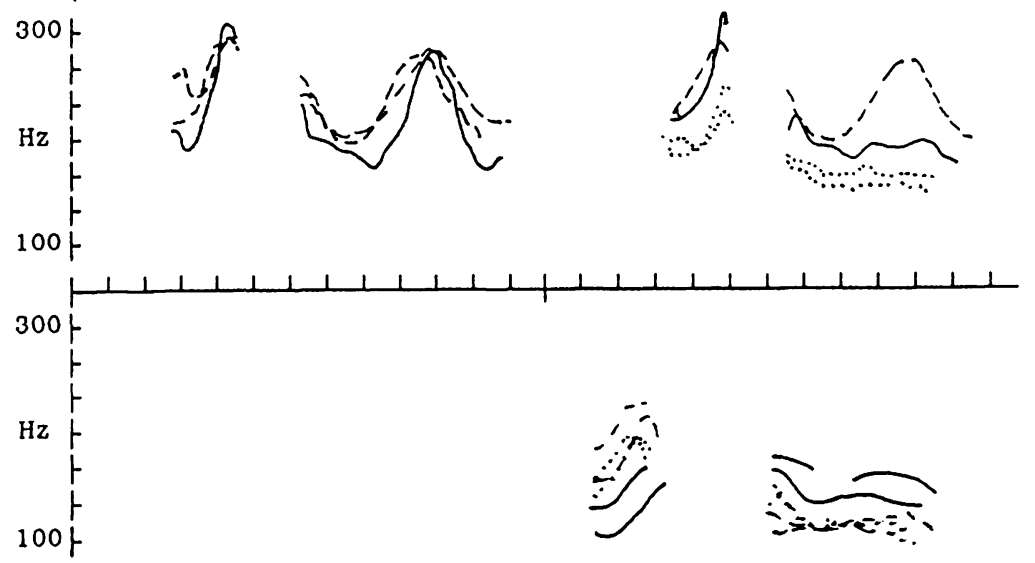

$1+12.11 .01 .00 *-11.8 .60 .70$

$--2+12.0 .891 .00 *+12.31 .01 .00 *-13.31 .0 .94$

$\ldots . .3-12.0 . .80 *-11.8 .80 .80$

$4-14.3 .33 .55 *-12.6 .45 .33$

$-5-14.10 .0 .18 *-13.0 .180 .0$

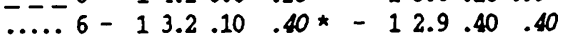




\section{LITERATUR}

Altmann, H. (1984): Linguistische Aspekte der Intonation am Beispiel Satzmodus. In: Forschungsberichte des Instituts für Phonetik und Sprachliche Kommunikation der Universität München (FIPKM) 19. S.132-152.

Altmann, H. (1987): Zur Problematik der Konstitution von Satzmodi als Formtypen. In: Meibauer, J. (Hg.) (1987): Satzmodus zwischen Grammatik und Pragmatik. Tübingen. S.22-56.

Altmann, H. (Hg.) (1988): Intonationsforschungen. Tübingen.

Batliner, A. (1988): Produktion und Prädiktion. Die Rolle intonatorischer und anderer Merkmale bei der Bestimmung des Satzmodus. In: Altmann, H. (Hg.) (1988). S.207-221.

Batliner, A. (1989a): Fokus, Modus und die große Zahl. Zur intonatorischen Indizierung des Fokus im Deutschen. (In diesem Band)

Batliner, A. (1989b). Wieviel Halbtöne braucht die Frage? Merkmale, Dimsisionen, Kategorien. (In diesem Band)

Batliner, A. (1989c). Ein einfaches Modell der Frageintonation und seine Folgen. (in Vorbereitung)

Batliner, A./Nöth, E. (1989). The prediction of focus. Proceedings of the European Conference on Speech Communication and Technology, Paris, 2628 September 1989. (Im Druck)

Batliner, A./Nöth, E./Lang, R./Stallwitz, G. (1989): Zur Klassifikation von Fragen und Nicht-Fragen anhand intonatorischer Merkmale. (Erscheint in: Tagungsband der 15. Gemeinschaftstagung der Deutschen Arbeitsgemeinschaft für Akustik (DAGA), 13.-16. März 1989.)

Luukko-Vinchenzo, L. (1988): Formen von Fragen und Funktionen von Fragesätzen. Eine deutsch-finnische kontrastive Studie unter besonderer Berücksichtigung der Intonation. Tübingen.

Oppenrieder, W. (1988a): Intonation und Identifikation. Kategorisierungstests zur kontextfreien Identifikation von Satzmodi. In: Altmann, H. (Hg.) (1988): S.153-167.

Oppenrieder, W. (1988b): Intonatorische Kennzeichnung von Satzmodi. In: Altmann, H. (Hg.) (1988). S.169-205.

Oppenrieder, W. (1989a): Selbständige Verb-Letzt-Sätze: Ihr Platz im Satzmodussystem und ihre intonatorische Kennzeichnung. (In diesem Band)

Oppenrieder, W. (1989b): Fokus, Fokusprojektion und ihre intonatorische Kennzeichnung. (In diesem Band)

Oppenrieder, W. (1989c). Deklination und Satzmodus. (In diesem Band) 
Nöth, E./Batliner, A./Lang, R./Oppenrieder, w. (1987): Automatische Grundfrequenzanalyse und Satzmodusdifferenzierung. In: Tillmann, H.G./Willée G. (Hgg.) (1987): Analyse und Synthese gesprochener Sprache. Hildesheim etc. S.59-66.

Scholz, U. (1989): Wunschsätze im Deutschen - formale und funktionale $\mathrm{Be}-$ schreibung. Satztypen mit Verberst- und Verbletztstellung. Ms. 